\title{
Conceptual Design Report
}

\section{NUCLEAR MATERIALS STORAGEECEIVED FACILITY RENOVATION

Part V - Structural / Seismic Investigation Section A Report

Existing Conditions Calculations/

Supporting Information

\section{Los Alamos}

National Laboratory

July 14, 1995 


\section{DISCLAMMIER}

Portions of this document may be illegible in electronic image products. Images are produced from the best available original document. 
FOR

LNE ITEM PROJECT

NUCLEAR MATERIALS STORAGE FACIUTYY RENOVATION

AT THE

LOS ALAMOS NATIONAL LABORATORY

Project Identification Number 11818

Technical Area 55

Building PF-41

Prepared by

ICF KAISER ENGINEERS, INC.

1900 Diamond Drive

Los Alamos, NM 87544

and

University of California

Los Alamos National Laboratory
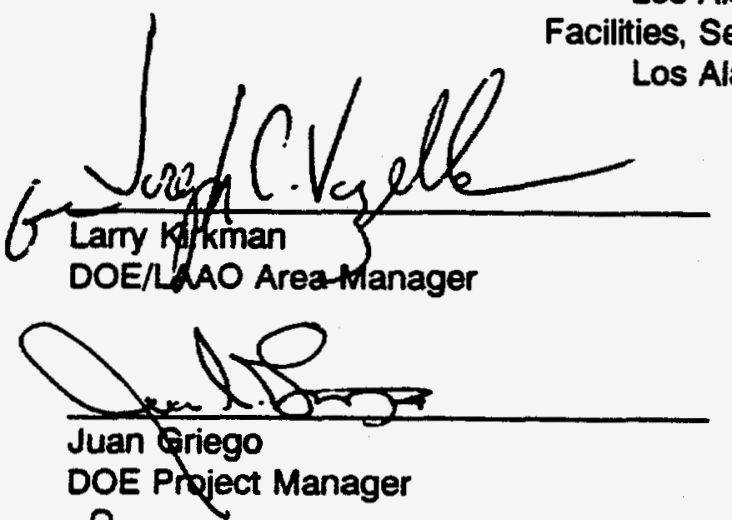

T. J. Trapp

Nuclear Materiais Program Manager

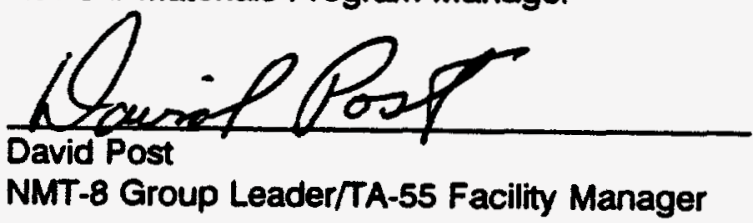

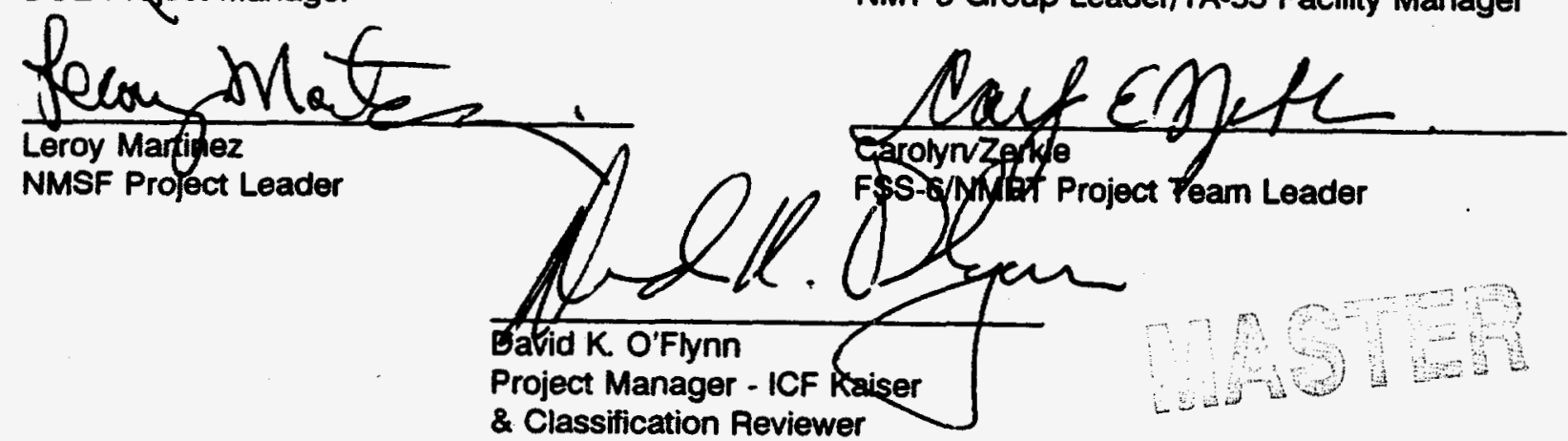

DISCLAIMER

This report was prepared as an account of work sponsored by an agency of the United States Government. Neither the United States Government nor any agency thereof, nor any of their employees, makes any warranty, express or implied, or assumes any legal liability or responsibility for the accuracy, completeness, or usefulness of any information, apparatus, product, or process disclosed, or represents that its use would not infringe privately owned rights. Reference herein to any specific commercial product, process, or service by trade name, trademark, manufacturer, or otherwise does not necessarily constitute or imply its endorsement, recommendation, or favoring by the United States Government or any agency thereof. The views and opinions of authors expressed herein do not necessarily state or reflect those of the United States Government or any agency thereof. 
TABLE OF CONTENTS

PART I - DESIGN CONCEPT

A. GENERAL DESCRIPTION OF PROJECT

B. JUSTIFICATION

C. RELATIONSHIP TO OTHER PROJECTS

D. ALTERNATIVES

E. DESIGN CONCEPT
SECTION

\section{A}

B

C

D

E

1. Project Design Description

2. Energy Conservation Analysis

3. Utility Assessment

4. Environmental Considerations

5. Facility and Equipment Maintenance and Operation Considerations

6. Safety Considerations

7. Security Considerations

8. Site Development Plan Coordination

9. Outline Specifications and Standards

10. Space Program

F. QUALITY ASSURANCE

G. SCHEDULES

H. KEY MILESTONES

I. COST ESTIMATES AND CONTINGENCY

J. ACRONYMS AND ABBREVIATIONS

PART II - PROJECT MANAGEMENT

A. PROJECT MANAGEMENT ORGANIZATION A

B. PROJECT MANAGEMENT ELEMENTS B 


\section{PART \|I - PROJECT MANAGEMENT (Cont'd)}

C. PROJECT CONTROLS AND REPORTING SYSTEM

D. SPECIAL PLANS

\section{PART III - SUPPLEMENTAL INFORMATION}

A. RENEWABLE ENERGY SYSTEMS ANALYSIS

B. CALCULATIONS AND SUPPLEMENTAL DATA

1. Civil/Miscellaneous Structural

2. Architectural

3. Mechanical

4. Electrical

5. Special Facilities and Storage Array Equipment

6. Thermal Analysis

7. Criticality

8. Radiological / Shielding

9. Material Handling Data

10. Process Flow

C. PRELMMINARY FIRE HAZARDS ANALYSIS

D. EXISTING CONDITION SURVEY

E. DRAFT Q/A PLAN

PART IV - CONCEPTUAL DRAWINGS

PART V - STRUCTURAL / SEISMIC INVESTIGATION
A. EXISTING CONDITION
B. RENOVATION

\section{SECTION}

C

D

A

B

C

D

E
A
B

PART VI - ALTERNATIVES STUDY 
PART VII - ESTIMATE DATA

SECTION

BASIS AND ASSUMPTIONS

TRACKING EXAMPLE

CONVENTIONAL ACQUISITION STRATEGY

A. TEC AND TPC SUMMARIES A

B. CONTINGENCY ANALYSIS B

C. ESCALATION ANALYSIS C

D. LABORATORY PROCUREMENT BURDEN D

E. OTHER PROJECT COSTS

F. FIELD OVERHEAD AND EXPENSES $\quad F$

G. ED\&1 G

H. A\&E COST OF SERVICES H

I. DETAILED CONSTRUCTION ANALYSIS

DESIGN/BUILD ACQUISITION STRATEGY

A. TEC AND TPC SUMMARIES A

B. CONTINGENCY ANALYSIS B

C. ESCALATION ANALYSIS C C

D. LABORATORY PROCUREMENT BURDEN D

E. OTHER PROJECT COSTS

F. FIELD OVERHEAD AND EXPENSES F

G. ED\&I G 
NMSF Conceptual Design Report July 14, 1995

Page iv

H. A\&E COST OF SERVICES H

I. DETAILED CONSTRUCTION ANALYSIS I

SUPPORTING INFORMATION 
REVISION NO. 3

$7 / 14 / 95$

\section{F I N A L}

\section{PRELIMINARY STRUCTURAL EVALUATION OF THE NMSF FACILITY LOS ALAMOS NATIONAL LABORATORY \\ LOS ALAMOS, NEW MEXICO}

Prepared By

ICF Kaiser Engineers, Inc.

JULY 1995 


\section{EXECUTIVE SUMMARY}

The existing Nuciear Material Storage Facility (NMSF), PF-41, is located in Technical Area TA-55 at the Los Alamos National Laboratory in the State of New Mexico. Original design documents for the facility are incomplete; original design calculations, specifications, and criteria are not available. This report and associated calculations document a preliminary structural seismic evaluation of the existing NMSF.

A potential change of function for the facility is being explored. The change involves incorporating naturalconvection-cooled dry storage capabilities for high-level radioactive material within the boundaries of the existing NMSF structure. A conceptual structural modification to the existing facility has been developed. The conceptual structural modification is intended to satisfy the upgraded functional and operational requirements that the dry storage facility would be subjected to.

The existing structure and the modified structure are evaluated using simplified, conservative methods. Input loads are consistent with those specified for Moderate Hazard $(\mathrm{MH})$ facilities with Performance Category 3 Classification, per Department of Energy Standard DOE-STD-1020-94.

The simplified, conservative methods used in the current preliminary structural seismic evaluation are consistent with the conceptual design stage of the structural modifications to the existing NMSF building. A significantly more detailed and complete analysis and evaluation is required for full compliance with current DOE requirements. Detailed analysis is expected to take place at the Title Design stage for the NMSF facility.

The results of this preliminary evaluation indicate that the as-built NMSF building structure could be shown to be adequate to withstand the loads specified by current DOE standards for Performance Category 3 structures.

This preliminary evaluation also shows that the conceptual structural modifications envisioned for the proposed change of function of the NMSF building are feasible, and could be implemented so that the resulting structure adequately meets DOE requirements for Performance Category 3 structures.

Most of the structural strengthening effort required is concentrated in the west wall of the structure, in the detailing of the wall along line No. 2, and in the roof over the administrative area. 
TABLE OF CONTENTS

1.0 INTRODUCTION $\ldots \ldots \ldots \ldots \ldots \ldots \ldots \ldots \ldots \ldots \ldots \ldots \ldots \ldots \ldots \ldots \ldots \ldots \ldots$

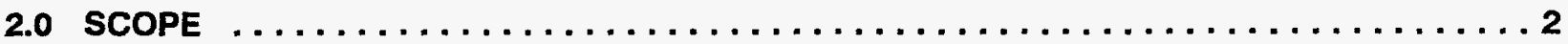

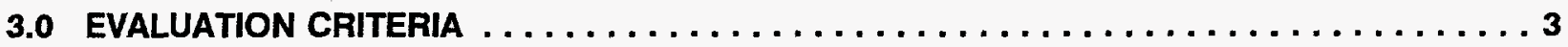

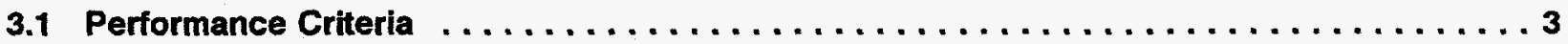

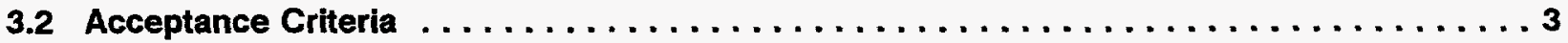

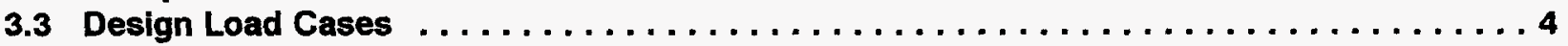

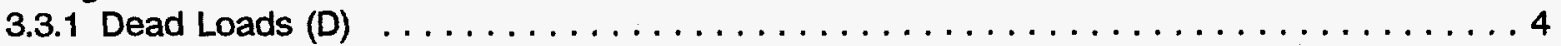

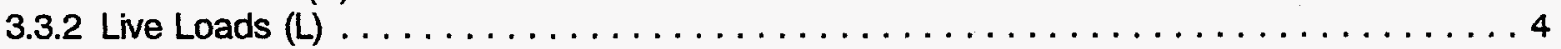

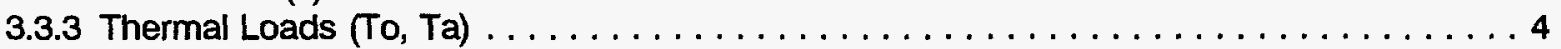

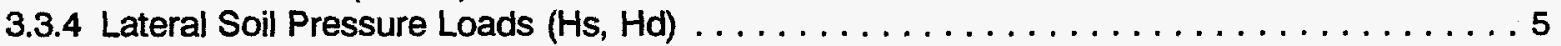

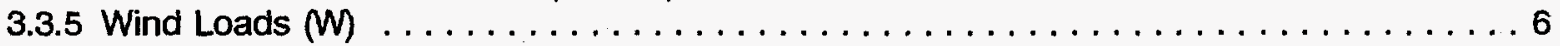

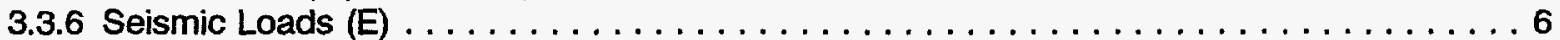

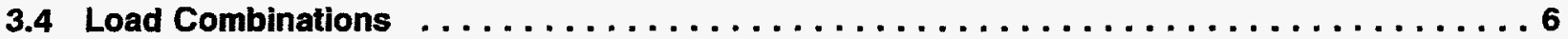

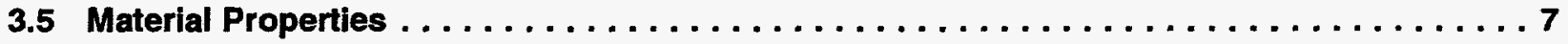

3.6 Damping $\ldots \ldots \ldots \ldots \ldots \ldots \ldots \ldots \ldots \ldots \ldots \ldots \ldots \ldots \ldots \ldots \ldots \ldots \ldots \ldots$

4.0 STRUCTURAL EVALUATION OF AS-BUILT STRUCTURE $\ldots \ldots \ldots \ldots \ldots \ldots \ldots \ldots$

4.1 Description of NMSF Structure $\ldots \ldots \ldots \ldots \ldots \ldots \ldots \ldots \ldots \ldots \ldots \ldots \ldots \ldots \ldots$

4.2 Modeling Approach $\ldots \ldots \ldots \ldots \ldots \ldots \ldots \ldots \ldots \ldots \ldots \ldots \ldots \ldots \ldots \ldots$

4.3 Analysis Procedure $\ldots \ldots \ldots \ldots \ldots \ldots \ldots \ldots \ldots \ldots \ldots \ldots \ldots \ldots \ldots \ldots \ldots$

4.3.1 Two-Dimensional Analysis of Exterior Walls $\ldots \ldots \ldots \ldots \ldots \ldots \ldots \ldots \ldots \ldots$

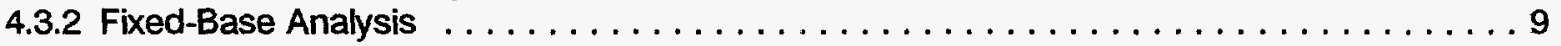

4.3.3 Soil-Structure Interaction (SSI) Effects $\ldots \ldots \ldots \ldots \ldots \ldots \ldots \ldots \ldots \ldots \ldots$

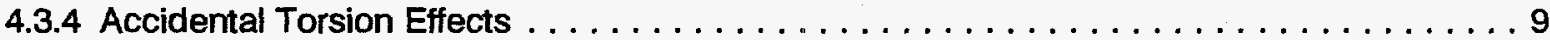

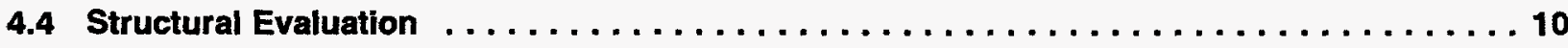

5.0 STRUCTURAL EVALUATION OF THE MODIFIED STRUCTURE $\ldots \ldots \ldots \ldots \ldots \ldots \ldots \ldots$

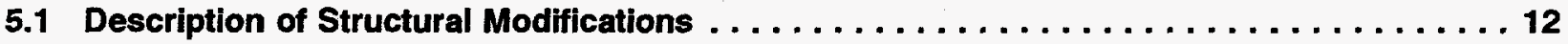

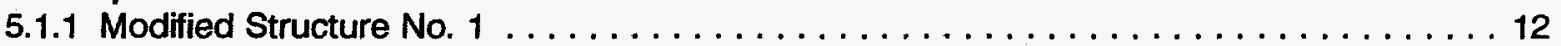

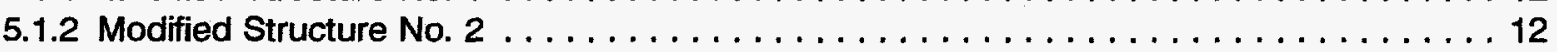

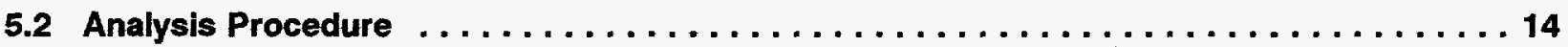

5.2.1 Two-Dimensional Analysis of Perimeter Walls $\ldots \ldots \ldots \ldots \ldots \ldots \ldots \ldots \ldots \ldots \ldots$

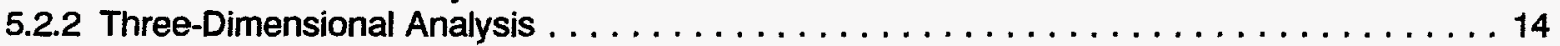

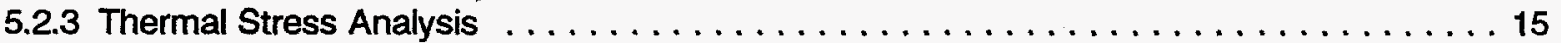

5.2.4 Two-Dimensional Analysis of Modified Structure No. $2 \ldots \ldots \ldots \ldots \ldots \ldots \ldots \ldots$

5.3 Structural Evaluation Results . . . . . . . . . . . . . . . . . . . . 16

6.0 EVALUATION OF NMSF/TUNNEL INTERACTION $\ldots \ldots \ldots \ldots \ldots \ldots \ldots \ldots \ldots \ldots$

7.0 CONCLUDING REMARKS AND RECOMMENDATIONS $\ldots \ldots \ldots \ldots \ldots \ldots \ldots \ldots \ldots$

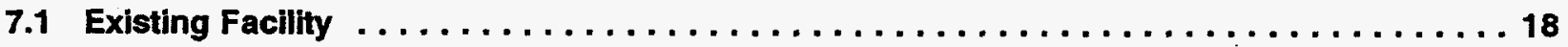


NMSF Conceptual Design Report

July 14, 1995

Page ii

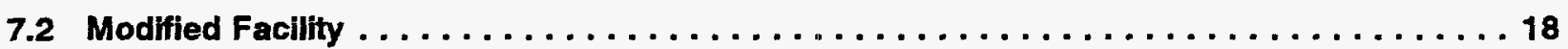

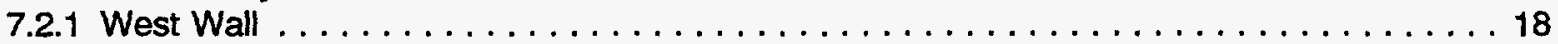

7.2.2 Precast Beams . . . . . . . . . . . . . . . . . . . . . . . . 19

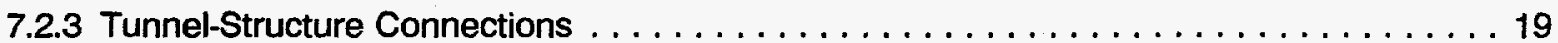

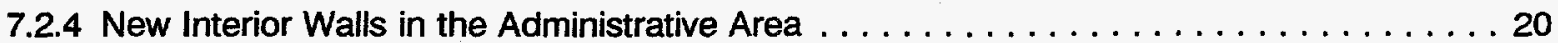

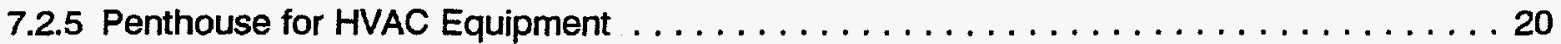

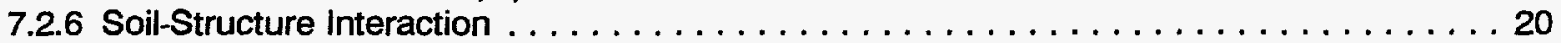

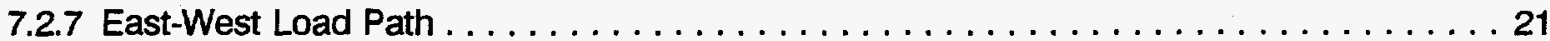

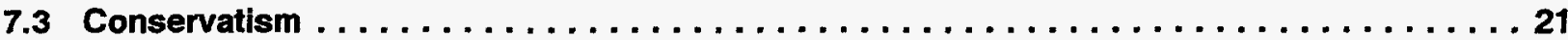

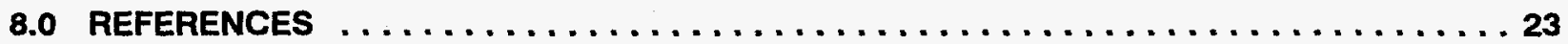

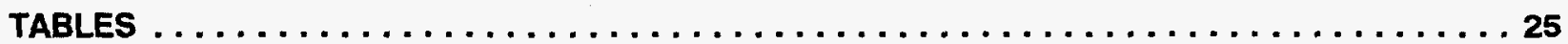

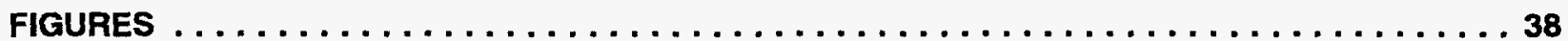




\subsection{INTRODUCTION}

The existing Nuclear Material Storage Facility (NMSF), PF-41, is located in Technical Area TA-55 at the Los Alamos National Laboratory in the State of New Mexico. Original design documents for the facility are incomplete; original design calculations, specifications, and criteria are not available. This report and associated calculations document a preliminary structural seismic evaluation of the existing NMSF.

A potential change of function for the facility is being explored. The change involves incorporating naturalconvection-cooled dry storage capabilities for high-level radioactive material within the boundaries of the existing NMSF structure. A conceptual structural modification to the existing facility has been developed. The conceptual structural modification is intended to satisfy the upgraded functional and operational requirements that the dry storage facility would be subjected to.

The existing structure and the modified structure are evaluated using simplified, conservative methods. Input loads are consistent with those specified for Moderate Hazard (MH) facilities with Performance Category 3 classification, per Department of Energy Standard DOE-STD-1020-94. Compliance with current DOE/LANL structural evaluation guidelines $(1,2)$, as described in Section 3.0 of this report, is examined.

This report and the associated calculations document the criteria, procedure, and results of structural evaluation for the NMSF building in its "as-built" configuration per the original design drawings by Burns and Roe Pacific (14), and the NMSF building in its modified configuration per ICF Kaiser preliminary drawings $(15,16)$ and final 100\% Draft Conceptual Design Report $(25)$.

For details of engineering calculations, refer to ICF Kaiser Calculation Numbers 42552-S-0001 and 4552-S-0002 $(12,13)$. 


\subsection{SCOPE}

As part of the current scope work, the existing "as-built" and conceptually modified NMSF buildings are evaluated for compliance with current seismic structural DOE evaluation guidelines using simplified, conservative procedures. Requirements are described in Section 3.0 of this report. Potential problem areas are identified, and possible corrective concepts are provided.

The structural evaluations make use of simplified mathematical models of the building. Sufficient detail is included to address the adequacy of only the major load-carrying structural components of the NMSF facility.

The scope of seismic evaluation is in accordance with the guidelines of the LANL Structural Standard (1), DOE Standard 1020-94 (2), and other required industry standards including UBC (4) and ASCE-7 (3). In the current evaluation, considerations are given to accidental torsion loads, soil-structure interaction, lateral soil pressure, and thermal stresses (for the modified structure only). The magnitude of the loads and the considerations for their combined effects are described in Section 3.0.

The analysis procedure and results of evaluation of the as-built structure are presented in Section 4.0 of this report. The structural analysis and evaluation results of the modified NMSF building are included in Section 5.0. This evaluation addresses the conceptual stage of the proposed structural modifications to the NMSF building. The structural evaluations are on a sampling basis and involve the main structural members of the building. A significantly more detailed analysis and complete evaluation of all structural components of the building is expected during evaluation of the final stage of the design.

A preliminary structural evaluation of the existing buried access tunnel, and its interaction with the NMSF building, is also performed in this study. The evaluation procedure and results of the analysis are presented in Section 6.0 of this report.

The concluding remarks and a list of references are included in Sections 7.0 and 8.0, respectively. 


\subsection{EVALUATION CRITERIA}

\subsection{Performance Criteria}

The as-built NMSF building shall be evaluated in accordance with the guidelines of DOE Standard 102094. On the basis of the use foreseen for this structure, Performance Category 3 is applicable to the existing Structures, Systems, and Components (SSC).

The performance goals for this Category 3 structure during and after occurrence of a postulated earthquake event include:

- Occupant safety,

- Continued operation, and

- Confinement of hazardous material.

Quantitatively, this performance requirement corresponds to a Hazard Exceedance Probability of $5 \times 10^{-4}$ (Return Period of 2000 years).

\subsection{Acceptance Criteria}

The acceptability of the response of the structural components of the NMSF building is evaluated on a demand-versus-capacity basis. The total demand on structural components consists of non-seismic loads (Dns) such as dead loads, live loads, and seismic loads (Dsi):

$$
\text { Dti }=\text { Dsi }+ \text { Dns }
$$

where:

$$
\begin{array}{ll}
\text { Dti } & =\text { Total demand } \\
\text { Dns } & =\text { Stresses due to non-seismic loads } \\
\text { Dsi } & =\text { Inelastic seismic SF (DS/FU) } \\
\text { Ds } & =\text { Elastic seismic demand due to seismic input } \\
\text { Fu } & =\text { Inelastic energy absorption factor } \\
& =1.75 \text { For Shear Wall In-plane Flexure } \\
& =1.5 \text { For Shear Wall In-plane Shear } \\
& =1.75 \text { For Shear Wall Out-of-plane Flexure } \\
\text { SF } & =1.0 \text { For Shear Wall Out-of-plane Shear } \\
& =\text { Scale factor }=1.0 \text { for PC-3 }
\end{array}
$$

These Fu values are the upper limits allowed, and are dependent upon the quality of the detailing that has been used and the consistency of the detailing with the current UBC provisions. Lower numbers shall be used for as-built components that are not in compliance with current detailing practice. An Fu factor of unity was used in this evaluation. This is a conservative assessment, given the lack of documentation and quality assurance for the original design and construction of the NMSF. 
The capacity $(\mathrm{Cc})$ of concrete components of the NMSF can be determined based on the ultimate strength provisions of ACl-318 or Chapter 26 of UBC-94(4). The capacity is considered adequate if it exceeds the demand (i.e., the $\mathrm{D} / \mathrm{C}$ ratio is greater than or equal to one).

\subsection{Design Load Cases}

In this section, the loading conditions and combinations used for this evaluation are described.

\subsubsection{Dead Loads (D)}

The dead loads consist of the following components:

\section{Structural Dead Weight:}

These loads are estimated based on the geometric dimensions given in the Burns \& Roe drawings (14). The following weight densities are used:

$\begin{array}{lll}\text { Reinforced concrete } & : & 150 \mathrm{pcf} \\ \text { Steel } & : & 490 \mathrm{pcf} \\ \text { Backfill soil } & : & 110 \mathrm{pcf}\end{array}$

\section{Equipment Weight:}

No specific equipment weight was accounted for in evaluation of the as-built structure.

The proposed modifications to the NMSF building include addition of a 20-ton bridge crane to operate between the first floor walls on lines 1 and 2 . The weight and mass of the crane are accounted for in the evaluation of the modified NMSF building.

\section{Future Loads:}

An extra 10 psf is assumed on all floors and roofs for future dead loads (1).

\section{Snow Loads:}

A minimum of 30 psf is assumed, per specifications of LANL Facility Engineering Standard (1).

\subsubsection{Live Loads (L)}

250 pounds per square foot of live loads shall be used for all first floor and basement floor areas, per UBC specification for storage areas (4).

\subsubsection{Thermal Loads (To, Ta)}

Thermal loads are not considered in the evaluation of the as-built structure.

The following thermal gradients are used in evaluation of the modified NMSF structure: 


\section{Operating condition:}

20.F: Across the east and west walls and the charge deck during normal operation (To).

Accident condition:

$40^{\circ} \mathrm{F}$ : Across the east and west walls and the charge deck during postulated accidental loss of cooling system (Ta).

\section{Both conditions:}

13F: Across the basement floor slab during operating as well as accidental conditions (To \& Ta).

16 ${ }^{\circ}$ : Across the 1 st floor walls and roof slab during operating as well as accidental conditions (To \& Ta).

The specified thermal load criteria were based on preliminary estimates provided by LANL staff (17). The thermal criteria are believed to be consenvative because they were based on a highly unlikely accident scenario: the cooling air flow to the facility is assumed to be completely stopped for a period of one year.

\subsubsection{Lateral Soil Pressure Loads (Hs,Hd)}

Lateral soil pressures are computed using the following properties for the backfill material:

$\begin{array}{llc}\text { Weight Density } & : & 110 \mathrm{pcf} \\ \text { Angle of internal Friction } & : & 40 \mathrm{deg} . \\ \text { Surcharge } & : & 250 \mathrm{psf}\end{array}$

The properties are estimated on the basis of information provided in references (6) and (21).

Due to lack of sufficient geotechnical information, and in lieu of sophisticated SSI analysis, the distribution of the lateral soil pressures on the NMSF walls is estimated using different conservative pressure distribution patterns.

For static pressure, two patterns are used:

1) A trapezoidal distribution as suggested by Dames \& Moore for NMSF site material (5); and

2) A standard triangular distribution typically used for granular backfill.

For dynamic soil pressure distribution, two load patterns are used:

1) A uniform distribution as recommended in references (6) and (7); and

2) An inverted triangular distribution as suggested by ASCE 4-86 (8).

As a result, a total of four different combinations of the static and dynamic lateral soil pressure loads are used to evaluate the out-of-plane response of NMSF's perimeter walls to dynamic lateral soil pressure. 


\subsubsection{Wind Loads $(W)$}

The structure is partially buried. Due to its limited exposure area and its low height, wind loads are ignored in the evaluation. Wind loads will be considered at the Title Design stage.

\subsubsection{Seismic Loads (E)}

The seismic loads used in this evaluation are based on the recommendations of reference (1) and recent seismic hazard evaluation work by Woodward Clyde as follows:

Peak Ground Acceleration (PGA): $\quad 0.30 \mathrm{~g}$

Response Spectral Shape:

US NRC Regulatory Guide 1.60 spectra shape. (A plot of the seismic spectrum is shown in Figure 3.1.)

The seismic hazard study for the general area around the NMSF building site, being completed by Woodward Clyde, was not available in time for use in the current evaluation.

The February 1975 Woodward Clyde Seismic Hazard Evaluation Report for LANL shows (Figure 10-19) the recommended DBE horizontal response spectra for a 2000-year return period. The US NRC Reg. Guide 1.60 spectral shape resembles the new Woodward Clyde recommended spectral shape better than the original (1975) NUREG/CR-0098 spectral shape. Also, it has been the experience of the project team at other sites that the 1975 NUREG/CR-0098 spectral shape does not hold up as well to regulatory review.

In view of the above, LANL and the project team have agreed on the use of the Reg. Guide 1.60 spectra at this time.

The February 1975 Woodward Clyde Seismic Hazard Evaluation Report for LANL shows (Figure 8-55) the recommended vertical to horizontal spectral ratio as ranging from 1.6 to 0.55 . Some controversy continues to cloud this issue. Some of Woodward Clyde's work shows the possibility of ratios as high as 2.0 for high frequencies at near-source sites. Other experts consider the vertical effects to be nonconcurrent with the horizontal and of limited duration. A sequencing effect might mitigate the impact of the vertical input motion, and a more standard $2 / 3$ of the horizontal might be applicable.

A simplified vertical evaluation using a vertical to horizontal spectral ratio of 1.0 has been included in the calculations. The report has been enhanced accordingly.

\subsection{Load Combinations}

The NMSF building is evaluated for the following load combinations:

Seismic load combinations per DOE Standard 1020-94 (three orthogonal components of ground motion are considered):

1) $\mathrm{D}+\mathrm{L}+\mathrm{Hs}+\mathrm{Hd}+\mathrm{To}+\mathrm{E}$

2) $\mathrm{D}+\mathrm{L}+\mathrm{Hs}+\mathrm{Hd}+\mathrm{To}-\mathrm{E}$ 
Non-seismic load combinations:

$$
\text { 3) } 1.4 \mathrm{D}+1.7 \mathrm{~L}+1.7 \mathrm{Hs}+1.4 \mathrm{Ta}
$$

The thermal loads (To,Ta) were not considered in evaluation of the as-built structure (the current structure is being used as office space).

\subsection{Material Properties}

The following properties are used to compute the strength and stiffness properties of the structural components of the NMSF building:

\section{Concrete:}

Compressive strength

Poisson's ratio

Steel:

Reinforcing steel, ASTM A615, Grade 60 Structural Steel, ASTM A36

Welding

Modulus of elasticity, Es

Poisson's ratio

$$
\begin{aligned}
& \text { fc }=4,000 \mathrm{psi} \\
& \mathrm{vc}=0.17
\end{aligned}
$$

$$
\begin{aligned}
& \text { fy }=60 \mathrm{ksi} \\
& \mathrm{fy}=36 \mathrm{ksi} \\
& E 7018 \\
& \text { Es }=29,500 \mathrm{ksi} \\
& \text { vs }=0.25
\end{aligned}
$$

Reference (18) provides some insight into the as-is condition of the structure. The tested concrete strength ranges from 5,780 to 9,070 psi. The concrete cover over the reinforcing steel varied with an average of 3.6 inches.

\subsection{Damping}

DOE Standard 1020-94 (2) specifies structural damping ratios corresponding to the level of structural response expected to occur during the postulated earthquake event. For a Performance Category 3 reinforced concrete structure such as the NMSF building, the guidelines allow a damping ratio of up to $10 \%$ of critical damping if stresses are high enough. A damping ratio of $7 \%$ was used in this evaluation; this might result in some conservatism. At the Title Design stage, analysis iterations should be performed to match the damping value used to the stress level obtained for the structure. 
NMSF Conceptual Design Report

July 14, 1995

Page 8

\subsection{STRUCTURAL EVALUATION OF AS-BUILT STRUCTURE}

\subsection{Description of NMSF Structure}

The existing NMSF building is a cast-in-place concrete shear wall structure which is partially embedded in the ground. The western portion of the building, referred to as the MAA/Storage area, is a two-story structure with its basement floor completely embedded in the ground. The second story of the western portion (from the first floor to the roof) is completely backfilled with soil on the north, west, and south sides of the building. The eastern portion of the NMSF building, constructed on the finished grade, provides space for administrative functions, a mechanical equipment room, and safe, secure transport (SST) garage space. The continuous first floor slab, roof, and shear walls in the east-west direction join the eastern and western portions of the NMSF building together with no construction joint in between. The basic geometric characteristics of the as-built NMSF building are shown in Figure 4.1.

\subsection{Modeling Approach}

The NMSF building is categorized as a Performance Category 3 structure. Therefore, it is required (2) that a dynamic analysis be performed for seismic evaluation. Additionally, considering the geometrical irregularities and the embedment of a significant portion of the building in the ground, a simplified threedimensional finite-element model, including only the major structural elements, was developed. The model incorporates rigid diaphragms to simplify the evaluation for the effects of accidental torsion and soilstructure interaction. The out-of-plane response of the typical exterior wall sections of the embedded portion of the NMSF building to the seismically-induced dynamic soil pressure was also analyzed using a separate two-dimensional model.

The vertical analyses for static and dynamic loads were also performed using simplified two-dimensional models.

\subsection{Analysis Procedure}

In order to ascertain the basic behavior of the structure and help focus the analysis, very simplistic evaluations were initially performed. The understanding of the structure gained in the initial evaluations helped develop subsequent models that addressed areas of structural concern.

\subsubsection{Two-Dimensional Analysis of Exterior Walls}

The objective of the two-dimensional analysis of the exterior walls was to perform a preliminary investigation of the performance of these walls under static and dynamic lateral soil pressures alone. A one-foot slice of a typical wall section was selected and analyzed using the SAP-90 program. The lateral loads consisted of static as well as dynamic pressures. A more detailed description of the soil pressure loads and their assumed distribution along the height of the walls is provided in Section 3 of this report.

Results from the analysis indicated that the walls in general are capable of withstanding the out-of-plane applied loads.

The results from the two-dimensional analysis are mostly superseded by the more accurate results from the three-dimensional analysis. For details on the procedure and results of the two-dimensional analysis, refer to the calculations (12). 


\subsubsection{Fixed-Base Analysis}

The objective of this analysis was to study the dynamic characteristics of the building without consideration of the embedment effects of the surrounding soil. A three-dimensional finite-element model of the NMSF building was developed using the SAP90 (10) computer program. The exterior walls, major interior walls, roof, and slabs of the building were idealized using the shell elements of SAP90. The computer-generated plots of the finite element model are presented in Figures 4.2 to 4.4.

An eigen-analysis of the fixed base three-dimensional model of the NMSF, described above, was performed to extract the natural frequencies and mode shapes of the building. The results of this analysis are summarized in Table 4.1. The fundamental frequency of the fixed base structure was found to be 13.4 Hertz.

\subsubsection{Soil-Structure Interaction (SSI) Effects}

To account for SSI effects, the fixed-base three-dimensional model of the NMSF building was revised by adding foundation impedances for three translational and three rotational (two rocking and torsion) degrees of freedom. The foundation impedance for each degree of freedom typically consists of a pair of springs and dashpots representing the stiffness and the damping characteristics of the soil medium respectively. In this evaluation, only the soil springs (no dashpots) were utilized in the model to account for the SSI effects on the natural frequency shift of the building. No time-history analyses were performed in this simplified evaluation. The procedures recommended in reference (8) were used to develop the SSI model.

To account for embedment effects, the soil-springs constants were calculated separately for the western and eastern portions of the NMSF building. The embedment effects of the western portion of the building were accounted for using approximate methods given in reference (11). The embedment above the first floor was neglected, conservatively, to account for potential loss of lateral support during an earthquake (8). The stiffness values used to represent the foundation impedances in the SSI model are summarized in Table 4.2.

A frequency analysis of the SSI model was performed to compute the modal properties of the complete soil-structure model. The results of this analysis are summarized in Table 4.3. The fundamental natural frequency of the SSI model was $11.4 \mathrm{~Hz}$, indicating a frequency shift of about $2.6 \mathrm{Hertz}$ from the fixedbase frequency of $14.0 \mathrm{~Hz}$ due to the additional flexibility provided by soil-foundation springs.

It should be noted that SSI analyses in full conformance with current DOE requirements are to be performed for best-estimate, upper-bound, and lower-bound soil properties. In the current conceptual design effort, only best-estimate soil properties are used. Title Design should address the full range of soil properties.

\subsubsection{Accidental Torsion Effects}

Analysis for accidental torsion was performed by modifying the three-dimensional finite-element model of the NMSF building used in the SSI analysis. The floor mass, mass moments of inertia, and center-of-mass coordinates were first computed for the basement floor, first floor, and roof. The computed masses were then located away from the calculated center of mass so that the resulting eccentricity between the centers of mass and rigidity was effectively increased by $5 \%$ of the corresponding floor dimension. Arbitrarily rigid and massless beams were used to connect the lumped floor mass to the wall top nodes 
to simulate rigid diaphragms for all floors and the roof. Care was exercised to ensure that the numerical stability of the model was not compromised by the artificially "rigid" beams.

A frequency analysis of this model was performed to study the effects of accidental torsion on the building response. Table 4.4 summarizes the results of the frequency analysis. A comparison of Tables 4.3 and 4.4 shows that a 5\% eccentricity of floor mass has a minimal impact on the dynamic characteristics and response of the building. The computer-generated plots of the fundamental natural modes resulting from this analysis are shown in Figures 4.5 through 4.7.

This model was used to carry out the seismic evaluation of the NMSF building subjected to the postulated earthquake. A response spectra analysis was performed using a simultaneous application of the seismic spectra in north/south, east/west, and vertical directions. The PGA of the vertical component of the earthquake was set to $2 / 3$ and to $100 \%$ of the horizontal for the analysis of modified structure No. 1 and modified structure No. 2, respectively.

\subsection{Structural Evaluation}

The response spectrum analysis results were combined with those due to dead loads, live loads, and lateral soil pressure to obtain the total demand on the structure for the load combinations described in Section 3.4. The capacity of the walls was computed in accordance with the provisions of Chapter 26 of the UBC Code (4).

The summary demand/capacity ratios for in-plane and out-of-plane response of the walls of the NMSF building are summarized in Tables 4.5 and 4.6, respectively. The resulting $D / C$ ratios are below the allowable limits.

The computed in-plane shear demand on the shear walls of the NMSF was used to conservatively evaluate the in-plane shear response of the roof and floor slabs. It was assumed that the shear stress is uniformly distributed along the length of the shear walls. This assumption is deemed reasonable, based on an effective length equal to $80 \%$ of the actual length of the walls. The computed shear per unit length of walls was compared with the shear capacity of the adjoining roof or floor slab. The maximum shear demand computed for the entire NMSF building was 18.1 kips/F.L. The capacity of the $12^{n}$ thick concrete slab is conservatively estimated to be $18.2 \mathrm{k} / \mathrm{L} . \mathrm{F}$.

It is therefore concluded that there is adequate margin for safety against in-plane shear failure in the $12^{n}$ concrete slab of the MAA roof and the concrete floor slabs.

The evaluation of the vertical response and resulting out-of-plane demand on roof slabs and floor slabs was accomplished using a 2-D model of a east-west strip of the building. This model and resulting data are explained in further detail in Section 5.

The simplified conservative methods used in the current preliminary structural seismic evaluation are consistent with the conceptual design stage of the structural modifications to the existing NMSF building. A significantly more detailed and complete analysis and evaluation is required for full compliance with current DOE requirements. Detailed analysis is expected to take place at the Title Design stage for the NMSF facility. 
NMSF Conceptual Design Report

July 14, 1995

Page 11

The results of this preliminary evaluation indicate that the as-built NMSF building structure could be shown to be adequate to withstand the loads specified by current DOE standards for Performance Category 3 structures. 


\subsection{STRUCTURAL EVALUATION OF THE MODIFIED STRUCTURE}

During this conceptual design effort, the change in function postulated for the structure resulted in a new physical configuration.

The new physical configuration developed as a result of airflow, shielding, criticality, security access, and operational requirements. The structural considerations had to accommodate evolving requirements that were given greater priority. The physical configuration evolved in a continuous manner and two modified conditions (No. 1 and No. 2) are reported in detail.

\subsection{Description of Structural Modifications}

\subsubsection{Modified Structure No. 1}

The proposed modifications to the as-built structure of the NMSF facility include the following major items:

1) The first floor slab between lines 1 and 3 is removed.

2) A new 30-inch-thick charge deck is added at about 3 feet below the existing first floor level between lines 1 and 2 .

3) The north/south wall on line 1.5 in the basement is removed.

4) The existing mezzanine slab between lines $B$ and $C$ is removed.

5) A new mezzanine floor slab is added at 10 feet above the basement slab between lines 2 and 3.

6) New concrete and concrete-block walls are introduced in the administrative area. The attached calculation in Section 8.4 addresses these walls. Care should be exercised in the detailing of these walls. The new walls should be restrained laterally at top and bottom. The lateral restraint at the top should allow for relative vertical displacement between the top of the walls or partitions, and the roof and supporting precast beams.

This modified configuration is based on references (15) and (16). Modified structure No. 1 was extensively investigated, and is reported in Tables 4.7 to 4.9 and in Figures 4.11 to 4.19.

The analysis results indicated potential vulnerabilities to the structure because of the increased vertical span of the west wall, the new crane loads, and the conservatively estimated thermal loads.

At a late stage in the project, additional shielding requirements resulted in the incorporation of additional walls. Although additional walls would increase the structural margins of safety for the structure, it was deemed appropriate to revise the mathematical model and develop a model for Modified Structure No. 2 .

\subsubsection{Modified Structure No. 2}

The analysis for Modified Structure No. 2 has a confirmatory value because the new modifications considered for the most part increase available structural margins. 
This modified configuration is based on reference (25) and is reported in Tables 4.10 to 4.14 and Figures 4.20 to 4.24 .

The model for Modified Structure No. 2 included the following:

8) Two additional 1.5-ft-thick walls were added in a north/south direction between the basement floor and the charge deck. These walls (15) were included in the threedimensional SAP90 (10) model. The impact of these additional walls is an increase in the rigidity and strength of the lateral load-resisting system of the NMSF building in the northsouth direction. These new walls are included and accounted for in the two-dimensional thermal analysis presented in Section 5.2.3 of this report, and the 2-D models for vertical and horizontal analysis reported in 5.2.4.

9) A lightweight mechanical penthouse structure is introduced over the southeast corner of the facility. This penthouse will house unclassified, non-safety mechanical equipment.

10) Steel grating is being added to protect the intake vent $(26 \mathrm{k})$ and the stack outlet vent $(26 \mathrm{k})$. These vents will have to be adequately supported in order not to compromise the safety of the NMSF facility.

11) Buttresses are introduced to strengthen the west wall in the modified structure. The west wall (line 1) in the modified structure requires strengthening because the vertical span above the charge deck increased by 3 feet. This west wall in the modified structure also supports a new crane and accessory rail, and new thermal loads.

12) At the time of this effort, consideration was being given to the potential need to upgrade the NMSF roof over the administrative area for security reasons. In order to harden the roof and prevent or delay penetration by an explosive device, it might become necessary to increase the roof thickness to 8 inches. The capacity of the existing roof precast beams to withstand an additional load appears to be limited. The actual additional thickness requirements are not clear at this time. If substantial slab thickening is required, it could be accommodated by:

1) introducing intermediate beams between the existing $T$ beams,

2) using the current roof slab as part of the formwork system for the facility, and, after removing the existing concrete topping, casting a thicker roof slab that would effectively increase the moment arm for the existing post-tensioning tendons and consequently increase the moment capacity of the existing precast beams, and

3) providing for shear transfer between the old and the added concrete slab by means of closely spaced studs. 


\subsection{Analysis Procedure}

In order to ascertain the basic behavior of the structure and help focus the analysis effort, very simplistic evaluations were initially performed. The understanding of the structure gained in the initial evaluations helped develop subsequent models that addressed areas of structural concern.

\subsubsection{Two-Dimensional Analysis of Perimeter Walls}

The objective of the two-dimensional analysis of the exterior walls was to provide a preliminary investigation of the performance of these walls due to static and dynamic lateral soil pressures alone. A one-foot slice of a typical wall section was selected and analyzed using the SAP-90 program. The lateral loads consisted of static as well as dynamic pressures. A more detailed description of the soil pressure loads and their assumed distribution along the height of the walls is provided in Section 3 of this report.

The results from this analysis indicate that the walls in general are capable of withstanding the applied loads without major modifications, with the exception of the wall on line 1 between the charge deck and the roof. The vertical span of this wall has been increased due to removal of the existing first floor slab. The wall is subjected to new additional stresses from the new bridge crane and from thermal loads which are consistent with the revised function for the facility and were not accounted for in the original design of the wall. The three-dimensional analysis, presented in the next section, was used to generate more realistic estimates for the out-of-plane forces in the walls.

The results from this analysis are mostly superseded by the results from three-dimensional analyses. For more information on the procedure and results of the two-dimensional analyses, refer to the calculations (13).

\subsubsection{Three-Dimensional Analysis}

The three-dimensional analyses were performed using the three-dimensional SAP90 model. The threedimensional model of the as-built NMSF structure, described in Section 4.0 of this report, was first modified to reflect the major structural modifications to the NMSF building. Figures $4.11,4.12$, and 4.13 depict the finite element model of the modified structure. The less important changes to interior walls and equipment are not accounted for at this point.

The modified NMSF model (NMSF05) was then analyzed to extract natural frequencies and mode shapes of the NMSF structure at its modified configuration. The result of the frequency analysis is summarized in Table 4.7. A comparison of the contents of Tables 4.4 and 4.7 indicates that the proposed modifications have increased the stiffness of the structure, resulting in higher natural frequencies. Plots of the fundamental mode shapes of the modified structure are shown in Figures 4.14 through 4.19.

Similar to the analysis of the as-built structure described in Section 4.0, the modified structure was subjected to the combined effects of dead loads, live loads, lateral soil pressure, and earthquake loads as described in Sections 3.0 and 4.0 of this report.

The modified NMSF model No. 2 (NMSF06) provides some confirmatory analyses reported in Tables 4.10 and 4.11. 
Additional information regarding vertical amplified response of the structure and horizontal load path across the wall along line 2 is provided by developing a 2-D model reported in Section 5.2.4.

For a detailed description of the analysis results, refer to the supporting engineering calculations (13).

\subsubsection{Thermal Stress Analysis}

A simplified preliminary analysis was performed to account for the stresses due to the thermal gradients across the walls and slabs surrounding the nuclear material storage area located in the basement of the NMSF building. The thermal load criteria are described in Section 3.3.3.

A two-dimensional east/west slice of the building was modeled for this analysis using the SAP-90 program. The out-of-plane moments and shear forces resulting from thermal analysis were combined with those from three-dimensional analysis to arrive at the total demand on the walls affected by thermal loads. The forces due to thermal loads appear to be very severe for the walls surrounding the storage area, and as such control the design of these elements. A more accurate and less conservative estimate of the thermal gradients through the concrete walls and slabs surrounding the stored nuclear material needs to be developed at the Title Design stage. Realistic accident scenarios need to be considered.

For a more detailed description of the thermal analysis procedure and results, refer to the supporting engineering calculations (13).

\subsubsection{Two-Dimensional Analysis of Modified Structure No. 2}

The resulting configuration for the modified NMSF results in a very adequate structure in the north-south direction. There is a positive load path and a substantial number of shear walls in the north-south direction. The east-west direction of the structure is not as adequately configured. There is a limited number of east-west shear walls, and the charge deck loads do not have a direct path to the east-west shear walls. Openings required for cooling air flow cannot be disrupted with shear wall elements because of potential air stagnation problems. Furthermore, operational considerations prevent the introduction of north-south shear walls immediately east of the charge deck.

The increased local demands on the west wall because of increased vertical spans, crane loads, and thermal loads are resolved by the introduction of buttresses as shown in Table 4.11. The wall along line no. 2 has to provide a horizontal load path between the charge deck and the slab between lines 2 and 3 at the original first floor level.

In order to better determine the out-of-place demand on the line 2 wall, a 2-D strip model (Figure 4.24) was developed. This is a very conservative model for the purposes of calculating the out-of-place demand for the wall on line 2 because it neglects all shear walls perpendicular to this line.

The north-south walls act as beams in the out-of-plane direction. This model provides an upper bound estimate for the out-of-plane demand on the wall on line 2.

This 2-D model was also used for a cursory vertical analyses of the as-built as well as the modified structure.

For a more detailed description of the 2-D model of modified structure No. 2, and the results of the analysis, refer to the supporting engineering calculations (13). 


\subsection{Structural Evaluation Results}

The response spectrum analysis results were combined with those due to dead loads, live loads, and lateral soil pressure to obtain the total demand on the structure for the load combinations described in Section 3.4. The capacity of the walls was computed using the ultimate capacity provisions of Chapter 26 of the UBC (4).

A summary of the Demand and Capacity $(D / C)$ ratios for in-plane shear of the major walls is presented in Table 4.8. Results show that the $D / C$ ratios for the modified structure are all within allowable limits. Based on the field test results for the compressive strength of the concrete in the NMSF building (18), the actual margin of safety is significantly higher than that tabulated.

The summary of the $\mathrm{D} / \mathrm{C}$ ratios for out-of-plane response of major walls is presented in Table 4.9. Only the walls that are subjected to significant out-of-plane loads are analyzed using the computer model. The secondary interior walls are checked for adequacy using hand calculations. The results show that the $\mathrm{D} / \mathrm{C}$ ratios for the walls in the modified structure are all within acceptable limits without need for additional strengthening, with the exception of the wall on line 1 (west wall). The new wall added between the existing columns on line 2 can easily be designed to meet the new demands. The existing wall on line 1 , however, does not have sufficient strength to withstand the new additional crane and thermal loads and added lateral loads because the vertical span of the wall is also increased by 3 feet. Strengthening of this wall can be accomplished by introducing exterior buttresses.

A retrofit concept using buttresses is provided in the ICF KE engineering calculation supporting this report (13). It should be noted that, although the proposed retrofit scheme was not evaluated for the full extent of the design load combinations required by applicable codes, it is believed to be adequate, qualitatively. More refined solution designs are expected during the Title Design stage.

It should be noted that refined SSI analyses and realistic thermal analyses may possibly help minimize the strengthening requirements for this wall.

The summary of the Demand/Capacity $(D / C)$ ratios for confirmatory in-plane shear for modified structure No. 2 (3-D model) is provided in Table 4-10.

The summary of the Demand/Capacity $(D / C)$ ratios for out-of-plane response of floors and roofs is presented in Table 4.11. This table, when compared with Table 4.9, shows the localized relief provided by the buttresses.

There are extraordinary demands in the out-of-plane direction for the wall along line 2. (See the results of the conservative 2-D model in Table 4.12). It is expected that refined analysis at the Title Design stage will show a reduced demand. In any event, the conservative demand estimated at this point (almost $4 \sqrt{ } f^{\prime} c$ ) requires that the line along line 2 be detailed as a beam with shear reinforcement in the out-of-plane direction.

The vertical analysis is performed using a simplified 2-D strip model. This simplified approach is conservative in that it ignores the two-way slab action that is likely to further increase the slab capacity, especially in the vicinity of the east-west walls. Results are provided in Table 4.13. 


\subsection{EVALUATION OF NMSF/TUNNEL INTERACTION}

The existing NMSF structure is connected to three tunnels at the lowest elevation (el. 7281). These belowgrade connections were examined to determine whether their behavior during a seismic event would be acceptable.

During a seismic event, the tunnels, being fully embedded in soil and much lighter than the NMSF structure, will tend to ride with the soil and be subjected to stresses consistent with seismic soil strains. The NMSF structure, being significantly larger, more rigid, and only partially buried, will interact with the soil and deform in a manner consistent with the resulting soil-structure interaction. The difference in behavior between the NMSF structure and the tunnels would result in seismically-induced stresses at the interface.

A site inspection was performed, and structural drawings were reviewed to determine whether a flexible connection, or at least a construction joint, was introduced at the interface during original design and construction. It was determined that the existing connection was designed and constructed monolithically.

A simplified conservative evaluation was performed and is provided in Section 8.3 of the attached calculations. The methodology used is provided in references (19) and (20). Soil strains introduced in the tunnel and differential seismic anchor movement effects were conservatively estimated and calculated, and the resulting concrete strains were obtained.

The simplified evaluation performed indicates that the tunnel-structure connections will undergo moderate tensile concrete cracking but no compressive crushing. Steel reinforcement will remain below yield. These results indicate that there is no collapse potential for the tunnels and their connection to the structure. It should, however, be noted that the tensile cracking expected could contribute to air and moisture infiltration in the tunnels, as they are envisioned to be maintained at a constant negative pressure.

Consideration should be made of impact to operations and HVAC system demands if tensile cracking and air and moisture infiltration take place during a seismic event. If the facility operating conditions allow it, repair of tensile concrete cracks is not a significant construction effort. 
NMSF Conceptual Design Report

July 14, 1995

Page 18

\subsection{CONCLUDING REMARKS AND RECOMMENDATIONS}

\subsection{Existing Facility}

The simplified conservative methods used in the current preliminary structural seismic evaluation are consistent with the conceptual design stage of the structural modifications to the existing NMSF building. A significantly more detailed and complete analysis and evaluation is required for full compliance with current DOE requirements. Detailed analysis is expected to take place at the Title Design stage for the NMSF facility.

The results of this preliminary evaluation indicate that the as-built NMSF building structure could be shown to be, in general, adequate to withstand the loads specified by current DOE standards for Performance Category 3 structures, with the exception noted below.

One outstanding weakness of the existing facility is the lack of positive connection between the precast beams and the existing support walls. This deficiency can easily be corrected by providing safety restraints at the connections.

\subsection{Modified Facility}

The simplified conservative methods used in the current preliminary structural seismic evaluation are consistent with the conceptual design stage of the structural modifications to the existing NMSF building. A significantly more detailed and complete analysis and evaluation is required for full compliance with current DOE requirements. Detailed analysis is expected to take place at the Title Design stage for the NMSF facility.

A summary of the demand and capacity ratios for in-plane shear of the major walls is presented in Table 4.8 and 4.10. Results show that the $D / C$ ratios for the modified structure are all within allowable limits. Based on the field test results for the compressive strength of the concrete in the NMSF building (18), the actual margin of safety is significantly higher than that tabulated.

The summary of the $\mathrm{D} / \mathrm{C}$ ratios for out-of-plane response of major walls is presented in Table 4.9 and 4.11. Only the walls that are subjected to significant out-of-plane loads are analyzed using the computer model. The secondary interior walls are checked for adequacy using hand calculations.

The north and south exterior walls were evaluated and are reported in Tables 4.5 and 4.6. Please note that these walls do not have to support soil pressures above el. $7302.0^{\prime}$. In the modified structure, the soil bearing against the south wall is removed to provide clearance for air circulation through the new intake grate. Also note that these walls do not have the long unsupported horizontal spans and crane loads that the west wall has.

\subsubsection{West Wall}

The results show that the $D / C$ ratios for the walls in the modified structure are all within acceptable limits without need for additional strengthening, with the exception of the wall on line 1 (west wall). The new wall added between the existing columns on line 2 can be designed to perform as a beam in the out-of-plane direction to meet the new demands. The existing wall on line 1, however, does not have sufficient strength because: 
1) the vertical span of the wall has been increased by 3 feet,

2) the wall is now supporting a new crane and accessory rail, and

3) the new function envisioned for the modified facility results in thermal loads that were not considered in the original design.

Strengthening this wall can be accomplished by adding exterior buttresses.

A retrofit concept is provided in the ICF KE engineering calculation supporting this report (13) (see Section 8.5 of the attached calculations). It should be noted that, although the proposed retrofit scheme was not evaluated for the whole extent of the design load combinations set forth in Section 3.0, it is believed to be qualitatively adequate. More refined solution designs are expected during the Title Design stage.

It should be noted that refined SSI analyses and realistic thermal analyses may possibly help minimize the strengthening requirements for this wall.

\subsubsection{Precast Beams}

At the time of this effort, consideration was being given to the potential need to upgrade the NMSF roof over the administrative area for security reasons (security concerns apply to the MAA area only). In order to harden the roof and prevent or delay penetration by an explosive device, it might become necessary to increase the roof thickness to 8 inches. The capacity of the existing roof precast beams to withstand an additional load appears to be limited. The actual additional thickness requirements are not clear at this time. If substantial slab thickening is required, it could be accommodated by:

1) introducing intermediate beams between the existing $T$ beams,

2) using the current roof slab as part of the formwork system for the facility, and, after removing the existing concrete topping, casting a thicker roof slab that would effectively increase the moment arm for the existing post-tensioning tendons and consequently increase the moment capacity of the existing precast beams, and

3) providing for shear transfer between the old and the added concrete slab by means of closely spaced studs.

Details are provided in the calculation in Section 8.1, attached.

\subsubsection{Tunnel-Structure Connections}

The simplified evaluation performed indicates that the tunnel-structure connections will undergo moderate tensile concrete cracking but no compressive crushing. Steel reinforcement will remain below yield. These results indicate that there is no collapse potential for the tunnels and their connection to the structure. It should, however, be noted that the tensile cracking expected could contribute to air and moisture infiltration in the tunnels, as they are envisioned to be maintained at a constant negative pressure. A more realistic evaluation, using 3-D SSI analysis of the tunnel/NMSF structure, will be included in the Title Design stage. 


\subsubsection{New Interior Walls in the Administrative Area}

As shown in the drawings for the modified structure, new concrete and concrete block walls are being introduced in the administrative area. The attached calculation in Section 8.4 addresses these walls. Care should be exercised in the detailing of these walls. The new walls should be restrained laterally at top and bottom. The lateral restraint at the top should allow for relative vertical displacement between the top of the walls or partitions, and the roof and supporting precast beams.

The existing precast beams are likely to be detailed for a moment demand consistent with simplysupported conditions. Any accidental intermediate support could introduce a negative moment overstress.

\subsubsection{Penthouse for HVAC Equipment}

In the modified structure, it is envisioned that some of the non-safety-related equipment will be placed outside the boundaries of the existing facility because of space limitations.

For the purposes of protecting this equipment from weather, a lightweight steel structure has been conceptualized. Section 8.2 of the attached calculations provides a preliminary design for this enclosure. The calculation also provides a check on the adequacy of the existing structure to support the new penthouse.

\subsubsection{Soil-Structure Interaction}

In this conceptual design stage, only a simplified SSI evaluation has been performed. To account for SSI effects, the fixed-base, three-dimensional model of the NMSF building was revised by adding foundation impedances for three translational and three rotational (two rocking and torsion) degrees of freedom. The foundation impedance for each degree of freedom typically consists of a pair of springs and dashpots representing the stiffness and the damping characteristics of the soil medium, respectively. In this evaluation only the soil springs (no dashpots) were utilized in the model to account for the SSI effects on the natural frequency shift for the building. No time history analyses were performed in this simplified evaluation. The procedures recommended in reference (8) were used to develop the SSI model.

To account for embedment effects, the soil springs constants were calculated for the western and eastern portions of the NMSF building separately. The embedment effects of the western portion of the building were accounted for using approximate methods given in reference (11). The embedment above the first floor was neglected, conservatively, to account for potential loss of lateral support during an earthquake (8). The stiffness values used to represent the foundation impedances in the SSI model are summarized in Table 4.2.

A frequency analysis of the SSI model was performed to compute modal properties of the complete soilstructure model. The results of this analysis are summarized in Table 4.3. The fundamental natural frequency of the SSI model was $11.4 \mathrm{~Hz}$, indicating a frequency shift of about $2.6 \mathrm{~Hz}$ from the fixed base frequency of $14.0 \mathrm{~Hz}$ due to the additional flexibility provided by soil foundation springs.

It should be noted that SSI analyses in full conformance with current DOE requirements are to be performed for best-estimate, upper-bound, and lower-bound soil properties. In the current conceptual design effort, only best-estimate soil properties are used. Title Design should address the full range of soil properties. 
It is recommended that a site-specific geotechnical investigation be pursued in support of the Title Design effort. The experimental determination of high-strain soil properties should be pursued to avoid potential challenges to the adequacy of the Title Design.

\subsubsection{East-West Load Path}

The east-west load path is compromised because of the reduced number of east-west shear walls and the openings around the charge deck.

The increased local demands on the west wall because of increased vertical spans, crane loads, and thermal loads are resolved by the introduction of buttresses as shown in Table 4.11. The wall along line 2 has to provide a horizontal load path between the charge deck and the slab between lines 2 and 3 at the original first floor level.

In order to better determine the out-of-plane demand on the line 2 wall, a 2-D strip model (Figure 4.24) was developed. This is a very conservative model for the purposes of calculating the out-of-plane demand for the wall on line 2 because it neglects all shear walls perpendicular to this line.

The north-south walls act as beams in the out-of-plane direction. This model provides an upper bound estimate for the out-of-plane demand on the wall on line 2, and indicates a need to design and detail the wall on line 2 as a beam for out-of-plane shear.

This 2-D model was also used for a cursory vertical analysis of the as-built as well as the modified structure.

For a more detailed description of the 2-D model of modified structure No. 2, and the results, refer to the supporting engineering calculations (13).

\subsection{Conservatism}

The actual margins of safety for the NMSF building in its as-built and proposed modified configurations could be significantly higher than those obtained from this evaluation. A list of conservative assumptions implicit in this evaluation can be summarized as follows:

1) The energy absorption coefficient of Fu was assumed to be equal to 1.0 throughout this evaluation. The allowable Fu values range between 1.0 to 1.75 depending on the quality of construction, per specifications of the DOE-STD-1020-94 (2).

2) Structural damping was assumed to be $7 \%$ of critical damping. The computed D/C ratios indicate that a higher value may be justified, per the recommendations of reference (2).

3) Field test results (18) indicate a much higher compression strength for the concrete (in excess of 7,000 psi) than the value of $4,000 \mathrm{psi}$ used in this evaluation. Also, no credit was taken for the reinforcing steel in computing in-plane shear capacity of the walls. As a result, the margin of safety for the in-plane shear capacity of the walls is significantly higher than those shown in Tables 4.5 and 4.9.

4) The dynamic soil pressure on the perimeter walls conservatively overestimates the actual soil pressures. The passive soil pressures generated on the perimeter walls opposing the 
ground motion were excluded, resulting in conservative results for the in-plane shear loads on the main shear walls.

5) The preliminary thermal loading criteria (Section 3.3.3) is believed to be conservative. Heat transfer analyses performed to obtain thermal gradients across the concrete walls of the material storage area are based on a very conservative accident scenario in which cooling air flow to the facility is completely disrupted for a very extended period of time. Considering the controlling role of the thermal stresses on the total out-of-plane bending demand on these walls, it is important that a more accurate measure of the thermal demands be at hand at the time of Title Design.

6) The fieid tests reported in reference (18) have also determined that the concrete cover over reinforcing steel is greater than that reflected on the design drawings. An increased concrete cover and a reduced moment capacity have been considered in the capacity calculations.

7) The shear capacity determination is conservative in that reinforcing was neglected. Higher margins of safety are available and could be demonstrated with further analysis.

8) The east-west 2-D model neglects the contribution to lateral strength provided by the east-west walls and buttresses.

9) SSI radiation damping has not been taken advantage of. 


\subsection{REFERENCES}

(1) Los Alamos Facilities Engineering Standards, Volume 5, "Structural Standards Manual," Revision 4, November 1994.

(2) "Natural Phenomena Hazards Design and Evaluation Criteria for Department of Energy Facilities," DOE Standard DOE-STD-1020-94, April 1994.

(3) American Society of Civil Engineers, "Minimum Design Loads for Buildings and Other Structures," ASCE/ANSI Standard 7-93.

(4) International Congress of Building Officials, "Uniform Building Code" (UBC), 1994 edition.

(5) Dames \& Moore Geotechnical report, 1972. (Most complete title available.)

(6) EPRI Report NP-6041-SL, Rev 1. (Most complete title available.)

(7) "Soil Dynamics," by Shamsher Parkash, McGraw Hill, 1981.

(8) American Society of Civil Engineers, "ASCE-4-86, Seismic Analysis of Safety-Related Nuclear Structures and Commentary on Standard for Seismic Analysis of Safety Related Nuclear Structures," 1986.

(9) U. S. Nuclear Regulatory Commission, "Nuclear Regulatony Guide NUREG-0098".

(10) "A Series of Computer Programs for Static and Dynamic Finite Element Analysis of Structures," SAP90 User's Manual, Computers and Structures, Inc., Berkeley, California.

(11) "Soil-Structure Interaction in Time Domain," by Wolf.

(12) "Structural Evaluation of the NMSF at LANL, As-Built Structure," ICF Kaiser Calculation Number 41552-S-0001, rev 0, March 1995.

(13) "Structural Evaluation of the NMSF at LANL, Modified Structure," ICF Kaiser Caiculation Number 41552-S-0002, rev 0, March 1995.

(14) As-Built Construction Drawings for the Nuclear Materials Storage Facility, prepared by Burns and Roe Pacific, Inc, dated October 18, 1985. As-Built as of July 10, 1985. Lab Job No. 6481-55, LANL Drawing No. C-44952. Specific sheets listed below:

DOE DWG. NO's LA-ZJ-B.A. 1 through LA-ZJ-B.A.25 and DOE DWG. NO's LA-ZJ-B.S. 1 through LAZJ-B.S.11

(15) ICF Kaiser Preliminary (0\%) Architectural Drawings of the Modified NMSF Facility, Sheets A1 through A5, dated Jan. 13, 1995.

(16) Marked-up Preliminary (0\%) ICF Kaiser drawing sheet A2. Transmitted to ICF Kaiser Oakland office on Feb. 12, 1995. 
(17) Notes from Thad Knight of LANL to Pepe Vallenas of ICF Kaiser, dated Feb. 8, 1995.

(18) "Condition Survey of Nuclear Material Storage Facility, Los Alamos Laboratory, Los Alamos, New Mexico," by Construction Technology Laboratory, Inc., January 1995.

(19) American Society of Civil Engineers, ASCE Structural Division Committee on Nuclear Structures and Materials, "Seismic Response of Buried Pipes and Structural Components," 1983.

(20) Atalay M. B. "Seismic Analysis and Design of Buried Piping." Lifeline Earthquake Engineering, Third U.S. Conference, Los Angeles, CA, August 22-23, 1991.

(21) Goen, L. K., "Soil Structure Interaction Analysis," PF-4-ESA 13-2B-001.

(22) American Society of Civil Engineers, "ASCE 4-86, Seismic Analysis of Safety Related Structures and Commentary for Seismic Analysis fo Safety Related Structures," 1986.

(23) Anmerican Concrete Institute, "ACl 349-90, Code Requirements for Nuclear Safety Related Concrete Structures," 1990.

(24) American Institute of Steel Construction, "AISC N690-84, Specification for the Design, Fabrication, and Erection of Steel-Safety Related Structures for Nuclear Facilities," 1984.

(25) ICF Kaiser 100\% Draft Architectural Drawings of the Modified NMSF Facility, Sheets A1-A5, May 1995. 
NMSF Conceptual Design Report

TABLE 4.1 FREQUENCY ANALYSIS, FIXED BASE MODEL, AS-BUILT STRUCTURE (NMSFO)

\begin{tabular}{|c|c|c|c|c|}
\hline Mode & $\begin{array}{c}\text { Frequency } \\
\text { Hz }\end{array}$ & $\begin{array}{c}\text { X-Direction } \\
\text { Mass Percent }\end{array}$ & $\begin{array}{c}\text { Y-Direction } \\
\text { Mass Percent }\end{array}$ & $\begin{array}{c}\text { Z-Direction } \\
\text { Mass Percent }\end{array}$ \\
\hline 1 & 14.0 & 73.3 & 0.0 & 0.0 \\
\hline 2 & 15.7 & 73.3 & 1.1 & 0.0 \\
\hline 3 & 17.4 & 73.6 & 1.2 & 0.0 \\
\hline 4 & 17.8 & 73.7 & 1.2 & 0.0 \\
\hline 5 & 19.4 & 73.7 & 71.7 & 0.0 \\
\hline 6 & 25.8 & 78.8 & 71.8 & 0.0 \\
\hline 7 & 31.2 & 80.8 & 80.7 & 0.2 \\
\hline 8 & 33.2 & 81.1 & 81.9 & 2.5 \\
\hline 9 & 36.2 & 92.5 & 83.9 & 2.6 \\
\hline
\end{tabular}


TABLE 4.2 FOUNDATION SOIL SPRING CONSTANTS (Units: Kips,Ft)

\begin{tabular}{|l|l|c|c|c|c|c||}
\hline \multicolumn{1}{|c|}{ Foundation } & \multicolumn{1}{|c|}{$\mathrm{Kx} \mathrm{(*)}$} & $\mathrm{Ky}$ & $\mathrm{Kz}$ & $\mathrm{Kxx}$ & $\mathrm{Kyy}$ & $\mathrm{Kzz}$ \\
\hline Basement (lines 1 to 3) & $259 \mathrm{G}\left(^{* *}\right)$ & $272 \mathrm{G}$ & $340 \mathrm{G}$ & $3.67 \mathrm{E} 5 \mathrm{G}$ & $2.15 \mathrm{E} 6 \mathrm{G}$ & $1.15 \mathrm{E} 6 \mathrm{G}$ \\
\hline 1st Floor (line 3 to 8) & $317 \mathrm{G}$ & $300 \mathrm{G}$ & $214 \mathrm{G}$ & $2.53 \mathrm{E} 6 \mathrm{G}$ & $1.25 \mathrm{E} 6 \mathrm{G}$ & $1.60 \mathrm{E} 7 \mathrm{G}$ \\
\hline
\end{tabular}

Notes:

$\begin{array}{lll}\left(^{*}\right) \text { - Coordinate System: } & \text { X-dir } & \text { East/West } \\ & \text { Y-dir } & \text { North/South } \\ & \text { Z-dir } & \text { Vertical }\end{array}$

$\left.{ }^{(* *}\right)-G=6.1 E 3 \mathrm{Kips} / \mathrm{SF}$ (Best Estimate Soil Shear Modulus) 
NMSF Conceptual Design Report

July 14, 1995

Page 27

TABLE 4.3 FREQUENCY ANALYSIS, FIXED BASE MODEL WITH SOIL SPRINGS, AS-BUILT STRUCTURE (NMSF01)

\begin{tabular}{|c|c|c|c|c|}
\hline Mode & $\begin{array}{c}\text { Frequency } \\
\text { Hz }\end{array}$ & $\begin{array}{c}\text { X-Direction } \\
\text { Mass Percent }\end{array}$ & $\begin{array}{c}\text { Y-Direction } \\
\text { Mass Percent }\end{array}$ & $\begin{array}{c}\text { Z-Direction } \\
\text { Mass Percent }\end{array}$ \\
\hline 1 & 11.4 & 92.3 & 0.2 & 0.2 \\
\hline 2 & 11.7 & 92.6 & 87.8 & 0.3 \\
\hline 3 & 14.9 & 92.9 & 87.9 & 98.6 \\
\hline 4 & 16.4 & 92.9 & 88.0 & 98.7 \\
\hline 5 & 17.4 & 93.0 & 88.2 & 98.8 \\
\hline 6 & 18.0 & 93.0 & 96.7 & 98.9 \\
\hline 7 & 23.2 & 96.3 & 96.7 & 99.0 \\
\hline 8 & 23.7 & 96.3 & 98.8 & 99.0 \\
\hline 9 & 28.0 & 96.9 & 98.8 & 99.9 \\
\hline
\end{tabular}


NMSF Conceptual Design Report July 14, 1995

Page 28

TABLE 4.4 FREQUENCY ANALYSIS, SSI MODEL WITH MASS ECCENTRICITY FOR ACCIDENTAL TORSION, AS-BUILT STRUCTURE (NMSF04)

\begin{tabular}{|c|c|c|c|c|}
\hline Mode & $\begin{array}{c}\text { Frequency } \\
\mathrm{Hz}\end{array}$ & $\begin{array}{c}\text { X-Direction } \\
\text { Mass Percent }\end{array}$ & $\begin{array}{c}\text { Y-Direction } \\
\text { Mass Percent }\end{array}$ & $\begin{array}{c}\text { Z-Direction } \\
\text { Mass Percent }\end{array}$ \\
\hline 1 & 11.7 & 93.4 & 0 & 0 \\
\hline 2 & 11.8 & 94.0 & 92.8 & 1 \\
\hline 3 & 12.3 & 94.0 & 93.8 & 99.7 \\
\hline 4 & 17.7 & 94.0 & 98.7 & 99.9 \\
\hline 5 & 21.1 & 97.8 & 98.8 & 99.9 \\
\hline 6 & 26.5 & 99.1 & 98.8 & 99.9 \\
\hline 7 & 28.7 & 99.7 & 98.8 & 99.9 \\
\hline 8 & 36.8 & 99.7 & 100 & 99.9 \\
\hline 9 & 41.5 & 100 & 100 & 99.9 \\
\hline
\end{tabular}


TABLE 4.5 SUMMARY OF WALL IN-PLANE SHEAR DEMAND/CAPACITY RATIOS FOR AS-BUILT STRUCTURE

\begin{tabular}{|c|c|c|c|c|}
\hline $\begin{array}{c}\text { Wall } \\
\text { ID }\end{array}$ & $\begin{array}{c}\text { Shear } \\
\text { Demand } \\
\text { (Kips) }\end{array}$ & $\begin{array}{c}\text { Shear } \\
\text { Capacity } \\
\text { (Kips) }\end{array}$ & $\begin{array}{c}\text { Shear } \\
\text { D/C }\end{array}$ & Wall Location \\
\hline 3 & 822 & 2763 & .30 & Line 1 Basement \\
\hline 6 & 868 & 2671 & .33 & Line 3 Basement \\
\hline 8 & 167 & 434 & .38 & Line 5 Basement \\
\hline 9 & 1216 & 1239 & .98 & Line B Basement \\
\hline 12 & 788 & 1022 & .77 & Line G Basement \\
\hline 4 & 485 & 1594 & .30 & Line 1+ Basement \\
\hline 5 & 432 & 1310 & .33 & Line 2 Basement \\
\hline 1 & 84 & 284 & .30 & Line 2 Basement \\
\hline 10 & 189 & 235 & .80 & Line C Basement \\
\hline 11 & 264 & 358 & .74 & Line F Basement \\
\hline 19 & 1477 & 2450 & .60 & Line 1 1st Floor \\
\hline 34 & 692 & 881 & .79 & Line G 1st Floor \\
\hline 27 & 695 & 1006 & .69 & Line B 1st Floor \\
\hline 21 & 1333 & 1638 & .81 & Line 3 1st Floor \\
\hline 20 & 222 & 377 & .59 & Line 2 1st Floor \\
\hline 29 & 325 & 364 & .89 & Line $C$ ist Floor \\
\hline 28 & 758 & 991 & .76 & Line B 1st Floor \\
\hline 25 & 996 & 1367 & .73 & Line 7 1st Floor \\
\hline 35 & 736 & 966 & .76 & Line $\mathrm{G}$ 1st Floor \\
\hline 38 & 745 & 991 & .75 & Line A 1st Floor \\
\hline 32 & 253 & 358 & .71 & Line $F$ 1st Floor \\
\hline
\end{tabular}


NMSF Conceptual Design Report

July 14,1995

Page 30

TABLE 4.6 SUMMARY OF WALL OUT-OF-PLANE SHEAR AND BENDING DEMAND/CAPACITY RATIOS FOR AS-BUILT STRUCTURE

\begin{tabular}{|c|c|c|c|c|c|c|c|}
\hline $\begin{array}{c}\text { Wall } \\
\text { ID }\end{array}$ & $\begin{array}{l}\text { Shear } \\
\text { Demand } \\
\text { (Kips) }\end{array}$ & $\begin{array}{l}\text { Shear } \\
\text { Capacity } \\
\text { (Kips) }\end{array}$ & $\begin{array}{l}\text { SHR } \\
\text { D/C }\end{array}$ & $\begin{array}{c}\text { Moment } \\
\text { Demand } \\
(k-f t)\end{array}$ & $\begin{array}{c}\text { Moment } \\
\text { Capacity } \\
(k-f t) \\
\end{array}$ & $\begin{array}{c}\text { MOM } \\
\text { D/C }\end{array}$ & $\begin{array}{c}\text { Wall } \\
\text { Location }\end{array}$ \\
\hline 3 & 17.5 & 21.3 & .82 & $\begin{array}{r}(+) 55.0 \\
(-) 30.1\end{array}$ & $\begin{array}{l}108 \\
116\end{array}$ & $\begin{array}{l}.51 \\
.26\end{array}$ & West Wall Basement \\
\hline 19 & 14.9 & 18.7 & .80 & $\begin{array}{r}(+) 32.3 \\
(-) 16.9 \\
\end{array}$ & $\begin{array}{l}60 \\
26 \\
\end{array}$ & $\begin{array}{l}.54 \\
.65 \\
\end{array}$ & $\begin{array}{c}\text { West Wall } \\
\text { 1st Floor }\end{array}$ \\
\hline 12 & 17.5 & 21.3 & .82 & $\begin{array}{r}(+) 5.2 \\
(-) 3.0\end{array}$ & $\begin{array}{r}78 \\
232 \\
\end{array}$ & $\begin{array}{l}.07 \\
.01\end{array}$ & $\begin{array}{l}\text { South Wall } \\
\text { Basement }\end{array}$ \\
\hline 34 & 14.9 & 19 & .78 & $\begin{array}{r}(+) 5.2 \\
(-) 3.0\end{array}$ & $\begin{array}{r}78 \\
232\end{array}$ & $\begin{array}{l}.07 \\
.01\end{array}$ & $\begin{array}{l}\text { South Wall } \\
\text { 1st Floor }\end{array}$ \\
\hline 9 & 17.5 & 21.3 & .82 & $\begin{array}{r}(+) 13.6 \\
(-)+1.4\end{array}$ & $\begin{array}{r}78 \\
232 \\
\end{array}$ & $\begin{array}{l}.17 \\
.05\end{array}$ & $\begin{array}{l}\text { North Wall } \\
\text { Basement }\end{array}$ \\
\hline 27 & 14.9 & 19 & .78 & $\begin{array}{r}(+) 16.5 \\
(-) 31.5 \\
\end{array}$ & $\begin{array}{l}42 \\
24 \\
\end{array}$ & $\begin{array}{r}.39 \\
1.31 \\
\end{array}$ & $\begin{array}{l}\text { North Wall } \\
\text { 1st Floor }\end{array}$ \\
\hline Others & $\mathrm{n} / \mathrm{a}$ & $\mathrm{n} / \mathrm{a}$ & $\mathrm{n} / \mathrm{a}$ & $\begin{array}{r}(+) 5.2 \\
(-) 6.9\end{array}$ & & & $\begin{array}{l}\text { All Other Walls Mostly } \\
\text { Interior Walls, O.K. By } \\
\text { Inspection }\end{array}$ \\
\hline
\end{tabular}


NMSF Conceptual Design Report

July 14, 1995

Page 31

TABLE 4.7 FREQUENCY ANALYSIS, SSI MODEL WITH MASS ECCENTRICITY FOR ACCIDENTAL TORSION, MODIFIED STRUCTURE (NMSF05)

\begin{tabular}{|c|r|r|r|c||}
\hline Mode & $\begin{array}{c}\text { Frequency } \\
\text { (Hz) }\end{array}$ & $\begin{array}{c}\text { X-Direction } \\
\text { Mass (Percent) }\end{array}$ & $\begin{array}{c}\text { Y-Direction } \\
\text { Mass (Percent) }\end{array}$ & $\begin{array}{c}\text { Z-Direction } \\
\text { Mass (Percent) }\end{array}$ \\
\hline 1 & 9.9 & 80.6 & 0.2 & 16.4 \\
\hline 2 & 10.0 & 80.8 & 91.9 & 20.9 \\
\hline 3 & 10.2 & 97.6 & 96.4 & 99.2 \\
\hline 4 & 15.3 & 97.6 & 99.5 & 99.4 \\
\hline 5 & 18.1 & 97.6 & 99.6 & 99.4 \\
\hline 6 & 19.4 & 99.0 & 99.6 & 99.9 \\
\hline 7 & 25.8 & 99.9 & 99.6 & 99.9 \\
\hline 8 & 31.6 & 100. & 99.6 & 99.9 \\
\hline 9 & 40.5 & 100. & 99.9 & 99.9 \\
\hline \hline
\end{tabular}


TABLE 4.8 SUMMARY OF WALL IN-PLANE SHEAR DEMAND/CAPACITY RATIOS FOR MODIFIED STRUCTURE NO. 1

\begin{tabular}{|c|c|c|c|c|}
\hline $\begin{array}{l}\text { Wall } \\
\text { ID }\end{array}$ & $\begin{array}{l}\text { Shear } \\
\text { Demand } \\
\text { (Kips) }\end{array}$ & $\begin{array}{l}\text { Shear } \\
\text { Capacity } \\
\text { (Kips) }\end{array}$ & $\begin{array}{c}\text { Shear } \\
D / C\end{array}$ & Wall Location \\
\hline 1 & 1629 & 4847 & 0.34 & Basement-line 1 \\
\hline 2 & 131 & 339 & 0.39 & Basement-line 1 \\
\hline 4 & 124 & 301 & 0.41 & Basement-line 2 \\
\hline 5 & 1770 & 2424 & 0.73 & Basement-line 2 \\
\hline 6 & 1080 & 2671 & 0.40 & Basement-line 3 \\
\hline 9 & 1315 & 1239 & 1.06 & Basement-line B \\
\hline 12 & 837 & 994 & 0.84 & Basement-line $\mathbf{G}$ \\
\hline 8 & 215 & 550 & 0.39 & Basement-line 4 \\
\hline 10 & 230 & 235 & 0.98 & Basement-line D \\
\hline 3 & 70 & 550 & 0.13 & Basement - S. Baffle \\
\hline 33 & 279 & 296 & 0.94 & Basement- line $F$ \\
\hline 11 & 227 & 296 & 0.77 & Basement-line C \\
\hline 7 & 274 & 550 & 0.50 & Basement- N. Baffle \\
\hline 29 & 250 & 279 & 0.90 & Buttress \\
\hline 21 & 1072 & 2302 & 0.47 & 1st flr-line 3 \\
\hline 28 & 791 & 991 & 0.80 & 1st flr-line B \\
\hline 30 & 578 & 812 & 0.71 & 1st fir-line $\mathrm{E}$ \\
\hline 35 & 751 & 996 & 0.78 & 1st flr-line $G$ \\
\hline 18 & 509 & 550 & 0.93 & Basement-line $F$ \\
\hline 36 & 114 & 158 & 0.72 & 1st flr-line $\mathrm{C}$ \\
\hline 25 & 826 & 1371 & 0.60 & 1st fir-line 7 \\
\hline 38 & 721 & 991 & 0.73 & 1st fir-line $\mathrm{A}$ \\
\hline
\end{tabular}


TABLE 4.9 SUMMARY OF WALL OUT-OF-PLANE SHEAR AND BENDING DEMAND/CAPACITY RATIOS FOR MODIFIED STRUCTURE NO. 1

\begin{tabular}{|c|c|c|c|c|c|c|c|c||}
\hline $\begin{array}{c}\text { Wall } \\
\text { ID }\end{array}$ & $\begin{array}{c}\text { Shear } \\
\text { Demand } \\
\text { (Kips) }\end{array}$ & $\begin{array}{c}\text { Shear } \\
\text { Capacity } \\
\text { (Kips) }\end{array}$ & $\begin{array}{c}\text { SHR } \\
\text { D/C }\end{array}$ & $\begin{array}{c}\text { Moment } \\
\text { Demand } \\
\text { (k-ft) }\end{array}$ & $\begin{array}{c}\text { Moment } \\
\text { Capacity } \\
\text { (k-ft) }\end{array}$ & $\begin{array}{c}\text { MOM } \\
\text { D/C }\end{array}$ & $\begin{array}{c}\text { Wall } \\
\text { Location }\end{array}$ & Notes \\
\hline 1 & 15.4 & 21.3 & 0.72 & 279 & 356 & 0.78 & $\begin{array}{c}\text { Basement } \\
\text { line 1 }\end{array}$ & New \\
\hline 5 & N/A & N/A & N/A & 201 & 287 & 0.70 & $\begin{array}{c}\text { Basement } \\
\text { line 2 }\end{array}$ & New \\
\hline 9 & 15.4 & 21.3 & 0.72 & 44 & 78 & 0.56 & $\begin{array}{c}\text { Basement } \\
\text { lines B\&G }\end{array}$ & Existing \\
\hline 19 & 19.7 & 18.7 & 1.07 & 129 & 60 & 2.15 & $\begin{array}{c}\text { 1st-FIr line } \\
1\end{array}$ & $(1)$ \\
\hline 27 & 19.7 & 21.3 & 0.92 & 26 & 24 & 1.08 & $\begin{array}{c}\text { 1st Flr-lines } \\
\text { B\&G }\end{array}$ & Existing \\
\hline Others & negligible & N/A & N/A & 10 & N/A & $\begin{array}{c}\text { negli- } \\
\text { gible }\end{array}$ & $\begin{array}{c}\star \\
\text { ( }) \text { O.K. By } \\
\text { Inspection }\end{array}$ & \\
\hline
\end{tabular}

NOTES:

(1) This segment of the west wall is determined to be inadequate at as-built conditions to meet the additional demands due to a large span caused by lowering the first floor slab and addition of the new bridge crane. A conceptual upgrade is provided in the supporting calculations, Section 8.5 (13).

N/A Not Available 
NMSF Conceptual Design Report

July 14, 1995

Page 34

TABLE 4.10 SUMMARY OF WALL IN-PLANE SHEAR DEMAND/CAPACITY RATIOS FOR MODIFIED STRUCTURE NO. 2, 3-D MODEL

\begin{tabular}{|c|c|c|c|c||}
\hline Wall ID & $\begin{array}{c}\text { Shear } \\
\text { Demand } \\
\text { (Kips) }\end{array}$ & $\begin{array}{c}\text { Shear } \\
\text { Capacity } \\
\text { (Kips) }\end{array}$ & $\begin{array}{c}\text { Shear } \\
\text { D/C }\end{array}$ & Wall Location \\
\hline 1 & 1416 & 4847 & 0.29 & Basement-line 1 \\
\hline 2 & 181 & 339 & 0.53 & Basement-line 1 \\
\hline 4 & 126 & 301 & 0.42 & Basement-line 2 \\
\hline 5 & 1705 & 2424 & 0.70 & Basement-line 2 \\
\hline 9 & 1518 & 1239 & 1.24 & Basement-line B \\
\hline 12 & 994 & 994 & 0.97 & Basement-line G \\
\hline
\end{tabular}


TABLE 4.11 SUMMARY OF WALL OUT-OF-PLANE BENDING DEMAND/CAPACITY RATIOS FOR MODIFIED STRUCTURE NO. 2, 3-D MODEL

\begin{tabular}{|c|c|c|c|c|c|}
\hline $\begin{array}{l}\text { Wall } \\
\text { ID }\end{array}$ & $\begin{array}{c}\text { Moment } \\
\text { Demand } \\
(k-f t)\end{array}$ & $\begin{array}{c}\text { Moment } \\
\text { Capacity } \\
\text { (k-ft) }\end{array}$ & $\begin{array}{l}\text { MOM } \\
\text { D/C }\end{array}$ & $\begin{array}{c}\text { Wall } \\
\text { Location }\end{array}$ & Notes \\
\hline 1 & 281 & 356 & 0.79 & $\begin{array}{c}\text { Basement } \\
\text { line } 1\end{array}$ & New \\
\hline 5 & 206 & 287 & 0.72 & $\begin{array}{l}\text { Basement } \\
\text { line } 2\end{array}$ & New \\
\hline 9 & 49 & 78 & 0.63 & $\begin{array}{l}\text { Basement } \\
\text { lines } B \& G\end{array}$ & Existing \\
\hline 19 & 75 & 60 & 1.25 & $\begin{array}{c}\text { 1st-Flr line } \\
1\end{array}$ & $\begin{array}{c}(1) \\
\text { Modify }\end{array}$ \\
\hline 16 & 35 & 96 & 0.36 & $\begin{array}{c}\text { 1st-Flr line } \\
2\end{array}$ & New \\
\hline 27 & 46 & 42 & 1.10 & $\begin{array}{l}\text { 1st Fir-lines } \\
\text { B\&G }\end{array}$ & Existing \\
\hline Others & 10 & Small & Small & All others & $\begin{array}{c}\text { O.K. By } \\
\text { Inspection }\end{array}$ \\
\hline
\end{tabular}

NOTES:

(1) This segment of the west wall was determined to be inadequate at as-built conditions to meet the additional demands due to a large span caused by lowering the first floor slab and addition of the new bridge crane. A conceptual upgrade using buttresses is provided in the supporting calculations, Section 8.5 (13), and results in the lowered D/C ratio. 
TABLE 4.12 SUMMARY OF WALL OUT-OF-PLANE SHEAR AND BENDING DEMAND/CAPACITY RATIOS FROM HORIZONTAL ANALYSIS OF MODIFIED STRUCTURE NO. 2, 2-D MODEL

\begin{tabular}{|c|c|c|c|c|c|c|c|c|}
\hline $\begin{array}{l}\text { Wall } \\
\text { ID }\end{array}$ & $\begin{array}{c}\text { Shear } \\
\text { Demand } \\
\text { (Kips) }\end{array}$ & $\begin{array}{c}\text { Shear } \\
\text { Capacity } \\
\text { (Kips) }\end{array}$ & $\begin{array}{l}\text { SHR } \\
D / C\end{array}$ & $\begin{array}{c}\text { Moment } \\
\text { Demand } \\
\text { (k-ft) }\end{array}$ & $\begin{array}{c}\text { Moment } \\
\text { Capacity } \\
\text { (k-ft) }\end{array}$ & $\begin{array}{c}\text { MOM } \\
\text { D/C }\end{array}$ & $\begin{array}{c}\text { Wall } \\
\text { Location }\end{array}$ & Notes \\
\hline 1 & 5 & 21.3 & 0.23 & 253 & $356^{3}$ & 0.71 & $\begin{array}{c}\text { Basement } \\
\text { line } 1\end{array}$ & New \\
\hline 5 & 35 & 46 & 0.76 & 320 & $356^{3}$ & .90 & $\begin{array}{l}\text { Basement } \\
\text { line } 2\end{array}$ & $\begin{array}{c}\text { New } \\
\text { (3) }\end{array}$ \\
\hline 9 & $N / A$ & $N / A$ & N/A & N/A & N/A & N/A & $\begin{array}{l}\text { Basement } \\
\text { lines B\&G }\end{array}$ & Existing \\
\hline 19 & 6.6 & 18.7 & 0.35 & 112 & 60 & 1.87 & $\begin{array}{c}\text { 1st-Flr line } \\
1\end{array}$ & $\begin{array}{c}(1) \\
\text { Modify }\end{array}$ \\
\hline 16 & 15 & 18.7 & 0.80 & 135 & $135^{3}$ & 1.0 & $\begin{array}{c}\text { 1st-Fir line } \\
2\end{array}$ & $\begin{array}{l}\text { New } \\
\text { (2) }\end{array}$ \\
\hline 27 & $N / A$ & N/A & N/A & N/A & N/A & N/A & $\begin{array}{c}\text { 1st-Flr } \\
\text { lines } B \& G\end{array}$ & Existing \\
\hline
\end{tabular}

NOTES:

(1) This segment of the west wall is determined to be inadequate at as-built conditions to meet the additional demands due to a large span caused by lowering the first floor slab and addition of the new bridge crane. A conceptual upgrade is provided in the supporting calculations, Section 8.5 (13).

(2) The new wall will be designed as a beam out-of-plane with shear reinforcement and shear capacity of $4 \sqrt{ } f^{f} c$.

(3) Preliminary estimate design value.

N/A Not Available. 
TABLE 4.13 SUMMARY OF VERTICAL ANALYSIS RESULTS MODIFIED STRUCTURE NO. 2, 2-D MODEL

\begin{tabular}{||l|c|c|c|c|c|c|c||}
\hline \multirow{2}{*}{ Item } & \multicolumn{3}{|c|}{ Max Moment (Ft-K) } & \multicolumn{3}{c|}{ Max. Shear (Kips) } & \multirow{2}{*}{ Notes } \\
\cline { 2 - 8 } & Demand & Capacity & D/C & Demand & Capacity & D/C & \\
\hline MAA Roof Slab & 41.9 & 43.7 & 0.96 & 8.5 & 12.9 & 0.66 & Existing \\
\hline 1st Floor Slab & 30.5 & 25.3 & 1.21 & 7.9 & 12.9 & 0.61 & Existing \\
\hline Mezz. Floor Slab & 32.9 & 33.9 & .97 & 8.0 & 12.9 & 0.62 & $\begin{array}{c}\text { New } \\
\text { Design }\end{array}$ \\
\hline Charge Deck & 45.7 & 540 & 0.08 & 10.8 & 38.7 & 0.28 & $\begin{array}{c}\text { New } \\
\text { Design }\end{array}$ \\
\hline $\begin{array}{l}\text { 30' Span 18x38 } \\
\text { Beams @ MAA } \\
\text { Area Roof }\end{array}$ & 1476 & 1430 & 1.03 & 246 & 187 & 1.32 & $\begin{array}{c}\text { Existing } \\
(1)\end{array}$ \\
\hline $\begin{array}{l}\text { 30' Span 18x38 } \\
\text { Beams @ MAA } \\
\text { Area Roof }\end{array}$ & & & & 122 & 187 & 0.65 & $\begin{array}{c}\text { Existing } \\
(2)\end{array}$ \\
\hline
\end{tabular}

NOTES:

(1) Beam with topside soil in-place.

(2) Beam O.K. with topside soils removed. 


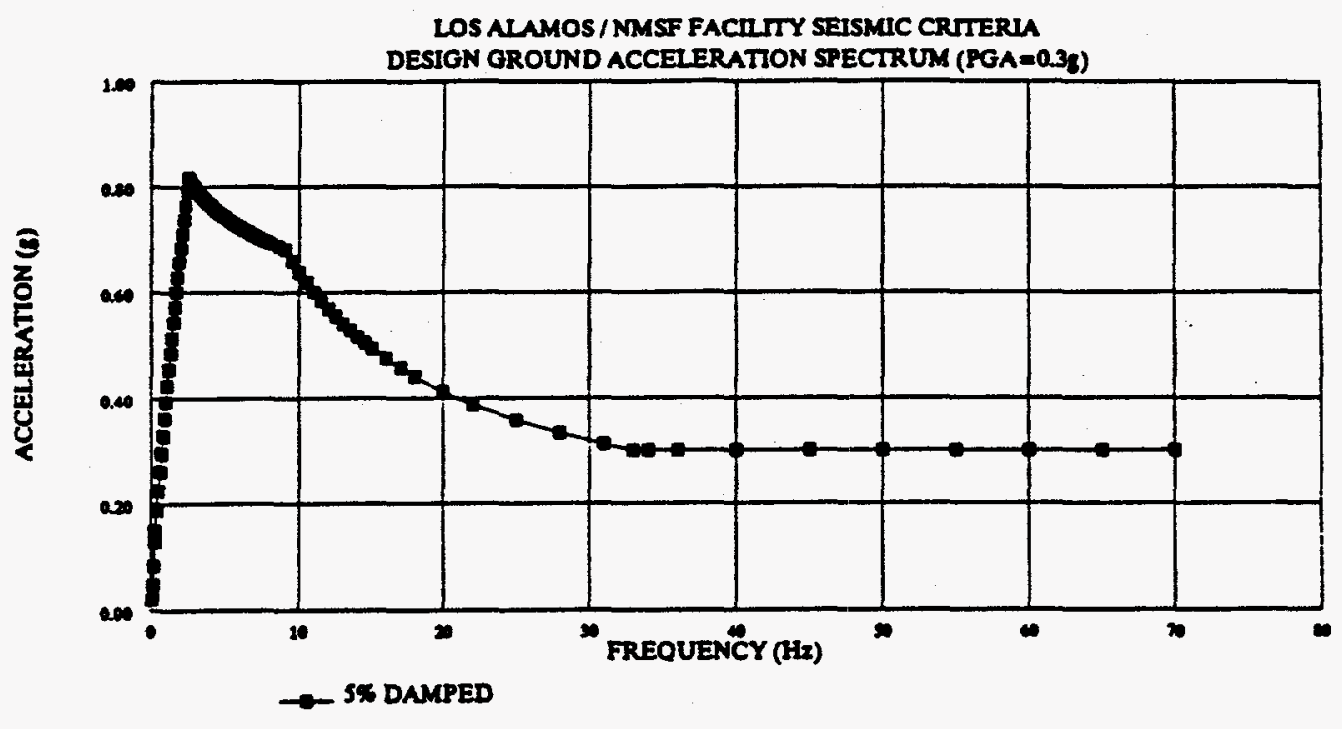

Figure 3 - Horizontal and Vertical Acceleration Response Spectrum 


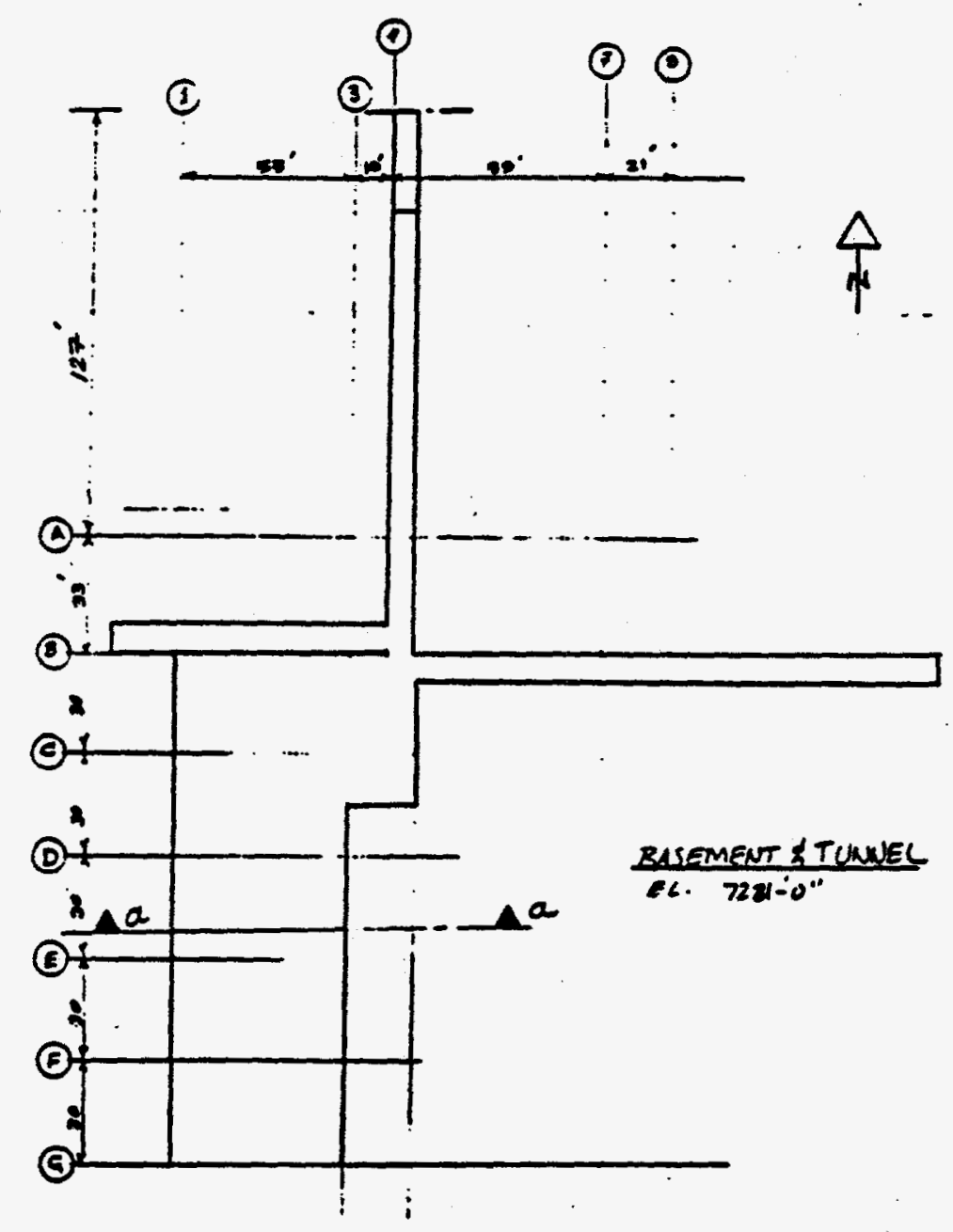

Figure 4.1 - Geometry of As-Bullt NMSF Bullding 


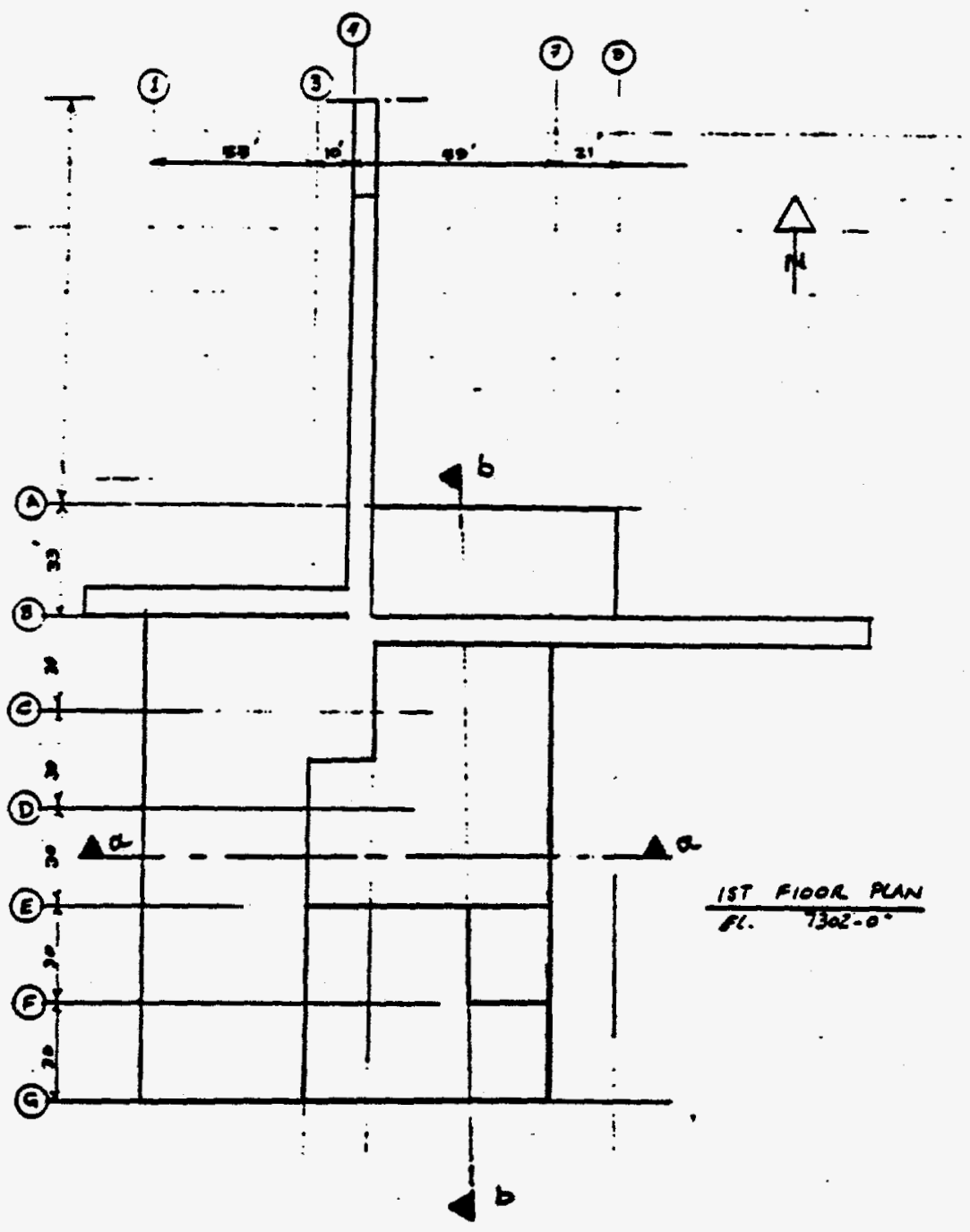

Figure 4.1 - Geometry of As-Bulit NMSF Bullding (Continued) 
NMSF Conceptual Design Report

July 14, 1995

Page 41

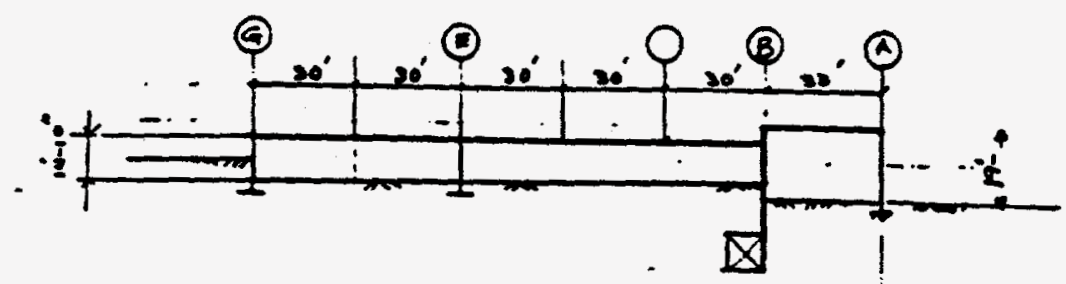

SECTION" $b$ '

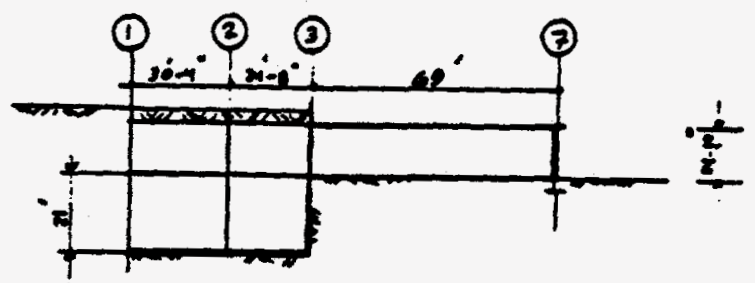

SETON ' $a$ '

Figure 4.1 - Geometry of As-Built NMSF Bullding (Continued) 


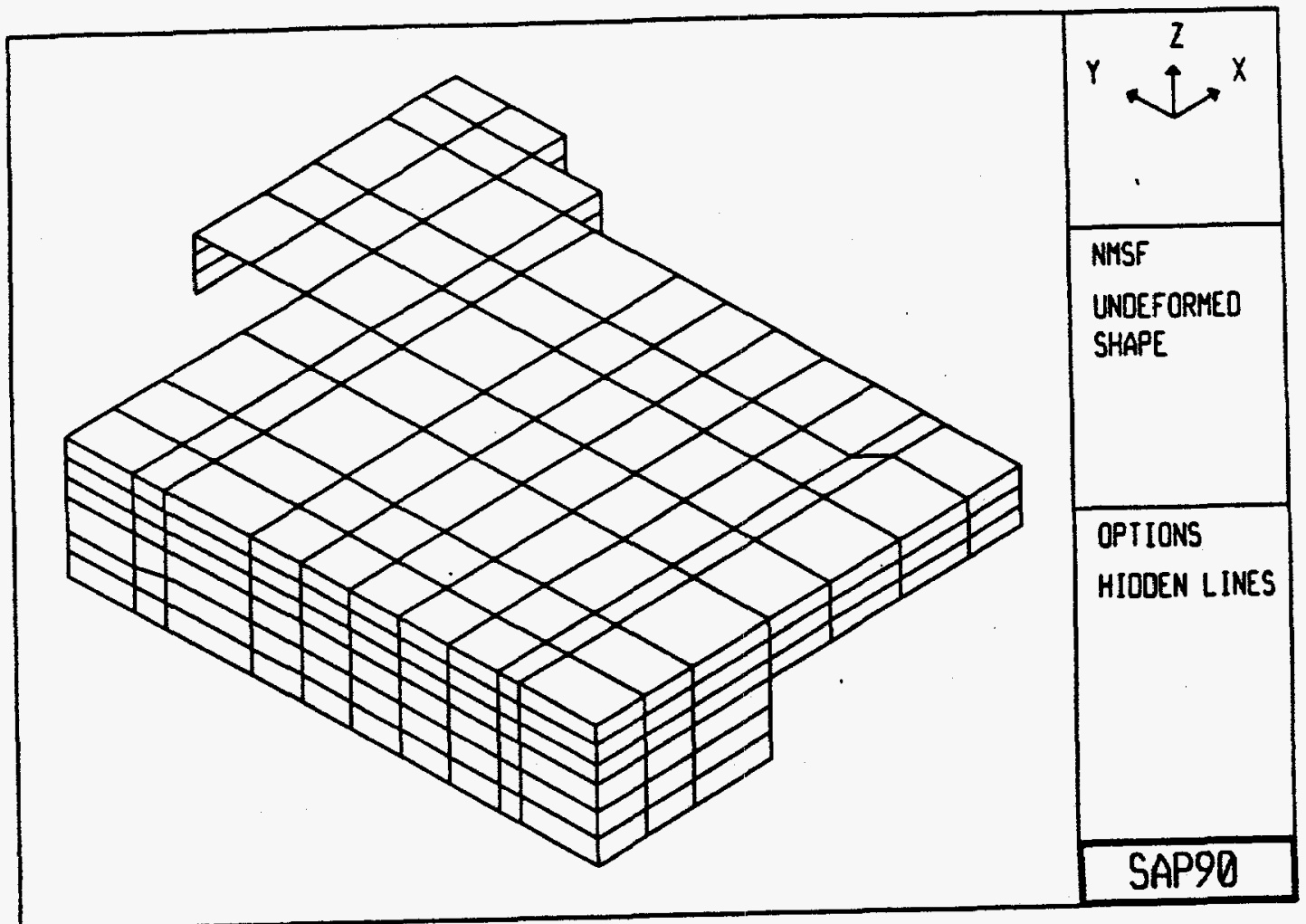

Figure 4.2 - MMSF Finite Element Model, Complete Model As-Built Structure 


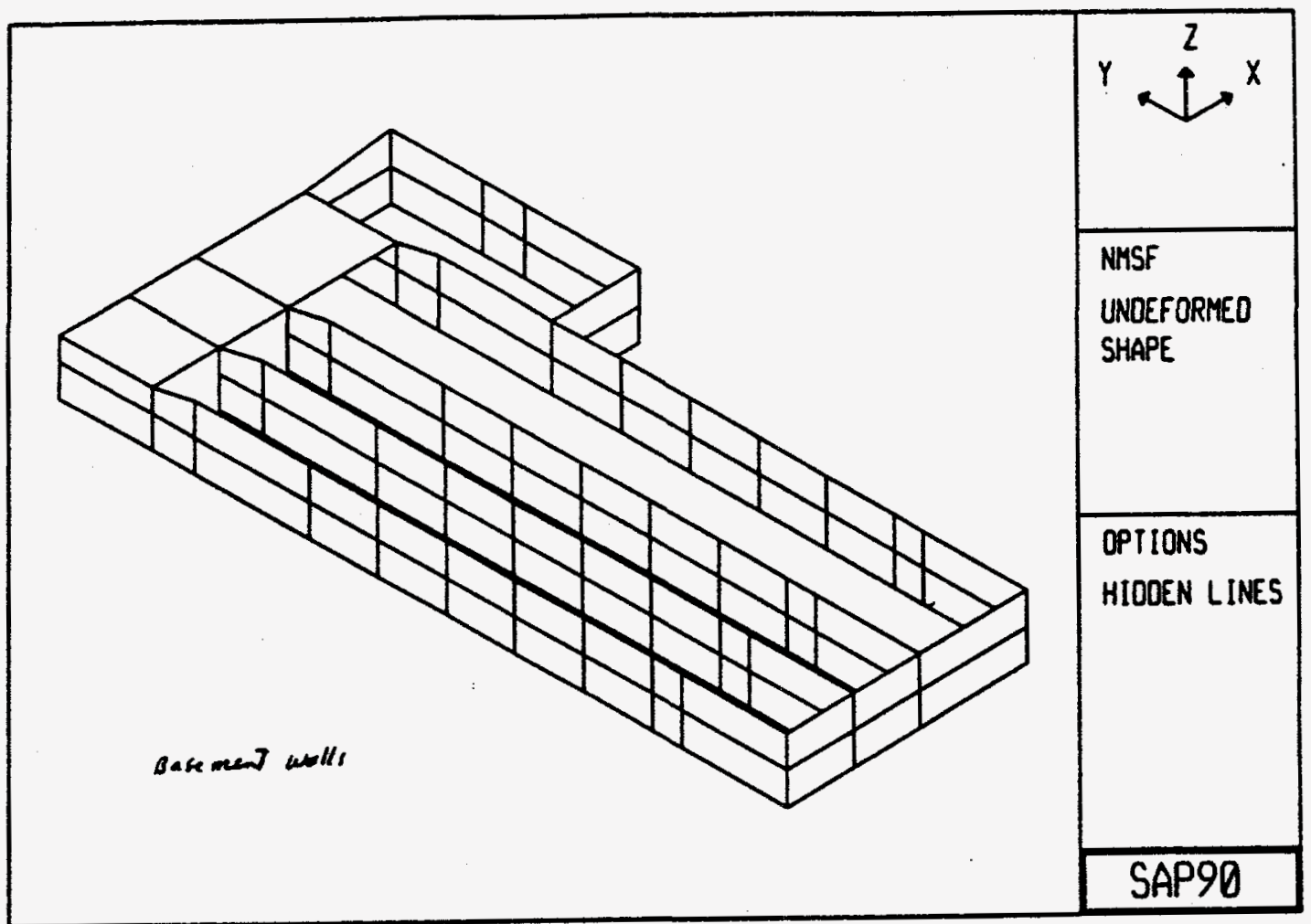

Flgure 4.3 - NMSF Finite Element Model, Basement Walls As-Built Structure 
NMSF Conceptual Design Report

July 14, 1995

Page 44

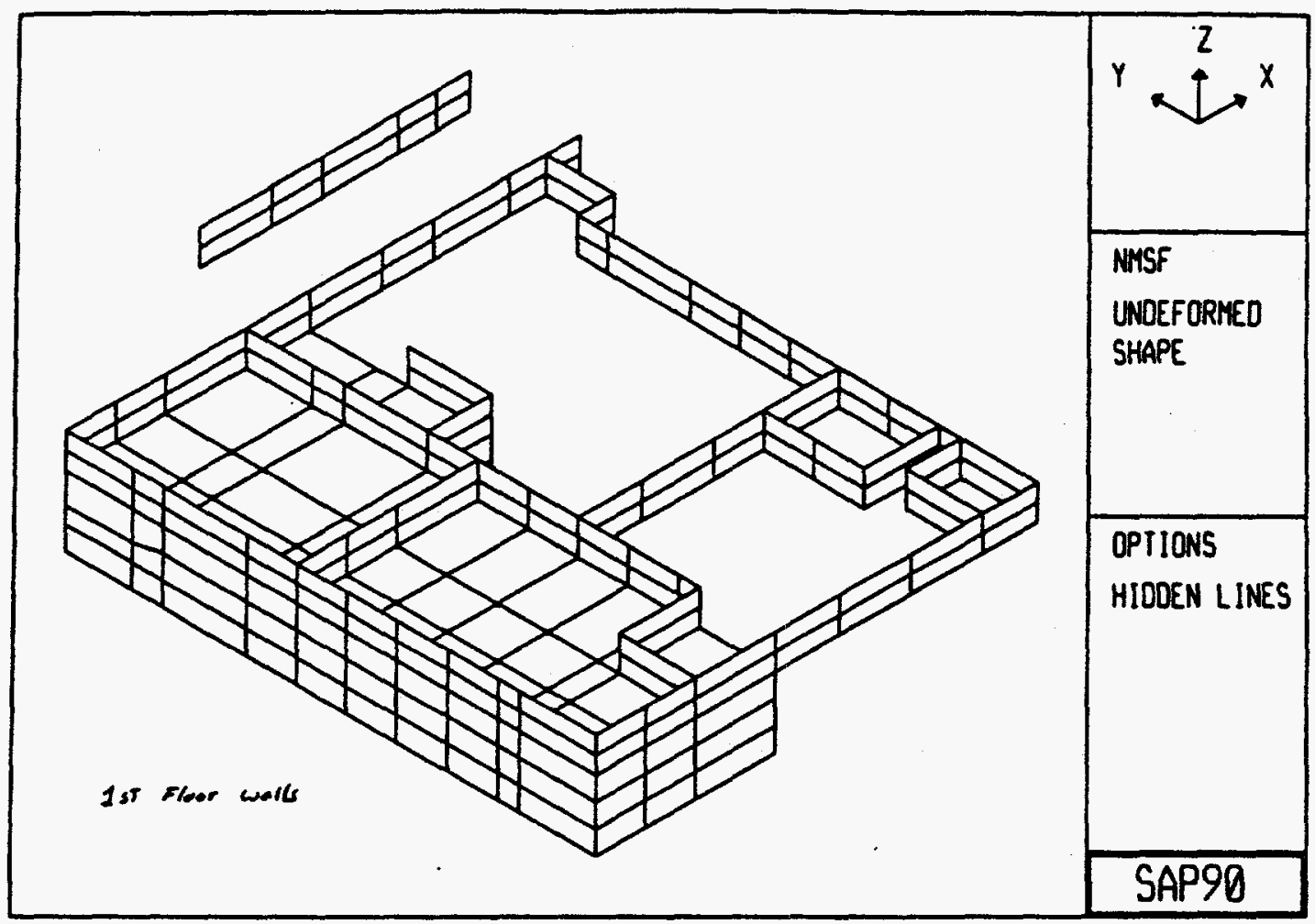

Figure 4.4 - NMSF Finite Element Model, First Floor Walls As-Built Structure 


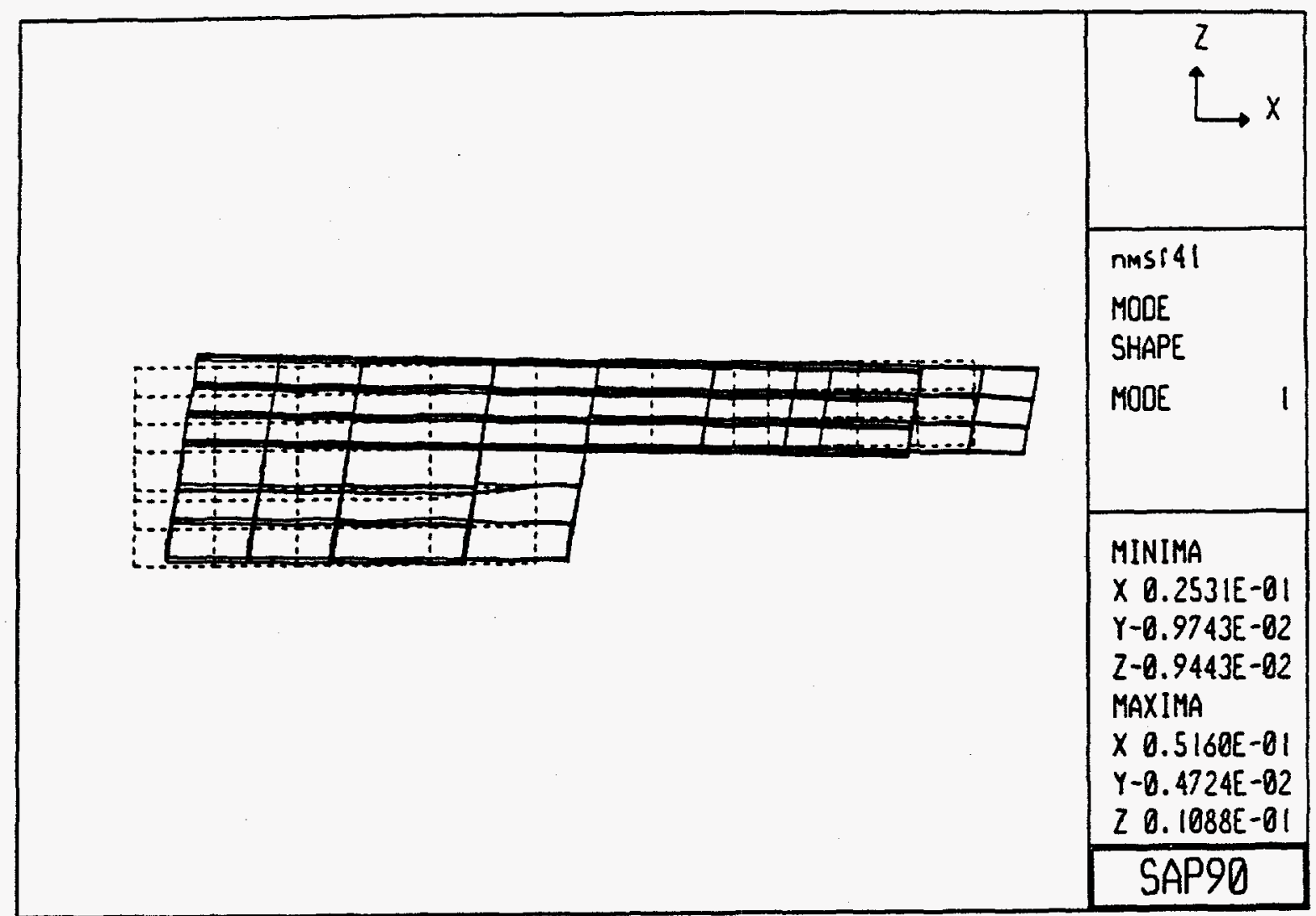

Figure 4.5 - Fundamental Mode of Vibration No. 1 As-Built Structure 


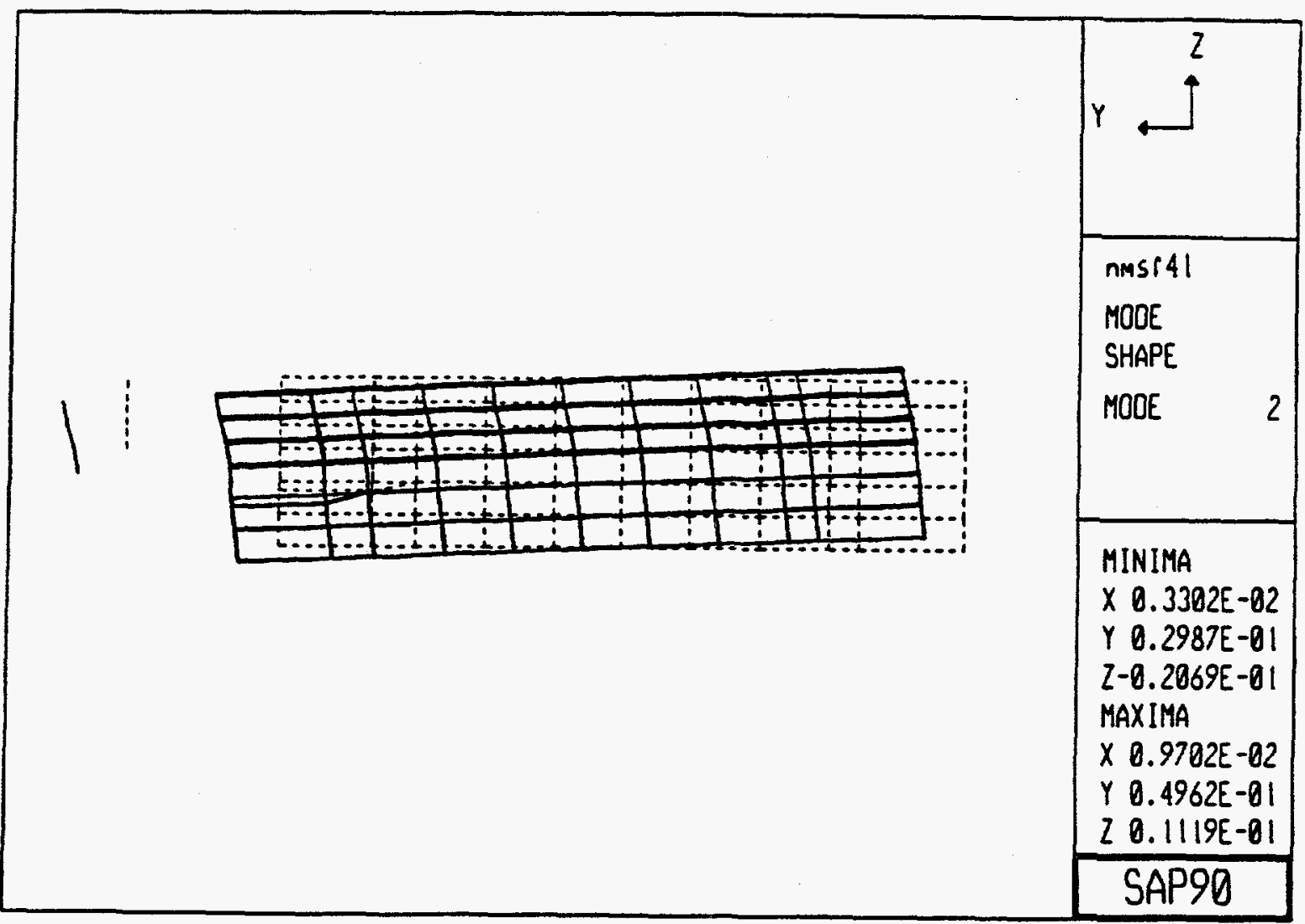

Figure 4.6 - Fundamental Mode of Vibration No. 2 As-Built Structure 
NMSF Conceptual Design Report

July 14, 1995

Page 47

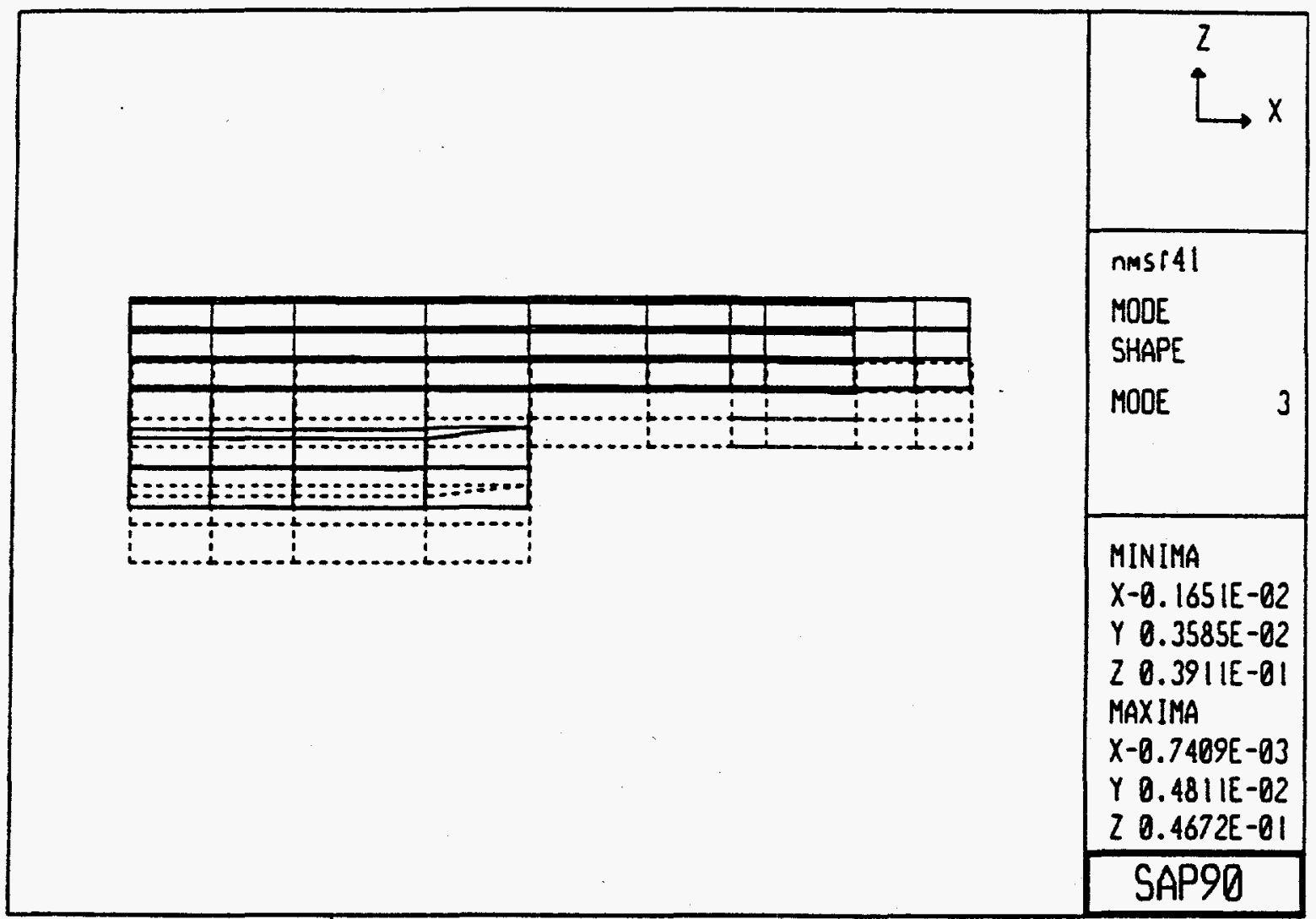

Figure 4.7 - Fundamental Mode of Vibration No. 3 As-Bullt Structure 
NMSF Conceptual Design Report

July 14, 1995

Page 48

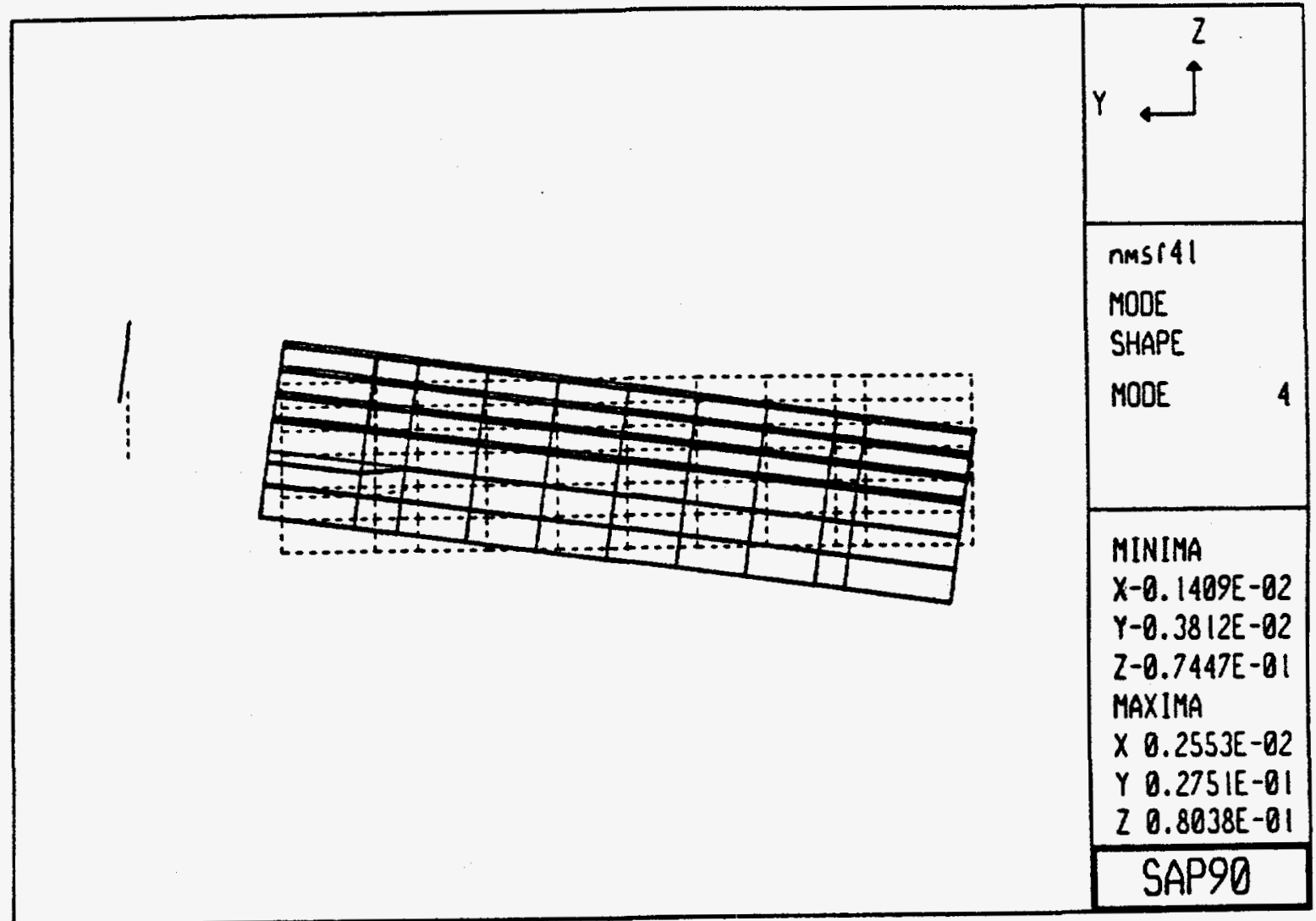

Figure 4.8 - Fundamental Mode of Vibration No. 4 As-Bullt Structure 


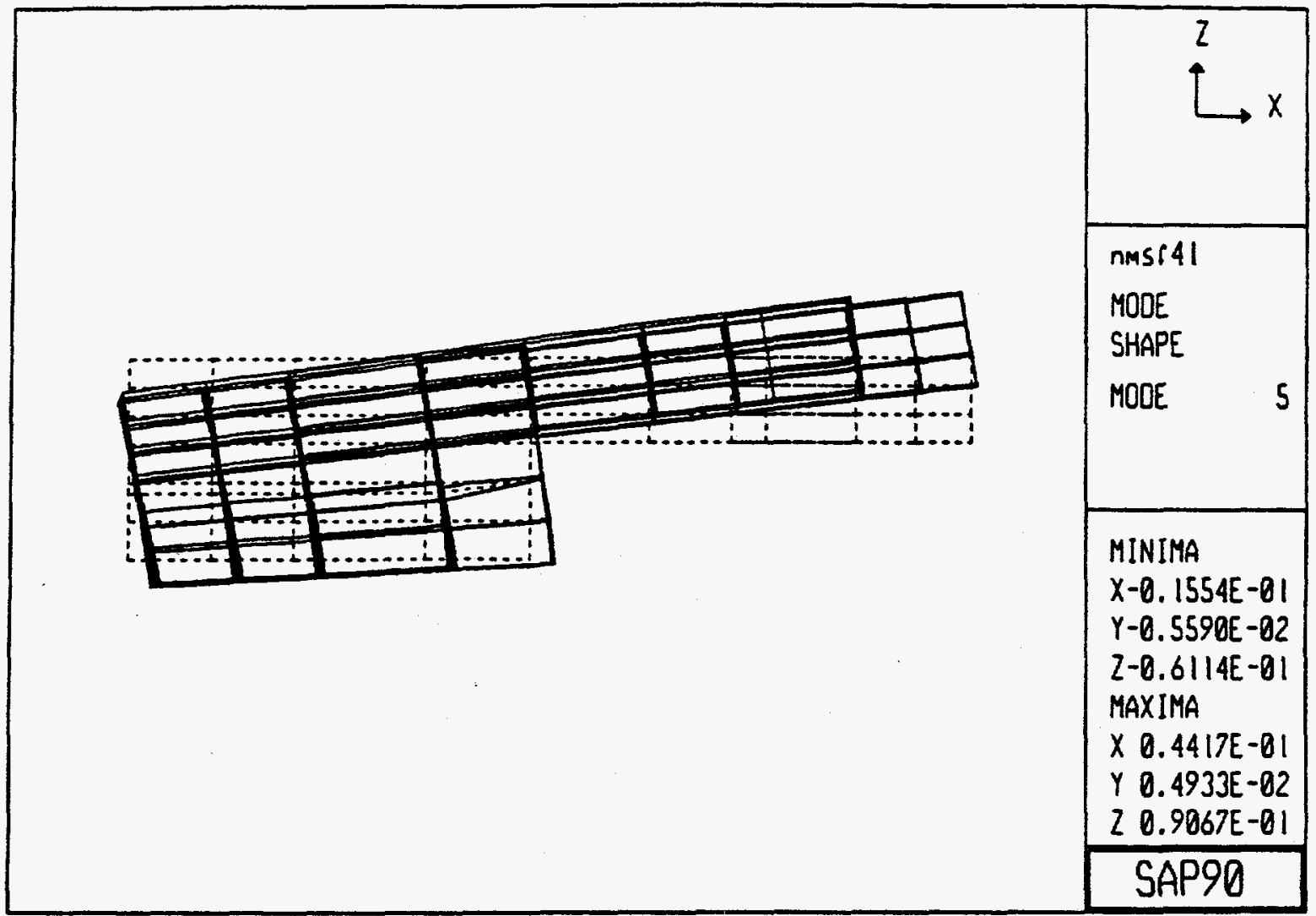

Figure 4.9 - Fundamental Mode of Vibration No. 5 As-Bullt Structure 


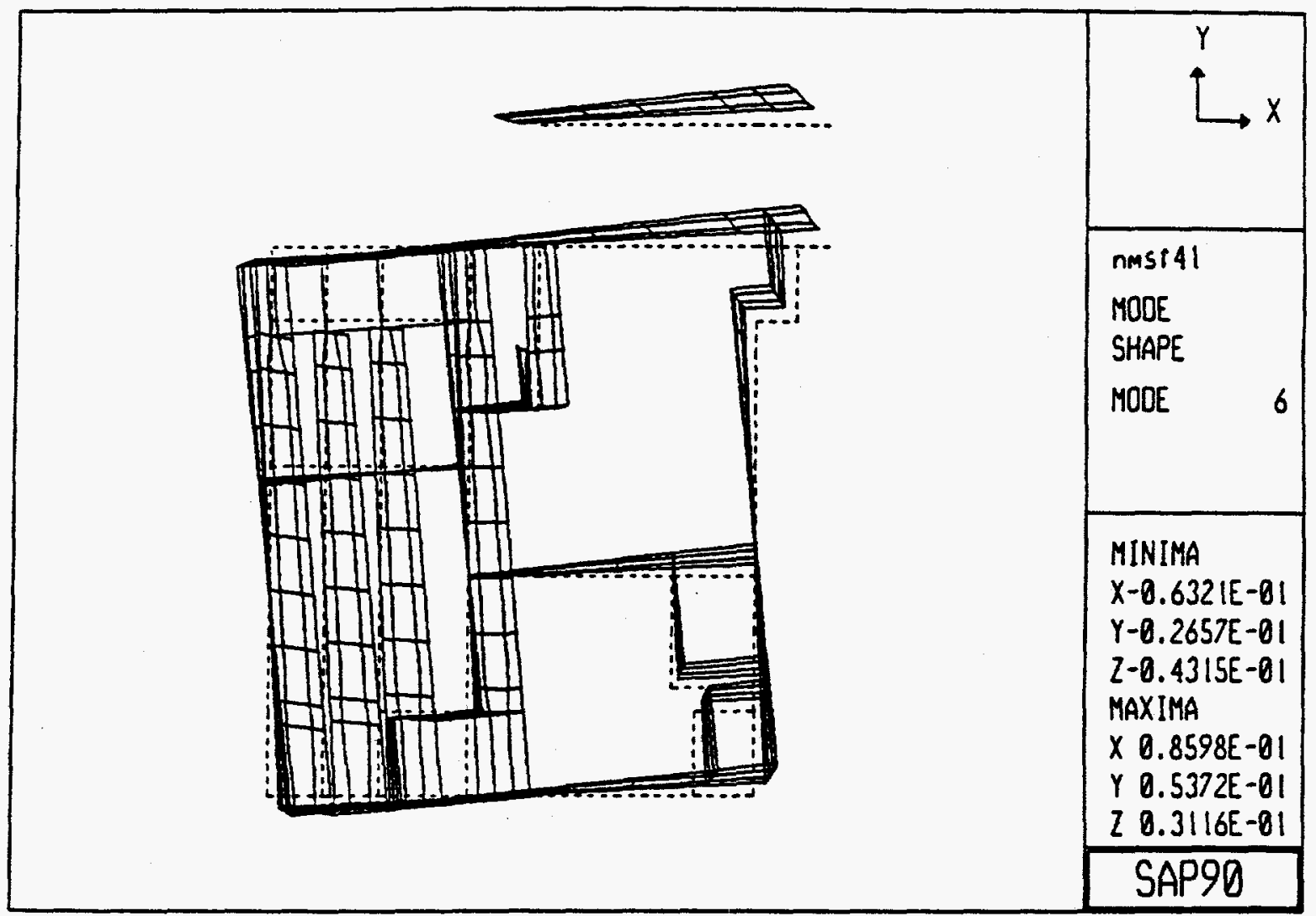

Figure 4.10 - Fundamental Mode of Vibration No. 6 As-Built Structure 


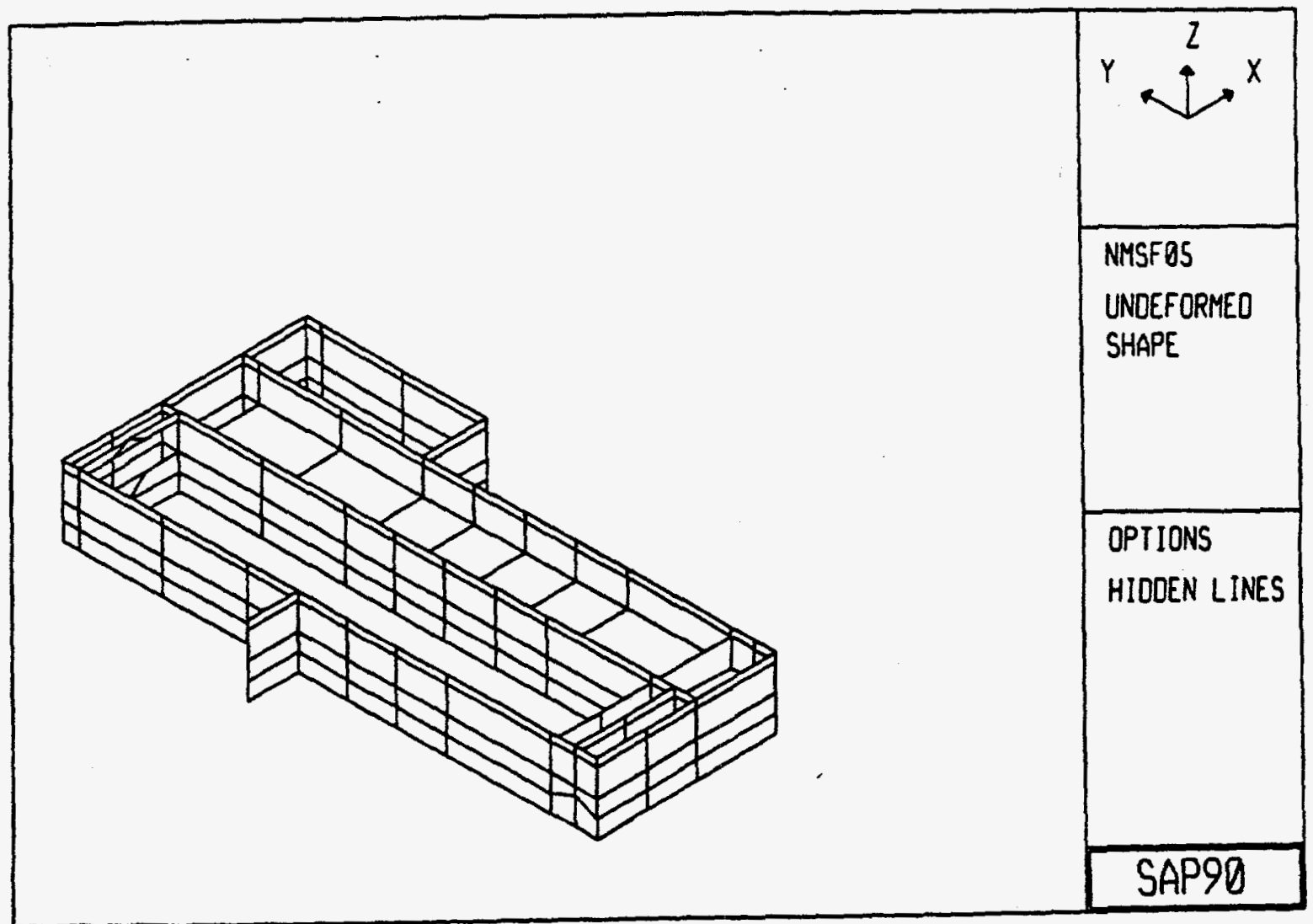

Figure 4.11 - NMSF Finite Element Model, Basement Walls Modifled Structure No. 1 


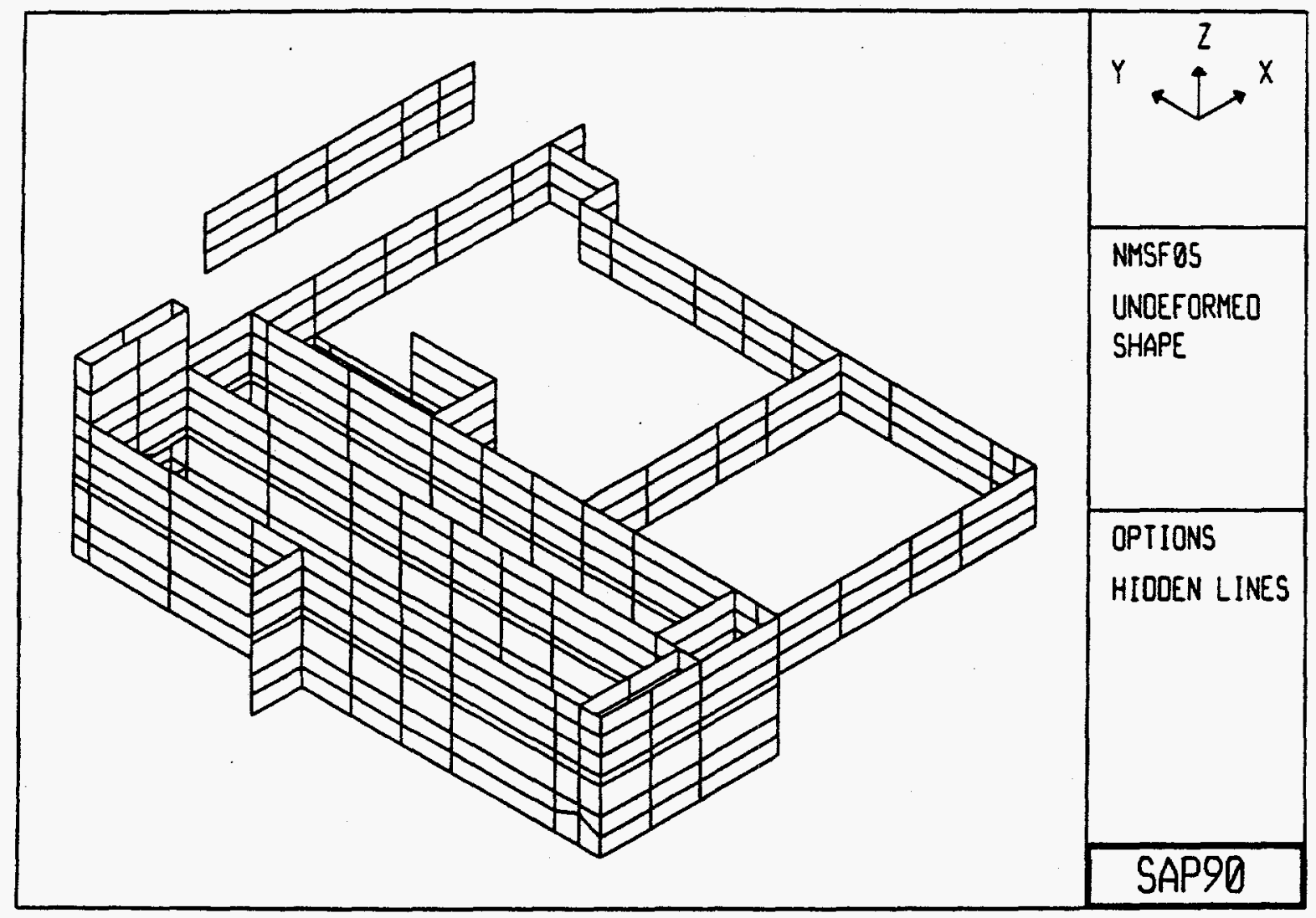

Figure 4.12 - NMSF Finite Element Model, First Floor Walls Modifled Structure No. 1 


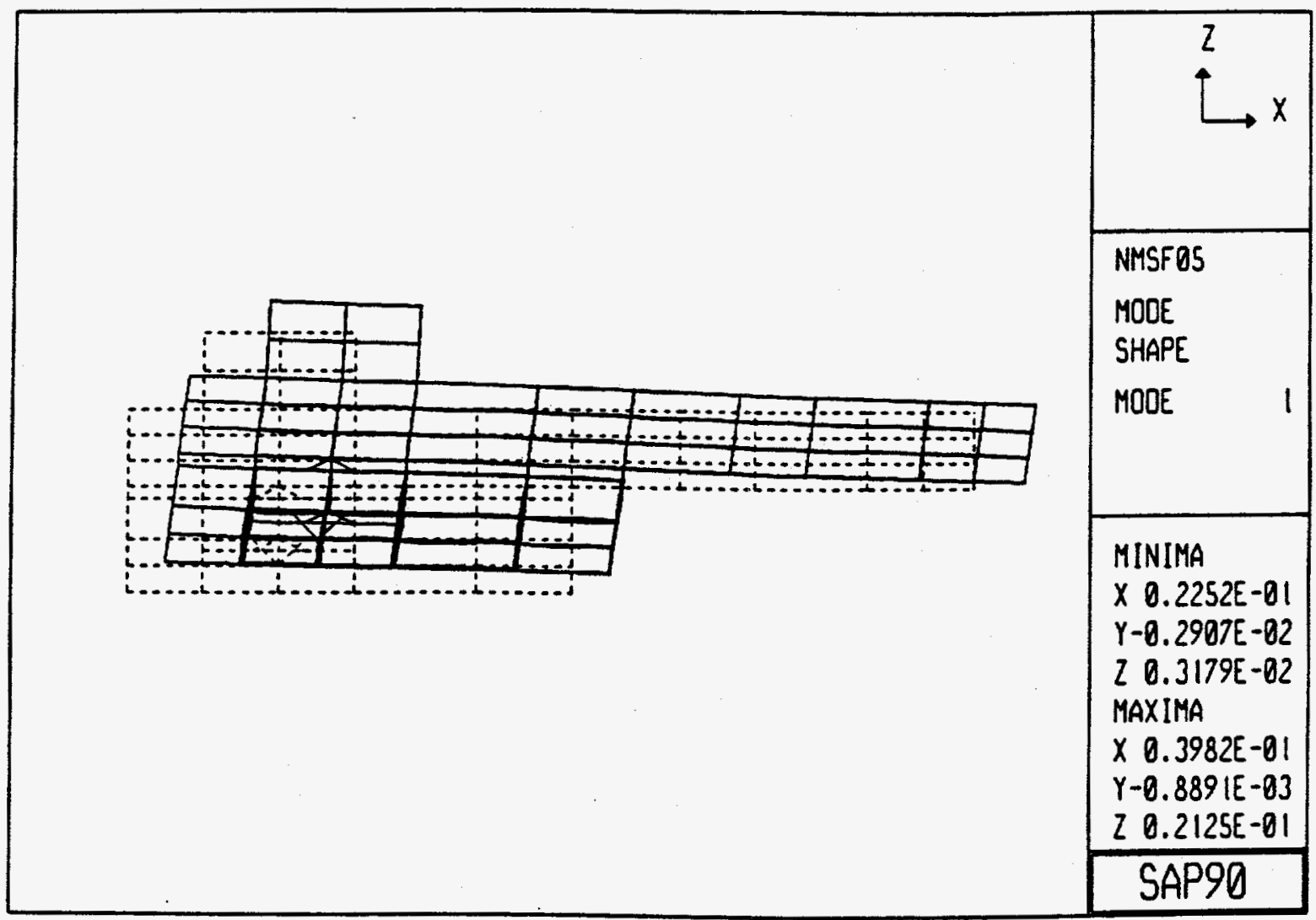

Figure 4.13 - Fundamental Mode of Vibration No. 1 Modifled Structure No. 1 
NMSF Conceptual Design Report

July 14, 1995

Pago 54

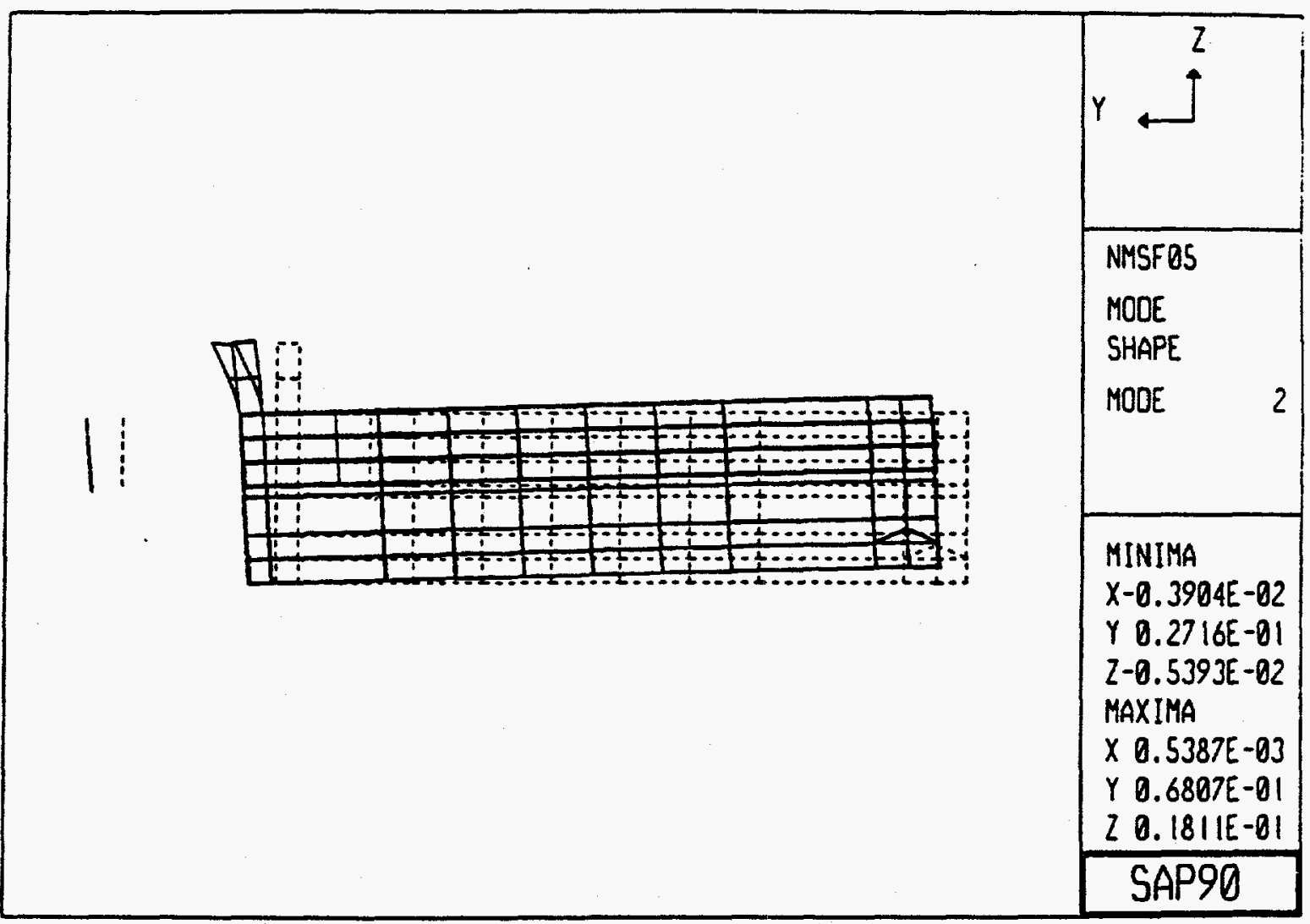

Figure 4.14 - Fundamental Mode of Vlbration No. 2 Modlfled Structure No. 1 


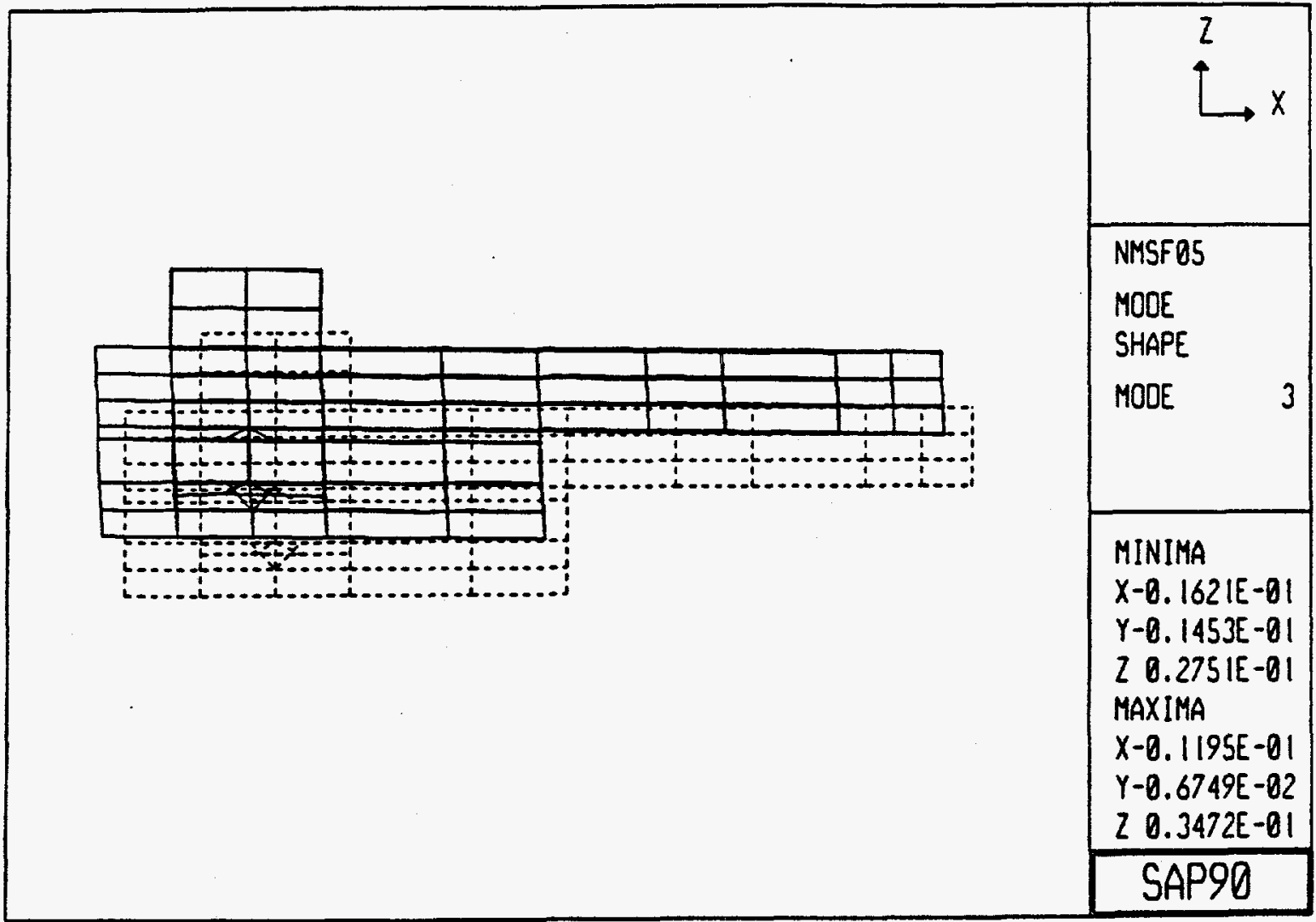

Figure 4.15 - Fundamental Mode of Vibration No. 3 Modified Structure No. 1 


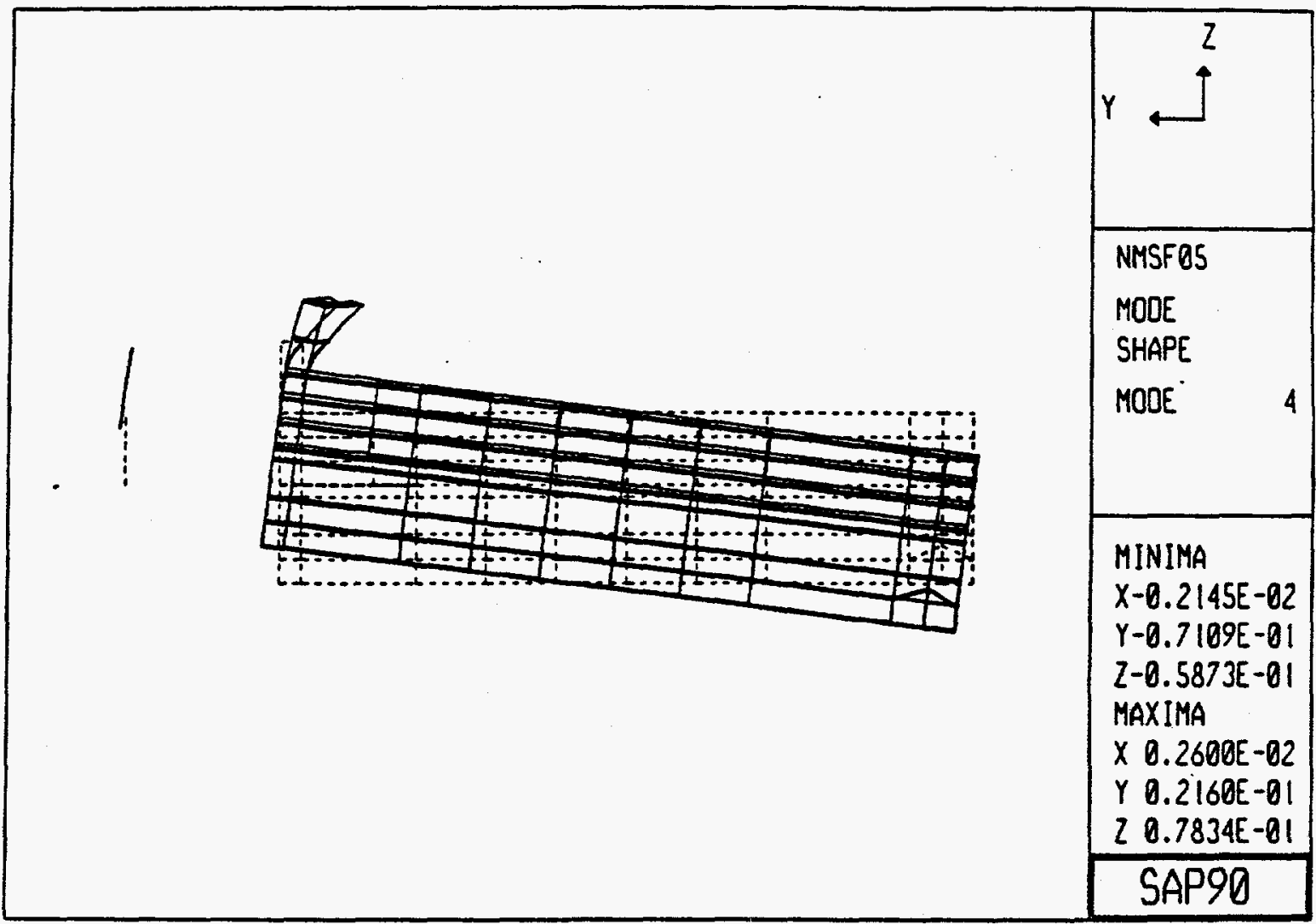

Figure 4.16 - Fundamental Mode of Vibration No. 4 Modifled Structure No. 1 


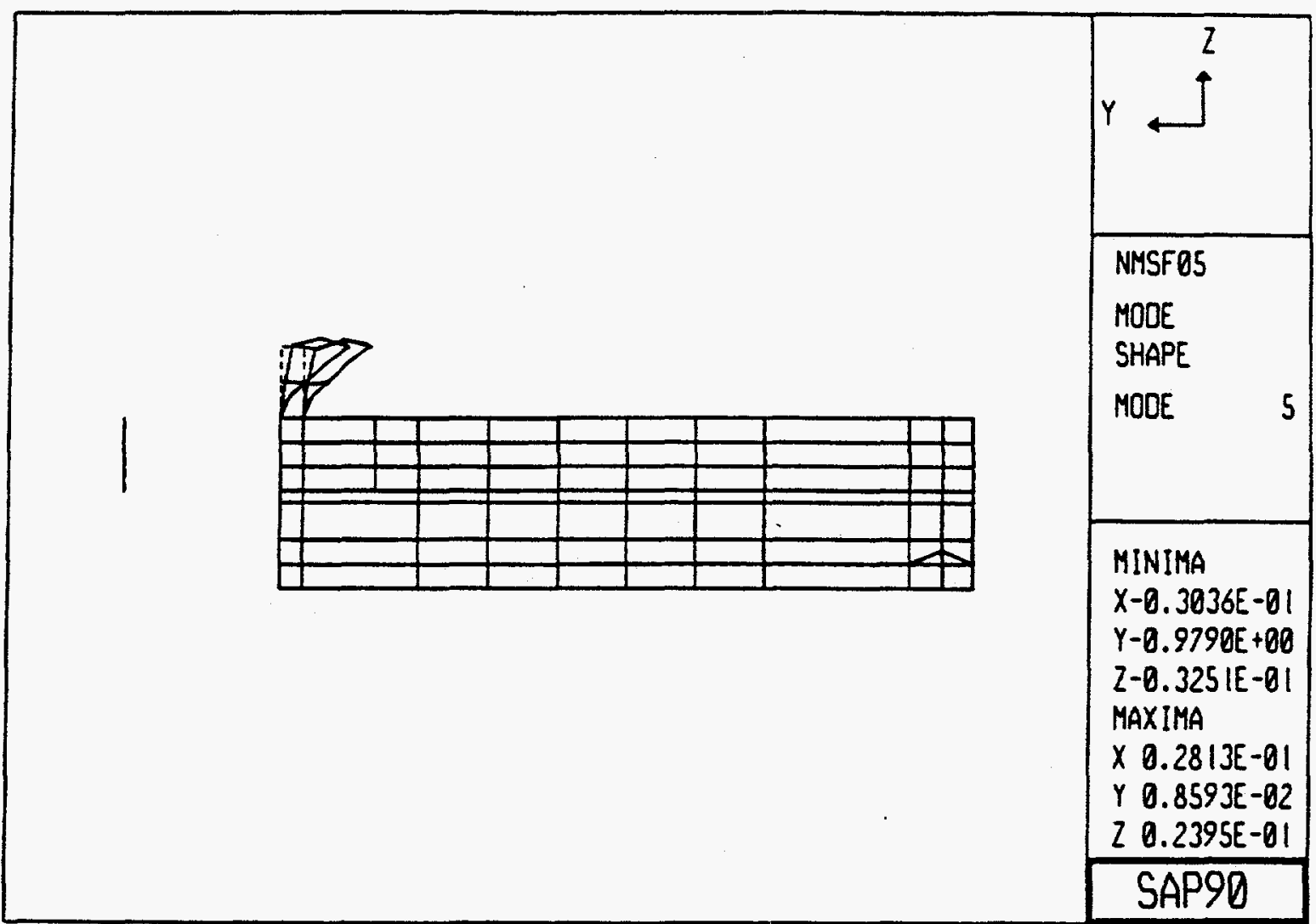

Figure 4.17 - Fundamental Mode of Vibration No. 5 Modifled Structure No. 1 


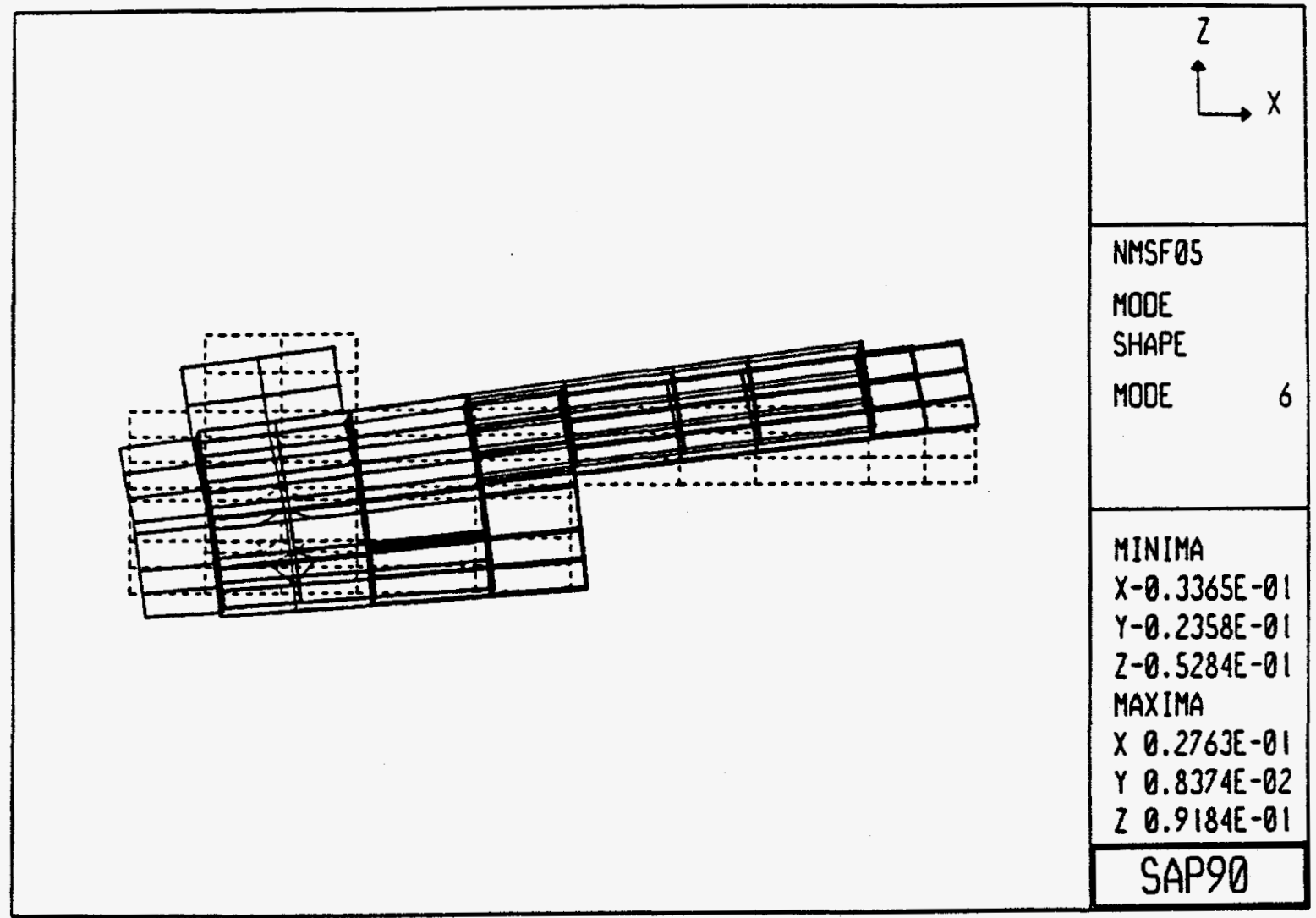

Figure 4.18 - Fundamental Mode of Vibration No. 6 Modified Structure No. 1 


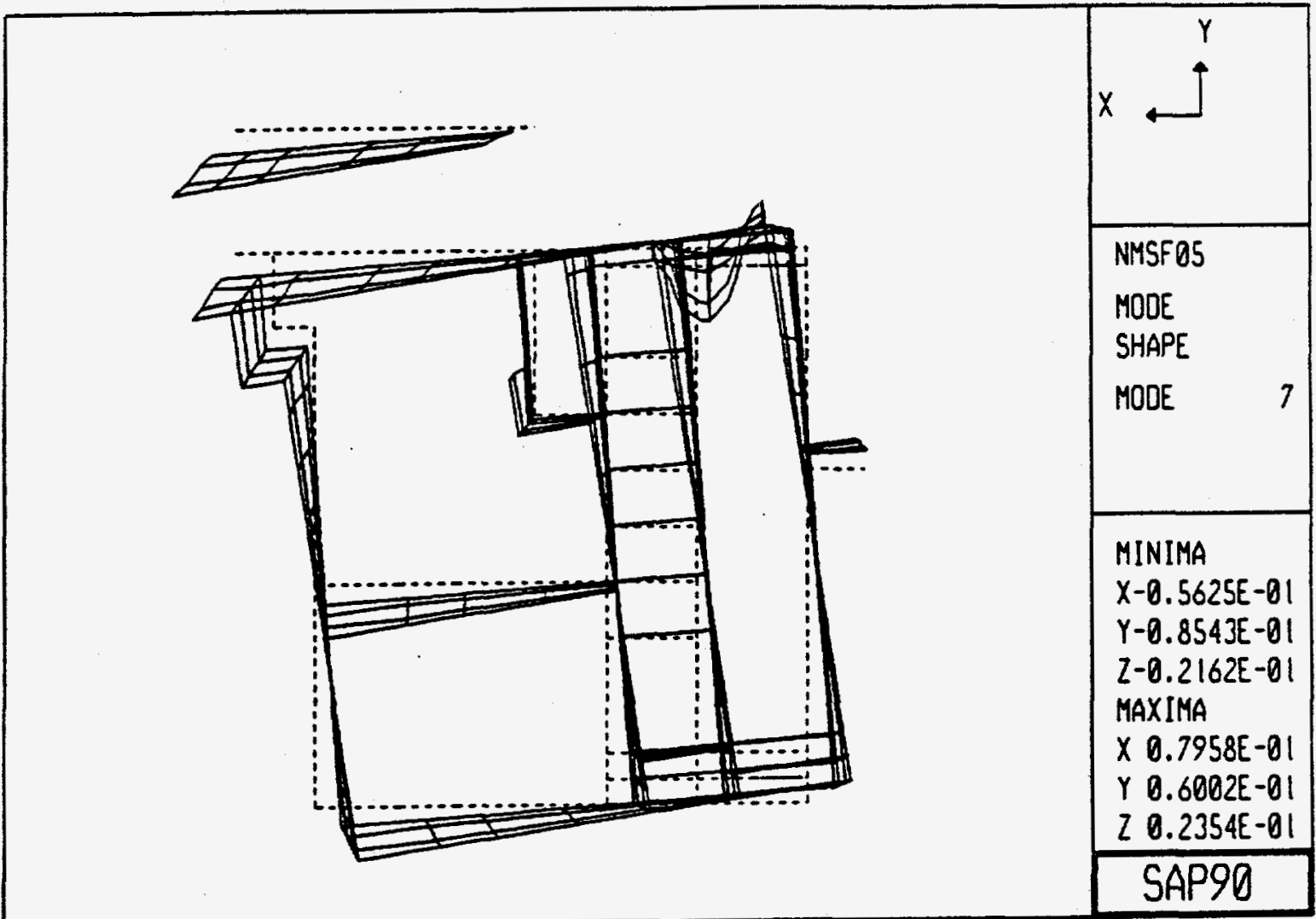

Figure 4.19 - Fundamental Mode of Vlbration No. 7 Modifled Structure No. 1 


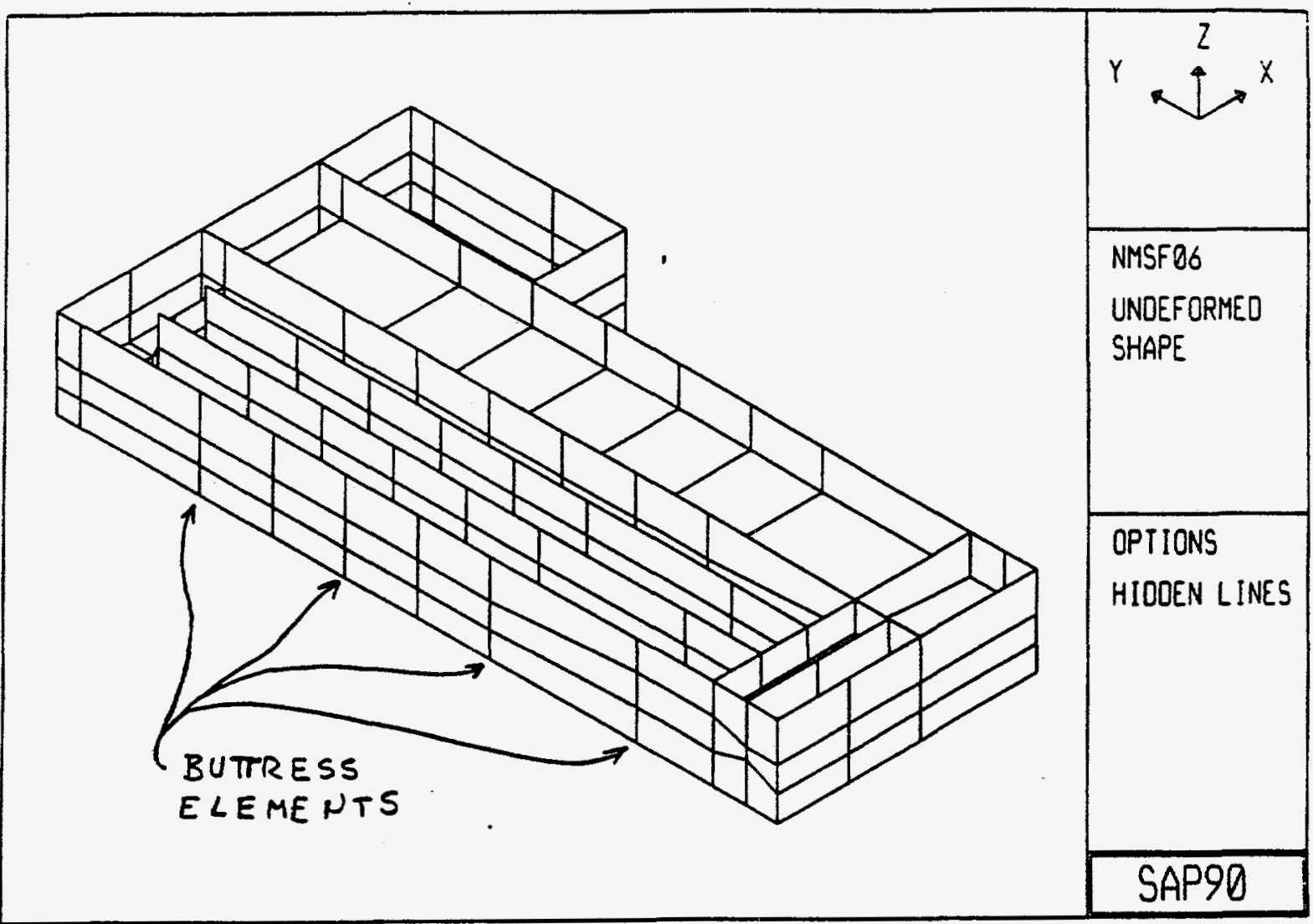

Figure 4.20 - NMSF Finite Element Model, Basement Walls Modifled Structure No. 2, 3-D Model 


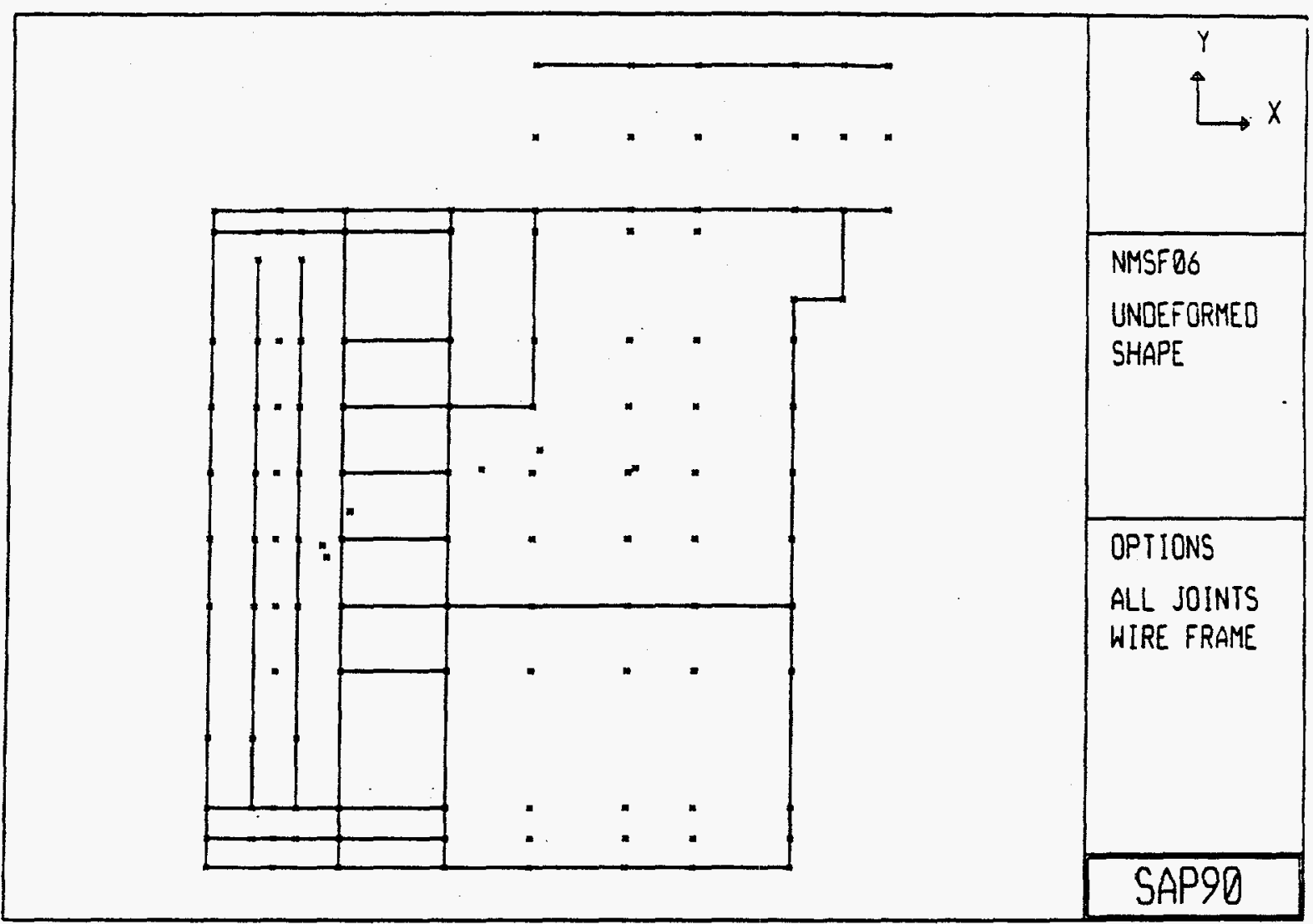

Figure 4.21 - NMSF Finite Element Model, First Floor Walls Modifled Structure No. 2, 3-D Model 


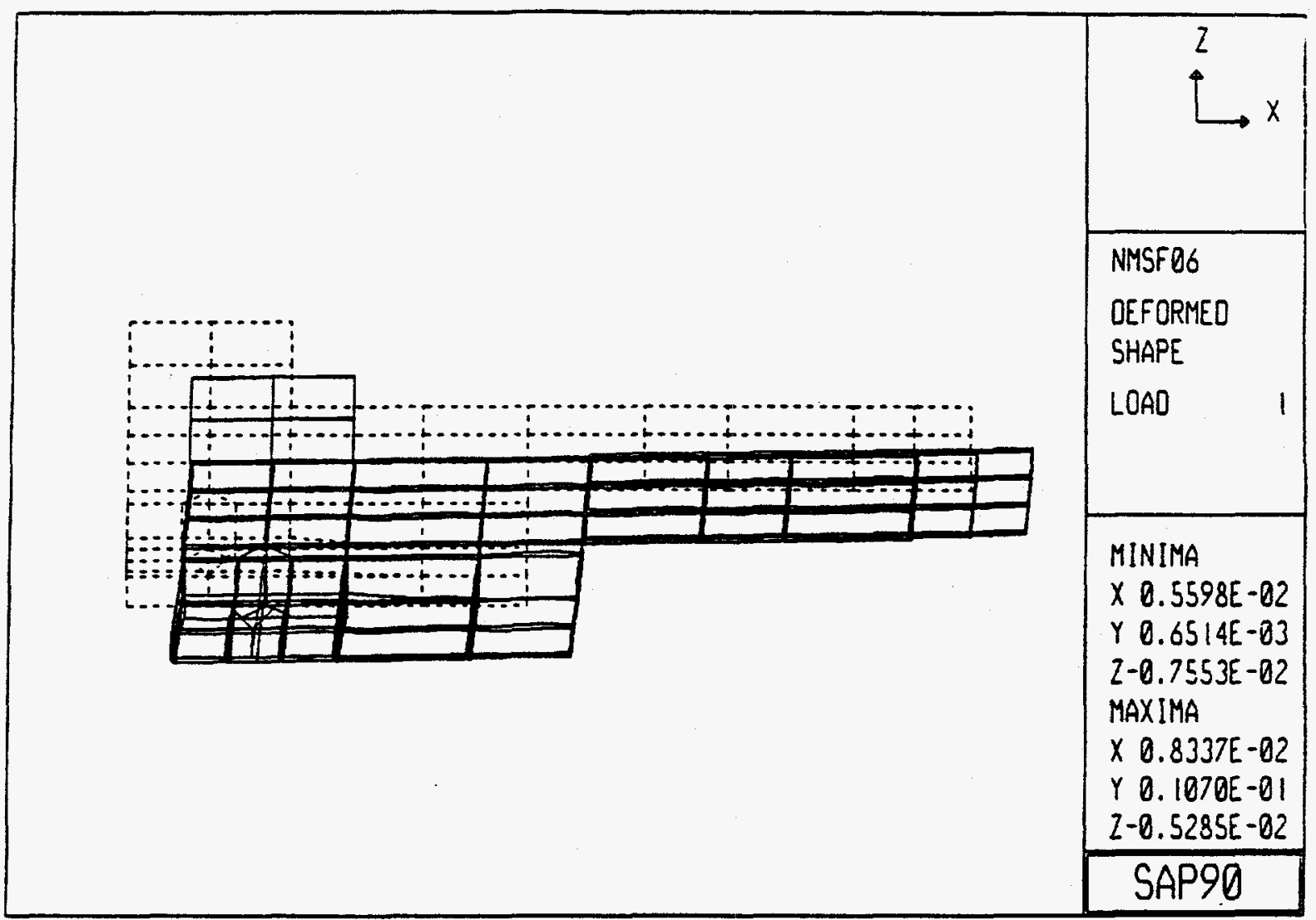

Figure 4.22 - Fundamental Mode of Vibration No. 1 Modifled Structure No. 2, 3-D Model 


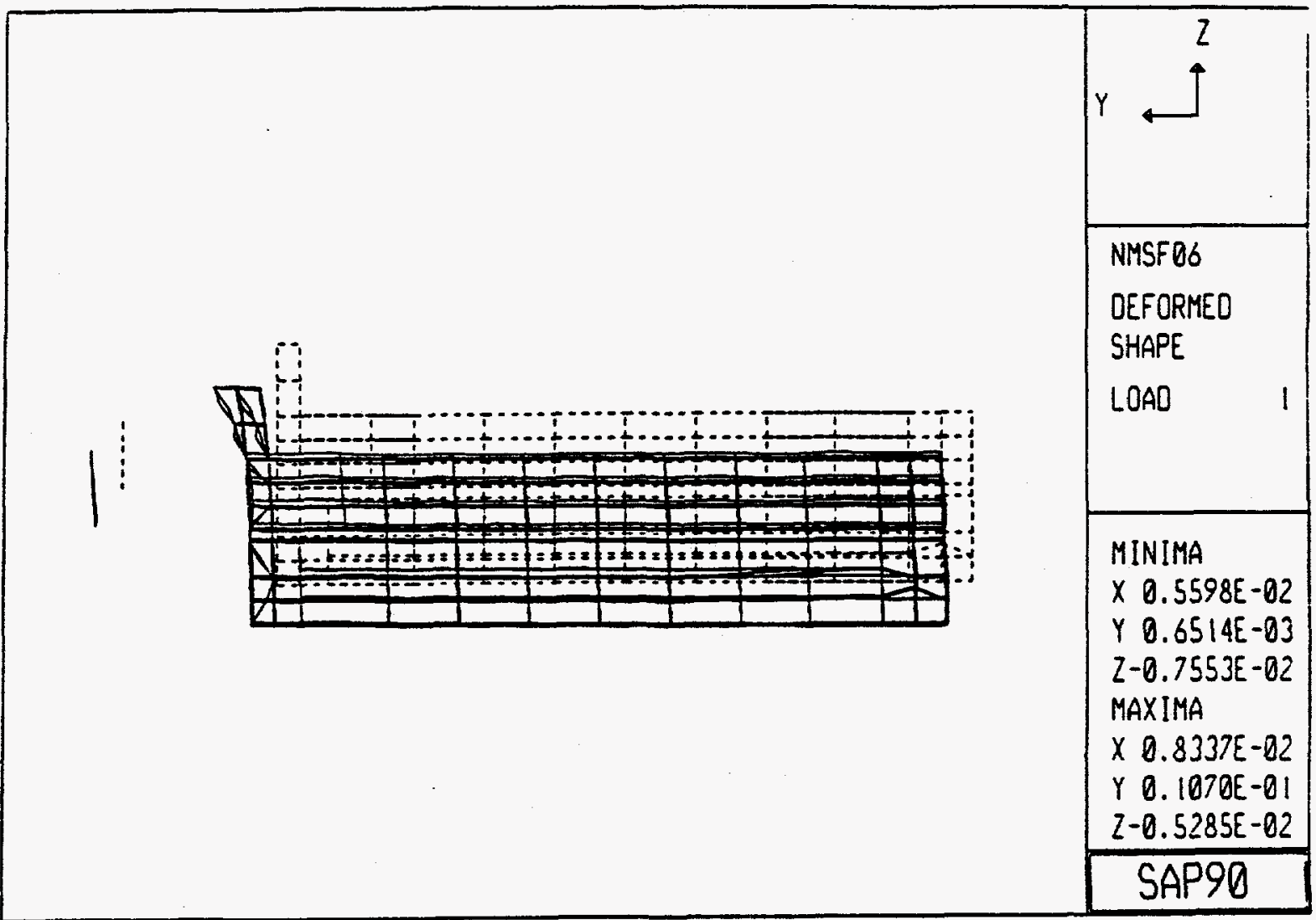

Figure 4.23 - Fundamental Mode of Vlbration No. 2 Modifled Structure No. 2, 3-D Model 


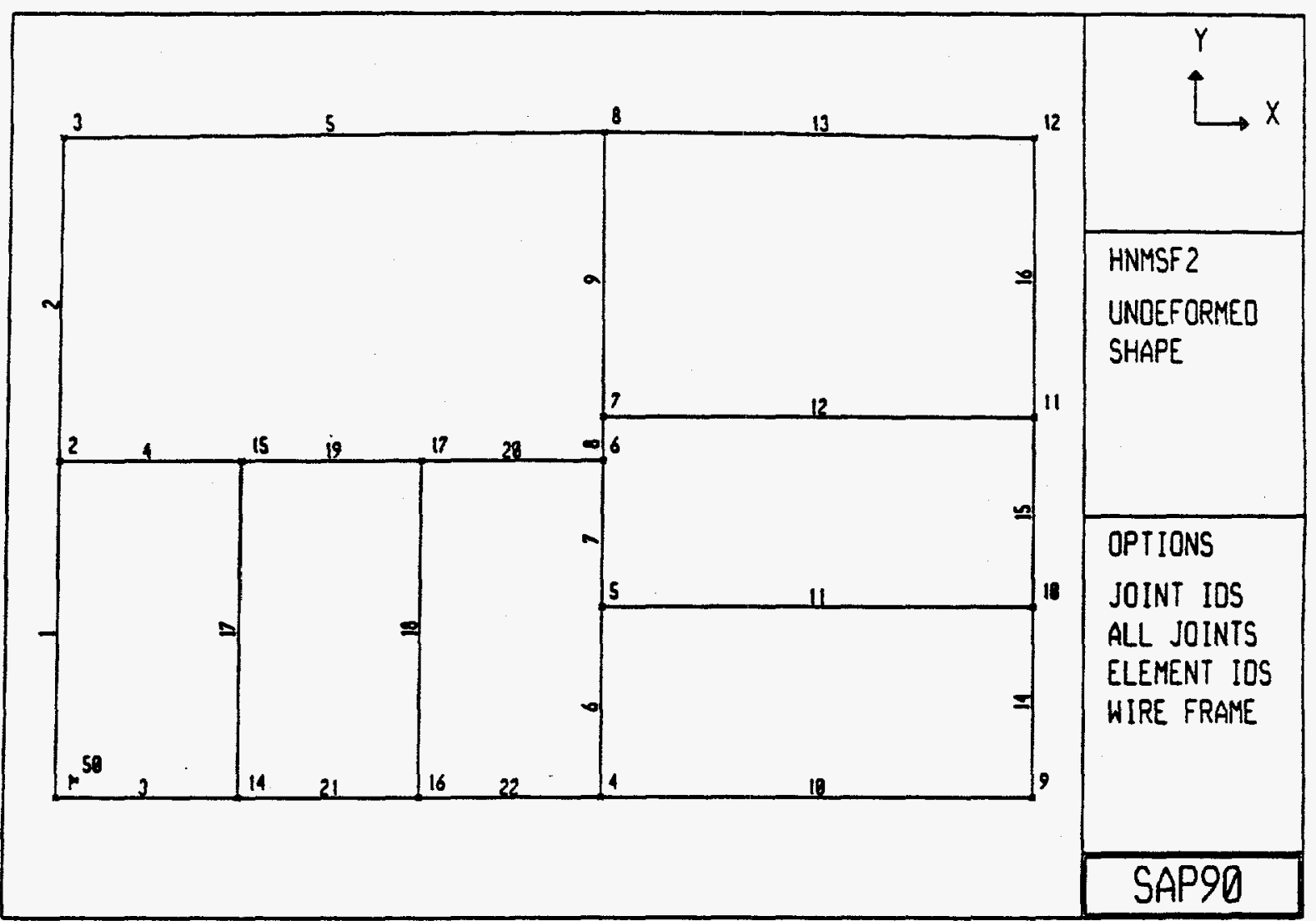

Figure 4.24 - NMSF 2-D Finite Element Model, Modffied Structure No. 2 


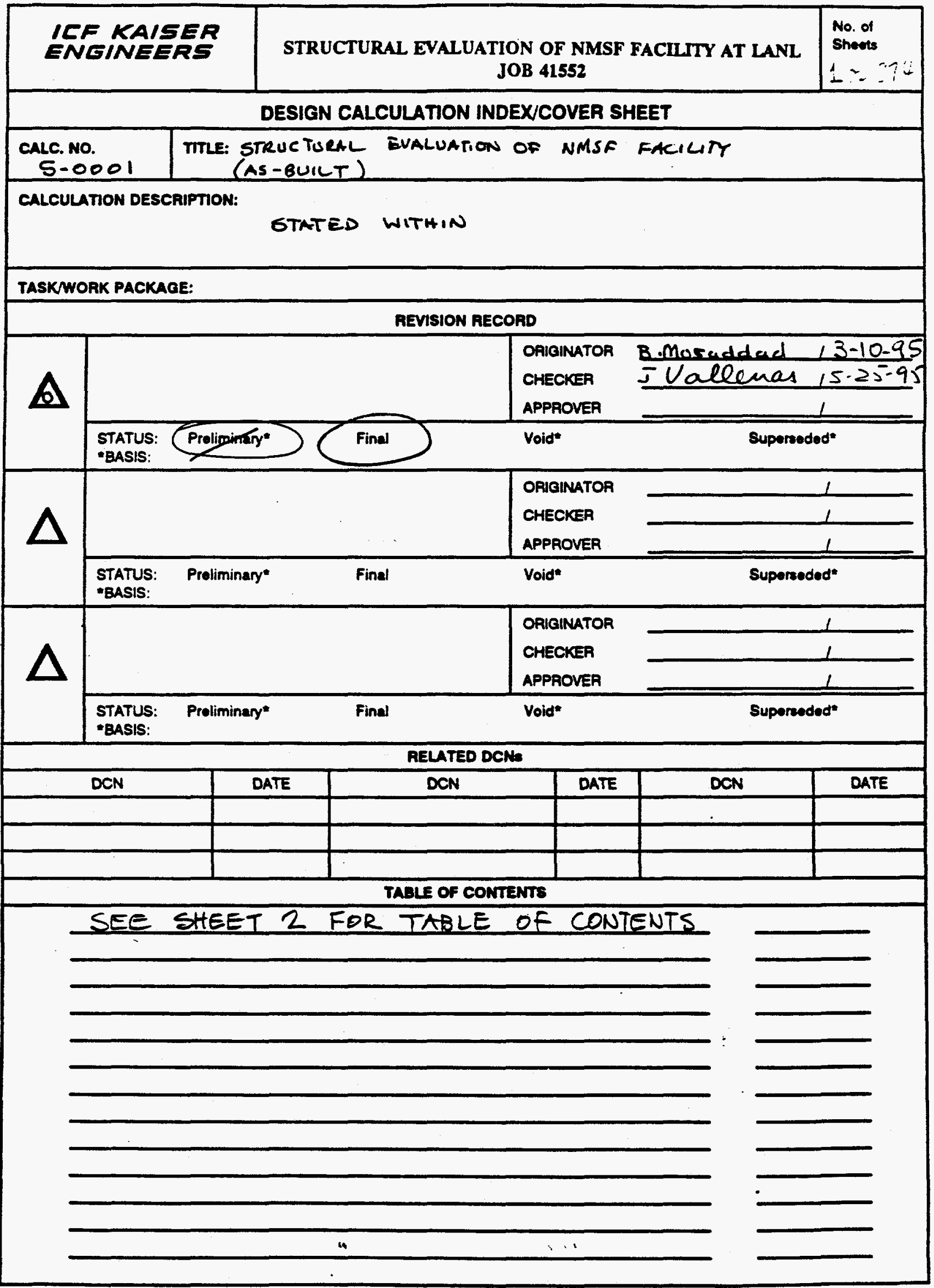

Form 3.3-1 


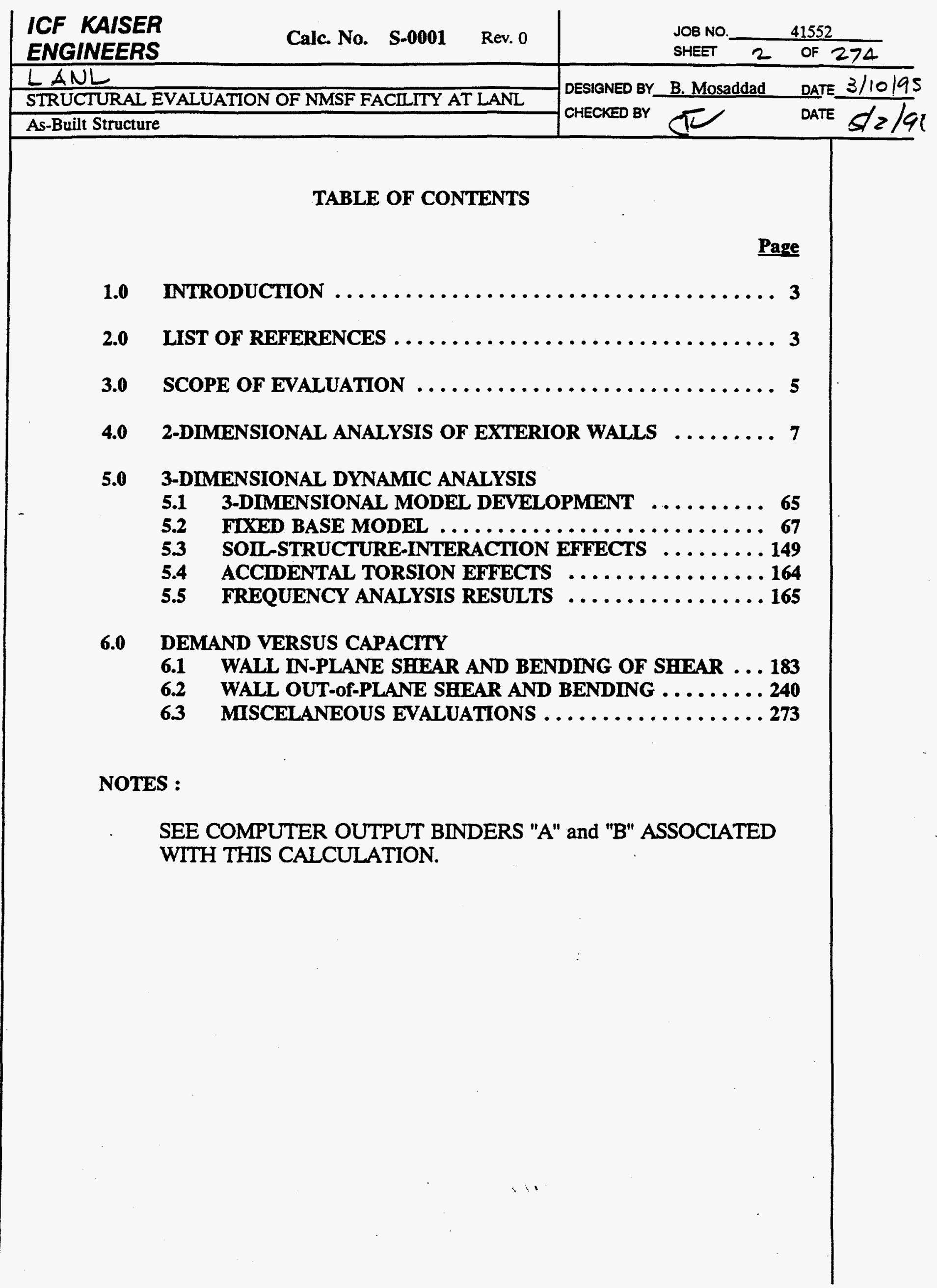




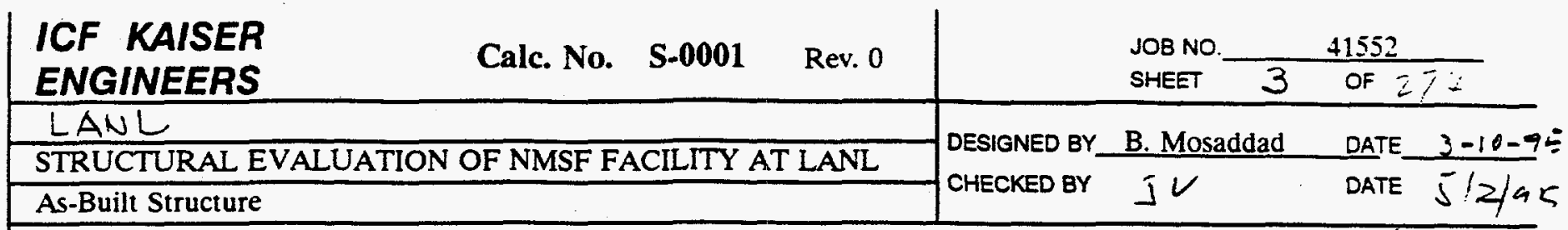

\subsection{INTRODUCTION}

The NMSF building consists of a two story storage area and a single story section including mechanical and administration area. The 1st floor of the storage area continues on grade to form the administration building, See figure 1. The basement of the storage building is buried in the soil. The 1st floor walls of the storage area is also completely embedded on east side, north side and south side of the building.

This calculation documents the details of the analysis procedure and structural evaluation of the As-built NMSF building in accordance with the LANL, DOE and associated industry standard design codes $(1,2,3,4)$. For a complete presentation of the evaluation criteria and presentation of the summary of evaluation results refer to the report titled:

Seinunic,

"Structural Evaluation of the NMSF Building at LANL"

\subsection{LIST OF REFERENCES}

(1) Los Alamos Facilities Engineering Standard- Structural, 1993

(2) "Natural Phenomena Hazards Design and Evaluation Criteria for Department of Energy Facilities", DOE Standard DOE-STD-1020-94, April 1994.

(3) "Minimum Design Loads for Buildings and Other Structures", ASCE Standard ASCE-7:-93

(4) Uniform Building Code (UBC), 1994 edition.

(5) Dames \& Moor Geotechnical report, 1972

(6) EPRI Report NP-6041-SL Rev 1.

(7) Soil Dynamics, By Shamsher Parkash

(8) "Seismic Analysis of Safety-Related Nuclear Structures and Commentary on Standard for Seismic Analysis of Safety Related Nuclear Structures", ASCE Standard ASCE-4-86, September 1986.

(9) Nuclear Regulatory Guide NUREG-098

(10) "A Series of Computer Programs for Static and Dynamic Finite Element Analysis of Structures", SAP90 User's Manual, Computers and 


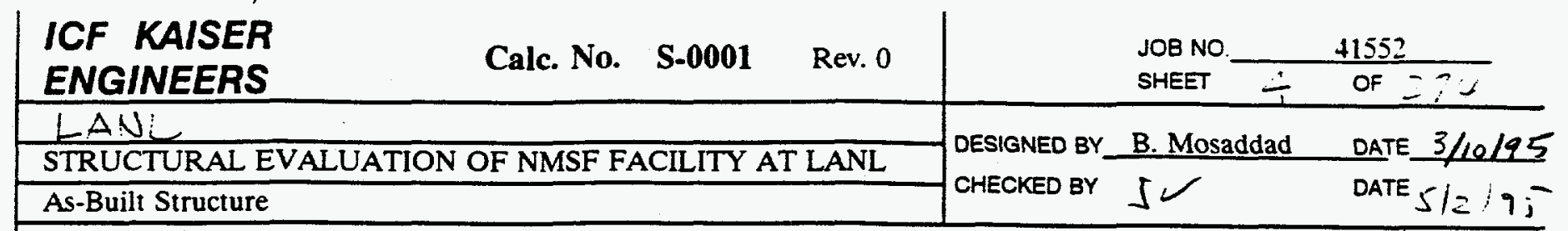

Structures, Inc., Berkeley, California.

(11) Soil-Structure-Interaction in Time Domain, by Wolf.

(12) Burns and Roe Drawings

DOE DRG. NO.: LA-ZJ-B.A.1 Through LA-ZJ-B.A.25 and

DOE DRG NO.: LA-ZJ-B.S.1 Through LA-ZJ-B.S.11

NOTE

a complete reference UBT cAN bE FOUND MI SECTION A OF part - preqiminlart report.

THE LBS PROVIDED HERE IS FOR INFORMATION ONLY AND COMPETE CTAATIOUS ARE NOT provides 


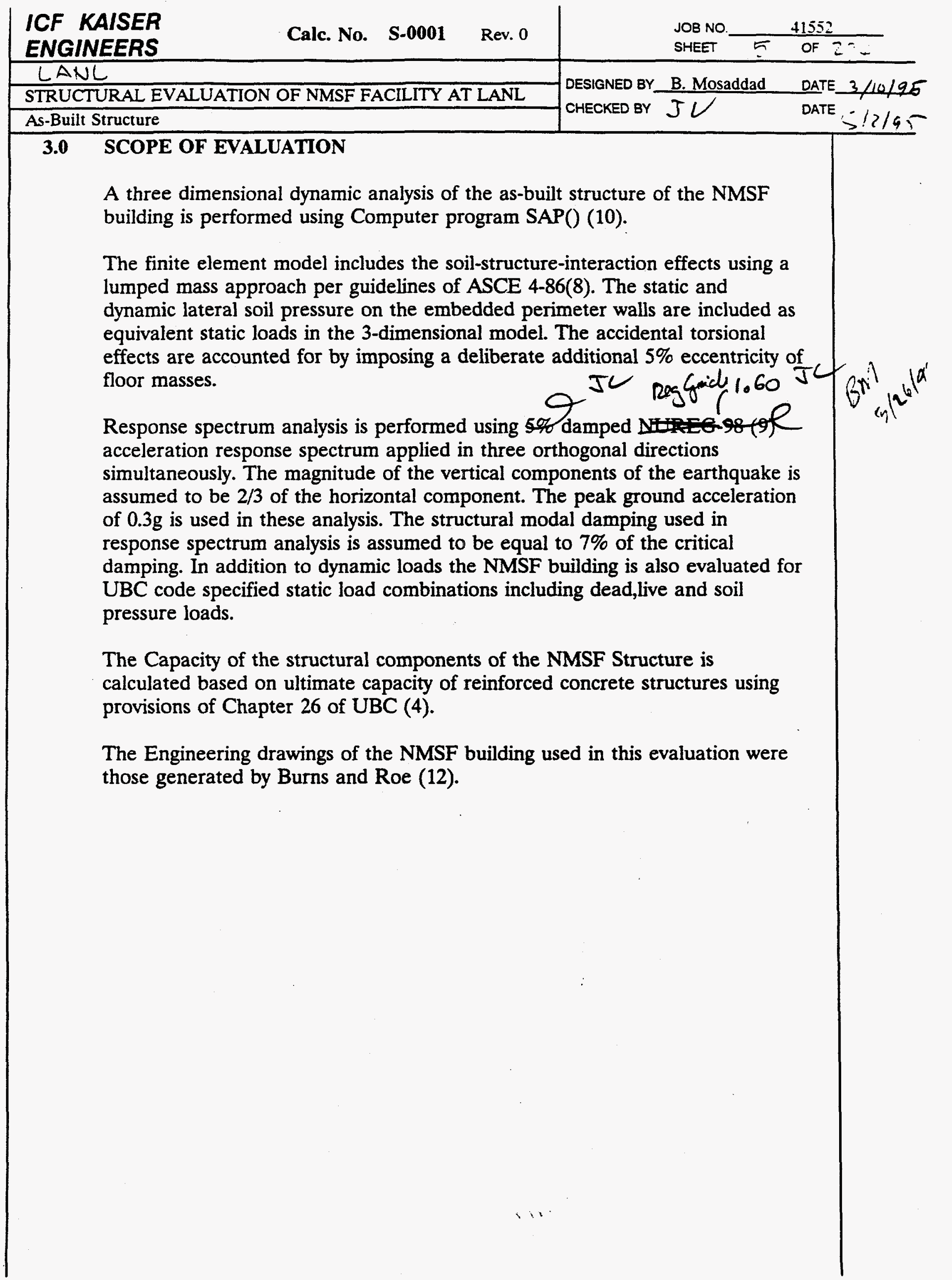




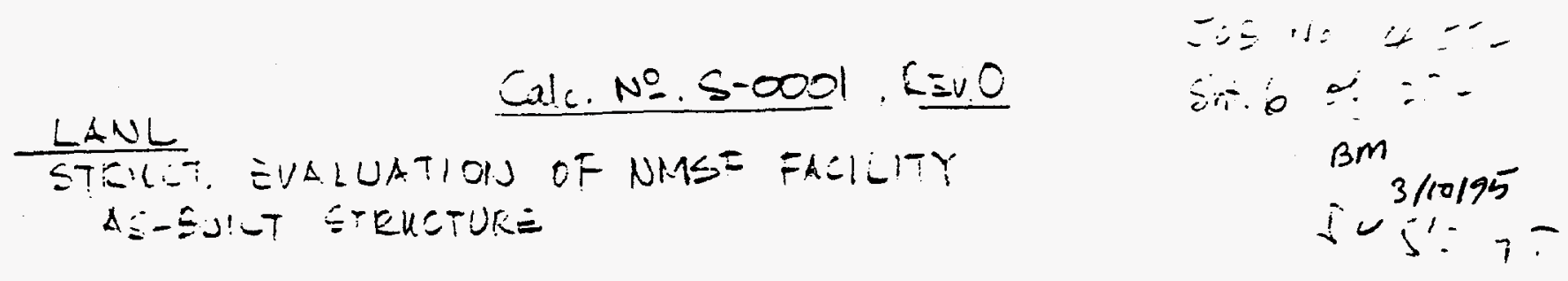

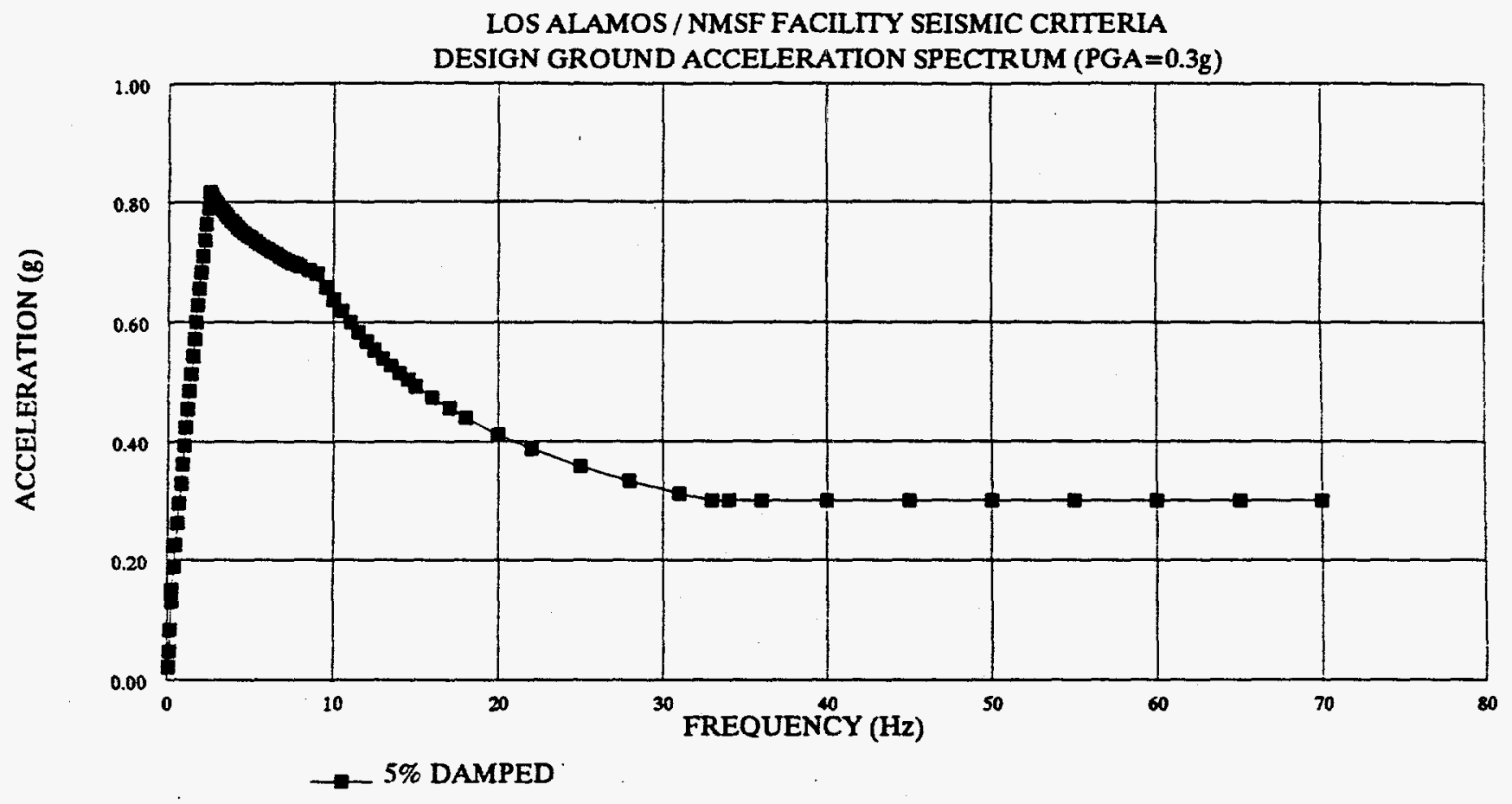




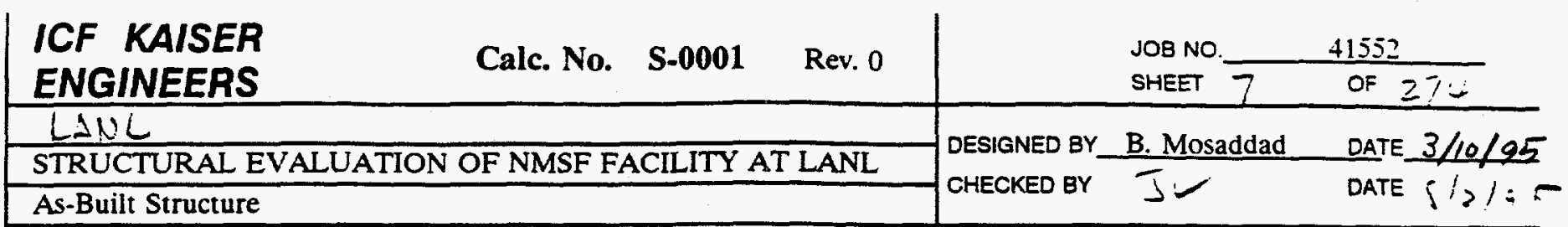

\subsection{2-DIMENSIONAL ANALYSIS OF EXTERIOR WALLS}

The purpose of this analysis was to perform a preliminary evaluation of the exterior walls of the NMSF building that are buried in the ground and backfilled with soil on for sides of the western portion of the building.

In this analysis a 1 foot slice of the walls are modeled using beam elements of Computer program SAP90. The loadings consist of static and dynamic soil pressures. The dynamic soil pressure is estimated using Mononobe-Okabe Formulas from reference provided by reference $(6,7,8)$ using a PGA of equal to $0.3 \mathrm{~g}$.

A summary of computed demands and capacities of different walls are tabulated on next page. The results indicate that the exterior walls have adequate strength to withstand the lateral soil pressure caused by the postulated DBE earthquake. 

KAISER (alc. No. $\div-000 /$ kew. 0
ENGINEERS
JOB NO. 41552-056-03 SHEET \& OF $27 \%$

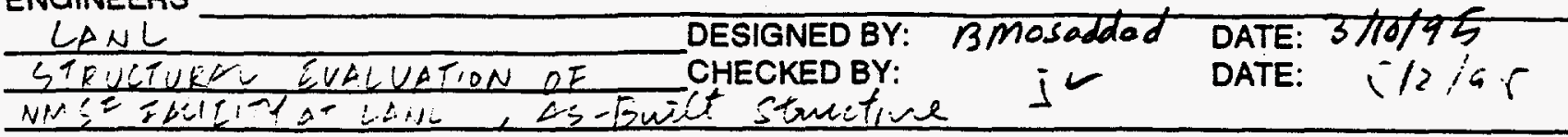

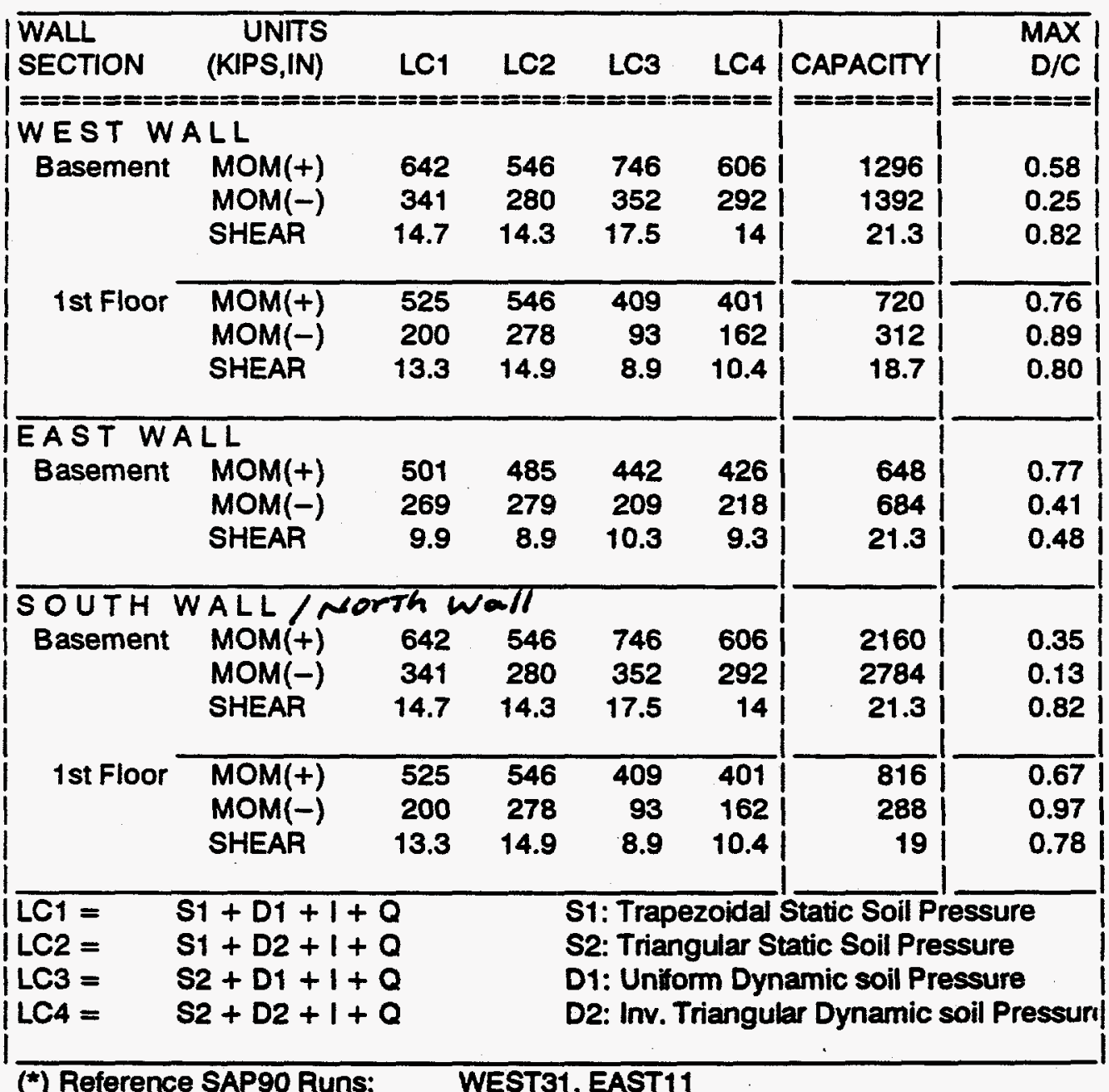




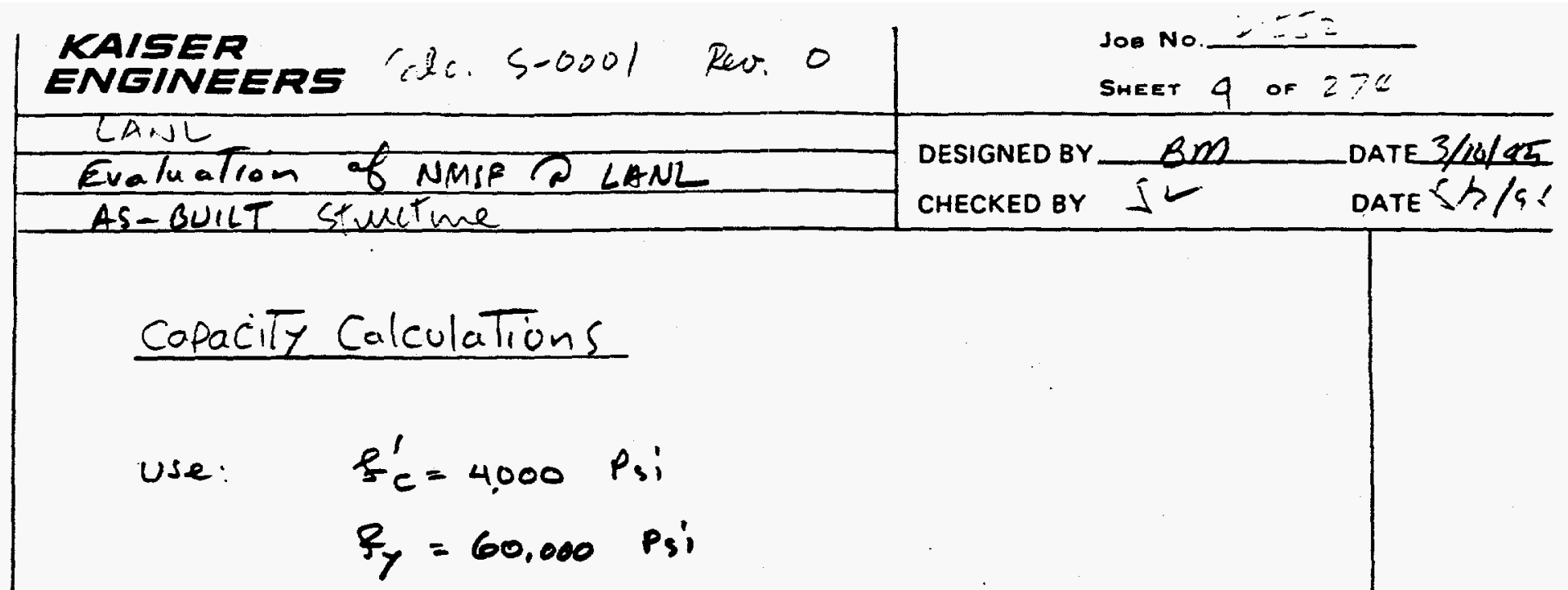

1) West wall, Basement.

Flexure:

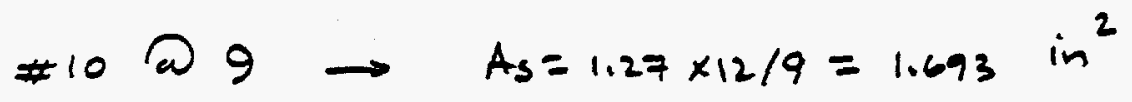

$$
\begin{aligned}
& b=12^{\prime \prime} \\
& \alpha=18-1.5=16.5^{\prime \prime} \\
& S=\frac{1.693}{(12 \times 16.5)}=.0086 \\
& a=A_{3} f_{7} / .185 B_{c}^{\prime} b \\
& =\frac{1.693 \times 60}{.85 \times 4 \times 12} \doteq 2.49 \\
& \phi=0.9 \rightarrow \\
& M_{u}=.90 \times 1.693 \times 60\left(16.5-\frac{2.49}{2}\right) \\
& =1395 \mathrm{Kin} / \mathrm{K} . \\
& V_{c}=2 \sqrt{f^{\prime}} \cdot b_{w} d-1 \\
& =2 \sqrt{4000} \times 12 \times 16.5 \\
& =25 \mathrm{kipl} / \mathrm{L} . \mathrm{F} \text {. } \\
& V_{v}=.85 \times 25=21.3^{k}
\end{aligned}
$$

shear: 


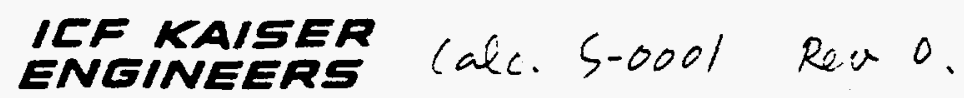

Sos No. $->5=$

LANL EVALUATION $Q$ N NMSF FAULIYAT LAOL Ac- Built Stuotans

DESIGNED BY

SMEET 10 OF $27 \%$

WESI WALL

REF : DOE DRG NO.

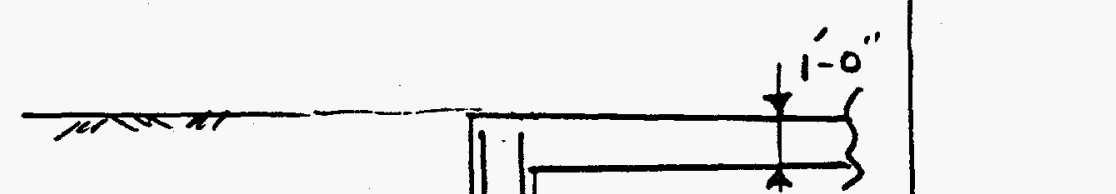
$\angle A-2 J-B .5 .8 .1$ DATE $3 / 10 / 9$ CHECKED BY IV DATE IS:S:
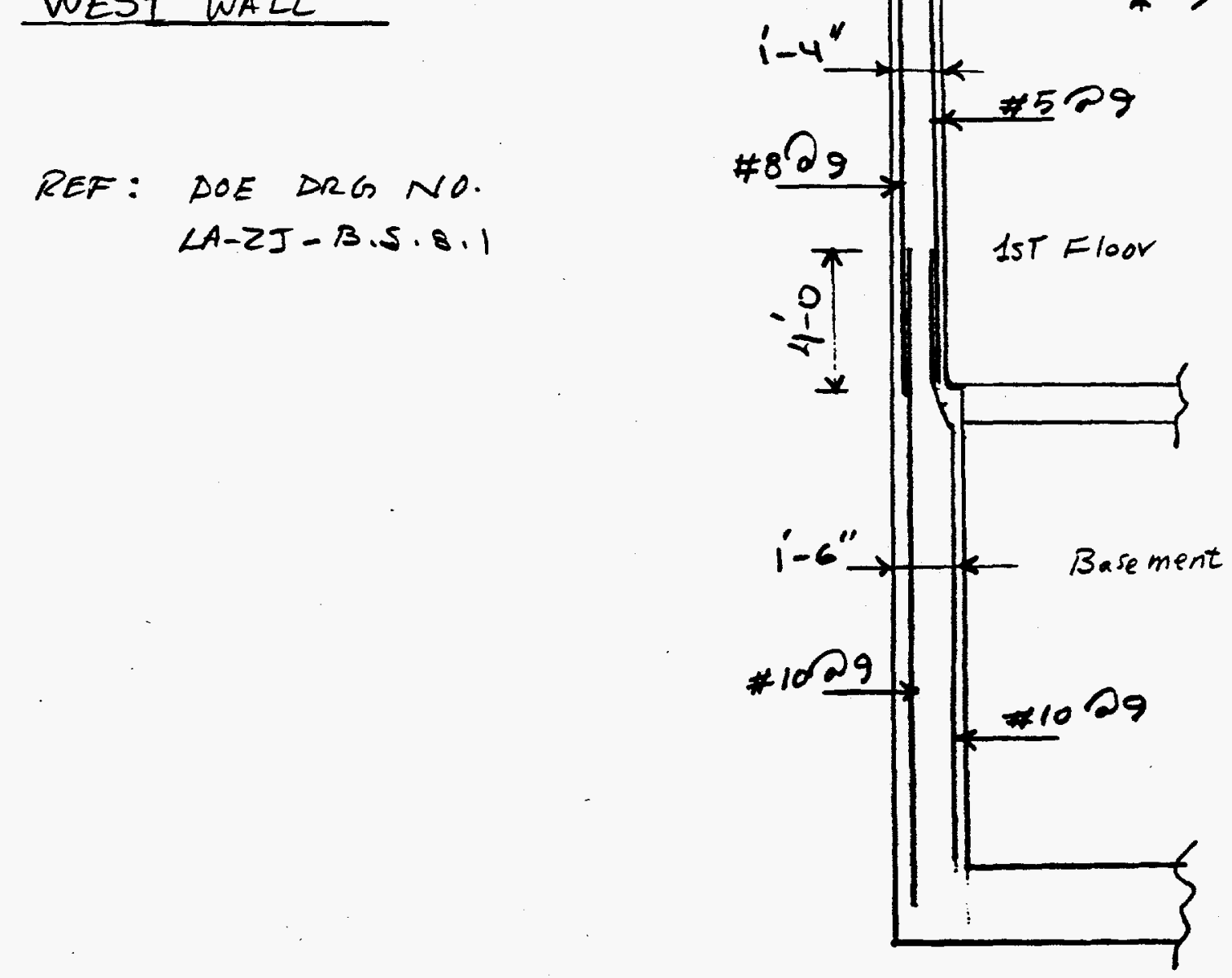

$2=:$ 


\begin{tabular}{|c|c|c|c|}
\hline $\begin{array}{l}\text { KAISER } \\
\text { ENGINEERS }\end{array}$ & Calc. S-0001 Rev. 0 & & No. $\frac{1115=2}{11 \text { or } 274}$ \\
\hline $\begin{array}{l}\text { LANL } \\
\text { STRUCTURAL EUAL } \\
\text { LAUL AO - Bu }\end{array}$ & $10 \mathrm{~N}$ OF NMSF FE & $\begin{array}{l}\text { DESIGNED BY } \beta M \\
\text { CHECKED BY } \bar{T}\end{array}$ & DATE $3 / 10 / 25$ \\
\hline
\end{tabular}

EAST WALL

REF: $\begin{aligned} & \triangle O E \text { DRG } M O \\ & \angle A-Z J-B-S-8.1\end{aligned}$

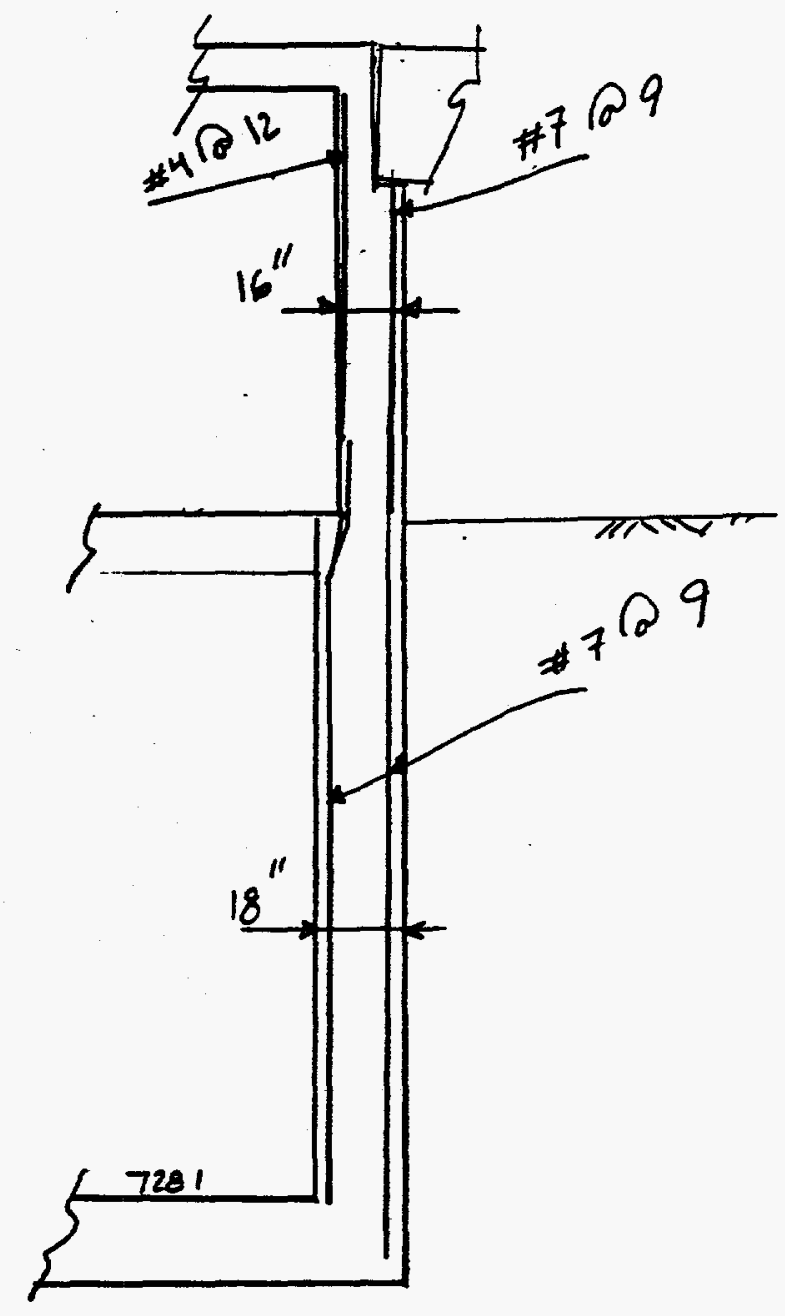

$\therefore$ 
KAISER

(alc. $4-0001$, Rev. 0

JOB NO.

41552-056-03

SHEET $1 \equiv$ OF $27 i$

ENGINEERS

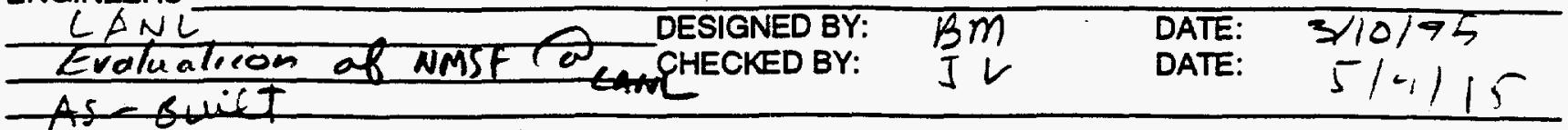

SUMMARY OF WALL CAPACITY CALCULATIONS

(AS BUILT)

\begin{tabular}{|c|c|c|c|c|c|c|}
\hline$\left\{\begin{array}{l}\overline{\text { WALL }} \\
\text { SECTION } \\
=============== \\
\text { WEST WALL } \\
\text { Basement } \\
\end{array}\right.$ & $\begin{array}{l}\text { Rebar } \\
===== \\
\# 10 @ 9 \\
\# 10 @ 9\end{array}$ & $\begin{array}{r}\text { UNITS: } \\
\text { AS } \\
=====\end{array}$ & $===$ & $\begin{array}{l}2.49 \\
2.49\end{array}$ & $\begin{array}{l}\text { CAPACITY } \\
\text { U: (K,FT) } \\
======\end{array}$ & $\begin{array}{l}\text { Comments } \\
=========\end{array}$ \\
\hline $\begin{array}{rr}\text { 1st Floor } & \mathrm{Mu}(+) \\
\mathrm{Mu}(-) \\
\mathrm{Vc}\end{array}$ & $\begin{array}{l}\text { \#8@9 } \\
\text { \#5@9 }\end{array}$ & $\begin{array}{l}1.053 \\
0.413\end{array}$ & $\begin{array}{l}13.5 \\
14.5 \\
14.5\end{array}$ & $\begin{array}{l}1.55 \\
0.61\end{array}$ & $\begin{array}{r}60 \\
26 \\
18.7\end{array}$ & \\
\hline \begin{tabular}{|ll} 
EAST WALL & \\
Basement & Mu(t) \\
& $M u(-)$ \\
& Vc
\end{tabular} & $\begin{array}{l}\text { \#7@9 } \\
\text { \#7@9 }\end{array}$ & $\begin{array}{l}0.8 \\
0.8\end{array}$ & $\begin{array}{l}15.5 \\
16.5 \\
16.5\end{array}$ & $\begin{array}{l}1.18 \\
1.18\end{array}$ & $\begin{array}{r}54 \\
57 \\
21.3\end{array}$ & \\
\hline \begin{tabular}{|cc} 
SOUTH WALL \\
Basement & $M u(+)$ \\
& $M u(+)$ \\
& $M u(-)$ \\
& Vc
\end{tabular} & $\begin{array}{l}\text { \#10@5 } \\
\text { \#9@10 } \\
\text { \#11@5 }\end{array}$ & $\begin{array}{r}3.048 \\
1.2 \\
3.744\end{array}$ & $\begin{array}{l}15.4 \\
15.4 \\
16.5 \\
16.5\end{array}$ & $\begin{array}{l}4.48 \\
1.76 \\
5.51\end{array}$ & $\begin{array}{r}180 \\
78 \\
232 \\
21.3\end{array}$ & $\begin{array}{l}\text { @ Bottom half } \\
@ \text { Top half }\end{array}$ \\
\hline $\begin{array}{l}\mathrm{Mu(t)} \\
\mathrm{Mu}(+) \\
\mathrm{Mu}(-) \\
\mathrm{V} c\end{array}$ & $\begin{array}{l}\text { \#9@10 } \\
\# 7 @ 10 \\
\# 5 @ 10\end{array}$ & $\begin{array}{r}1.2 \\
0.72 \\
0.372\end{array}$ & $\begin{array}{l}13.5 \\
13.5 \\
14.7 \\
14.7\end{array}$ & $\begin{array}{l}1.76 \\
1.06 \\
0.55\end{array}$ & $\begin{array}{r}68 \\
42 \\
24 \\
19.0\end{array}$ & $\begin{array}{l}\text { @ Bottom half } \\
@ \text { Top half }\end{array}$ \\
\hline$f^{\prime} c=$ & & $f y=$ & & & & \\
\hline $\begin{array}{l}M u=0.90 * A s * f s^{*} \\
V c=0.85 * 2 * \text { Sqrt(f }\end{array}$ & $\begin{array}{l}-a / 2) / \\
* b w\end{array}$ & & $\overline{A S}$ & $\pi(.8$ & $\left.f^{\prime} c^{\star} b\right)$ & \\
\hline
\end{tabular}




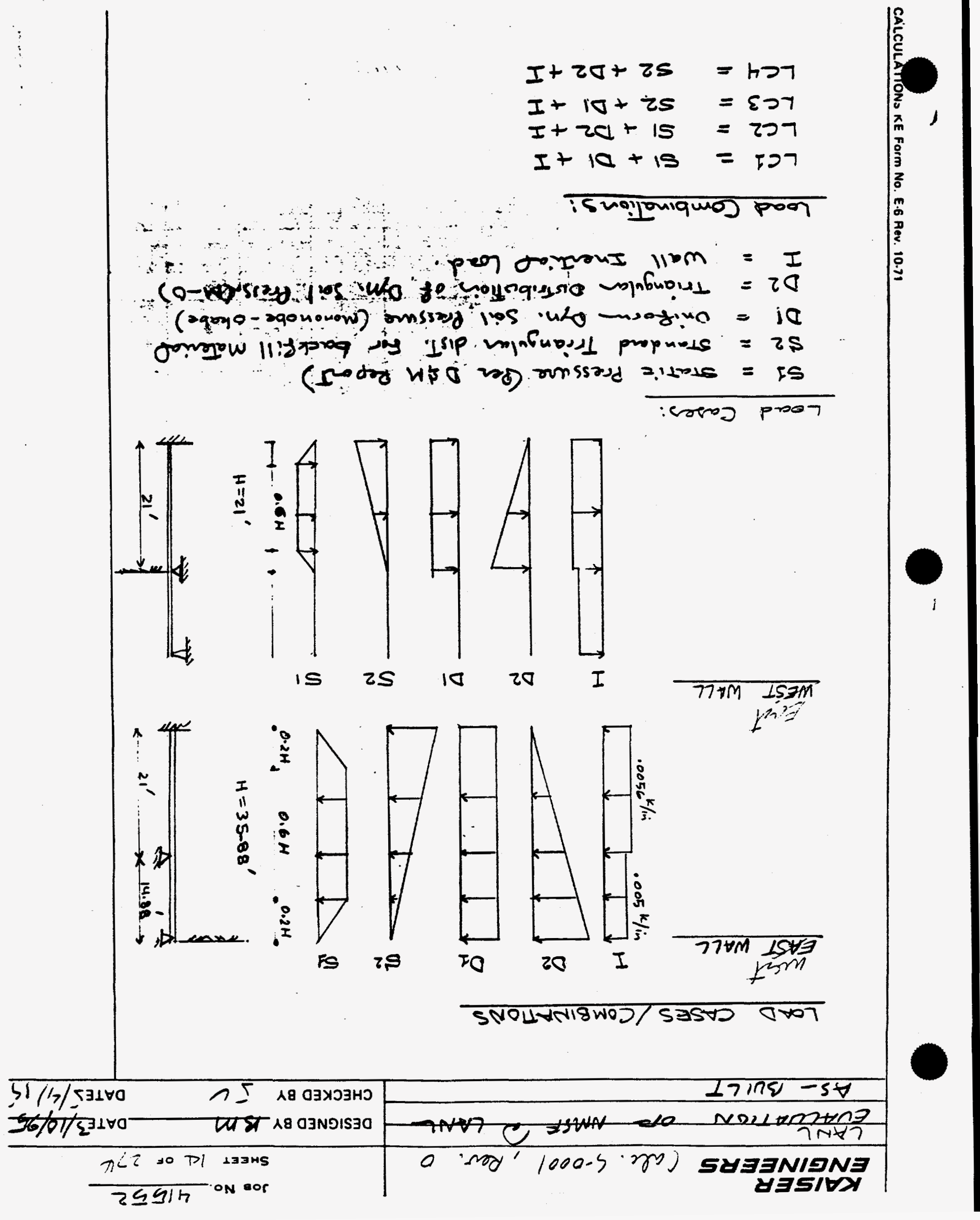


\begin{tabular}{c} 
KAISER \\
ENGINE \\
\hline LAN \\
\hline STRUcTURAL \\
\hline AS-BUil \\
SI:
\end{tabular}

$$
\begin{aligned}
S 1 & =25 \mathrm{H} \quad \text { (REF. } \\
& =25 \times 35.88=898 \mathrm{lb} / \mathrm{Fr} \simeq 900 \mathrm{lb} / \mathrm{FT}=\frac{75 \mathrm{~b} / \mathrm{in}}{\text { Eat wall }} \\
S 1 & =25 \times 21=525 \mathrm{lb} / \mathrm{FT}=\frac{44 \mathrm{~b} / \mathrm{in}}{\text { West wall }}
\end{aligned}
$$

$\leq 2$ :

$$
\begin{aligned}
& 52=8 . H \cdot k_{a} \quad \gamma=110 \text { PF } \\
& k_{a}=0.3 \text { (conservative) } \\
& S 2=110 \times 35188 \times .3=1184 \mathrm{lb} / \mathrm{kT}=\frac{100 \mathrm{lb} / \mathrm{in}}{\text { WoW. }} . \\
& S 2=110 \times 21 \times .3=693 \mathrm{k} / \mathrm{fT} \simeq 5 \mathrm{Bb} / \mathrm{m}
\end{aligned}
$$

DI:

East wale :

west

soil
Total Din. Loud $=15,447$ ib

$$
D 2=\frac{15,447}{35,88 \times 12}=3616 / \mathrm{in}
$$

Total Don. Load = 45716 .

$$
D 2=\frac{5457}{21 \times 12}=21 \mathrm{lb} / \mathrm{in}
$$




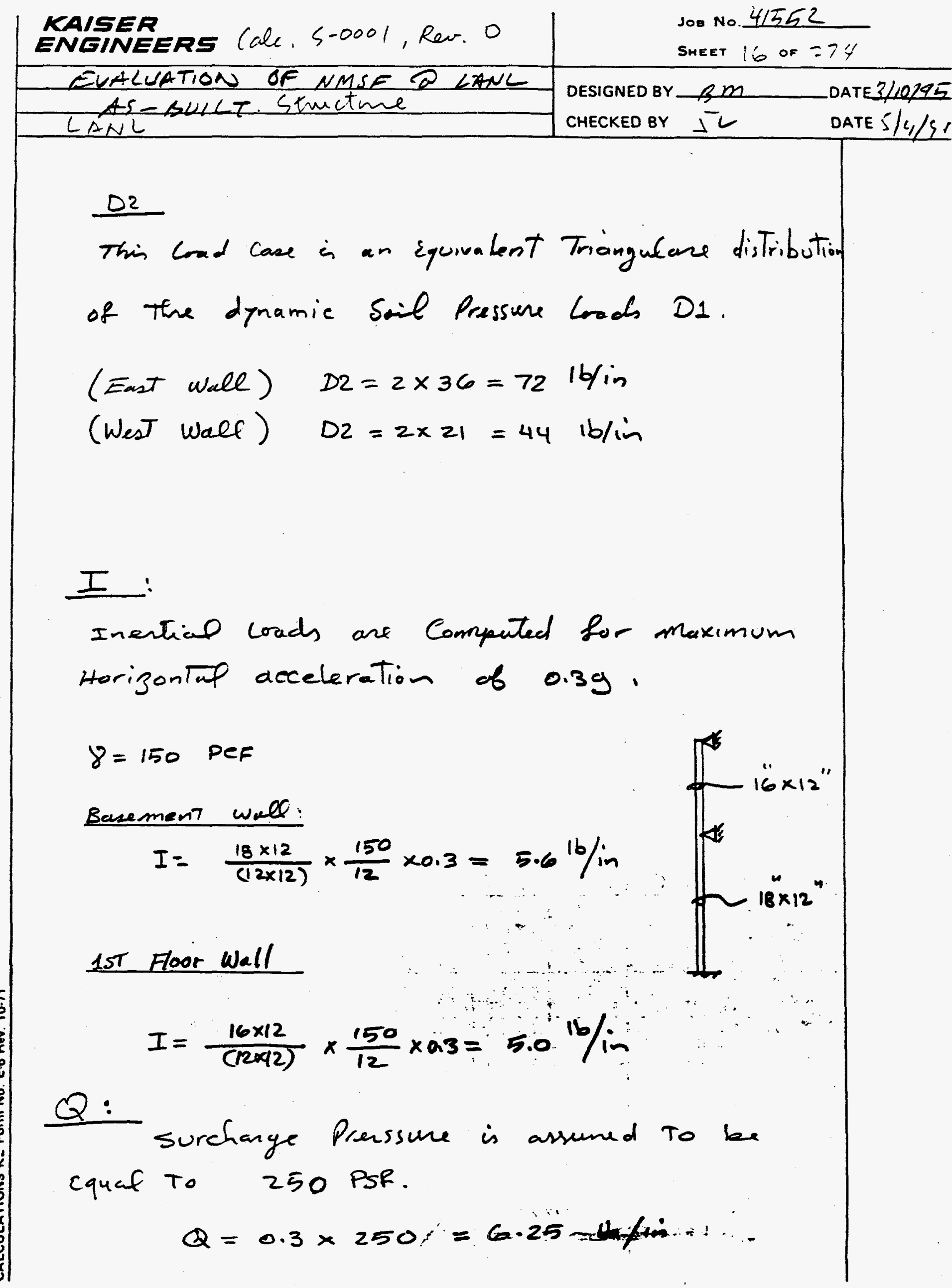




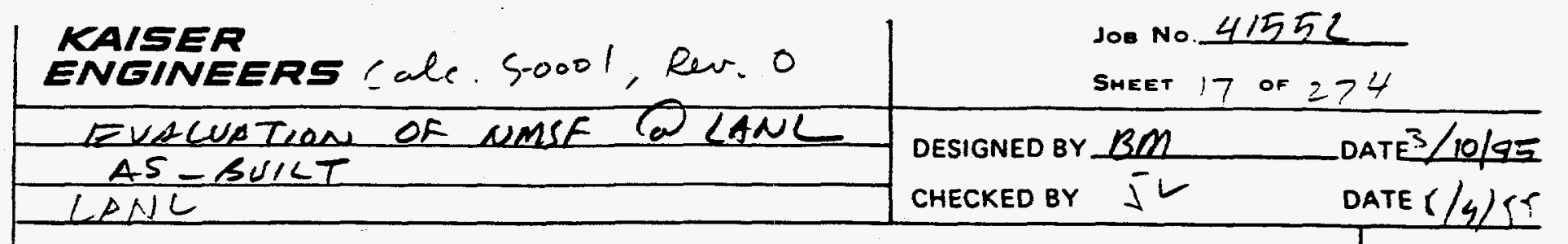

Dynamic Soil Pressure (Mononobe Okabe) (Sample Calces To check SPread sheeT)

$$
\begin{aligned}
& \beta=C^{\circ}=\delta=0 \cdot \frac{\pi}{180}=0 \text { Rad. } \\
& \phi=40 \times \frac{\pi}{180}=.698 \mathrm{Rad} . \\
& 8=110 \mathrm{PCf} \\
& H=18 \mathrm{FT} . \\
& K h=K v=0.5 \mathrm{~g}
\end{aligned}
$$

I) ACE $4-86$

$$
\begin{aligned}
& \psi=\operatorname{Tan}^{-1}\left(\frac{k h}{1-k_{v}}\right)=0.785 \\
& P_{a c}=\frac{H^{2}}{2} \cdot \gamma \cdot\left(1-k_{v}\right) \frac{\operatorname{Cos}^{2}(\phi-\psi-\beta)}{\operatorname{Cos} \gamma \cdot \operatorname{Cos}^{2} \beta \cdot \operatorname{Cos}(\delta+\beta-\psi) \times A}
\end{aligned}
$$

where.

$$
A=\left[1+\left(\frac{-\sin (\phi+\delta) \cdot \sin (\phi-i-\psi)}{\cos (\beta-i) \cdot \cos (\delta+\beta+\psi)}\right)^{1 / 2}\right]^{2}
$$

Total Pressure:

$$
\begin{aligned}
& P_{a e}=10773 \mathrm{lb} / \mathrm{L} . \mathrm{F}_{\mathrm{P}} \longrightarrow p_{d a}=10733 /(\mathrm{H} / 2)=1197 \mathrm{PSF} \\
& \text { (Assuming Triangular Hist) } \\
& \begin{aligned}
P_{a} & \left.=\frac{H^{2}}{2} \cdot \gamma \cdot \frac{\operatorname{Cos}^{2}(\phi-\beta)}{\operatorname{Cos}^{2} \beta \cdot \operatorname{Con}(\delta+\beta) \cdot\left[1+\left(\frac{\operatorname{Sin}(\phi+\delta) \cdot \operatorname{Sin}(\phi-i)}{\operatorname{Cos}(\beta-i) \cdot \operatorname{Cos}(\delta+\beta)}\right)^{5}\right]^{2}}\right]^{2} \\
& =3875
\end{aligned} \\
& k_{a}=3875 /(\mu / 2)=431 \text { PSF }
\end{aligned}
$$




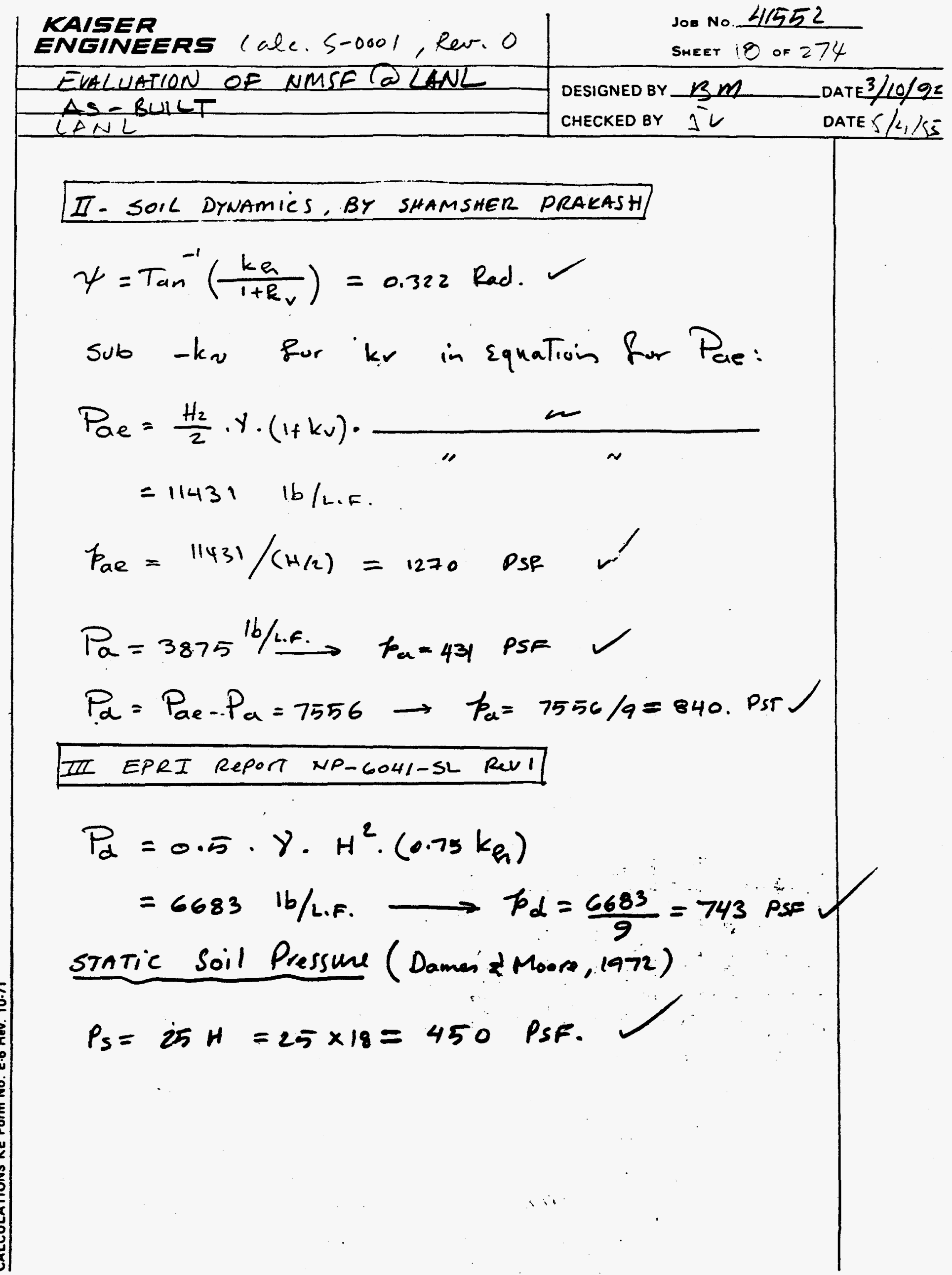




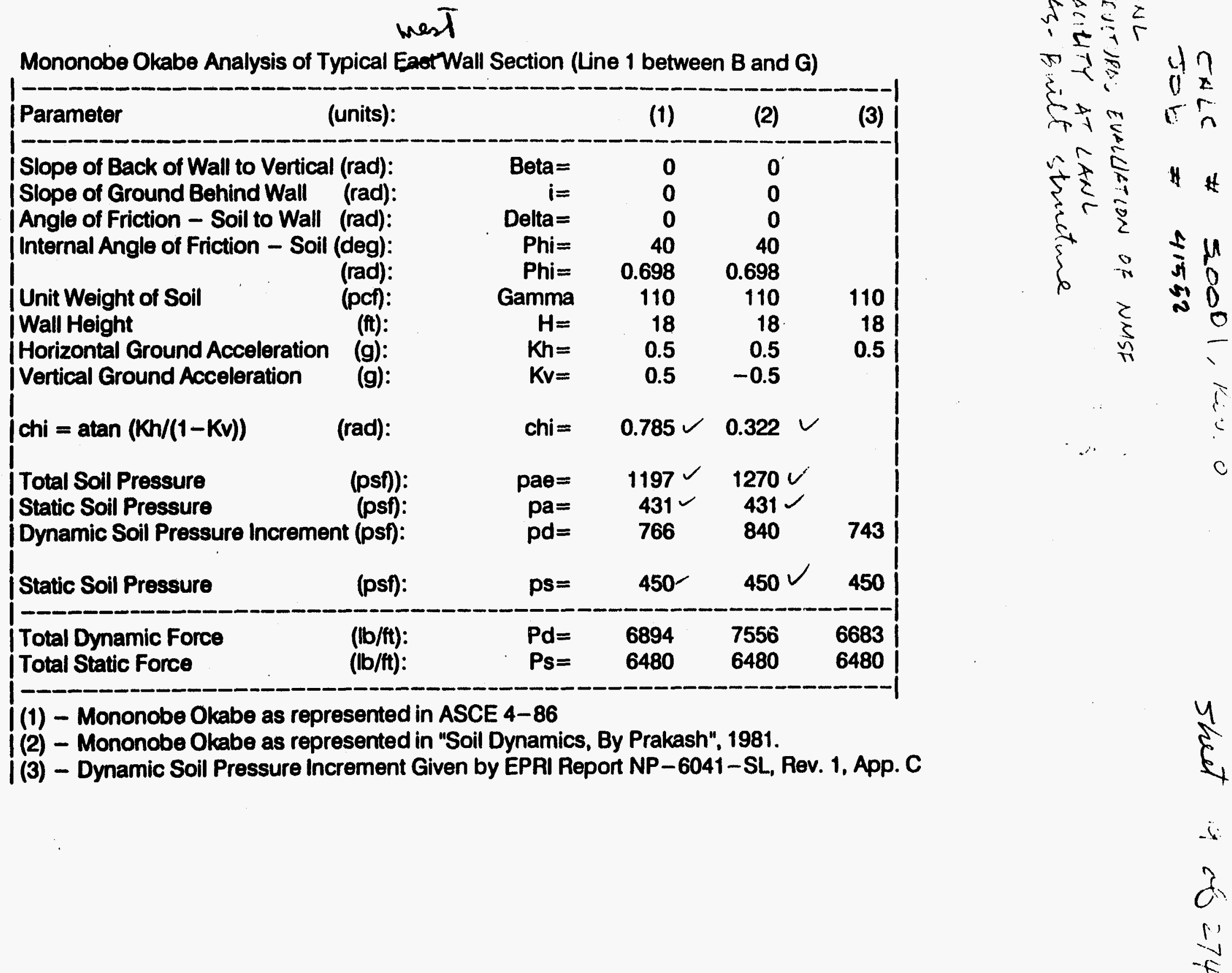




\begin{tabular}{|c|c|c|c|c|}
\hline Parameter & & (1) & (2) & (3) \\
\hline $\begin{array}{l}\text { Slope of Back of Wall to Vertical (rad): } \\
\text { Slope of Ground Behind Wall (rad): } \\
\text { Angle of Friction - Soil to Wall (rad): } \\
\text { Internal Angle of Friction - Soil (deg): } \\
\text { (rad): }\end{array}$ & $\begin{array}{r}\text { Beta }= \\
i= \\
\text { Delta }= \\
\text { Phi }= \\
\text { Phi }=\end{array}$ & $\begin{array}{r}0 \\
0 \\
0 \\
40 \\
0.698\end{array}$ & $\begin{array}{r}0 \\
0 \\
0 \\
40 \\
0.698\end{array}$ & \\
\hline Unit Weight of Soil & Gamma & 110 & 110 & 110 \\
\hline Wall Hoight & $H=$ & 35.9 & 35.9 & 35.9 \\
\hline $\begin{array}{ll}\text { Horizontal Ground Acceleration } & \text { (g): } \\
\text { Vertical Ground Acceleration } & \text { (g): }\end{array}$ & $\begin{array}{l}\mathrm{Kh}= \\
\mathrm{K} \mathbf{v}=\end{array}$ & $\begin{array}{l}0.3 \\
0.2\end{array}$ & $\begin{array}{r}0.3 \\
-0.2\end{array}$ & 0.3 \\
\hline$c h i=\operatorname{atan}(K h /(1-K v))$ & chi $=$ & 0.359 & 0.245 & \\
\hline $\begin{array}{lr}\text { Total Soil Pressure } & \text { (psf)): } \\
\text { Static Soil Pressure } & \text { (psf): } \\
\text { Dynamic Soil Pressure Increment (psf): }\end{array}$ & $\begin{array}{r}p a e= \\
p a= \\
p d=\end{array}$ & $\begin{array}{r}1467 \\
859 \\
608\end{array}$ & $\begin{array}{r}1719 \\
859 \\
861\end{array}$ & 889 \\
\hline Static Soil Pressure & $p s=$ & 897.5 & 897.5 & 897.5 \\
\hline $\begin{array}{l}\text { Total Dynamic Force } \\
\text { Total Static Force }\end{array}$ & $\begin{array}{l}P d= \\
P s=\end{array}$ & $\begin{array}{l}10914 \\
25776\end{array}$ & $\frac{(15447)}{25776}$ & $\begin{array}{l}15949 \\
25776\end{array}$ \\
\hline
\end{tabular}




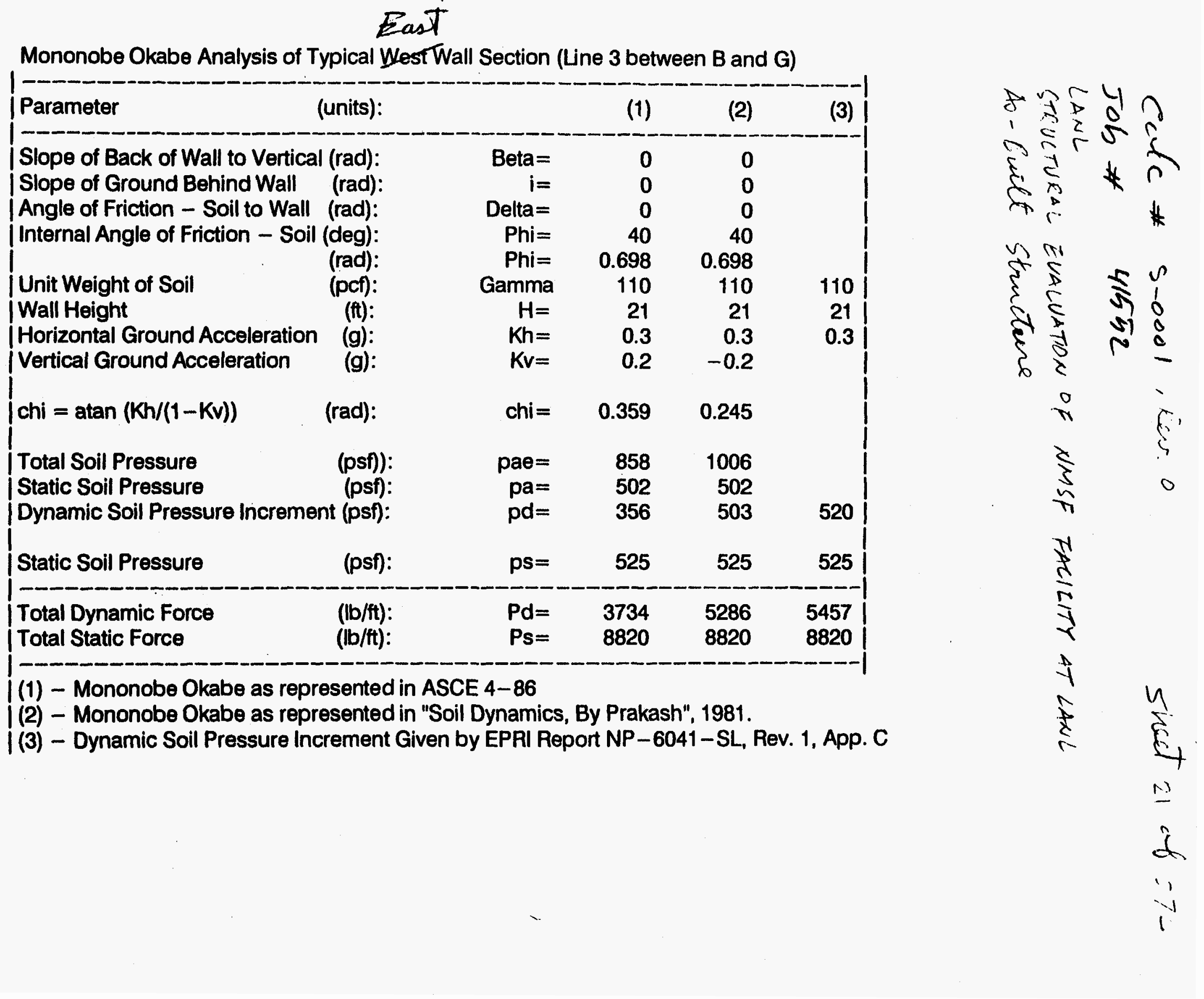


Tob*

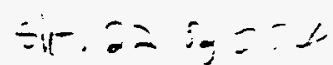

ANALYSIS OF WEST WALL SECTION - MONONOBE OKABE DYNAMIC SOIL PRESSURE (WEST3I) C C C -REV. DATE -FILE-- - DESCRIPTION-

C
SYSTEM
$\mathrm{L}=6$

JOINTS

$1 \quad X=0$

$3 \quad x=0$

$12-7-94$

$12-09-94$

$12-12-94$

$12-14-94$

B. MOSADDAD

$\mathrm{BM}$

$\mathrm{BM}$

BM
WEST1 ORIGINAL

WEST2 ADD TRIANGULAR DYN. PRESSURE

WEST3 ADD TRIANGULAR STATIC PRESSURE WEST31 ADD SURCHARGE ( $1=6)$

$5 \quad X=0 \quad Y=35.88$

$50 \quad X=1 \quad Y=0$

RESTRAINTS

$152 \mathrm{R}=0,0,1,1,1,0$

$1 \quad R=1,1,1,1,1,1$

$3 \quad \mathrm{R}=1,0,1,1,1,0$

$5 \quad R=1,0,1,1,1,0$

$50 \quad R=1,1,1,1,1,1$

\section{FRAME}

$N M=2 \quad N L=10 \quad N S E C=8$

$1 \mathrm{SH}=\mathrm{R} \quad \mathrm{T}=18,12 \mathrm{E}=3600$.

$2 \mathrm{SH}=\mathrm{R} T=16,12 \mathrm{E}=3600$.

C $0.2 * 35.88 / 21=0.34,(.8 * 35.88-21) / 14.88=0.52$

1 TRAP $=0,0,0,-.34,0.075,0,-1 ., 0.075,0$ :STATIC-TRAP BSMNT

TRAP $=0,0.075,0,-.52,0.075,0,-1,0,0$ :STATIC-TARP 1ST FLR

$L=0,0.036,0$
$L=0,0.0056,0$ :DYN. SOIL PRESSURE, UNIFORM

$5 \hat{W L}=0,0.0050,0$

6 TRAP $=0,0,0,-1,0.042,0$

7 TRAP $=0,0.042,0,-1,0.072,0$

8 TRAP $=0,0.10,0,-1,0.04,0$

:INERTIA - BASEMENT

:INERTIA- IST FLR

:DYN SOIL PRESSURE TRIANGULAR BSMNT

9 TRAP $=0,0.04,0,-1 \ldots, 0,0$ :DYN SOIL PRESSURE TRIANGUIAR IST FLR :STATIC- TRIANGULAR BSMNT :STATIC- TRIANGULAR IST FLR

C - . . $q=250$ PSF SURCHARGE, $w=k A \times q=0.3 * 0.250 / 12=0.0063 \mathrm{~K} / \mathrm{IN}$

10. $\mathrm{WL}=0,0.0063,0$
$1 \quad 1 \quad 3 \quad M=1,1 \quad L P=0,50 \quad N S L=1,3,4,6,8,10$
$253 \quad M=2,2 \quad L P=0,50$ NSL $=2,3,5,7,9,10$

COMBO

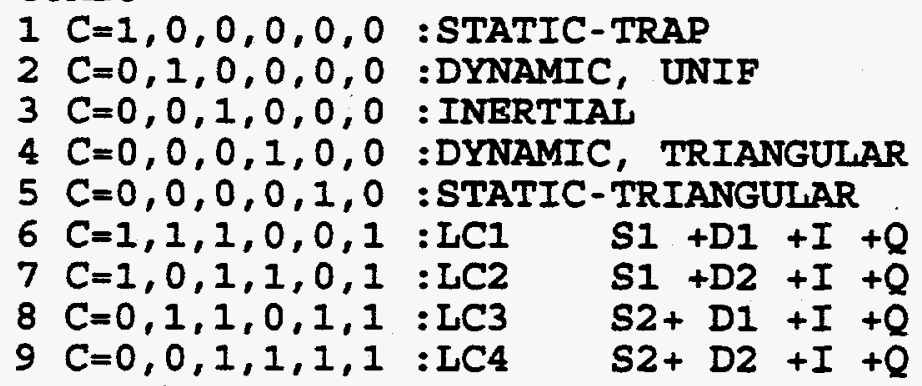




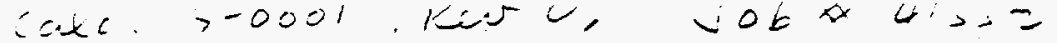

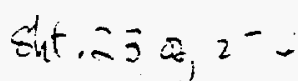

\begin{tabular}{|c|c|c|c|}
\hline $\begin{array}{r}x \\
+4 \\
\end{array}$ & 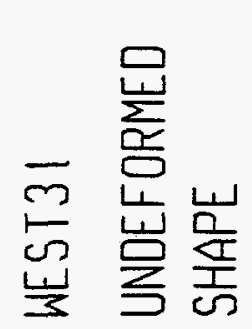 & 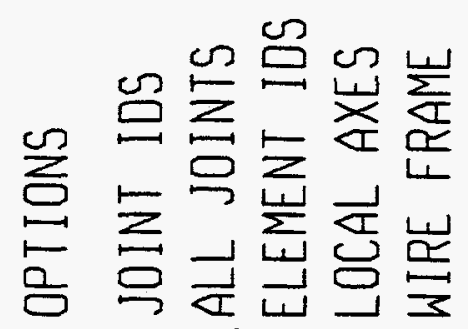 & $\frac{s}{a}$ \\
\hline
\end{tabular}

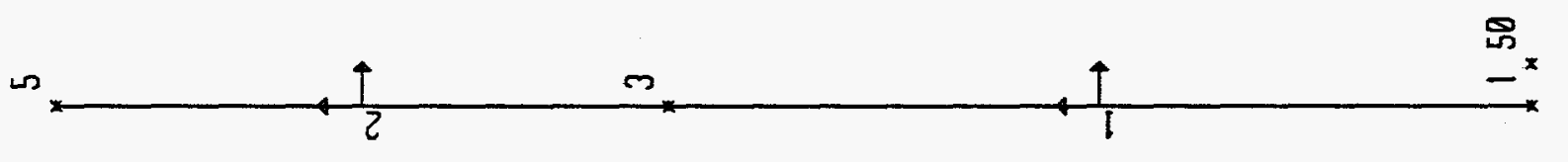


lake $\$ 5-0001, k 50, \quad 506 \times 41552$

$\sin =-2=27$

\begin{tabular}{|c|c|c|c|c|c|}
\hline $\begin{array}{r}4 \\
-4 \\
\end{array}$ & $\begin{array}{l}\bar{m} \\
m \\
m\end{array}$ & 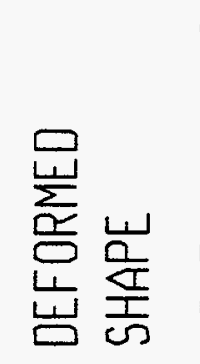 & 导 & 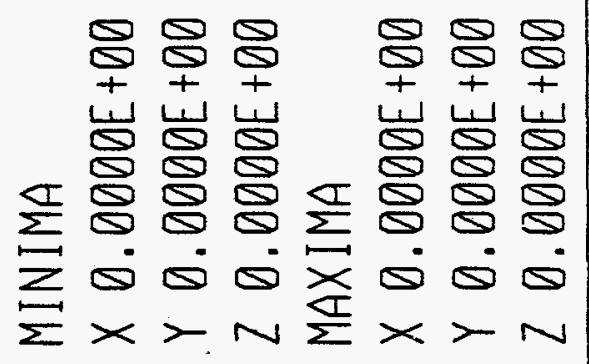 & $\frac{s}{\partial}$ \\
\hline
\end{tabular}


lale S-0001, Res. 0, job* 415-

As rimet

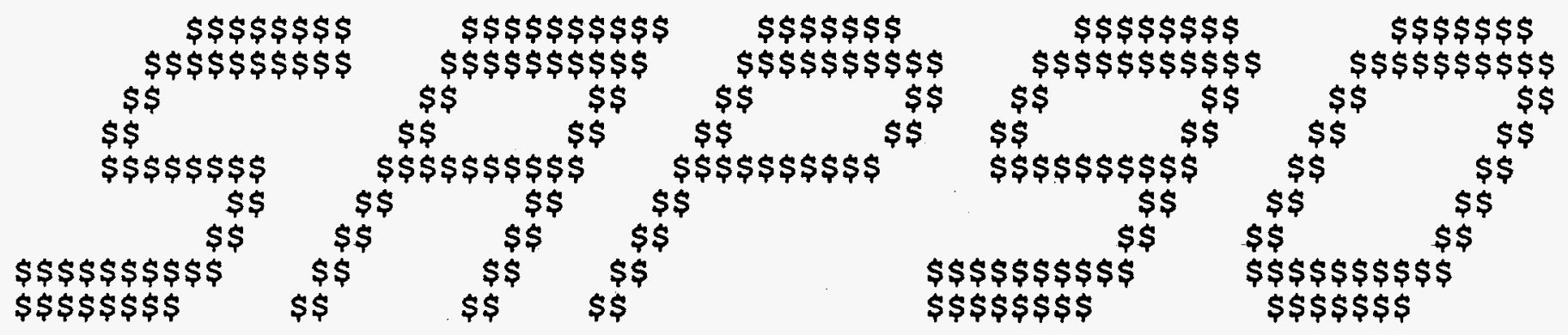

STRUCTURAL ANALYSIS PROGRAMS

VERSION P5.40

Copyright (C) 1978-1992

EDWARD L. WILSON

All rights reserved 
lalc. S-0001, Rev. 0, Job * 4:5-52 5 st. $200=7$

C S I / S A P 90 - - FINITE ELEMENT ANALYSIS OF STRUCTURES PAGE 1 ANALYSIS OF WEST WALL SECTION - MONONOBE OKABE DYNAMIC SOIL PRESSURE (

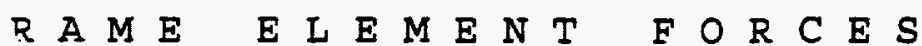

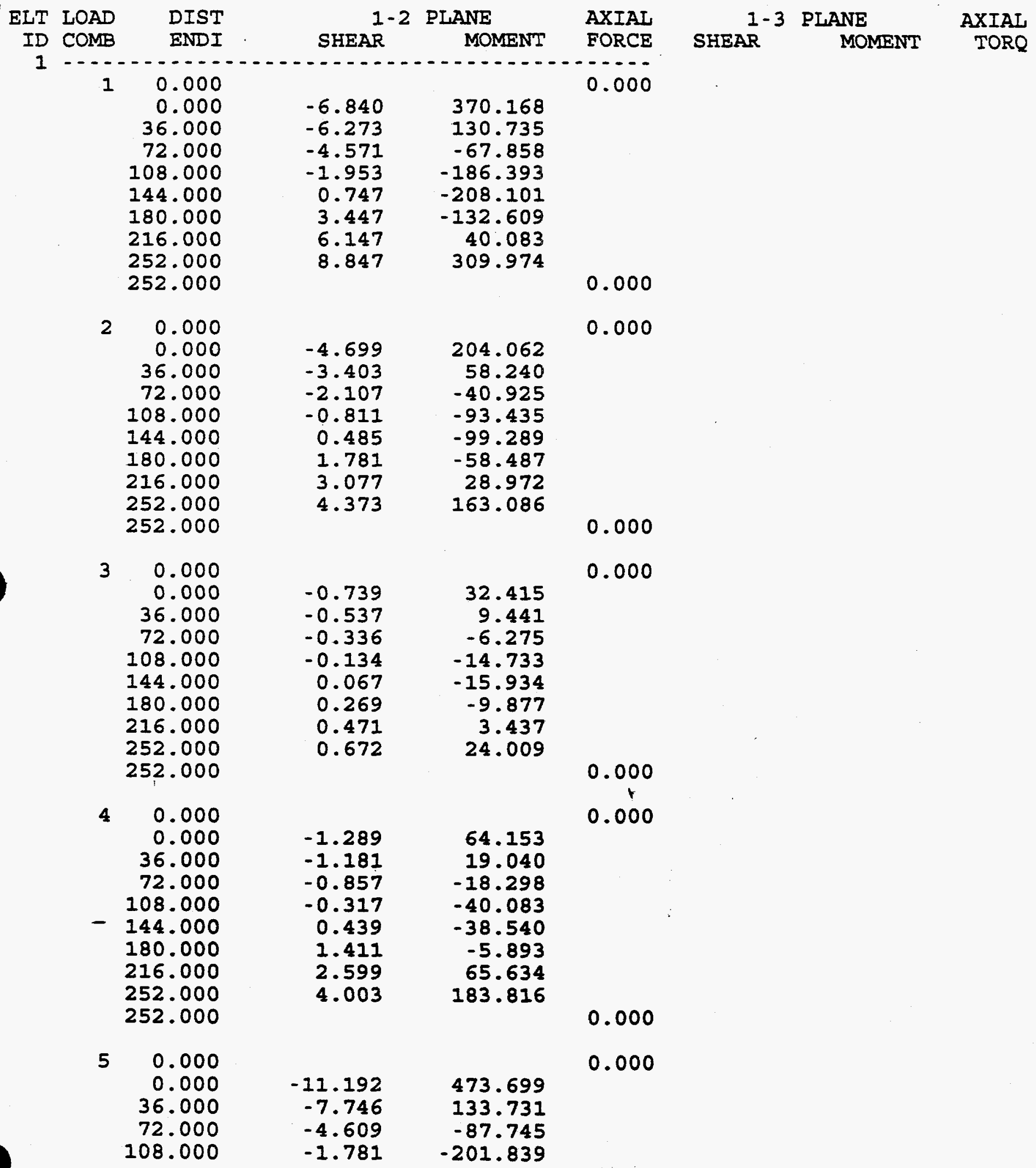


ale 5-000!, lier. 0, Tob $\$ 41552$

Sit. =? o- = -

C SI / SAP 90 - - FINITE ELEMENT ANALYSIS OF STRUCTURES PAGE 2 PROGRAM: SAP9 0/FILE:WEST31.F3F ANALYSIS OF WEST WALL SECTION - MONONOBE OKABE DYNAMIC SOIL PRESSURE ( Q A M E E L E M E N T FOR C E S

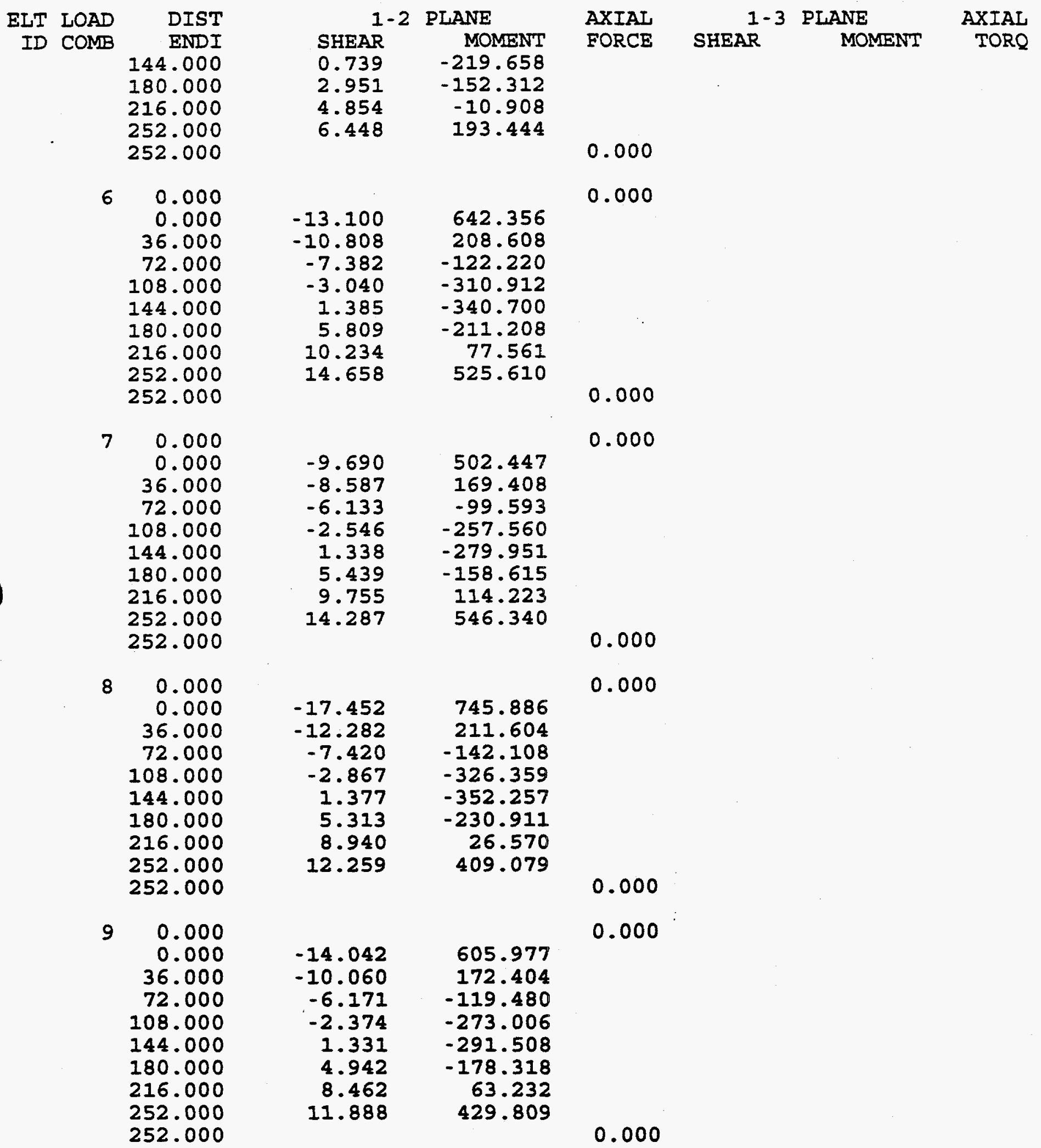




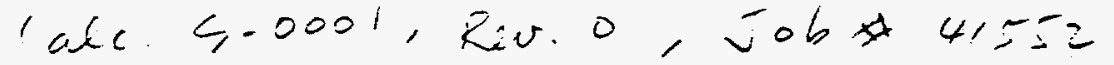

$-n^{+}=E \because-27$

C S I / S A P 90 - - FINITE ELEMENT ANALYSIS OF STRUCTURES PAGE 3 ANALYSIS OF WEST WALL SECTION - MONONOBE ORABE DYNAMIC SOIL PRESSURE (

R A M E E LE M EN T FOR C E S

\begin{tabular}{|c|c|c|c|c|c|c|}
\hline ELT 1 & $\begin{array}{l}\text { LOAD } \\
\text { COMB }\end{array}$ & SHEAR & $\begin{array}{l}\text { PLANE } \\
\text { MOMENT }\end{array}$ & $\begin{array}{l}\text { AXIAL } \\
\text { FORCE }\end{array}$ & $\begin{array}{cc}1-3 & \text { PLANE } \\
\text { SHEAR } & \text { MOMENT }\end{array}$ & $\begin{array}{l}\text { AXIAL } \\
\text { TORQ }\end{array}$ \\
\hline 2 & $\begin{array}{r}1 \quad 0.000 \\
0.000 \\
25.509 \\
51.017 \\
76.526 \\
102.034 \\
127.543 \\
153.051 \\
178.560 \\
178.560\end{array}$ & $\begin{array}{r}-7.918 \\
-6.005 \\
-4.091 \\
-2.178 \\
-0.302 \\
1.121 \\
1.976 \\
2.260\end{array}$ & $\begin{array}{r}309.974 \\
132.406 \\
3.638 \\
-76.327 \\
-107.605 \\
-95.944 \\
-55.234 \\
0.000\end{array}$ & 0.000 & & \\
\hline & $2 \quad \begin{array}{r}0.000 \\
0.000 \\
25.509 \\
51.017 \\
76.526 \\
102.034 \\
127.543 \\
153.051 \\
178.560 \\
178.560\end{array}$ & $\begin{array}{r}-4.127 \\
-3.209 \\
-2.291 \\
-1.372 \\
-0.454 \\
0.464 \\
1.382 \\
2.301\end{array}$ & $\begin{array}{r}163.086 \\
69.514 \\
-0.634 \\
-47.356 \\
-70.654 \\
-70.528 \\
-46.976 \\
0.000\end{array}$ & 0.000 & & \\
\hline & $\begin{array}{r}0.000 \\
0.000 \\
25.509 \\
51.017 \\
76.526 \\
102.034 \\
127.543 \\
153.051 \\
178.560 \\
178.560\end{array}$ & $\begin{array}{r}-0.581 \\
-0.453 \\
-0.326 \\
-0.198 \\
-0.071 \\
0.057 \\
0.184 \\
0.312\end{array}$ & $\begin{array}{r}24.009 \\
10.819 \\
0.882 \\
-5.801 \\
-9.231 \\
-9.407 \\
-6.330 \\
0.000\end{array}$ & 0.000 & & \\
\hline & $\begin{array}{r}0.000 \\
0.000 \\
25.509 \\
51.017 \\
76.526 \\
102.034 \\
127.543 \\
153.051 \\
178.560 \\
178.560\end{array}$ & $\begin{array}{r}-5.672 \\
-4.546 \\
-3.311 \\
-1.966 \\
-0.512 \\
1.051 \\
2.724 \\
4.506\end{array}$ & $\begin{array}{r}183.816 \\
53.261 \\
-47.177 \\
-114.708 \\
-146.545 \\
-139.899 \\
-91.980 \\
0.000\end{array}$ & 0.000 & & \\
\hline & $5 \quad \begin{array}{r}0.000 \\
0.000 \\
25.509 \\
51.017 \\
76.526\end{array}$ & $\begin{array}{l}-3.464 \\
-2.517 \\
-1.715 \\
-1.059\end{array}$ & $\begin{array}{r}193.444 \\
117.472 \\
63.810 \\
28.739\end{array}$ & 0.000 & & \\
\hline
\end{tabular}


Gixe, S-0001, Rer.0, Vob * 41552

C S I / S A P 90 - - FINITE ELEMENT ANALYSIS OF STRUCTURES PAGE 4 ANALYSIS OF WEST WALL SECTION - MONONOBE OKABE DYNAMIC SOIL PRESSURE (

R A M E E L E M E N T FO R C E S

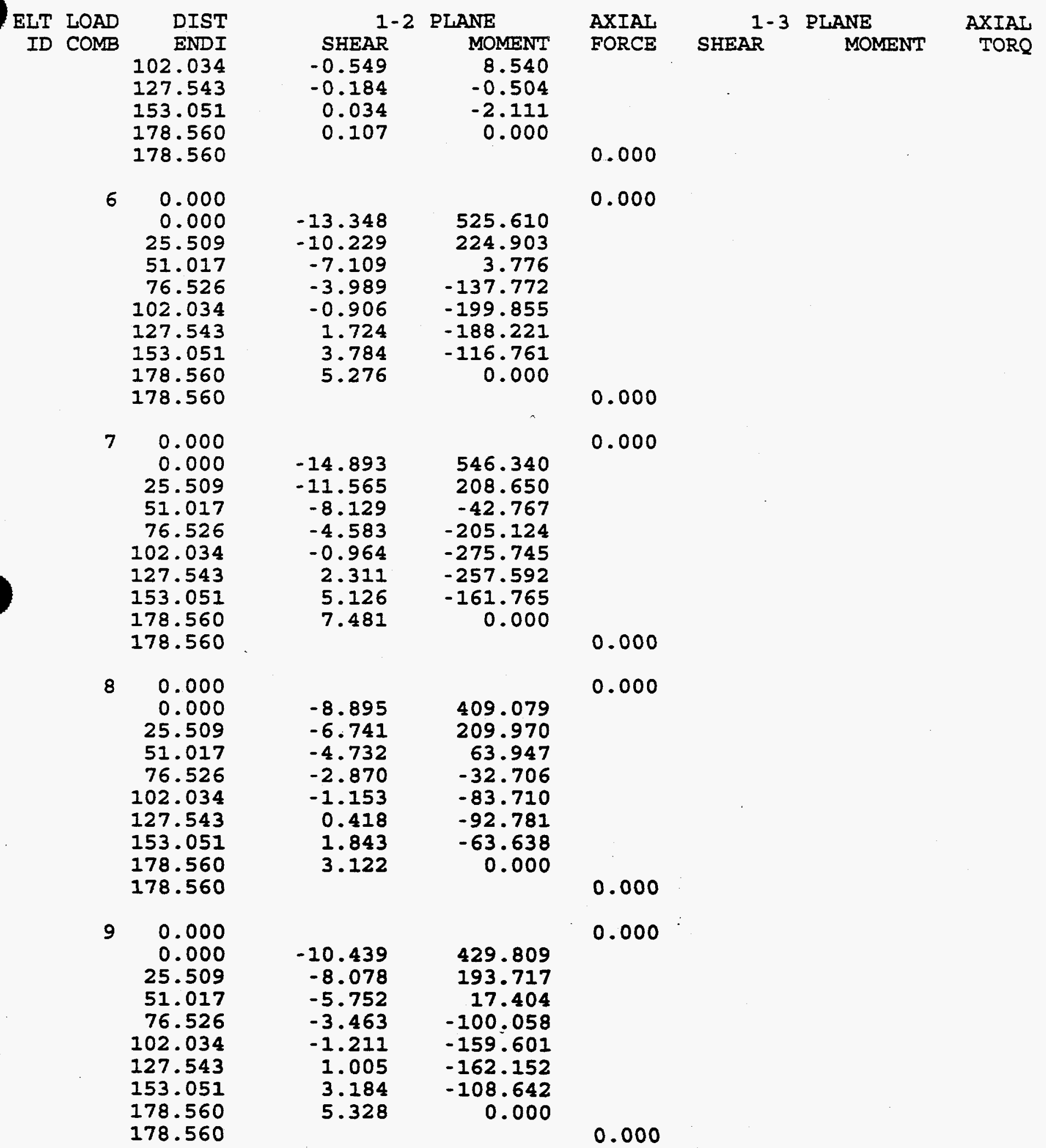


Calc. 5-0001, kevro, Tob \&41552

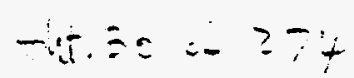

\begin{tabular}{|c|c|c|c|c|c|c|}
\hline $\begin{array}{r}x \\
+4 \\
\end{array}$ & $\begin{array}{l}\bar{m} \\
m \\
\frac{\omega}{3}\end{array}$ & 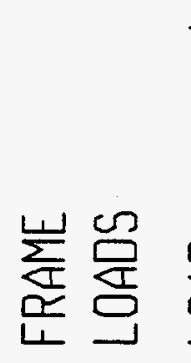 & 足 & 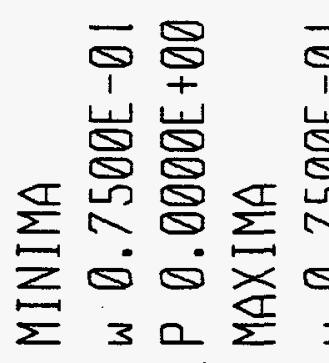 & 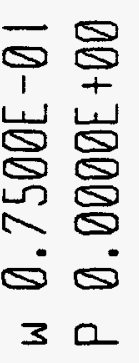 & $\frac{\substack{2 \\
0}}{2}$ \\
\hline
\end{tabular}




\begin{tabular}{|c|c|c|c|c|c|}
\hline $\begin{array}{r}x \\
+4\end{array}$ & $\begin{array}{l}\bar{m} \\
\underline{w} \\
\underline{3}\end{array}$ & 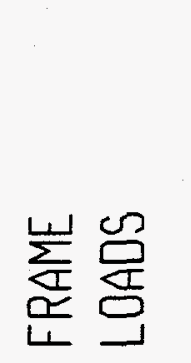 & $\begin{array}{c}\sim \\
\square \\
\square\end{array}$ & 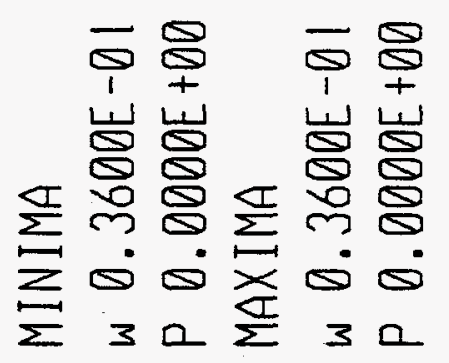 & $\frac{\sqrt{2}}{\frac{1}{2}}$ \\
\hline
\end{tabular}


rale S-0001, Rev. 0. Job 4155z sit. $320=4$

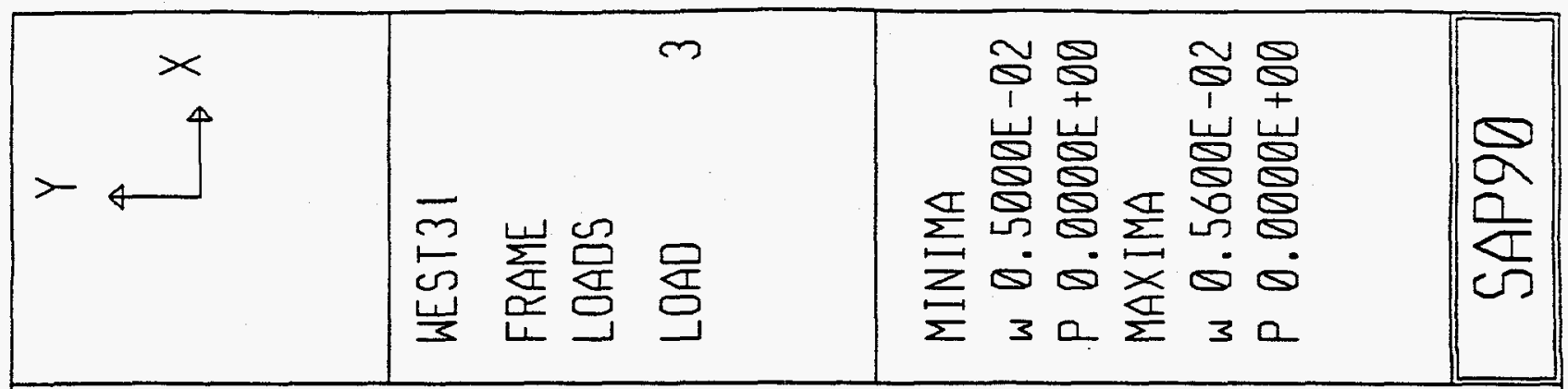


(alc. s,-0001, Rev. o, Got $\approx 415=2$

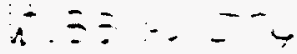

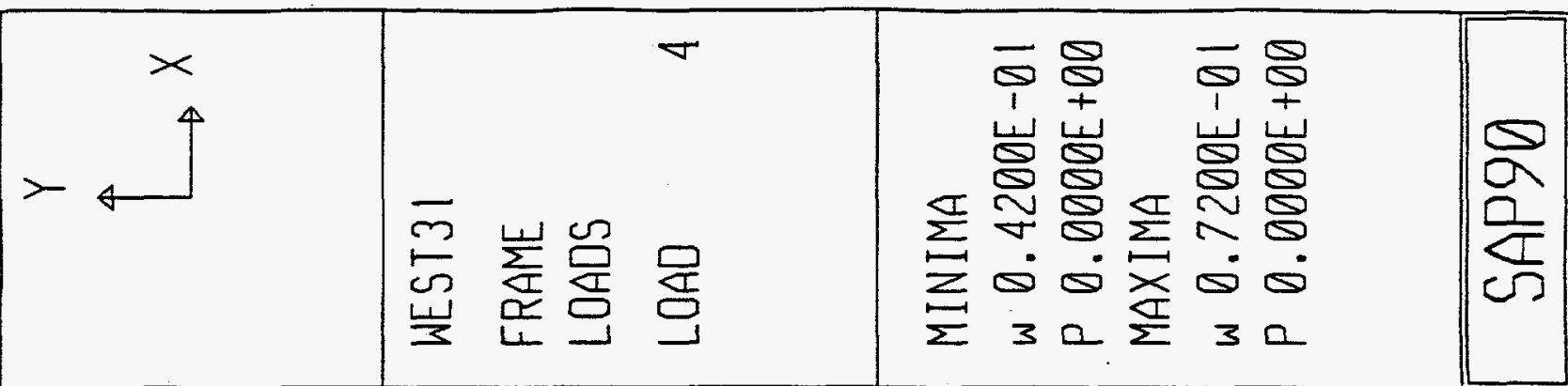


Cale. S-0001, Rew 0, Job HISE

Sut 5s: $=70$

\begin{tabular}{|c|c|c|c|c|}
\hline $\begin{array}{r}x \\
+4 \\
\end{array}$ & $\begin{array}{l}\bar{m} \\
\frac{m}{3} \\
\frac{\mu}{3}\end{array}$ & 岸号宽 & 导 & 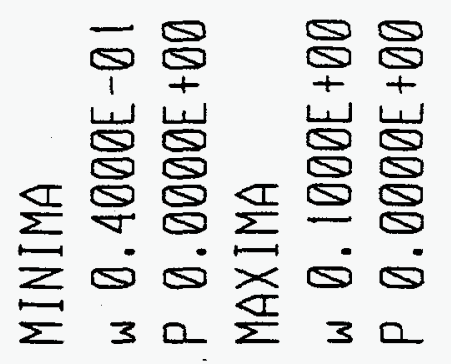 \\
\hline
\end{tabular}


Saxc. S-0001, Rer. o, Tok 41552 sit. 350.17

\begin{tabular}{|c|c|c|c|}
\hline $\begin{array}{r}x \\
4 \\
\end{array}$ & 学崖总是 & 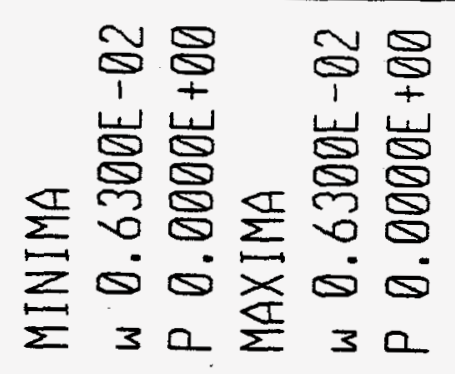 & $\frac{s}{\frac{2}{2}}$ \\
\hline
\end{tabular}

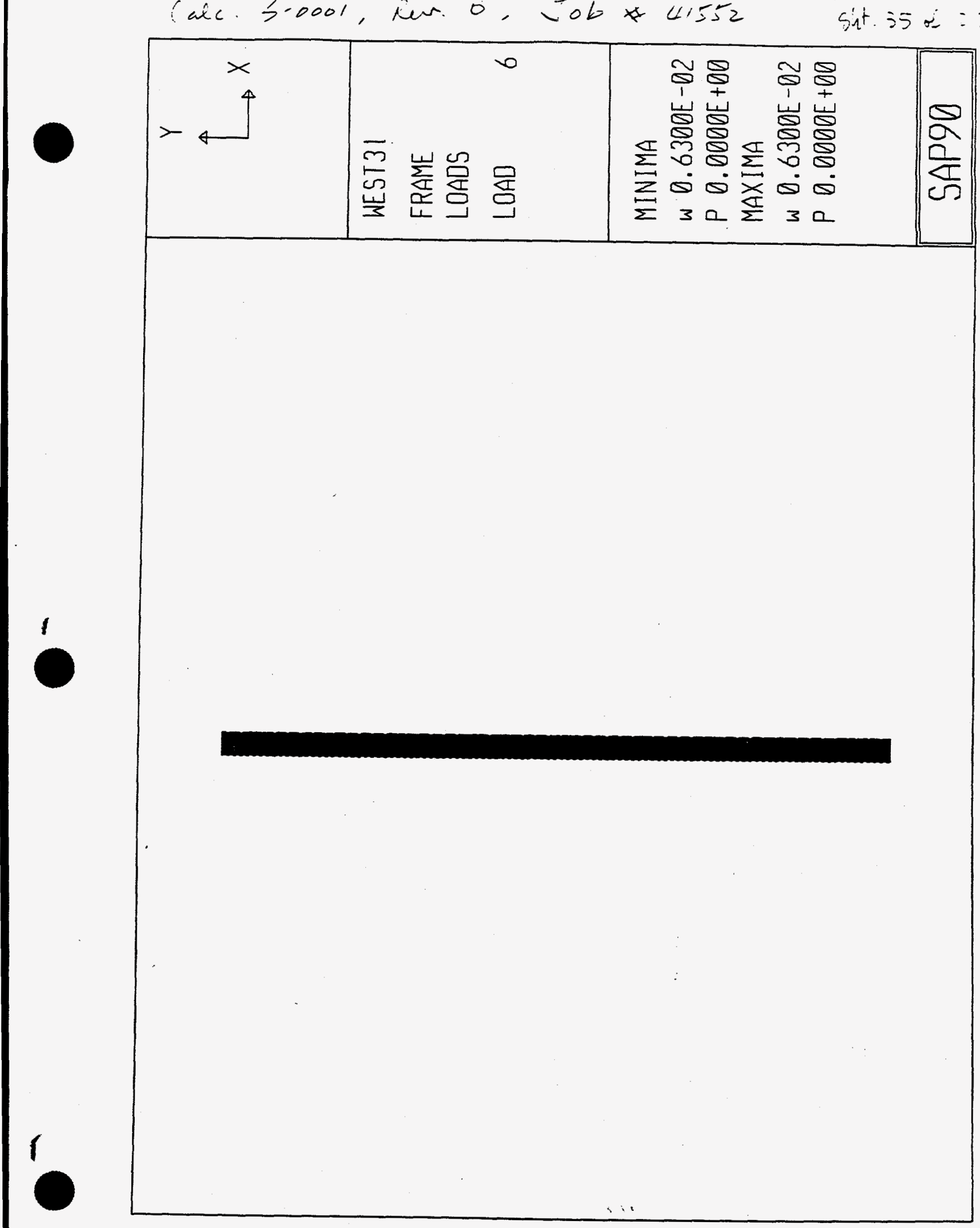


Latc. S.0001, kis. o, vot 4/552

ditt 的 = zit

\begin{tabular}{|c|c|c|c|c|c|}
\hline$>\longleftarrow$ & $\begin{array}{l}\bar{m} \\
\bar{m} \\
\underline{\mu}\end{array}$ & 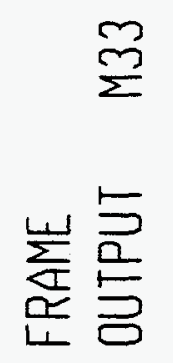 & 名 & 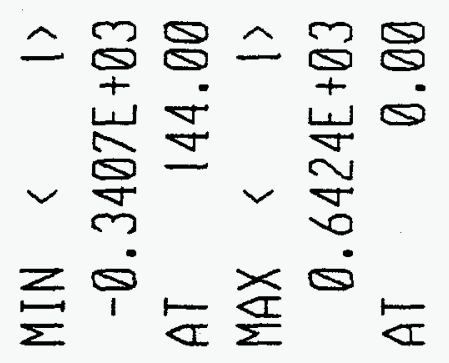 & $\frac{S}{\frac{S}{a}} \frac{a}{\sigma}$ \\
\hline
\end{tabular}

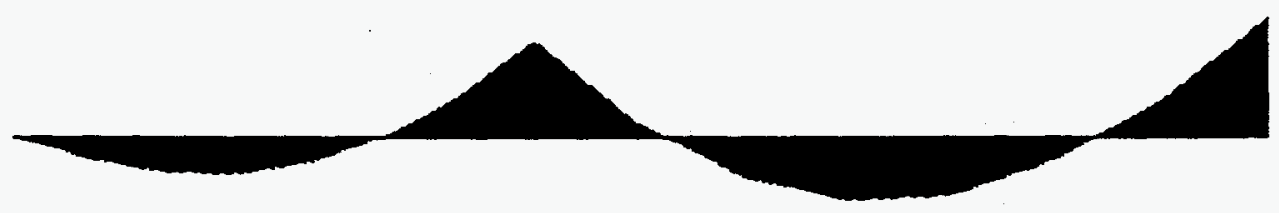




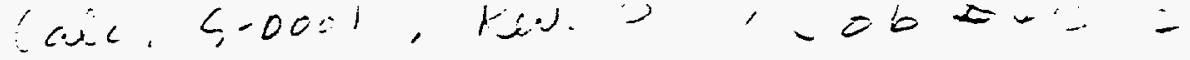

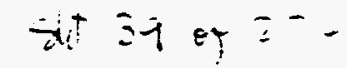

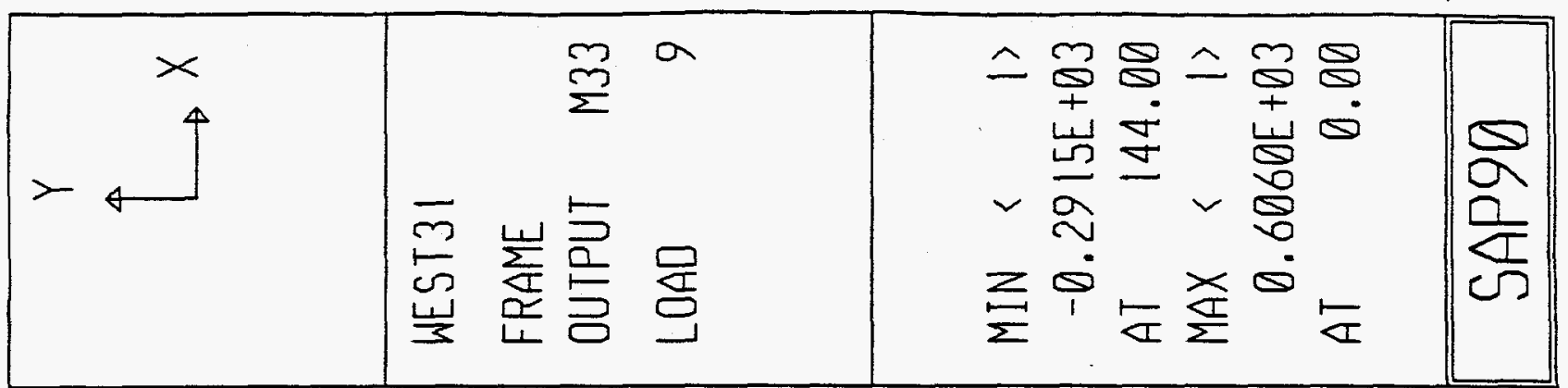

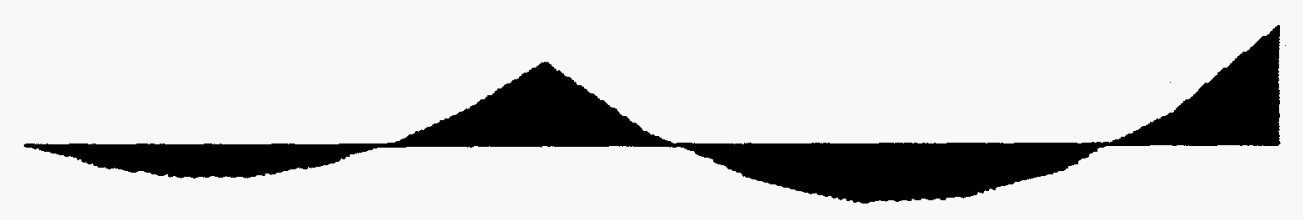




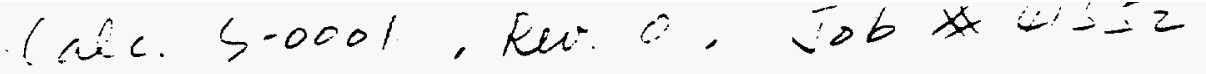

$s-\therefore=\cdots$

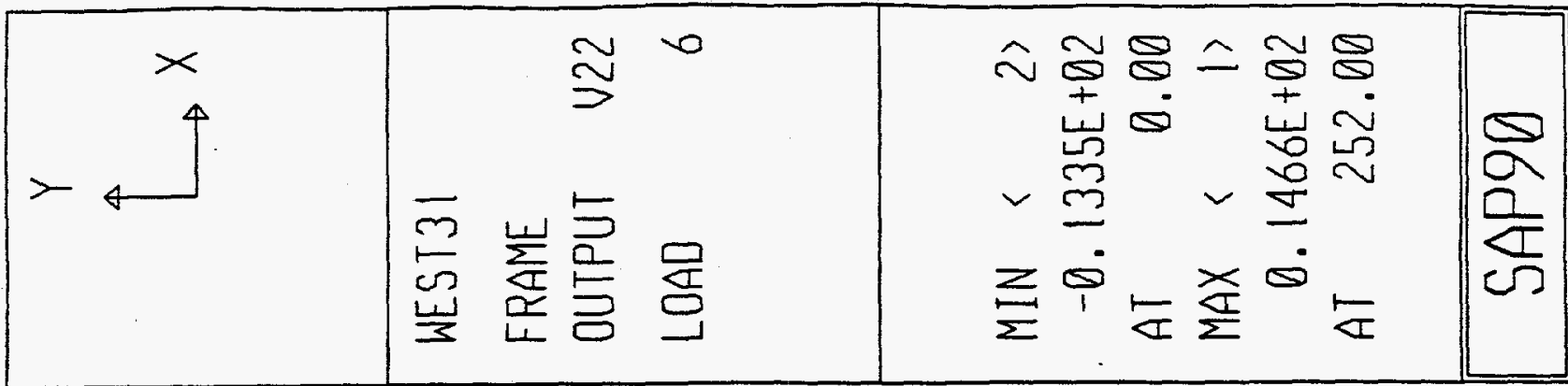

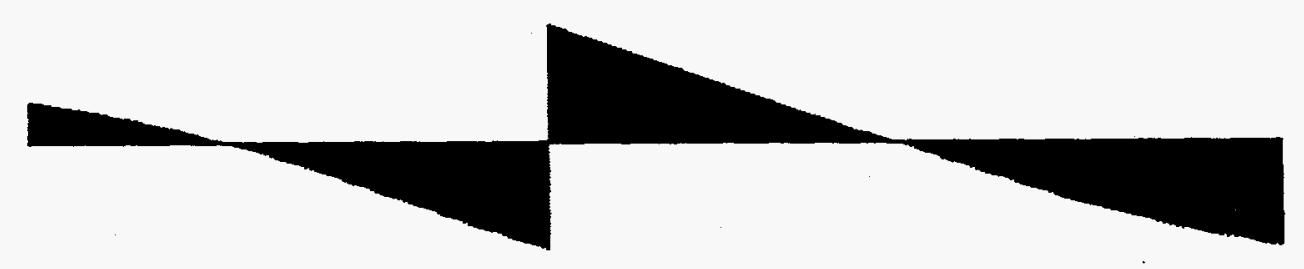




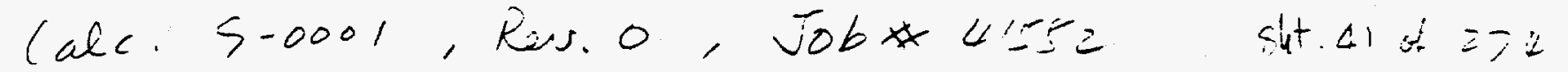

\begin{tabular}{|c|c|c|}
\hline$>\leftarrow$ & 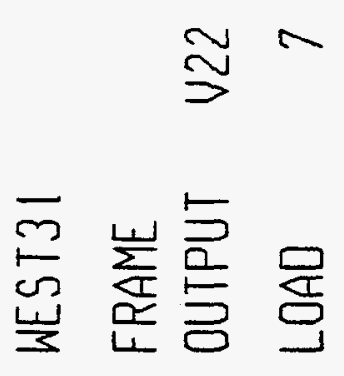 & 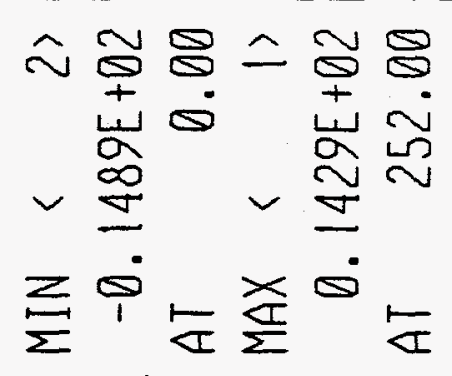 \\
\hline
\end{tabular}

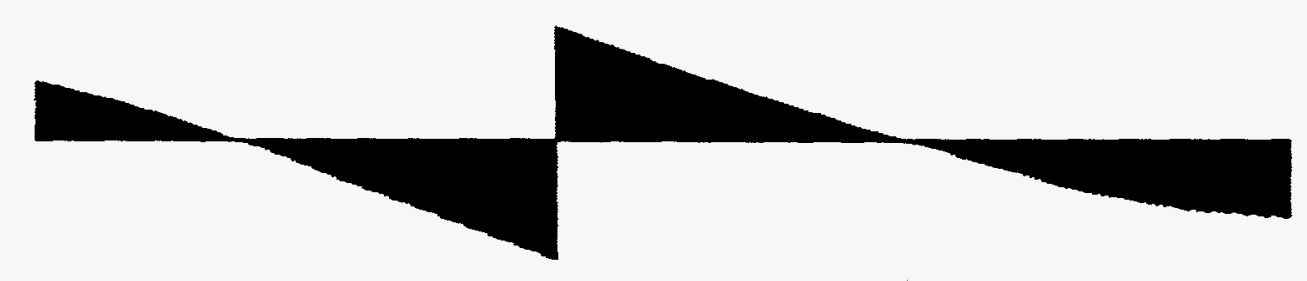


Call 5-0001, Rev.0, Jo6 $04155=$ sht $4=0$

\begin{tabular}{|c|c|c|c|}
\hline$x$ & 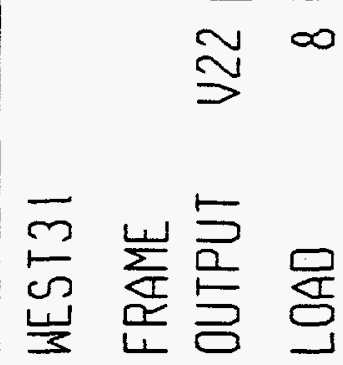 & 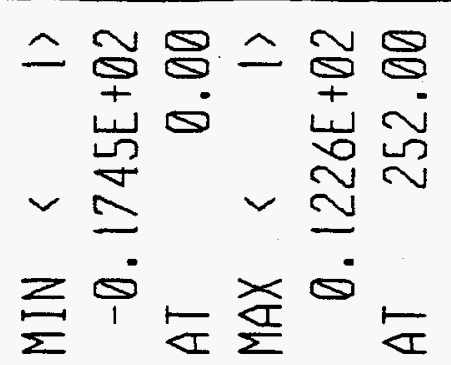 & $\mid \frac{S}{\sigma}$ \\
\hline
\end{tabular}


Cale. S-0001, Rev. 0, Job $\$ 4155=$

$\operatorname{sit} 4 \equiv+2$

\begin{tabular}{|c|c|c|c|c|c|c|}
\hline $\begin{array}{r}x \\
+4 \\
\end{array}$ & $\begin{array}{l}\frac{m}{5} \\
\frac{\omega}{3}\end{array}$ & 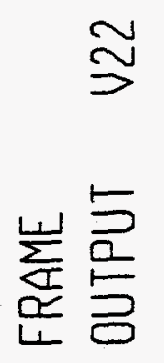 & 롬 & 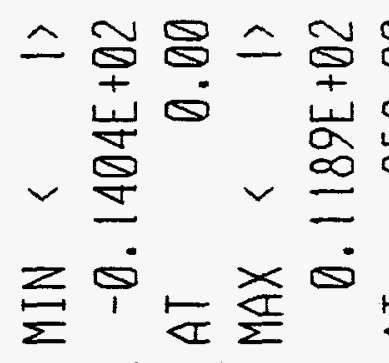 & 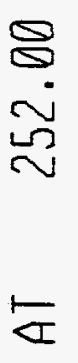 & $\frac{8}{2}$ \\
\hline
\end{tabular}

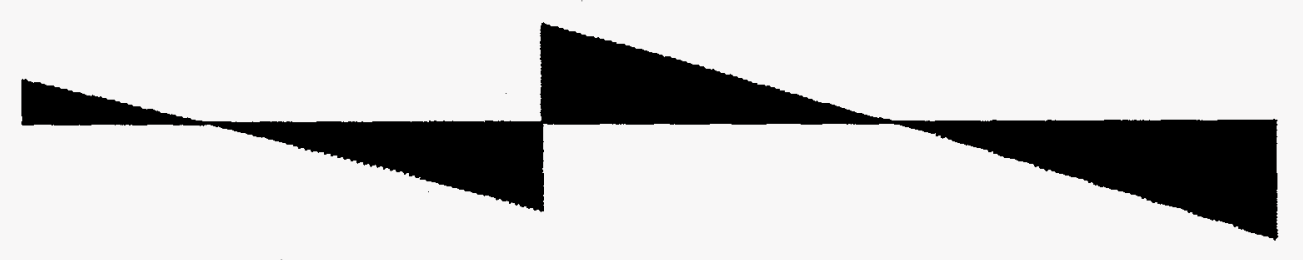


(afe $5-0001, k e), 0,006 \times 45$

ANALYSIS OF EAST WALI SECTION - MONONOBE OKABE DYNAMIC SOIL PRESSURE (EASTI1) C C.-...-REV. DATE-.....-

C $\quad 12-7-94$

$12-09-94$

B.MOSADDAD

WEST1

DESCRIPTION

$12-09-94$

BM

$12-12-94$

BM

WEST2

ORIGINAI

$12-14-94$

$\mathrm{BM}$

EAST1

ADD TRIANGULAR DYN. PRESSURE

EAST WALL MODEL

$\mathrm{BM}$

EAST11 ADD SURCHARGE LOAD $(\mathrm{L}=6)$

C.

SYSTEM

$I=6$

JOINTS

$\begin{array}{llll}1 & X=0 & Y=0 & S=12 \\ 3 & X=0 & Y=21 & \\ 5 & X=0 & Y=35.88 & \\ 50 & X=1 & Y=0\end{array}$

RESTRAINTS

$152 R=0,0,1,1,1,0$

$1 \quad \mathrm{R}=1,1,1,1,1,1$

$3 \quad R=1,0,1,1,1,0$

$5 \quad \mathrm{R}=1,0,1,1,1,0$

$50 \quad R=1,1,1,1,1,1$

\section{FRAME}

$\mathrm{NM}=2 \mathrm{NL}=6 \quad \mathrm{NSEC}=8$

$1 \mathrm{SH}=\mathrm{R} \quad \mathrm{T}=18,12 \mathrm{E}=3600$.

$2 \mathrm{SH}=\mathrm{R} \mathrm{T}=16,12 \mathrm{E}=3600$.

1. TRAP $=0,0,0,-.2,0.044,0,-.8,0.044,0,-1 ., 0,0 \quad$ :STATIC-TRAP

$T_{\mathrm{J}}=0,0.021,0$ $\mu=0,0.0056,0$

:DYN. SOII PRESSURE, UNIFORM

RAP $=0,0,0,-1,0.042,0$ :DY. SOIL PRESSURE TRIANGULAR

IIRAP $=0, .058,0,-1,0,0$

C

$6 \mathrm{WL}=0,0.0063,0$

$\begin{array}{lllll}1 & 1 & 3 & M=1,1 & L P=0,50 \\ 2 & 3 & 5 & M=2,2 & L P=0,50\end{array}$

\section{COMBO}

$1 \mathrm{C}=1,0,0,0,0,0$ :STATIC-TRAP

$2 \mathrm{C}=0,1,0,0,0,0$ :DYNAMIC, UNIF

$3 \mathrm{C}=0,0,1,0,0,0$ : INERTIAL

$4 C=0,0,0,1,0,0$ :DYNAMIC, TRIANGULAR

$5 \mathrm{C}=0,0,0,0,1,0$ :STATIC-TRIANGULAR

$6 \mathrm{C}=1,1,1,0,0,1: L C 1$

$7 \mathrm{C}=1,0,1,1,0,1: L C 2$

$8 \mathrm{C}=0,1,1,0,1,1: L C 3$

$\mathrm{S} 1+\mathrm{D} 1+\mathrm{I}+\mathrm{Q}$

$S 1+D 2+I+Q$

$\mathrm{S} 2+\mathrm{D} 1+\mathrm{I}+\mathrm{Q}$

$9 \mathrm{C}=0,0,1,1,1,1: L C 4$

$\mathrm{S} 2+\mathrm{D} 2+\mathrm{I}+\mathrm{Q}$ 
' a.c. S-0001, kev. 0, Job $\$ 4,52$

$\operatorname{lij}<\equiv=-$

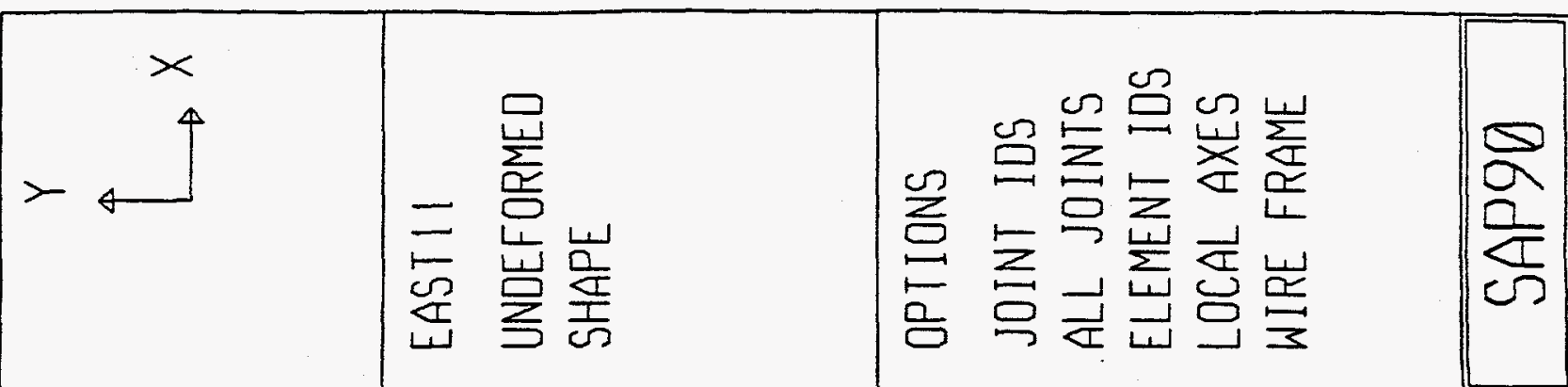

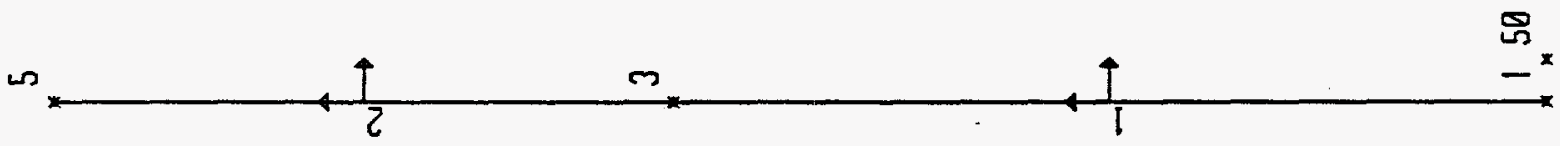


(aic.s-0001, kis. $0,706 \times 4552$

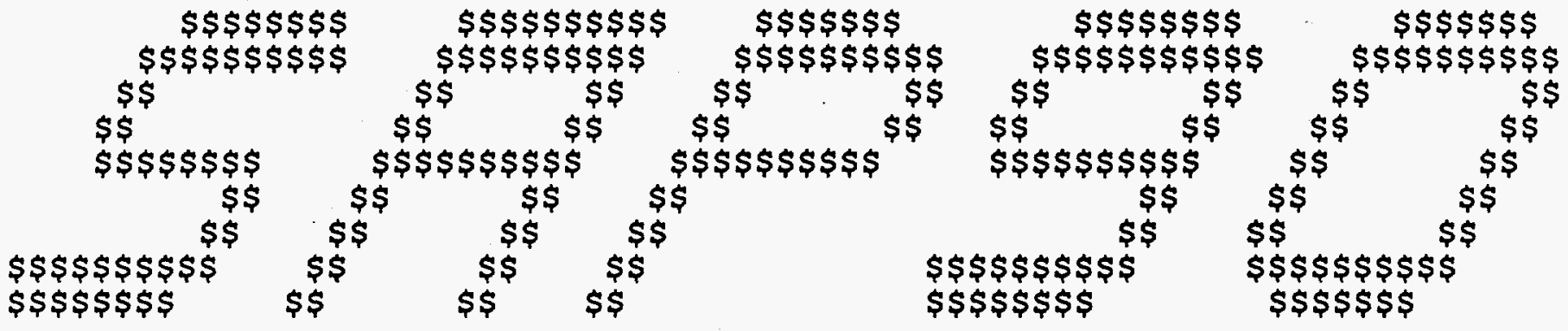

STRUCTURAL ANALYSIS PROGRAMS

VERSION P5.40

Copyright (C) 1978-1992

EDWARD L. WILSON

All rights reserved 
C S I / S A P 90 - - FINITE ELEMENT ANALYSIS OF STRUCTURES PAGE 1 ANALYSIS OF EAST WALL SECTION - MONONOBE ORABE DYNAMIC SOIL PRESSURE (

R A M E E L E M E N T FOR C E S

\begin{tabular}{|c|c|c|c|c|c|c|c|}
\hline ELT & $\begin{array}{l}\text { LOAD } \\
\text { COMB }\end{array}$ & $\begin{array}{r}1 \\
\text { SHEAR }\end{array}$ & $\begin{array}{l}\text { PLANE } \\
\text { MOMENT }\end{array}$ & $\begin{array}{l}\text { AXIAL } \\
\text { FORCE }\end{array}$ & SHEAR & $\begin{array}{l}\text { PLANE } \\
\text { MOMENT }\end{array}$ & $\begin{array}{r}\text { AXIAL } \\
\text { TORQ }\end{array}$ \\
\hline 1 & $\begin{array}{r}1 \\
0.000 \\
0.000 \\
36.000 \\
72.000 \\
108.000 \\
144.000 \\
180.000 \\
216.000 \\
252.000 \\
252.000\end{array}$ & $\begin{array}{r}-5.168 \\
-4.603 \\
-3.109 \\
-1.525 \\
0.059 \\
1.643 \\
3.136 \\
3.702\end{array}$ & $\begin{array}{r}277.172 \\
97.902 \\
-42.102 \\
-125.517 \\
-151.909 \\
-121.276 \\
-34.053 \\
92.434\end{array}$ & 0.000 & & & . \\
\hline & $2 \begin{array}{r}0.000 \\
0.000 \\
36.000 \\
72.000 \\
108.000 \\
144.000 \\
180.000 \\
216.000 \\
252.000 \\
252.000\end{array}$ & $\begin{array}{r}-3.023 \\
-2.267 \\
-1.511 \\
-0.755 \\
0.001 \\
0.757 \\
1.513 \\
2.269\end{array}$ & $\begin{array}{r}142.550 \\
47.329 \\
-20.676 \\
-61.465 \\
-75.038 \\
-61.395 \\
-20.536 \\
47.539\end{array}$ & 0.000 & & & \\
\hline & $\begin{array}{r}0.000 \\
0.000 \\
36.000 \\
72.000 \\
108.000 \\
144.000 \\
180.000 \\
216.000 \\
252.000 \\
252.000\end{array}$ & $\begin{array}{r}-0.806 \\
-0.605 \\
-0.403 \\
-0.201 \\
0.000 \\
0.202 \\
0.403 \\
0.605\end{array}$ & $\begin{array}{r}38.013 \\
12.621 \\
-5.514 \\
-16.391 \\
-20.010 \\
-16.372 \\
-5.476 \\
12.677\end{array}$ & 0.000 & & & \\
\hline & $\begin{array}{r}0.000 \\
0.000 \\
36.000 \\
72.000 \\
108.000 \\
144.000 \\
180.000 \\
216.000 \\
252.000 \\
252.000\end{array}$ & $\begin{array}{r}-2.042 \\
-1.934 \\
-1.610 \\
-1.070 \\
-0.314 \\
0.658 \\
1.846 \\
3.250\end{array}$ & $\begin{array}{r}126.857 \\
54.655 \\
-9.771 \\
-58.645 \\
-84.191 \\
-78.634 \\
-34.196 \\
56.898\end{array}$ & $\begin{array}{l}0.000 \\
0.000\end{array}$ & & & \\
\hline & $\begin{array}{r}0.000 \\
0.000 \\
36.000 \\
72.000 \\
108.000\end{array}$ & $\begin{array}{l}-5.530 \\
-3.591 \\
-1.951 \\
-0.608\end{array}$ & $\begin{array}{r}218.526 \\
55.243 \\
-43.611 \\
-88.774\end{array}$ & 0.000 & & & \\
\hline
\end{tabular}


lake. 50001, kis. $0,506 \times 4552$

C S I / S A P 90 - - FINITE ELEMENT ANALYSIS OF STRUCTURES PAGE 2 ANALYSIS OF EAST WALL SECTION - MONONOBE ORABE DYNAMIC SOIL PRESSURE (

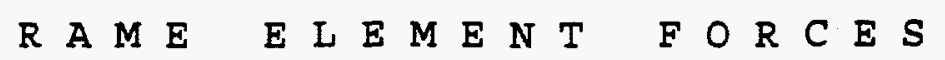

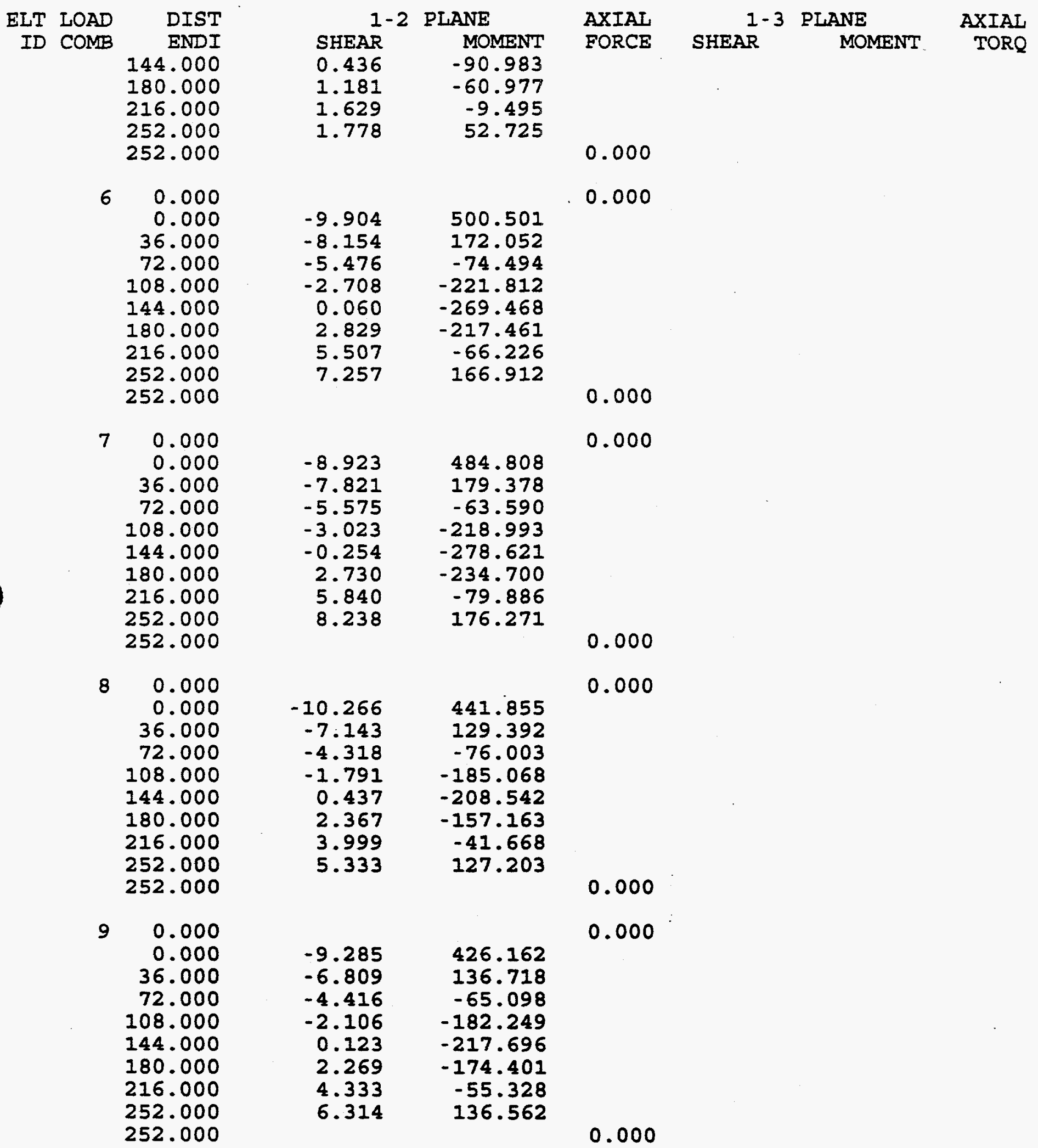


C S I / S A P 90 - - FINITE ELEMENT ANALYSIS OF STRUCTURES PAGE 3 ANALYSIS OF EAST WALL SECTION - MONONOBE OKABE DYNAMIC SOIL PRESSURE ( $\begin{array}{lllllllllllllll}R & \text { A } & \text { E L E M E T }\end{array}$

\begin{tabular}{|c|c|c|c|c|c|c|c|}
\hline $\begin{array}{l}\text { ELT } \\
\text { ID }\end{array}$ & $\begin{array}{l}\text { LOAD } \\
\text { COMB }\end{array}$ & SHEAR & $\begin{array}{l}\text { PLANE } \\
\text { MOMENT }\end{array}$ & $\begin{array}{l}\text { AXIAL } \\
\text { FORCE }\end{array}$ & SHEAR & $\begin{array}{l}\text { PLANE } \\
\text { MOMENT }\end{array}$ & $\begin{array}{r}\text { AXIAL } \\
\text { TORQ }\end{array}$ \\
\hline 2 & $\begin{array}{r}1.000 \\
0.000 \\
25.509 \\
51.017 \\
76.526 \\
102.034 \\
127.543 \\
153.051 \\
178.560 \\
178.560\end{array}$ & $\begin{array}{l}-0.518 \\
-0.518 \\
-0.518 \\
-0.518 \\
-0.518 \\
-0.518 \\
-0.518 \\
-0.518\end{array}$ & $\begin{array}{r}92.434 \\
79.229 \\
66.024 \\
52.820 \\
39.615 \\
26.410 \\
13.205 \\
0.000\end{array}$ & 0.000 & & & \\
\hline & $2 \begin{array}{r}0.000 \\
0.000 \\
25.509 \\
51.017 \\
76.526 \\
102.034 \\
127.543 \\
153.051 \\
178.560 \\
178.560\end{array}$ & $\begin{array}{l}-0.266 \\
-0.266 \\
-0.266 \\
-0.266 \\
-0.266 \\
-0.266 \\
-0.266 \\
-0.266\end{array}$ & $\begin{array}{r}47.539 \\
40.748 \\
33.957 \\
27.165 \\
20.374 \\
13.583 \\
6.791 \\
0.000\end{array}$ & 0.000 & & & \\
\hline & $\begin{array}{r}0.000 \\
0.000 \\
25.509 \\
51.017 \\
76.526 \\
102.034 \\
127.543 \\
153.051 \\
178.560 \\
178.560\end{array}$ & $\begin{array}{l}-0.071 \\
-0.071 \\
-0.071 \\
-0.071 \\
-0.071 \\
-0.071 \\
-0.071 \\
-0.071\end{array}$ & $\begin{array}{r}12.677 \\
10.866 \\
9.055 \\
7.244 \\
5.433 \\
3.622 \\
1.811 \\
0.000\end{array}$ & 0.000 & & & \\
\hline & $\begin{array}{r}0.000 \\
0.000 \\
25.509 \\
51.017 \\
76.526 \\
102.034 \\
127.543 \\
153.051 \\
178.560 \\
178.560\end{array}$ & $\begin{array}{l}-0.319 \\
-0.319 \\
-0.319 \\
-0.319 \\
-0.319 \\
-0.319 \\
-0.319 \\
-0.319\end{array}$ & $\begin{array}{r}56.898 \\
48.770 \\
40.641 \\
32.513 \\
24.385 \\
16.257 \\
8.128 \\
0.000\end{array}$ & 0.000 & & & \\
\hline & $\begin{array}{r}0.000 \\
0.000 \\
25.509 \\
51.017 \\
76.526\end{array}$ & $\begin{array}{l}-0.295 \\
-0.295 \\
-0.295 \\
-0.295\end{array}$ & $\begin{array}{l}52.725 \\
45.193 \\
37.661 \\
30.129\end{array}$ & 0.000 & & & \\
\hline
\end{tabular}




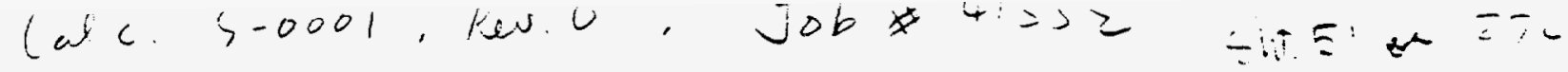

\begin{tabular}{|c|c|c|c|c|c|}
\hline $\begin{array}{r}x \\
4 \\
\end{array}$ & $=$ & 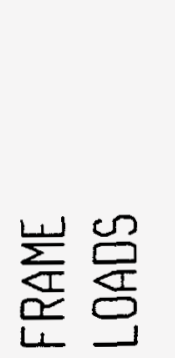 & 号 & 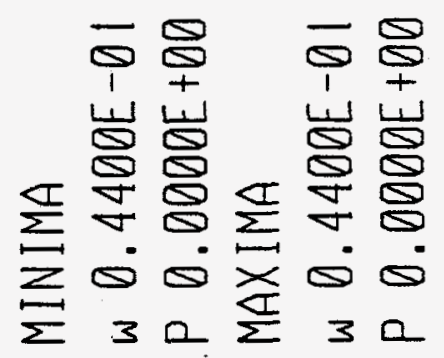 & $\frac{5}{2}$ \\
\hline
\end{tabular}


(cite $>-0001$, his. $0,000, c i s=$

Ent. $=z \cdots z-$ -

\begin{tabular}{|c|c|c|c|c|c|c|c|c|}
\hline $\begin{array}{r}x \\
-4\end{array}$ & $\begin{array}{l}E \\
E \\
\sum_{1}^{\prime}\end{array}$ & 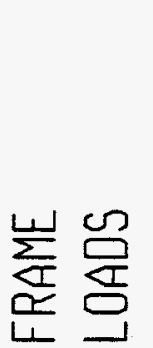 & 异 & $\underset{\sum}{\sum}$ & 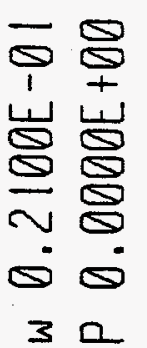 & 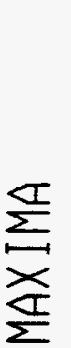 & 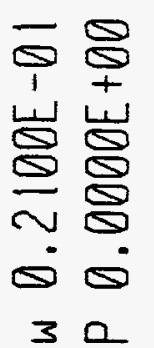 & $\frac{1}{2 \pi}$ \\
\hline
\end{tabular}


$\therefore \therefore 5,000 !$, Ker. 0 , Jo6 41552

HA. $5 \equiv$ of $=0$

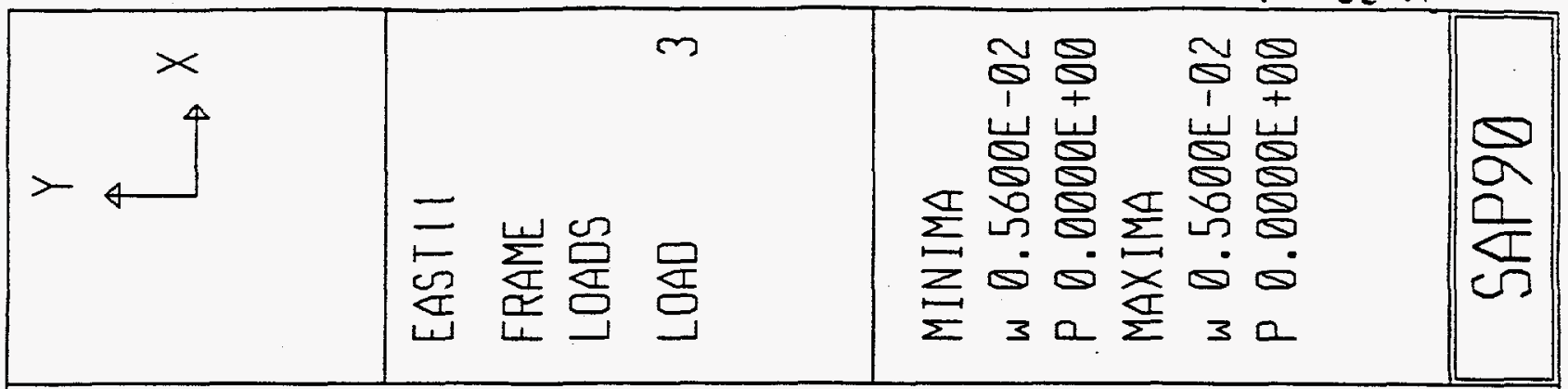


(axe. s-000i, 1e.5.0, Job $1, j-2$

$\dot{S}=\dot{i}=-$

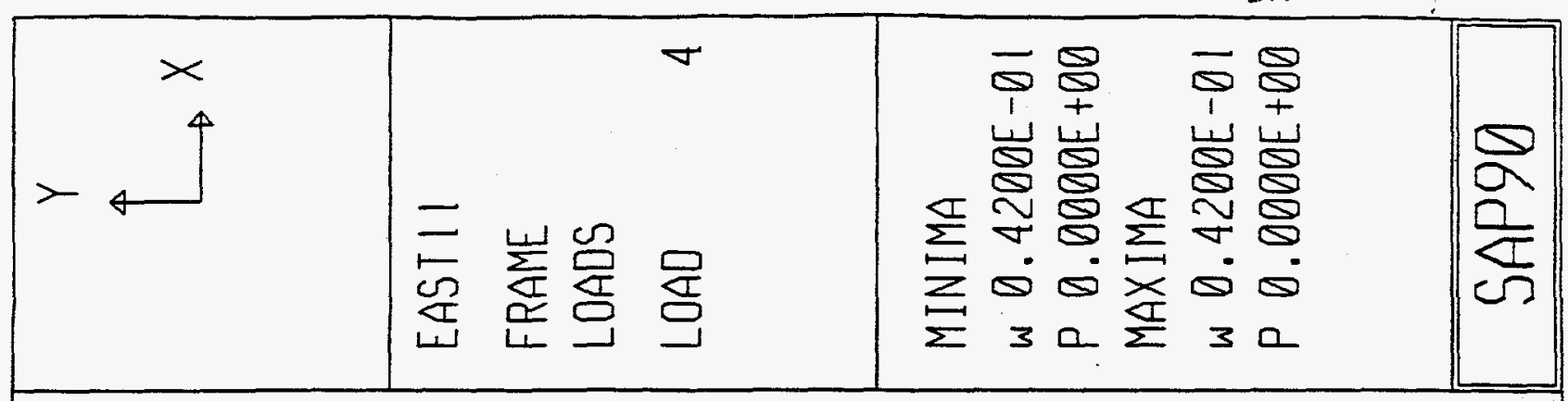




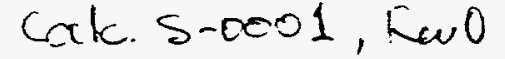
Job 41552

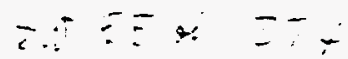

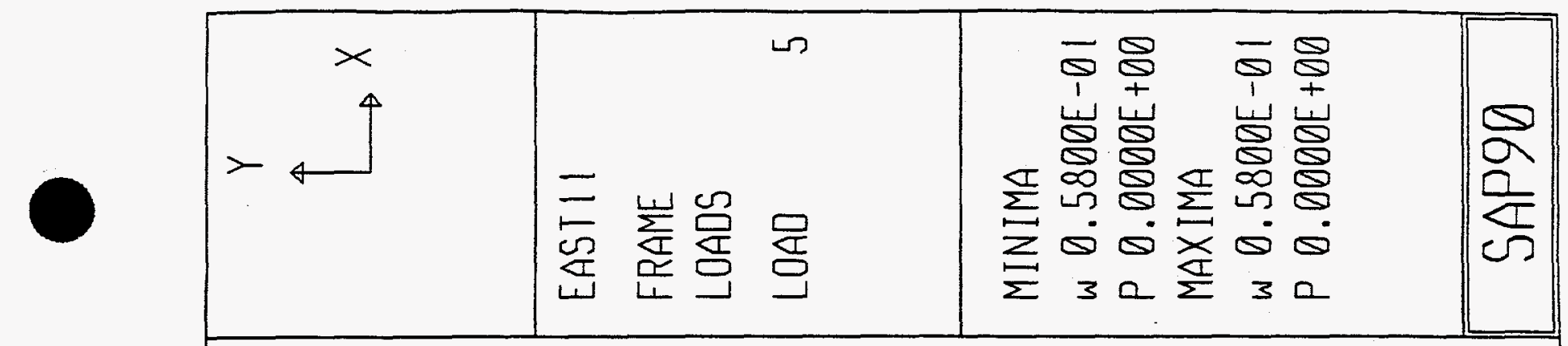




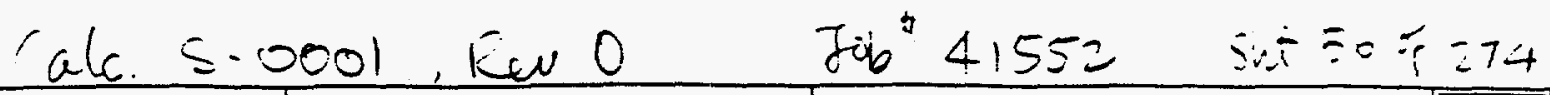

\begin{tabular}{|c|c|c|c|c|c|c|c|}
\hline $4_{4}^{x}$ & $\begin{array}{l}E \\
\sum \\
E\end{array}$ & 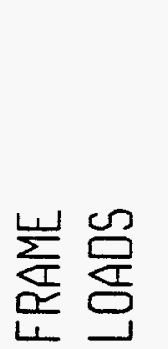 & $\infty$ & $\sum_{\sum}^{\sum}$ & 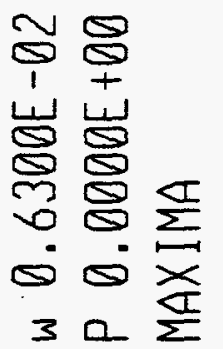 & 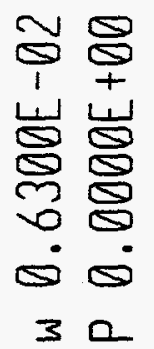 & $\sqrt{2}$ \\
\hline
\end{tabular}


$\mathrm{Calc}^{-1} \mathrm{~S}-0001$, Rew. O

Tod

$4155=\quad \therefore t=-\alpha=-$

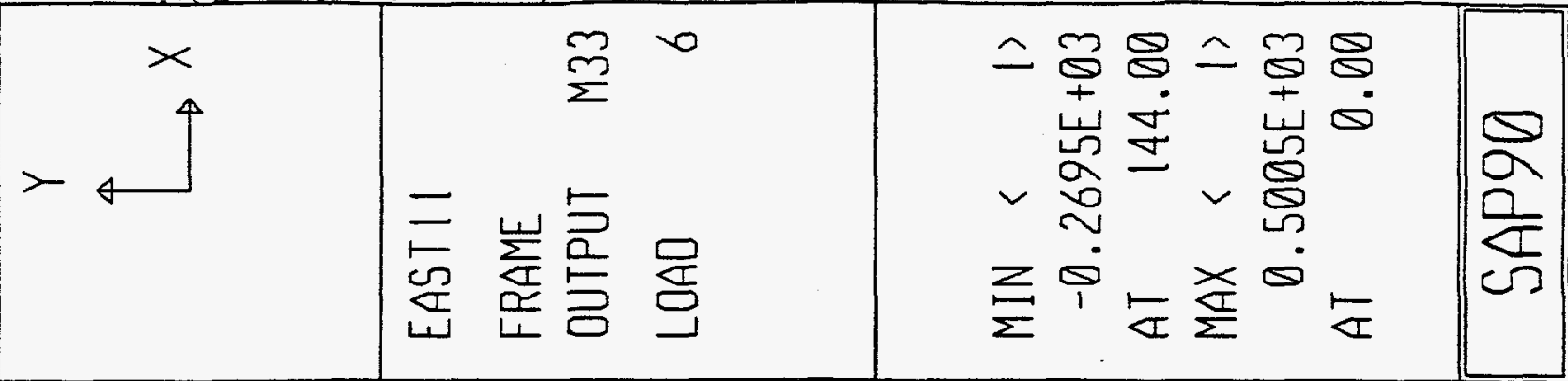




\begin{tabular}{|c|c|c|c|}
\hline Cale & Fu. 0 & ${ }^{2} b^{*} 4155=$ & \\
\hline$>\quad \begin{array}{r}x \\
4\end{array}$ & 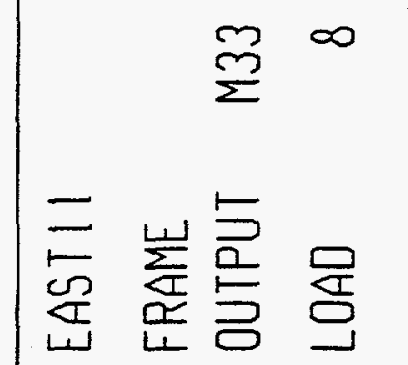 & 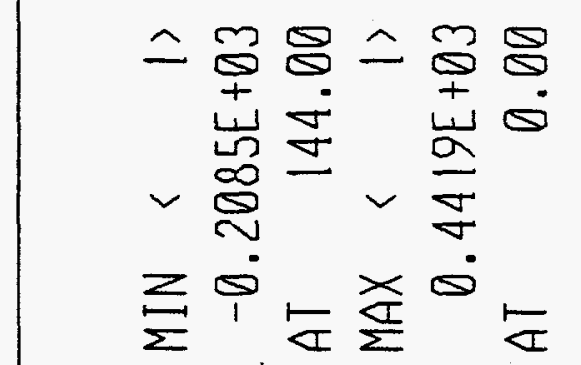 & $\frac{\widehat{\partial}}{\frac{\sigma}{\sigma}}$ \\
\hline
\end{tabular}


late S-soo1, ful 0 jok $4155=$

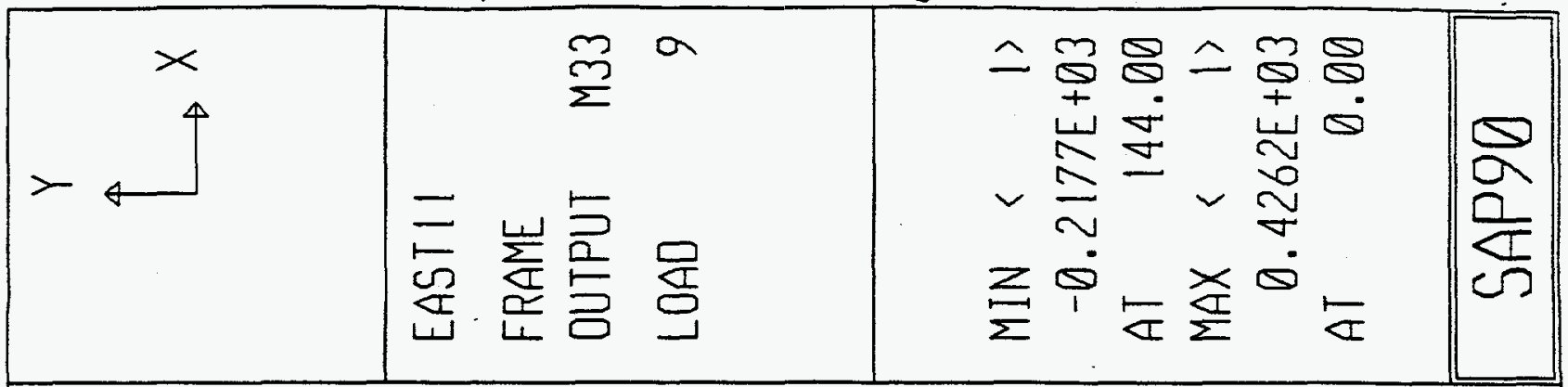


Calc S-D001, Revo job $4155=$ Sht ol os:-1

\begin{tabular}{|c|c|c|}
\hline$L_{4}$ & 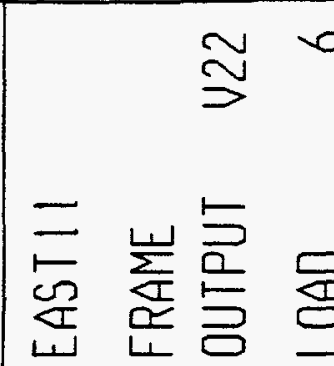 & 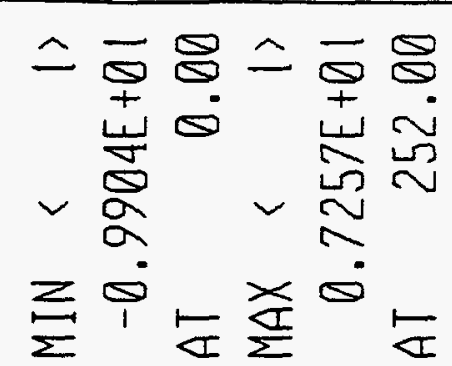 \\
\hline
\end{tabular}

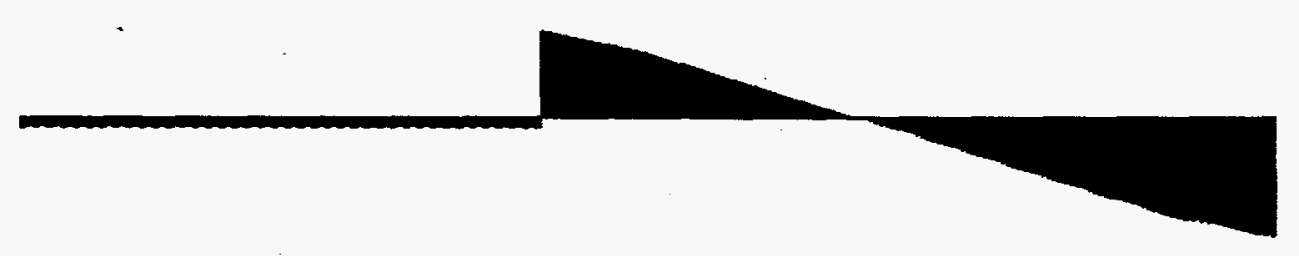


Calc. $\leqslant-0001$, Fw. 0 Fob $4155=$ sht $t=0$ t. $=-\cdot$

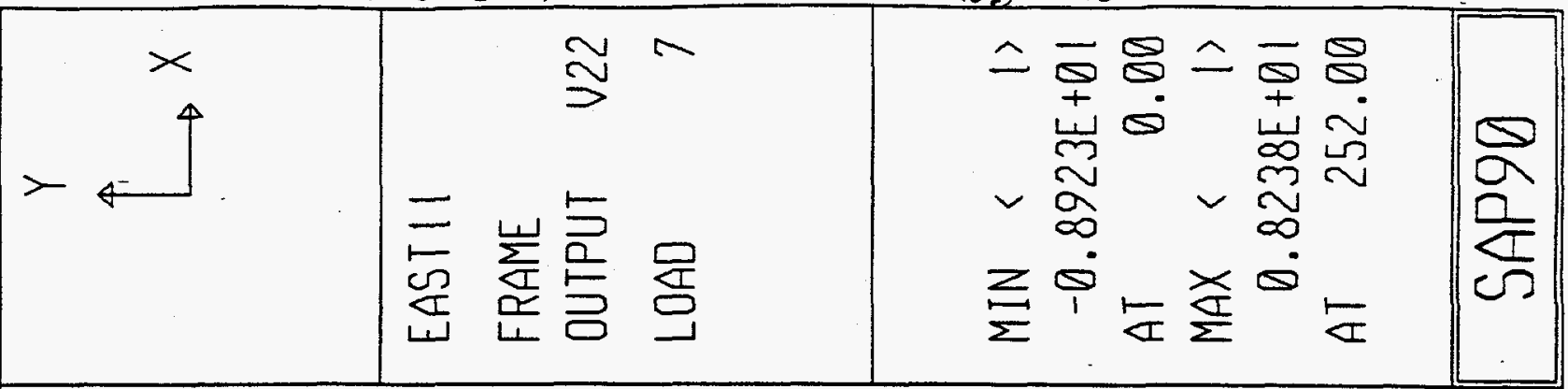

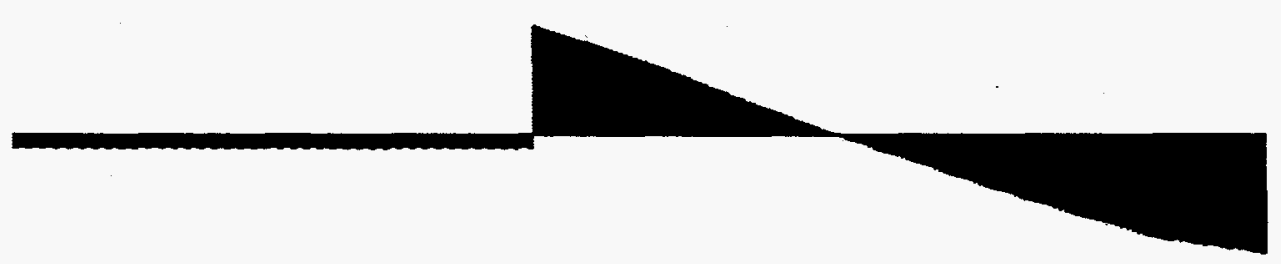


Calc S-0001, Rev 0 Job $4155=$ sht $636=-1$

\begin{tabular}{|c|c|c|}
\hline$>\leftarrow$ & 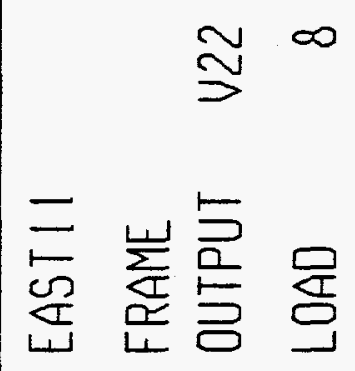 & 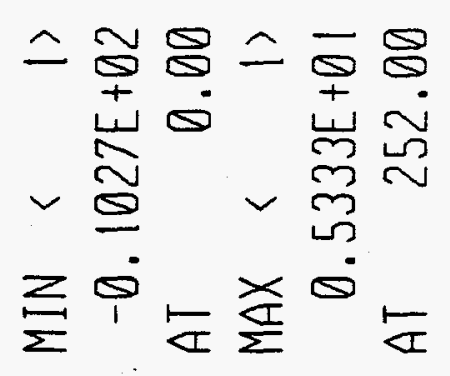 \\
\hline
\end{tabular}

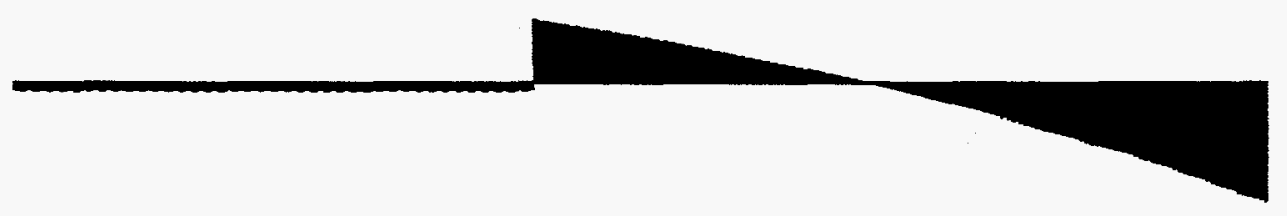


Cale. S-0001. Rev 0 Jok $4155=$ $i h^{+}-4$ y. $=-$

\begin{tabular}{|c|c|c|}
\hline$>\longleftarrow$ & 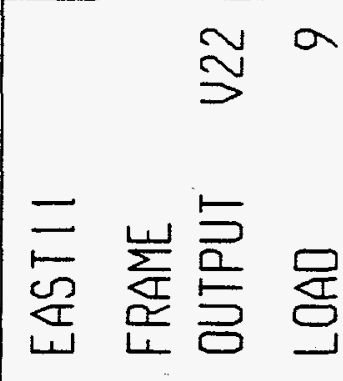 & 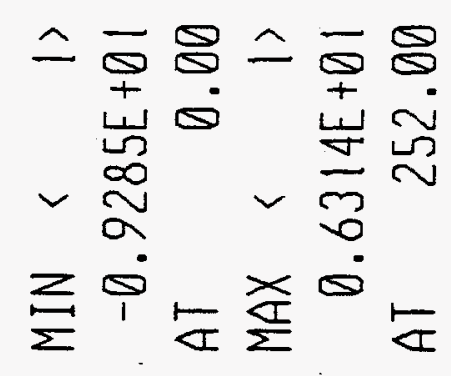 \\
\hline
\end{tabular}

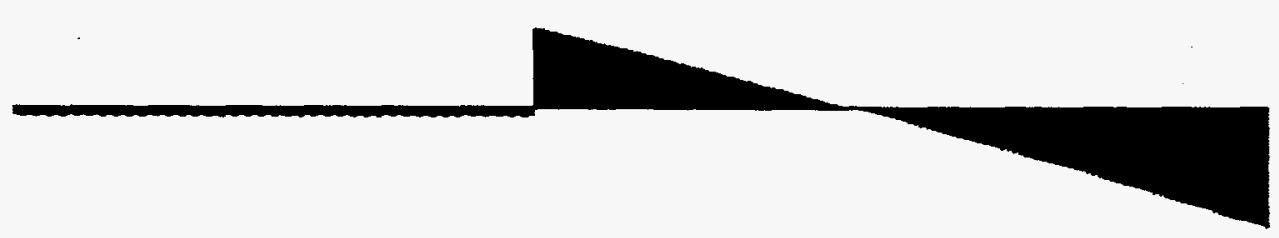




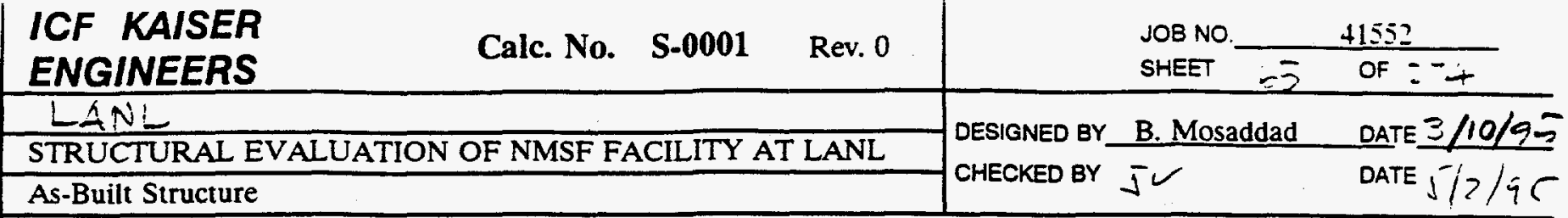

5.0 THREE DIMENSIONAL DYNAMIC ANALYSIS

\section{C5.1 3-DIMENSIONAL MODEL DEVELOPMENT}

The 3-D model is constructed using shell element to represent the major exterior and interior walls of the NMSF building. Several different versions of the model was utilized in this evaluation are described below.

\section{NMSF0:}

This model represent the fixed base configuration of the building without consideration for the surrounding soil, embedment and lateral soil pressure. The mass of building is included as a distributed mass density of the shell elements. This model was used to compute fundamental natural frequencies and the mode shapes of the fixed base structure.

\section{NMSF01:}

This version of the model included soil springs representing the foundation impedance functions. This model was used to compute the fundamental natural frequencies and the mode shapes of the building taking into account the SSI effects.

\section{NMSF41:}

This is the version of the model that was use to carry out the final set of dynamic analyses used in the structural evaluation of the building.

The following features are added to the NMSF01 model to create NMSF41:

1) The floor masses, including the tributary wall masses, are calculated for each floor and placed at a distance equal to $5 \%$ of the building dimensions away from the center of rigidity to account for accidental torsion loads.

2) The floor mass, located at the floor levels are connected to the corresponding wall nodes using rigid links, simulating a rigid diaphragm at basement, 1st floor and roof elevations.

3) The static and dynamic lateral soil pressures $(\mathrm{Hs}+\mathrm{Hd})$ are applied at the exterior walls in the West and North sides. The passive soil pressures on the east and south walls are neglected, conservatively.

4) This model was used to compute the member stresses due to seismic load combinations: 


\begin{tabular}{|l|}
\hline LANL \\
\hline STRUCTURAL EVALUATION OF NMSF FACILITY AT LANL
\end{tabular}

As-Built Structure

$$
\begin{array}{ll}
\mathrm{D}+\mathrm{L}+\mathrm{Hs}+\mathrm{Hd}+\mathrm{E} \\
\text { and } & \mathrm{D}+\mathrm{L}+\mathrm{Hs}+\mathrm{Hd}-\mathrm{E}
\end{array}
$$

NMSF42:

This model, essentially the same as NMSF41, was used to carry out analysis for the static load case of:

$$
1.4 \mathrm{D}+1.7 \mathrm{~L}+1.7 \mathrm{Hs}
$$




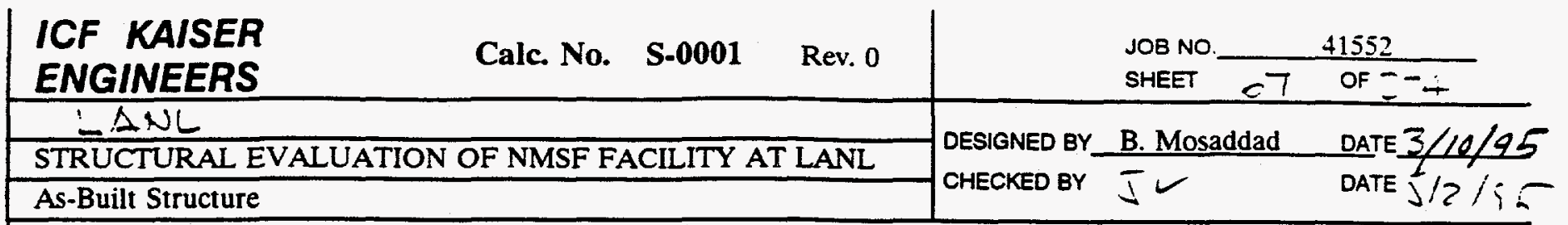

5.2 FIXED BASE MODEL

i: 


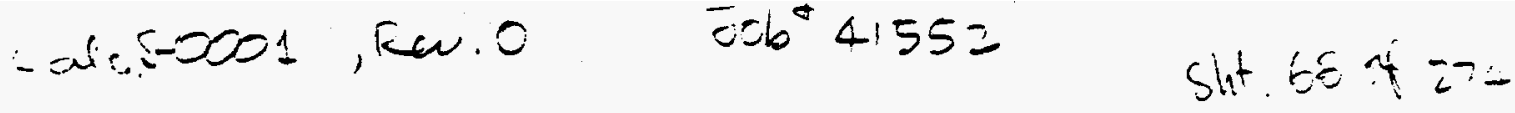

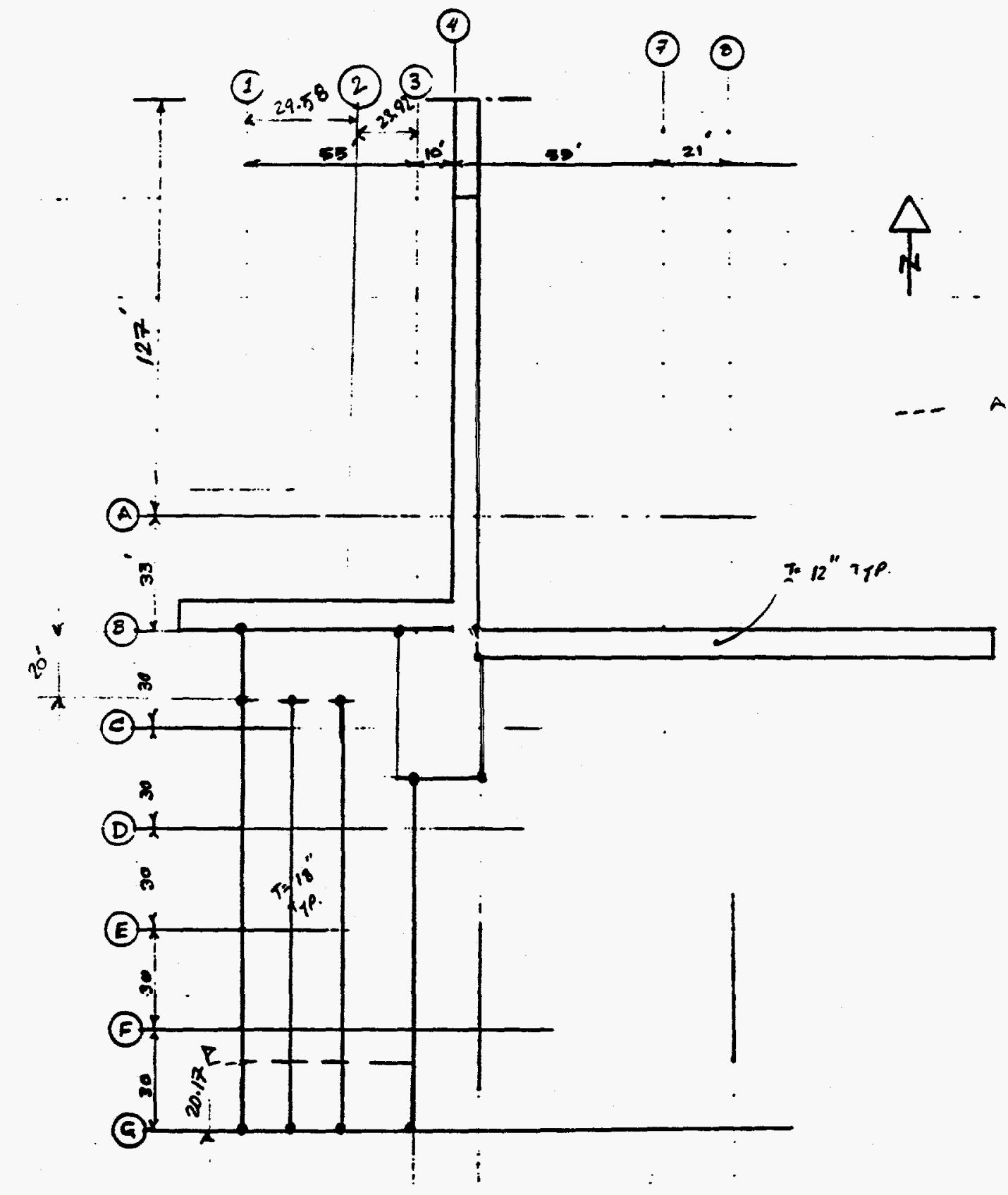

$\frac{\text { Easement \& Tunnel Floor }}{\text { ELEV. }}$ 
$C_{x l c} \leqslant-c 021$, Rev.0 job 41552

sint $6: 20=7$

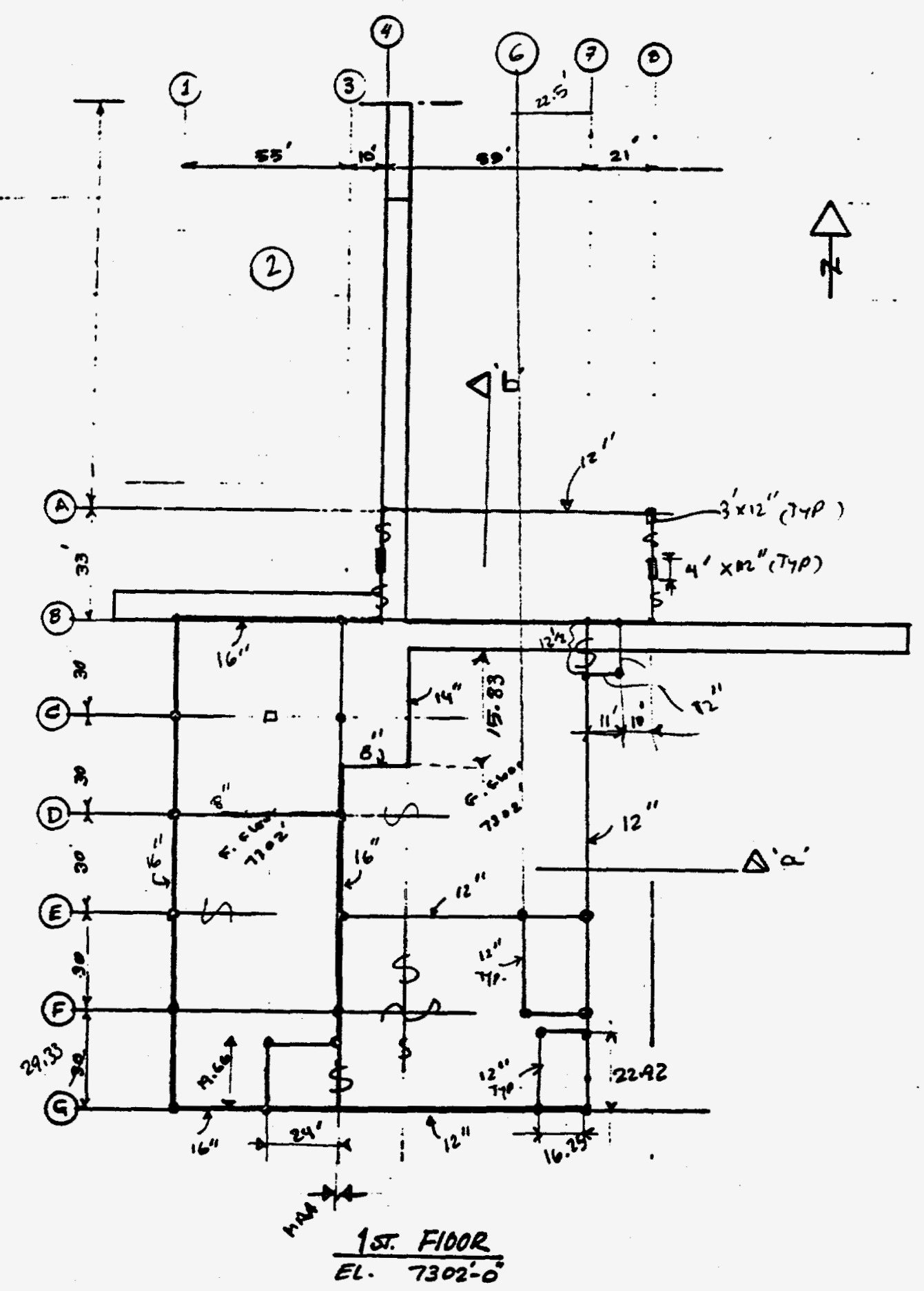


Calc. $\leqslant-5001$, Rew $0 \quad J 0 b^{\frac{7}{4}} 4155=\quad$ Sht .70 si : $: 74$

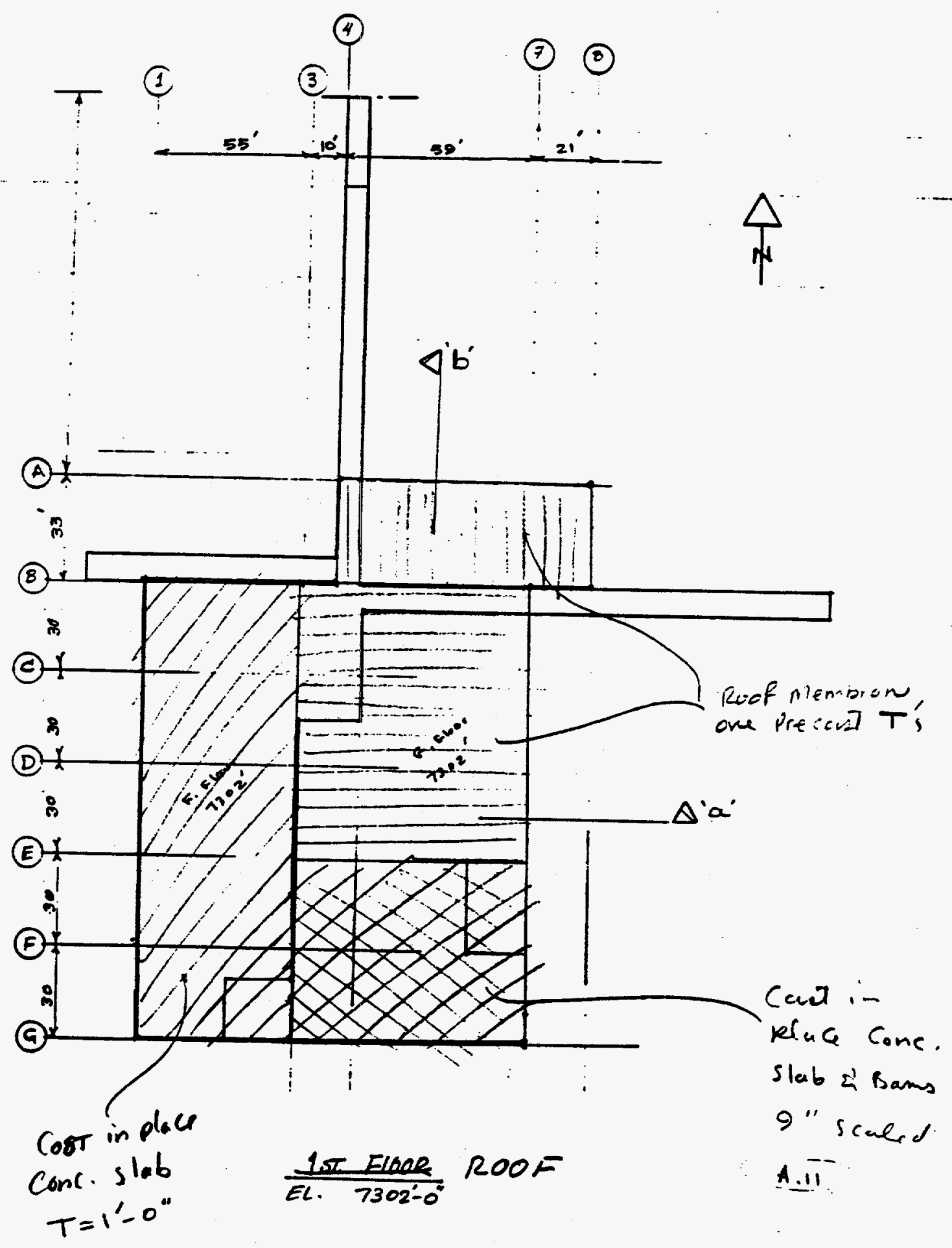


Calc. $s-5001$, Lew. 0 , Tob $4155=$

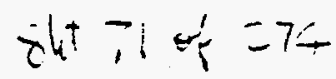

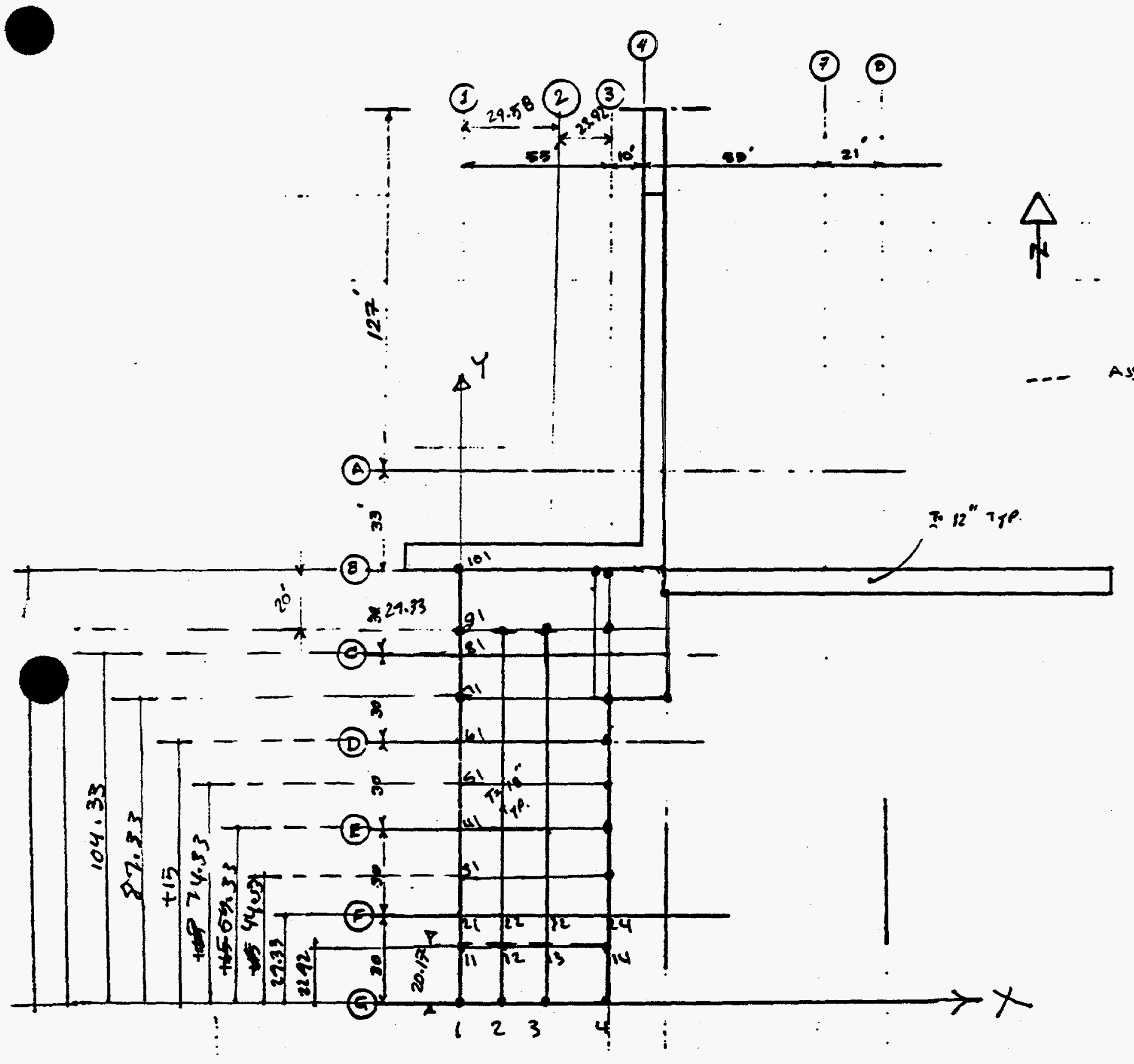

$$
\frac{\text { Besement \& Tunnel Floor }}{\text { ELEV. }}
$$

Badement - Foundation $\rightarrow 1000 \longrightarrow 1999$ To Ist fles 1ST Flom $\rightarrow$ Roof 2000 


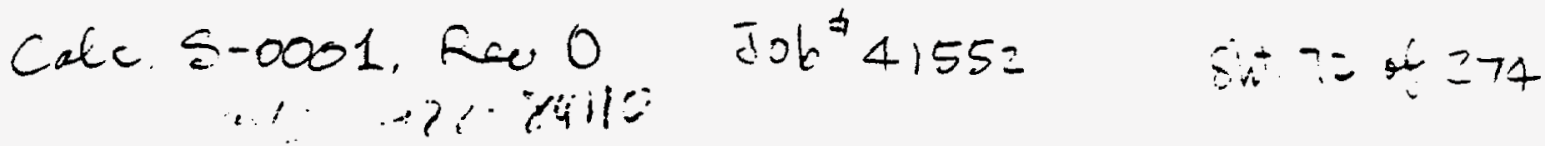

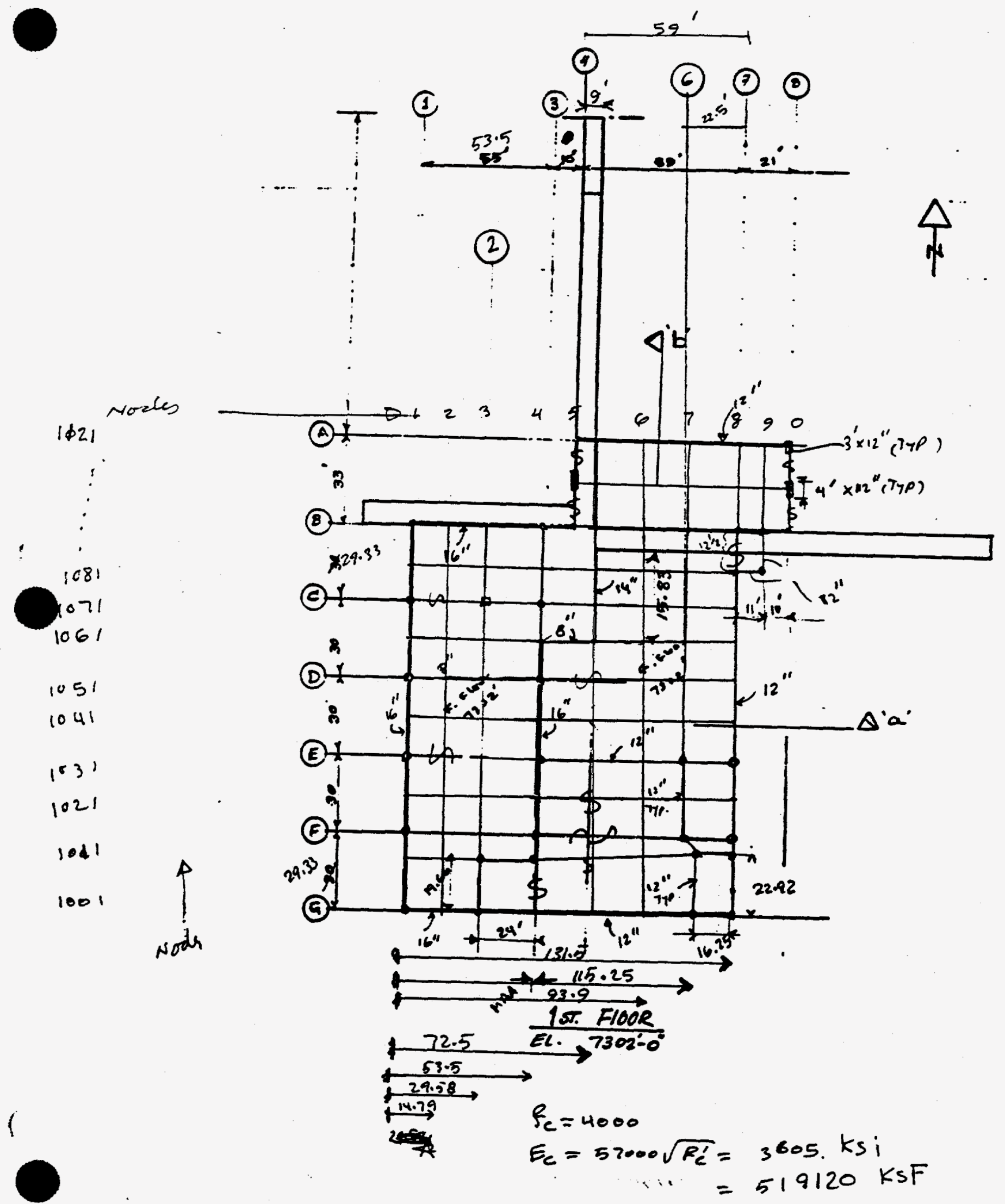




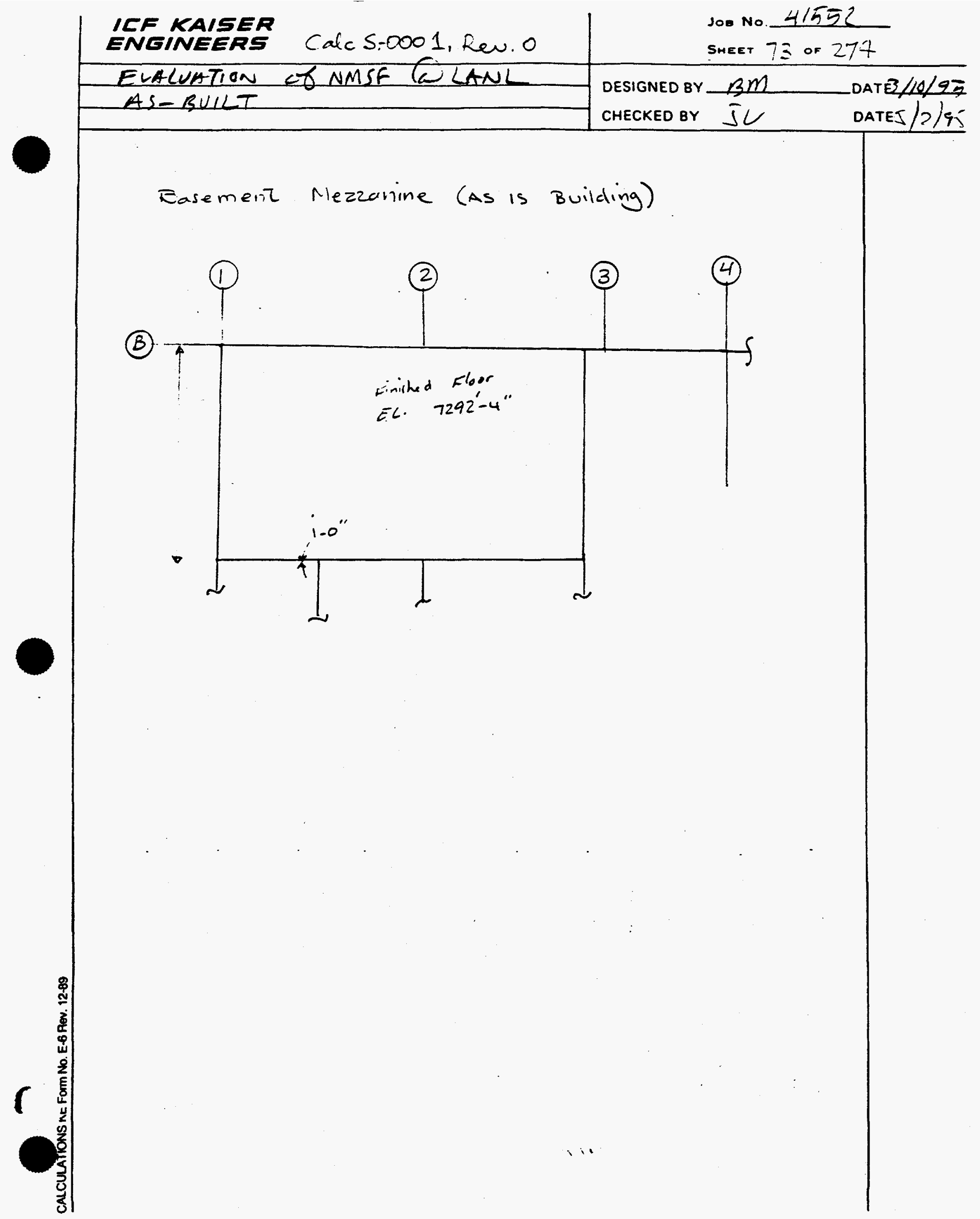



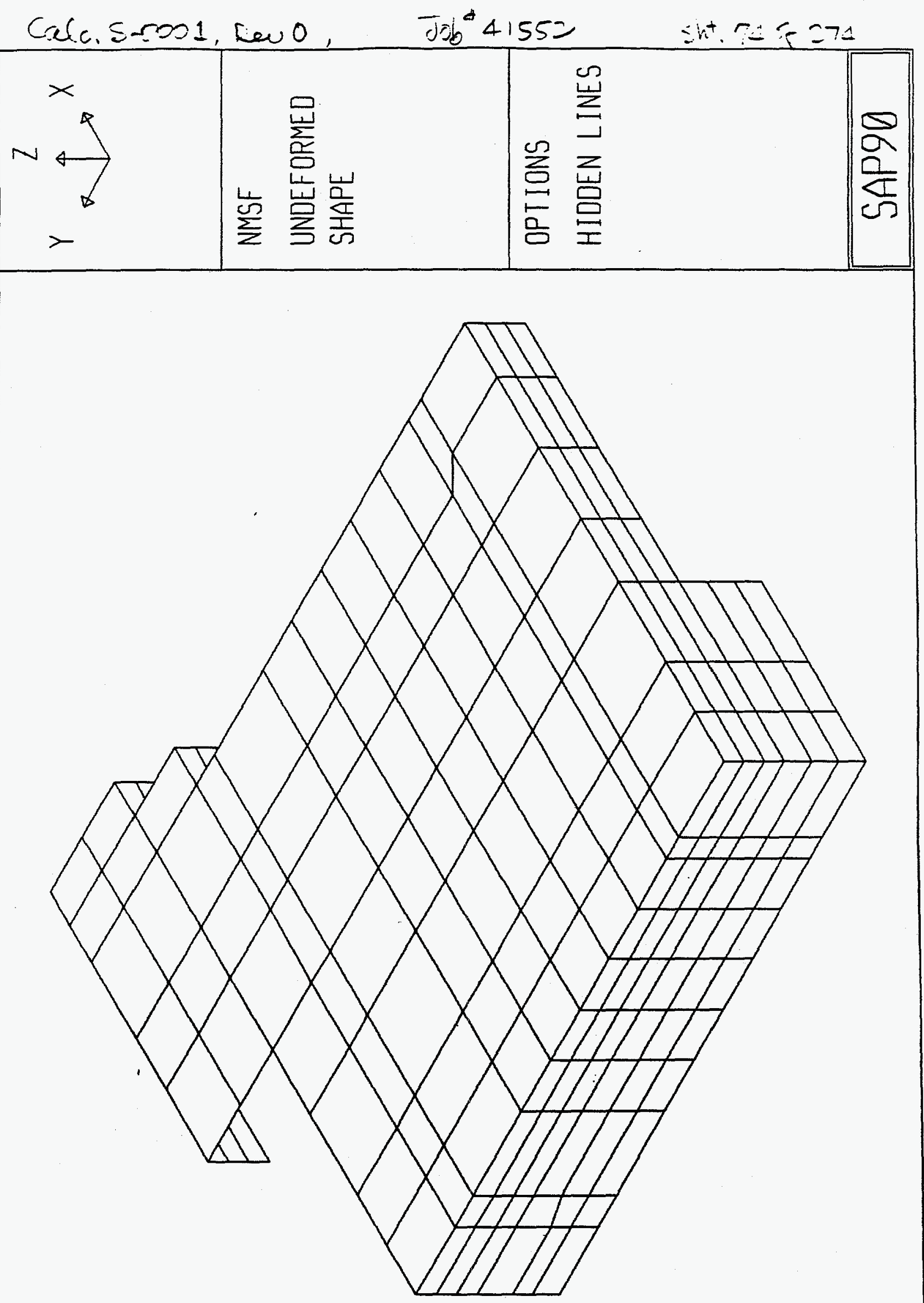
Calc. Stcos1, Fue. 0 Job 41552 is 75 st $=74$

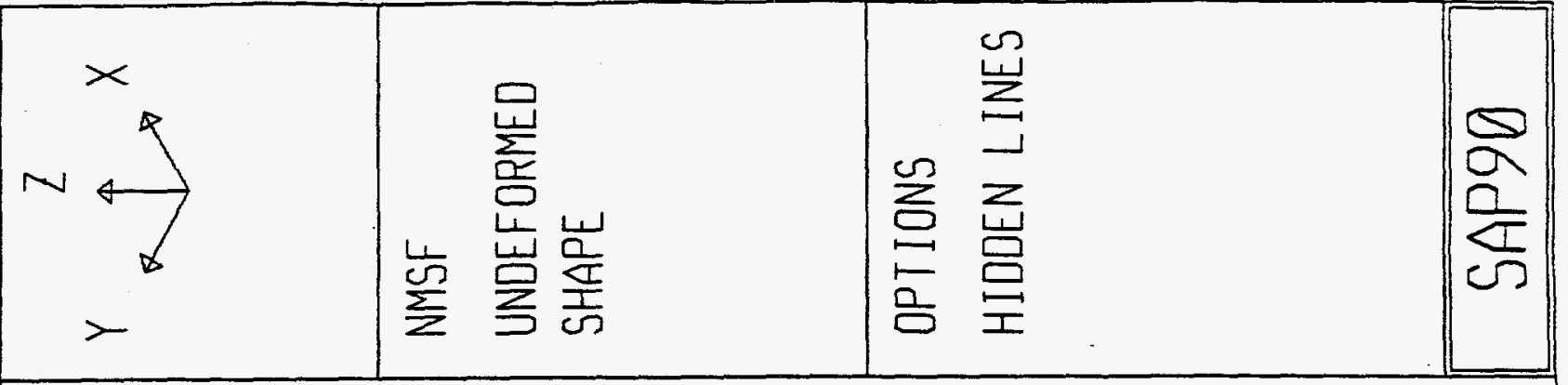

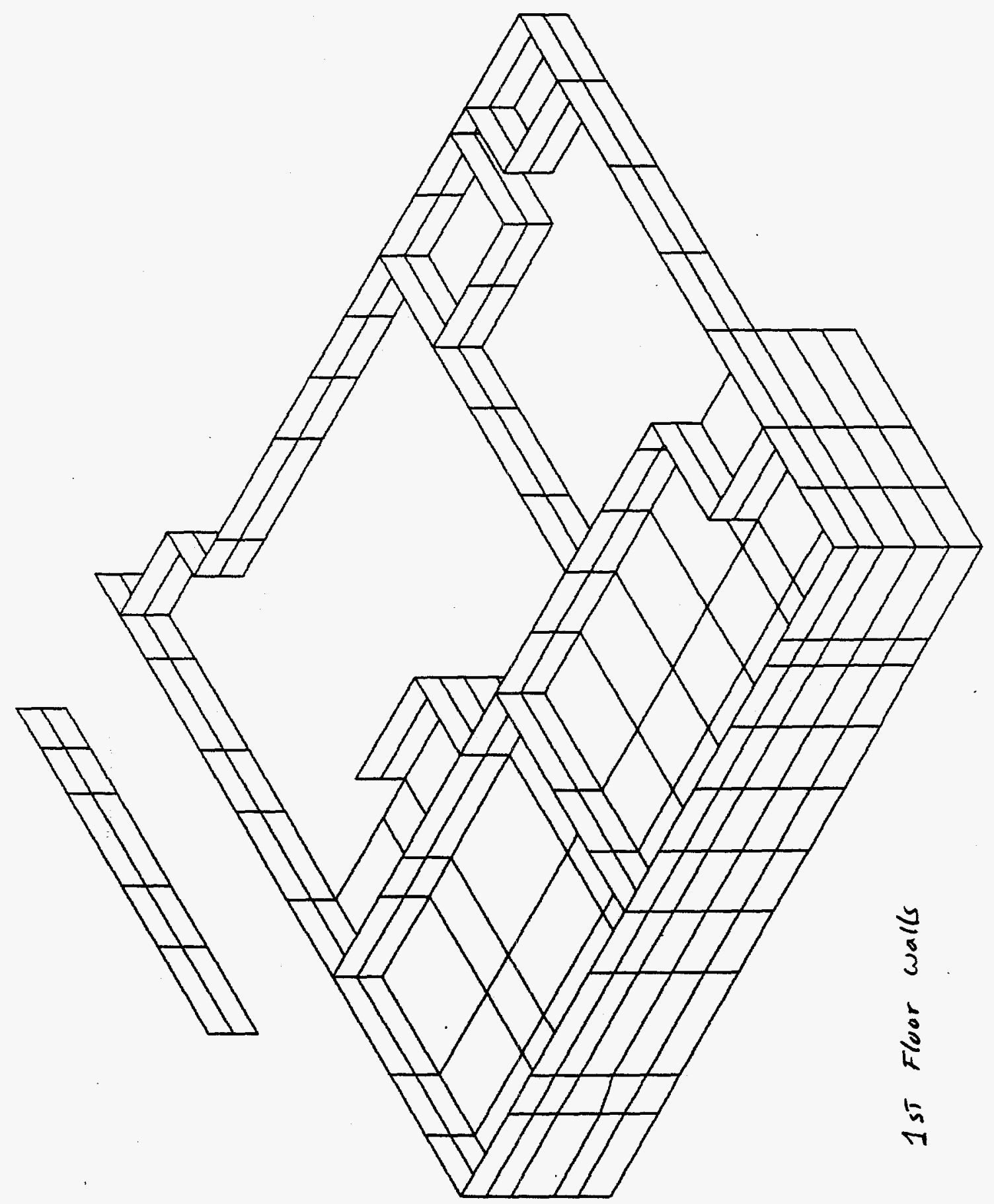


Calc. S-0001, far. $0 \quad$ job" $41552 \quad$ int.70 of $=71$

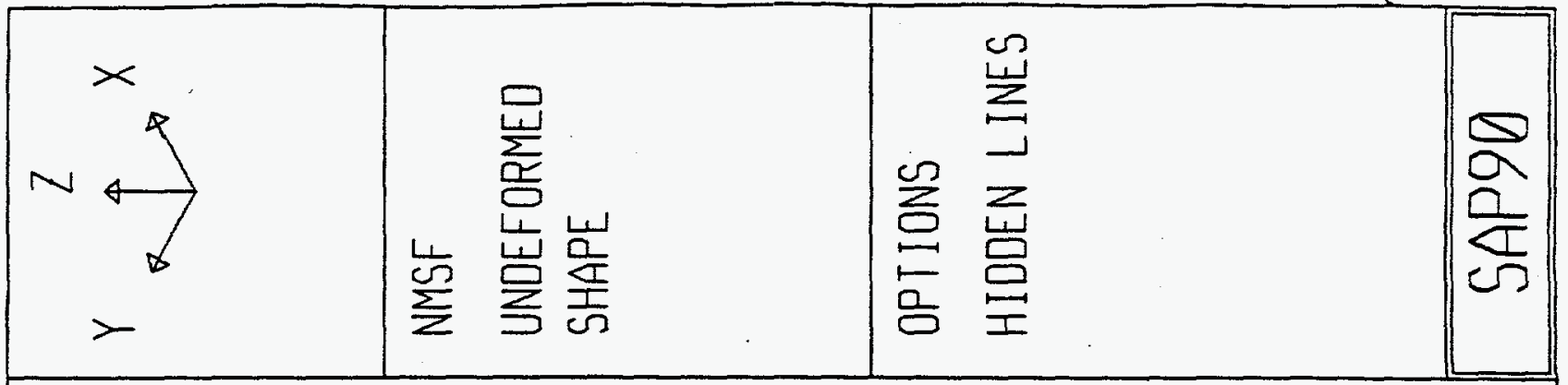

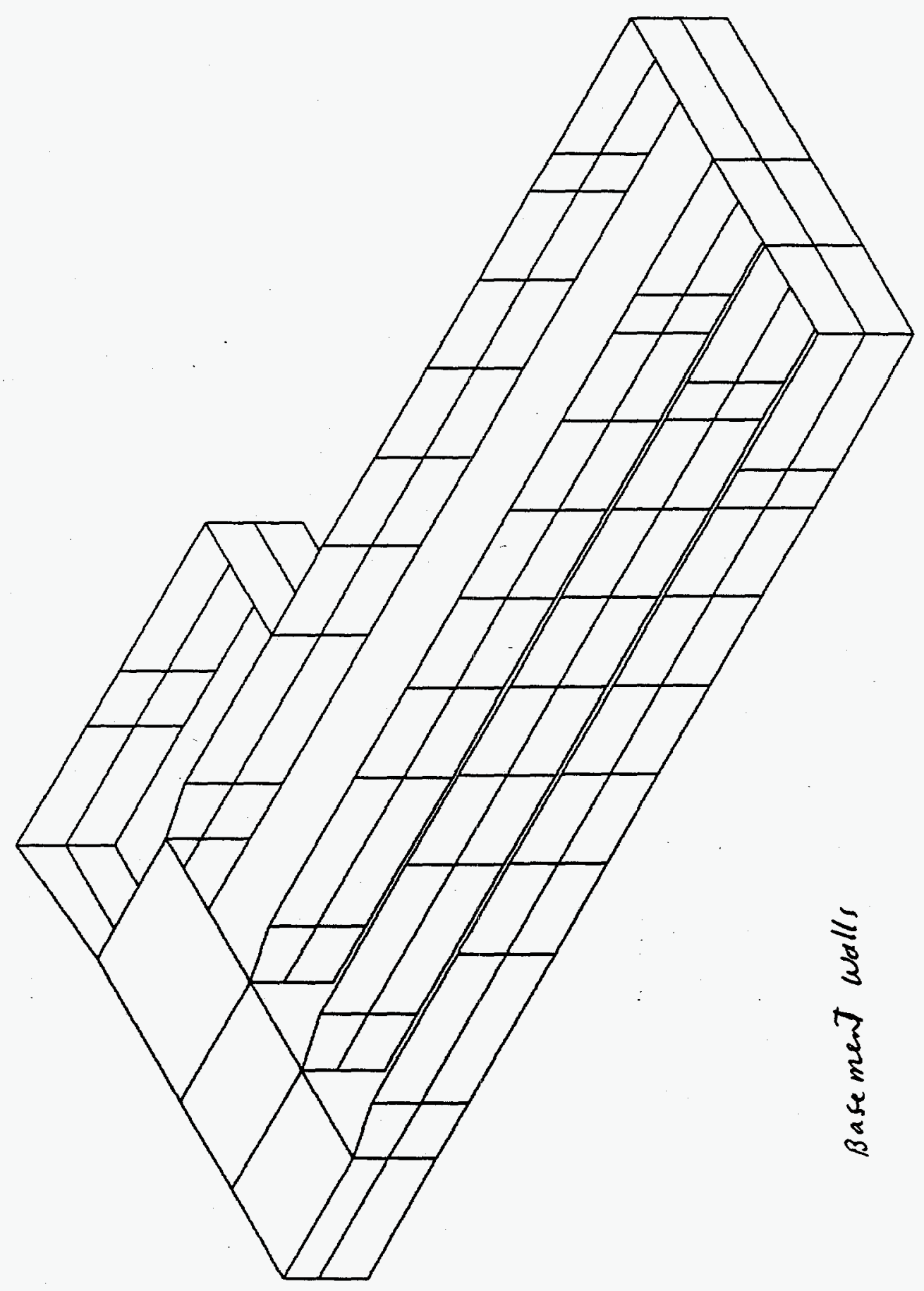

ara 


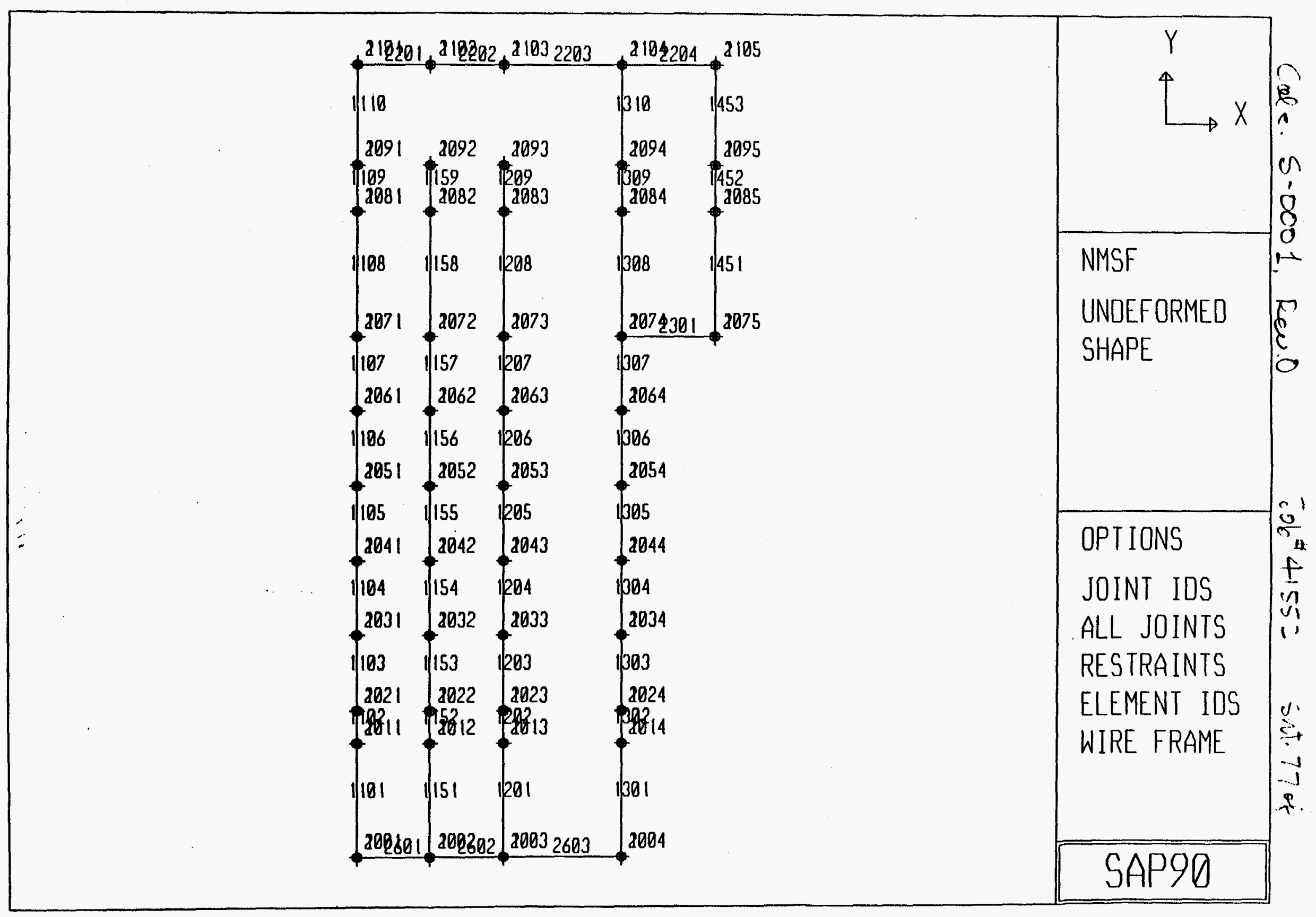




\section{$0^{-}$}

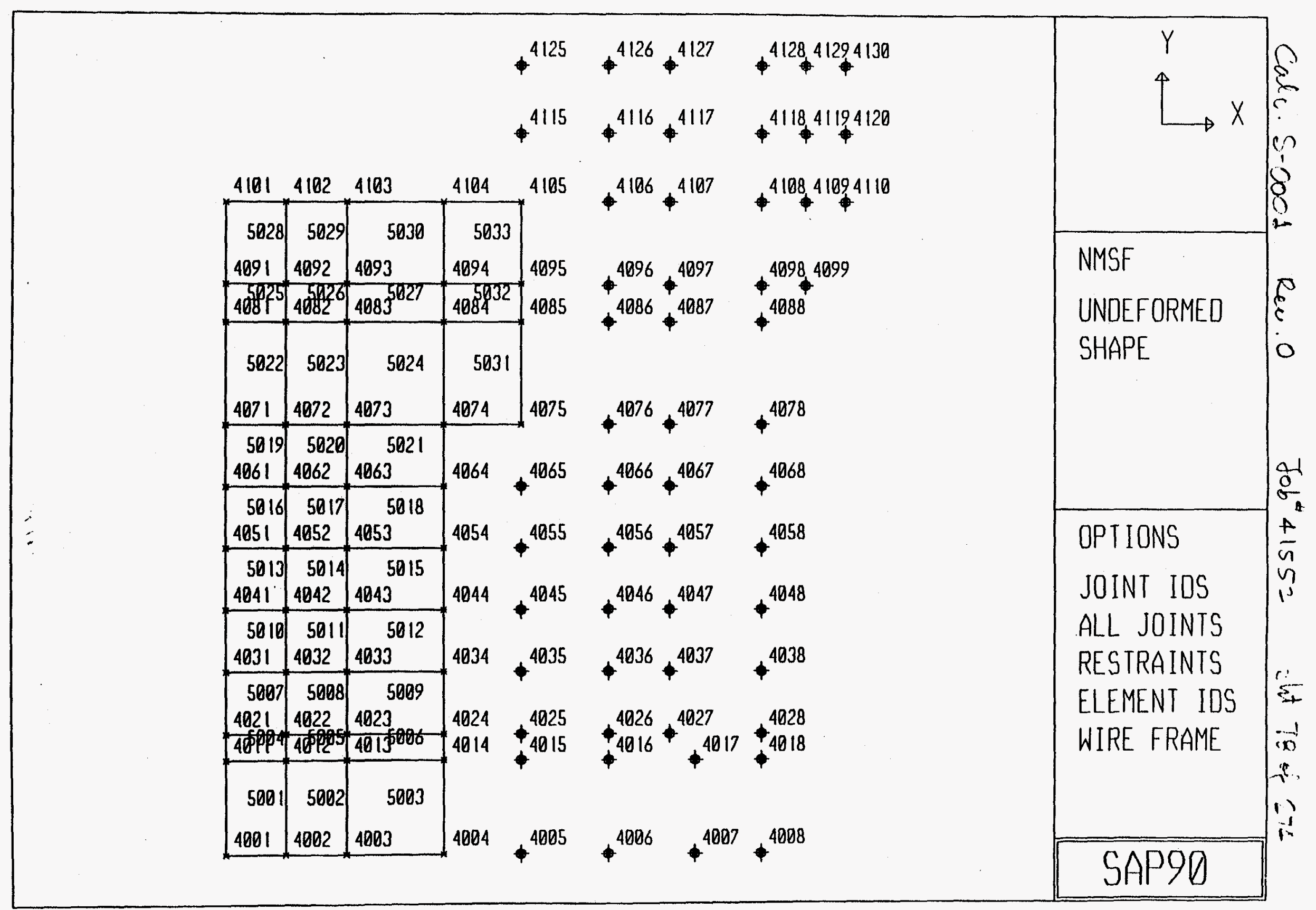


Calc $\leqslant-\infty 001$. hen. 0 $v_{0 b}=4155=$

Sint $70 \%=7$

\begin{tabular}{|c|c|c|c|}
\hline $\begin{array}{r}\times \\
+4\end{array}$ & 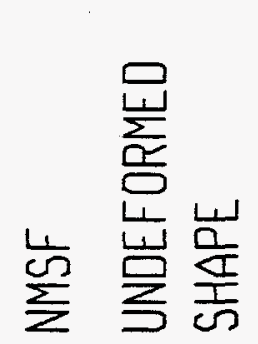 & 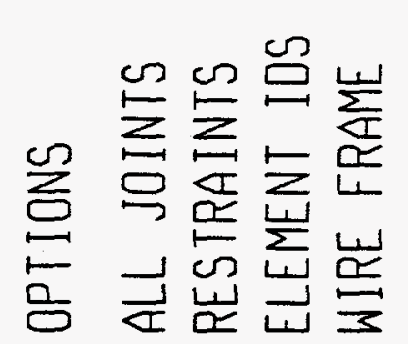 & $\sum_{\frac{1}{6}}^{\frac{1}{c}}$ \\
\hline
\end{tabular}

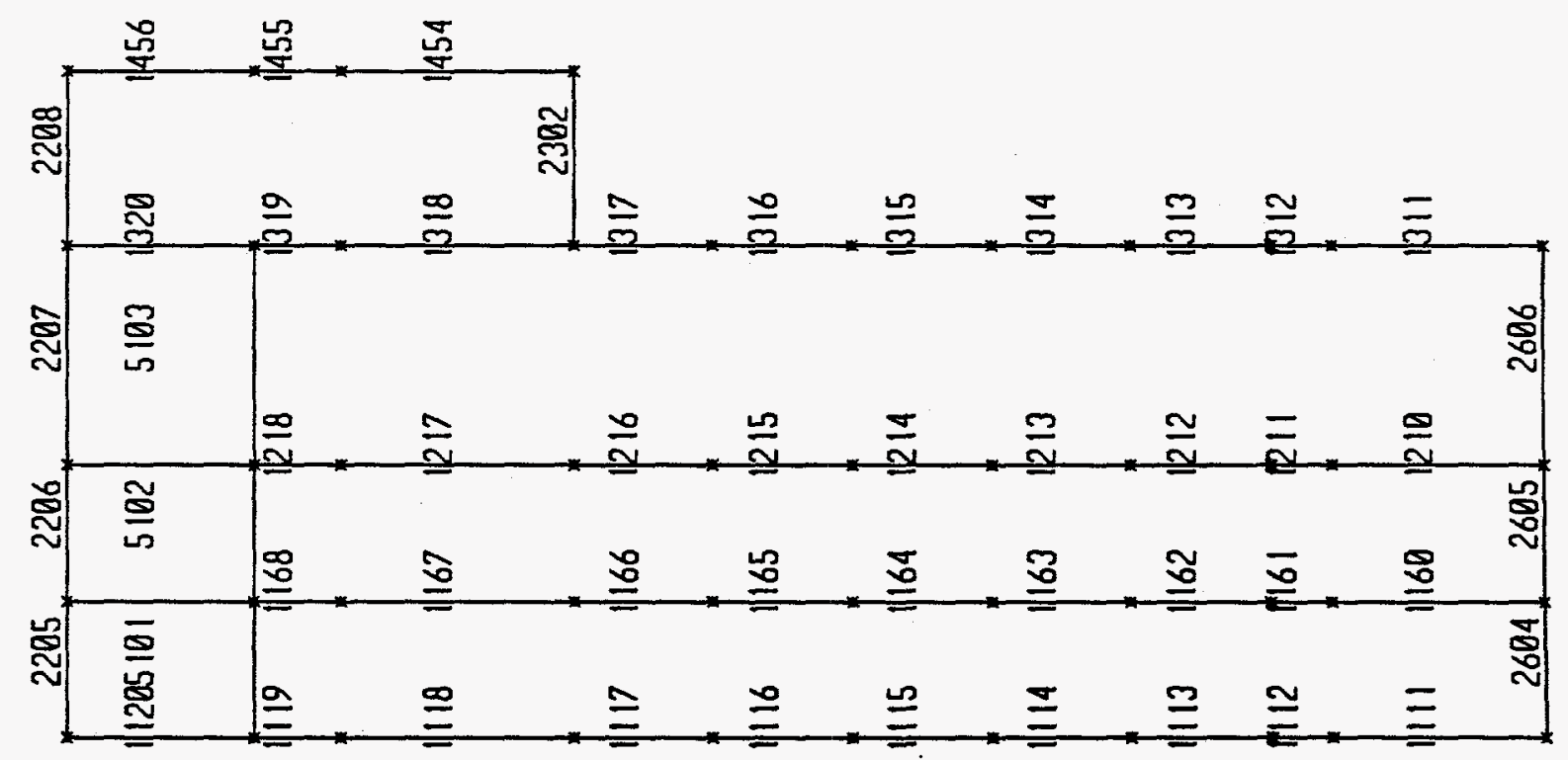


Calc. S-0001, few. 0

Tof $415=$

$\sin 500=?$

\begin{tabular}{|c|c|c|c|}
\hline $\begin{array}{r}x \\
+\quad 4 \\
\end{array}$ & 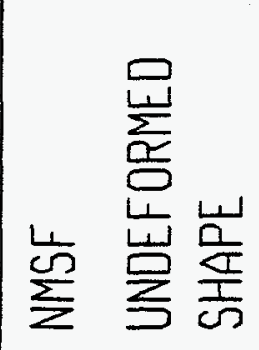 & 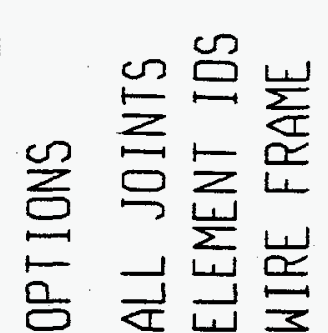 & $\mid \frac{5}{\frac{5}{6}}$ \\
\hline
\end{tabular}

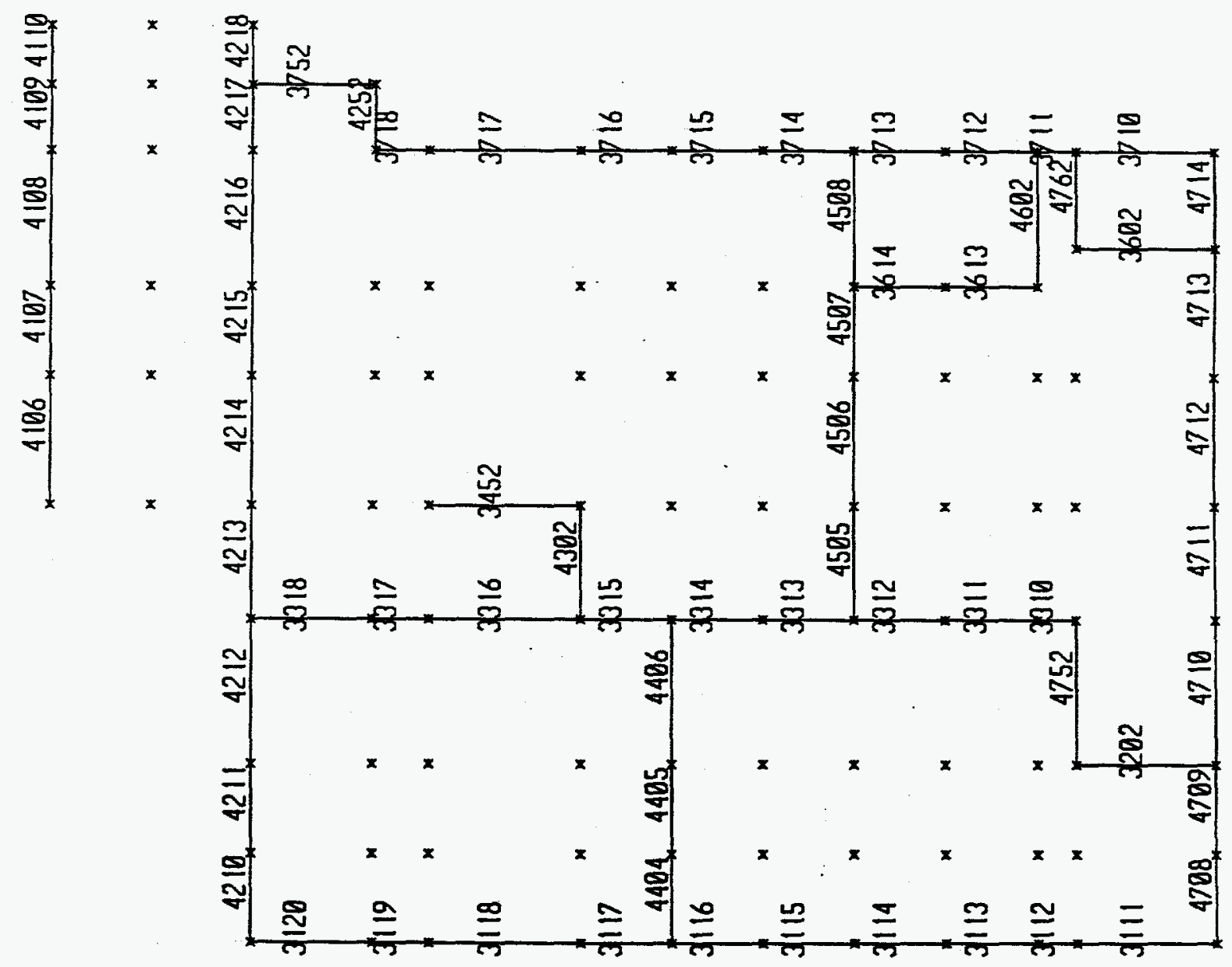



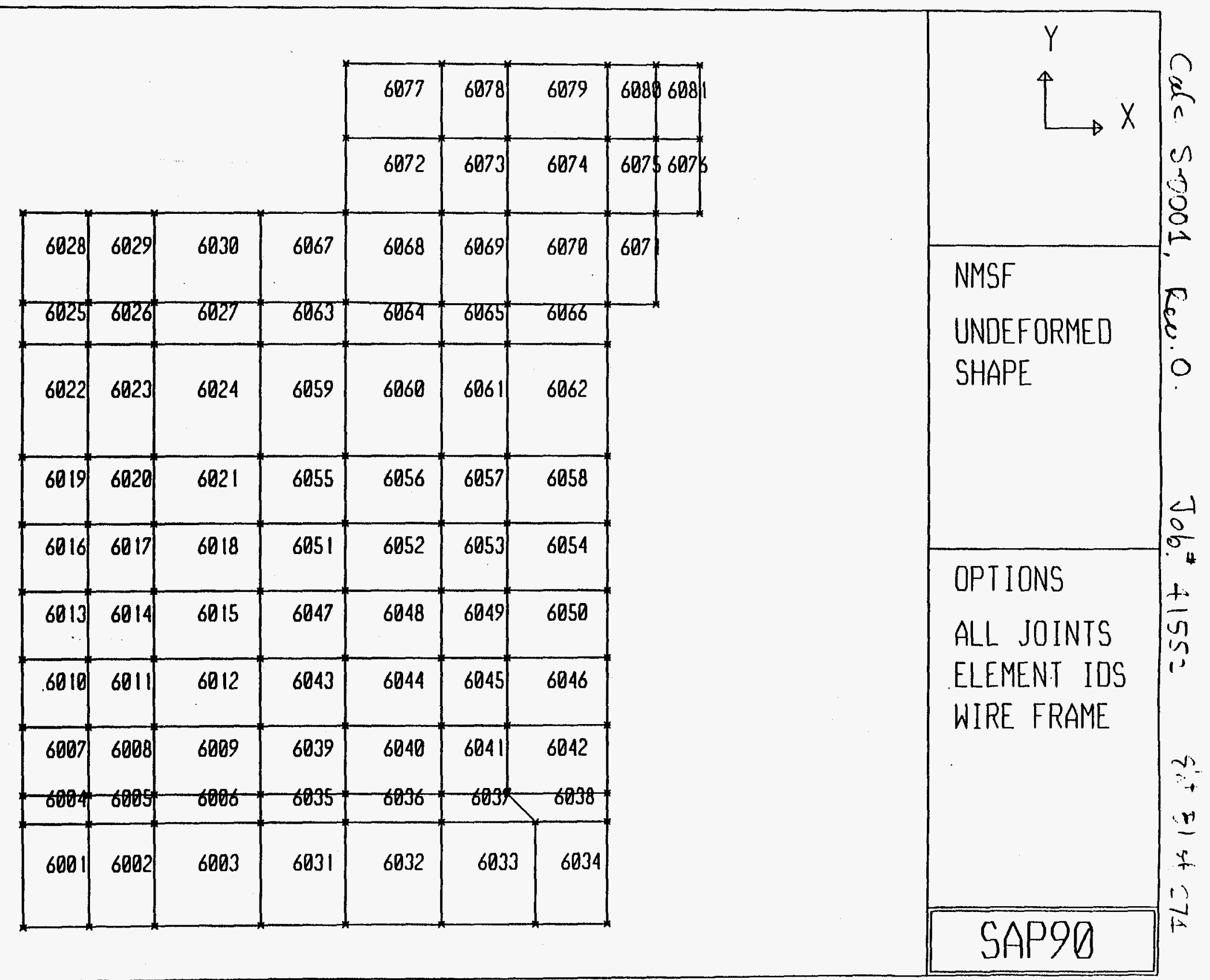


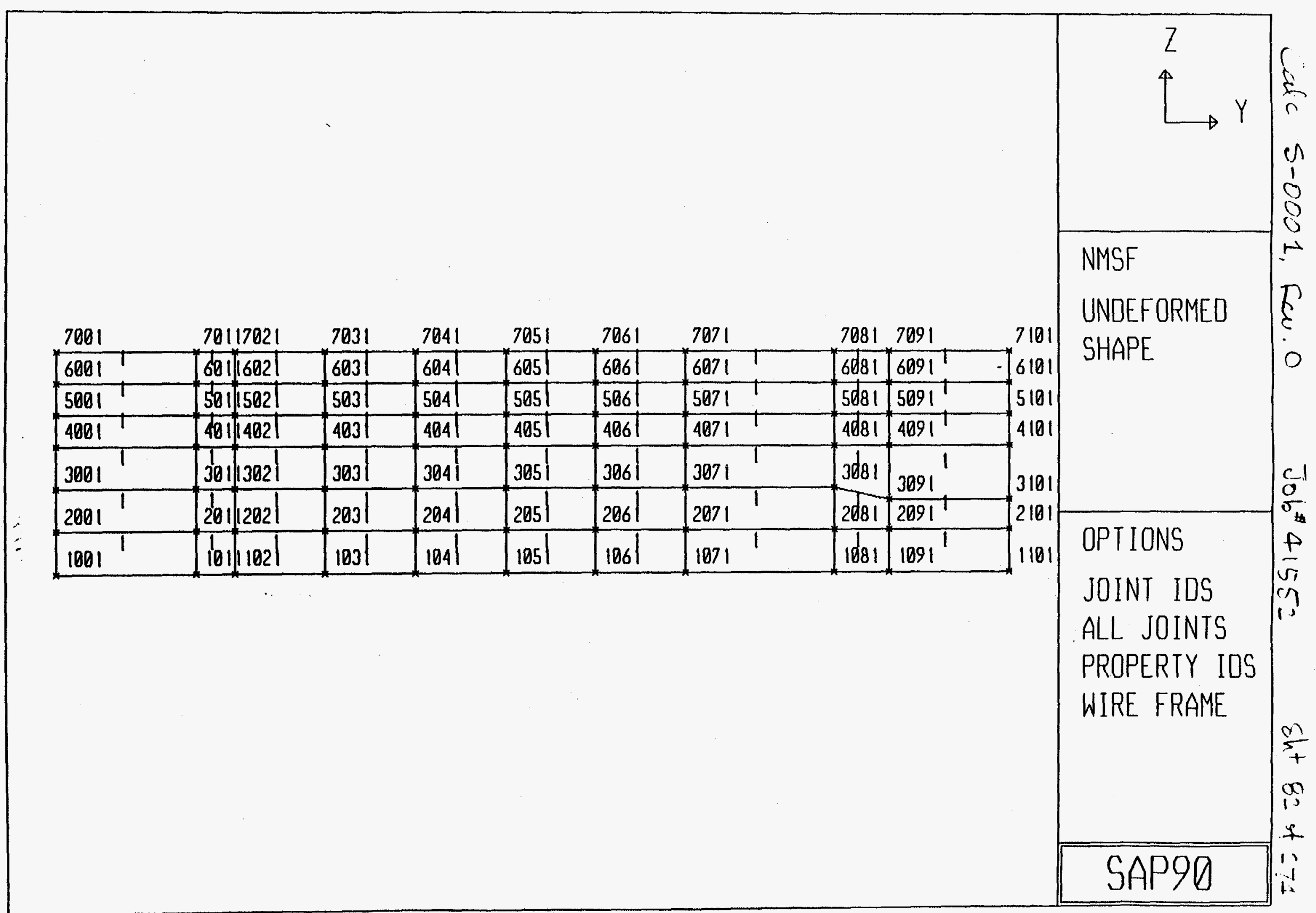




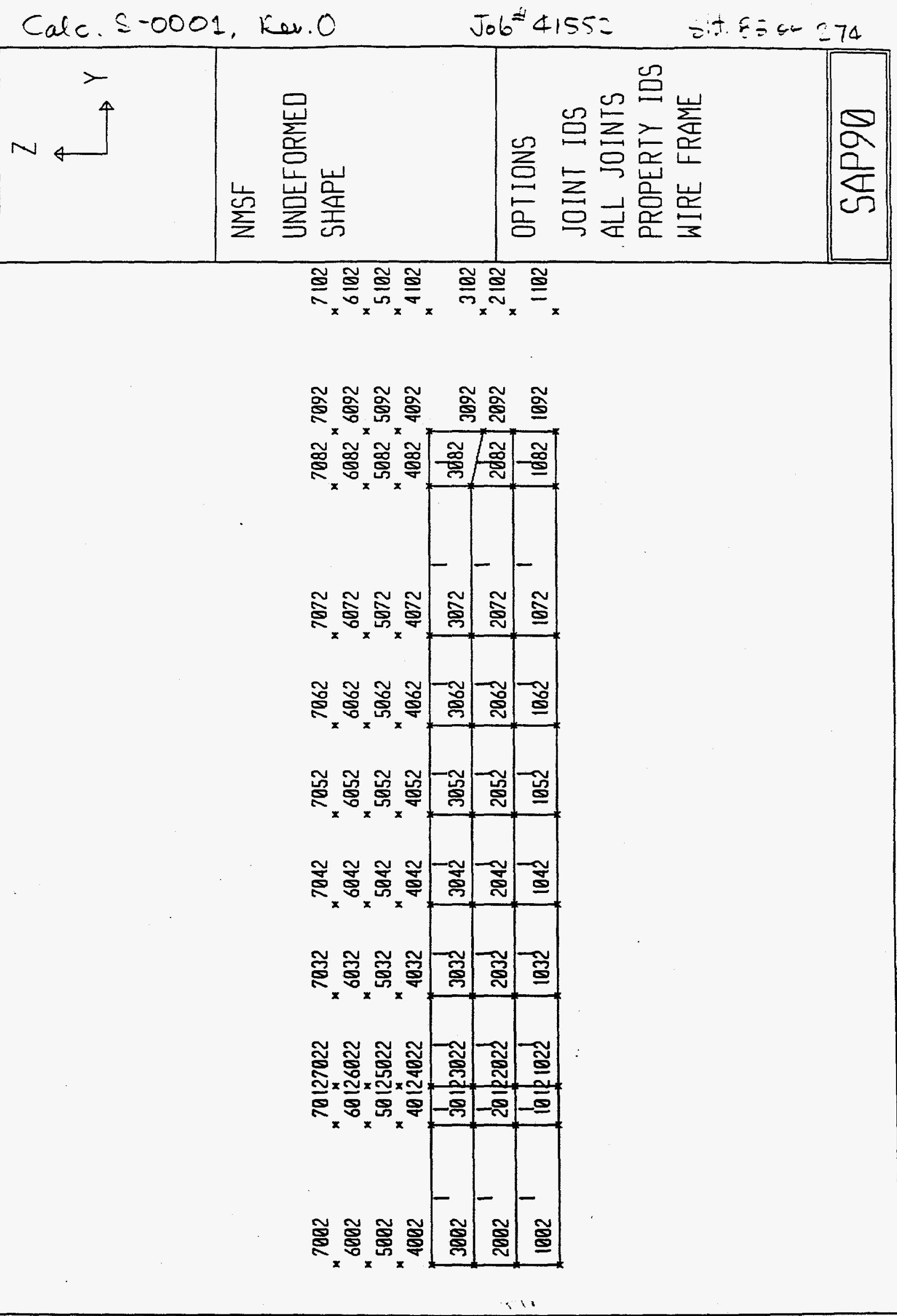


Calc $5-00=1$. Rev. $0 \quad$ Job $4155=$ Sht $84 \leqslant-71$

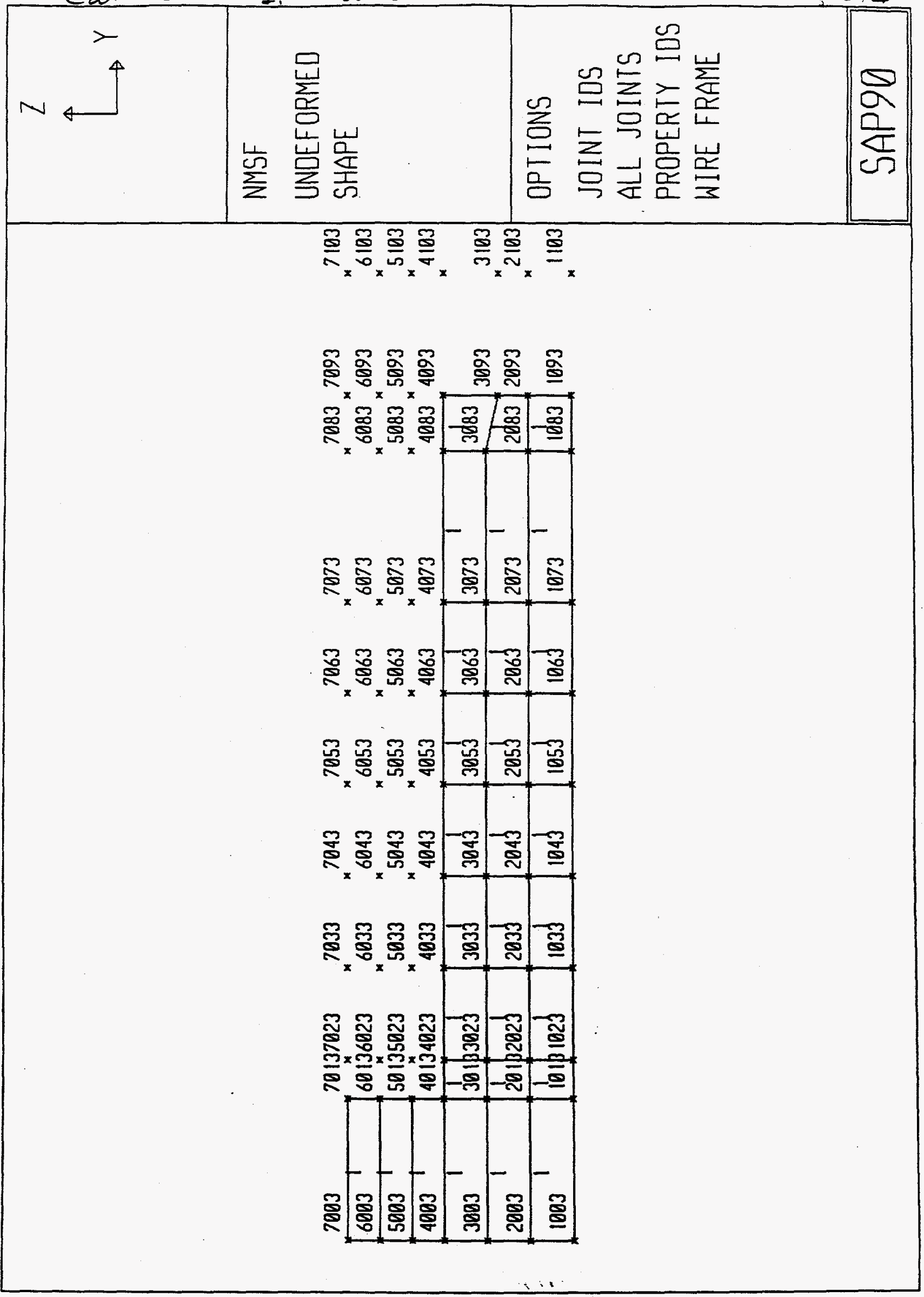




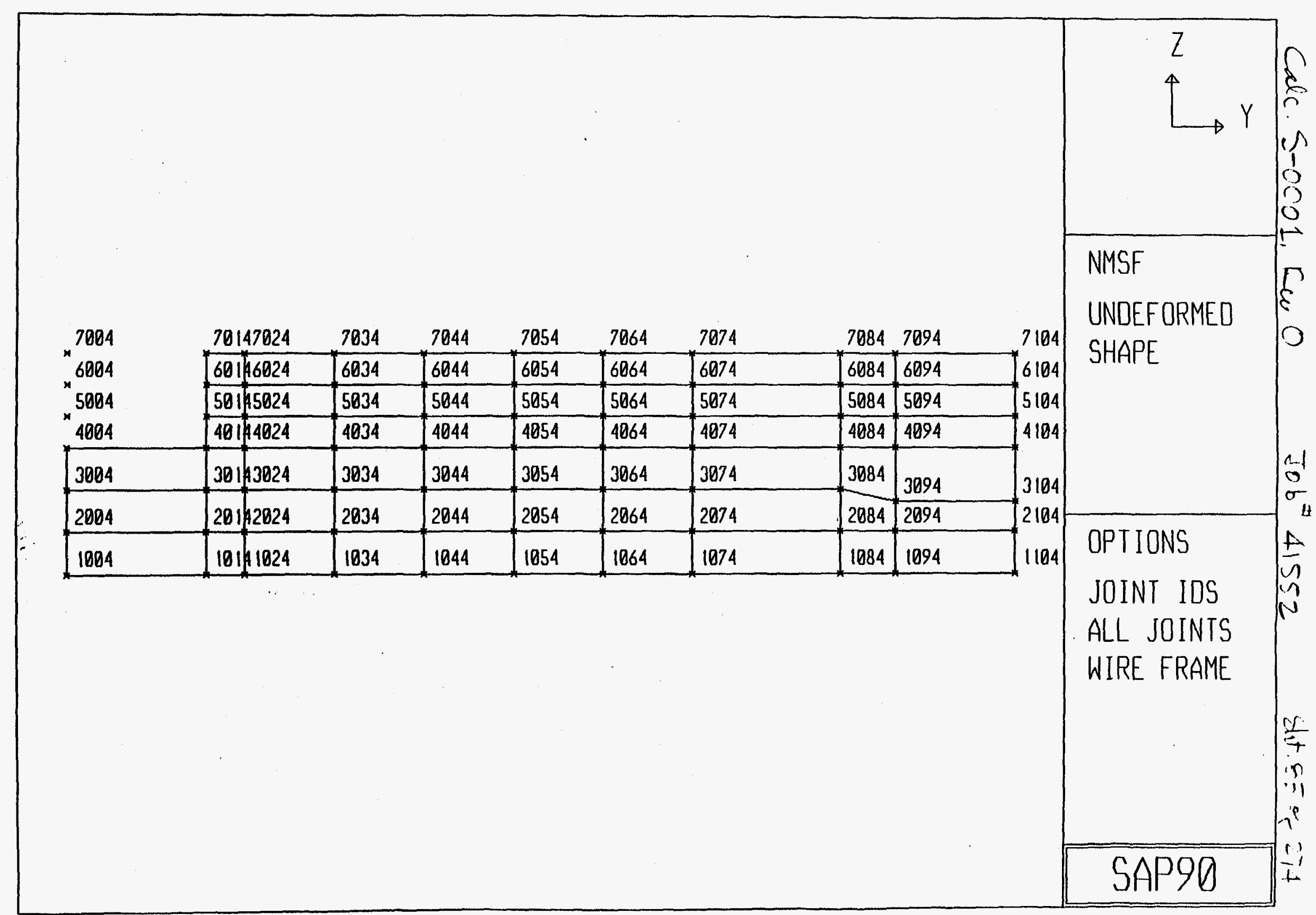




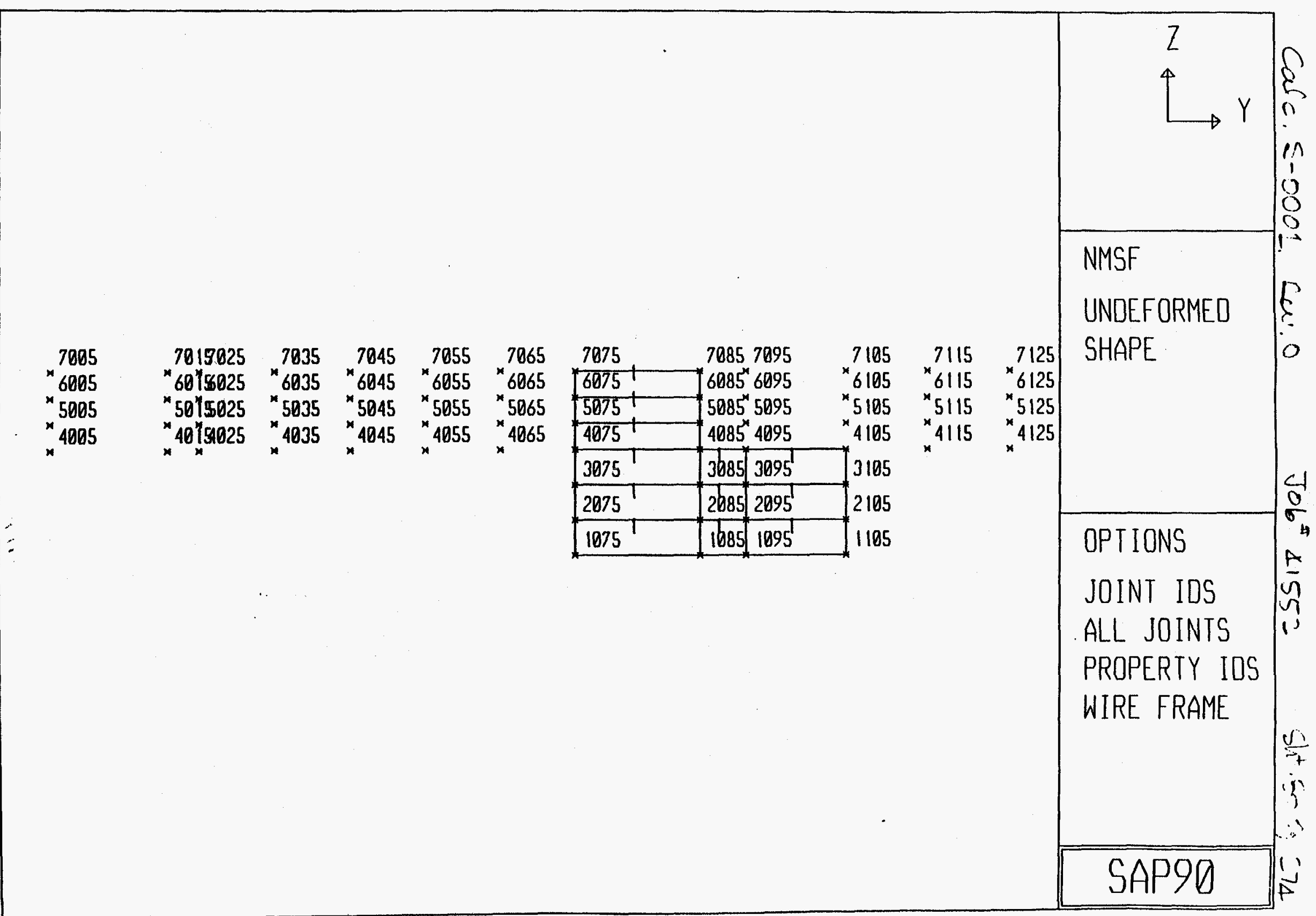


Calc. S-0001, Rew 0 cob $4155=$ the $\leqq-=-$

\begin{tabular}{|c|c|c|c|}
\hline $\begin{array}{l}> \\
N \\
\end{array}$ & 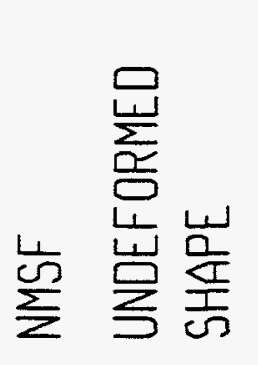 & 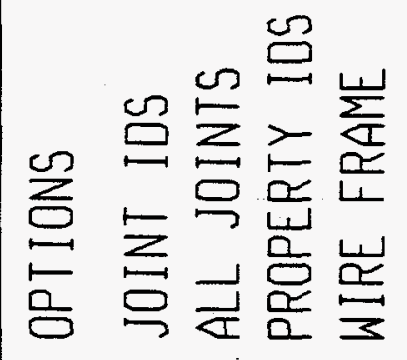 & 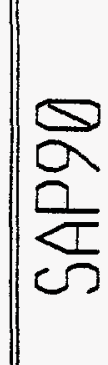 \\
\hline
\end{tabular}

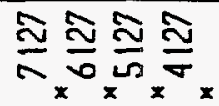

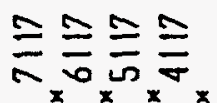

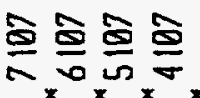

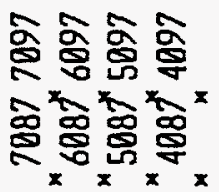

商商高产

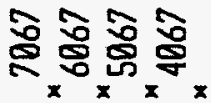

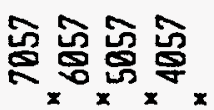

产㐔高

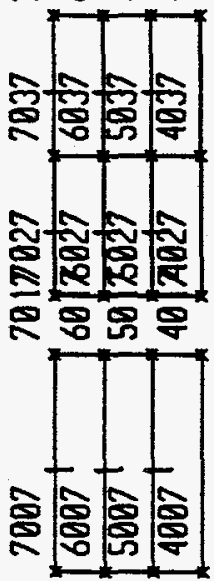




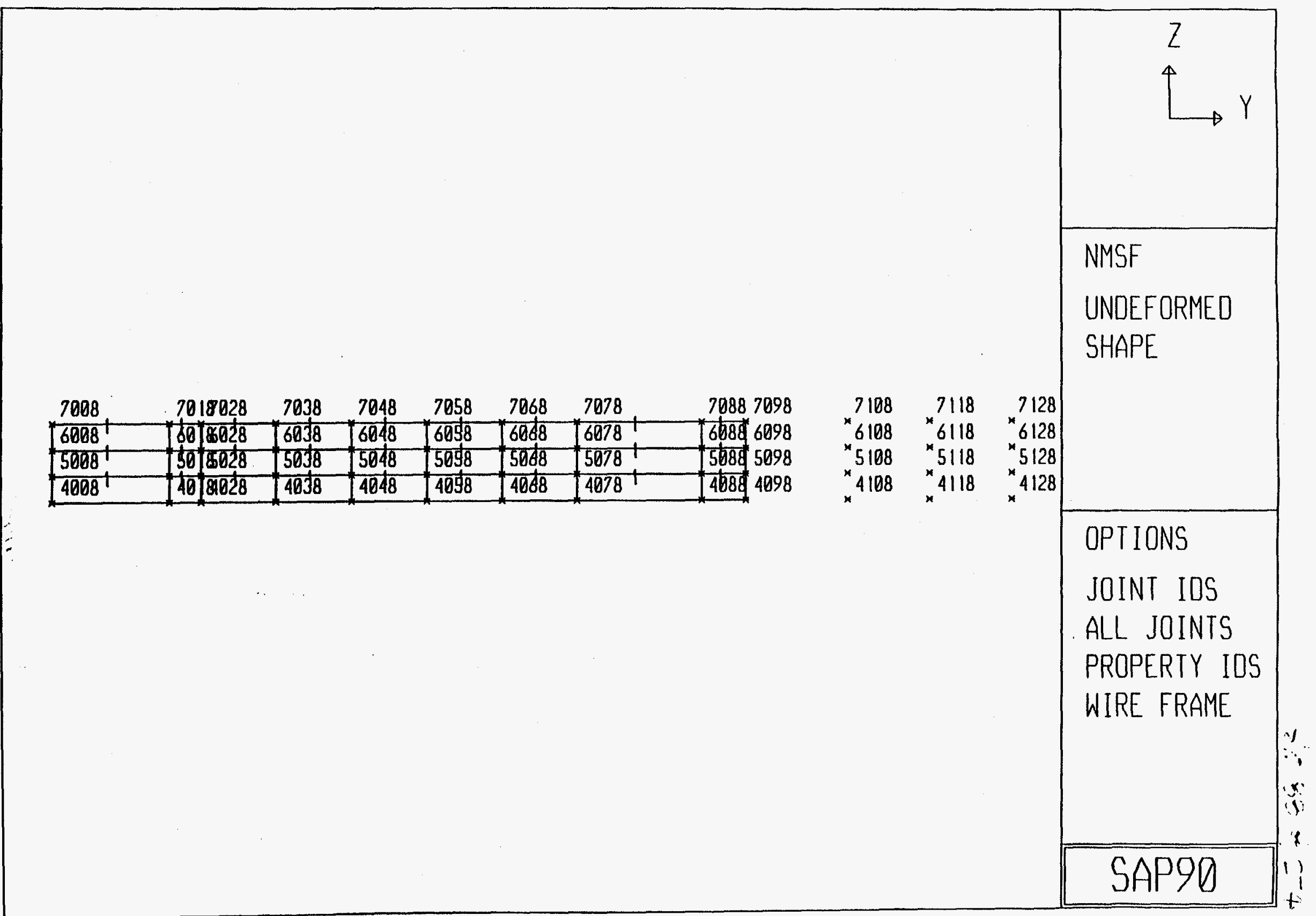


$=-1=x=74$

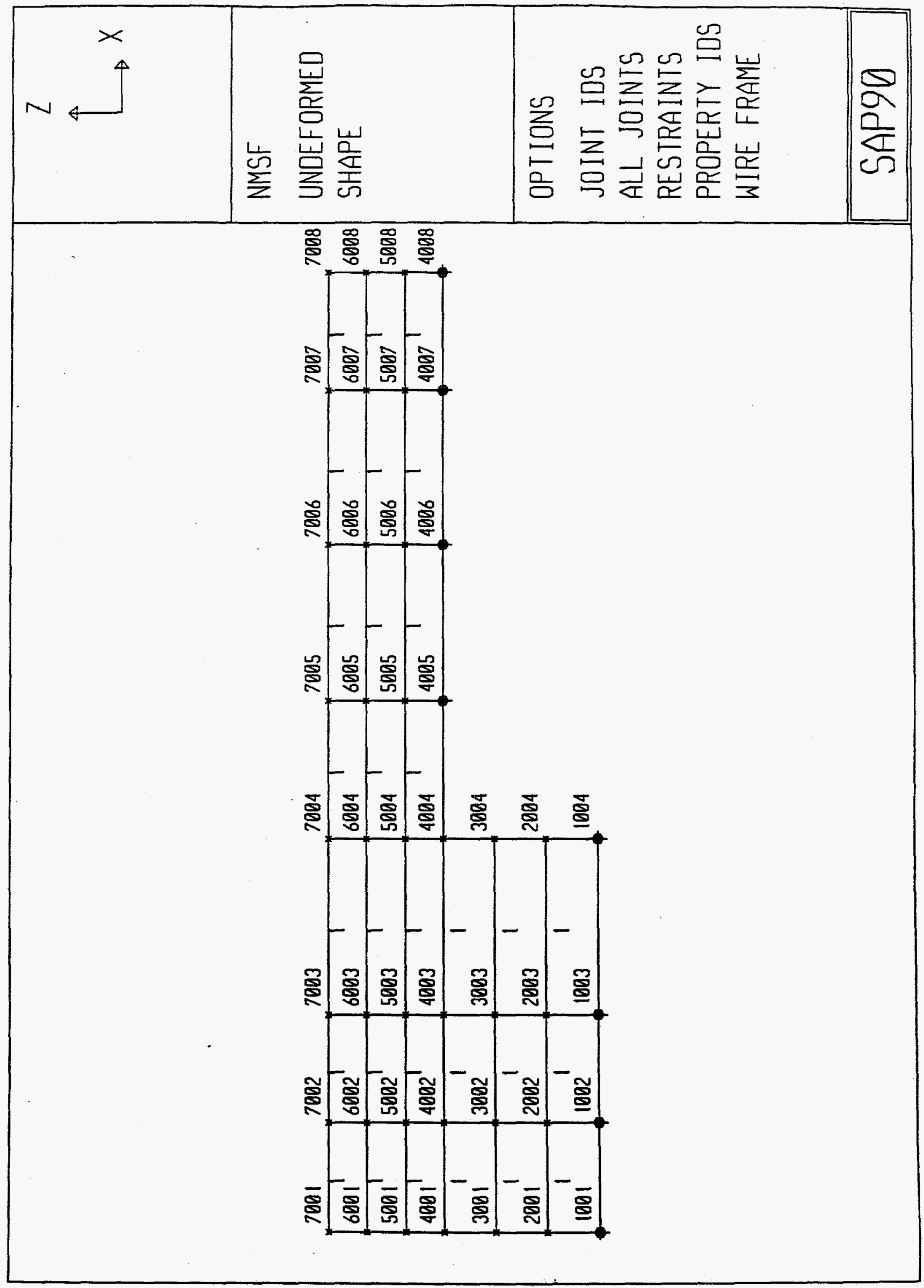


$21+11=-2=7$

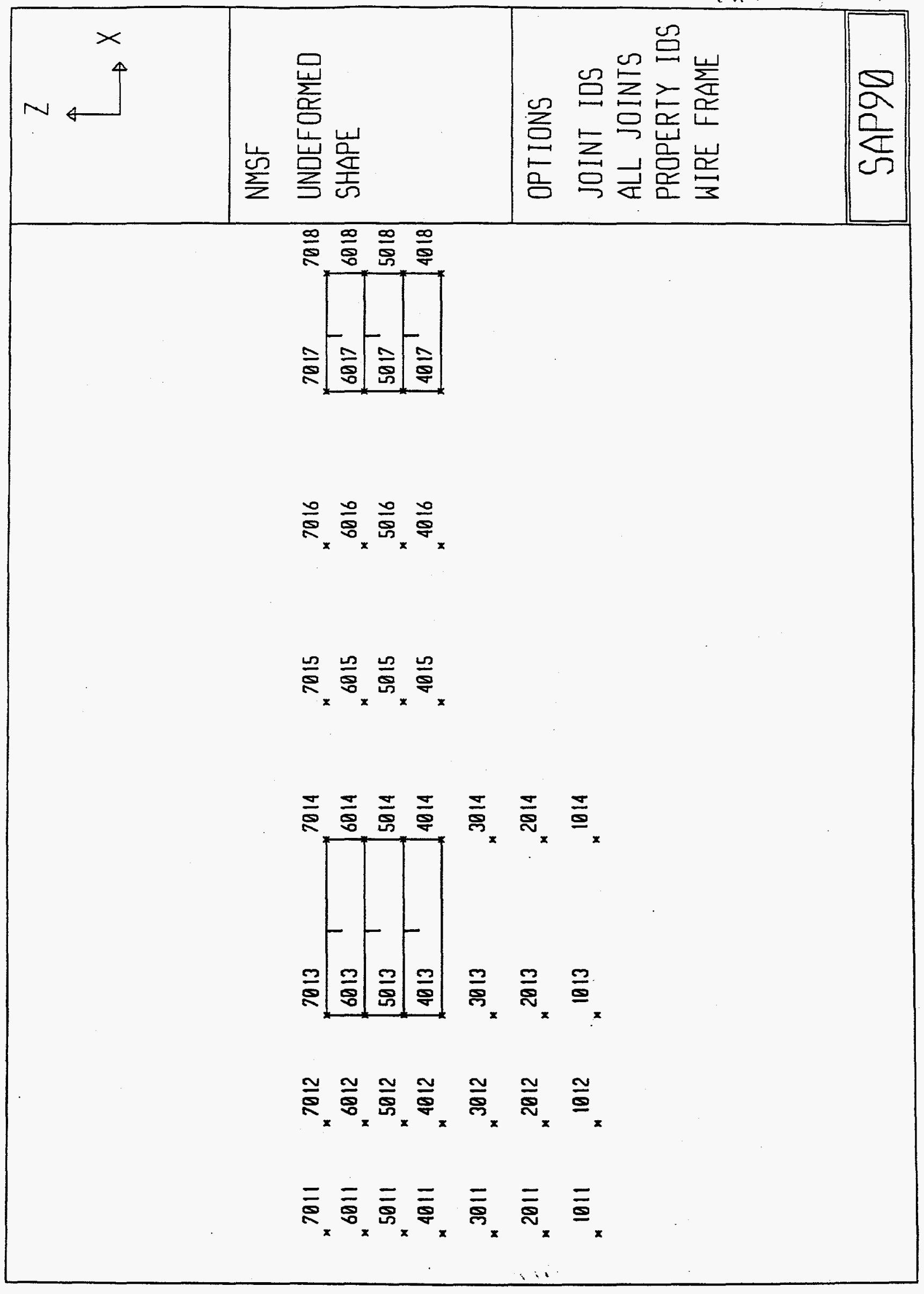




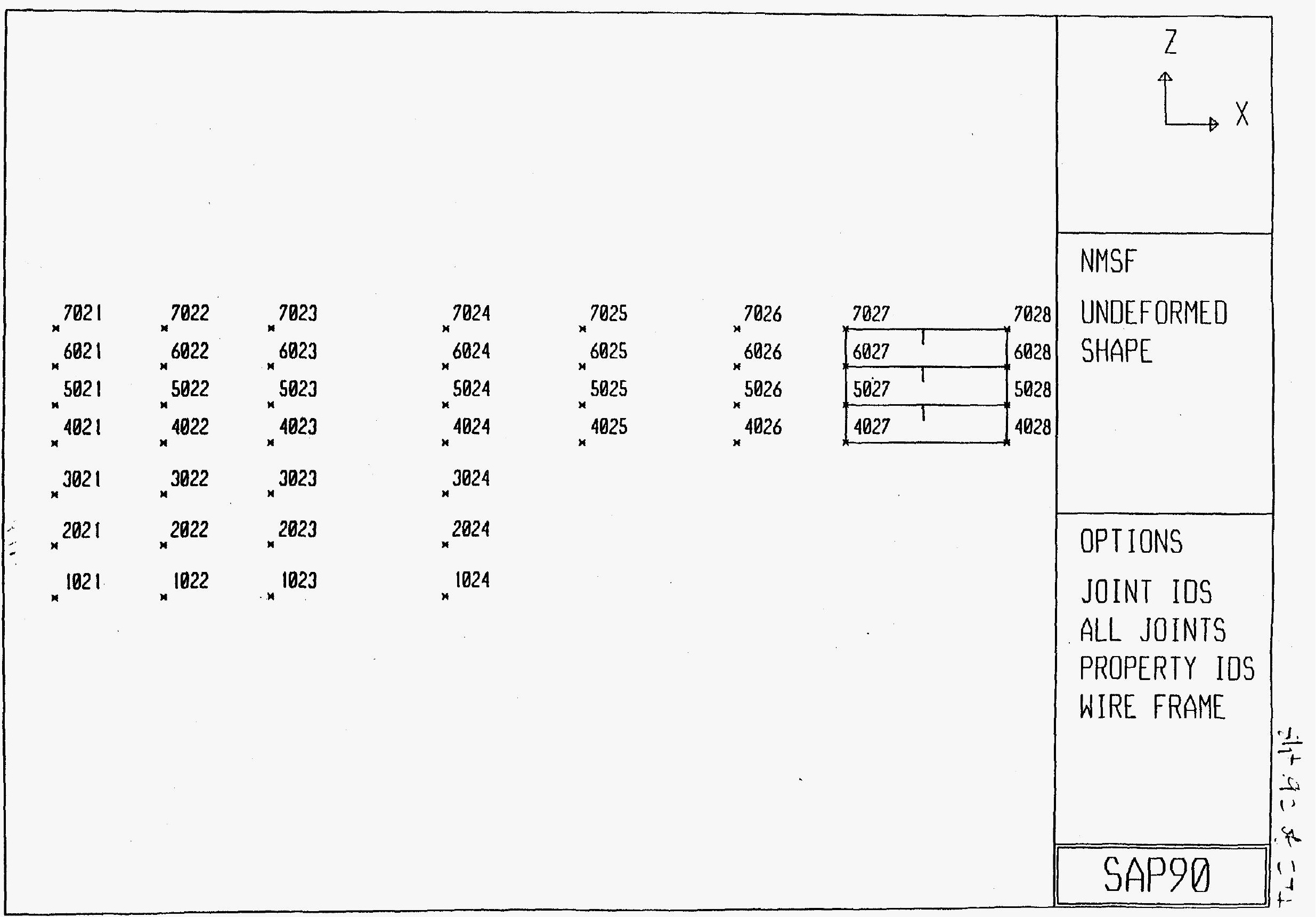




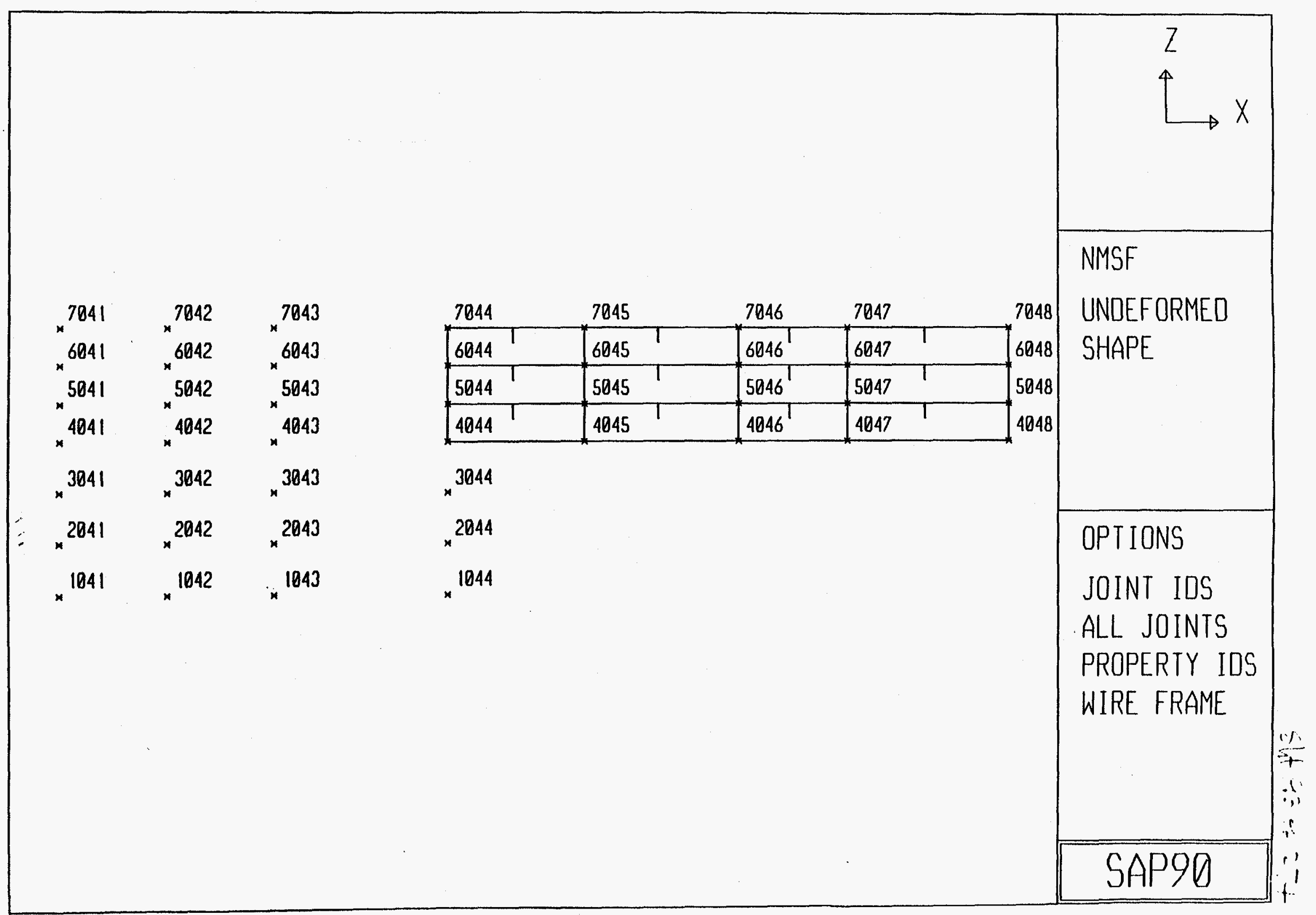




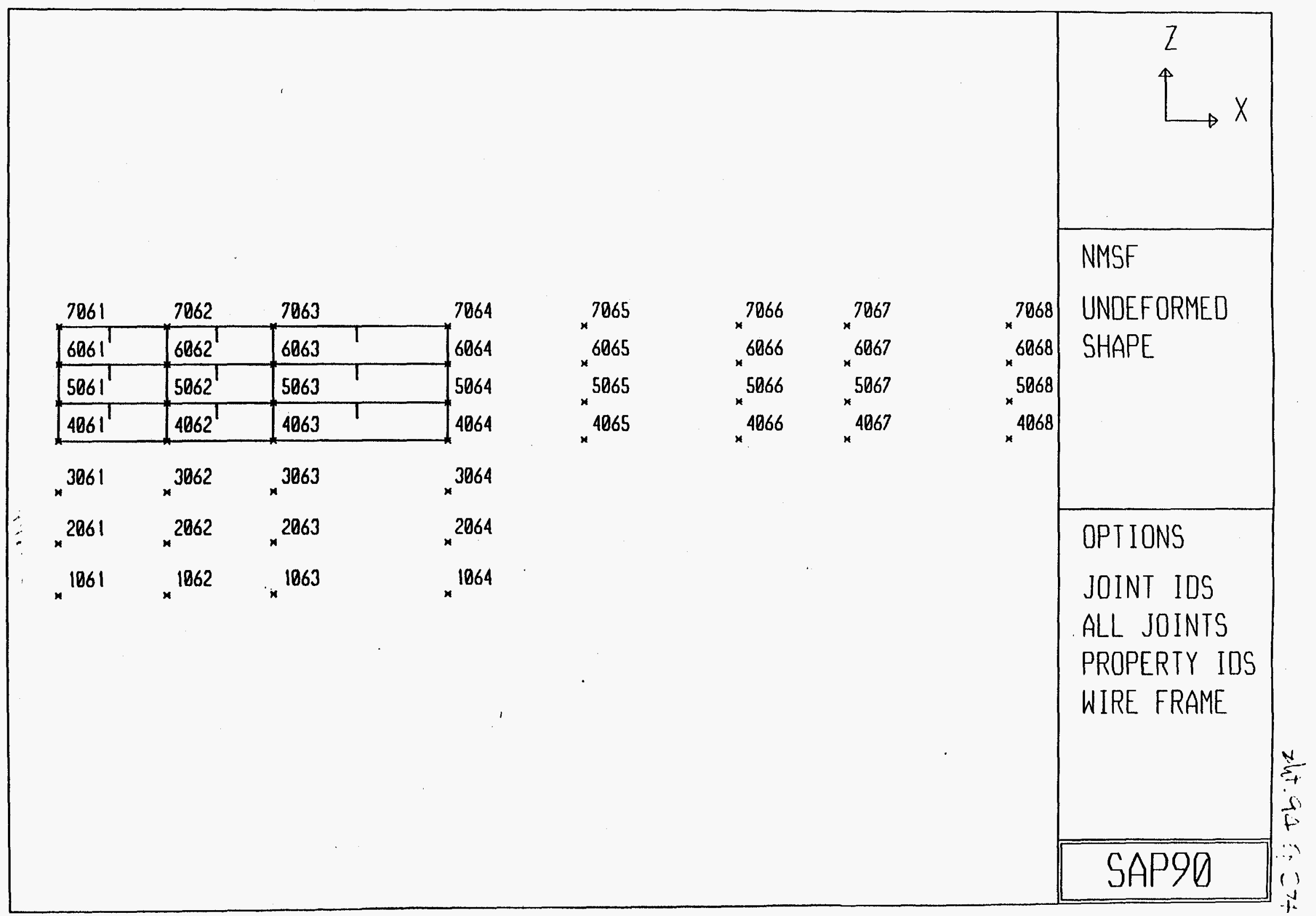




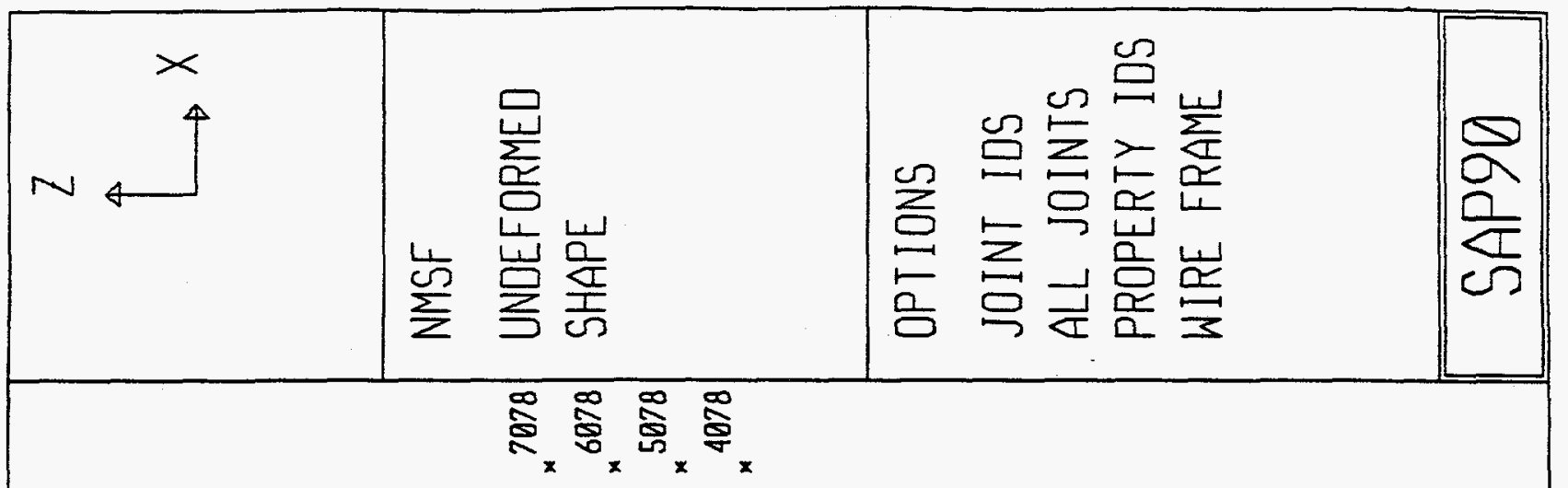

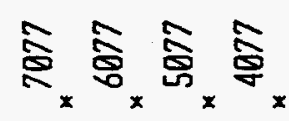

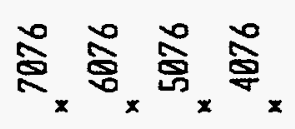

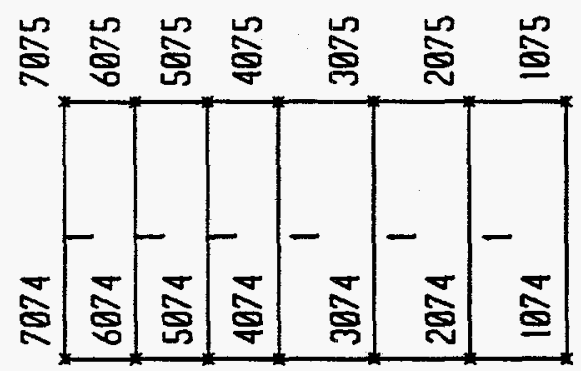

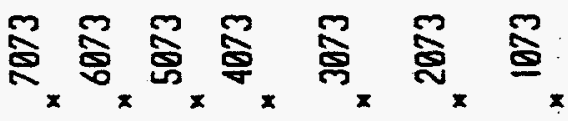

尺.

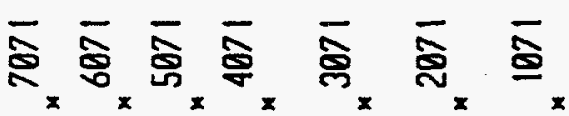




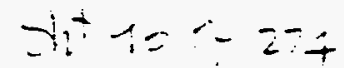

\begin{tabular}{|c|c|c|c|}
\hline$N$ & 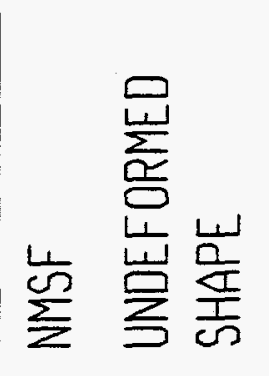 & 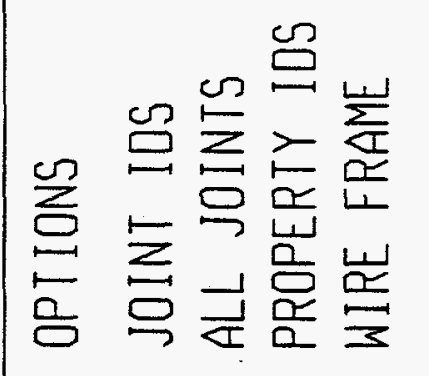 & $\begin{array}{l}\frac{s}{a} \\
\frac{a}{\pi} \\
c\end{array}$ \\
\hline \multicolumn{4}{|c|}{ 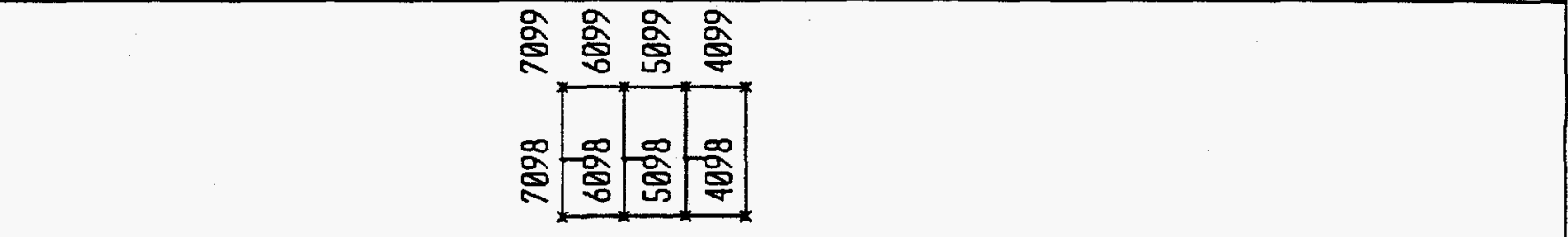 } \\
\hline & 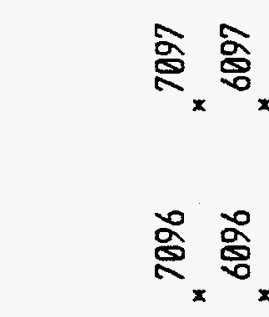 & & 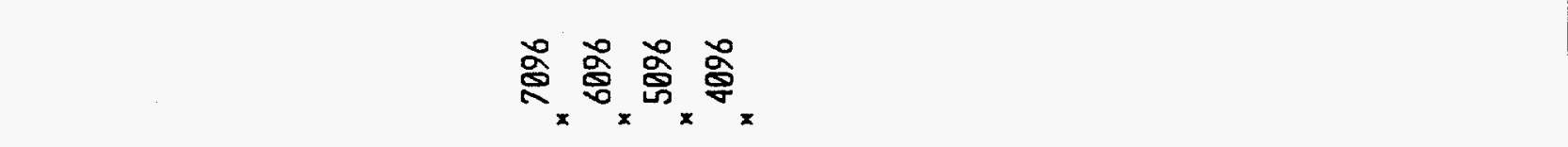 \\
\hline & 哭 & 葵 & \\
\hline & 总 & ${ }_{x}^{\sigma} \underset{x}{\sigma}$ & \\
\hline & g. & 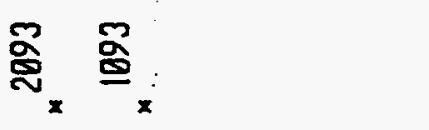 & \\
\hline & 令 & 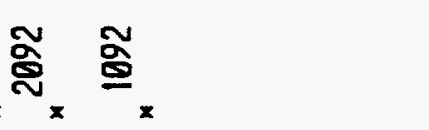 & \\
\hline & $\overline{\mathrm{g}} \overline{\mathrm{g}}$ & $\overline{\mathrm{a}}_{x} \overline{\mathrm{g}}_{\mathrm{x}}$ & \\
\hline
\end{tabular}


$8097 \div=7+$

\begin{tabular}{|c|c|c|c|}
\hline$N_{4}^{x}$ & 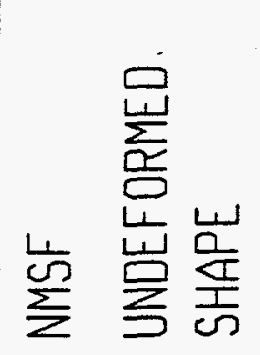 & 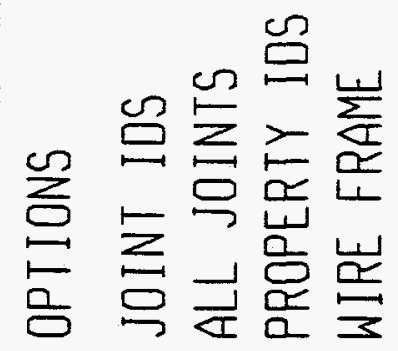 & $\frac{5}{2}$ \\
\hline
\end{tabular}

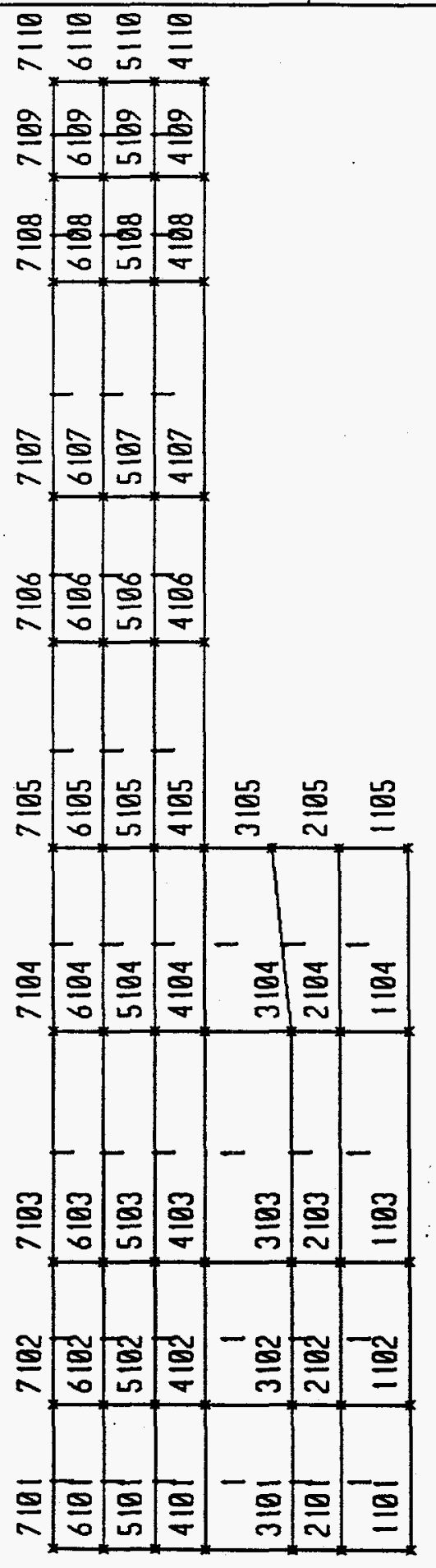




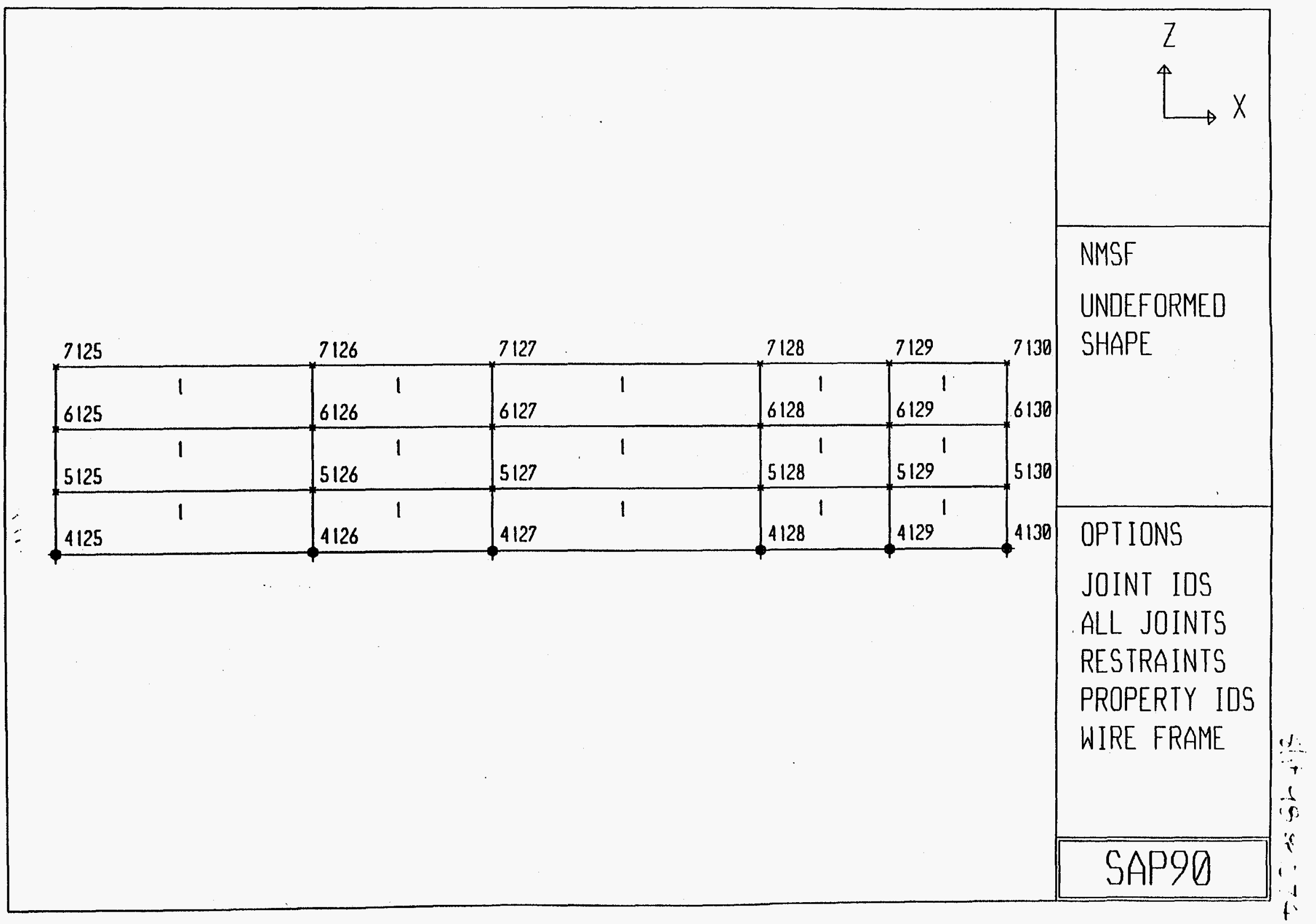




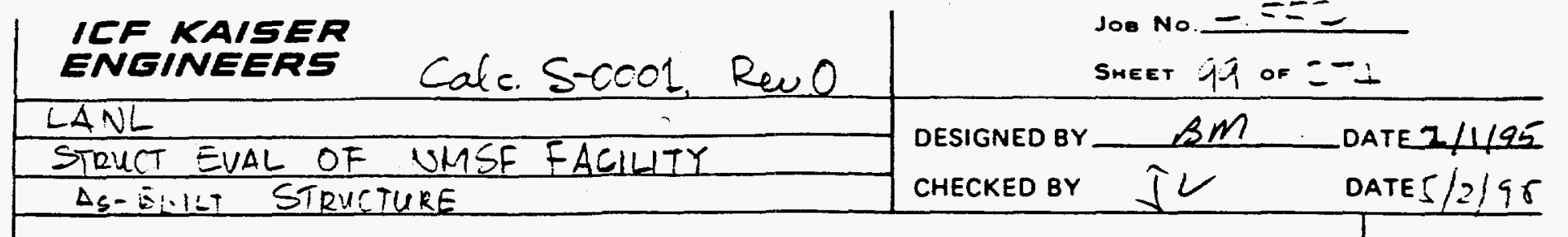

Effective shear wall thickness.

openings in the wall are accounted for in calculation of Rigidity of the wall by assuming an effective Thickness based on equal volume of Concrete.

The same affections wall thicknesses are used later in calculation of wall capacities. This method is justified based on the fact that no credit is taken for the reinforcing steel in the wall for their is plane shear capacities and sill a confortall margin of safety is obtained by the calculated Dumand/eapacity Ratios. 


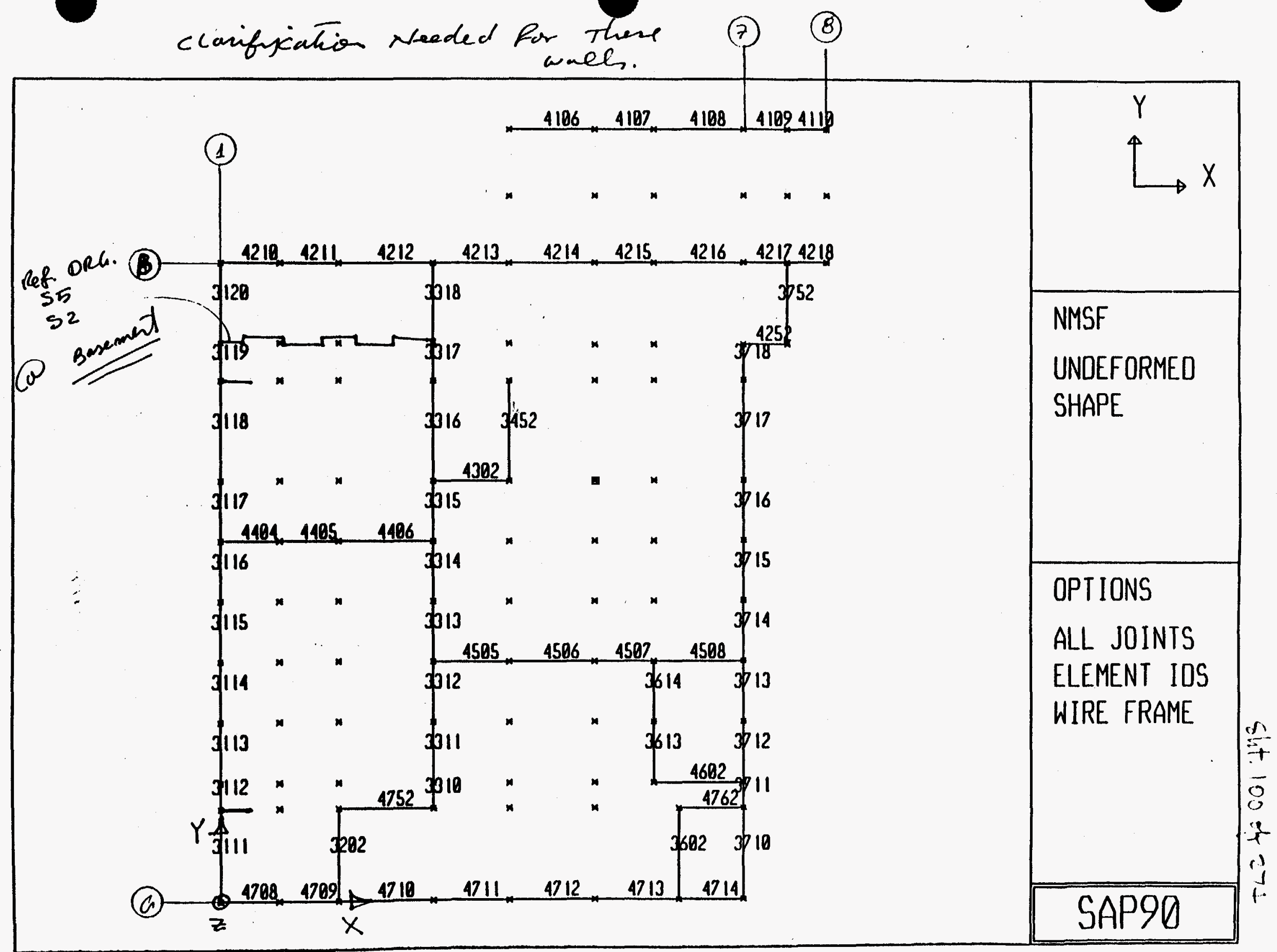




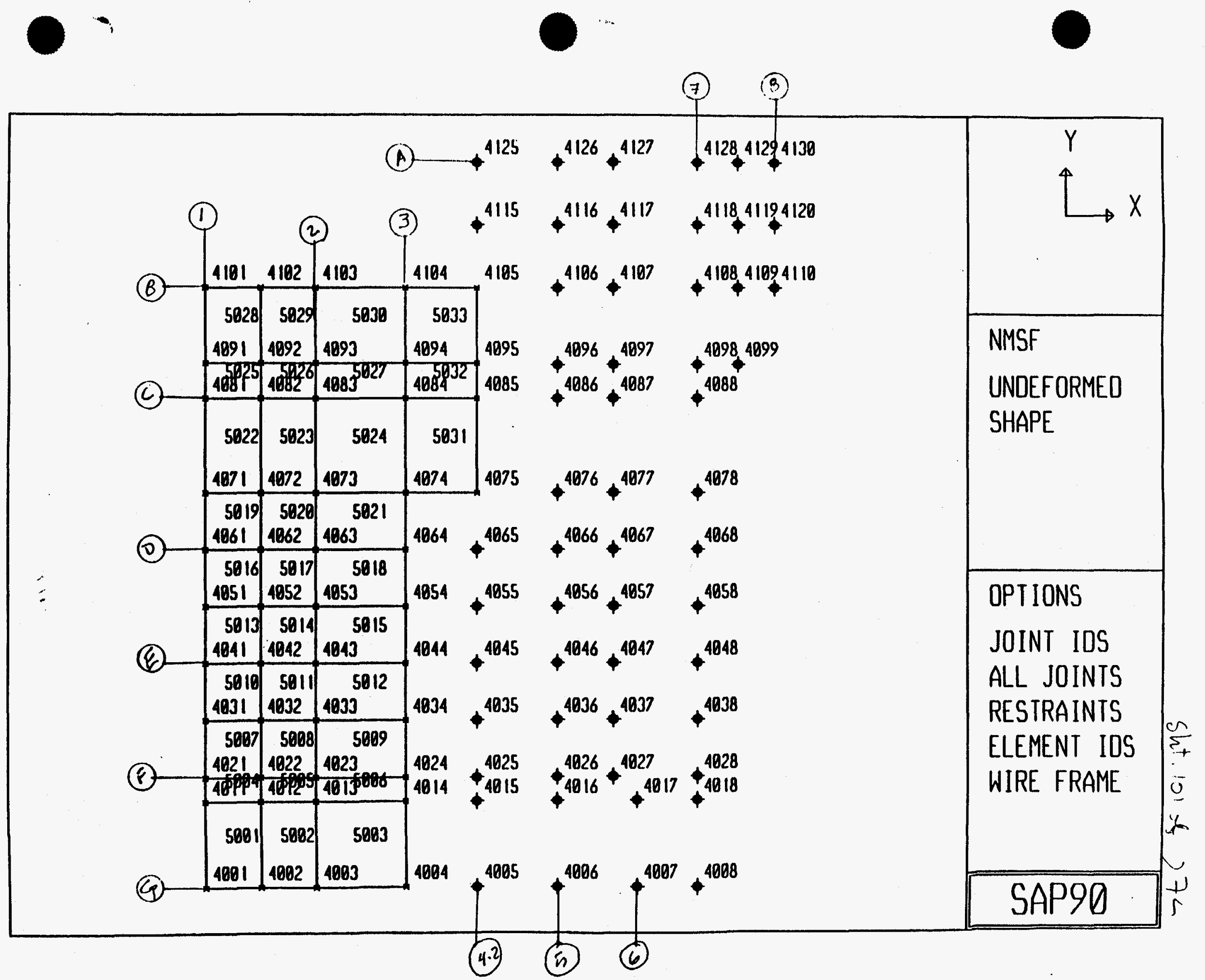




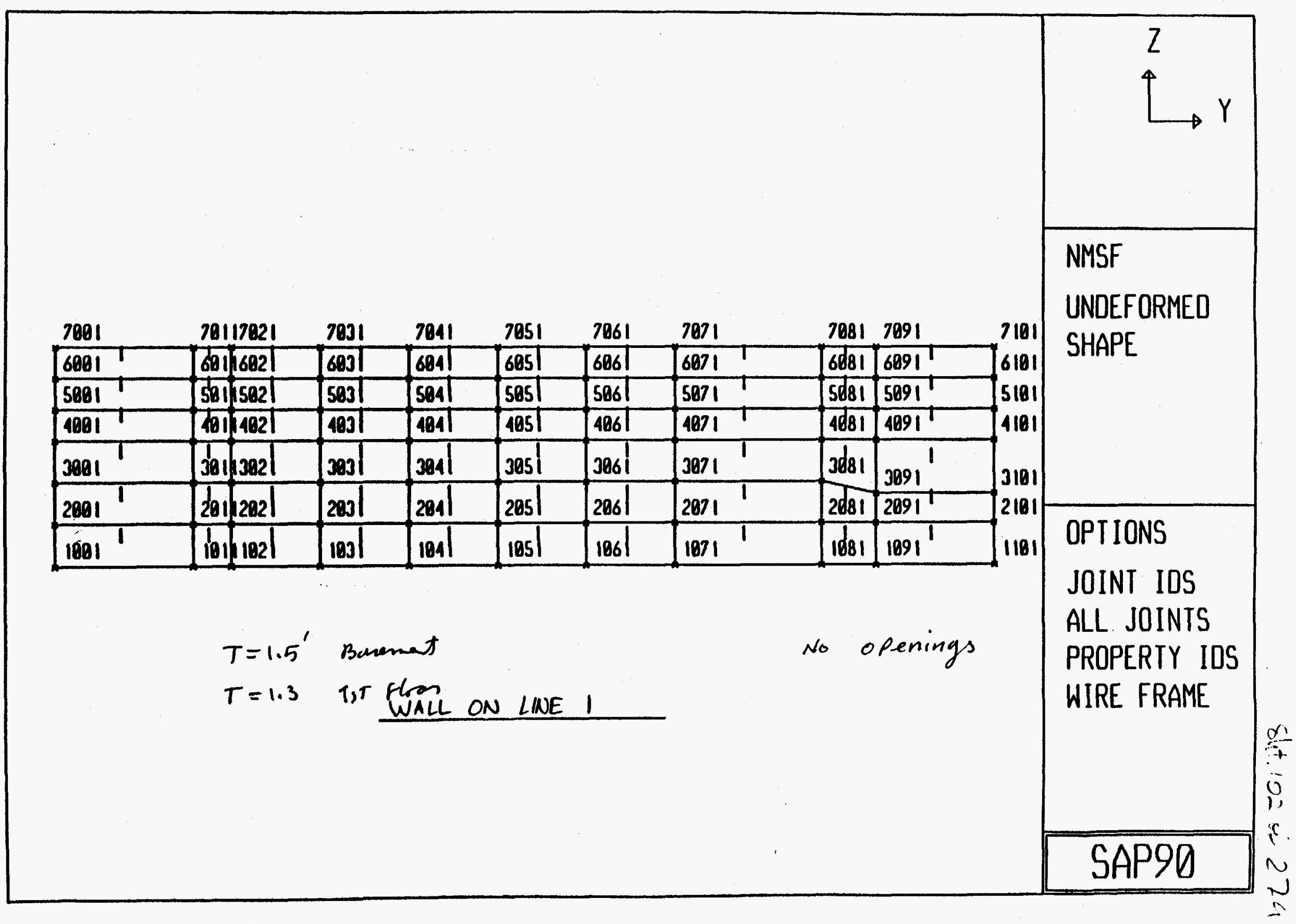


$-215=4$

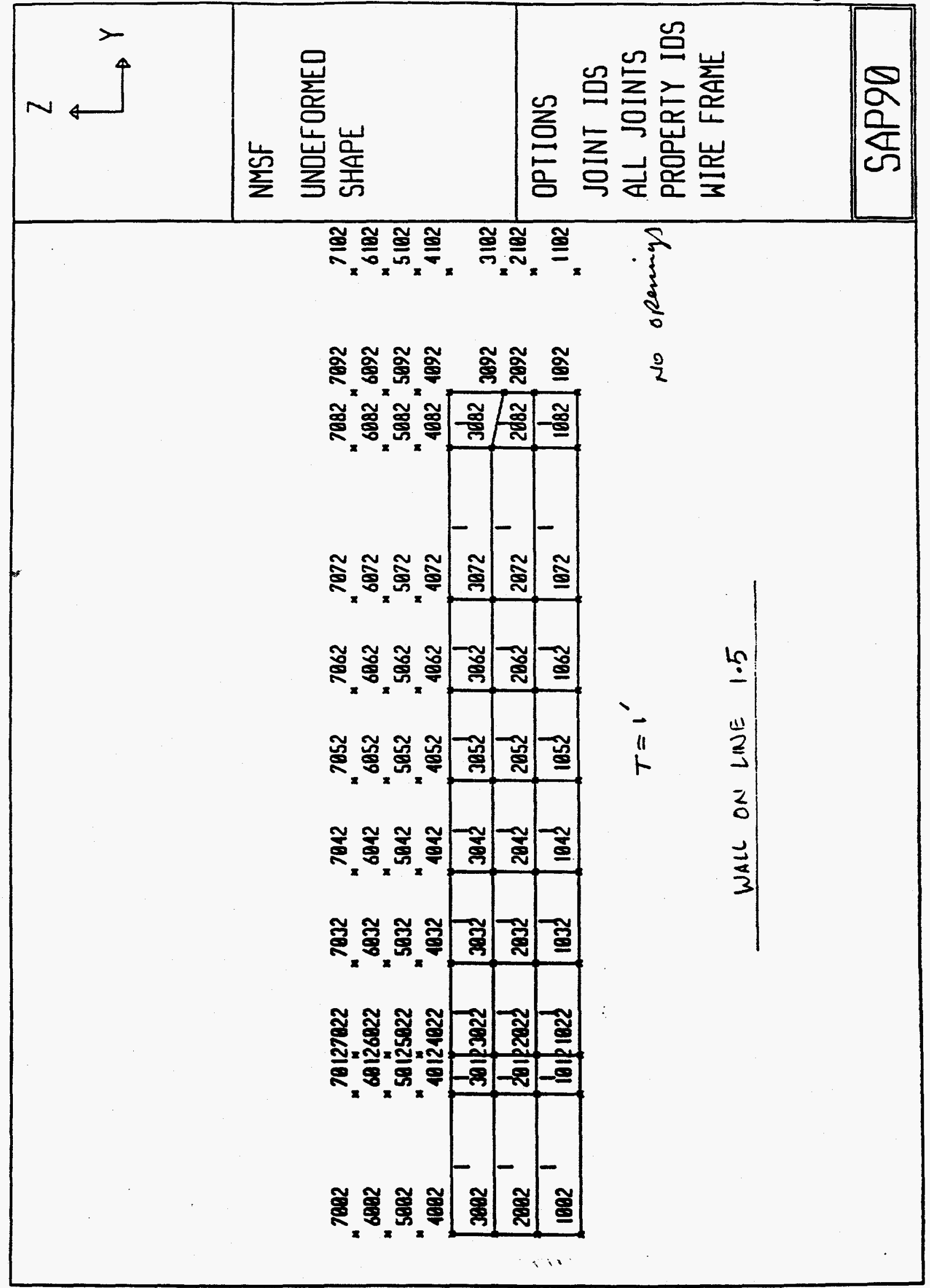


-1nt 104

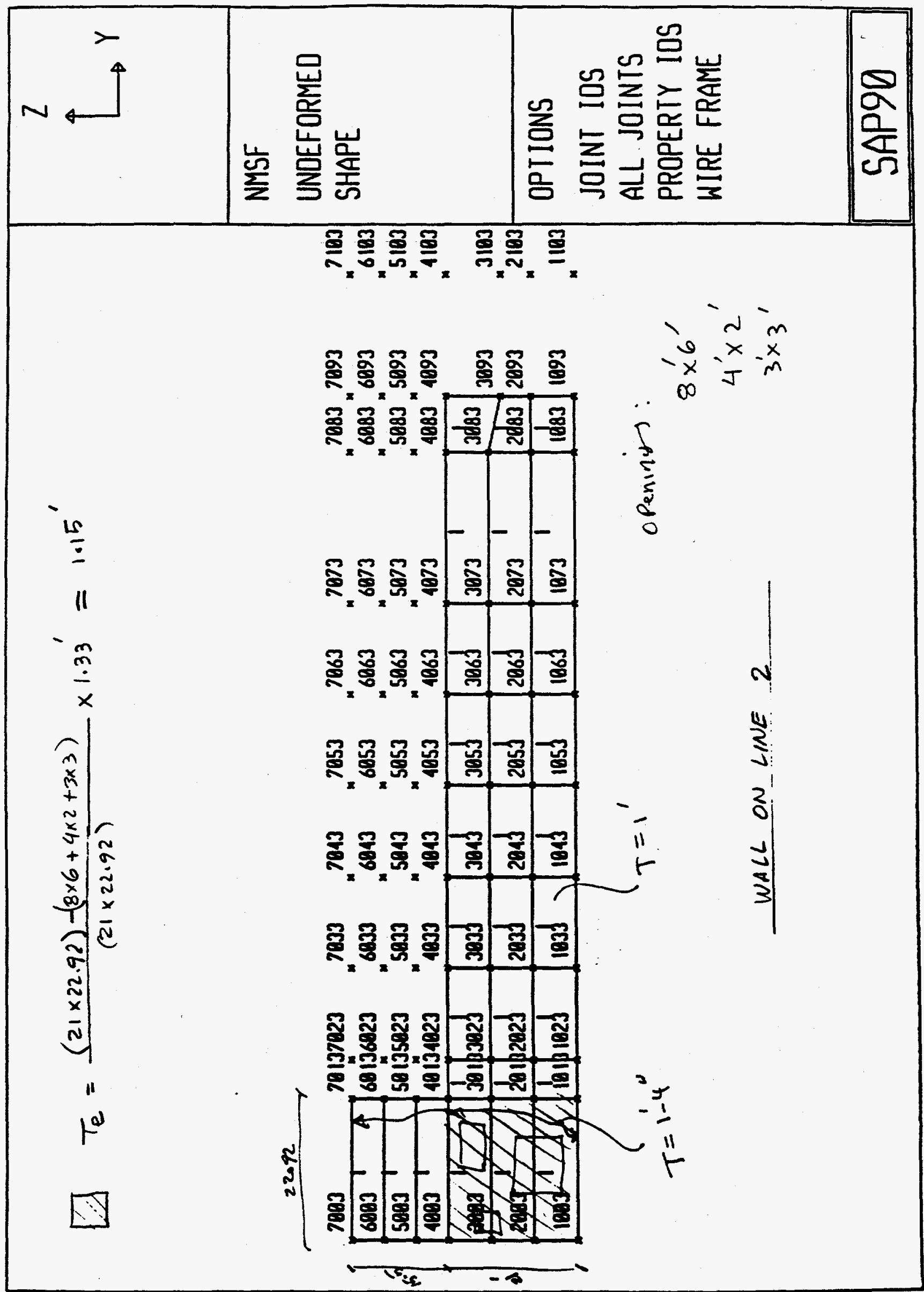




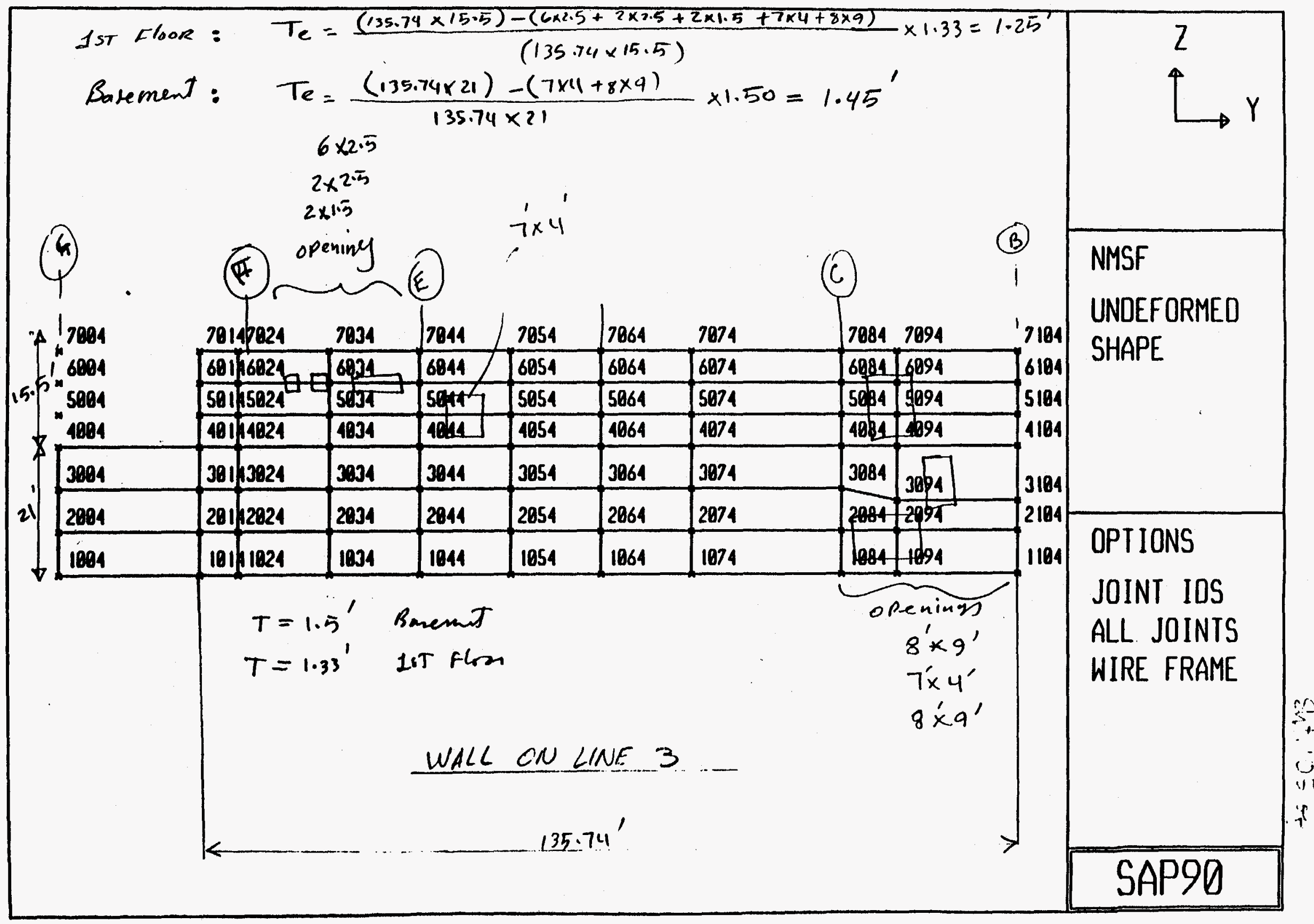




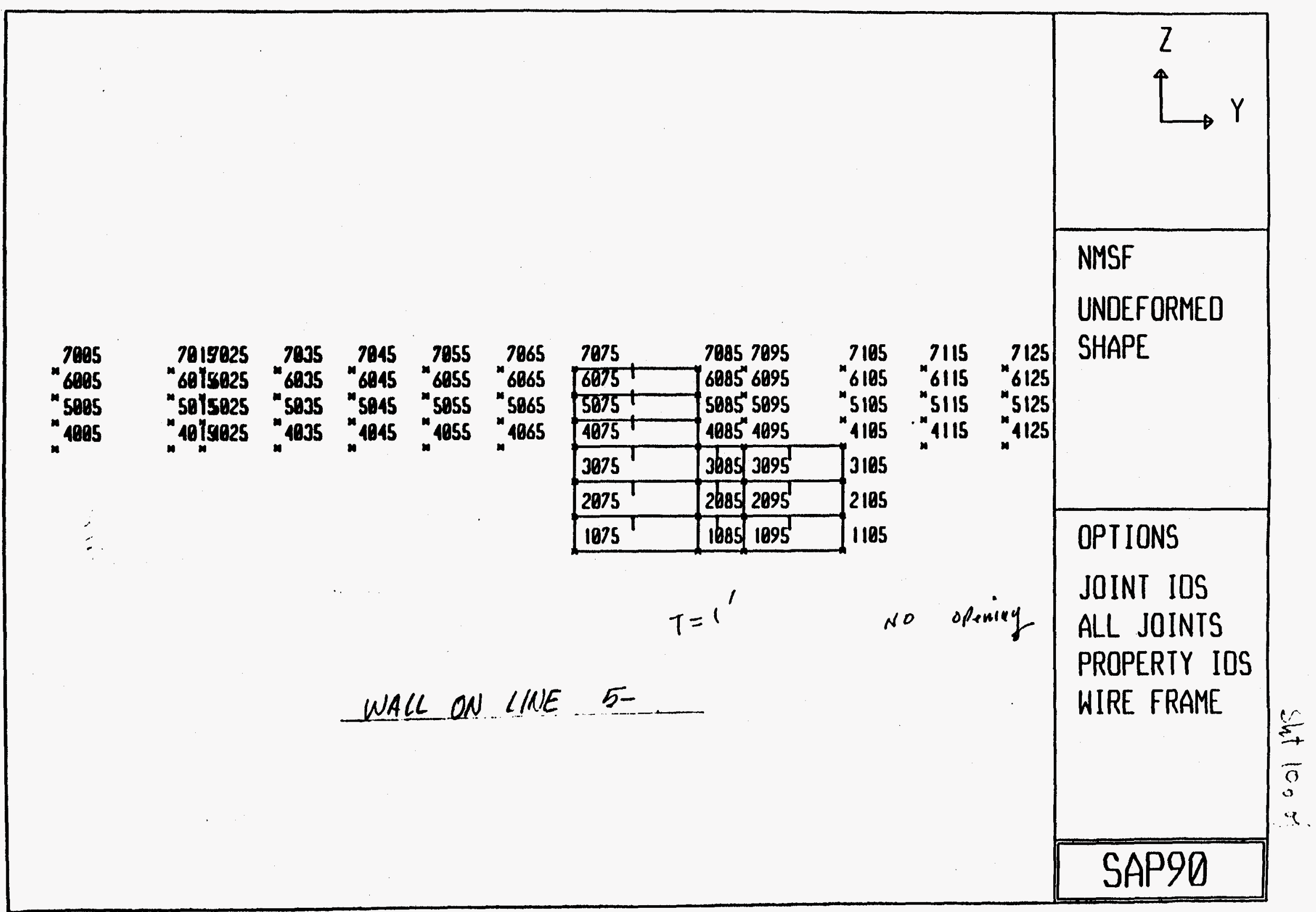




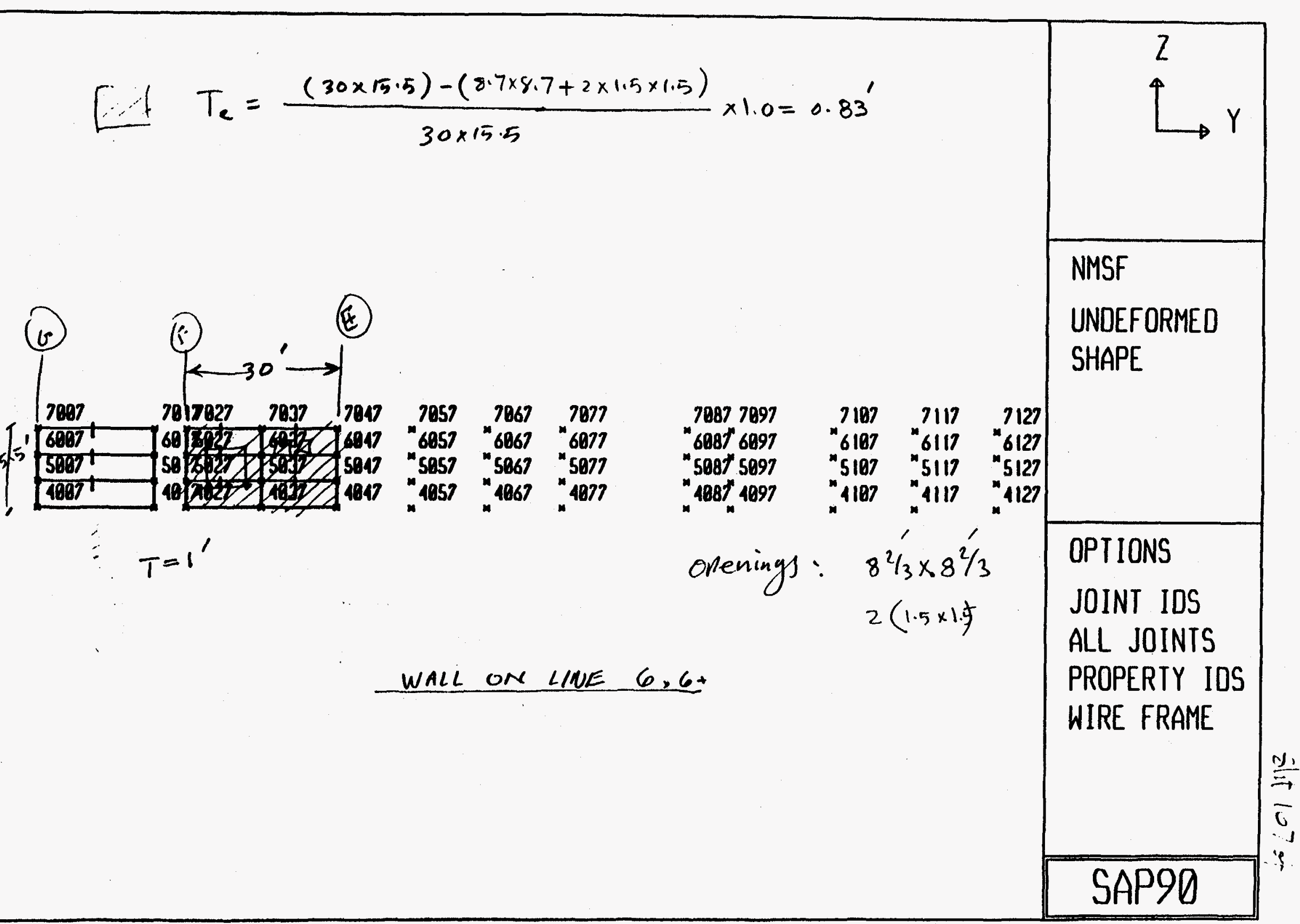




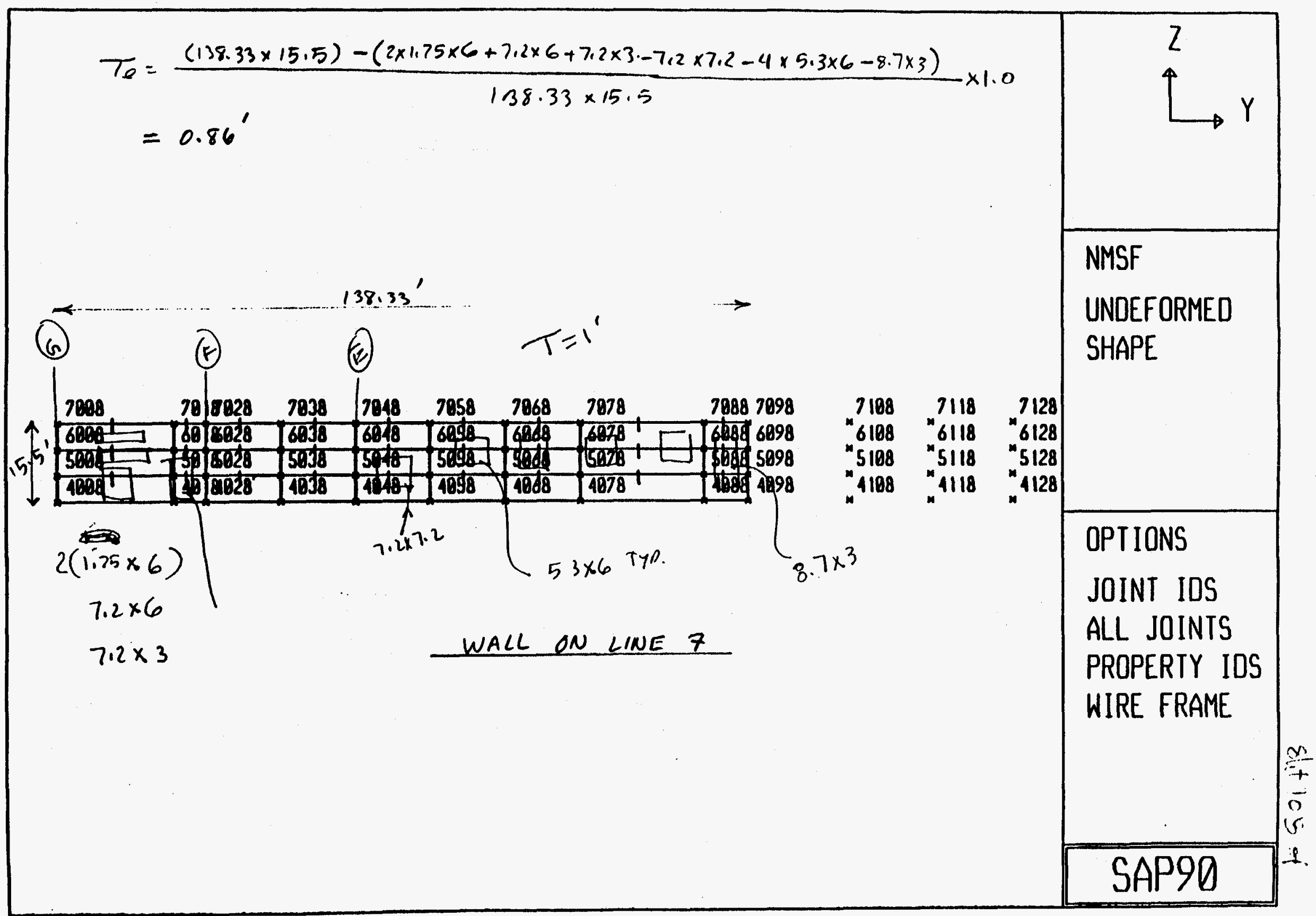




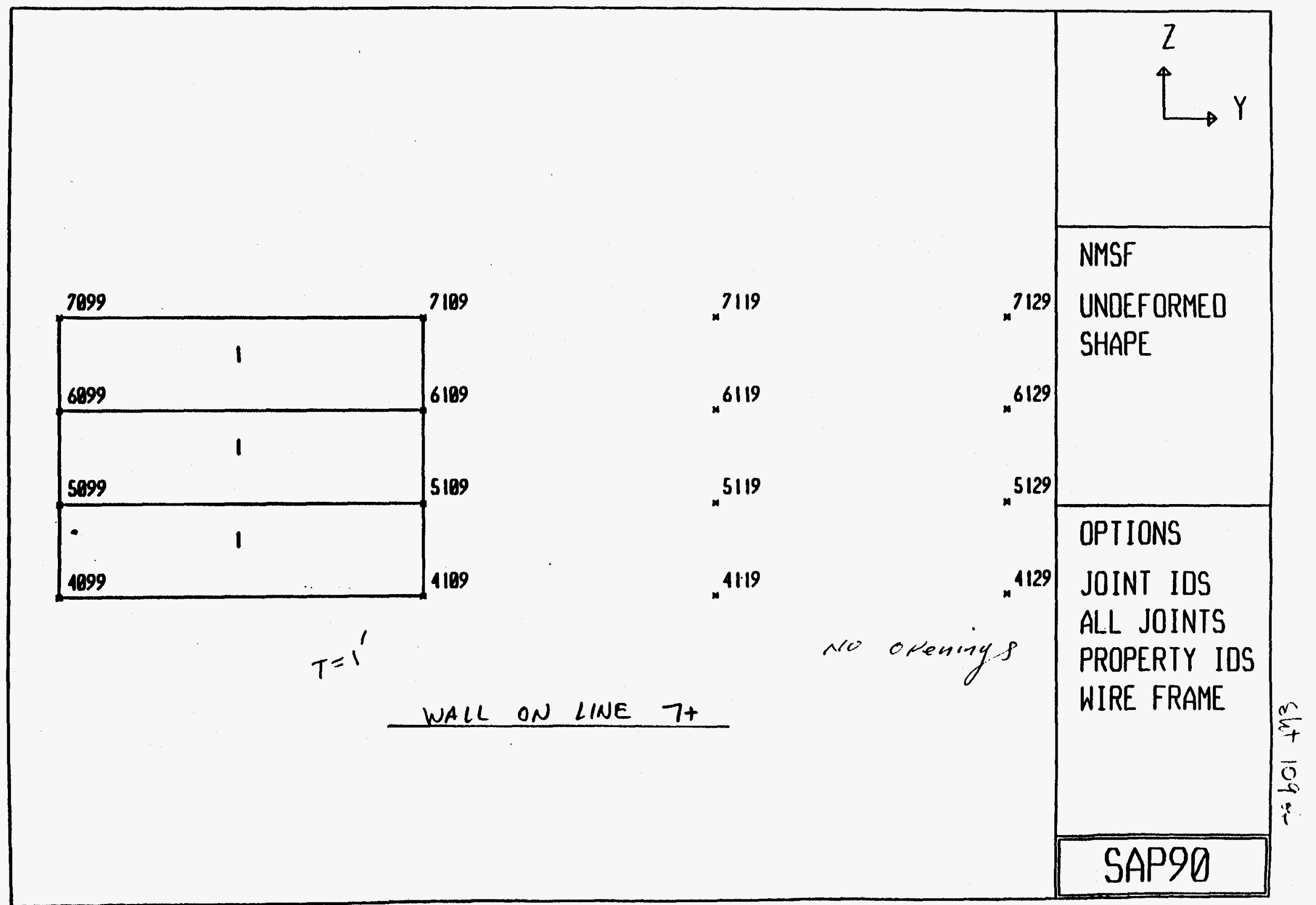


sht. $110 \%$

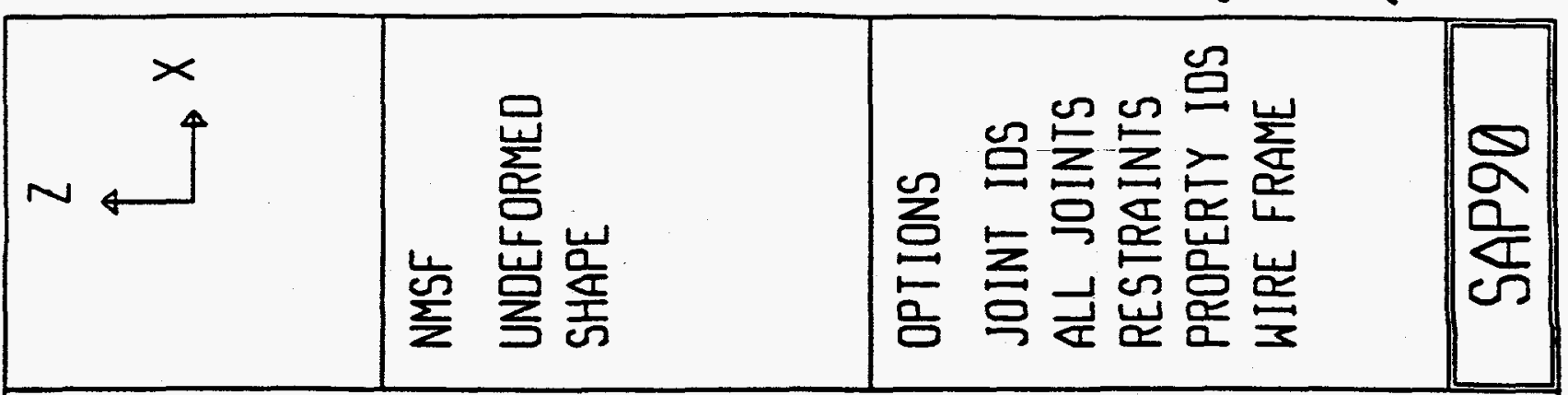

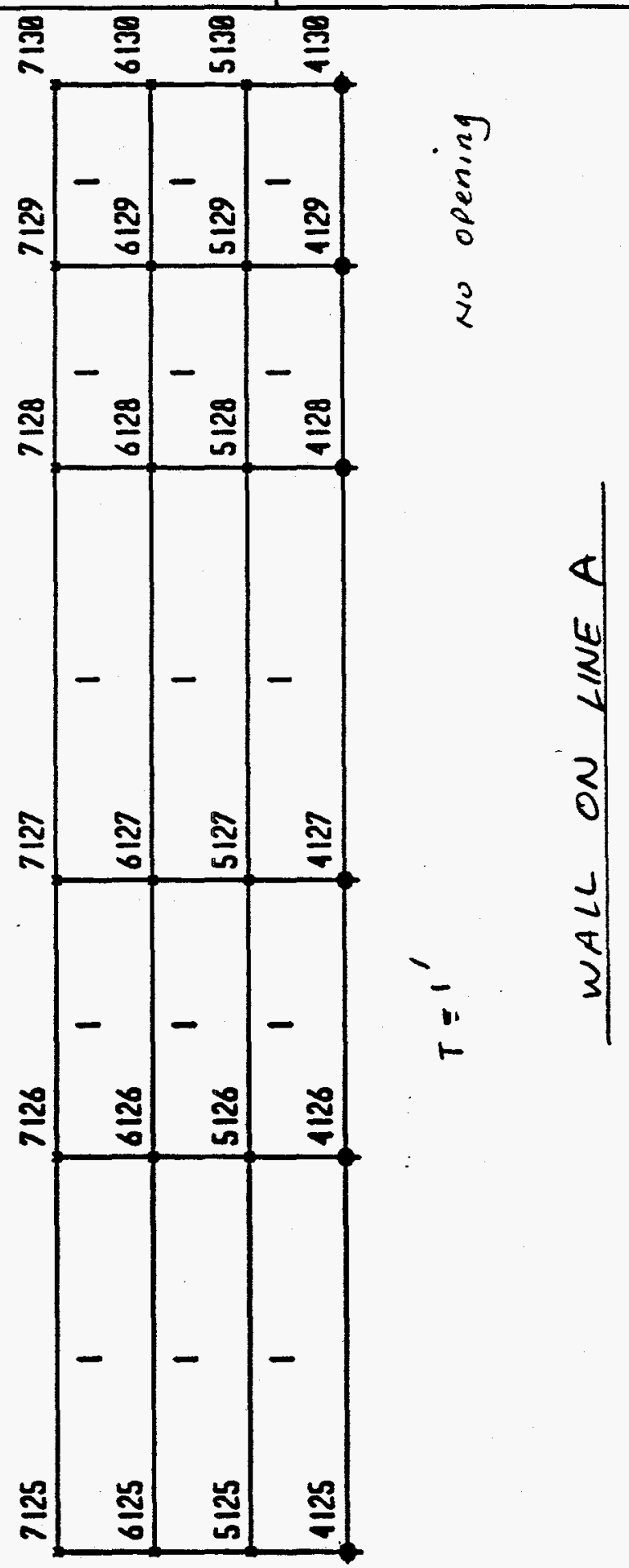




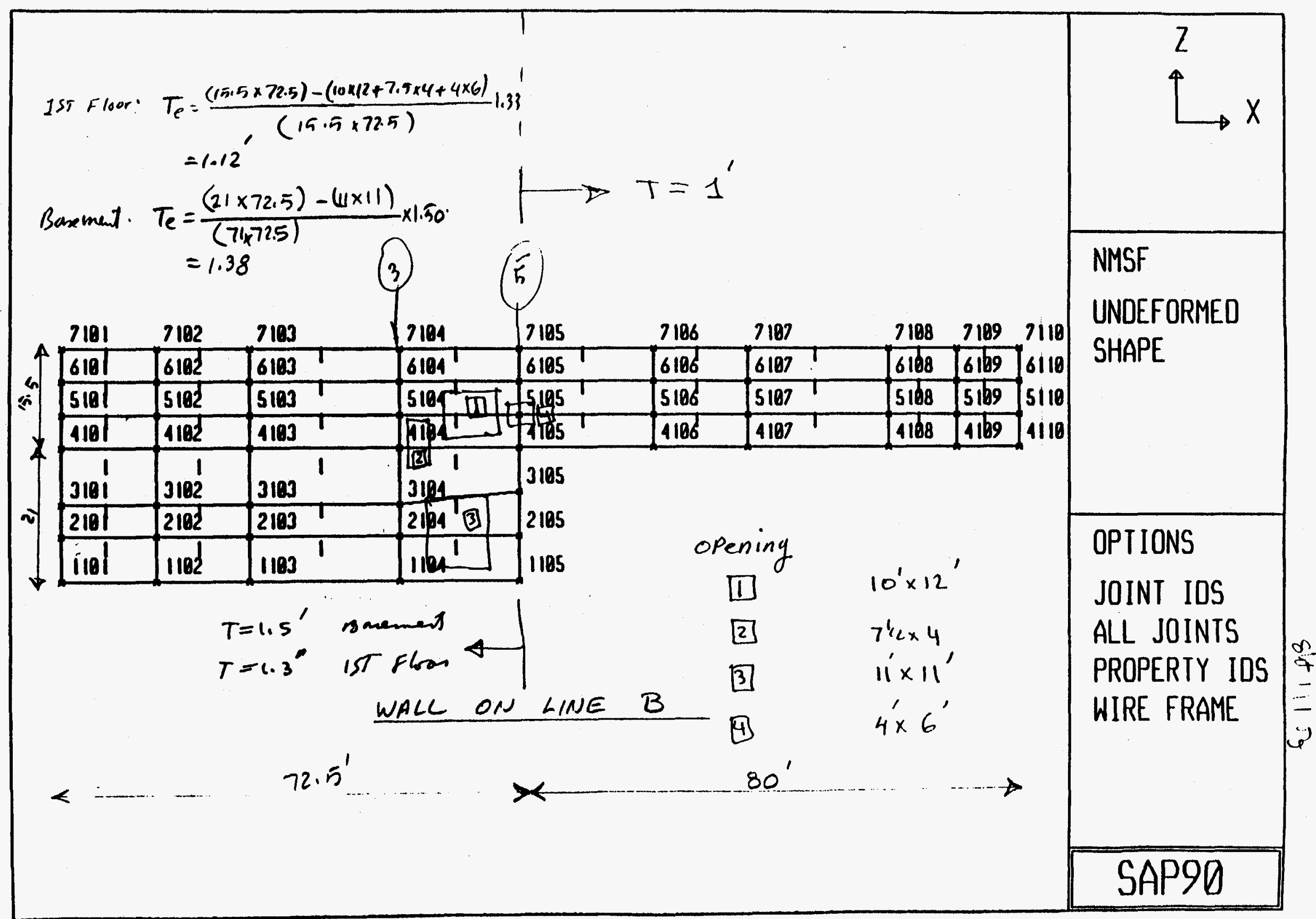




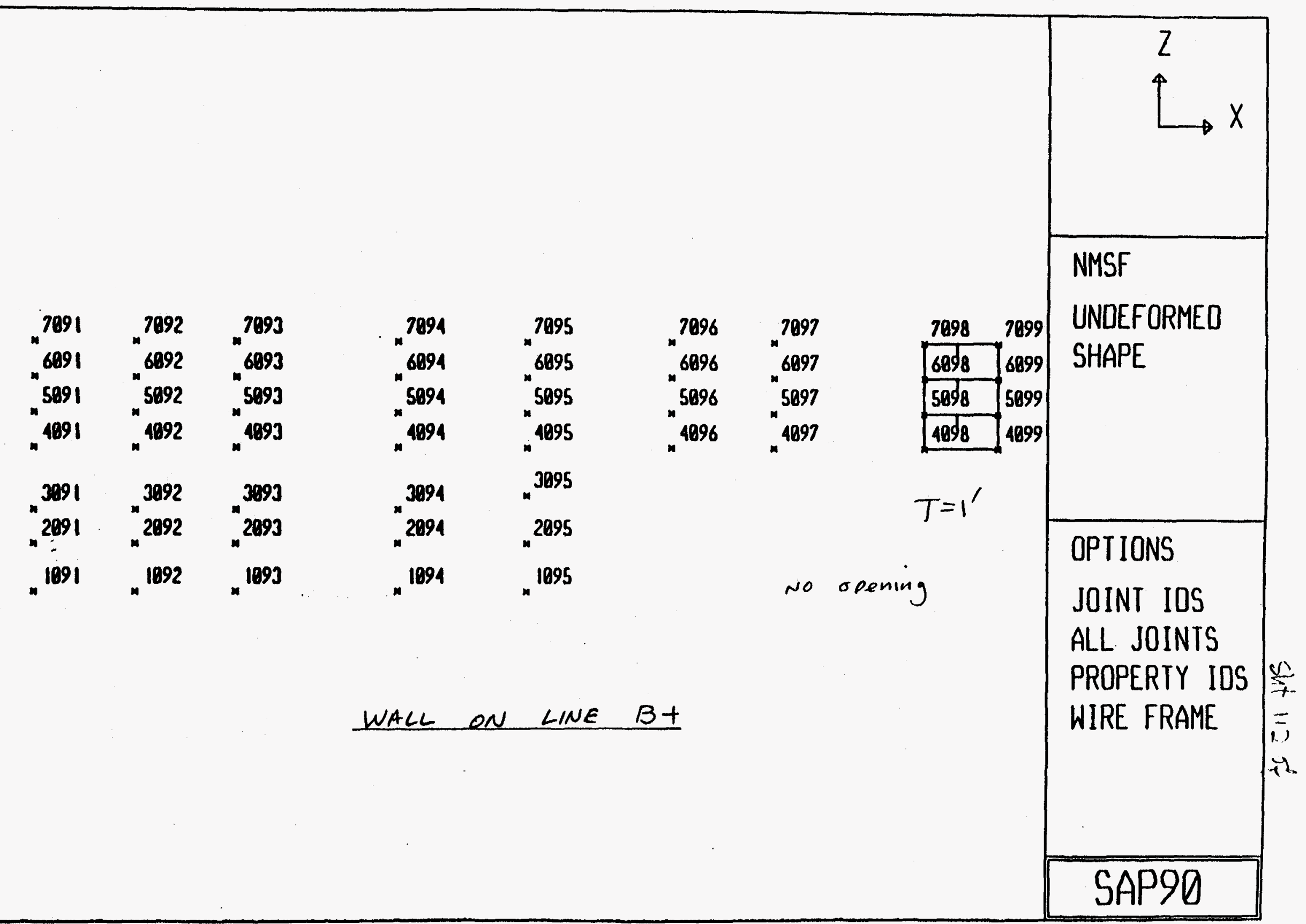




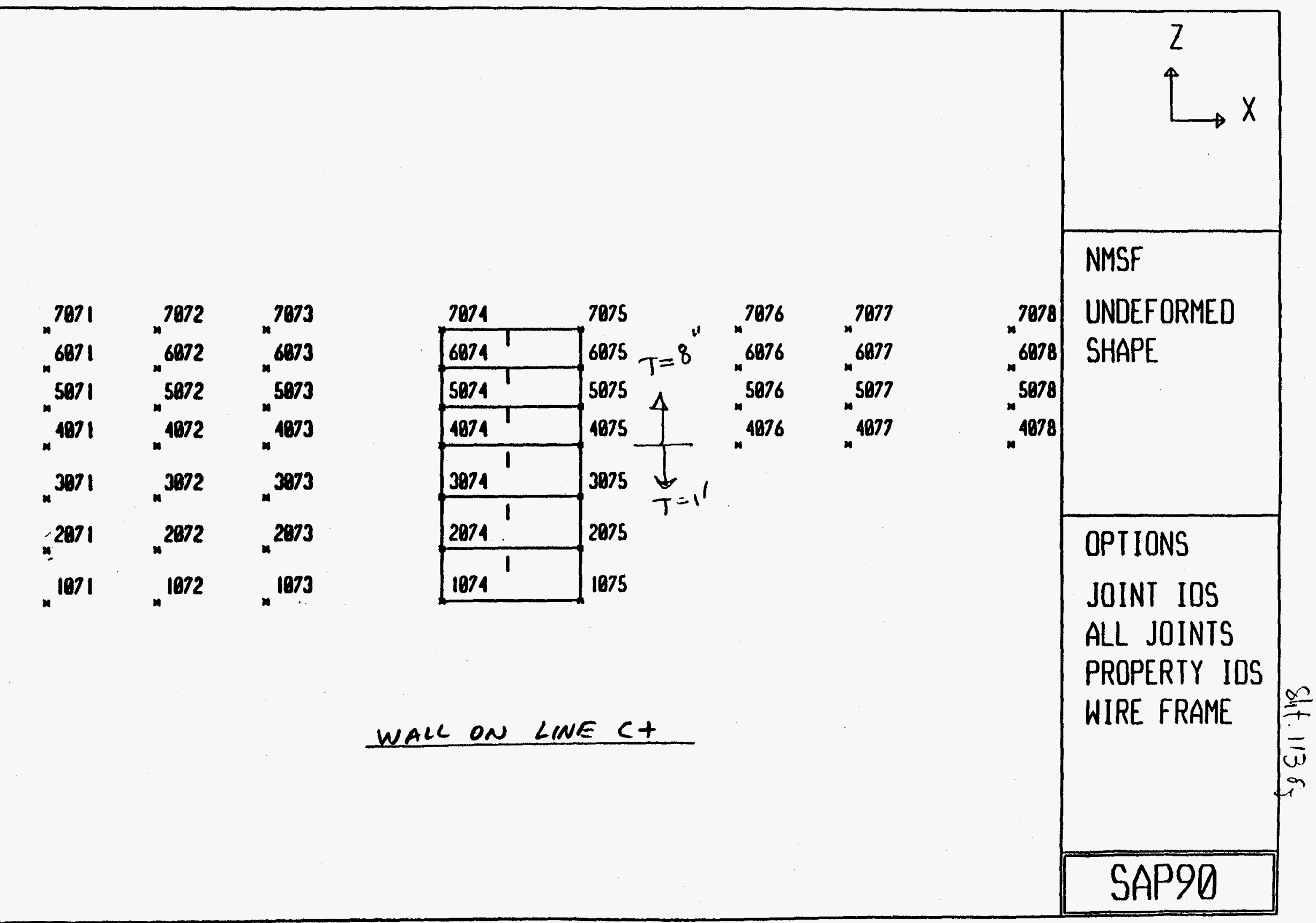




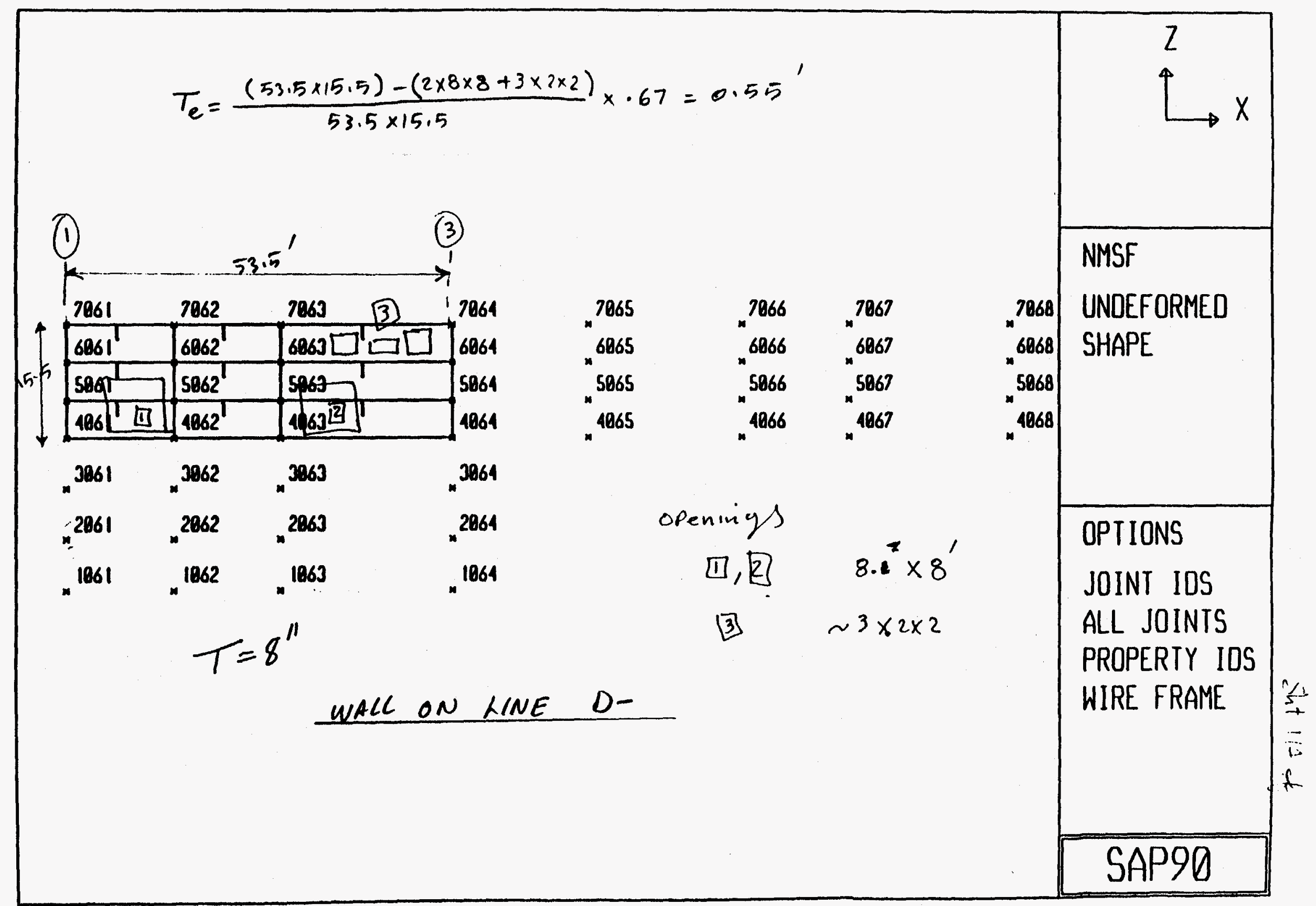


$2+150$

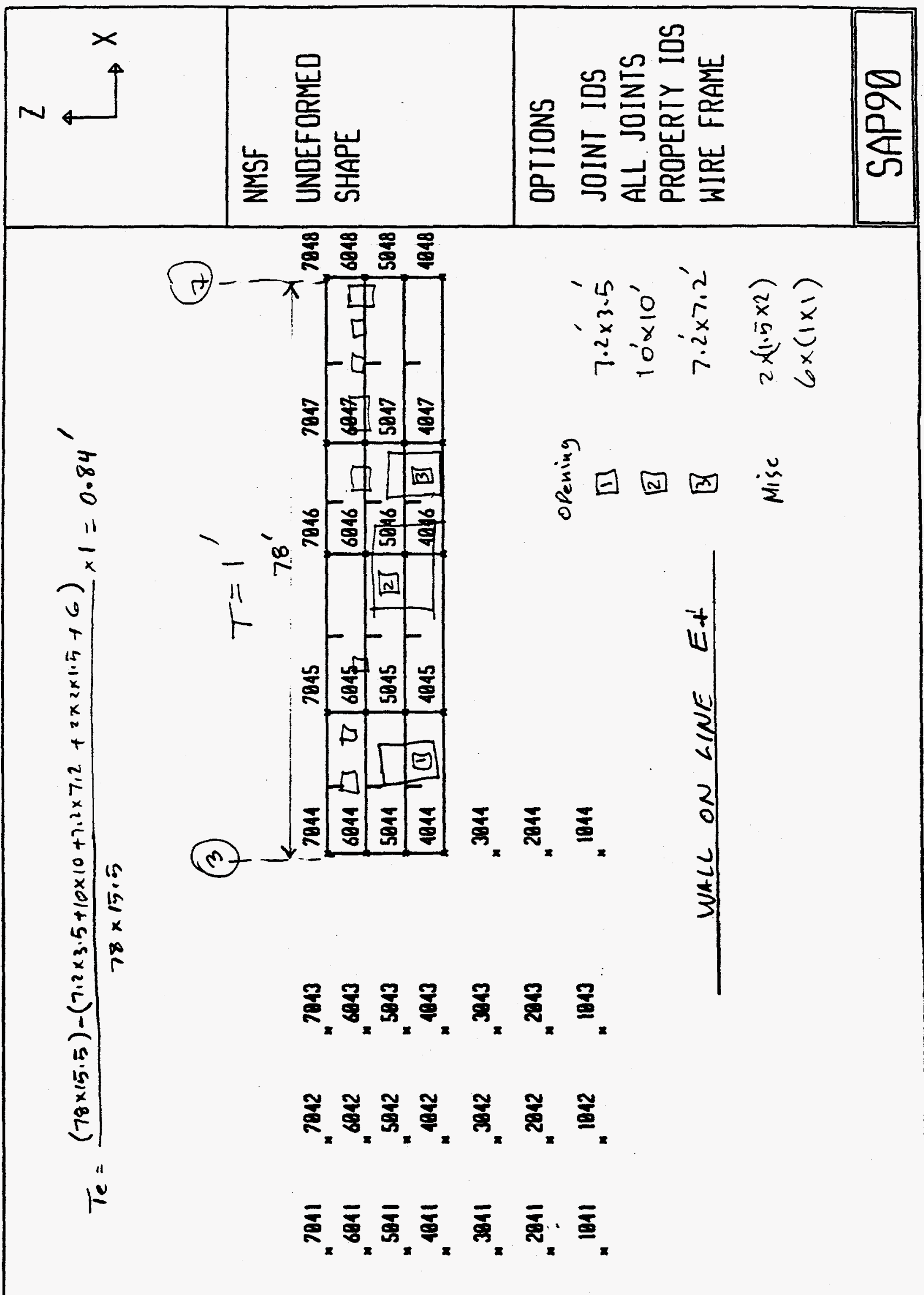




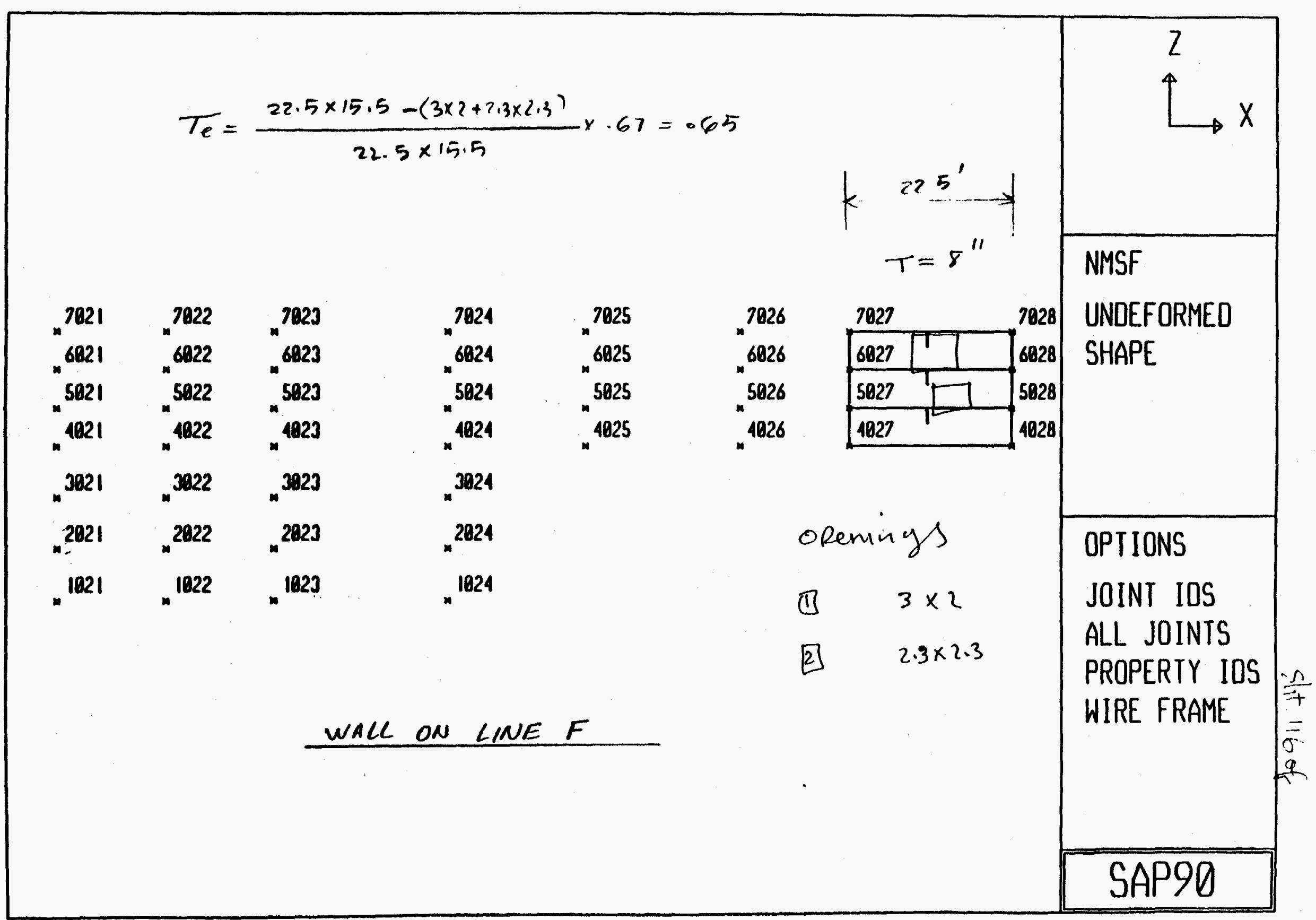


$-19.117$

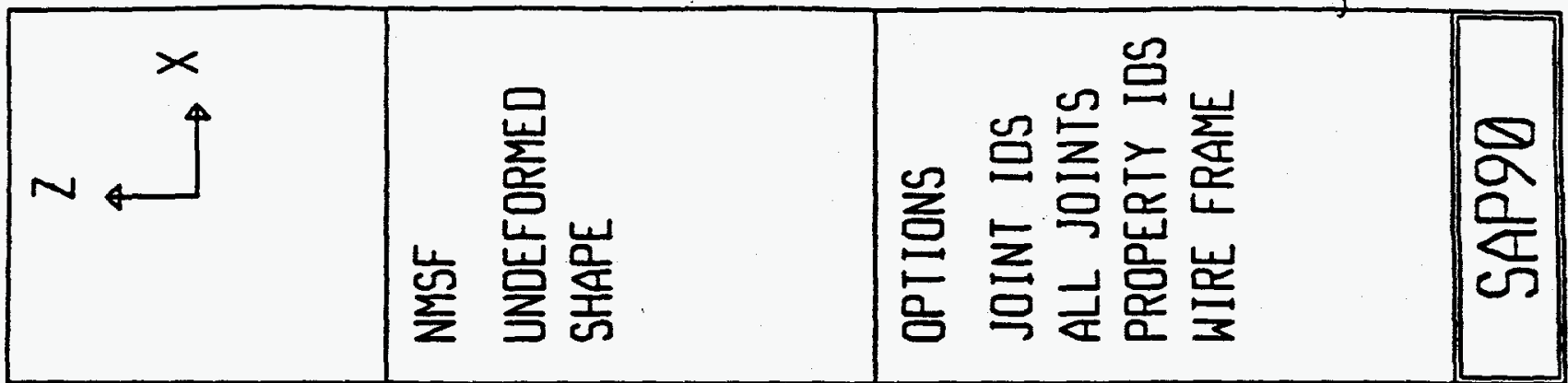
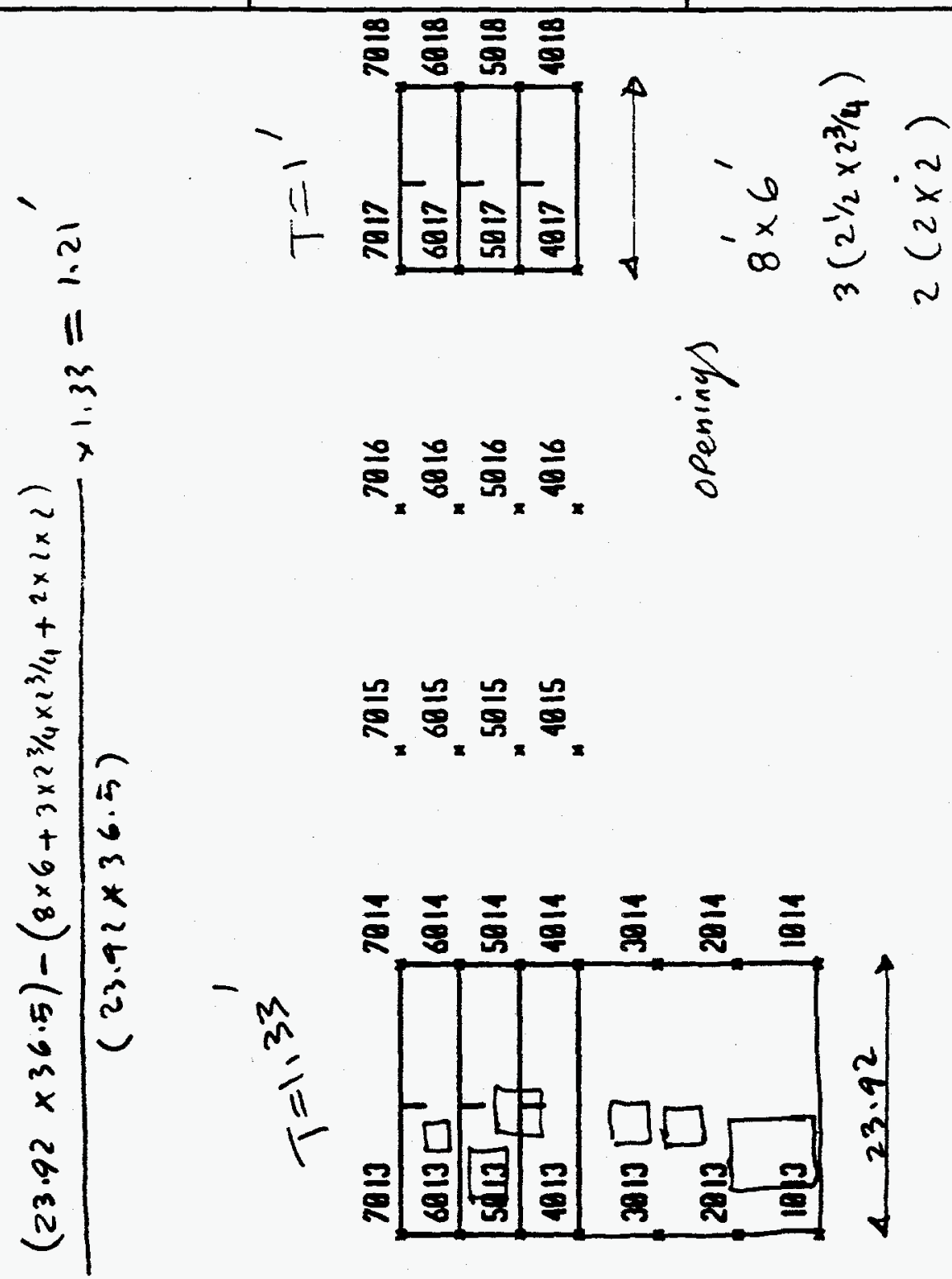

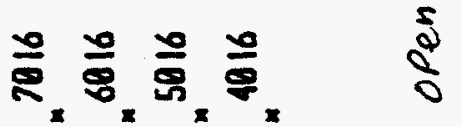

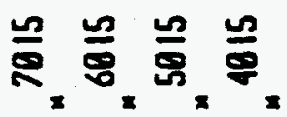

$\frac{5}{\frac{5}{2}}$

$r^{\prime \prime}$

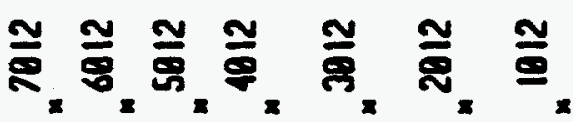

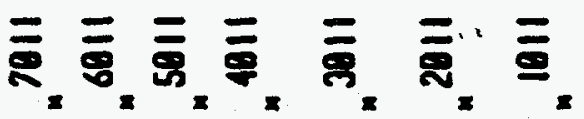




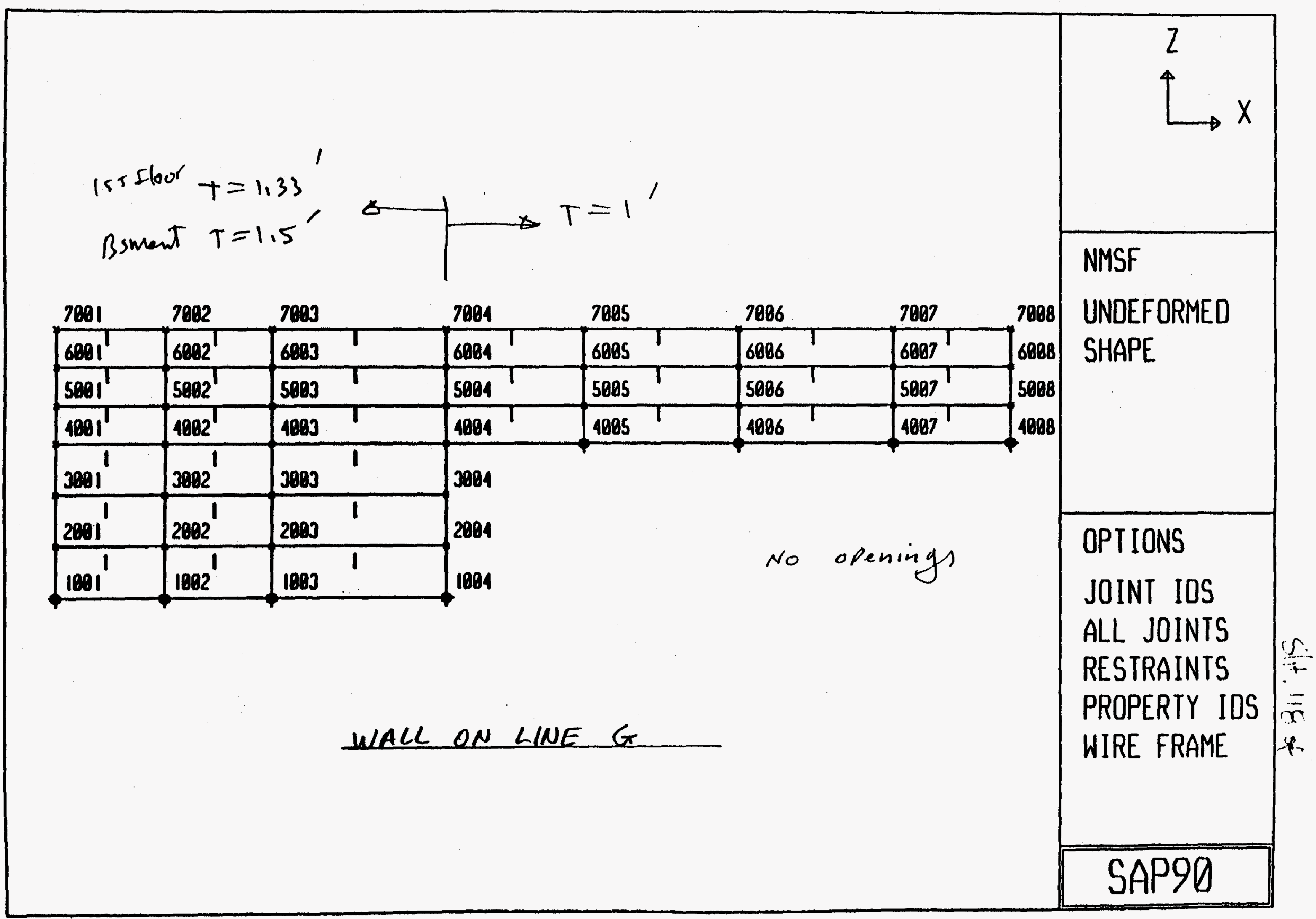


Cprof jounoy

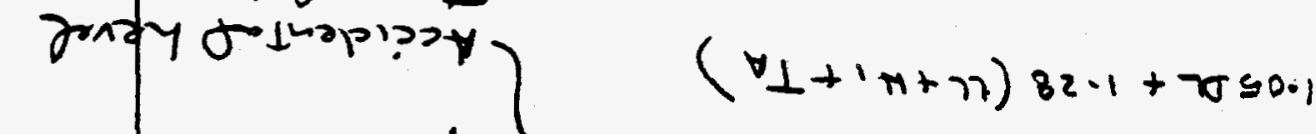

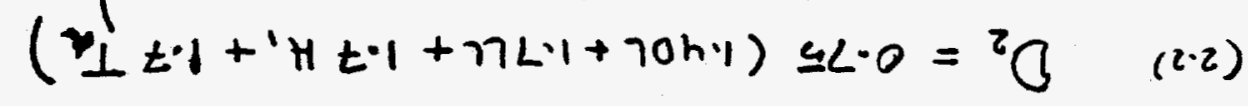

$$
1<1+: H<1+77<1+70 h \cdot 1=1(1 \cdot 2)
$$

$(9240$ '16-28n) sugipaniqmos prof 2 imsias-woN (z
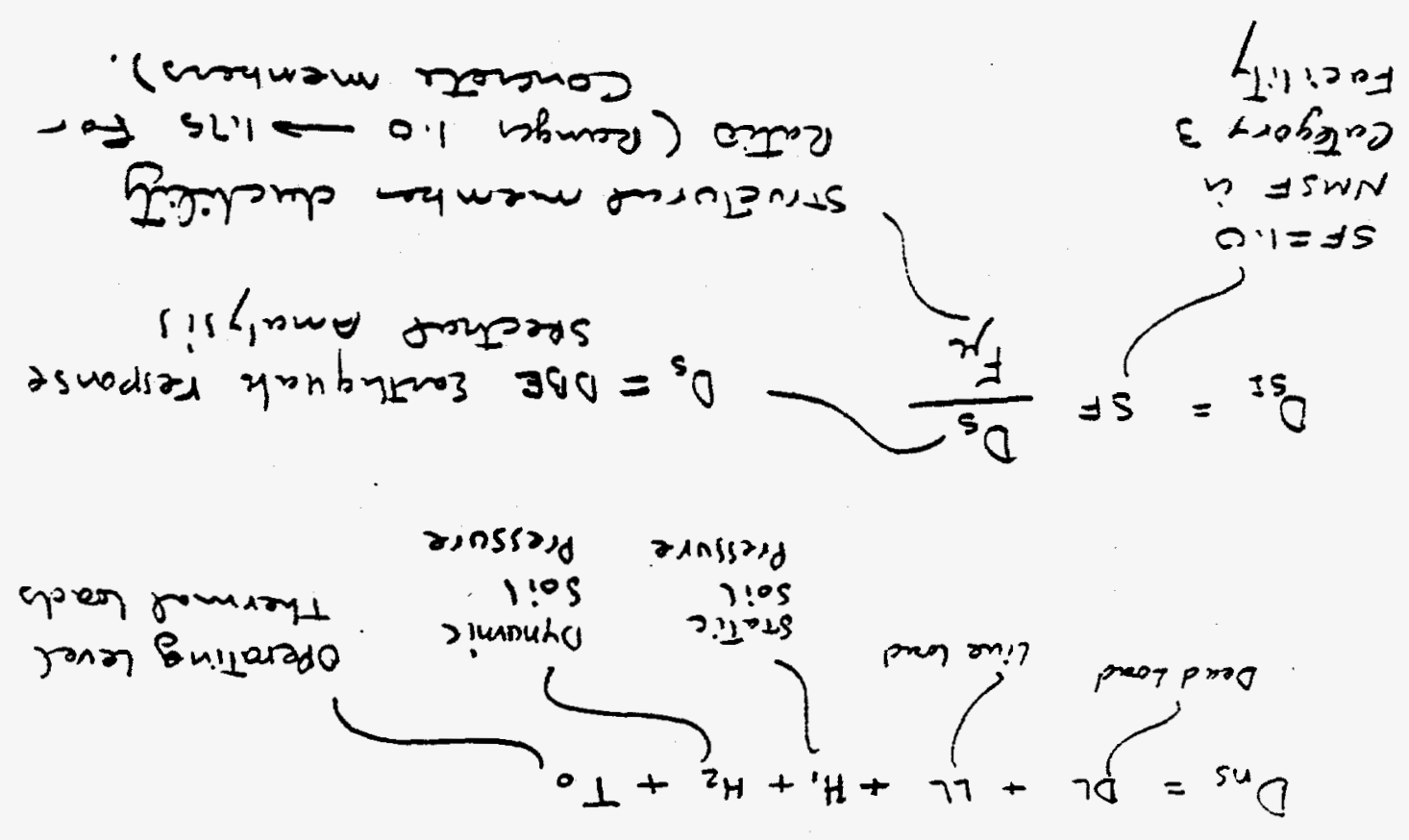

$$
{ }^{r s} Q+{ }^{s u} Q=a
$$

\begin{tabular}{|c|c|}
\hline 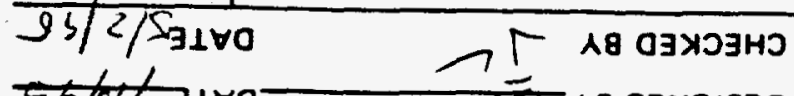 & $3 x n+2 n z i s \quad 17108-$ \\
\hline CUタ $1803 \mathrm{NOIS} 30$ & $7 N+7 m, \quad 354 N$ ग0 \\
\hline 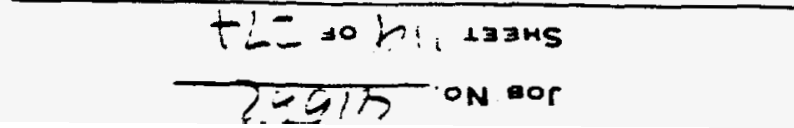 & a my i \\
\hline
\end{tabular}

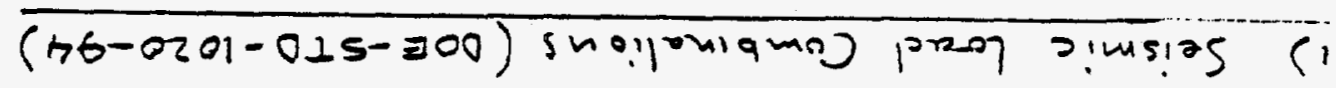




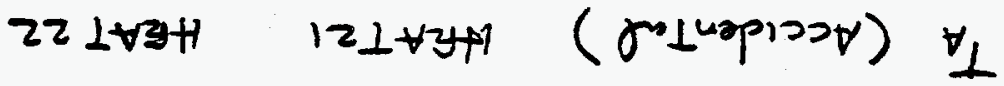

$$
\begin{aligned}
& \text { - (Bulpiado) }{ }^{2}
\end{aligned}
$$

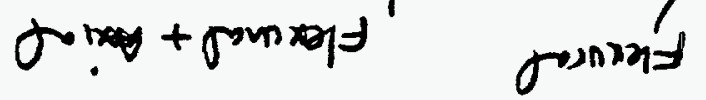

$$
\begin{aligned}
& \text { sistinnt somizuL }
\end{aligned}
$$

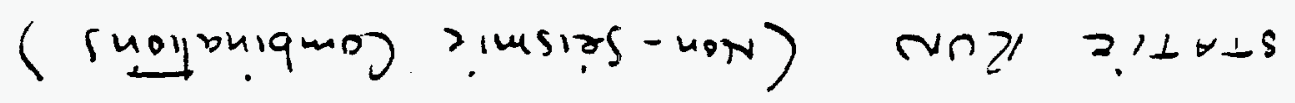

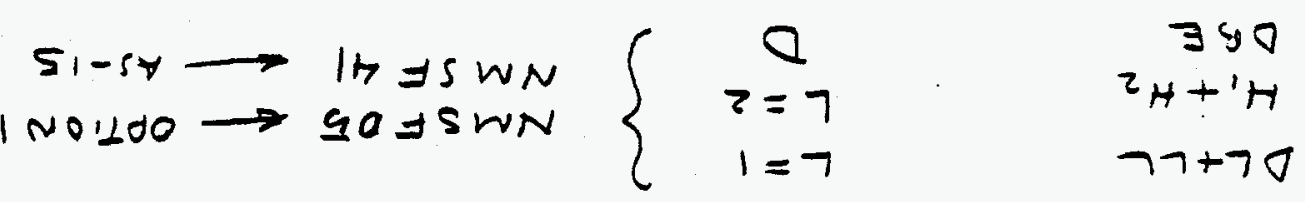

\begin{tabular}{|c|c|c|}
\hline \multirow{2}{*}{ 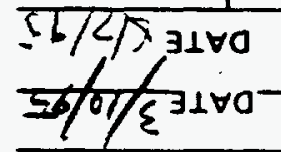 } & \multirow{2}{*}{ 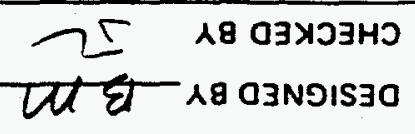 } & \\
\hline & & 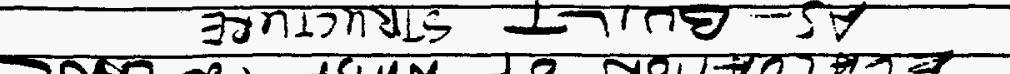 \\
\hline & 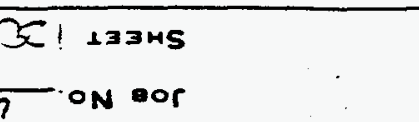 & 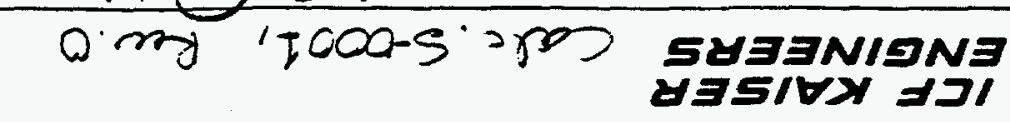 \\
\hline
\end{tabular}

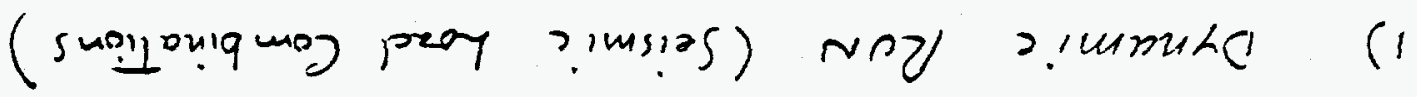$$
\text { snod } 0,0 d H
$$ 


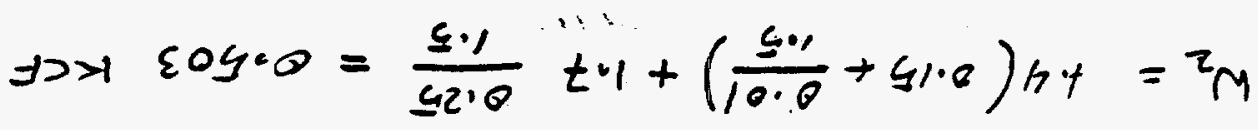

$\neg \neg ட \cdot 1+70 h \cdot 1$ pronainbz

$\Rightarrow \supset>\quad \varepsilon \varepsilon \varepsilon \cdot 0=\frac{\xi \cdot 1}{s 2 \cdot 0+10 \cdot 0}+\xi 1.0=\mathrm{m}$

$77+70$ peyoninbs

$91900.0=2.2 \varepsilon /\left(\frac{5.1}{52.0 \times 52.0+10.0}+41.0\right)=2 M$

suro ssow rugoninby

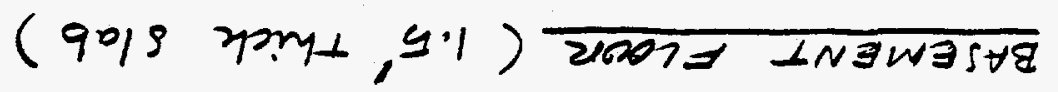

SISATENH DIVHNLO

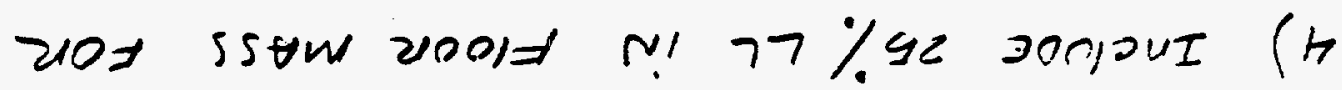

ySO OE

$\nabla+07$ MONS $(\varepsilon$

I5d $01 \quad 0+0) 7+40$ ganlnd (2

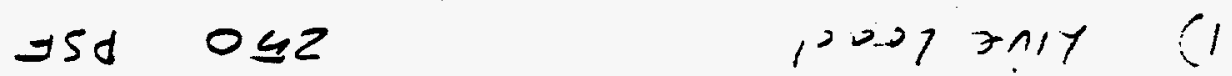

$\overline{25 / 2 / S 3170 \quad \sim 5 \quad 1803 \times 23 H 0}$

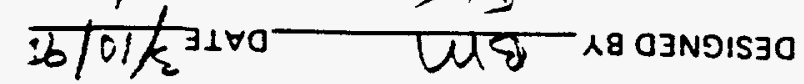

sossolv' qoory miy 'yoroy pood

DLZ オ0 に1 133HS

\begin{tabular}{|c|c|c|c|}
\hline 。 & 001 & $\sim$ & $\sim$ \\
\hline$\sim$ & ObH & $v$ & N \\
\hline IDd & 091 & Gisure & $\operatorname{lm}$ \\
\hline
\end{tabular}




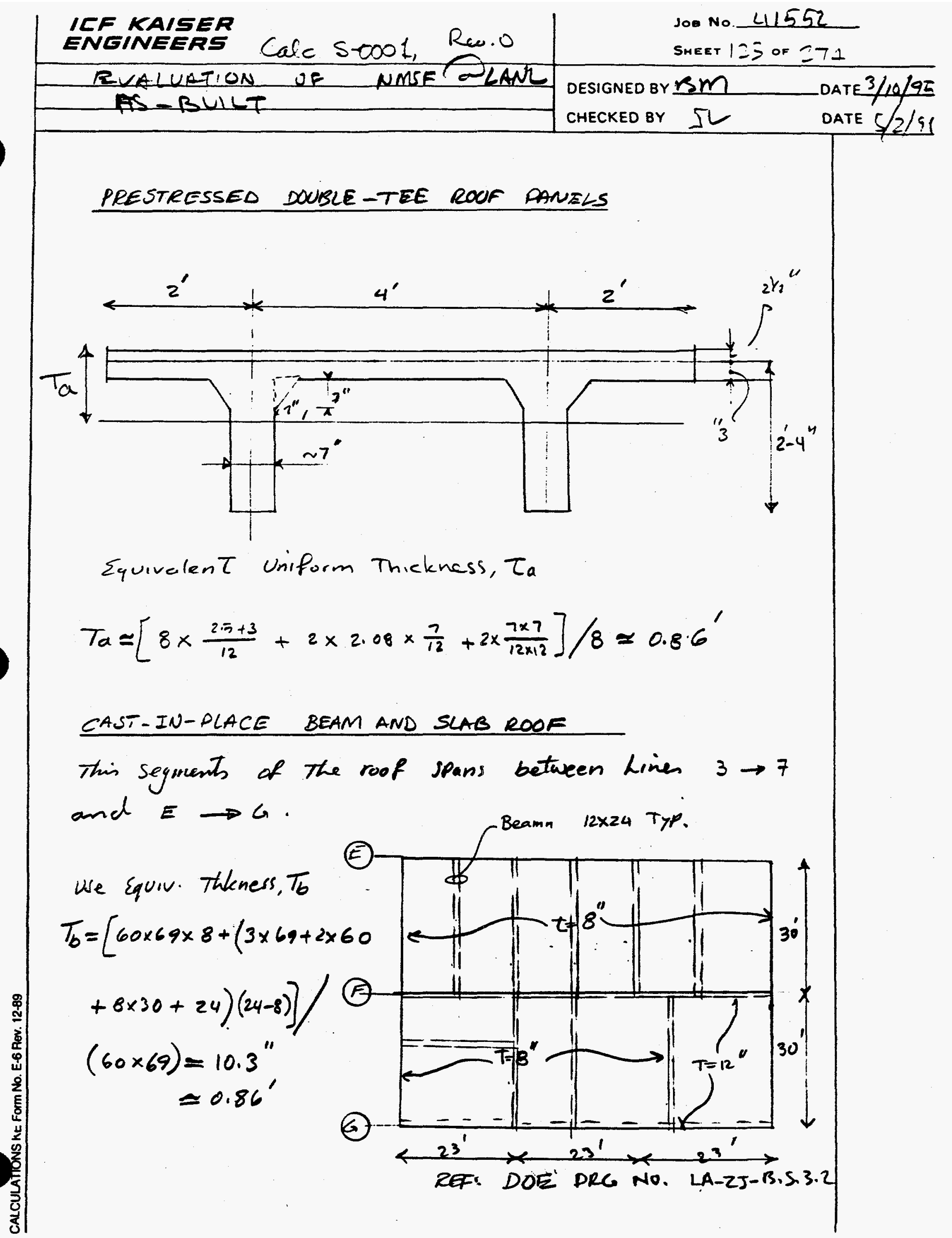




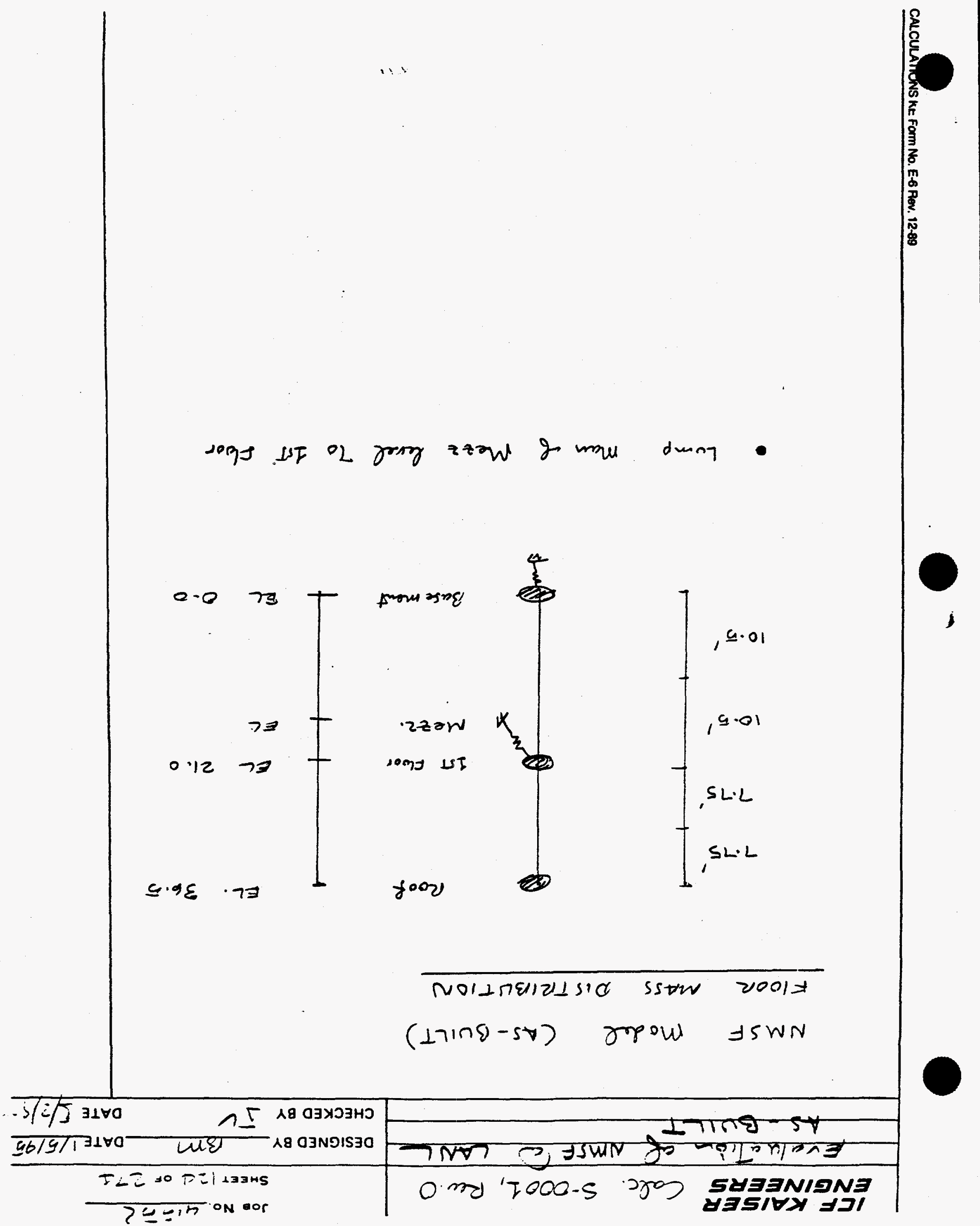




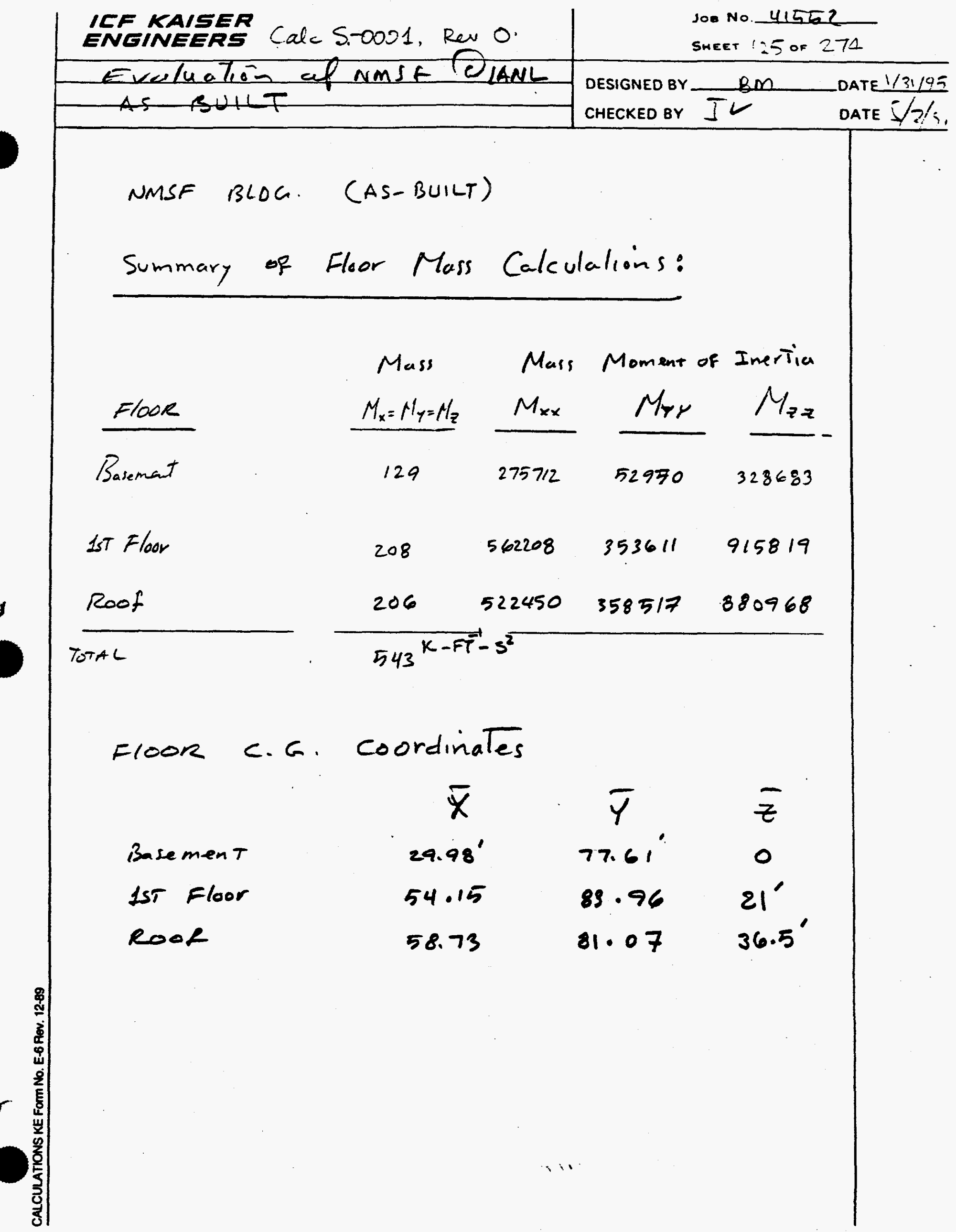




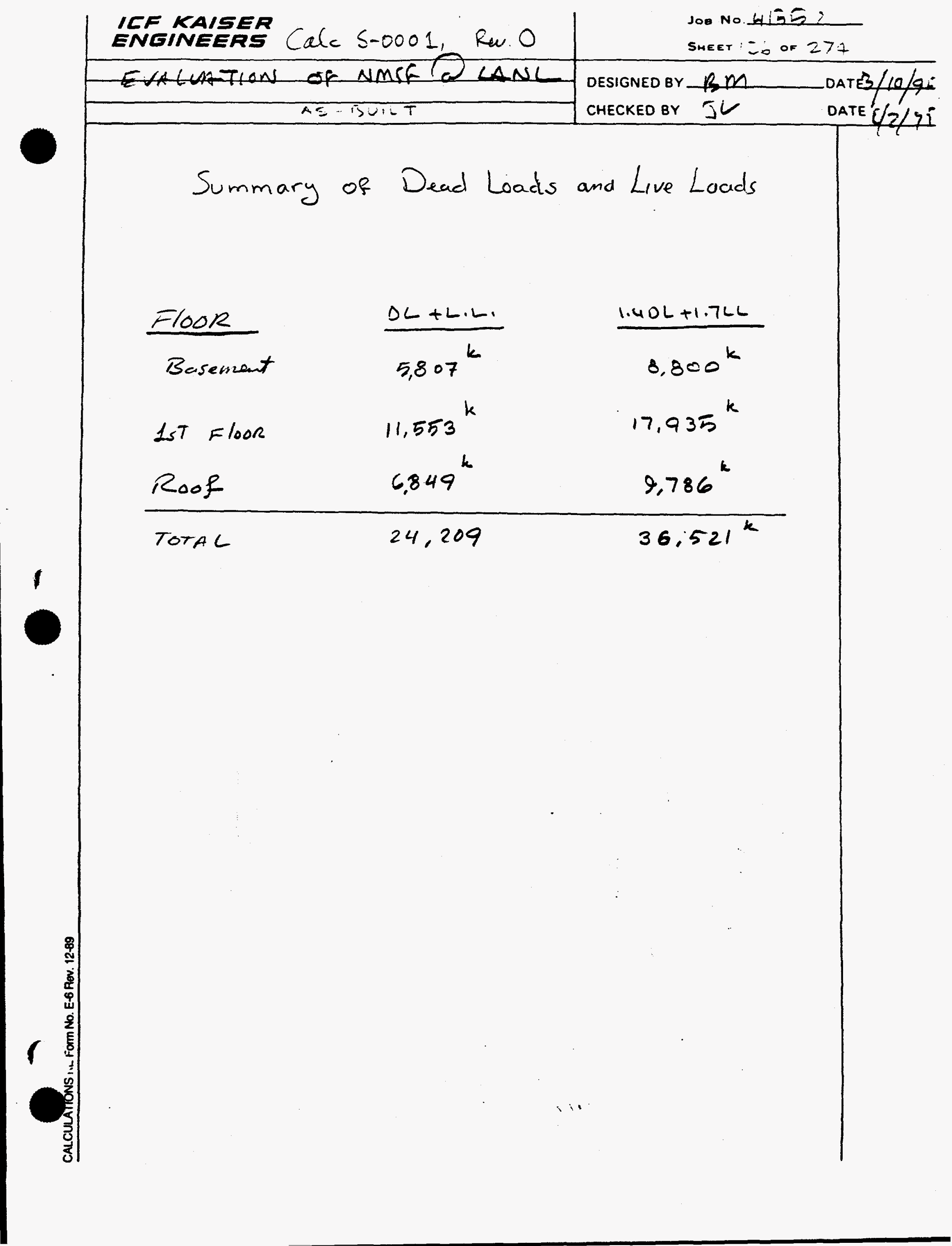




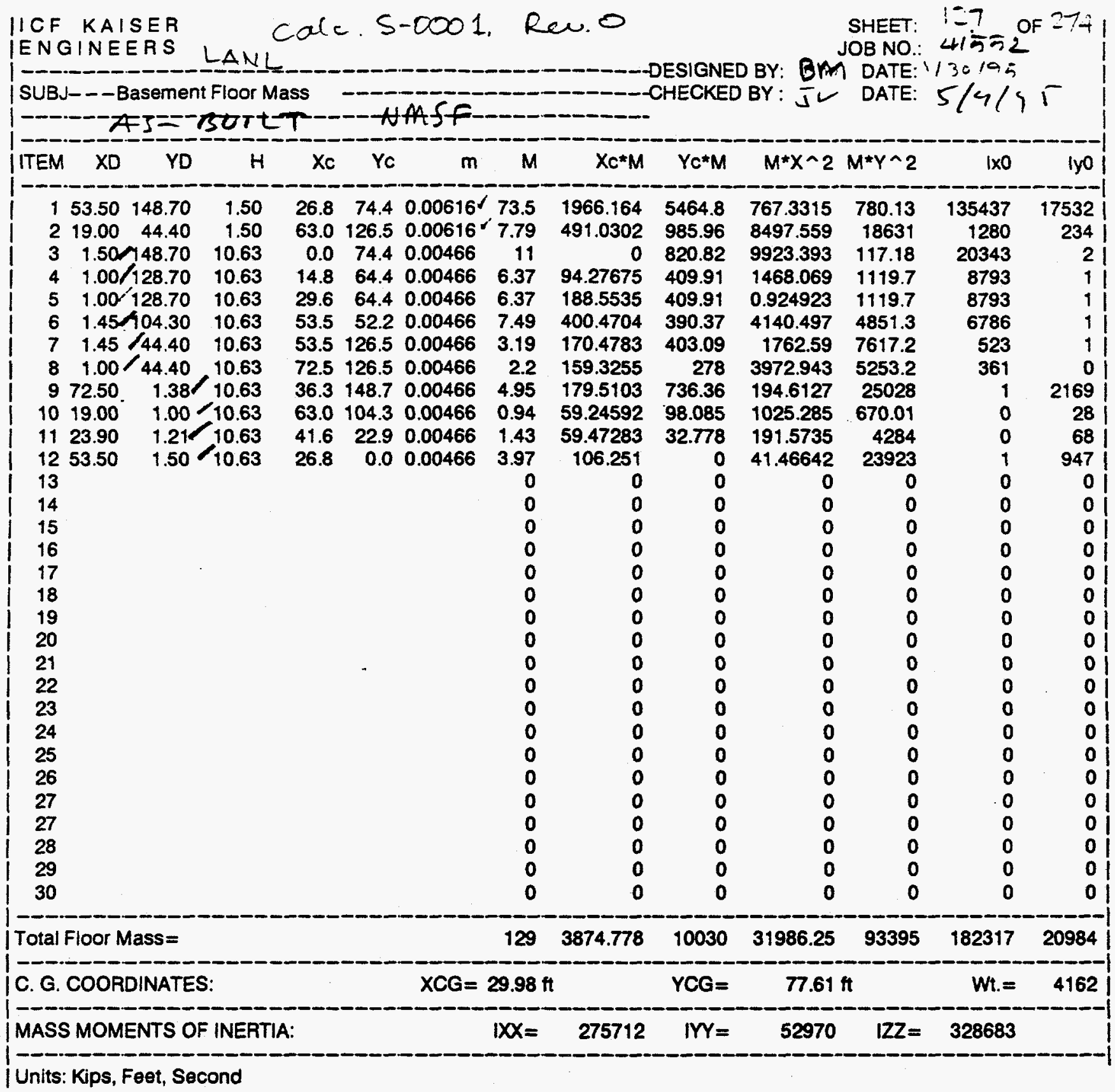


IICF KAISER IENGINEERS Cal C. S-O001, Rer.

Calc. S-0001, Rev. O

SHEET: 125 OF $27+1$

| SUBJ- - Basement Floor (DL+LL) -

CHECKED BY: IL

DATE: $3-1-950$

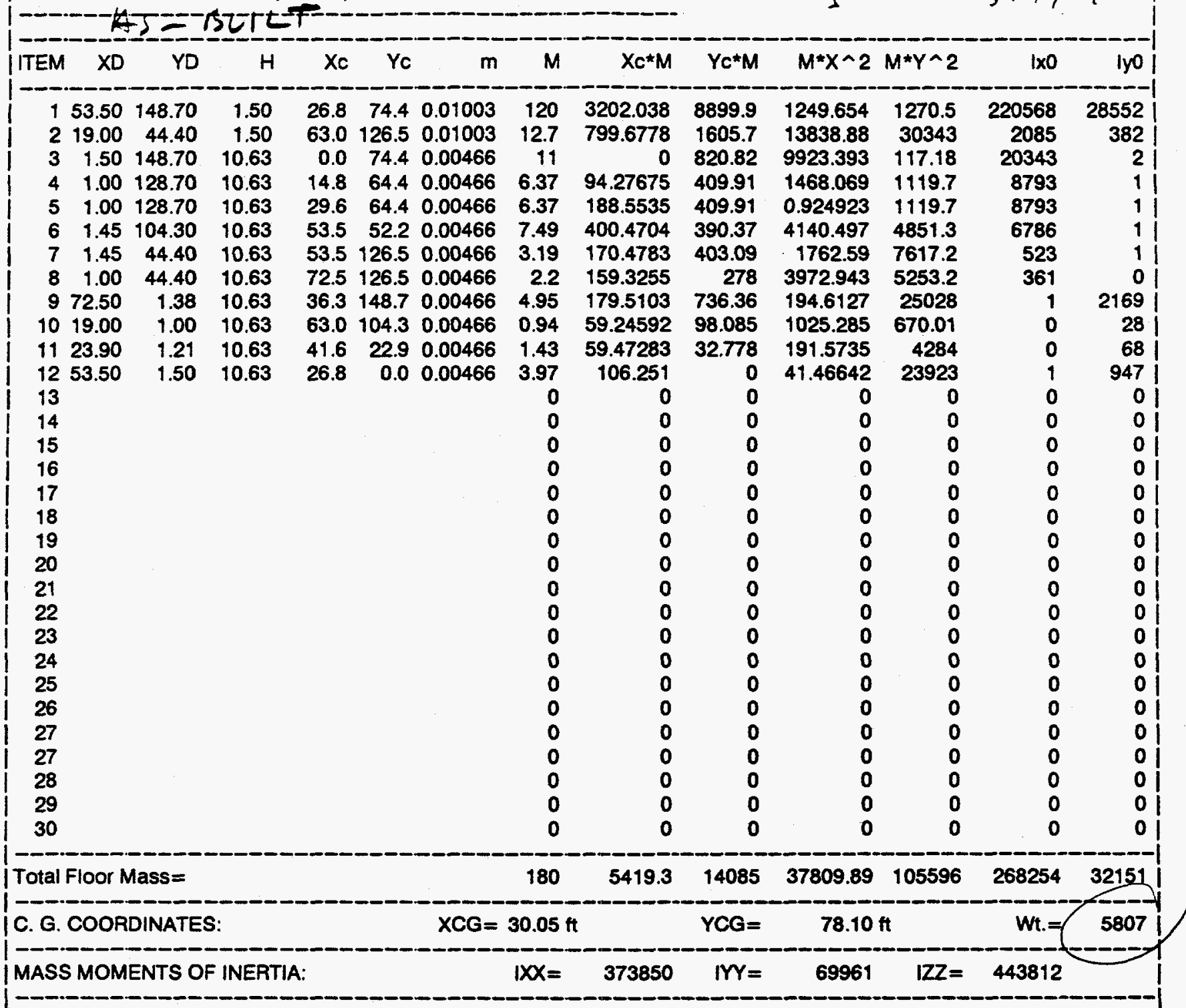

Units: Kips, Feet, Second 

IICF KAISER CalC. S-COD1, ReU. 0
IENGINEERS

SHEET: $1=-1$ OF 27 -

JOB NO.: 41552

ISUBJ---Basement Floor (1.4DL + 1.7LL)-_-_-_-_-CHECKED BY: $\backsim$ DATE: $[/ 4 / 9 r$

\begin{tabular}{|c|c|c|c|c|c|c|c|c|c|c|c|c|c|}
\hline ITEM & $X D$ & YD & $H$ & Xc & Yo & $m$ & $M$ & $X c^{*} M$ & $Y C^{\star} M$ & $M * X \wedge 2$ & $M^{\star} Y \wedge 2$ & $1 \times 0$ & Iyo \\
\hline & $\begin{array}{l}53.50 \\
19.00\end{array}$ & $\begin{array}{r}148.70 \\
44.40\end{array}$ & $\begin{array}{l}1.50 \\
1.50\end{array}$ & $\begin{array}{l}26.8 \\
63.0\end{array}$ & $\begin{array}{r}74.4 \\
126.5\end{array}$ & $\begin{array}{l}0.01562 \\
0.01562\end{array}$ & $\begin{array}{r}186 \\
19.8\end{array}$ & $\begin{array}{l}4986.455 \\
1245.319\end{array}$ & $\begin{array}{r}13860 \\
2500.5\end{array}$ & $\begin{array}{l}1946.056 \\
21550.95\end{array}$ & $\begin{array}{r}1978.5 \\
47252\end{array}$ & $\begin{array}{r}343486 \\
3247\end{array}$ & $\begin{array}{r}44463 \\
595\end{array}$ \\
\hline 3 & 1.50 & 148.70 & 10.63 & 0.0 & 74.4 & 0.00652 & 15.5 & 0 & 1149.1 & 13892.75 & 164.05 & 28480 & 3 \\
\hline 4 & 1.00 & 128.70 & 10.63 & 14.8 & 64.4 & 0.00652 & 8.92 & 131.9874 & 573.88 & 2055.297 & 1567.5 & 12310 & 1 \\
\hline 5 & 1.00 & 128.70 & 10.63 & 29.6 & 64.4 & 0.00652 & 8.92 & 263.9749 & 573.88 & 1.294892 & 1567.5 & 12310 & 1 \\
\hline 6 & 1.45 & 104.30 & 10.63 & 53.5 & 52.2 & 0.00652 & 10.5 & 560.6586 & 546.51 & 5796.696 & 6791.9 & 9500 & 2 \\
\hline 7 & 1.45 & 44.40 & 10.63 & 53.5 & 126.5 & 0.00652 & 4.46 & 238.6696 & 564.33 & 2467.625 & 10664 & 733 & 1 \\
\hline 8 & 1.00 & 44.40 & 10.63 & 72.5 & 126.5 & 0.00652 & 3.08 & 223.0557 & 389.19 & 5562.121 & 7354.5 & 505 & 0 \\
\hline 9 & 72.50 & 1.38 & 10.63 & 36.3 & 148.7 & 0.00652 & 6.93 & 251.3145 & 1030.9 & 272.4577 & 35039 & 1 & 3037 \\
\hline 10 & $: 9.00$ & 1.00 & 10.63 & 63.0 & 104.3 & 0.00652 & 1.32 & 82.94429 & 137.32 & 1435.399 & 938.02 & 0 & 40 \\
\hline 11 & 23.90 & 1.21 & 10.63 & 41.6 & 22.9 & 0.00652 & 2 & 83.26197 & 45.889 & 268.203 & 5997.6 & 0 & 95 \\
\hline 12 & 53.50 & 1.50 & 10.63 & 26.8 & 0.0 & 0.00652 & 5.56 & 148.7514 & 0 & 58.05299 & 33493 & 1 & 1326 \\
\hline 13 & & & & & & & 0 & 0 & 0 & 0 & 0 & 0 & 0 \\
\hline 14 & & & & & & & 0 & 0 & 0 & 0 & 0 & 0 & 0 \\
\hline 15 & & & & & & & 0 & 0 & 0 & 0 & 0 & 0 & 0 \\
\hline 16 & & & & & & & 0 & 0 & 0 & 0 & 0 & 0 & 0 \\
\hline 17 & & & & & & & 0 & 0 & 0 & 0 & 0 & 0 & 0 \\
\hline 18 & & & & & & & 0 & 0 & 0 & 0 & 0 & 0 & 0 \\
\hline 19 & & & & & & & 0 & 0 & 0 & 0 & 0 & 0 & 0 \\
\hline 20 & & & & & & & 0 & 0 & 0 & 0 & 0 & 0 & 0 \\
\hline 21 & & & & & & & 0 & 0 & 0 & 0 & 0 & 0 & 0 \\
\hline 22 & & & & & & & 0 & 0 & 0 & 0 & 0 & 0 & 0 \\
\hline 23 & & & & & & & 0 & 0 & 0 & 0 & 0 & 0 & 0 \\
\hline 24 & & & & & & & 0 & 0 & 0 & 0 & 0 & 0 & 0 \\
\hline 25 & & & & & & & 0 & 0 & 0 & 0 & o & 0 & \\
\hline 26 & & & & & & & 0 & 0 & 0 & 0 & 0 & 0 & 0 \\
\hline 27 & & & & & & & 0 & 0 & 0 & 0 & 0 & 0 & 0 \\
\hline 27 & & & & & & & 0 & 0 & 0 & 0 & 0 & 0 & 0 \\
\hline 28 & & & & & & & 0 & 0 & 0 & 0 & o & 0 & 0 \\
\hline 29 & & & & & & & 0 & 0 & 0 & 0 & 0 & 0 & \\
\hline 30 & & & & & & & 0 & 0 & 0 & 0 & 0 & 0 & 0 \\
\hline Total F & Floor $\mathrm{A}$ & Mass= & & & & & 273 & 8216.392 & 21371 & 55306.91 & 152807 & 410573 & 49563 \\
\hline C. G. & COOR & ZDINATE & & & & $X C G=$ & 30.06 & & $Y C G=$ & 78.20 & & $\mathbf{W t} .=$ & 00 \\
\hline MASS & MON & AENTS & NEI & & & & $|x| x=$ & 563380 & $M Y=$ & 104869 & $1 Z Z=$ & 668250 & \\
\hline
\end{tabular}

Units: Kips, Feet, Second 


$$
\text { Salc S-0001, Rav. O }
$$

IICF KAISER

SHEET: : : : OF

IENGINEERS 'Li:

JOB NO: 4

SUBJECT: - - First Floor Mass

-

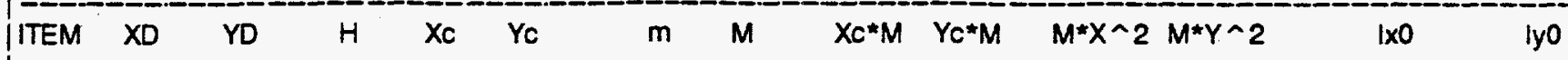

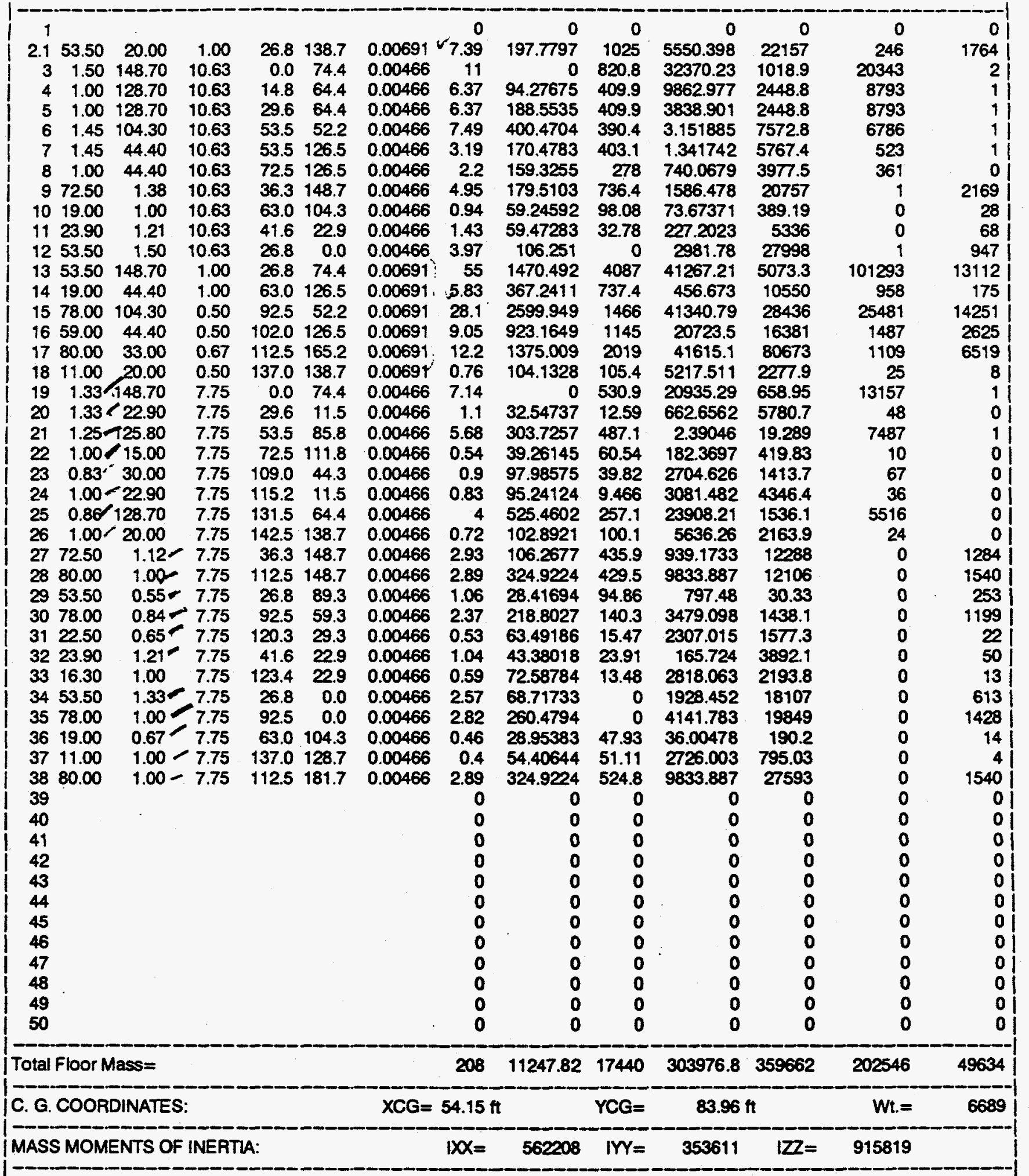

Units: Kips, Feet, Second 
ll CF KaISER Cale S-Do01. Rev.0

IENGINEERS

LANL

SHEET: $1 \equiv 1 \quad$ OF=7

JOB NO: 4 प⿺辶万丂 2

I SUBJECT: - - First Floor (DL+LL) - - D CHECKED BY:5L DATE: $5 / 4 / 95$

\begin{tabular}{|c|c|c|c|c|c|c|c|c|c|c|c|c|c|}
\hline ITEM & $X$ & YD & H & $x c$ & Yc & $m$ & $M$ & $X c * M$ & $Y C^{\star} M$ & $M^{\star} X^{\wedge} 2$ & $M * Y \wedge 2$ & $1 \times 0$ & lyo \\
\hline 1 & & & & & & & 0 & 0 & 0 & 0 & 0 & 0 & 0 \\
\hline 2.1 & 53.50 & 20.00 & 1.00 & 26.8 & 138.7 & 0.01273 & 13.6 & 364.448 & 1890 & 10227.7 & 40829 & 454 & 3250 \\
\hline 3 & 1.50 & 148.70 & 10.63 & 0.0 & 74.4 & 0.00466 & 11 & & 820.8 & 32370.23 & 1018.9 & 20343 & 2 \\
\hline 4 & 1.00 & 128.70 & 10.63 & 14.8 & 64.4 & 0.00466 & 6.37 & 94.27675 & 409.9 & 9862.977 & 2448.8 & 8793 & 1 \\
\hline 5 & 1.00 & 128.70 & 10.63 & 29.6 & 64.4 & 0.00466 & 6.37 & 188.5535 & 409.9 & 3838.901 & 2448.8 & 8793 & 1 \\
\hline 6 & 1.45 & 104.30 & 10.63 & 53.5 & 52.2 & 0.00466 & 7.49 & 400.4704 & 390.4 & 3.151885 & 7572.8 & 6786 & 1 \\
\hline 7 & 1.45 & 44.40 & 10.63 & 53.5 & 126.5 & 0.00466 & 3.19 & 170.4783 & 403.1 & 1.341742 & 5767.4 & 523 & 1 \\
\hline 8 & 1.00 & 44.40 & 10.63 & 72.5 & 126.5 & 0.00466 & 2.2 & 159.3255 & 278 & 740.0679 & 3977.5 & 361 & 0 \\
\hline 9 & 72.50 & 1.38 & 10.63 & 36.3 & 148.7 & 0.00466 & 4.95 & 179.5103 & 736.4 & 1586.478 & 20757 & 1 & 2169 \\
\hline 10 & 19.00 & 1.00 & 10.63 & 63.0 & 104.3 & 0.00466 & 0.94 & 59.24592 & 98.08 & 73.67371 & 389.19 & 0 & 28 \\
\hline 11 & 23.90 & 1.21 & 10.63 & 41.6 & 22.9 & 0.00466 & 1.43 & 59.47283 & 32.78 & 227.2023 & 5336 & 0 & 68 \\
\hline 12 & 53.50 & 1.50 & 10.63 & 26.8 & 0.0 & 0.00466 & 3.97 & 106.251 & & 2981.78 & 27998 & 1 & 947 \\
\hline 13 & 53.50 & 148.70 & 1.00 & 26.8 & 74.4 & 0.01273 & 101 & 2709.671 & 7531 & 76042.95 & 9348.6 & 186652 & 24161 \\
\hline 14 & 19.00 & 44.40 & 1.00 & 63.0 & 126.5 & 0.01273 & 10.7 & 676.7139 & 1359 & 841.5098 & 19441 & 1765 & 323 \\
\hline 15 & 78.00 & 104.30 & 0.50 & 92.5 & 52.2 & 0.02081 & 84.6 & 7829.059 & 4414 & 124486.9 & 85626 & 76728 & 42912 \\
\hline 16 & 59.00 & 44.40 & 0.50 & 102.0 & 126.5 & 0.02081 & 27.3 & 2779.867 & 3448 & 62403.33 & 49327 & 4477 & 7906 \\
\hline 17 & 80.00 & 33.00 & 0.67 & 112.5 & 165.2 & 0.01671 & 29.6 & 3325.109 & 4883 & 100635.6 & 195087 & 2682 & 15763 \\
\hline 18 & 11.00 & 20.00 & 0.50 & 137.0 & 138.7 & 0.02081 & 2.29 & 313.5683 & 317.5 & 15711.16 & 6859.2 & 76 & 23 \\
\hline 19 & 1.33 & 148.70 & 7.75 & 0.0 & 74.4 & 0.00466 & 7.14 & 0 & 530.9 & 20935.29 & 658.95 & 13157 & 1 \\
\hline 20 & 1.33 & 22.90 & 7.75 & 29.6 & 11.5 & 0.00466 & 1.1 & 32.54737 & 12.59 & 662.6562 & 5780.7 & 48 & 0 \\
\hline 21 & 1.25 & 125.80 & 7.75 & 53.5 & 85.8 & 0.00466 & 5.68 & 303.7257 & 487.1 & 2.39046 & 19.289 & 7487 & 1 \\
\hline 22 & 1.00 & 15.00 & 7.75 & 72.5 & 111.8 & 0.00466 & 0.54 & 39.26145 & 60.54 & 182.3697 & 419.83 & 10 & 0 \\
\hline 23 & 0.83 & 30.00 & 7.75 & 109.0 & 44.3 & 0.00466 & 0.9 & 97.98575 & 39.82 & 2704.626 & 1413.7 & 67 & 0 \\
\hline 24 & 1.00 & 22.90 & 7.75 & 115.2 & 11.5 & 0.00466 & 0.83 & 95.24124 & 9.466 & 3081.482 & 4346.4 & 36 & o \\
\hline 25 & 0.86 & 128.70 & 7.75 & 131.5 & 64.4 & 0.00466 & 4 & 525.4602 & 257.1 & 23908.21 & 1536.1 & 5516 & 0 \\
\hline 26 & 1.00 & 20.00 & 7.75 & 142.5 & 138.7 & 0.00466 & 0.72 & 102.8921 & 100.1 & 5636.26 & 2163.9 & 24 & 0 \\
\hline 27 & 72.50 & 1.12 & 7.75 & 36.3 & 148.7 & 0.00466 & 2.93 & 106.2677 & 435.9 & 939.1733 & 12288 & 0 & 1284 \\
\hline 28 & 80.00 & 1.00 & 7.75 & 112.5 & 148.7 & 0.00466 & 2.89 & 324.9224 & 429.5 & 9833.887 & 12106 & 0 & 1540 \\
\hline 29 & 53.50 & 0.55 & 7.75 & 26.8 & 89.3 & 0.00466 & 1.06 & 28.41694 & 94.86 & 797.48 & 30.33 & 0 & 253 \\
\hline 30 & 78.00 & 0.84 & 7.75 & 92.5 & 59.3 & 0.00466 & 2.37 & 218.8027 & 140.3 & 3479.098 & 1438.1 & 0 & 1199 \\
\hline 31 & 22.50 & 0.65 & 7.75 & 120.3 & 29.3 & 0.00466 & 0.53 & 63.49186 & 15.47 & 2307.015 & 1577.3 & 0 & 22 \\
\hline 32 & 23.90 & 1.21 & 7.75 & 41.6 & 22.9 & 0.00466 & 1.04 & 43.38018 & 23.91 & 165.724 & 3892.1 & 0 & 50 \\
\hline 33 & 16.30 & 1.00 & 7.75 & 123.4 & 22.9 & 0.00466 & 0.59 & 72.58784 & 13.48 & 2818.063 & 2193.8 & 0 & 13 \\
\hline 34 & 53.50 & 1.33 & 7.75 & 26.8 & 0.0 & 0.00466 & 2.57 & 68.71733 & 0 & 1928.452 & 18107 & 0 & 613 \\
\hline 35 & 78.00 & 1.00 & 7.75 & 92.5 & 0.0 & 0.00466 & 2.82 & 260.4794 & 0 & 4141.783 & 19849 & 0 & 1428 \\
\hline 36 & 19.00 & 0.67 & 7.75 & 63.0 & 104.3 & 0.00466 & 0.46 & 28.95383 & 47.93 & 36.00478 & 190.2 & 0 & 14 \\
\hline 37 & 11.00 & 1.00 & 7.75 & 137.0 & 128.7 & 0.00466 & 0.4 & 54.40644 & 51.11 & 2726.003 & 795.03 & 0 & 4 \\
\hline 38 & 80.00 & 1.00 & 7.75 & 112.5 & 181.7 & 0.00466 & 2.89 & 324.9224 & 524.8 & 9833.887 & 27593 & 0 & 1540 \\
\hline $\begin{array}{l}39 \\
40\end{array}$ & & & & & & & 0 & 0 & 0 & 0 & 0 & 0 & 0 \\
\hline $\begin{array}{l}40 \\
41\end{array}$ & & & & & & & 0 & 0 & 0 & 0 & 0 & 0 & \\
\hline 42 & & & & & & & $\begin{array}{l}0 \\
0\end{array}$ & $\begin{array}{l}0 \\
0\end{array}$ & $\begin{array}{l}0 \\
0\end{array}$ & $\begin{array}{l}0 \\
0\end{array}$ & $\begin{array}{l}0 \\
0\end{array}$ & $\begin{array}{l}0 \\
0\end{array}$ & $\begin{array}{l}0 \\
0\end{array}$ \\
\hline 43 & & & & & & & 0 & 0 & 0 & 0 & 0 & 0 & 0 \\
\hline 44 & & & & & & & 0 & 0 & 0 & 0 & 0 & 0 & 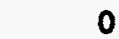 \\
\hline 45 & & & & & & & 0 & 0 & 0 & 0 & 0 & 0 & 0 \\
\hline 46 & & & & & & & 0 & 0 & $\mathbf{0}$ & 0 & 0 & 0 & 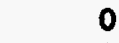 \\
\hline 47 & & & & & & & 0 & 0 & $\mathbf{0}$ & 0 & 0 & 0 & 0 \\
\hline 48 & & & & & & & 0 & 0 & 0 & 0 & 0 & 0 & 0 \\
\hline 49 & & & & & & & 0 & 0 & 0 & 0 & 0 & 0 & 0 \\
\hline 50 & & & & & & & 0 & 0 & 0 & 0 & 0 & 0 & 0 \\
\hline \multicolumn{6}{|c|}{ Total Floor Mass= } & & 359 & 22208.48 & 30696 & 538154.7 & 600632 & 344782 & 105519 \\
\hline \multicolumn{6}{|c|}{ C. G. COORDINATES: } & \multicolumn{3}{|c|}{$X C G=61.90 \mathrm{ft}$} & $Y C G=$ & \multicolumn{2}{|c|}{$85.55 \mathrm{ft}$} & $\mathbf{W} t=$ & 11553 \\
\hline \multicolumn{7}{|c|}{ MASS MOMENTS OF INERTIA: } & $\mid X X=$ & 945414 & $I Y Y=$ & 643674 & $\mathbf{1 Z Z}=$ & 1589088 & 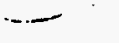 \\
\hline
\end{tabular}

|Units: Kips, Feet, Second 
IICF KAISER

Calc. S-0001, Cer. O

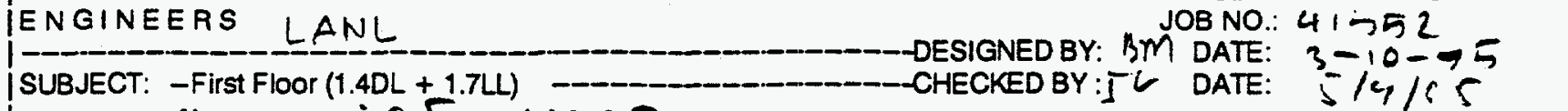

SHEET: $13=\quad O F: ? 4$

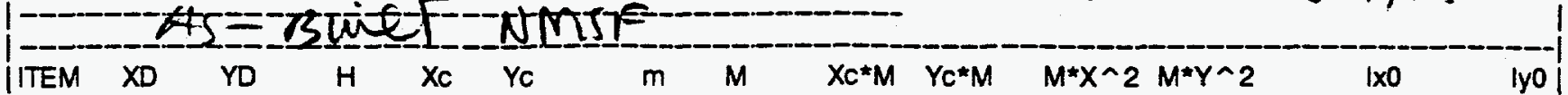

\begin{tabular}{|c|c|c|c|c|c|c|c|c|c|c|c|c|c|}
\hline & & & & & & & 0 & 0 & 0 & 0 & 0 & 0 & 0 \\
\hline 21 & 5350 & 2000 & 100 & 268 & 1387 & 0.02016 & 216 & 576.8945 & 2991 & 161897 & 64630 & 719 & 5144 \\
\hline 3 & 1.50 & 148.70 & 10.63 & 0.0 & 74.4 & 0.00652 & 15.5 & 0 & 1149 & 45318.32 & 1426.4 & 28480 & 3 \\
\hline 4 & 1.00 & 128.70 & 10.63 & 14.8 & 64.4 & 0.00652 & 8.92 & 131.9874 & 573.9 & 13808.17 & 3428.3 & 12310 & 1 \\
\hline 5 & 1.00 & 128.70 & 10.63 & 29.6 & 64.4 & 0.00652 & 8.92 & 263.9749 & 573.9 & 5374.461 & 3428.3 & 12310 & 1 \\
\hline 6 & 1.45 & 104.30 & 10.63 & 53.5 & 52.2 & 0.00652 & 10.5 & 560.6586 & 546.5 & 4.412639 & 10602 & 9500 & 2 \\
\hline 7 & 1.45 & 44.40 & 10.63 & 53.5 & 126.5 & 0.00652 & 4.46 & 238.6696 & 564.3 & 1.878439 & 8074.3 & 733 & 1 \\
\hline 8 & 1.00 & 44.40 & 10.63 & 72.5 & 126.5 & 0.00652 & 3.08 & 223.0557 & 389.2 & 1036.095 & 5568.5 & 505 & 0 \\
\hline 9 & 72.50 & 1.38 & 10.63 & 36.3 & 148.7 & 0.00652 & 6.93 & 251.3145 & 1031 & 2221.069 & 29060 & 1 & 3037 \\
\hline 10 & 19.00 & 1.00 & 10.63 & 63.0 & 104.3 & 0.00652 & 1.32 & 82.94429 & 137.3 & 103.1432 & 544.86 & 0 & 40 \\
\hline 11 & 23.90 & 1.21 & 10.63 & 41.6 & 22.9 & 0.00652 & 2 & 83.26197 & 45.89 & 318.0832 & 7470.4 & 0 & 95 \\
\hline 12 & 53.50 & 1.50 & 10.63 & 26.8 & 0.0 & 0.00652 & 5.56 & 148.7514 & 0 & 4174.492 & 39197 & 1 & 1326 \\
\hline 13 & 53.50 & 148.70 & 1.00 & 26.8 & 74.4 & 0.02016 & 160 & 4289.211 & 11922 & 120370.4 & 14798 & 295457 & 38245 \\
\hline 14 & 19.00 & 44.40 & 1.00 & 63.0 & 126.5 & 0.02016 & 17 & 1071.189 & 2151 & 1332.048 & 30774 & 2793 & 512 \\
\hline 15 & 78.00 & 104.30 & 0.50 & 92.5 & 52.2 & 0.03379 & 137 & 12713.46 & 7168 & 202151.8 & 139047 & 124597 & 69683 \\
\hline 16 & 59.00 & 44.40 & 0.50 & 102.0 & 126.5 & 0.03379 & 44.3 & 4514.173 & 5598 & 101335.6 & 80101 & 7270 & 12838 \\
\hline 17 & 80.00 & 33.00 & 0.67 & 112.5 & 165.2 & 0.02687 & 47.5 & 5346.922 & 7852 & 161826.4 & 313708 & 4313 & 25348 \\
\hline 18 & 11.00 & 20.00 & 0.50 & 137.0 & 138.7 & 0.03379 & 3.72 & 509.1975 & 515.5 & 25513.04 & 11139 & 124 & 37 \\
\hline 19 & 1.33 & 148.70 & 7.75 & 0.0 & 74.4 & 0.00652 & 10 & 0 & 743.2 & 29309.4 & 922.53 & 18419 & 1 \\
\hline 20 & 1.33 & 22.90 & 7.75 & 29.6 & 11.5 & 0.00652 & 1.54 & 45.56632 & 17.63 & 927.7186 & 8093 & 67 & 0 \\
\hline 21 & 1.25 & 125.80 & 7.75 & 53.5 & 85.8 & 0.00652 & 7.95 & 425.216 & 681.9 & 3.346644 & 27.004 & 10482 & 1 \\
\hline 22 & 1.00 & 15.00 & 7.75 & 72.5 & 111.8 & 0.00652 & 0.76 & 54.96603 & 84.76 & 255.3175 & 587.76 & 14 & 0 \\
\hline 23 & 0.83 & 30.00 & 7.75 & 109.0 & 44.3 & 0.00652 & 1.26 & 137.1801 & 55.75 & 3786.476 & 1979.2 & 94 & 0 \\
\hline 24 & 1.00 & 22.90 & 7.75 & 115.2 & 11.5 & 0.00652 & 1.16 & 133.3377 & 13.25 & 4314.074 & 6085 & 51 & 0 \\
\hline 25 & 0.86 & 128.70 & 7.75 & 131.5 & 64.4 & 0.00652 & 5.59 & 735.6443 & 360 & 33471.5 & 2150.6 & 7722 & 0 \\
\hline 26 & 1.00 & 20.00 & 7.75 & 142.5 & 138.7 & 0.00652 & 1.01 & 144.0489 & 140.2 & 7890.764 & 3029.4 & 34 & 0 \\
\hline 27 & 72.50 & 1.12 & 7.75 & 36.3 & 148.7 & 0.00652 & 4.1 & 148.7747 & 610.3 & 1314.843 & 17203 & 0 & 1798 \\
\hline 28 & 80.00 & 1.00 & 7.75 & 112.5 & 148.7 & 0.00652 & 4.04 & 454.8913 & 601.3 & 13767.44 & 16949 & 0 & 2157 \\
\hline 29 & 53.50 & 0.55 & 7.75 & 26.8 & 89.3 & 0.00652 & 1.49 & 39.78372 & 132.8 & 1116.472 & 42.461 & 0 & 355 \\
\hline 30 & 78.00 & 0.84 & 7.75 & 92.5 & 59.3 & 0.00652 & 3.31 & 306.3238 & 196.4 & 4870.737 & 2013.3 & 0 & 1679 \\
\hline 31 & 22.50 & 0.65 & 7.75 & 120.3 & 29.3 & 0.00652 & 0.74 & 88.8886 & 21.66 & 3229.821 & 2208.3 & 0 & 31 \\
\hline 32 & 23.90 & 1.21 & 7.75 & 41.6 & 22.9 & 0.00652 & 1.46 & 60.73226 & 33.47 & 232.0137 & 5449 & 0 & 70 \\
\hline 33 & 16.30 & 1.00 & 7.75 & 123.4 & 22.9 & 0.00652 & 0.82 & 101.623 & 18.87 & 3945.288 & 3071.3 & 0 & 18 \\
\hline 34 & 53.50 & 1.33 & 7.75 & 26.8 & 0.0 & 0.00652 & 3.6 & 96.20427 & 0 & 2699.832 & 25350 & 1 & 858 \\
\hline 35 & 78.00 & 1.00 & 7.75 & 92.5 & 0.0 & 0.00652 & 3.94 & 364.6712 & 0 & 5798.497 & 27789 & 0 & 1999 \\
\hline 36 & 19.00 & 0.67 & 7.75 & 63.0 & 104.3 & 0.00652 & 0.64 & 40.53536 & 67.11 & 50.40669 & 266.28 & 0 & 19 \\
\hline 37 & 11.00 & 1.00 & 7.75 & 137.0 & 128.7 & 0.00652 & 0.56 & 76.16902 & 71.55 & 3816.404 & 1113 & 0 & 6 \\
\hline 38 & 80.00 & 1.00 & 7.75 & 112.5 & 181.7 & 0.00652 & 4.04 & 454.8913 & 734.7 & 13767.44 & 38630 & 0 & 2157 \\
\hline 39 & & & & & & & 0 & 0 & 0 & 0 & 0 & 0 & 0 \\
\hline 40 & & & & & & & 0 & 0 & 0 & 0 & 0 & 0 & o \\
\hline 41 & & & & & & & 0 & 0 & 0 & 0 & 0 & 0 & 0 \\
\hline 42 & & & & & & & 0 & 0 & 0 & 0 & 0 & $\mathbf{0}$ & 0 \\
\hline 43 & & & & & & & 0 & 0 & 0 & 0 & 0 & 0 & 0 \\
\hline 44 & & & & & & & 0 & 0 & 0 & 0 & 0 & 0 & 0 \\
\hline 45 & & & & & & & 0 & 0 & 0 & 0 & 0 & 0 & 0 \\
\hline 46 & & & & & & & 0 & 0 & 0 & 0 & 0 & 0 & o \\
\hline 47 & & & & & & & 0 & 0 & 0 & 0 & 0 & 0 & 0 \\
\hline 48 & & & & & & & 0 & 0 & 0 & 0 & 0 & 0 & 0 \\
\hline 49 & & & & & & & 0 & 0 & 0 & 0 & 0 & 0 & 0 \\
\hline 50 & & & & & & & 0 & 0 & 0 & 0 & 0 & 0 & 0 \\
\hline \multicolumn{7}{|c|}{ Total Floor Mass= } & 557 & 34915.11 & 47793 & 835646.9 & 925956 & 536000 & 167462 \\
\hline \multicolumn{6}{|c|}{ C. G. COORDINATES: } & $X C G=$ & $62.68 \mathrm{ft}$ & & $Y C G=$ & $85.80 \mathrm{f}$ & & $\mathrm{Nt} .=$ & 17935 \\
\hline \multicolumn{7}{|c|}{ MASS MOMENTS OF INERTLA: } & $1 x \mid x=$ & 1461956 & $I Y Y=$ & 1003109 & $127=$ & 2465065 & \\
\hline
\end{tabular}

IUnits: Kips, Feet, Second 
IICF KAISER

Calc. S-0001, ken 0

IENGINEERS

LANL

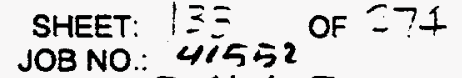

- DESIGNED BY: BMDATE: $3 / 1 / 95$

SSUBJECT: - - Roof Mass-_-_-_-CHECKED BY: Jレ DATE: $5 / 4 / 95$

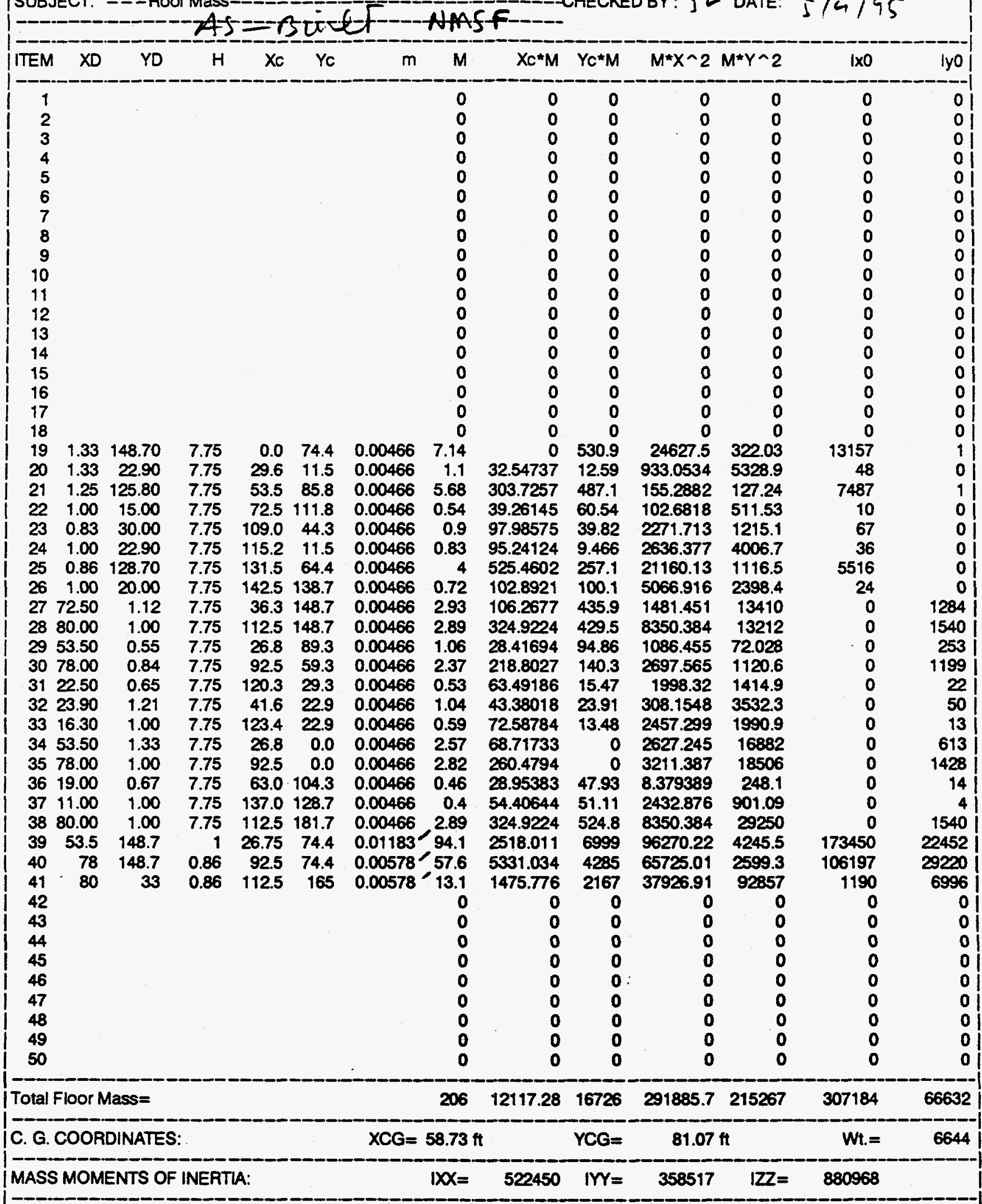

U Units: Kips, Feet, Second 
IICF KAISER

IENGINEERS LANL

SHEET: $1 \equiv-$

HIST, OF 274

SUBJECT: - - ROo (DL+LL) - DESIGNED BY: BM DATE: $3-1-9$

M DATE: $\quad 3-1-9=$

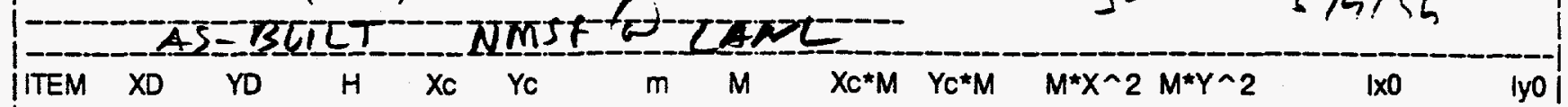

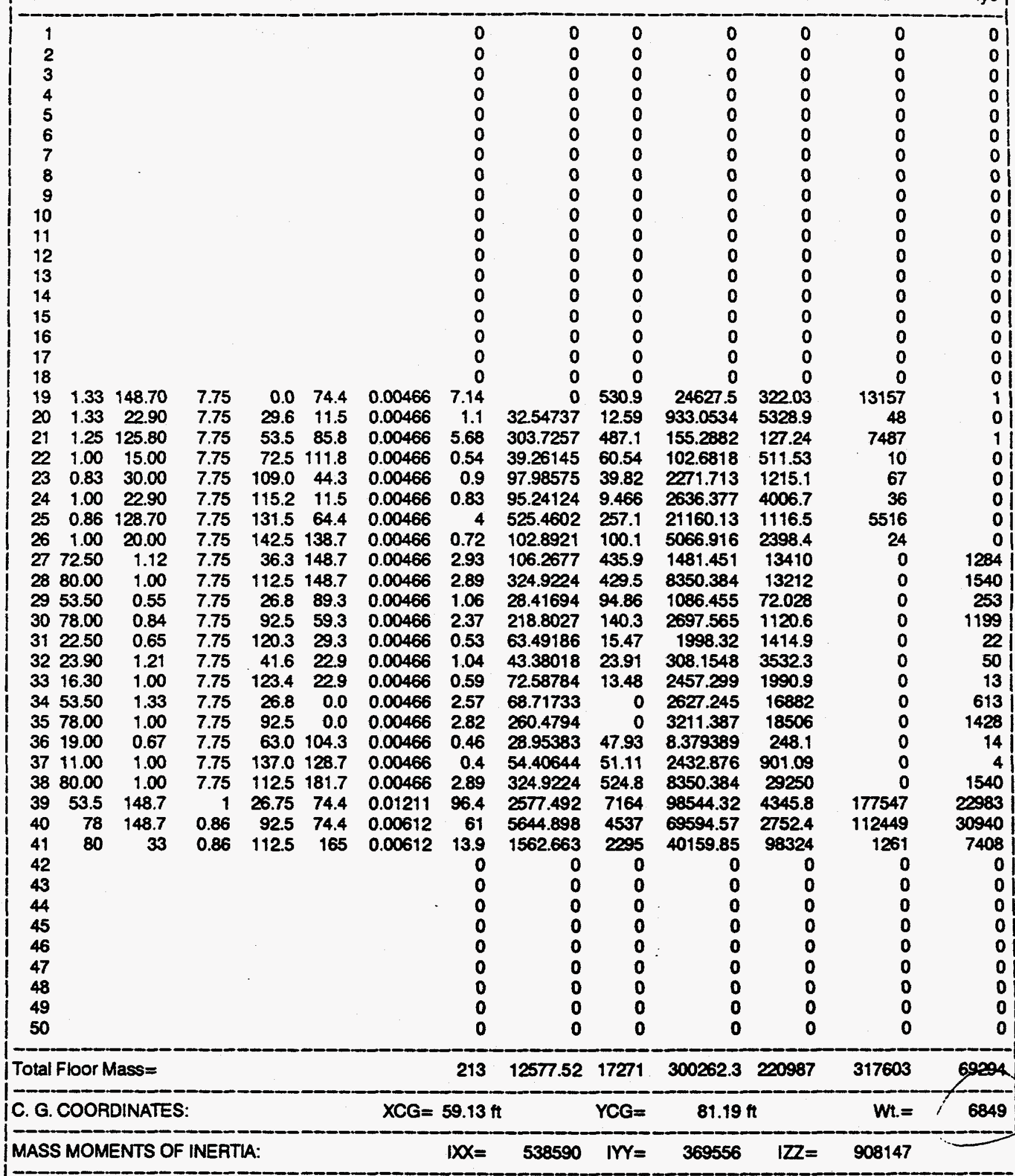

Units: Kips, Feet, Second 
SUBJECT: -Roof (1.4DL+1.7LL)

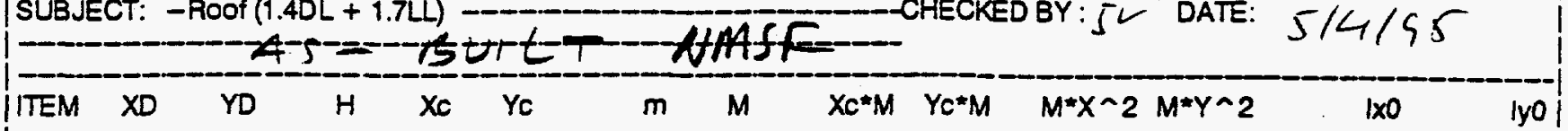

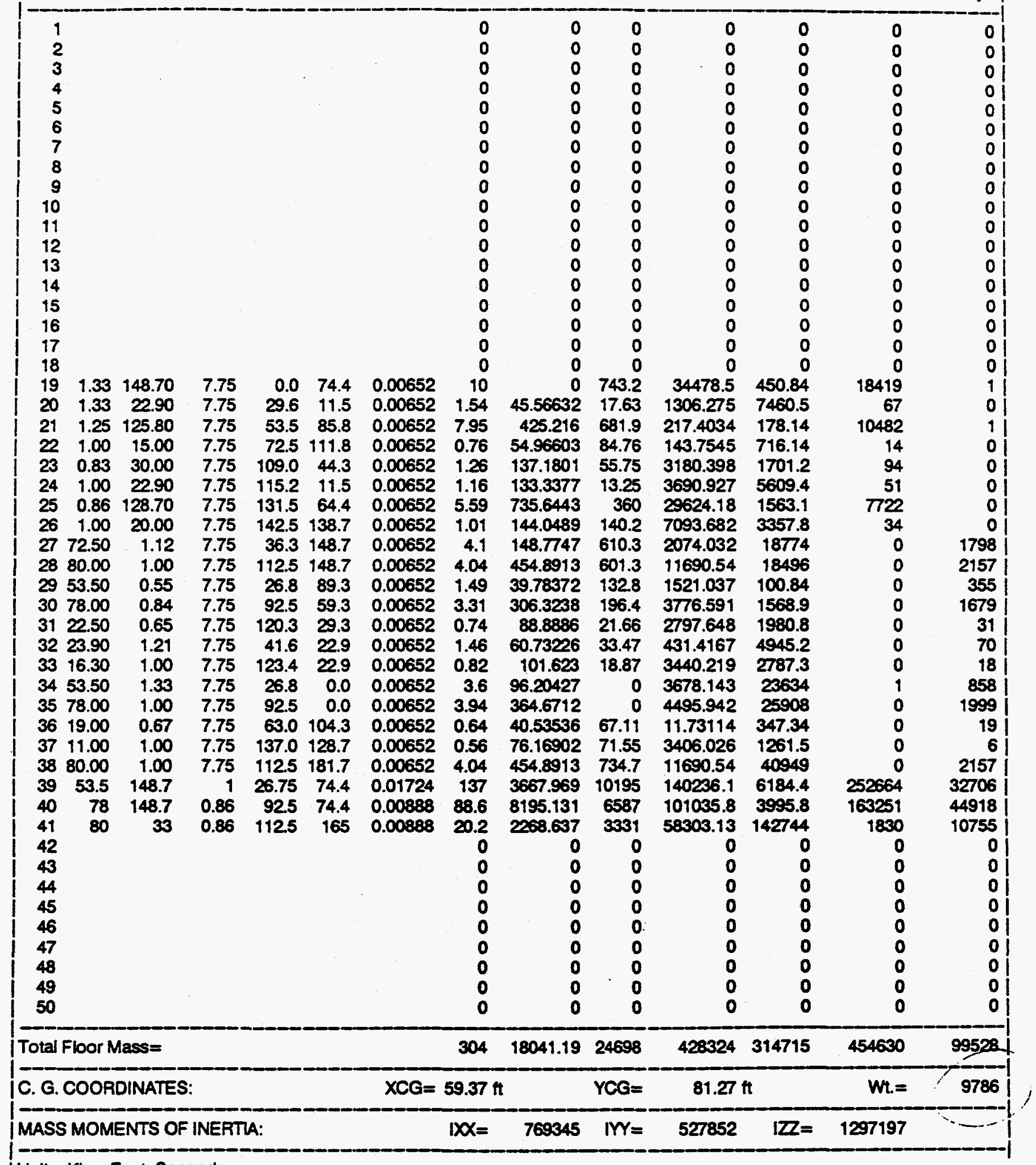

Units: Kips, Feet, Second 
str. $=0$ of -7

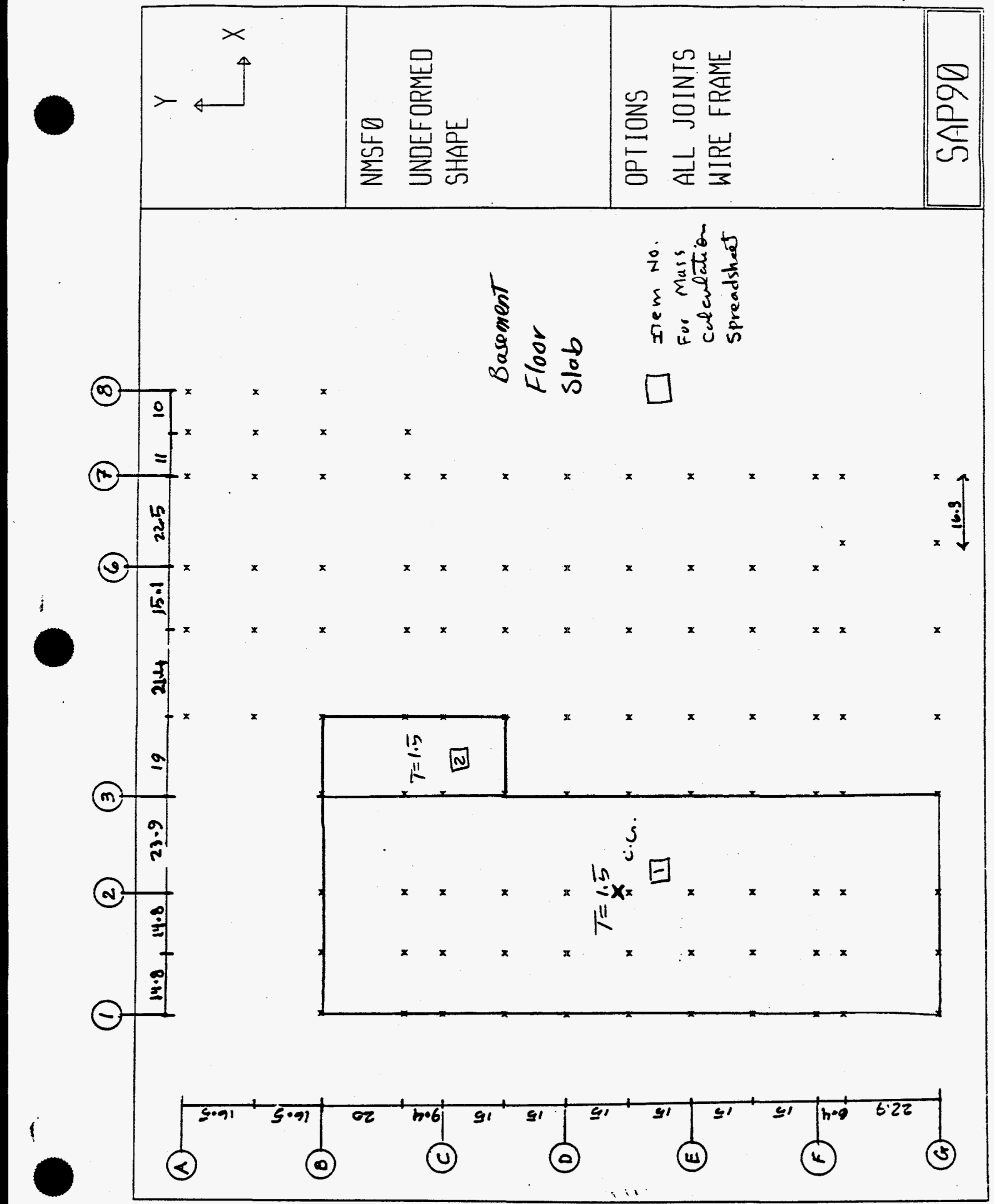




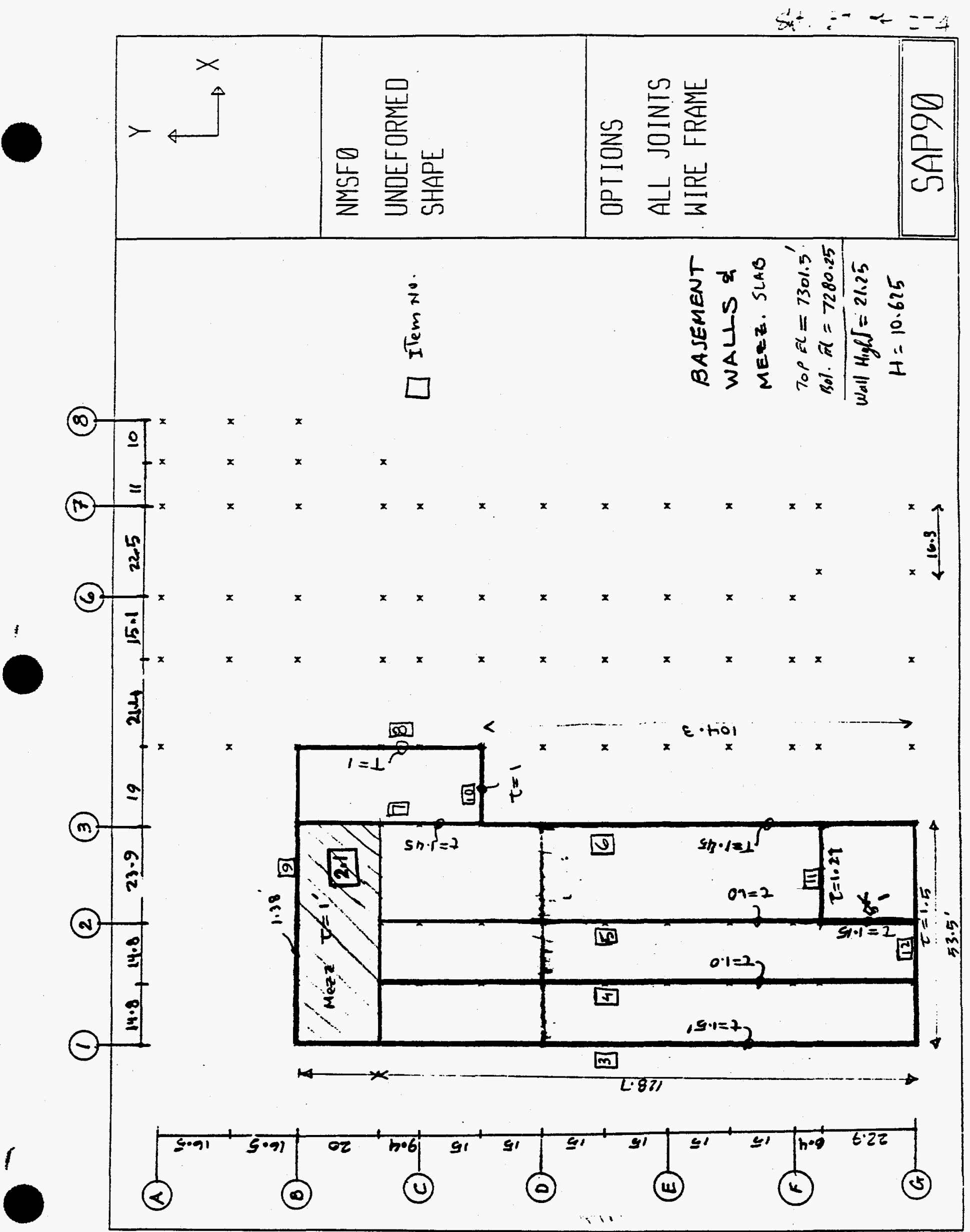


sit. $=1-74$
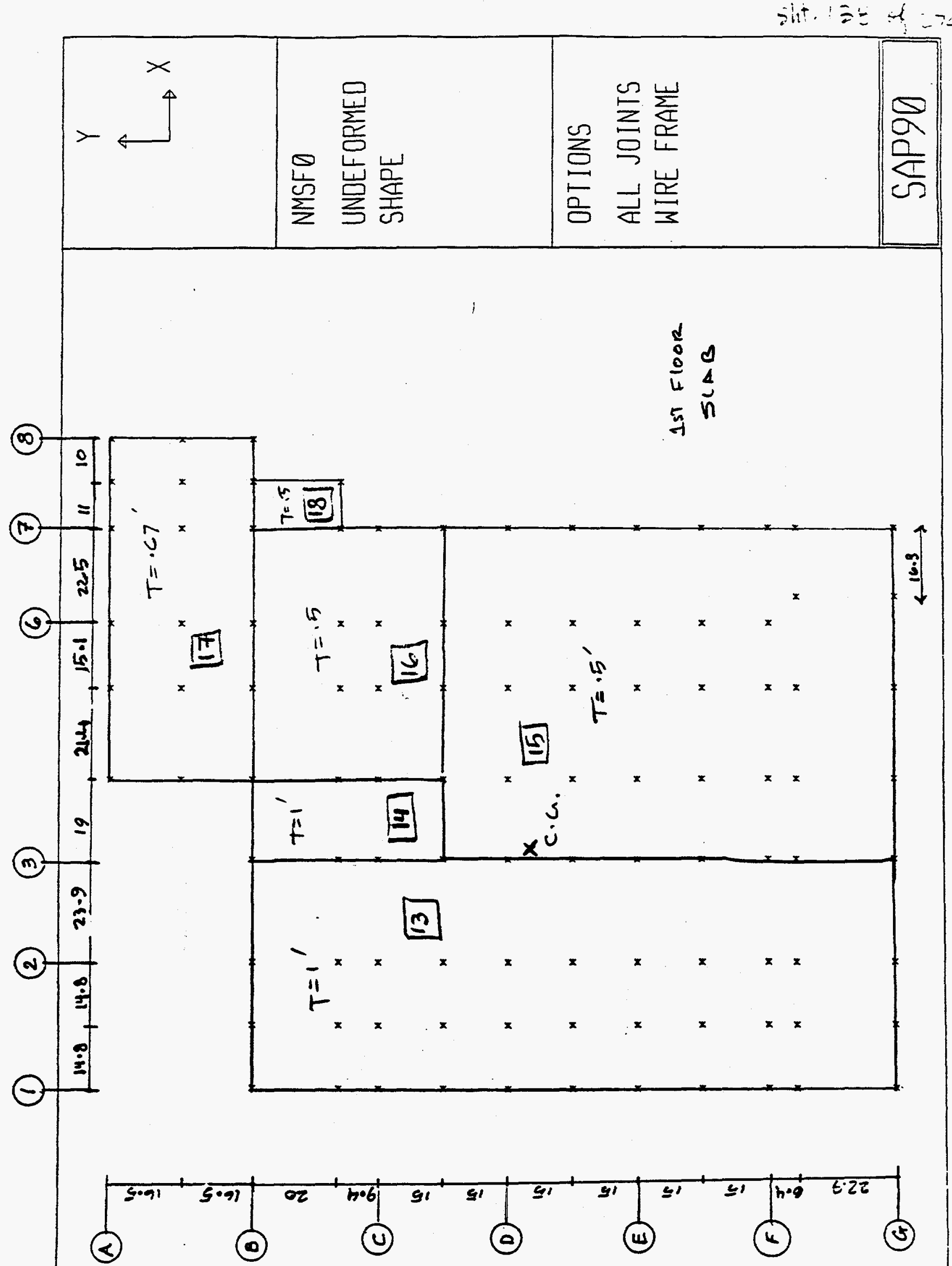


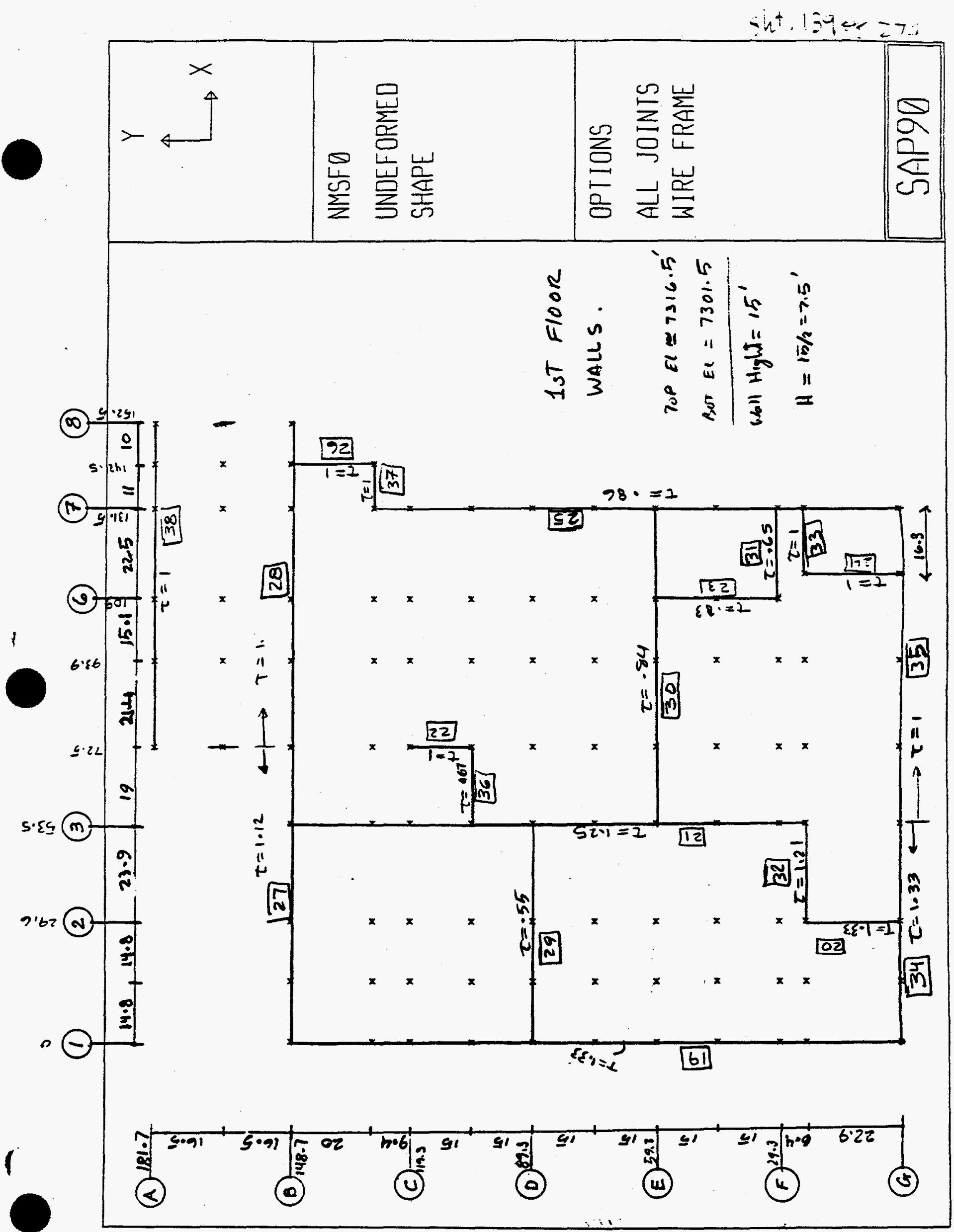




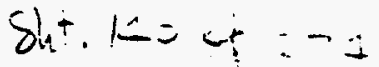

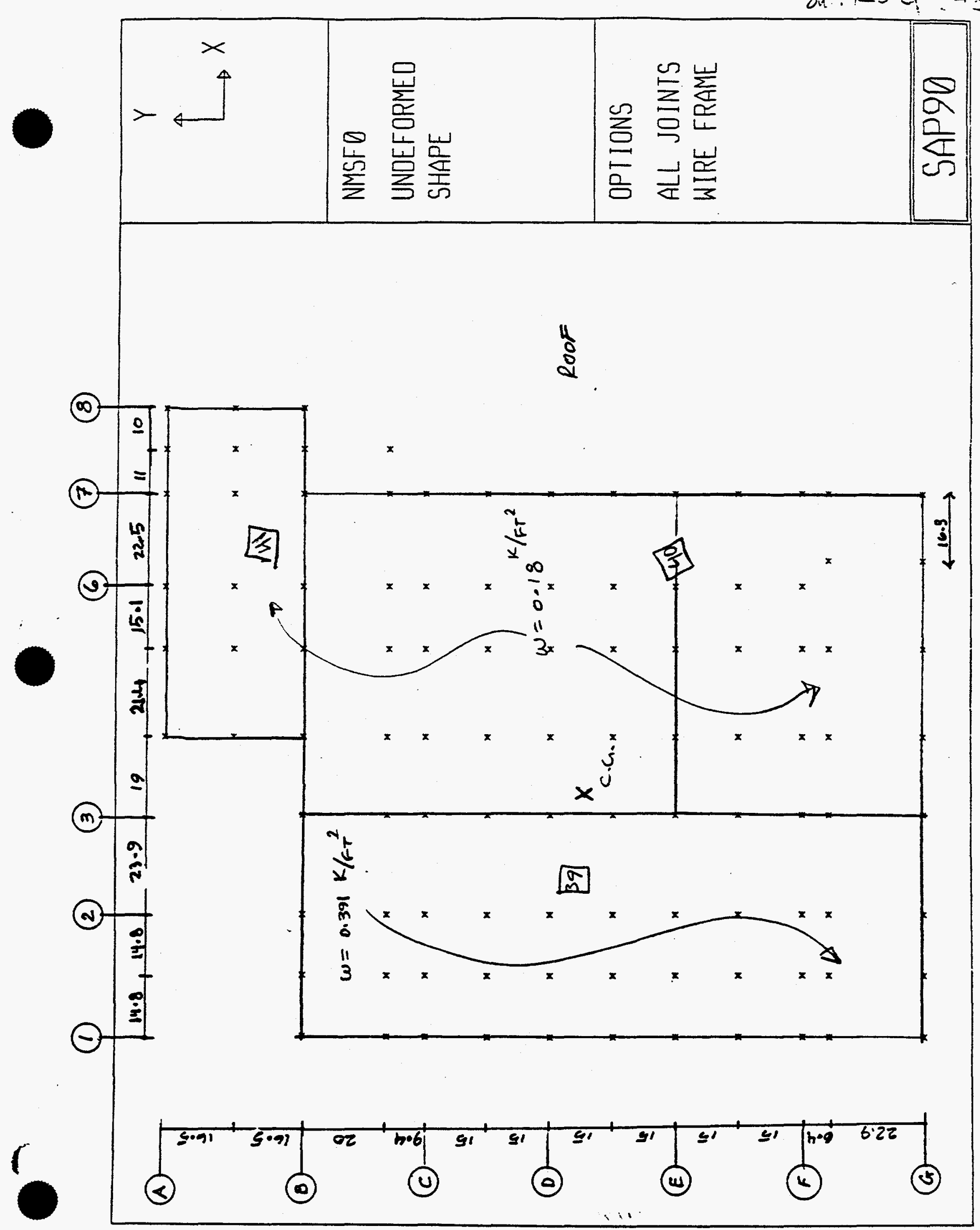




$$
\begin{aligned}
& \text { 8lk. } 141 \text {. } 274 \\
& \text { Job पान5? } \\
& \text { cate S-cicol }
\end{aligned}
$$
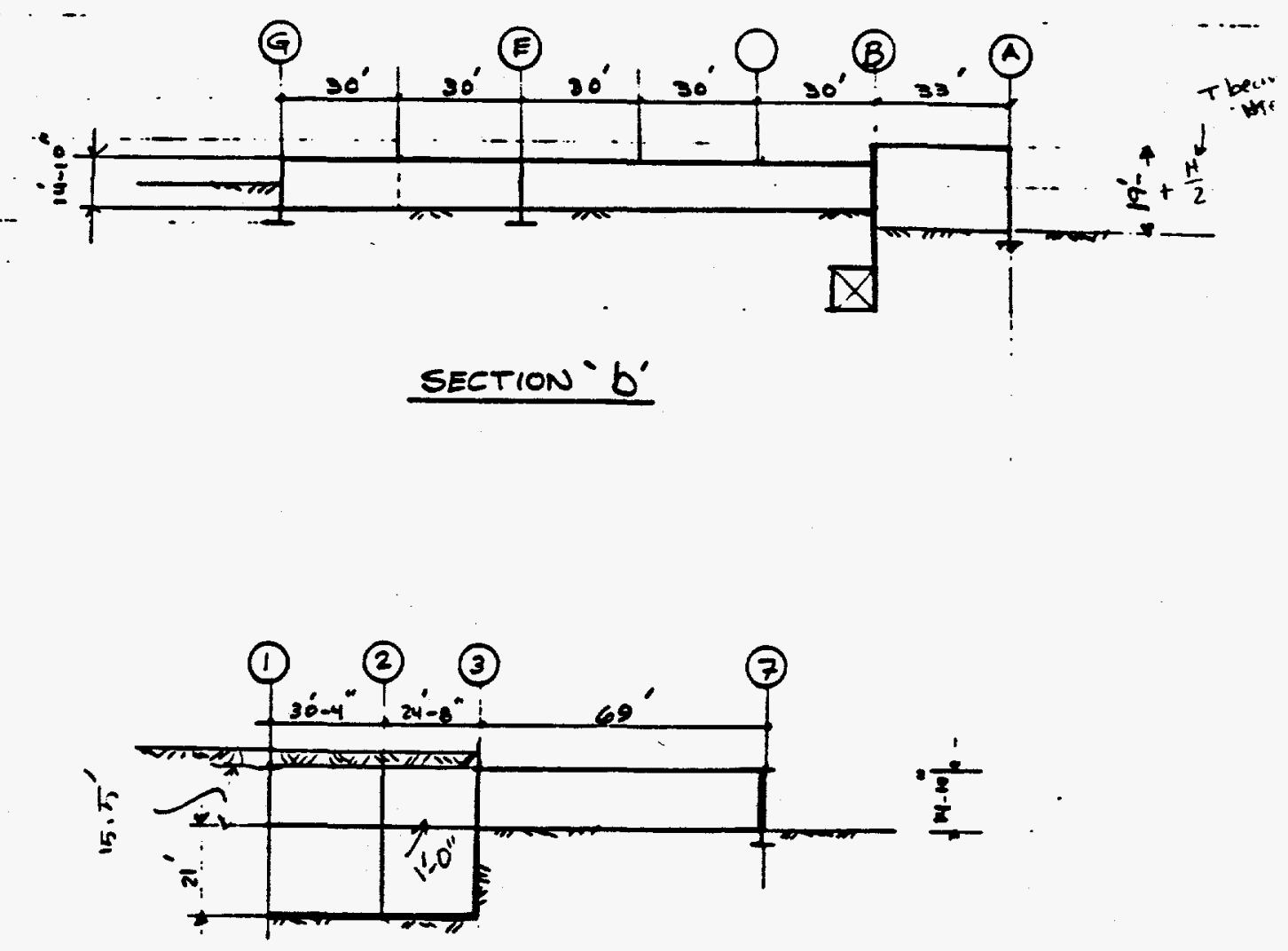

SECTION ' $a$ ' 


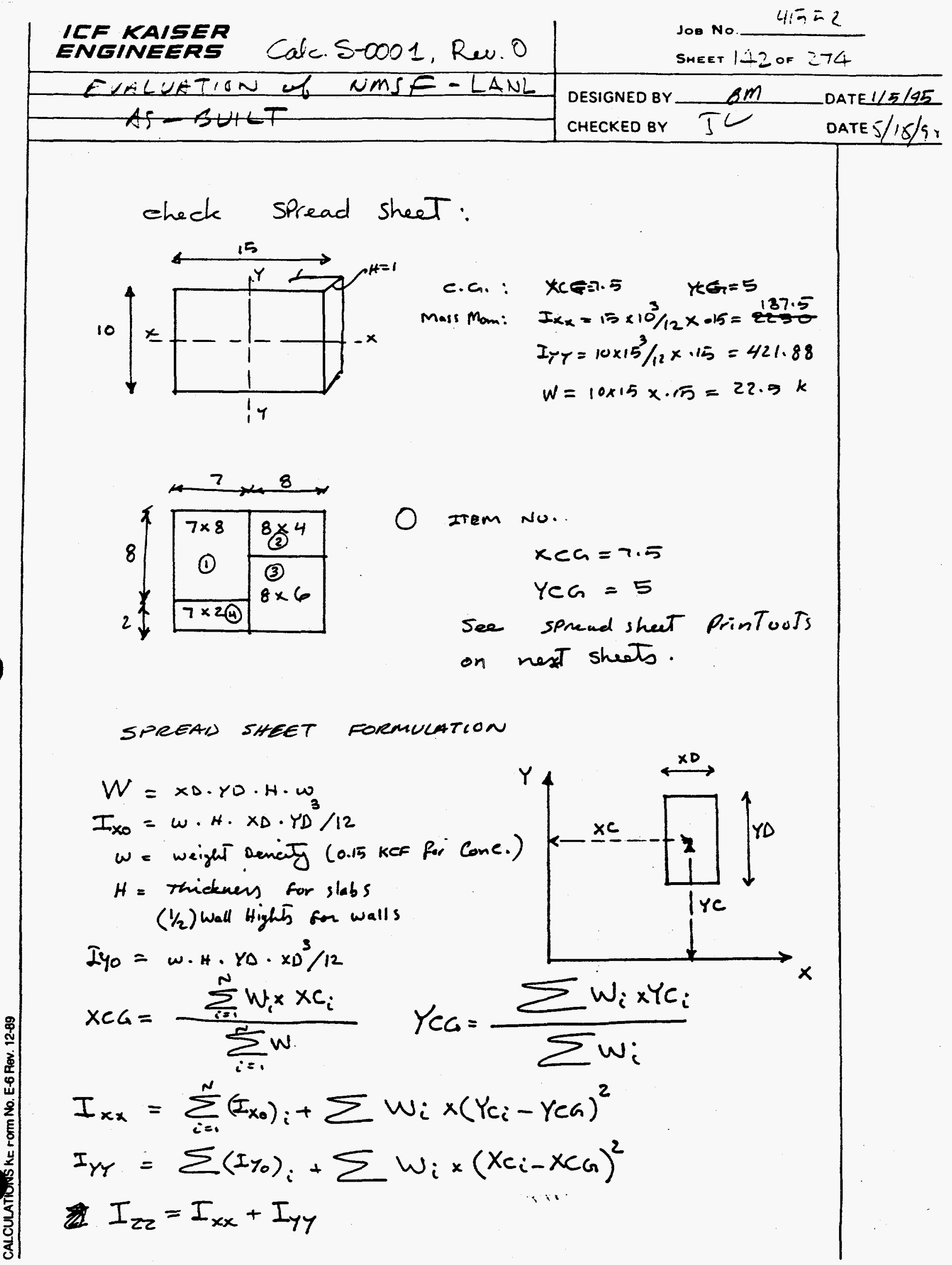




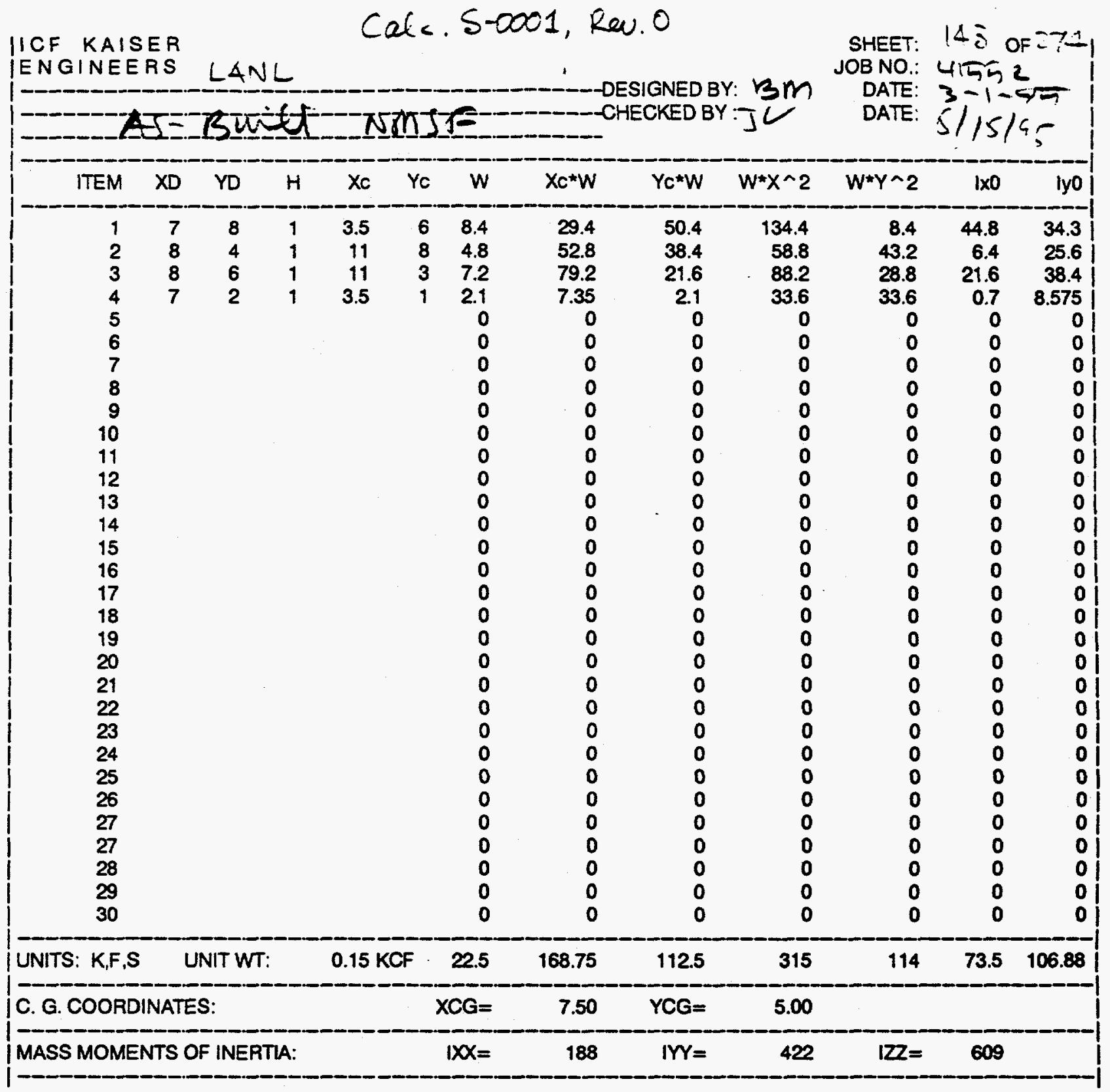




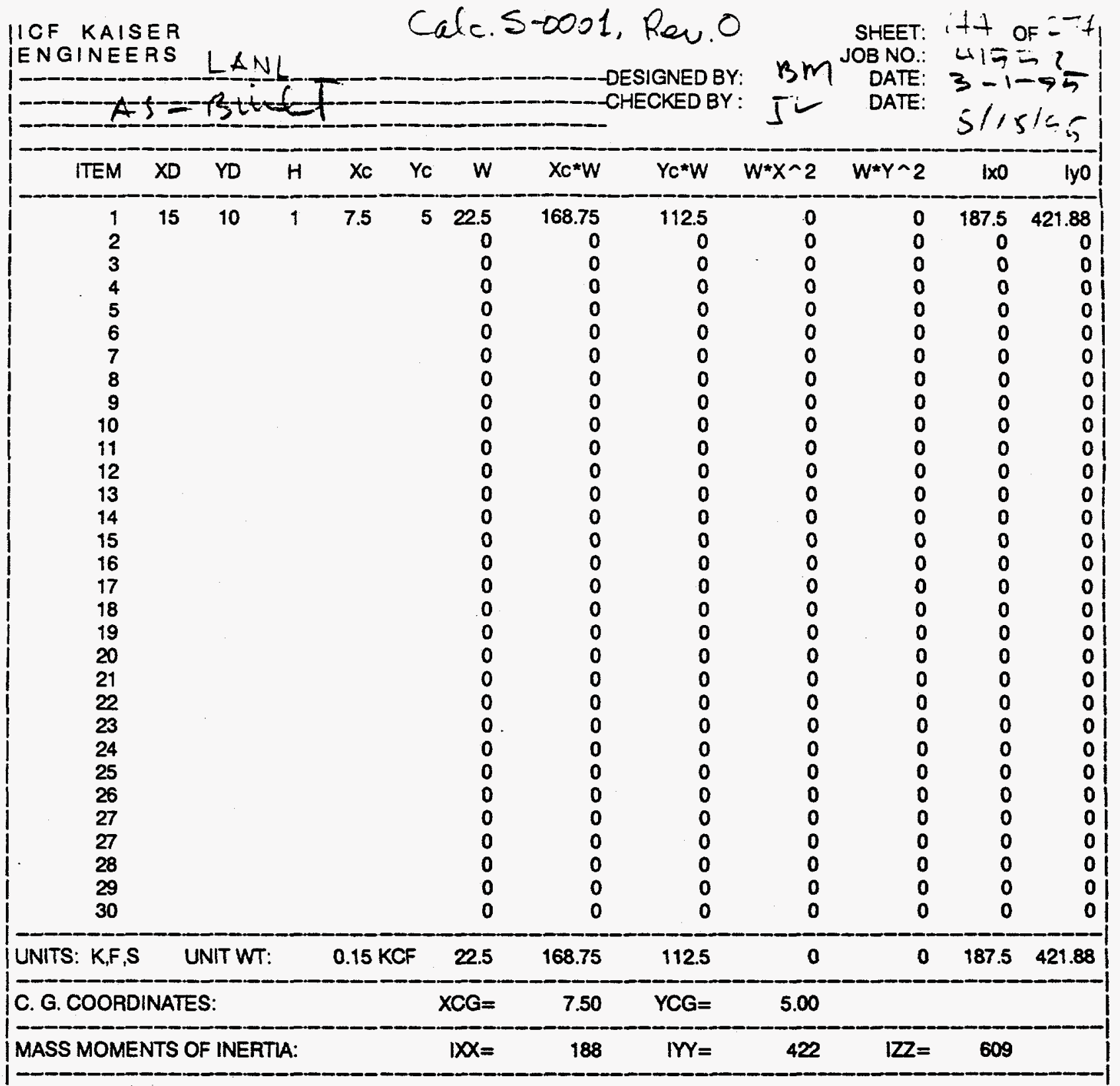



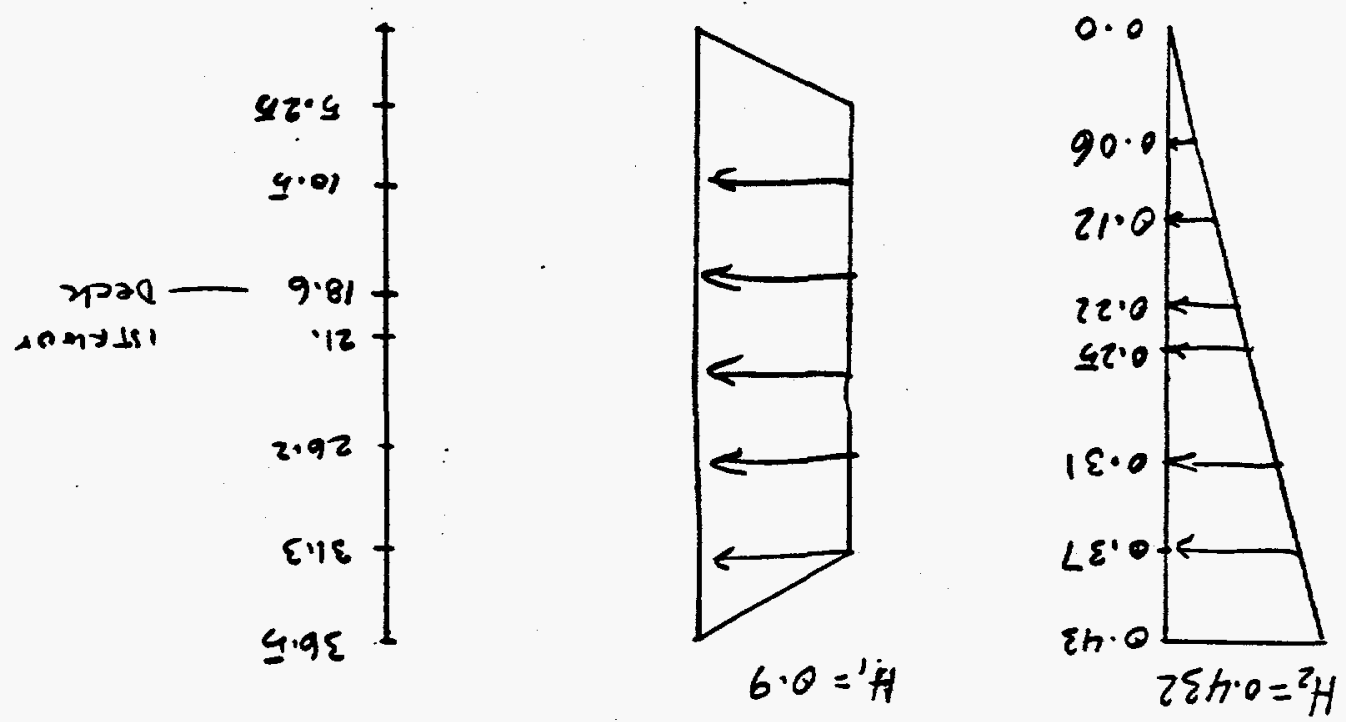

$($ SOJSWN ) 1-NOLde (2

(HS3W - S.9E
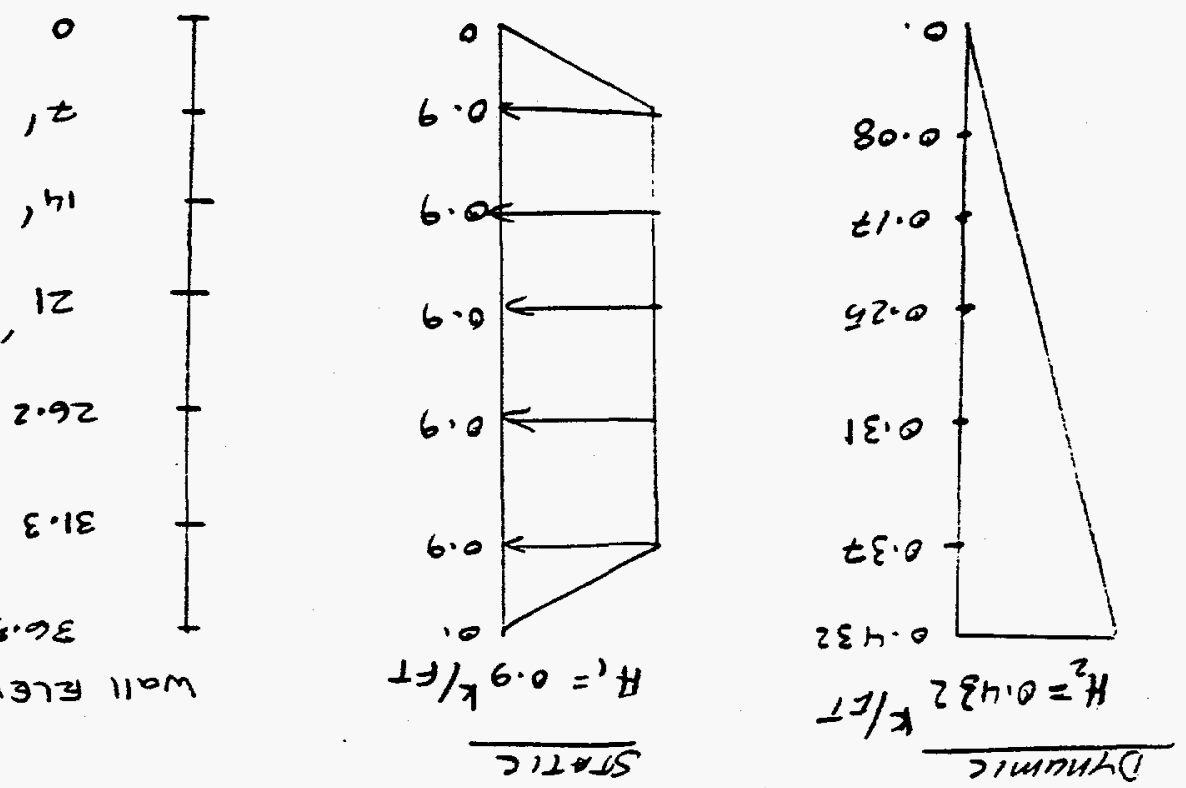

(ings $(\ln )$

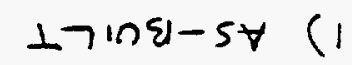

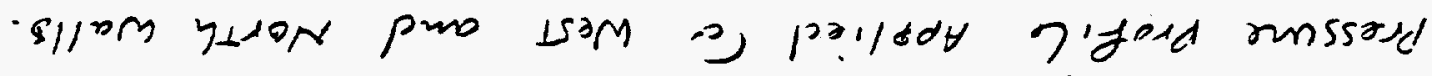

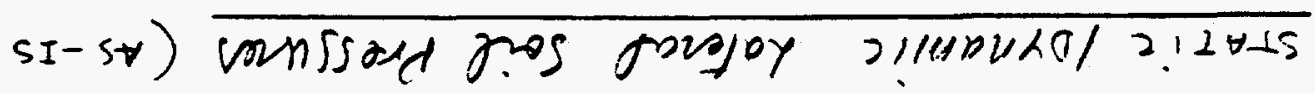

\begin{tabular}{|c|c|c|}
\hline $3 / 51 / 53170$ & $\wedge 80 \exists \times 2 \exists H \supset$ & $190140 /$ L Lingls \\
\hline 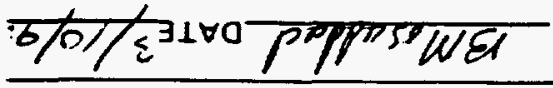 & 18 OZNOISFO & $7 N+7-0$ J \\
\hline 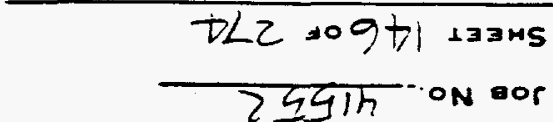 & & O mad I000-s' 'xps \\
\hline
\end{tabular}




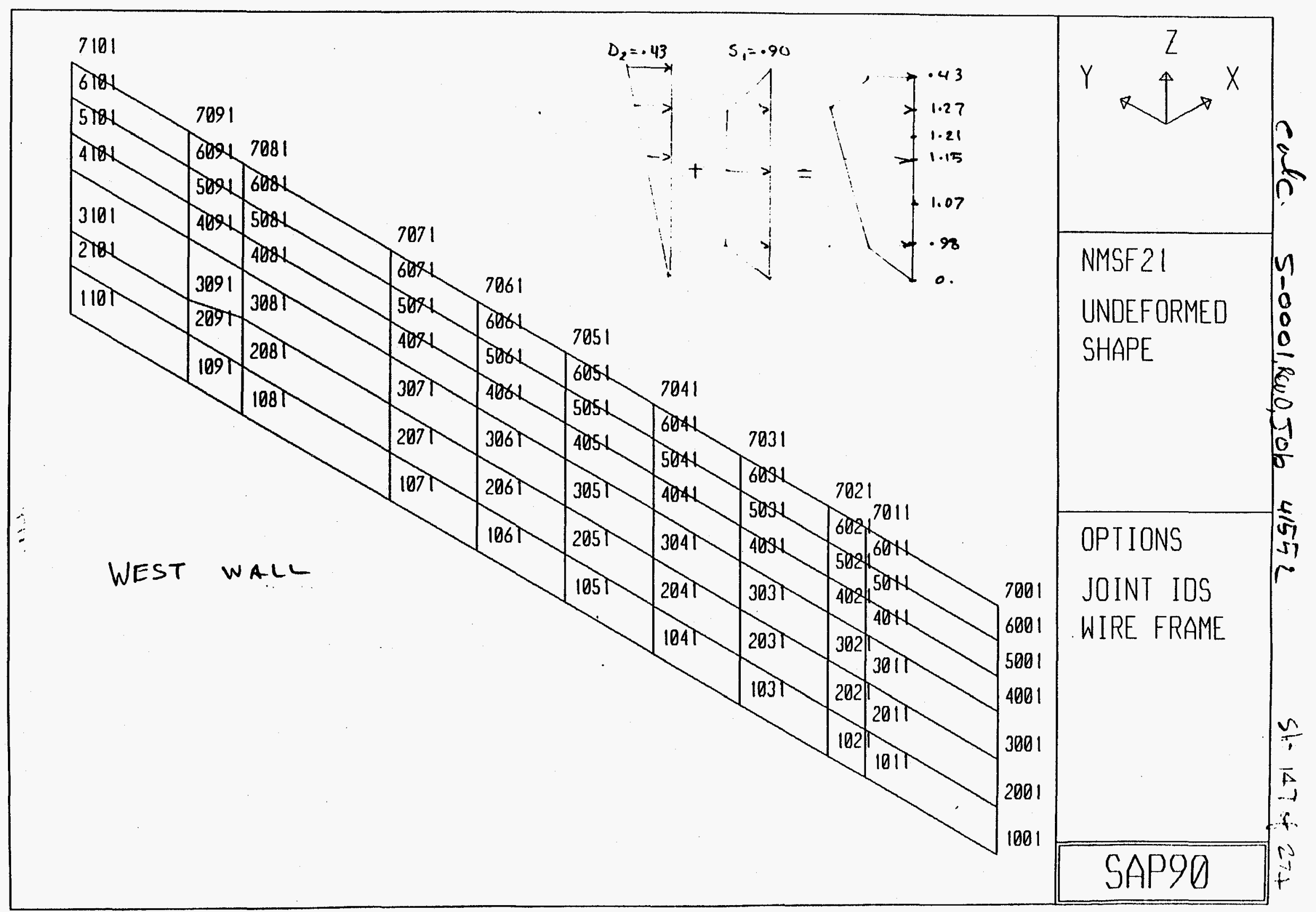




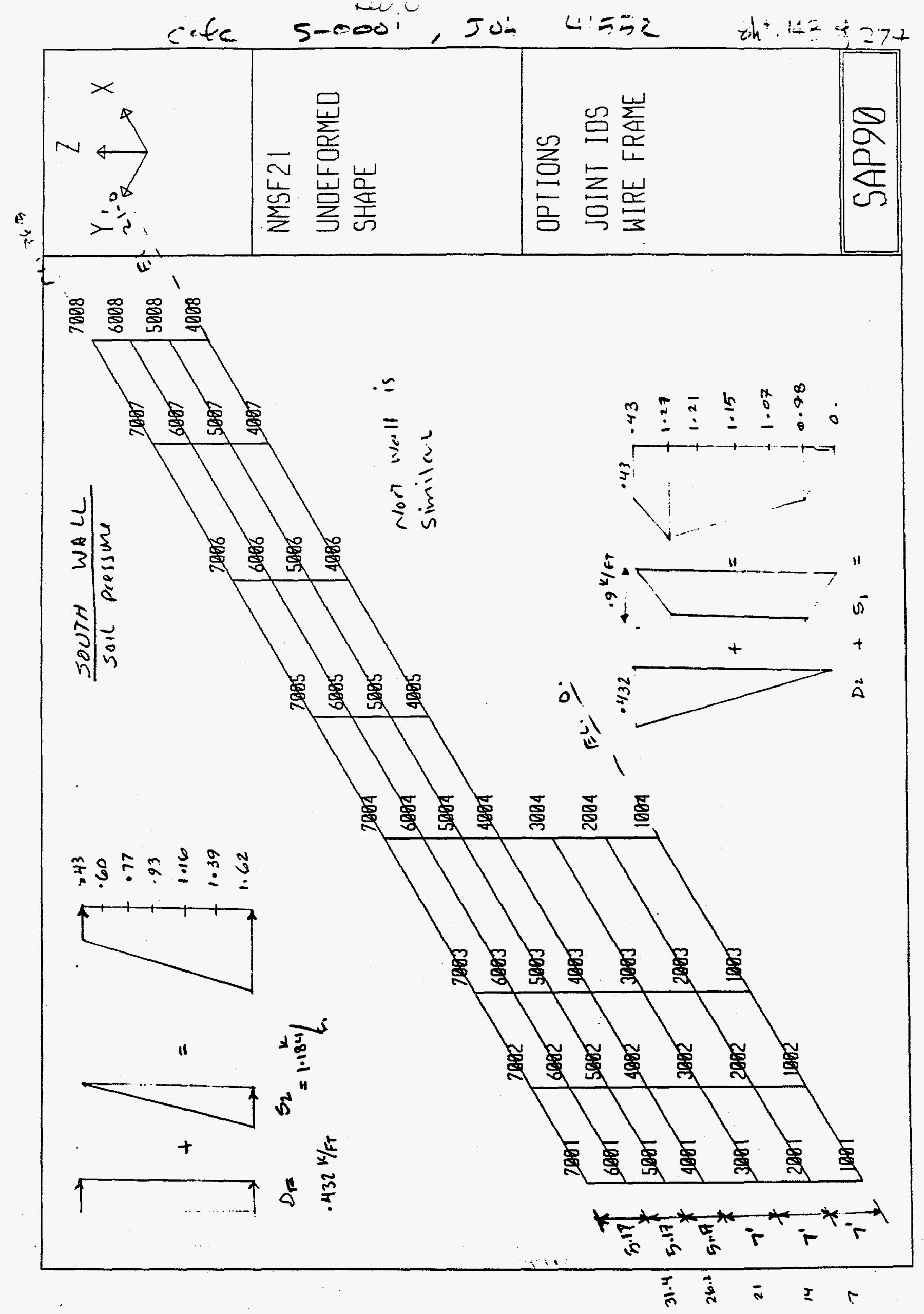


JOB NO SHEET 41552

\section{LANL}

STRUCTURAL EVALUATION OF NMSF FACILITY AT LANL

DESIGNED BY

As-Built Structure

CHECKED BY

B. Mosaddad

DATE $3 / 10 / 95$

5.3 SOIL-STRUCTURE-INTERACTION EFFECTS 

$A S-B U H T$

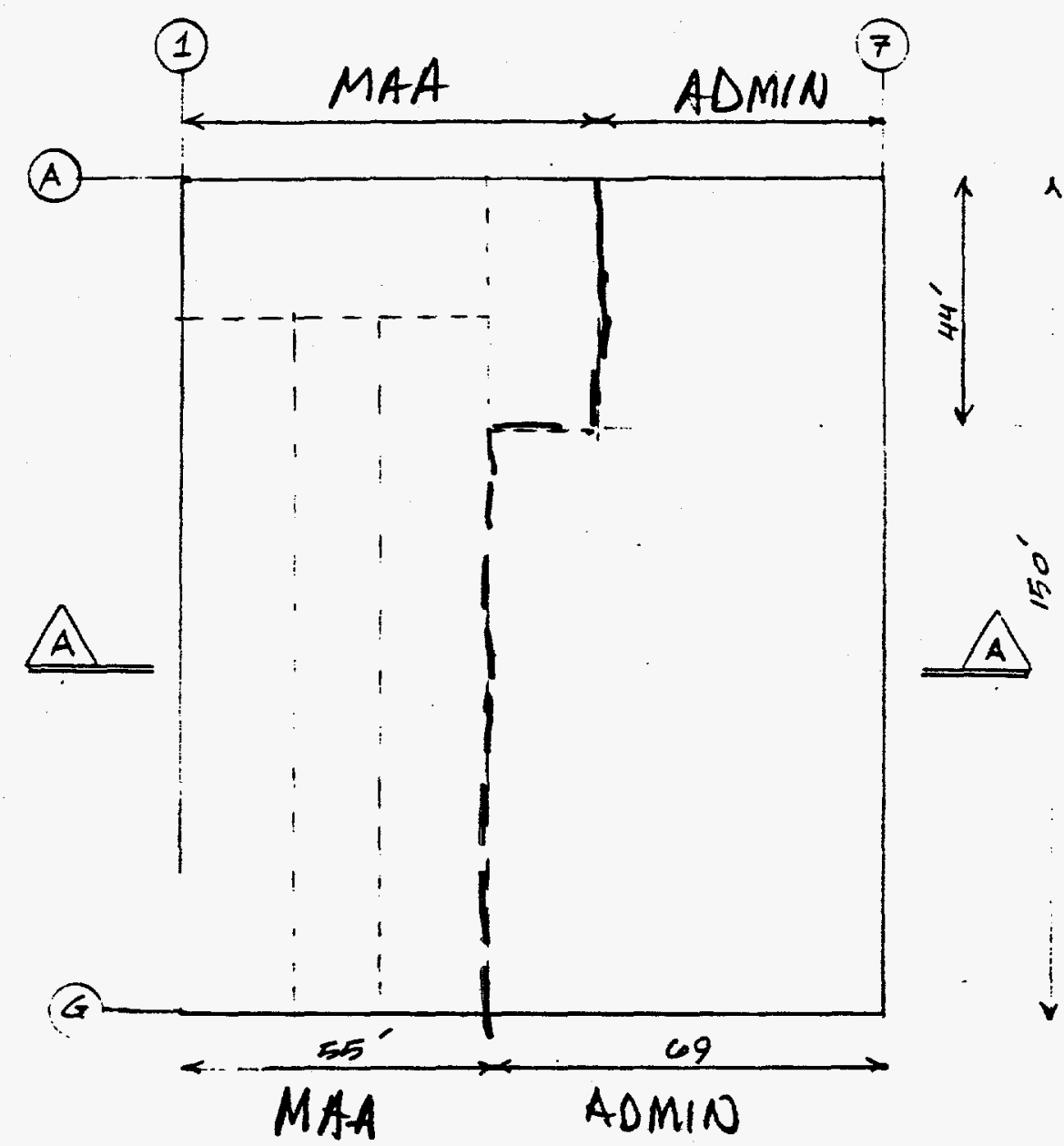

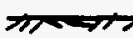
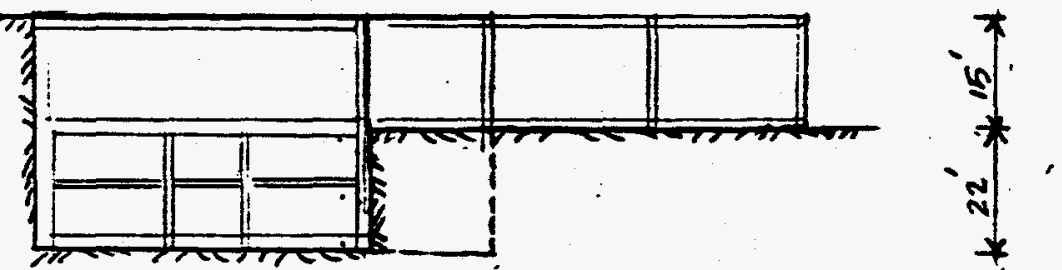

$A-A$ 


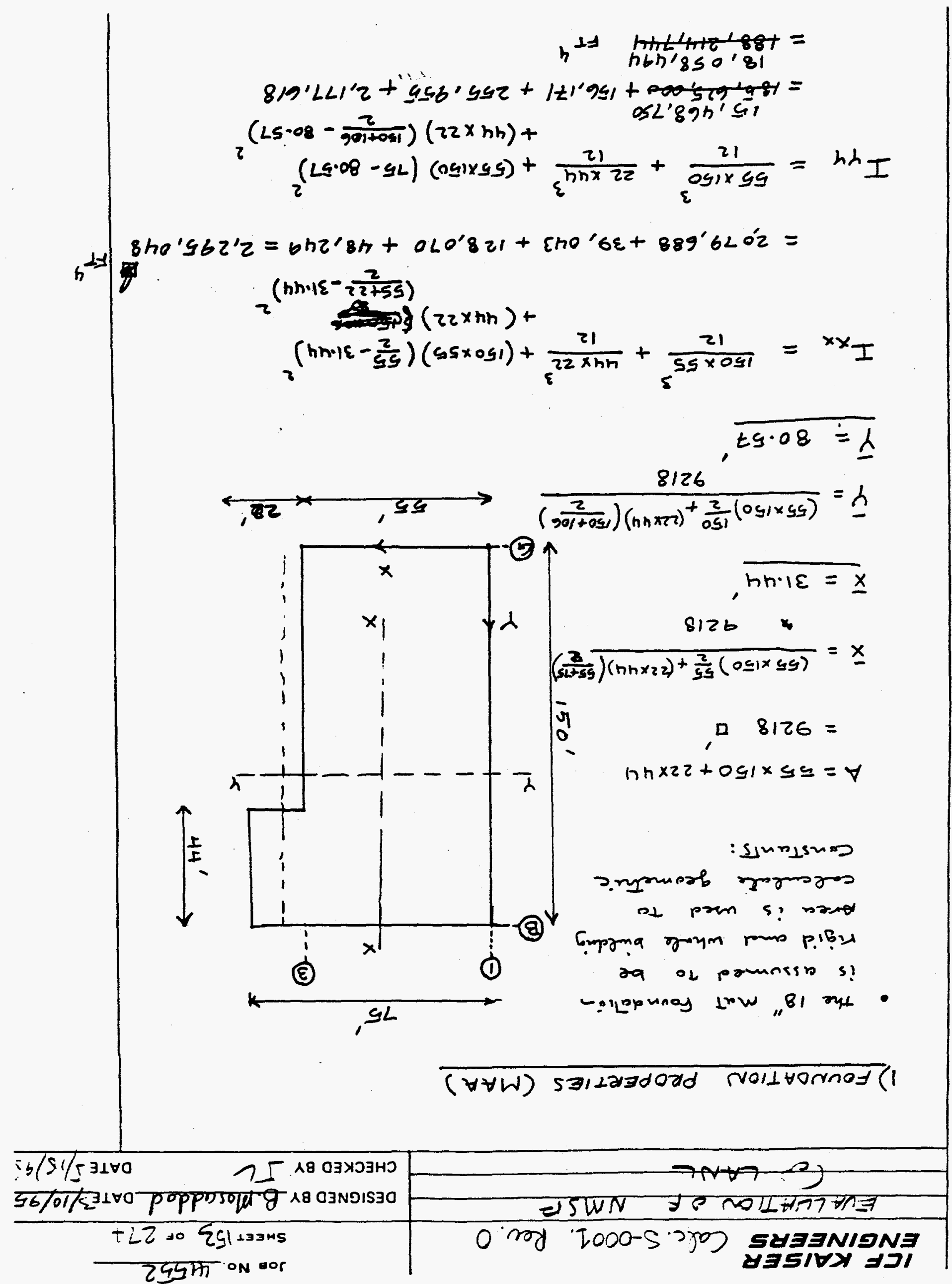




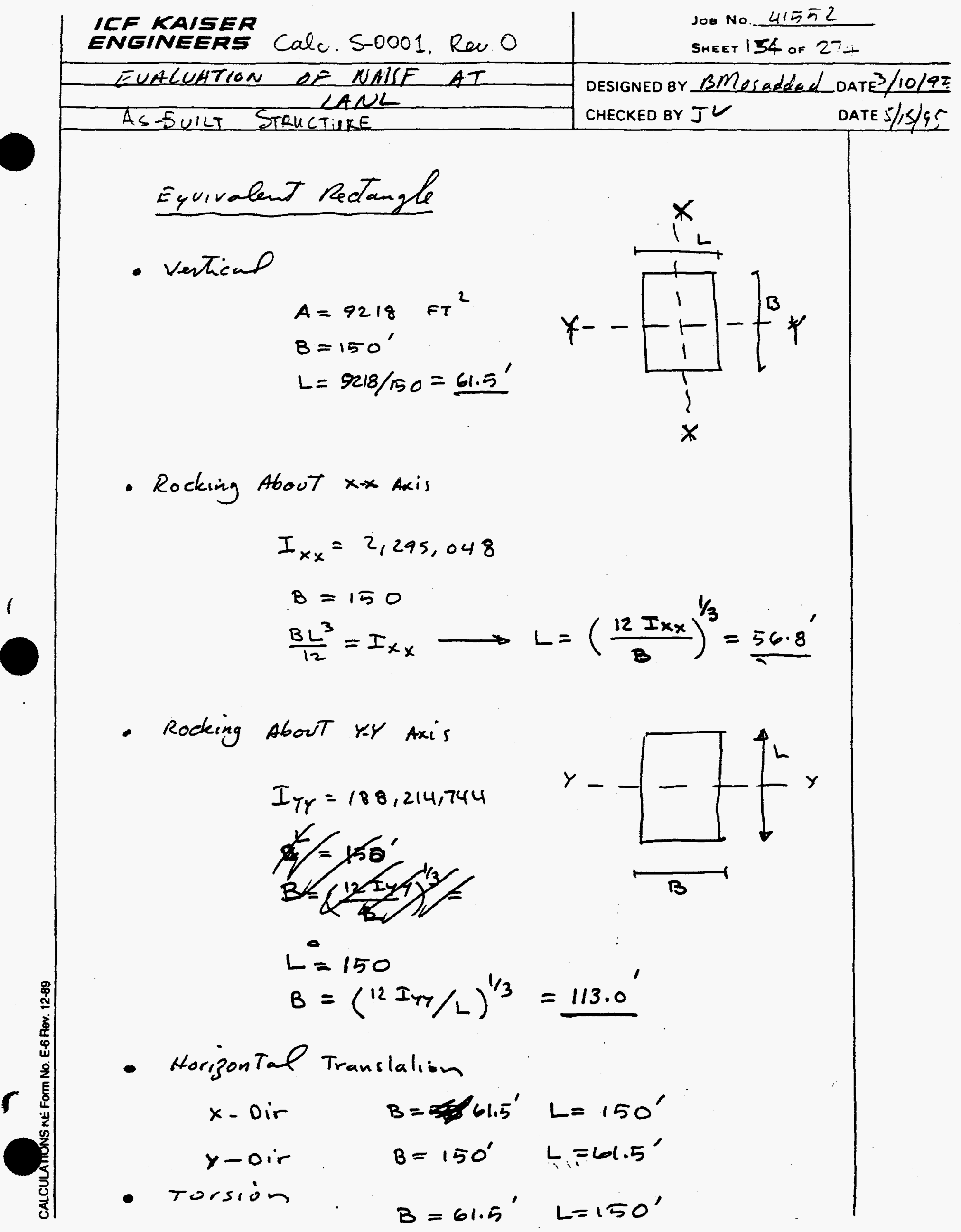




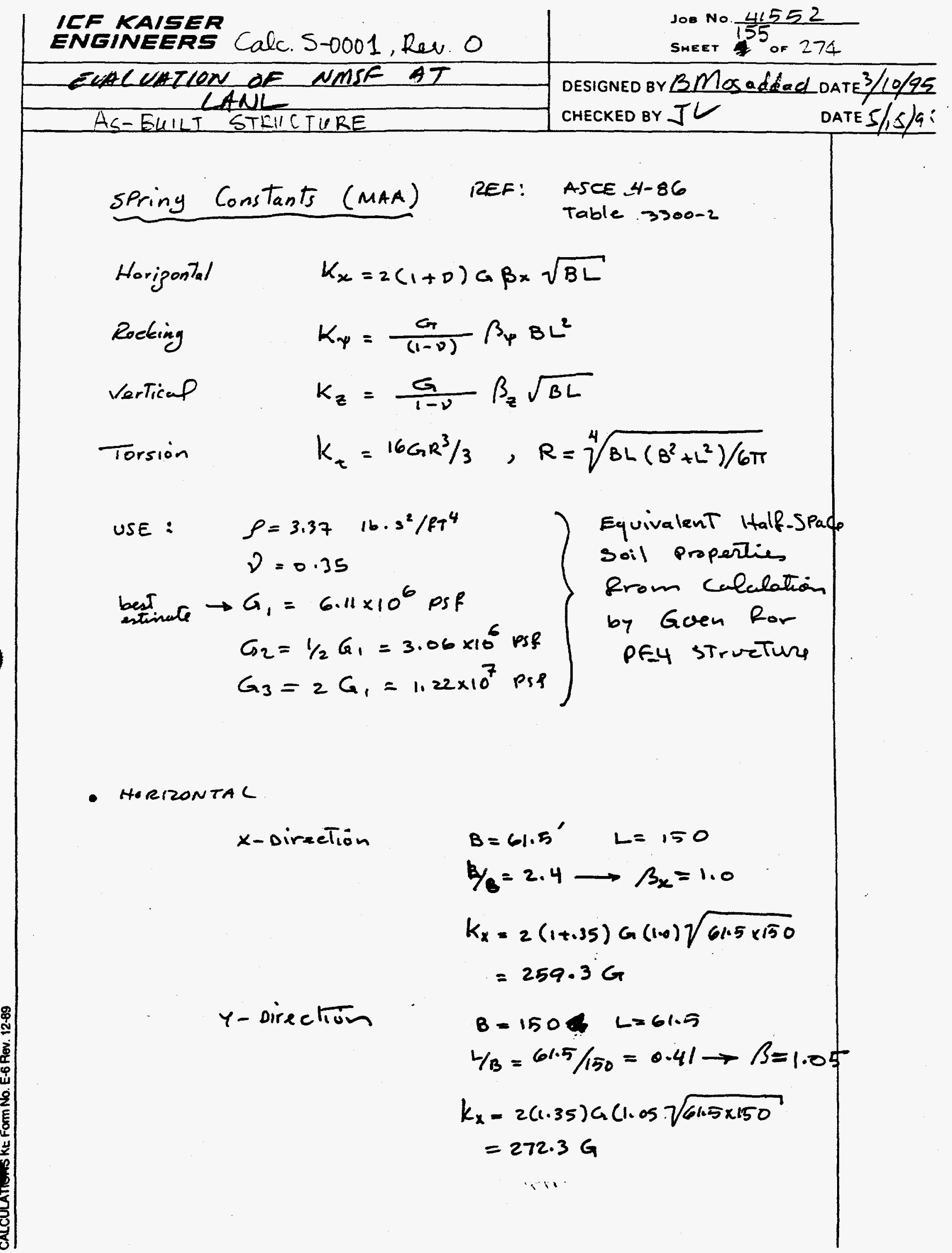




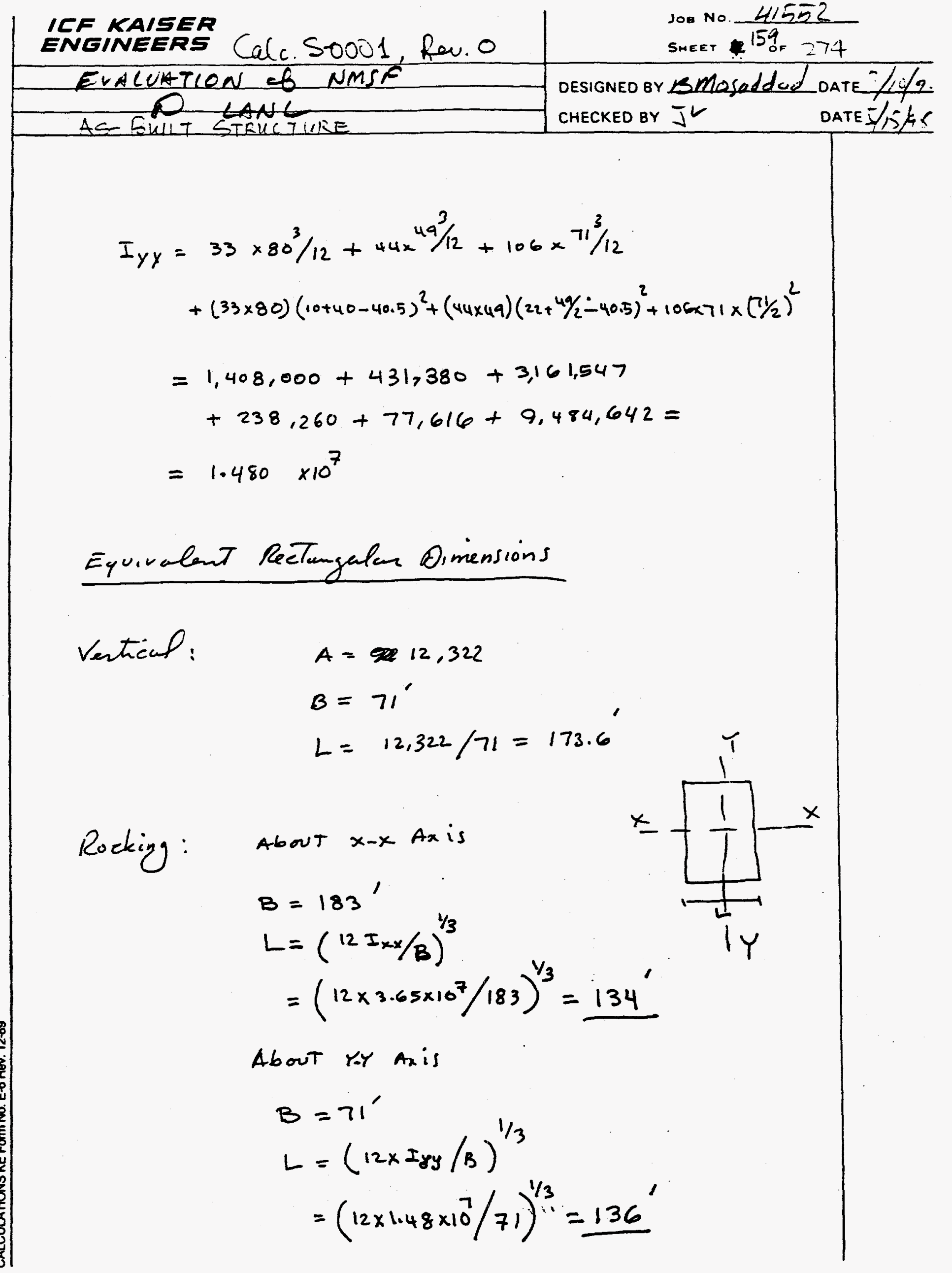




$$
\begin{aligned}
& ,^{01 \times \varepsilon s \cdot 2=} \\
& h \varepsilon \times \varepsilon 81 \times \operatorname{og} \cdot \times \frac{5 \varepsilon-1}{5}=x \\
& \text { c. } 0=A_{5}=L \cdot 0=8 / 7 \\
& h(1)=7, \varepsilon 81=8 \quad x-x+\text { sogt } \\
& \text { Guppoz - } \overrightarrow{\mathrm{N}} \\
& \text { DOE = } \\
& \operatorname{LxhLI}(0.1) \square(s \varepsilon \cdot 1)^{2}=x_{\lambda} \\
& 0.1=x_{8} 4
\end{aligned}
$$

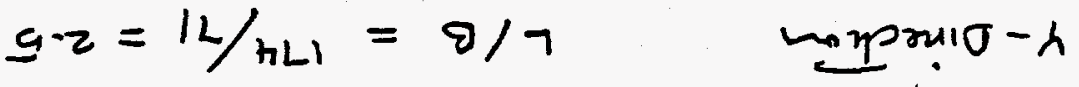

$$
\begin{aligned}
& \mapsto \cdot 21 \varepsilon= \\
& \operatorname{L9} \times\left\{21 / \Omega(90 \cdot 1) \rightarrow(5 \varepsilon \cdot+1)_{2}=x_{y}\right. \\
& 00-1=x / 2 t \varepsilon \cdot 0=\varepsilon / 7 \\
& \text { u.porato-x } \\
& \text { grinolinafl - } \\
& \text { Iundsuog } 5 \text { inds }
\end{aligned}
$$

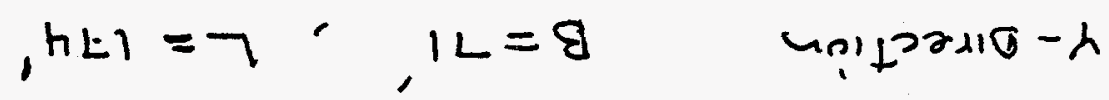

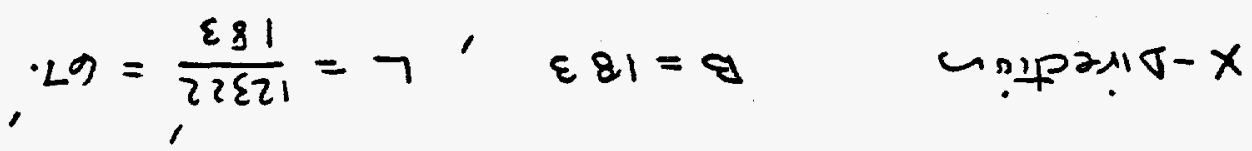

$$
\begin{aligned}
& \text {-Oyysung goluolison. }
\end{aligned}
$$

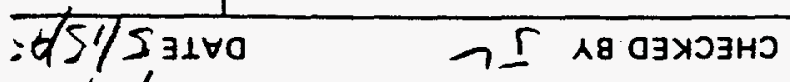

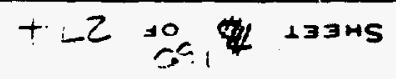

$$
\begin{aligned}
& \text { Tद्धाT ON BOr } \\
& 70 y+7 \text { wis } 1 \text { ing-50 }
\end{aligned}
$$

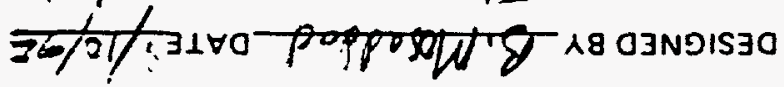

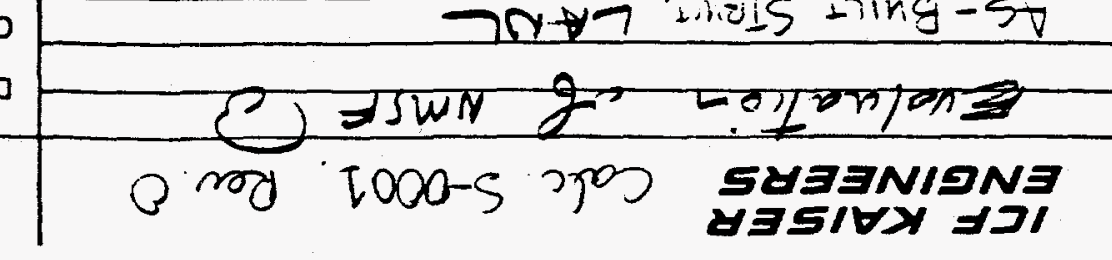




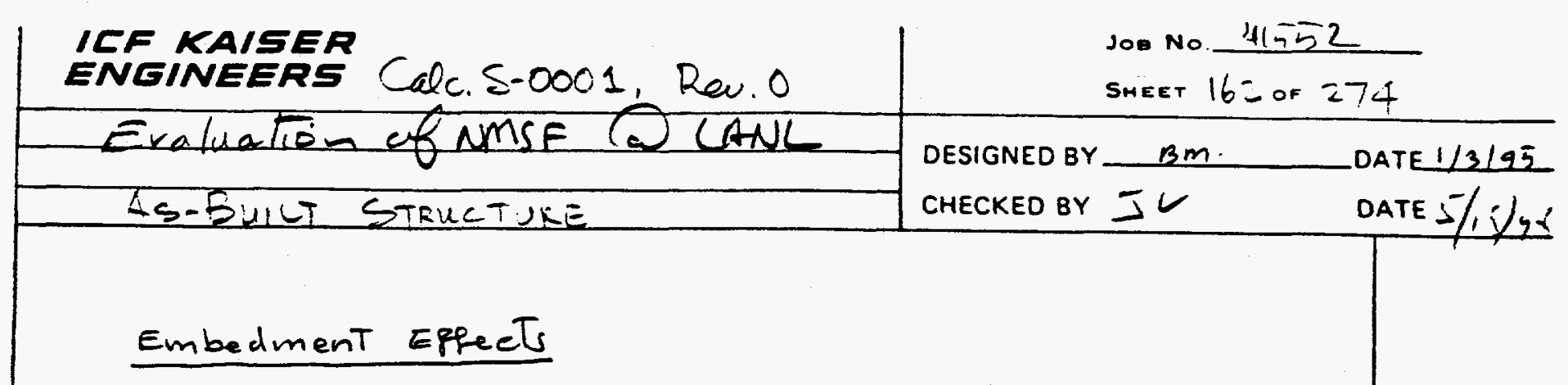

Compute an equivalent Embedment.

Assume:

- Mat portion Embedmat only 1/2 affective above dst. Floor. This in a Conservative assumption with respect to Computation of soil springs where the embedment above $15 T$ Floor was ignored (ALeE 4-86)

- use an equivalent Entedmet depth

- Compute soil spring sTiffren multiplies, kea "SSI in Time Domain." BY J.P. WOLF (1788, Bee 2.7)

$$
f_{h_{x}}=1+\left(.33+\frac{1.34}{1+e / b}\right)\left(\frac{e}{b}\right)^{0.8}
$$

where:

$$
\begin{aligned}
& e=\text { Embedment depth. } \\
& b=\text { roundatia width } \\
& l=\text { "length (along x-axis) }
\end{aligned}
$$

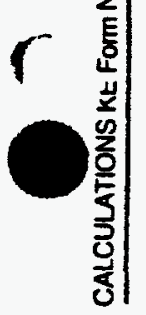

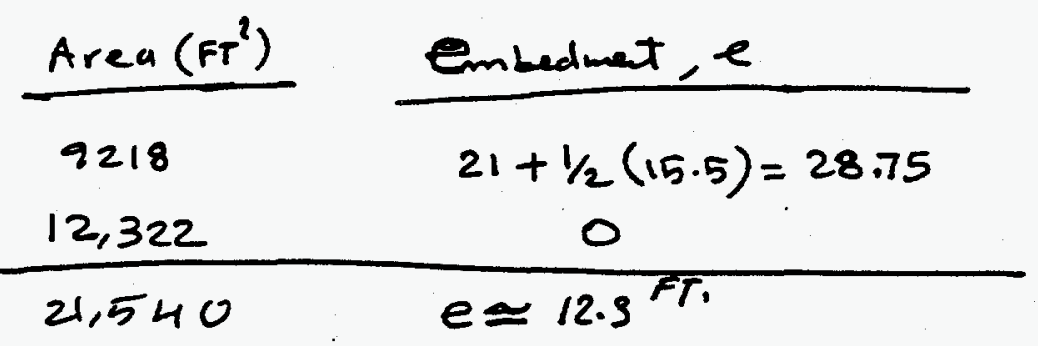


| ICF KAISER

JoB No.4155?

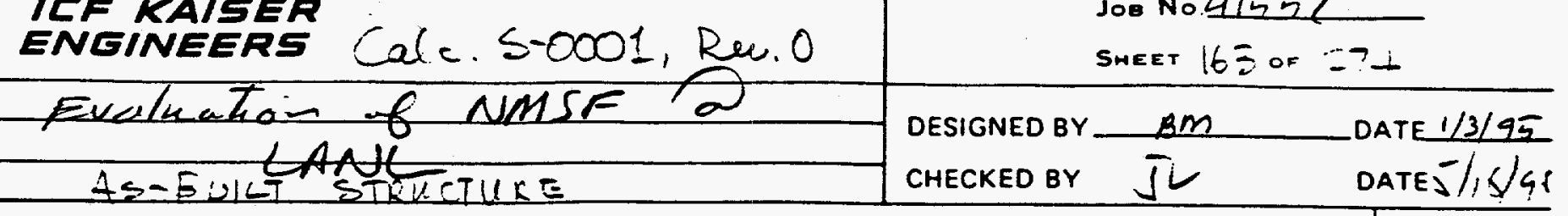

Embedmat Effects (Cont'd)

$$
\begin{aligned}
\begin{aligned}
f_{h x} & =1+\left(.33^{+} \frac{1.34}{1+55 / 150}\right)\left(\frac{12.3}{150}\right)^{.8}= \\
& =1+1.3105 \times .1352=1.177
\end{aligned} \\
\text { Impact of Freq. Shift } \simeq \sqrt{\frac{f}{h x}}=1.085
\end{aligned}
$$

Embedmeat results inte shift of Frequens Tuands higher Enequencier by $8 \%$.

1

reyligible! 


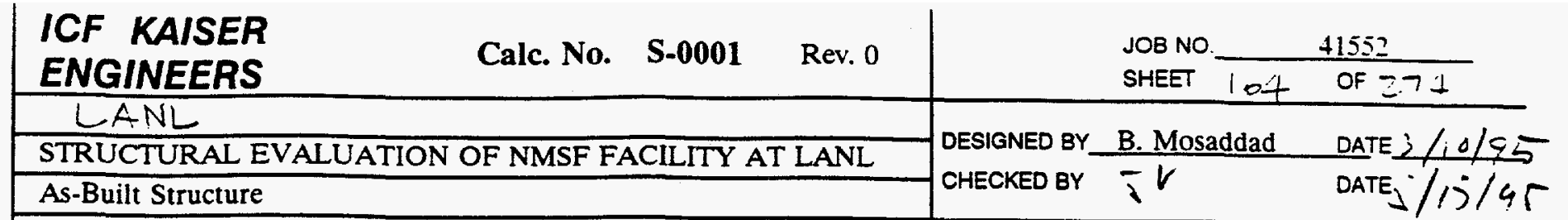

5.4 ACCIDENTAL TORSION EFFECTS

Accidentind Torsion d Effects are accounted for by moving the Computed Carter is yiseinty a nay from the carter of rigidity a each flow icu a distance equal to $5 \%$ if the overall Building dimension in the Cassesponding direction

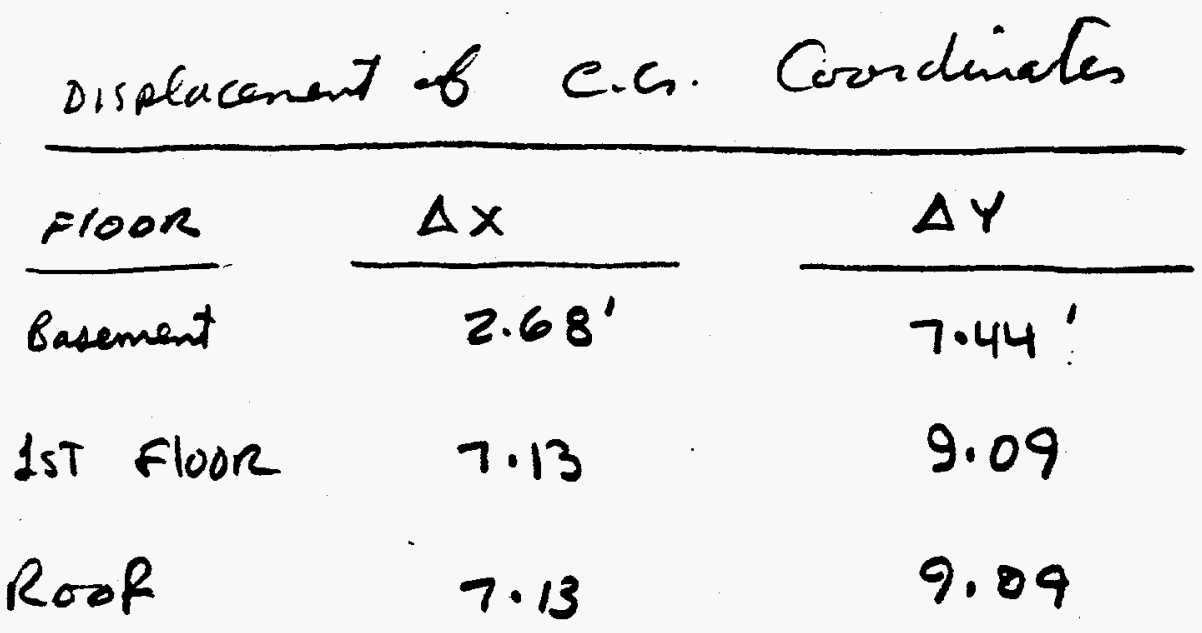




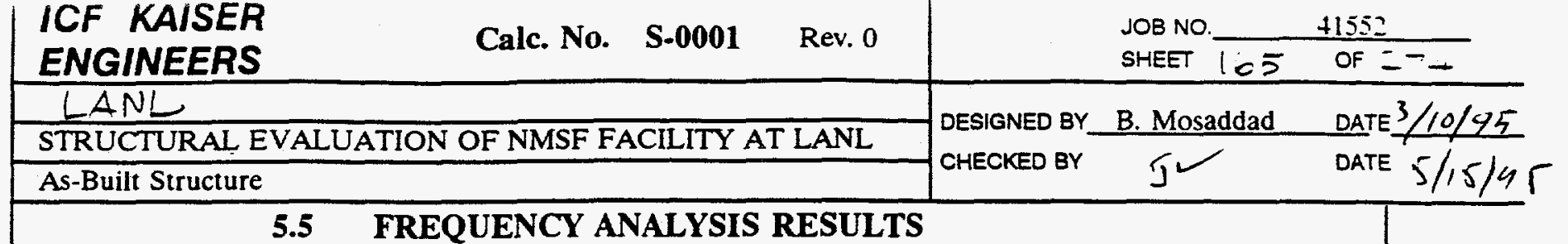

5.5 FREQUENCY ANALYSIS RESULTS

Fienew Volue Anclysin printerul Including Frequencies aund ithoss Pasticipation fuctors an printed on swent pages for the following sto9o models:

NMSF $\varnothing$ : rixed base NMSFDI: Include sss soil springs NMSFD3: Rigid Floon, No Torsion D iffects.

NMSF o 4: Rigid Floors, 5\% Accidentaf Mass Eecen. Included.

NMSF41: (Same as NurFo4) Mode shape plots. 


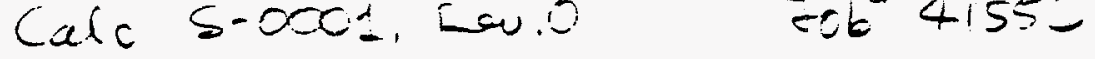

Sut. $\Rightarrow 50=7-$

C S I / S A P 90 - - FINITE ELEMENT ANALYSIS OF STRUCTURES PAGE 4

A R T I I PA T I G MASS - (percent)

$\begin{array}{rrrrrrr}\text { SODE } & \text { X-DIR } & \text { Y-DIR } & \text { Z-DIR } & \text { X-SUM } & Y \text {-SUM } & \text { Z-SUM } \\ 1 & 73.284 & 0.019 & 0.007 & 73.284 & 0.019 & 0.007 \\ 2 & 0.009 & 1.117 & 0.000 & 73.293 & 1.136 & 0.007 \\ 3 & 0.341 & 0.032 & 0.000 & 73.634 & 1.168 & 0.007 \\ 4 & 0.053 & 0.060 & 0.014 & 73.687 & 1.227 & 0.021 \\ 5 & 0.017 & 70.506 & 0.004 & 73.705 & 71.733 & 0.026 \\ 6 & 5.053 & 0.115 & 0.004 & 78.757 & 71.848 & 0.029 \\ 7 & 2.052 & 8.846 & 0.125 & 80.810 & 80.694 & 0.154 \\ 8 & 0.335 & 1.207 & 2.329 & 81.145 & 81.901 & 2.483 \\ 9 & 11.363 & 1.997 & 0.090 & 92.508 & 83.898 & 2.573 \\ 10 & 0.017 & 0.071 & 63.546 & 92.525 & 83.969 & 66.119\end{array}$


I GEN VALUES MODE EIGENVALUE (RAD/SEC) $* \star 2$ $0.509647 E+04$ $0.539964 E+04$ $0.880761 E+04$ $0.106612 \mathrm{E}+05$ $0.119197 E+05$ $0.128523 E+05$ $0.212757 \mathrm{E}+05$ $0.221158 E+05$ $0.309049 E+05$ $0.332912 \mathrm{E}+05$
A N D F R E

CIRCULAR FREQ (RAD/SEC)

$0.713896 E+02$

$0.734822 E+02$

$0.938489 \mathrm{E}+02$

$0.103253 E+03$

$0.109177 \mathrm{E}+03$

$0.113368 \mathrm{E}+03$

$0.145862 E+03$

$0.148714 \mathrm{E}+03$

$0.175798 \mathrm{E}+03$

$0.182459 \mathrm{E}+03$
UENCIES

FREQUENCY

(CYCLES/SEC)

11.362001

11.695063

14.936513

16.433257

17.376088

18.043056

23.214646

23.668550

27.979097

29.039234
PERIOD

(SEC)

0.088013

0.085506

0.066950

0.060852

0.057550

0.055423

0.043076

0.042250

0.035741

0.034436 

C S I / S A P $90-$ - FINITE ELEMENT ANALYSIS OF STRUCTURES PAGE 4

A R T I I P A T I N G MAS S - (percent)

$\begin{array}{rrrrrrr}\text { ODE } & \text { X-DIR } & Y \text {-DIR } & \text { Z-DIR } & \text { X-SUM } & Y \text {-SUM } & \text { Z-SUM } \\ 1 & 92.282 & 0.229 & 0.223 & 92.282 & 0.229 & 0.223 \\ 2 & 0.272 & 87.620 & 0.052 & 92.554 & 87.849 & 0.275 \\ 3 & 0.339 & 0.085 & 98.333 & 92.893 & 87.934 & 98.608 \\ 4 & 0.000 & 0.083 & 0.095 & 92.894 & 88.017 & 98.704 \\ 5 & 0.115 & 0.225 & 0.079 & 93.009 & 88.243 & 98.783 \\ 6 & 0.018 & 8.435 & 0.118 & 93.026 & 96.678 & 98.901 \\ 7 & 3.257 & 0.009 & 0.128 & 96.283 & 96.687 & 99.029 \\ 8 & 0.028 & 2.085 & 0.016 & 96.311 & 98.772 & 99.045 \\ 9 & 0.597 & 0.000 & 0.818 & 96.908 & 98.772 & 99.864 \\ 10 & 2.393 & 0.019 & 0.025 & 99.301 & 98.791 & 99.889\end{array}$


MODE EIGENVALUE CIRCULAR FREQ NUMBER (RAD/SEC) $* \star 2$ (RAD /SEC)

$\begin{array}{lll}1 & 0.534738 \mathrm{E}+04 & 0.731257 \mathrm{E}+02\end{array}$

$0.546127 \mathrm{E}+04 \quad 0.739004 \mathrm{E}+02$

$0.594000 \mathrm{E}+04 \quad 0.770714 \mathrm{E}+02$

$0.126489 \mathrm{E}+05 \quad 0.112467 \mathrm{E}+03$

$0.185804 \mathrm{E}+05 \quad 0.136310 \mathrm{E}+03$

$0.298748 \mathrm{E}+05 \quad 0.172843 \mathrm{E}+03$

$0.325896 \mathrm{E}+05 \quad 0.180526 \mathrm{E}+03$

$0.512355 \mathrm{E}+05 \quad 0.226353 \mathrm{E}+03$

$90.740126 \mathrm{E}+05 \quad 0.272053 \mathrm{E}+03$

$10 \quad 0.152804 E+06 \quad 0.390902 E+03$
FREQUENCY (CYCLES/SEC)

11.638325

11.761616

12.266295

17.899731

21.694400

27.508843

28.731589

36.025150

43.298508

62.213989
PERIOD

(SEC)

0.085923

0.085022

0.081524

0.055867

0.046095

0.036352

0.034805

0.027758

0.023095

I NMSFE3

- Rigid floor diaphragm

1

- No Acerdertal Mall eccelliez included

Compare with NMSFAI 
iéle S-0001. Fe. , Jor 41552

此: $7, q=70$

C S I

$S$ A P 9

FINITE

ELEMENT

ANAIYSIS OF STRUCTURES

PROGRAM: SAP90/FILE : NMSF03.RIT

A.R T I I P A T I G MASS - (percent)

ODE
1
2
3
4
5
6
7
8
9
10

$X-D I R$
89.940
1.546
2.597
0.021
3.793
1.852
0.034
0.003
0.215
0.000

Y-DIR

Z-DIR

1.858

2.437

89.975

95.017

4.622

0.192

0.005

0.315

0.000

0.019

0.003

0.000

1.234

0.000

0.005

0.000

0.000

0.154

X-SUM
89.940
91.486
94.083
94.103
97.896
99.748
99.781
99.785
99.999
99.999

Y - SUM

Z- SUM

2.131

92.106

94.106

98.728

1.858

4.295

99.312

99.504

98.733

98.734

98.737

99.819

99.838

99.838

99.838

$99.976 \quad 99.839$

$99.976 \quad 99.992$ 
Cit. $5-\infty 001$, Lew 0 os $4155=$

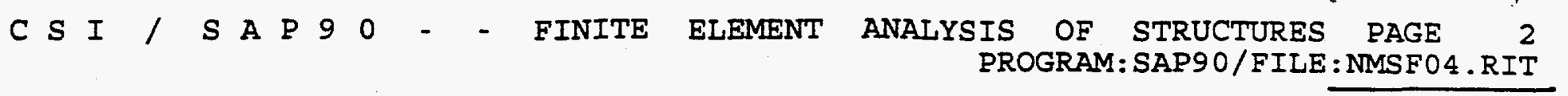

I GEN VALUES AN D FREQ U EN CI ES

MODE EIGENVALUE CIRCULAR FREQ NUMBER
FREQUENCY (CYCLES/SEC)

11.659858

11.778674

12.311752

17.650970

21. 112371

26.498063

28.712696

36.785228

41.528068

64.742695
PERIOD

(SEC)

0.085764

0.084899

0.081223

0.056654

0.047366

0.037739

0.034828

0.027185

0.024080

0.015446

Nouns $\$ 4$

adalitional

- Include $\triangle 5 \%$ Mass eccestinity

- Compar with DMSO $\Phi 3$ 


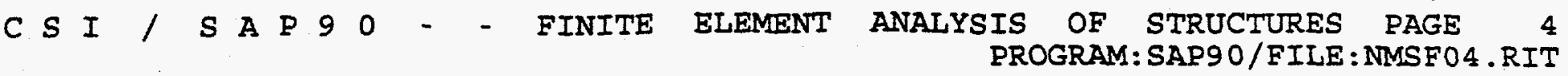

A R T I I P A T ING MASS - (percent)

$\begin{array}{rrrrrrr}\text { ODE } & \text { X-DIR } & Y-D I R & Z \text {-DIR } & X \text {-SUM } & Y \text {-SUM } & \text { Z-SUM } \\ 1 & 93.489 & 0.440 & 0.112 & 93.489 & 0.440 & 0.112 \\ 2 & 0.471 & 92.333 & 0.885 & 93.959 & 92.773 & 0.997 \\ 3 & 0.070 & 1.059 & 98.660 & 94.030 & 93.833 & 99.656 \\ 4 & 0.013 & 4.912 & 0.205 & 94.043 & 98.745 & 99.861 \\ 5 & 3.767 & 0.016 & 0.000 & 97.809 & 98.761 & 99.862 \\ 6 & 1.299 & 0.000 & 0.000 & 99.108 & 98.761 & 99.862 \\ 7 & 0.561 & 0.073 & 0.001 & 99.669 & 98.835 & 99.864 \\ 8 & 0.010 & 1.150 & 0.001 & 99.679 & 99.985 & 99.865 \\ 9 & 0.318 & 0.000 & 0.001 & 99.997 & 99.985 & 99.865 \\ 10 & 0.000 & 0.000 & 0.125 & 99.997 & 99.985 & 99.990\end{array}$


$\sin ^{-} .17$ of $=74$

\begin{tabular}{|c|c|c|}
\hline$N \leftarrow$ & 岁 & 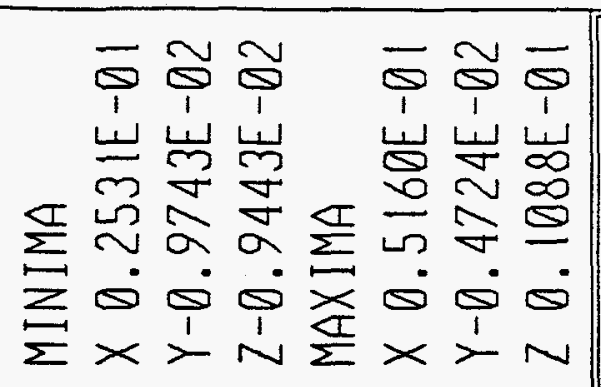 \\
\hline
\end{tabular}

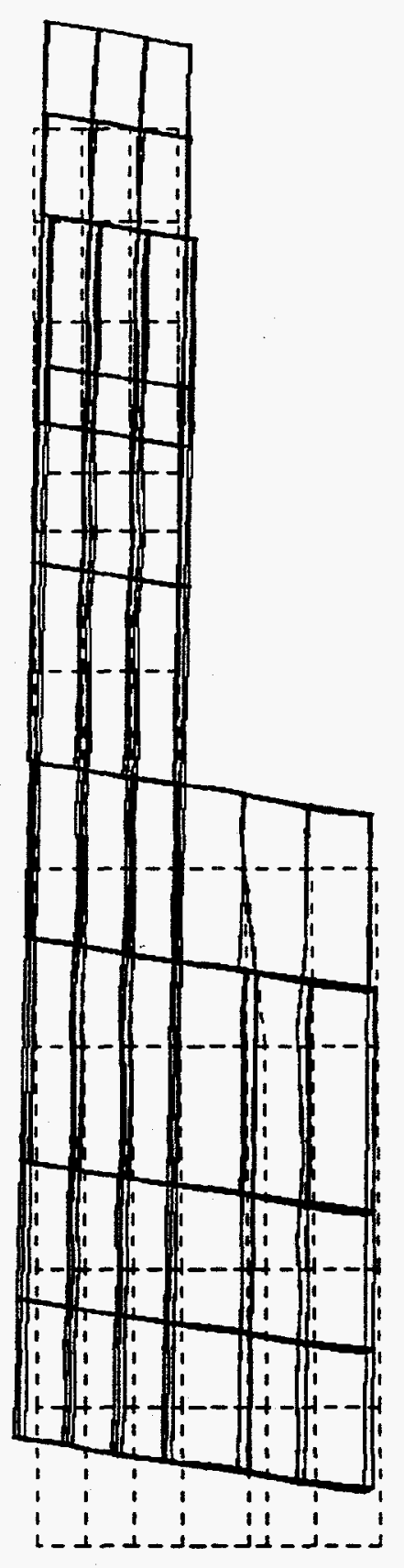




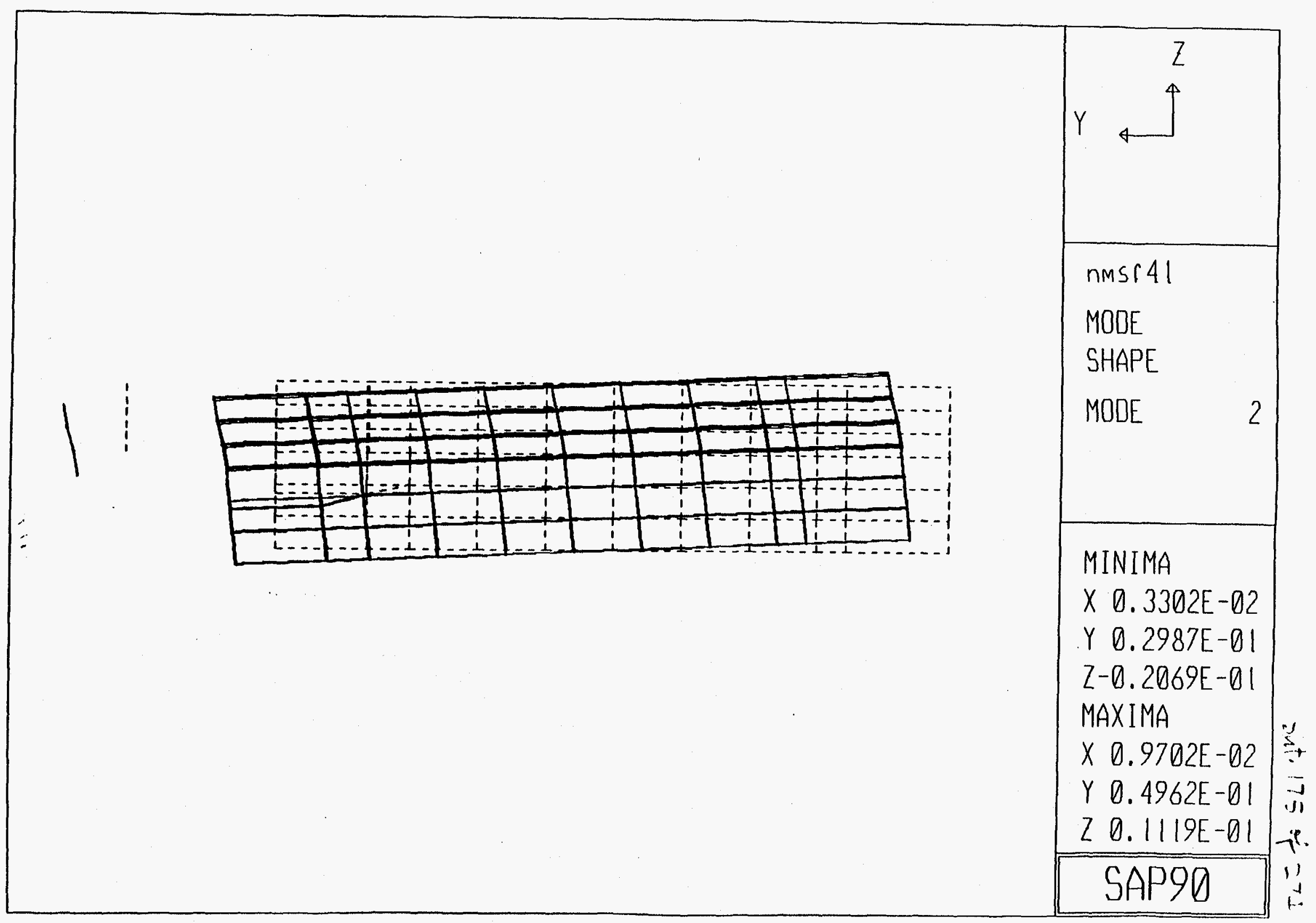


Sit. 176 of $=-$ -

\begin{tabular}{|c|c|c|c|c|c|}
\hline$N_{4}{ }^{x}$ & & 岸崖 & 岸 & 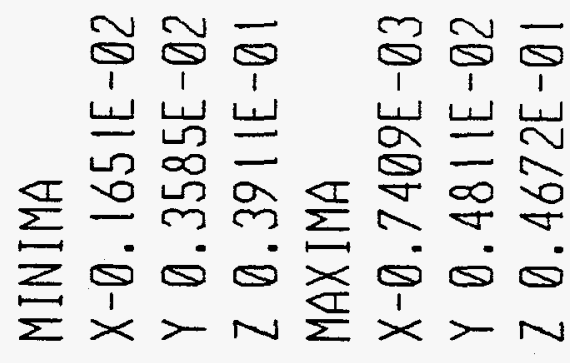 & $\frac{\vec{a}}{\frac{1}{2}}$ \\
\hline
\end{tabular}

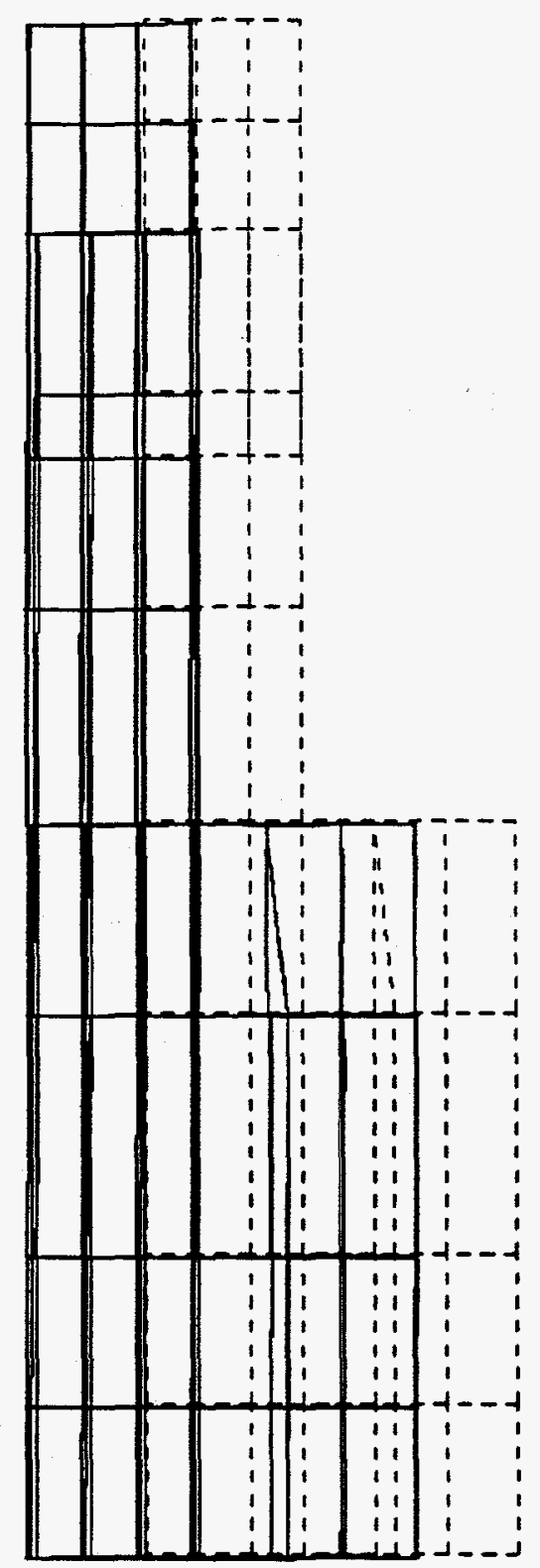


sit. $178+=0$

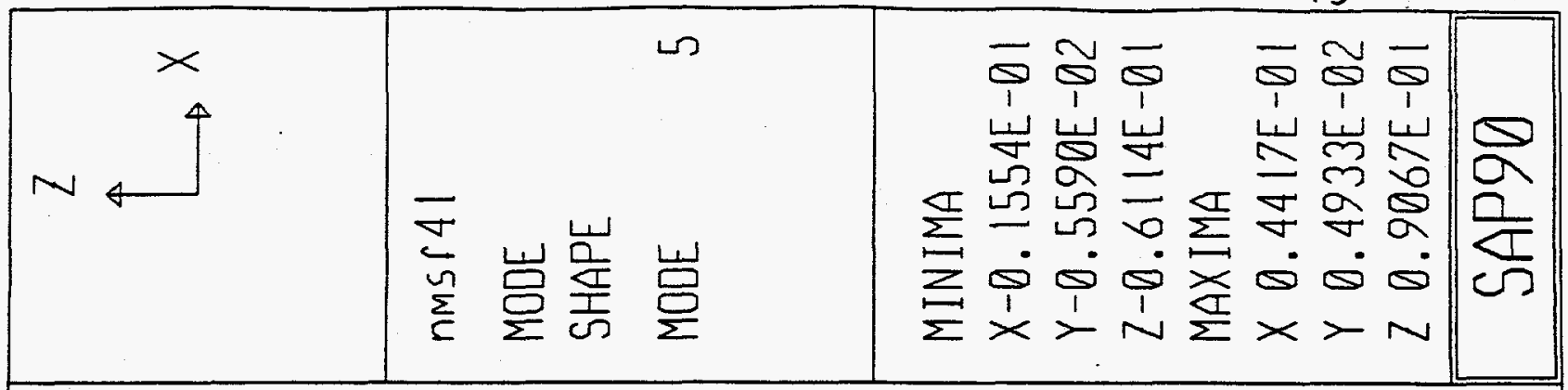

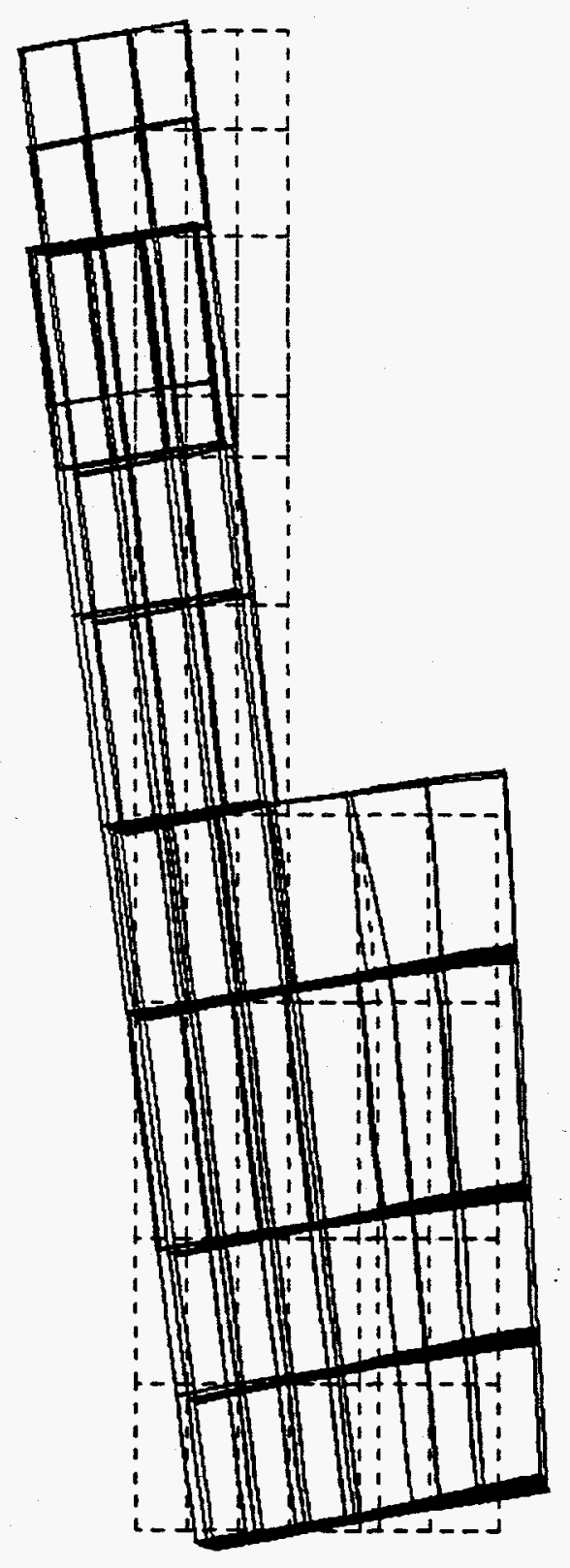




\begin{tabular}{|c|c|c|}
\hline$>4$ & 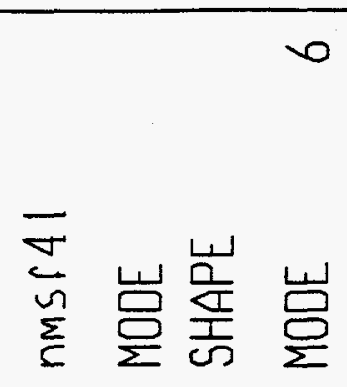 & 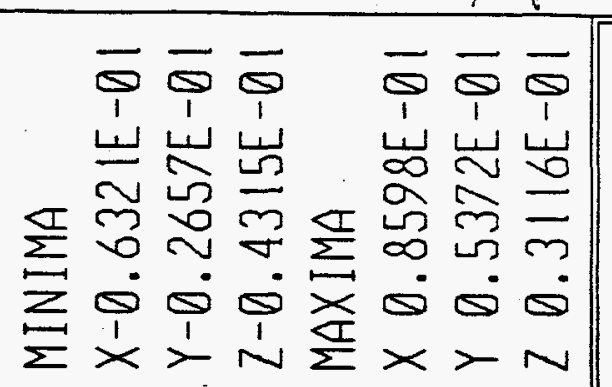 \\
\hline
\end{tabular}

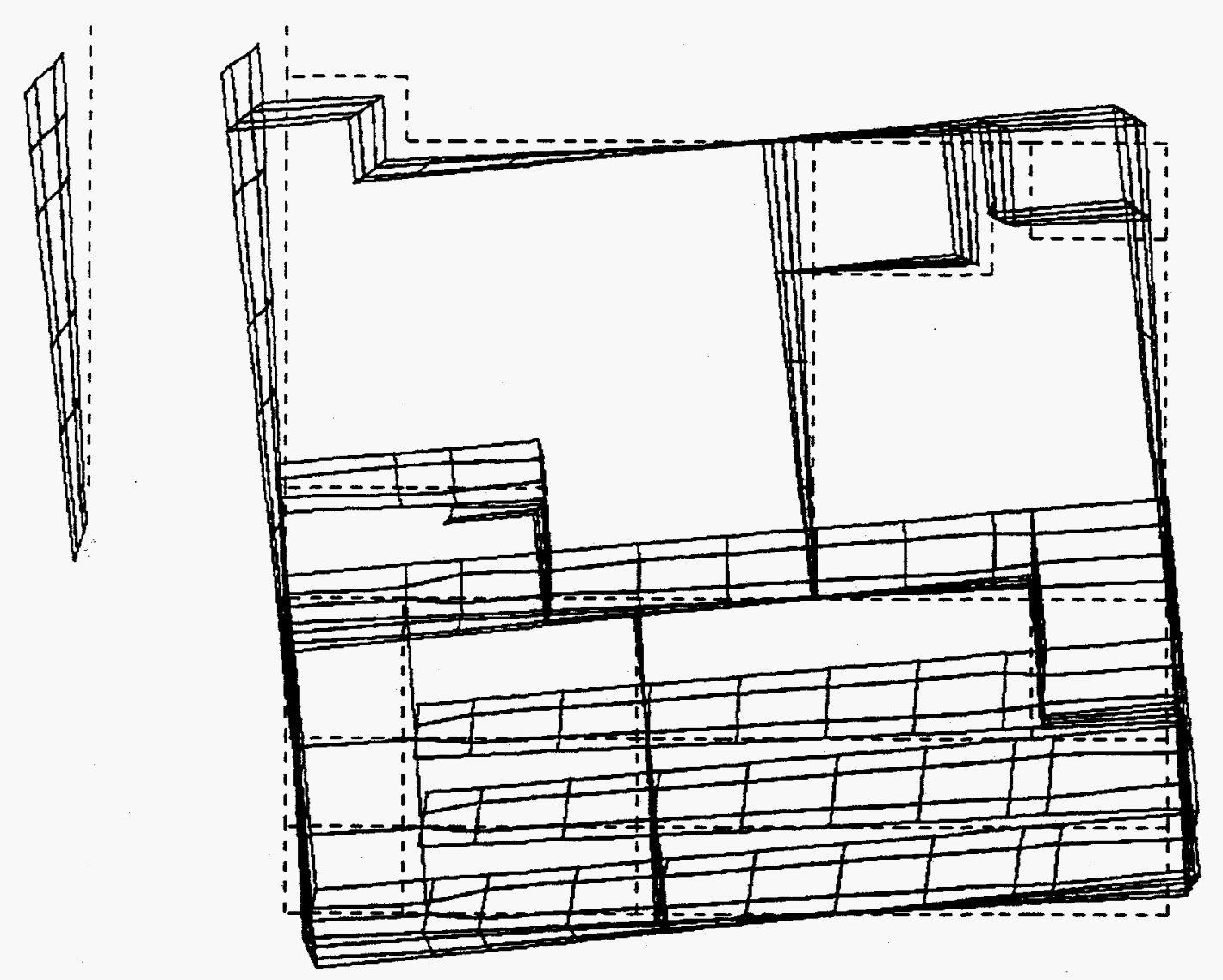


$\sin 180 t=-+$

\begin{tabular}{|c|c|c|}
\hline$>4$ & 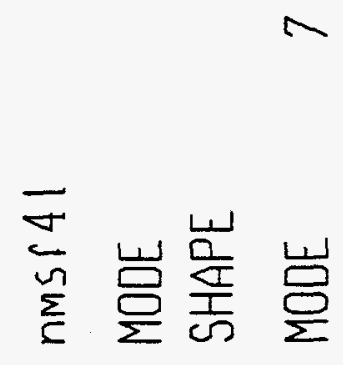 & 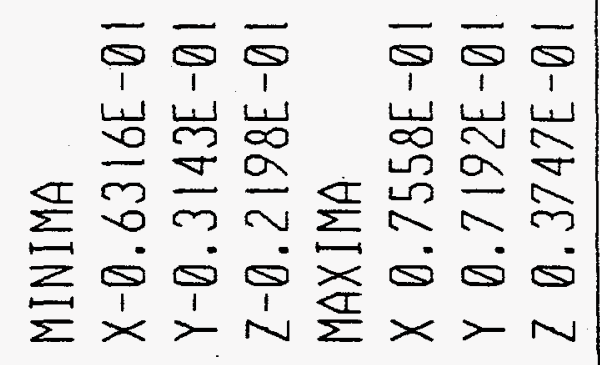 \\
\hline
\end{tabular}
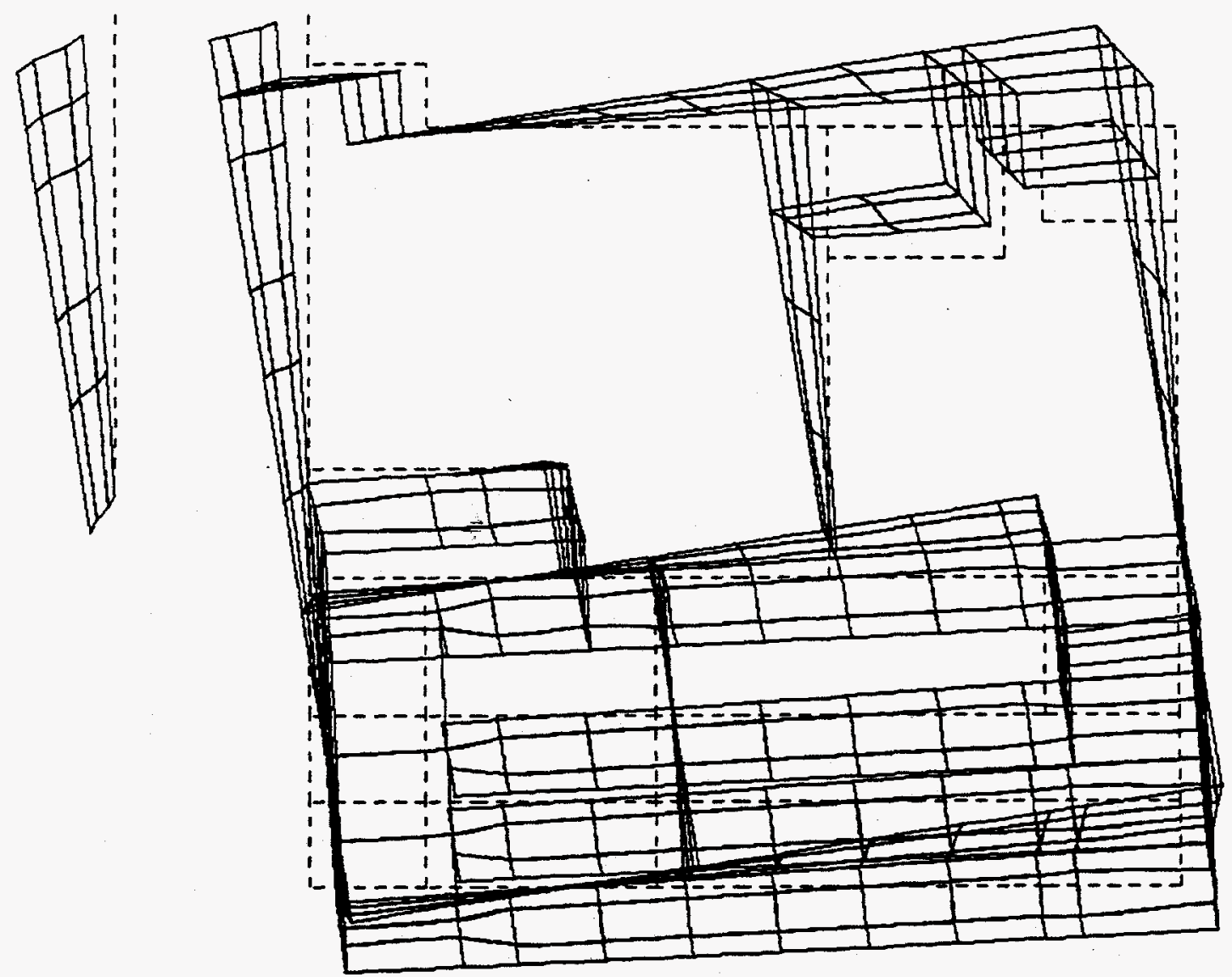
Sint $1315=$

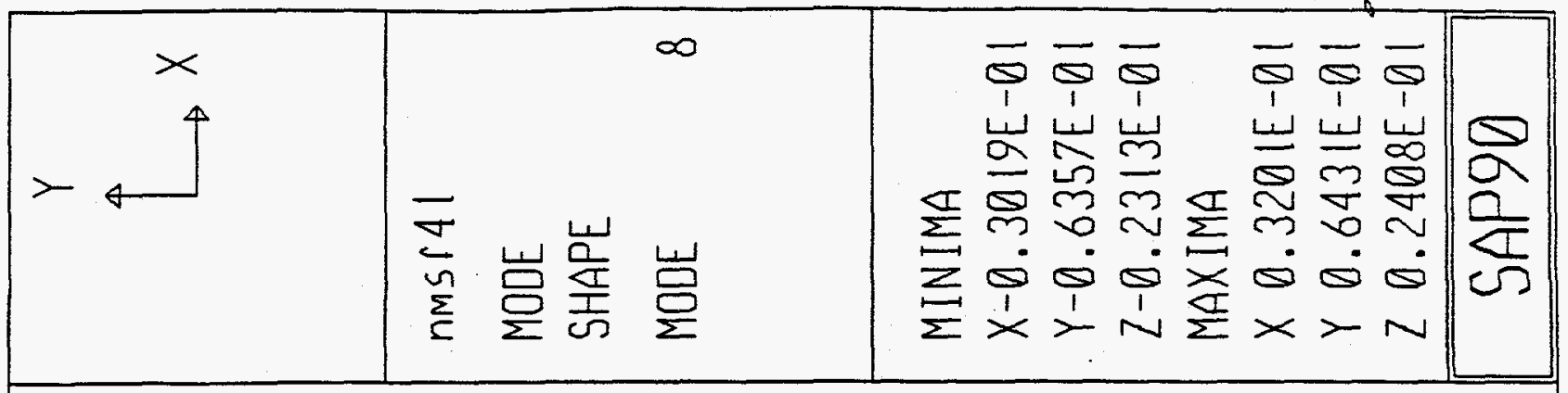
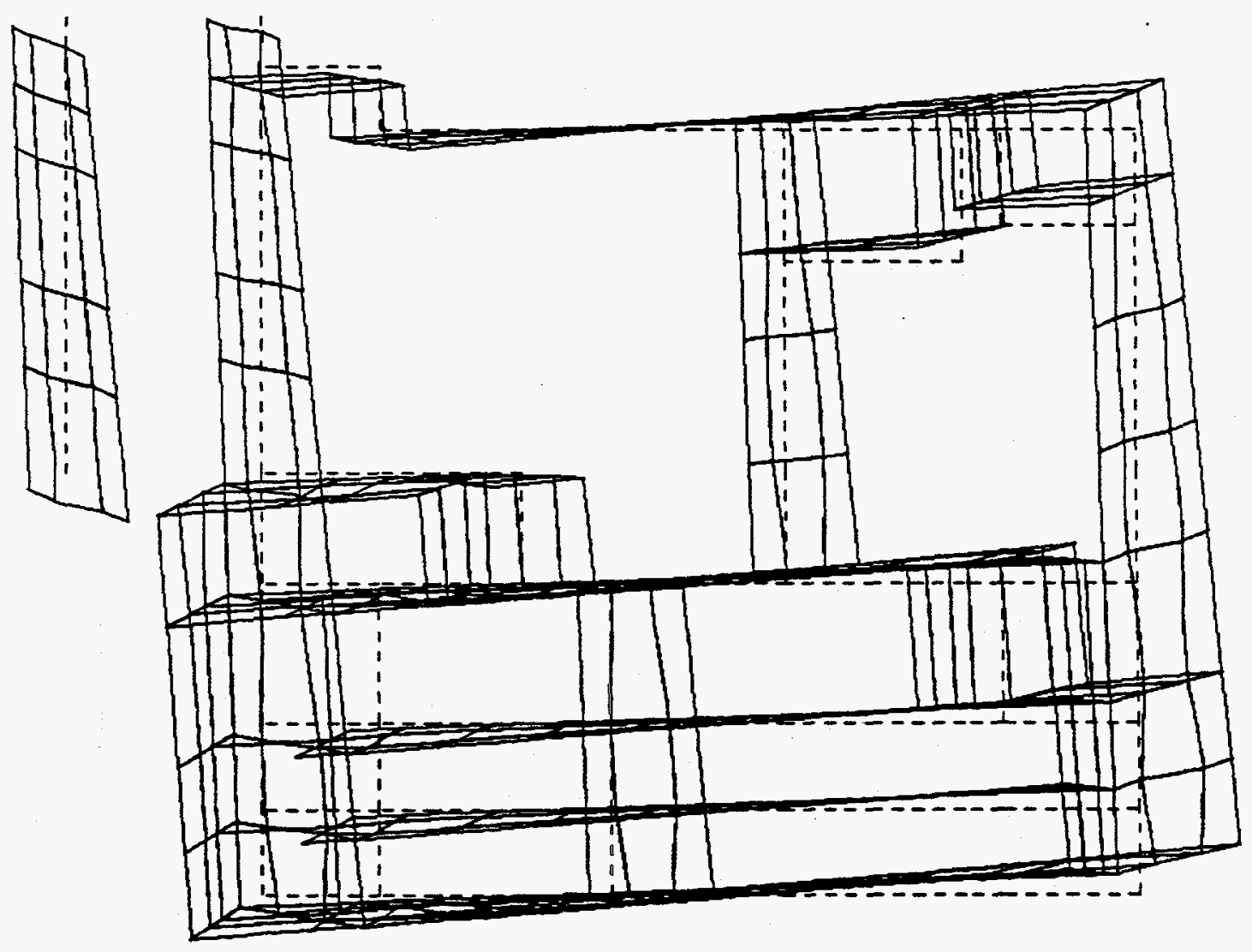
sint.18=

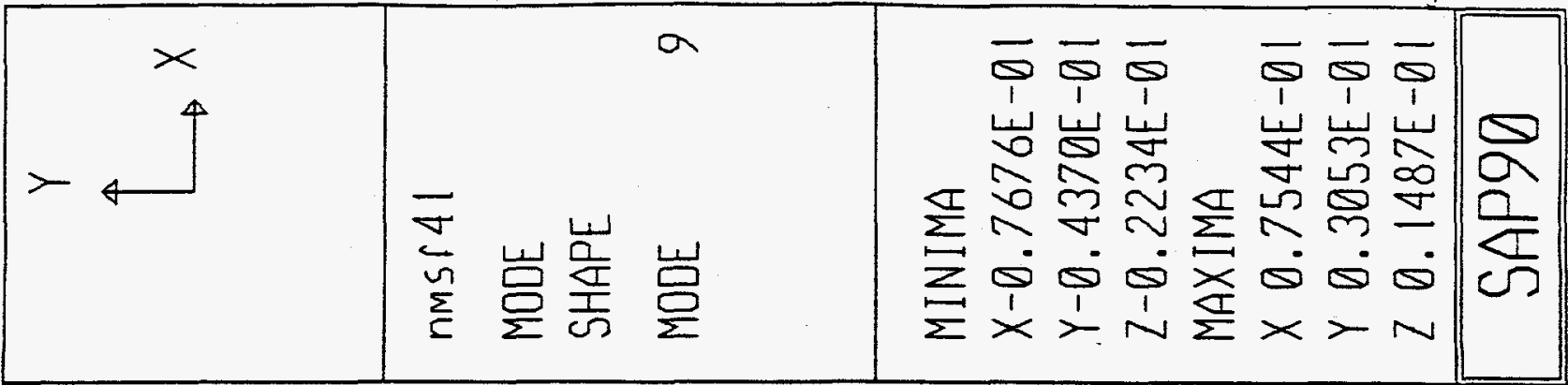

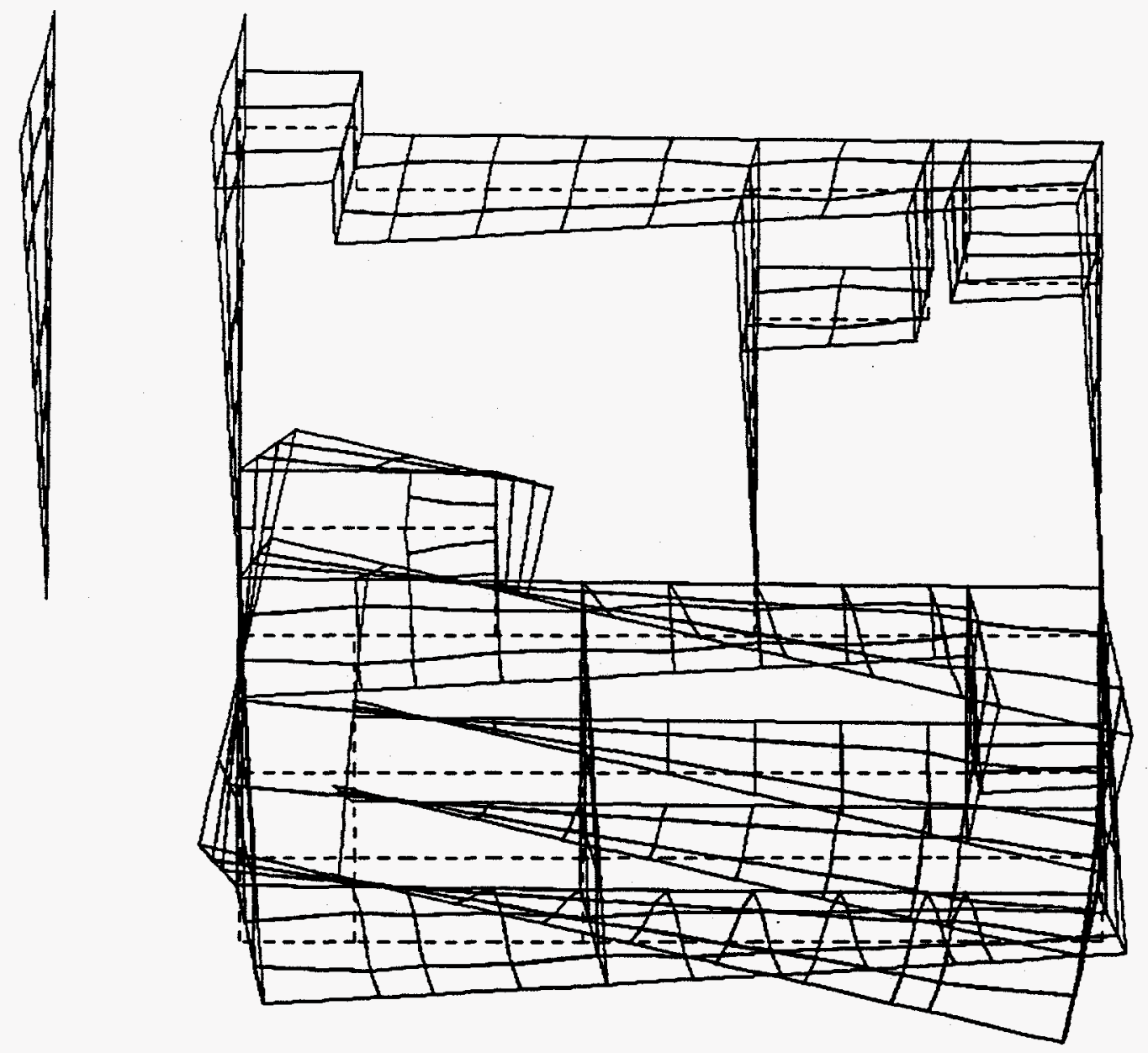


Calc. No. S-0001 Rev. 0

JOB NO. SHEET

133 OF $=71$

\begin{tabular}{|l|ll}
\hline$\leq \Delta N^{\prime} L$ & DESIGNED BY B. MOSaddad & DATE $3 / 10 / 95$ \\
\hline STRUCTURAL EVALUATION OF NMSF FACILITY AT LANL & CHECKED BY JU DATE $5 / 15 / 95$
\end{tabular}

6.0 DEMAND VERSUS CAPACITY

6.1 WALL IN-PLANE SHEAR AND BENDING OF SHEAR 


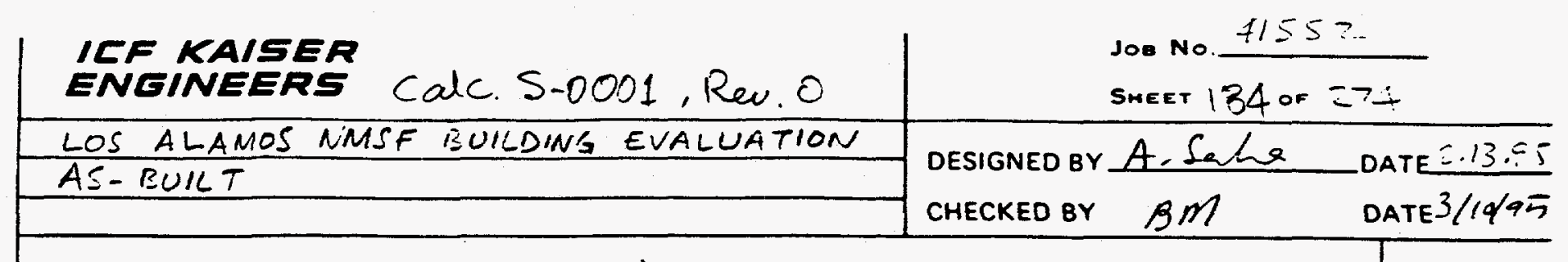

WALL SEGMENT 19 (Line (1)) Demand $V_{u}=1477^{\mathrm{K}}$ outside 2 Length $=150 \mathrm{ft}$., width $=1-4^{n}$, Vert. Reins. \#809 0.f. \#5@g I.F.

Height $h=15^{\prime}-0$ (first floor to roof). Inside $\rightarrow$

$$
\begin{aligned}
& M_{u}=1477 \times 15^{\prime} \text { conservative }=22,15 \mathrm{kft}^{\prime} \\
& A_{S}=(0.79+0.31) \times \frac{12}{9}=1.467 \mathrm{in}^{2} / \mathrm{st} \\
& 0.4 L=0.4 \times 150^{\prime}=60 \mathrm{ft} \text { Total } A_{S}=60 \times 1.467=88 \mathrm{in}^{2} . \\
& a=\frac{88 \times 60}{0.85 \times 4 \times 16}=97.1 \mathrm{in} . \\
& \phi M_{A}=0.9 \times 88 \times 60 \times\left(0.8 \times 150-\frac{97.1}{12 \times 2}\right)=551,000 \mathrm{kft}
\end{aligned}
$$

Basement

$$
\begin{aligned}
& \mathrm{Mu}_{\mathrm{u}}=22155+822 \times 21=39,417^{\mathrm{K}} \mathrm{ft} \\
& \begin{array}{l}
\text { Mu }=22155+822 \times 21=39,417 \\
\text { Width }=1^{\prime}-6^{\prime \prime} ; \text { vert. rein. \#10@9" Eff. Each }
\end{array} \\
& A_{S}=1.693 \times 2 \times 60=203 \mathrm{in}^{2} \\
& a=\frac{203 \times 60}{0.85 \times 4 \times 18}=199 \mathrm{in} \\
& \phi M_{n}=0.9 \times 203 \times 60 \times\left(0.8 \times 150-\frac{199}{12 \times 2}\right)=1,224,000^{\mathrm{kg}} \text {. }
\end{aligned}
$$

$\frac{\text { Demand }}{\text { Capacity }}=\frac{22,155}{551,000}=0.04$ for $1^{\text {st }}$ floor.

$$
=\frac{39,417}{1,224,000}=0.03 \text { for Basement. }
$$

Demand/capacity values are very low. .

$\therefore$ In plane overturning calculation for all other long walls is not required.

check overturning for short wall (wall segment 10) 
$\begin{array}{r}\text { IF KAISER } \\ \text { ENGINEERS Cal } \\ \hline \text { NOSE O CAN } \\ \hline \text { AS -BUILT } \\ \hline \text { Wall Segment } 10 ;\end{array}$

Jos No. 41552

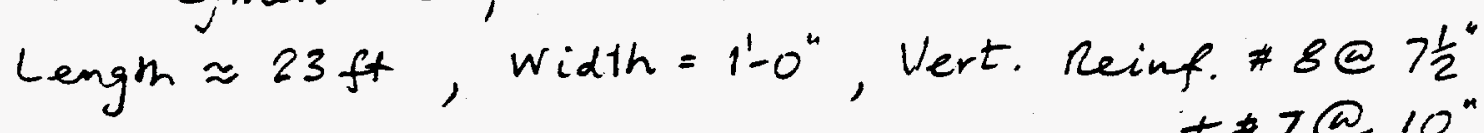

$$
A_{S}=0.6 \times 1.2+0.79 \times \frac{12}{7.5}=1.984 \mathrm{in}^{2} / \mathrm{ft} \text {. }
$$$$
\text { +71@10". }
$$

$0.4 L=0.4 \times 23=9.2 \mathrm{ft}$. Say 9'-0

Total $A_{S}=1.984 \times 9=17.856 \mathrm{in}^{2}$.

$$
\begin{aligned}
& a=\frac{17.856 \times 60}{0.85 \times 4 \times 12}=26.26 \mathrm{in} . \\
& \phi M_{n}=0.9 \times 17.856 \times 60 \times\left(0.8 \times 23-\frac{26.26}{2 \times 12}\right)=16,687^{\mathrm{kft}} \\
& V_{u}
\end{aligned}
$$

Demand $M_{U} \approx 189^{k} \times 21 \mathrm{ft}=3969^{\mathrm{kft}}$

$$
\frac{\text { Demand }}{\text { Capacity }}=\frac{3969}{16,687}=0.24<1.0
$$

By inspection, all other short walls are also 0.K. 
Job $4155=$

\begin{tabular}{|c|c|c|}
\hline $\begin{array}{l}\text { KAISER } \\
\text { ENGINEER Calc. S-0001, Reu. O }\end{array}$ & SHEET 186 & OF $=74$ \\
\hline SE D LANL & $\begin{array}{r}\text { BY: } \overline{B M} \\
\text { CHKD: TU }\end{array}$ & \\
\hline
\end{tabular}

\begin{tabular}{|c|c|c|c|c|c|}
\hline \multicolumn{6}{|c|}{ SUMMARY OF IN-PLANE SHEAR D/C RATIOS (AS-BUILT) } \\
\hline $\begin{array}{l}\text { WALL } \\
\text { SEGME }\end{array}$ & $\begin{array}{r}\text { LENGTH } \\
\text { L } \\
\text { (FEET) }\end{array}$ & $\begin{array}{r}\text { WIDTH } \\
\text { Be(*) } \\
\text { (FEET) }\end{array}$ & $\begin{array}{r}\text { CAPACIT } \\
(\mathrm{VC}) \\
(\mathrm{KIPS})\end{array}$ & $\begin{array}{r}\text { DEMAN } \\
\text { (VU) } \\
(\mathrm{KIPS})\end{array}$ & $\begin{array}{r}(D / C) \\
V u / N c\end{array}$ \\
\hline 3 & 148.7 & 1.5 & 2763 & 822 & 0.30 \\
\hline 6 & 148.7 & 1.45 & 2671 & 868 & 0.33 \\
\hline 8 & 35 & 1. & 434 & 167 & 0.39 \\
\hline 9 & 72.5 & 1.38 & 1239 & 1216 & 0.98 \\
\hline 12 & 55 & 1.5 & 1022 & 788 & 0.77 \\
\hline 4 & 128.7 & 1 & 1594 & 485 & 0.30 \\
\hline 5 & 105.8 & 1 & 1310 & 432 & 0.33 \\
\hline 1 & 22.9 & 1 & 284 & 84 & 0.30 \\
\hline 10 & 19 & 1 & 235 & 189 & 0.80 \\
\hline 11 & 23.9 & 1.21 & 358 & 264 & 0.74 \\
\hline 19 & 148.7 & 1.33 & 2450 & 1477 & 0.60 \\
\hline 34 & 53.5 & 1.33 & 881 & 692 & 0.79 \\
\hline 27 & 72.5 & 1.12 & 1006 & 695 & 0.69 \\
\hline 21 & 105.8 & 1.25 & 1638 & 1333 & 0.81 \\
\hline 20 & 22.9 & 1.33 & 377 & 222 & 0.59 \\
\hline 29 & 53.5 & 0.55 & 364 & 325 & 0.89 \\
\hline 28 & 80 & 1 & 991 & 758 & 0.76 \\
\hline 25 & 128.3 & 0.86 & 1367 & 996 & 0.73 \\
\hline 35 & 78 & 1 & 966 & 736 & 0.76 \\
\hline 38 & 80 & 1 & 991 & 745 & 0.75 \\
\hline 32 & 23.9 & 1.21 & 358 & 253 & 0.71 \\
\hline $\begin{array}{l}V C=.85 \\
V C=.8 \\
B e=E f\end{array}$ & $\begin{array}{l}\text { 'SQRT(f' } \\
c \star L^{\star} \\
\text { tive Wall }\end{array}$ & Innon & 108 & $\overline{S I}$ & \\
\hline
\end{tabular}


IEF KAISER

ENGINEERS Calc. S-0001, Rq O

LOS ALAMOS NMSF BUILDG EVALUATION AS-EUILT STRUCTURE soe No. $4157=$

SHEET 187 or 274 DESIGNED BY $6 M$ OATE ${ }^{2} / 1 \pi=$ CHECKED BY TU

WALL IN-PLANE SHEAR

WALL: 3 (LINE1-Basemed)

\begin{tabular}{|c|c|c|c|}
\hline $\begin{array}{l}\text { SHELL } \\
\text { ELEMENT }\end{array}$ & SOINT & $\begin{array}{l}\text { LCI } \\
F_{V}\end{array}$ & $\begin{array}{l}L C^{2} \\
F_{V}\end{array}$ \\
\hline \multirow{2}{*}{1101} & 1001 & 61 & -32 \\
\hline & 1011 & 57 & -38 \\
\hline \multirow{2}{*}{1102} & 1011 & 18 & -11 \\
\hline & 1021 & 5 & -25 \\
\hline \multirow{2}{*}{1103} & 1021 & 32 & -36 \\
\hline & $10 \equiv 1$ & 19 & -48 \\
\hline \multirow{2}{*}{1104} & 1031 & 33 & -33 \\
\hline & 1041 & 19 & -48 \\
\hline \multirow{2}{*}{1105} & 1041 & 34 & -33 \\
\hline & 1051 & 19 & -48 \\
\hline \multirow{2}{*}{1106} & $105:$ & 35 & -33 \\
\hline & 1061 & 18 & -49 \\
\hline \multirow{2}{*}{1107} & 1061 & 35 & -33 \\
\hline & 1071 & 17 & -49 \\
\hline \multirow{2}{*}{1108} & 107.1 & 35 & -33 \\
\hline & 1081 & 17 & -51 \\
\hline \multirow{2}{*}{1109} & 1081 & 27 & -19 \\
\hline & 1091 & 5 & -37 \\
\hline \multirow{2}{*}{1110} & 1091 & 19 & -73 \\
\hline & 1101 & $\cdot 3$ & -93 \\
\hline $\begin{array}{l}\text { WALL } \\
\text { BASE SHEAR }\end{array}$ & & 488 & 822 \\
\hline
\end{tabular}


ICF KAISER

ENEINEERS Calc. S-0001, Rew. O LOS ALAMOS NMSF BUILDC EVALUATION AS-BUILT STRUCTURE

A16 IH-WHE SHEAR

BALL 20

\begin{tabular}{|c|c|c|c|}
\hline $\begin{array}{l}\text { SHEL } \\
\text { ELEMENT }\end{array}$ & JOINT & $\begin{array}{l}\text { LCI } \\
F\end{array}$ & $\begin{array}{l}\text { LC2 } \\
F\end{array}$ \\
\hline \multirow{2}{*}{1301} & 1004 & 56 & -45 \\
\hline & 1084 & 46 & $-5 \pi$ \\
\hline \multirow{2}{*}{$\hat{i}$} & $121 i 1$ & 19 & $-1 !$ \\
\hline & 1024 & 8 & -26 \\
\hline \multirow{2}{*}{3} & 0211 & 38 & --3 \\
\hline & $C \leqq L 1$ & 27 & $-4 u$ \\
\hline \multirow{2}{*}{4} & iosu & ;8 & -31 \\
\hline & $104=$ & 26 & -44 \\
\hline \multirow{2}{*}{5} & .044 & 39 & -31 \\
\hline & 1054 & 26 & 44 \\
\hline \multirow{2}{*}{6} & 054 & 40 & -31 \\
\hline & 1064 & 25 & -45 \\
\hline \multirow{2}{*}{7} & 064 & 38 & -32 \\
\hline & 1074 & 22 & -47 \\
\hline \multirow{2}{*}{8} & 1074 & 39 & -33 \\
\hline & 1084 & 25 & -48 \\
\hline \multirow{2}{*}{9} & 1084 & 27 & -22 \\
\hline & 1094 & 8 & 36 \\
\hline \multirow{2}{*}{1310} & 1094 & 2 & -96 \\
\hline & 1104 & -20 & -114 \\
\hline $\begin{array}{l}\text { WALL } \\
\text { BAGE SHEAR }\end{array}$ & & 569 & 868 \\
\hline
\end{tabular}

$20 \cdot 6$
Jo. No. 1 ! 552

SHEET 188 of 277

DESIGNED BY

$3 \mathrm{rr}$ CHECKED BY

jr
DATE/ $/ 195$ DATE $Y / 1 / 9$ : 
ICF KAISER
ENEINEERS

1 soo No. $t==$

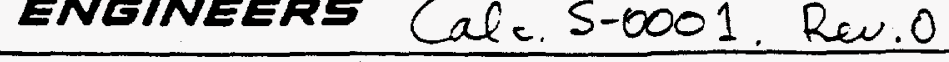

SMEET 189 or $=-+$

LOS ALAMOS NMSF BUILDC EVALUATION AS-BUILT STRUCTURE

DESIGNED BY $(2)$

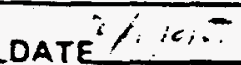

WALL IN-PLANE SHRAR

WALL: 9

\begin{tabular}{|c|c|c|c|}
\hline $\begin{array}{l}\text { SHEL } \\
\text { ELEMENT }\end{array}$ & SOINT & $\begin{array}{l}L C I \\
F_{x}\end{array}$ & $\begin{array}{l}\text { LC2 } \\
F_{x}\end{array}$ \\
\hline \multirow{2}{*}{2201} & 1101 & 84 & -104 \\
\hline & 1102 & 65 & -115 \\
\hline \multirow{2}{*}{2202} & 108 & $\therefore=$ & -122 \\
\hline & $111 \%$ & 45 & -137 \\
\hline \multirow{2}{*}{$=203$} & $116 \equiv$ & 99 & -200 \\
\hline & 1104 & 75 & -214 \\
\hline \multirow{2}{*}{2204} & 1104 & 61 & -143 \\
\hline & 1105 & 51 & -181 \\
\hline \multirow{4}{*}{ 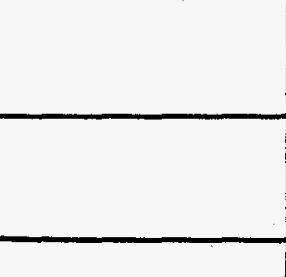 } & & & \\
\hline & & & \\
\hline & & & \\
\hline & & & \\
\hline 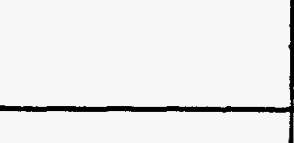 & & & \\
\hline \multirow{2}{*}{ 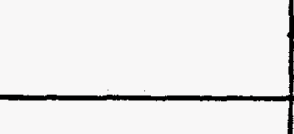 } & & & \\
\hline & & & \\
\hline 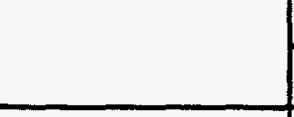 & & & \\
\hline 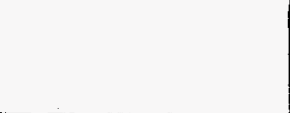 & & & \\
\hline \multirow{2}{*}{$\begin{array}{l}\text { WALL } \\
\text { BASE SHEAR }\end{array}$} & & 547 & 1216 \\
\hline & & T & 1210 \\
\hline
\end{tabular}


ICF KAISER

ENEINEERS Calc. S-0001. KUNO LOS ALAMOS NMSF BUILDC EVALUATION AS-BUHT STRUIT

WALL IN-PLANE SHEAR REF. SAPGO RNN: WALL: 8 21-10" EAST OF LINE 3 - BASEMENT

\begin{tabular}{|c|c|c|c|}
\hline $\begin{array}{l}\text { SHELL } \\
\text { ELEMENT }\end{array}$ & JOINT & $\begin{array}{l}L C I \\
F_{Y}\end{array}$ & $\begin{array}{l}L C^{2} \\
F_{Y}\end{array}$ \\
\hline \multirow{2}{*}{1451} & 1075 & 30 & -16 \\
\hline & 1085 & 20 & -26 \\
\hline \multirow{2}{*}{1452} & 1085 & 22 & -11 \\
\hline & 1025 & 9 & -23 \\
\hline \multirow{2}{*}{1453} & 1095 & 24 & -42 \\
\hline & 11.05 & 14 & -49 \\
\hline & & & \\
\hline & & & \\
\hline & & & \\
\hline & & & \\
\hline & & & \\
\hline & & & \\
\hline & & & \\
\hline & & & \\
\hline & & & \\
\hline & & & \\
\hline & & & \\
\hline WALL & & & \\
\hline BASE SHEAR & 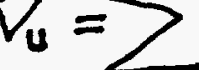 & 116 & -167 \\
\hline
\end{tabular}

Joe No $1:=$

SHEET 19C OF $=74$

DESIGNED BY AM


ICF KAIGER

ENGINEERS Calc. S-0001. Rev. 0 soo No. $1:=5=$

SMEET 191 of 274

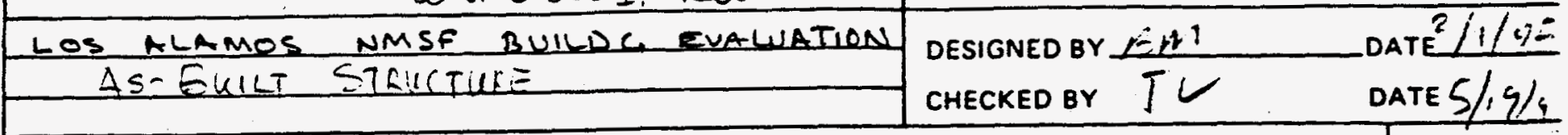

WALL IN-PLANE SHEAR

WALL: 12

\begin{tabular}{|c|c|c|c|}
\hline $\begin{array}{l}\text { SHEL } \\
\text { ELEMENT }\end{array}$ & SOINT & $\begin{array}{l}\text { LCI } \\
F_{x}\end{array}$ & $\begin{array}{c}L C^{2} \\
F_{x}\end{array}$ \\
\hline \multirow{2}{*}{2601} & 1001 & 43 & -108 \\
\hline & 1002 & 23 & -121 \\
\hline \multirow{2}{*}{2602} & 1002 & 53 & -105 \\
\hline & 1003 & 43 & -114 \\
\hline \multirow{2}{*}{2603} & 1003 & 80 & -165 \\
\hline & 1004 & 73 & -175 \\
\hline & & & \\
\hline & & & \\
\hline & & & \\
\hline & & & \\
\hline & & & \\
\hline & & & \\
\hline & & & \\
\hline & & & \\
\hline & & & \\
\hline & & & \\
\hline $\begin{array}{l}\text { WALL } \\
\text { BASE SHEAR }\end{array}$ & & 315 & 788 \\
\hline
\end{tabular}




\begin{tabular}{|c|c|c|c|}
\hline 486 & $h 62$ & $S=n$ & 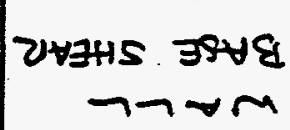 \\
\hline & & & \\
\hline $62-$ & $\pi$ & 26 & \multirow{2}{*}{6} \\
\hline $22-$ & $\varepsilon t$ & 28 & \\
\hline$s h-$ & 1 & 28 & \multirow{2}{*}{8} \\
\hline $8 \varepsilon-$ & 6 & 72 & \\
\hline $\ln \varepsilon-$ & 11 & 22 & \multirow{2}{*}{$L$} \\
\hline $22-$ & $h 2$ & 23 & \\
\hline $2 \varepsilon_{-}$ & $\sum 1$ & 79 & \multirow[t]{2}{*}{ g } \\
\hline $22-$ & $h z$ & 25 & \\
\hline $2 \varepsilon-$ & ता & 25 & \multirow{2}{*}{$G$} \\
\hline $2 \sqrt{z-}$ & $h 2$ & $2 n$ & \\
\hline $1 \varepsilon-$ & hI & $2 n$ & \multirow{2}{*}{ h } \\
\hline $22-$ & $\varepsilon z$ & $2 \xi$ & \\
\hline $2 \varepsilon-$ & $n 1$ & z \{ & \multirow{2}{*}{$\varepsilon$} \\
\hline$\varepsilon 2-$ & 23 & 22 & \\
\hline \pm 1 & 9 & 22 & \multirow[t]{2}{*}{$=$} \\
\hline$L-$ & 21 & 21 & \\
\hline $1 \varepsilon-$ & $t \varepsilon$ & 21 & \multirow{2}{*}{$\|4\|$} \\
\hline$h_{2}-$ & $O H$ & 2001 & \\
\hline $\begin{array}{l}r=1 \\
2 \supset 7\end{array}$ & $\begin{array}{l}h=1 \\
157\end{array}$ & INIOL & 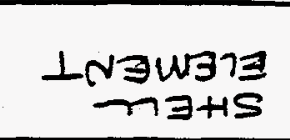 \\
\hline
\end{tabular}

\begin{tabular}{|c|c|c|}
\hline $35 / 6 / / 53100$ & $\Lambda \Gamma \wedge 8$ वэхОзно & \\
\hline$=\left(-91 / 2^{3}+0^{\circ}\right.$ & 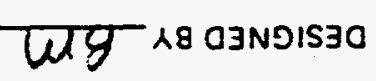 & 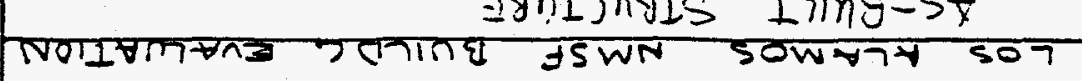 \\
\hline & & 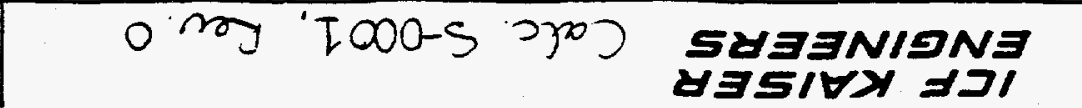 \\
\hline
\end{tabular}


ICF KAISER Calc. S-OOO1, RW. O

LOS ALAMOS NMSF BULLDC EvaLUATION AS-SMILT STRICTURE

WALL IN-PLANE SHEAR WALL: 5 LIME 2

\begin{tabular}{|c|c|c|c|}
\hline $\begin{array}{l}\text { SHEL } \\
\text { ELEMENT }\end{array}$ & SOINT & $\begin{array}{c}\text { LCI } \\
F_{Y}\end{array}$ & $\begin{array}{c}L^{2} 2 \\
F_{Y}\end{array}$ \\
\hline \multirow{2}{*}{1211} & 1013 & & -7 \\
\hline & 23 & & $-\sqrt{2}$ \\
\hline \multirow{2}{*}{2} & 23 & & -22 \\
\hline & 33 & & -31 \\
\hline \multirow{2}{*}{3} & 33 & & -22 \\
\hline & 4) & & -31 \\
\hline \multirow{2}{*}{4} & 43 & & -22 \\
\hline & 53 & & -31 \\
\hline \multirow[b]{2}{*}{5} & 53 & & -22 \\
\hline & 63 & & -32 \\
\hline \multirow{2}{*}{6} & 63 & & -22 \\
\hline & 73 & & -34 \\
\hline \multirow{2}{*}{7} & 73 & & -40 \\
\hline & 83 & & -47 \\
\hline \multirow{2}{*}{8} & 83 & & -24 \\
\hline & 93 & & -30 \\
\hline & & & \\
\hline & & & \\
\hline WคレL & & & \\
\hline BASE SHEAR & $\angle$ & & 432 \\
\hline
\end{tabular}

Jos No. $1==2$

SHEET 193 OF 271

DESIGNED BY $5 M$

CHECKED BY TC

REF. SAPOO RUN:
DATE $2 / 95$ DATES/9/95 
ILF KAISER

ENGINEERS Cale S-0001 Rev.0

sos No. $4:==$

SHEET 194 of 274

LOS NLAMOS NMSF BUILDC EVALUATION $15-E N I L T$ CTELTUK?

DESIGNED BY BWM CHECKED BY I $U$

REF. SAPGO RUN:

WALL IN-PLANE SHEAR Line 2

\begin{tabular}{|c|c|c|c|}
\hline $\begin{array}{l}\text { SHELU } \\
\text { ELEMENT }\end{array}$ & SOINT & $\begin{array}{l}F_{Y} \\
F_{Y}\end{array}$ & $\begin{array}{c}\text { LC2 } \\
F_{K}\end{array}$ \\
\hline \multirow{2}{*}{1201} & 1003 & 38 & -38 \\
\hline & 1013 & 33 & -46 \\
\hline & & & \\
\hline & & & \\
\hline & & & \\
\hline & & & \\
\hline & & & \\
\hline & & & \\
\hline & & & \\
\hline & & & \\
\hline & & & \\
\hline & & & \\
\hline & & & \\
\hline & & & \\
\hline & & & \\
\hline & & & \\
\hline & & & \\
\hline & & & \\
\hline & & & \\
\hline $\begin{array}{l}\text { WALL } \\
\text { BASE SHEAR }\end{array}$ & - & & $4^{4}$ \\
\hline
\end{tabular}




\section{ICF KAISER}

ENGINEERS Calc. S-0001, REW.O

LOS MLAMOS NMSF BUILDC EVALUTION

AS- BULT STRUCTURE

WALL IN-PLANE SHEAR WALL: 10 line $c$ -

\begin{tabular}{|c|c|c|c|}
\hline $\begin{array}{l}\text { SHEL } \\
\text { ELEMENT }\end{array}$ & SOINT & $\begin{array}{l}L C 1 \\
F_{x}\end{array}$ & $\begin{array}{c}L^{2} \\
F_{x}\end{array}$ \\
\hline \multirow{2}{*}{2301} & 1074 & 42 & -87 \\
\hline & 1075 & 38 & -102 \\
\hline & & & \\
\hline & & & \\
\hline & & & \\
\hline & & & \\
\hline & & & \\
\hline & & & \\
\hline & & & \\
\hline & & & \\
\hline & & & \\
\hline & & & \\
\hline & & & \\
\hline & & & \\
\hline & & & \\
\hline & & & \\
\hline & & & \\
\hline WALL & & & \\
\hline BASE SHE & & & 149 \\
\hline
\end{tabular}

soe No. $+15=$

SHEET 195 OF -74

DESIGNED BY BM

CHECKED BY T

REF. SAPGO RUN: 
ICF KAISER

ENGINEERS Cal. S-0001. Gen O

soe No. $+i==$

SMEet 196 of $=7+$ \begin{tabular}{c} 
LOS ALAMOS NMSF BUILDC EVALWATION \\
\hline SS-SUILT STRULTEE \\
\hline
\end{tabular}

DESIGNED BY BM

CHECKED BY IC

REF. SAPQO RNN:

WALL IN-PLANE SHEAR DATEZ/1/95 WALL: $I 1$ line $F$

\begin{tabular}{|c|c|c|c|}
\hline $\begin{array}{l}\text { SHEU } \\
\text { ELEMENT }\end{array}$ & JOINT & $\begin{array}{l}\text { LCI } \\
F_{x}\end{array}$ & $\begin{array}{c}L C_{2} \\
F_{x}\end{array}$ \\
\hline \multirow{2}{*}{2501} & 1013 & 67 & -130 \\
\hline & 1014 & 60 & -134 \\
\hline & & & \\
\hline & & & \\
\hline & & & \\
\hline & & & \\
\hline & & & \\
\hline & & & \\
\hline & & & \\
\hline & & & \\
\hline & & & \\
\hline & & & \\
\hline & & & \\
\hline & & & \\
\hline & & & \\
\hline & & & \\
\hline - & & & \\
\hline & & & \\
\hline & & & \\
\hline & & & \\
\hline $\begin{array}{l}\text { WALL } \\
\text { BASE SHEAR }\end{array}$ & 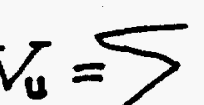 & & -264 \\
\hline
\end{tabular}


IEF KAISER

ENGINEERS ial C. S-0001, Rev.0

Joe No. $+1=5=$

SHEET 197 of $=74$

LOS ALAMOS NMSF BUILDC EVALUATION AS-SUILT STCKCTURE

DESIGNED BY $\notin . m$ DATE $2 / 1 / 95$ CHECKED BY $\bar{\jmath}$ DATE $2 / 1 / 4$

WALL IN-PLANE SHEAR

REF. SAPOO RUN: WALL: 19 line 1

\begin{tabular}{|c|c|c|c|}
\hline $\begin{array}{l}\text { SHELL } \\
\text { ELEMENT }\end{array}$ & SOINT & $\begin{array}{l}\text { LCI } \\
F_{Y}\end{array}$ & $\begin{array}{l}L E 2 \\
E Y\end{array}$ \\
\hline \multirow{2}{*}{3101} & 4001 & & -114 \\
\hline & 4011 & & -116 \\
\hline \multirow{2}{*}{2} & 11 & & -29 \\
\hline & 21 & & -38 \\
\hline \multirow{2}{*}{3} & 21 & & -73 \\
\hline & 31 & & -78 \\
\hline \multirow{2}{*}{4} & 31 & & -70 \\
\hline & 41 & & -77 \\
\hline \multirow{2}{*}{5} & 41 & & -70 \\
\hline & 51 & & -77 \\
\hline \multirow{2}{*}{6} & 51 & & -62 \\
\hline & 61 & & -72 \\
\hline \multirow{2}{*}{7} & 61 & & -63 \\
\hline & 71 & & -70 \\
\hline \multirow{2}{*}{8} & 71 & & -04 \\
\hline & 81 & & -73 \\
\hline \multirow{2}{*}{9} & 81 & & -39 \\
\hline & 91 &. & -51 \\
\hline \multirow{2}{*}{3110} & 91 & & -114 \\
\hline & 101 & & -122 \\
\hline WALL & - & & $:$ \\
\hline
\end{tabular}




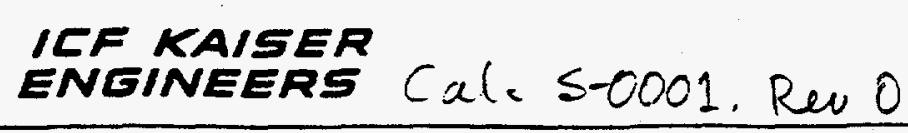

LOS ALAMOS NMSF BUILDC EVALUATION AS-SUILT STRUIT:IRE

WALL IN-PLANE SHEAR WALL: 34 Line $C$

\begin{tabular}{|c|c|c|c|}
\hline $\begin{array}{l}\text { SHELU } \\
\text { ELEMENT }\end{array}$ & SOINT & $\begin{array}{l}L^{\prime} \\
F_{x}\end{array}$ & $\begin{array}{l}L C^{2} \\
F_{x}\end{array}$ \\
\hline \multirow{2}{*}{4701} & 4002 & & -100 \\
\hline & 3 & & $-110^{\circ}$ \\
\hline \multirow{2}{*}{2} & 3 & & -92 \\
\hline & 4 & & -94 \\
\hline \multirow{2}{*}{3} & 4 & & -143 \\
\hline & 5 & & -153 \\
\hline & & & \\
\hline & & & \\
\hline & & & \\
\hline & & & \\
\hline & & & \\
\hline & & & \\
\hline & & & \\
\hline & & & \\
\hline & & & \\
\hline & & & \\
\hline & & & \\
\hline & & & \\
\hline WALL & 5 & & 1,02 \\
\hline BAGE SHEAR & & & 106 \\
\hline
\end{tabular}

REF. SAPQO RNN:
Jos No. $41===$

SMEET 198 of $=74$

DESIGNED BY $13 M$ DATE $\overline{2 / 1 / 95}$

CHECKED BY Ju

DATE $3 / 10 / 4$ 
IEF KAISER

ENGINEERS
Cale S-0001, Rev 0 NMSF BUILDC EVALUATION AS-SUILT STRUCTUEE

WALL IN-PLANE SHEAR WALL: 27 Line $B$

\begin{tabular}{|c|c|c|c|}
\hline $\begin{array}{l}\text { SHELL } \\
\text { ELEMENT }\end{array}$ & SOINT & $\begin{array}{l}\text { LCI } \\
F_{x}\end{array}$ & $\begin{array}{l}L C_{2} \\
F_{x}\end{array}$ \\
\hline \multirow{2}{*}{4201} & 4101 & & 54 \\
\hline & 2 & & 59 \\
\hline \multirow{2}{*}{2} & 2 & & 78 \\
\hline & 3 & & 85 \\
\hline \multirow{2}{*}{3} & 3 & & 115 \\
\hline & 4 & & 120 \\
\hline \multirow{2}{*}{4} & 4 & & 88 \\
\hline & 5 & & 96 \\
\hline & & & \\
\hline & & & \\
\hline & & & \\
\hline & & & \\
\hline & & & \\
\hline & & & \\
\hline & & & \\
\hline & & & \\
\hline & & & \\
\hline & & & \\
\hline & & & \\
\hline WALL & & & \\
\hline BASE SHEAR & & & \\
\hline
\end{tabular}

REF. SAPAO RNN:
Sos No. $\_==$

SHEET 199 of 274

DESIGNED BY $\mathrm{BM}$ DATE $2 / 1 / 95$

CHECKED BY JU DATE S/IJ 
IEF KAISER

ENGINEERS Cale.SOOO1, RQU.O

Joe No $-5=$

Sneet 200 or $=71$

LOS NLAMOS NMSF BUILDC EVALUATION AS-BULT STRVT $/ P E$

DESIGNED BY $\mathrm{BM}$

CHECKED BY IV

DATE/1/95

DATE $5 / 10 / 15$

WALL IN-PLANE SHEAR

REF. SAPGO RUN:

WALL: 21

\begin{tabular}{|c|c|c|c|}
\hline $\begin{array}{l}\text { SHEL } \\
\text { ELEMENT }\end{array}$ & SOINT & $\begin{array}{l}\text { LCI } \\
\text { FY }\end{array}$ & $\begin{array}{c}\sqrt[L C 2]{ } \\
F Y\end{array}$ \\
\hline \multirow{2}{*}{3301} & 4014 & $\neq 28$ & $78-25$ \\
\hline & 24 & +21 & $7-28$ \\
\hline \multirow{2}{*}{2} & 24 & $\$ 69$ & $-1-72$ \\
\hline & 34 & 62 & $-1-78$ \\
\hline \multirow{2}{*}{3} & 30 & 68 & $-8-23$ \\
\hline & 40 & 361 & $-4-79$ \\
\hline \multirow{2}{*}{4} & 414 & 36 & $-1-22$ \\
\hline & 54 & 62 & $-1-29$ \\
\hline \multirow[b]{2}{*}{5} & 50 & 69 & -22 \\
\hline & 44 & 261 & $-41-79$ \\
\hline \multirow{2}{*}{6} & 64 & 367 & $-6-71$ \\
\hline & 74 & 460 & $-5-80$ \\
\hline \multirow{2}{*}{7} & 74 & 370 & $-11-70$ \\
\hline & 84 & 65 & $-2-78$ \\
\hline \multirow[b]{2}{*}{8} & 80 & 50 & -38 \\
\hline & 74 & 737 & $7-52$ \\
\hline \multirow{2}{*}{9} & 94 & 2540 & $20-142$ \\
\hline & 104 & 31133 & $20-145$ \\
\hline & & & \\
\hline $\begin{array}{l}\text { WNLL } \\
\text { BASE SHEAR }\end{array}$ & & & \\
\hline
\end{tabular}

Use

Fy ralue. 
IEF KAISER

ENEINEERS Calc S-0001. Rev.0

SHEET 201 OF 274

LOS ALAMOS NMSF BUILDC EVALUATION $A S-E U I L T$ STRUCTURE

DESIGNED BY $B M$

DATE2/1/95

CHECKED BY ऽ DATE $5 / 10 \%$

WALL IN-PLANE SHEAR

REF. SAPAO RUN:

WALL: 21

\begin{tabular}{|c|c|c|c|}
\hline $\begin{array}{l}\text { SHELL } \\
\text { ELEMENT }\end{array}$ & SOINT & $\begin{array}{l}\text { LCI } \\
F_{Y}\end{array}$ & $\begin{array}{l}L_{2} \\
E_{Y}\end{array}$ \\
\hline \multirow{2}{*}{3301} & 4014 & 28 & $-2 \pi$ \\
\hline & 24 & 21 & -28 \\
\hline \multirow{2}{*}{2} & 24 & 69 & -72 \\
\hline & 34 & 62 & -78 \\
\hline \multirow{2}{*}{3} & 30 & 68 & -73 \\
\hline & 40 & 61 & -79 \\
\hline \multirow{2}{*}{4} & 44 & 68 & -72 \\
\hline & 54 & 62 & -79 \\
\hline \multirow{2}{*}{5} & 54 & 69 & -72 \\
\hline & 64 & 61 & -79 \\
\hline \multirow{2}{*}{6} & 64 & 67 & -71 \\
\hline & 74 & 60 & -80 \\
\hline \multirow{2}{*}{7} & 74 & 70 & -70 \\
\hline & 84 & 65 & -78 \\
\hline \multirow[b]{2}{*}{8} & 80 & 50 & -38 \\
\hline & 74 & 37 & -52 \\
\hline \multirow{2}{*}{9} & 94 & 40 & -142 \\
\hline & 104 & 33 & -145 \\
\hline & & & \\
\hline W & & & \\
\hline BASE SHE & & & \\
\hline
\end{tabular}


ICF KAISER

ENEINEERS Calc. S-0001, Rew. 0

Sos No.15

SHEET 2020F 274

LOS ALAMOS NMSF BUILDC EVALIATION AC-SUILT STRUCTURE

DESIGNED BY $\mathrm{BM}$

CHECKED BY Јレ

DATE $2 / 1 / 0 ;$ DATE $3 / 19$

WALL IN-PLANE SHEAR REF. SAPQO RNN:

WALL: 20 cine 2

\begin{tabular}{|c|c|c|c|}
\hline $\begin{array}{l}\text { SHELL } \\
\text { ELEMENT }\end{array}$ & JOINT & $\begin{array}{l}\text { LCl } \\
\text { FY }\end{array}$ & $\begin{array}{l}\text { LC2 } \\
F Y\end{array}$ \\
\hline \multirow{2}{*}{3201} & 4003 & 95 & -108 \\
\hline & 13 & 90 & -114 \\
\hline & & & \\
\hline & & & \\
\hline & & & \\
\hline & & & \\
\hline & & & \\
\hline & & & \\
\hline & & & \\
\hline & & & \\
\hline & & & \\
\hline & & & \\
\hline & & & \\
\hline & & & \\
\hline & & & \\
\hline & & & \\
\hline WRLL & & & $?$ \\
\hline BASE SHEAR & & & \\
\hline
\end{tabular}


IEF KAISER

ENGINEERS Calc. StO001, Reu 0

LOS HLAMOS NMSF BUILDC EVALUATION $\triangle S-B U L T$ STEUCTUR=

WALL IN-PLANE SHEAR
Joe No. A :S5=

SHEET 203 of 274 DESIGNED BY BM DATE $2 / 1 / 95$ CHECKED BY $Ј \checkmark$ DATE $\$ / 12 / 95$

REF. SAPAO RNN:

\begin{tabular}{|c|c|c|c|}
\hline $\begin{array}{l}\text { SHELL } \\
\text { ELEMENT }\end{array}$ & SOINT & $\begin{array}{l}\mathrm{LCI} \\
F_{x}\end{array}$ & $\begin{array}{l}L C^{2} \\
F_{x}\end{array}$ \\
\hline \multirow{2}{*}{4401} & 4061 & & -56 \\
\hline & 2 & & -66 \\
\hline \multirow{2}{*}{2} & 2 & & -41 \\
\hline & 3 & & -42 \\
\hline \multirow{2}{*}{3} & 3 & & -60 \\
\hline & 4 & & -60 \\
\hline & & & \\
\hline & & & \\
\hline & & & \\
\hline & & & \\
\hline & & & \\
\hline & & & \\
\hline & & & \\
\hline & & & \\
\hline & & & \\
\hline & & & \\
\hline & & & \\
\hline & & & \\
\hline BASE SHEAR & & & \\
\hline
\end{tabular}


WALL IN-PLANE SHEAR WALL: 28 line $B$

\begin{tabular}{|c|c|c|c|}
\hline $\begin{array}{l}\text { SHEL } \\
\text { ELEMENT }\end{array}$ & JOINT & $\begin{array}{l}\text { LCI } \\
F_{x}\end{array}$ & $\begin{array}{c}L C_{2} \\
F_{x}\end{array}$ \\
\hline \multirow{2}{*}{4221} & 4105 & & -100 \\
\hline & $\varphi$ & & -108 \\
\hline \multirow{2}{*}{2} & 4 & & -69 \\
\hline & 7 & & -75 \\
\hline \multirow{2}{*}{3} & 7 & & -104 \\
\hline & 8 & & -113 \\
\hline \multirow{2}{*}{4} & $e$ & & -50 \\
\hline & 9 & & -56 \\
\hline \multirow{2}{*}{5} & 9 & & -36 \\
\hline & 4110 & & -47 \\
\hline & & & \\
\hline & & & \\
\hline & & & \\
\hline & & & \\
\hline & & & \\
\hline & & & \\
\hline & & & \\
\hline & & & \\
\hline $\begin{array}{l}\text { WALL } \\
\text { BAGE SHEAR }\end{array}$ & & & 758 \\
\hline
\end{tabular}

REF. SAPQO RNN:

DESIGNED BY, $\mathrm{g} M$

CHECKED BY JU 
IEF KAISER

ENGINEERS Calc. Sto01, Rey 0 LOS ALAMOS NMSF BUILDC EVALUATION AS-6UILT STRICTURE

WALL IN-PLANE SHEAR WALL: 25 Line 7

\begin{tabular}{|c|c|c|c|}
\hline $\begin{array}{l}\text { SHELL } \\
\text { ELEMENT }\end{array}$ & SOINT & $\begin{array}{l}\text { LCI } \\
F_{Y}\end{array}$ & $\begin{array}{c}L C_{2} \\
F_{Y}\end{array}$ \\
\hline \multirow{2}{*}{3701} & 4008 & & 85 \\
\hline & 18 & & 87 \\
\hline \multirow[b]{2}{*}{2} & 18 & & 23 \\
\hline & 28 & & 26 \\
\hline \multirow{2}{*}{3} & 28 & & 58 \\
\hline & 30 & & 62 \\
\hline \multirow{2}{*}{4} & 38 & & 57 \\
\hline & 48 & & 60 \\
\hline \multirow{2}{*}{5} & 48 & & 56 \\
\hline & 58 & & 61 \\
\hline \multirow{2}{*}{6} & 58 & & 57 \\
\hline & 68 & & 61 \\
\hline \multirow{2}{*}{7} & 68 & & 56 \\
\hline & 98 & & 61 \\
\hline \multirow{2}{*}{8} & 78 & & 57 \\
\hline & 88 & & 62 \\
\hline \multirow{2}{*}{9} & 88 & & 31 \\
\hline & 98 & & 36 \\
\hline & & & \\
\hline WALL. & & & \\
\hline BASE SHEAR & & & \\
\hline
\end{tabular}

Jo: No. 4!55

SHEET 205 OF 271

DESIGNED BY BM CHECKED BY IV

REF. SAPGO RUN: 
ICF KAISER

ENGINEERS Calc. S-DOO 1, Rew. 0 LOS ALAMOS NMSF BUILDC EVALUATION $\triangle S-B U I L T$ STRICTURE

WALL IN-PLANE SHEAR WALL: 35

\begin{tabular}{|c|c|c|c|}
\hline $\begin{array}{l}\text { SHELL } \\
\text { ELEMENT }\end{array}$ & SOINT & $\begin{array}{c}\text { LCI } \\
F_{x}\end{array}$ & $\begin{array}{l}\text { LC2 } \\
F_{x}\end{array}$ \\
\hline \multirow{2}{*}{4711} & 4004 & & 89 \\
\hline & 5 & & 93 \\
\hline \multirow[b]{2}{*}{2} & 5 & & 99 \\
\hline & 6 & & 103 \\
\hline \multirow{2}{*}{3} & 6 & & 98 \\
\hline & 7 & & 102 \\
\hline \multirow{2}{*}{4} & 7 & & 73 \\
\hline & 8 & & 79 \\
\hline & & & \\
\hline & & & \\
\hline & & . & \\
\hline & & & \\
\hline & & & \\
\hline & & & \\
\hline & & & \\
\hline & & & \\
\hline & & & \\
\hline $\begin{array}{l}\text { WALL } \\
\text { BASE SHEAR }\end{array}$ & 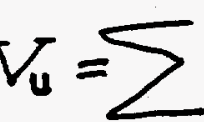 & & 736 \\
\hline
\end{tabular}

CHECKED BY

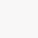

$$
\text { LINEG }
$$

Joe No. $1=5=$

SMEET 206 of $=7 i$

DESIGNEO BY BMI DATE $2 / 1 /$ IC

REF. SAPGO RUN: 
ICF KAISER

ENGINEERS Calc S-0001. Rew.O LOS ALAMOS NMSF BUILDC EVALUATION
AS-SUILT STCMSTIRE

WALL IN-PLANE SHEAR

Line $A$

\begin{tabular}{|c|c|c|c|}
\hline $\begin{array}{l}\text { SHEL } \\
\text { ELEMENT }\end{array}$ & SOINT & $\begin{array}{l}L_{C 1} \\
F_{x}\end{array}$ & $\begin{array}{l}L C^{2} \\
F_{x}\end{array}$ \\
\hline \multirow{2}{*}{4101} & 4125 & & 90 \\
\hline & $\varphi$ & & 96 \\
\hline \multirow{2}{*}{2} & 6 & & 69 \\
\hline & 7 & & 78 \\
\hline \multirow{2}{*}{3} & 7 & & 106 \\
\hline & 8 & & 113 \\
\hline \multirow{2}{*}{4} & 8 & & 50 \\
\hline & 9 & & 57 \\
\hline \multirow{4}{*}{5} & 9 & & 38 \\
\hline & 4130 & & 48 \\
\hline & & & \\
\hline & & . & \\
\hline & & & \\
\hline & & & \\
\hline & & & \\
\hline & & & \\
\hline & & & \\
\hline & & & \\
\hline & & & \\
\hline $\begin{array}{l}\text { WALL } \\
\text { BASE SHEAR }\end{array}$ & & & 745 \\
\hline
\end{tabular}
WALL: 38 soo No. $1 !=5=$

SHEET 207 or $=74$

DESIGNED BY $\beta M$

CHECKED BY JU

REF. SAPGO RNN: 
ICF NAISER

ENEINEERS
Jos No. $\perp 1 \leq==$

SHEET 208 OF $=7 f$
LOS ALAMOS NMSF BUILDC EVALUATION AS- SUILT CTRUTURE

WALL IN-PLANE SHEAR

$\operatorname{lin} F$

\begin{tabular}{|c|c|c|c|}
\hline $\begin{array}{l}\text { SHEL } \\
\text { ELEMENT }\end{array}$ & JOINT & $\begin{array}{l}L C I \\
E_{X}\end{array}$ & $\begin{array}{l}L C^{2} \\
F_{x}\end{array}$ \\
\hline \multirow{2}{*}{4751} & 4013 & & $12 \phi$ \\
\hline & 4014 & & 132 \\
\hline & & & \\
\hline & & & \\
\hline & & & \\
\hline & & & \\
\hline & & & \\
\hline & & & \\
\hline & & & \\
\hline & & & \\
\hline & & & \\
\hline & & . & \\
\hline & & & \\
\hline & & & \\
\hline & & & \\
\hline & & & \\
\hline & & & \\
\hline & & & \\
\hline & & & \\
\hline & & & \\
\hline WALL & $\leftarrow$ & & \\
\hline BASE SHEAR & & & \\
\hline
\end{tabular}

DESIGNED BY BM

CHECKED BY Iレ

REF. SAPAO RUN:
DATE $2 / 1 / 6$ DATE $5 / 10 / 95$ 
LOS ALAMOS NMSF BUILDING EVALUATION AS-SWLT STRUCTIRE

WALL IN-PLANE SHEAR

$D L=D E A D$ LOAD

LCI = LOAD CASE $1 \quad 1.4 \mathrm{DL}+1.7 \mathrm{L.L}$.

LL = LIVE LOAD

LC? = LUAD CASE 2

1.4 OL. +1.7 L.L $+1.7 \mathrm{H}$

$H=$ SOIL PRESS. AS-BUILT; WALL: 3 (LINEI - EASEMENT)

\begin{tabular}{|c|c|c|c|c|}
\hline $\begin{array}{l}\text { SHELL } \\
\text { ELEMENT }\end{array}$ & JOINT & $\begin{array}{l}\text { LCI } \\
F_{Y} \text { IN KIPS }\end{array}$ & $F_{Y}$ IN KIPS & REMARKS \\
\hline \multirow{2}{*}{1101} & 1001 & 18 & 26 & \\
\hline & 1011 & 3 & 16 & \\
\hline \multirow{2}{*}{1102} & 1011 & 10 & 7 & \\
\hline & 1021 & -9 & -15 & \\
\hline \multirow{2}{*}{1103} & 1021 & 11 & 0 & \\
\hline & 1031 & -9 & -21 & \\
\hline \multirow{2}{*}{1104} & 1031 & 13 & 3 & \\
\hline & 1041 & -10 & -20 & \\
\hline \multirow{2}{*}{1105} & 1041 & 14 & 4 & \\
\hline & 1051 & -10 & -21 & \\
\hline \multirow{2}{*}{1106} & 1051 & 15 & 5 & \\
\hline & 1061 & -11 & -22 & \\
\hline \multirow{2}{*}{1107} & 1061 & 16 & 5 & \\
\hline & 1071 & -13 & -24 & \\
\hline \multirow{2}{*}{1108} & 1071 & 16 & 5 & \\
\hline & 1081 & -14 & -25 & \\
\hline \multirow{2}{*}{1109} & 1081 & 16 & $B$ & \\
\hline & 1091 & -16 & -23 & \\
\hline \multirow{2}{*}{1110} & 1091 & 4 & -37 & \\
\hline & 1101 & -29 & -71 & \\
\hline & & & & \\
\hline & & & & \\
\hline $\begin{array}{l}\text { WALL } \\
\text { BASE SHEAR }\end{array}$ & $\Sigma$ & 15 & -200 & \\
\hline
\end{tabular}

REF. COMPUTER RUN NMSF 42 . FEF 
IEF KAISER

ENEINEERS CalC. S-COOL, ReV. O LOS ALAMOS NMSF BUILDING EVALUATION AS-BUILT STRUITURE

Jon No. 4552

SMEet 2 loof 271

DESIGNED BY A. Salne DATE 2.10 .95 CHECKED BY JV DATES/12/95

WALL IN-PLANE SHEAR

$D L=$ DEAD LOAD

LCI = LOAD CASE 1

1.4 DL. + 1.7 L.L.

LCZ = LOAD CASE 2

1.4 DL. + 1.7 L.L $-1.7 H$

LL = LIVE LOAD

$H=S O I L$ PRESS.

AS-BUILT; WALL: 6 (LINE 3 - BASEMENT)

\begin{tabular}{|c|c|c|c|c|}
\hline $\begin{array}{c}\text { SHELL } \\
\text { ELEMENT }\end{array}$ & JOINT & $\begin{array}{l}\text { LCI } \\
\text { FY IN KIPS }\end{array}$ & $\begin{array}{l}\text { LC2 } \\
\text { FY IN KIPS }\end{array}$ & REMARKS \\
\hline \multirow{2}{*}{1301} & 1004 & 16 & 12 & \\
\hline & 1014 & 2 & -4 & \\
\hline \multirow{2}{*}{1302} & 1014 & 9 & $B$ & \\
\hline & 1024 & -8 & -11 & \\
\hline \multirow{2}{*}{1303} & 1024 & 10 & 7 & \\
\hline & 1034 & -8 & -12 & \\
\hline \multirow{2}{*}{1304} & 1034 & 12 & 8 & \\
\hline & 1044 & -8 & -12 & \\
\hline \multirow{2}{*}{1305} & 1044 & 13 & 9 & \\
\hline & 1054 & -9 & -13 & \\
\hline \multirow{2}{*}{1306} & 1054 & 14 & 10 & \\
\hline & 1064 & -10 & -14 & \\
\hline \multirow{2}{*}{1307} & 1064 & 14 & 8 & \\
\hline & 1074 & -12 & -17 & \\
\hline \multirow{2}{*}{1308} & 1074 & 15 & 7 & \\
\hline & 1084 & -12 & -17 & \\
\hline \multirow{2}{*}{1309} & 1084 & 15 & 7 & \\
\hline & 1094 & -14 & -21 & \\
\hline \multirow{2}{*}{1310} & 1094 & 4 & -66 & \\
\hline & 1104 & -26 & -99 & \\
\hline & & & & \\
\hline & & & & \\
\hline & & & & \\
\hline $\begin{array}{c}\text { WALL } \\
\text { BASE SHEAR }\end{array}$ & $\Sigma$ & 17 & -210 & \\
\hline
\end{tabular}

REF. COMPUTER RUN NMSF42. FEF 


\begin{tabular}{c|l} 
LOS ALAMOS NMSF BUILDING EVALUATION & DESIGNED BY A. Sahg \\
\hline AS-SWILT STRIICTURE & CHECKED BY JL 2.10 .95 \\
\hline
\end{tabular}
WALL IN-PLANE SHEAR
$D L=D E A D$ LOAD
LCI = LOAD CASE 1
1.4 DL. + 1.7 L.L.
LC2 - LOAD CASE 2
1.4 OL. + 1.7 L.L $+1.7 \mathrm{H}$
LL = LIVE LOAD
$H=$ SOIL PRESS.

AS-BUILT; WALL: 9 (LINE B - BASEMENT)

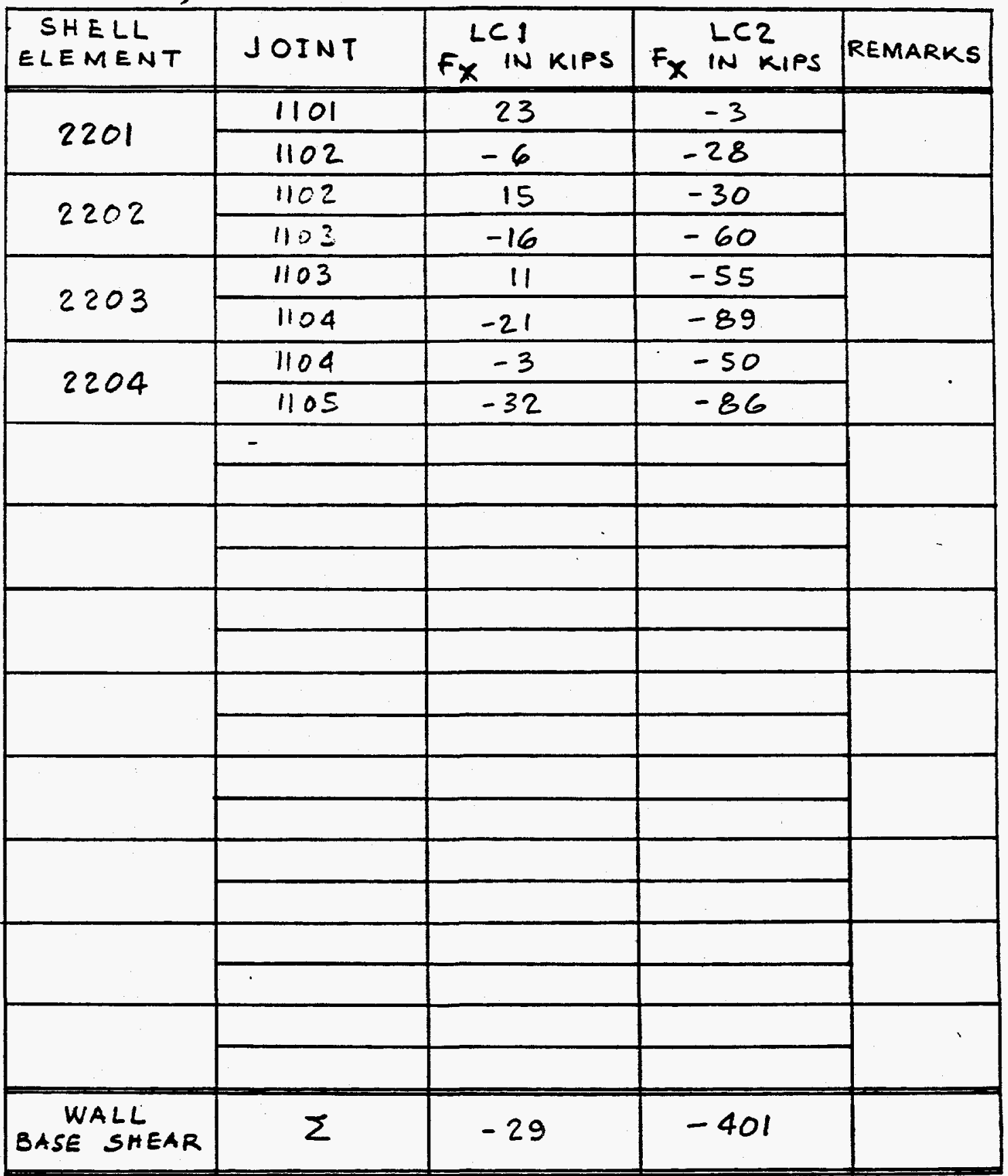

REF. COMPUTER RUN NMSF42. FEF 


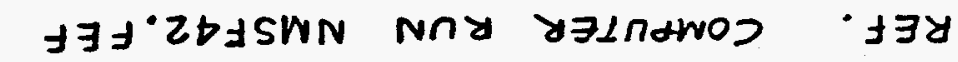

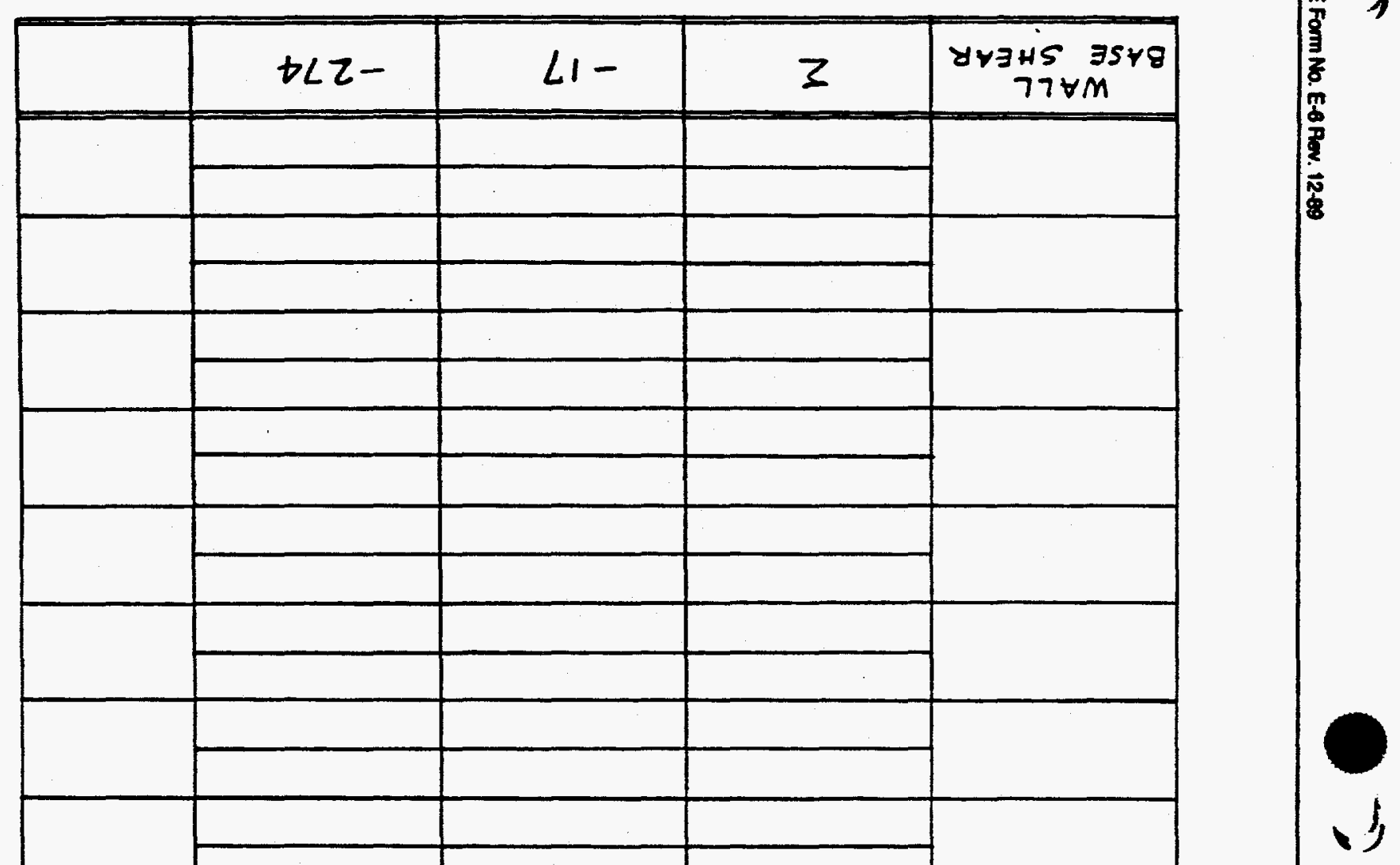

\begin{tabular}{|c|c|c|c|c|c|}
\hline \multirow{3}{*}{$\begin{array}{l}5661 / 53170 \\
56.01^{\prime} 23170\end{array}$} & \multirow{3}{*}{ 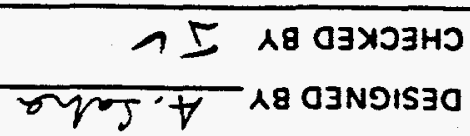 } & \multirow{2}{*}{\multicolumn{4}{|c|}{$20 n+3 n y+5$ 171ng-57 }} \\
\hline & & & & & \\
\hline & & 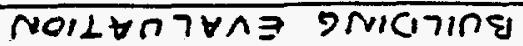 & ISWN & soW甘7Y & 507 \\
\hline
\end{tabular}


IEF KAISER

ENEINEERS Calc. S-OOC1. ReW. 0 LOS ALAMOS NMSF BUILDING EVALUATION $\triangle S-B U K T$ STRILTIEEE
Jo: No. $T_{r}=0$

SHEET 213 of $=74$

DESIGNED BY A.SAAL

DATE 2.10.95. CHECKED BY

WALL IN-PLANE SHEAR

$D L=D E A D$ LOAD

LCI = LOAD CASE $1 \quad 1.4 \mathrm{DL} .+1.7 \mathrm{~L} . \mathrm{L}$.

LCZ = LOAD CASE 2

1.4 DL. + 1.7 L.L. $+1.7 \mathrm{H}$

LL = LIVE LOAD

$H=S O I L$ PRESS.

AS-BUILT; WALL: 4 (LINE IS'EAST OF LINEI-BASEMENT)

\begin{tabular}{|c|c|c|c|c|}
\hline $\begin{aligned} \text { SHELL } \\
\text { ELEMENT }\end{aligned}$ & JOINT & $\begin{array}{l}\text { LCI } \\
F_{Y} \text { IN KIPS }\end{array}$ & $F_{Y}$ IN KIPS & REMARKS \\
\hline \multirow{2}{*}{1151} & 1002 & 11 & 15 & \\
\hline & 1012 & 1 & 6 & \\
\hline \multirow{2}{*}{1152} & 1012 & 7 & 5 & \\
\hline & 1022 & -6 & -9 & \\
\hline \multirow{2}{*}{1153} & 1022 & 7 & 1 & \\
\hline & 1032 & -6 & -12 & \\
\hline \multirow{2}{*}{1154} & 1032 & 8 & 3 & \\
\hline & 1042 & -6 & -12 & \\
\hline \multirow{2}{*}{1155} & 1042 & 9 & 3 & \\
\hline & 1052 & -7 & -13 & \\
\hline \multirow{2}{*}{1156} & 1052 & 10 & 4 & \\
\hline & 1062 & -8 & -13 & \\
\hline \multirow{2}{*}{1157} & 1062 & 10 & 3 & \\
\hline & 1072 & -9 & -16 & \\
\hline \multirow{2}{*}{1158} & 1072 & 7 & -19 & \\
\hline & 1082 & -11 & -32 & \\
\hline \multirow{2}{*}{1159} & 1082 & 3 & -13 & \\
\hline & 1092 & -14 & -25 & \\
\hline & & & & \\
\hline & & & & \\
\hline & & & & \\
\hline 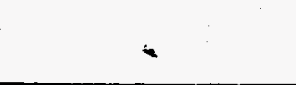 & & & & \\
\hline $\begin{array}{c}\text { WALL } \\
\text { BASE SHEAR }\end{array}$ & $\Sigma$ & 6 & -124 & \\
\hline
\end{tabular}

REF. COMPUTER RUN NMSF 42. FEF 

AS-EUILT STRIKTURE

WALL IN-PLANE SHEAR

$D L=D E A D$ LOAD

LCI = LOAD CASE $1 \quad 1.4 \mathrm{DL}+1.7$ L.L.

LL = LIVE LOAD

LCZ = LOAD CASE 2

1.4 DL. + 1.7 L.L + $1.7 H$

$H=S O I L$ PRESS.

AS-BUILT; WALL: 5 (LINE 2 - BASEMENT)

\begin{tabular}{|c|c|c|c|c|}
\hline $\begin{array}{c}\text { SHELL } \\
\text { ELEMENT }\end{array}$ & JOINT & $\begin{array}{c}\text { LCI } \\
F_{Y} \text { IN KIPS }\end{array}$ & Fy IN KIPS & REMARKS \\
\hline \multirow{2}{*}{$121 !$} & 1013 & 7 & 5 & \\
\hline & 1023 & -6 & -7 & \\
\hline \multirow{2}{*}{1212} & 1023 & 7 & 3 & \\
\hline & 1033 & -6 & -10 & \\
\hline \multirow{2}{*}{1213} & 1033 & 8 & 4 & \\
\hline & 1043 & -6 & -11 & \\
\hline \multirow{2}{*}{1214} & 1043 & 9 & 5 & \\
\hline & 1053 & -7 & -11 & \\
\hline \multirow{2}{*}{1215} & 1053 & 9 & 5 & \\
\hline & 1063 & -7 & -12 & \\
\hline \multirow{2}{*}{1216} & 1063 & 10 & 4 & \\
\hline & 1073 & -8 & -15 & \\
\hline \multirow{2}{*}{1217} & 1073 & 7 & -22 & \\
\hline & 1083 & -11 & -34 & \\
\hline \multirow{2}{*}{1218} & 1083 & 3 & -15 & \\
\hline & 1093 & -13 & -26 & \\
\hline & & & & \\
\hline & & & & \\
\hline & & & & \\
\hline & & & & \\
\hline & & & & \\
\hline & & & & \\
\hline $\begin{array}{l}\text { WALL } \\
\text { BASE SHEAR }\end{array}$ & $\Sigma$ & -4 & -137 & \\
\hline
\end{tabular}

REF. COMPUTER RUN NMSF 42. FEF 
IEF KAISER

ENGINEERS Calc. S-0001, Reu.0

\section{LOS ALAMOS NMSF BUILDING EVALUATION} AS 5 SUILT STRILTIIRE
Sos No. $\angle 1 \leq S 2$

SMEeT 215 of 274

DESIGNED BY A. Saha DATE $2 \cdot 10.95$ CHECKED BY T

$D L=D E A D$ LOAD

WALL IN-PLANE SHEAR

LCI = LOAD CASE $1 \quad 1.4 \mathrm{DL} .+1.7$ L.L.

LCZ = LOAD CASE 2

1.4 OL. + 1.7 L.L. $+1.7 H$

LL = LIVE LOAD

$H=S O I L$ PRESS. DATE : - : AS-BUILT; WALL:I (LINE 2 - BASEMENT)

\begin{tabular}{|l|l|l|l|l|}
\hline SHELL & JOINT & LCI & LCZ & REMARKS \\
\hline
\end{tabular}

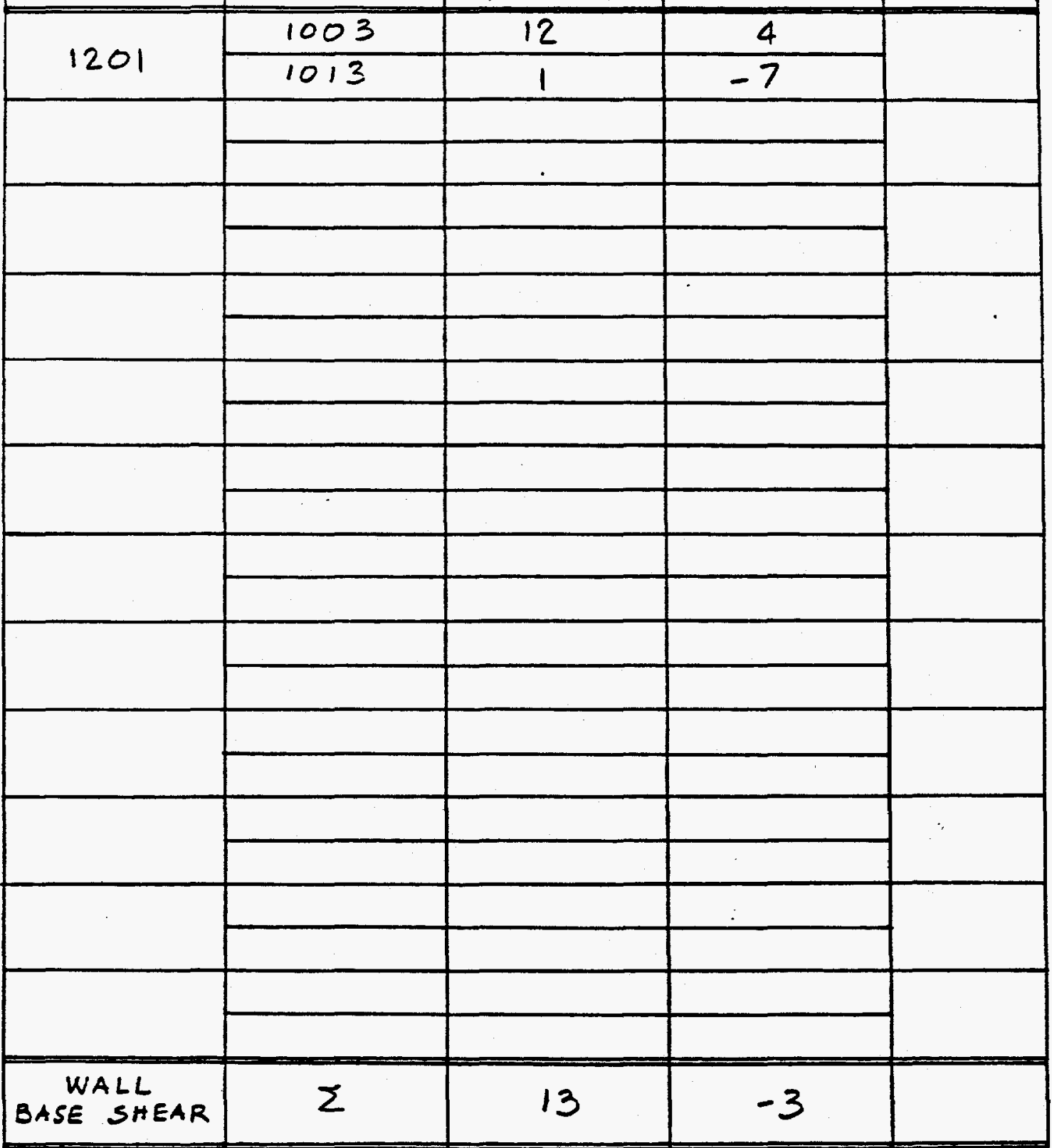

REF. COMPUTER RUN NMSF 42 . FEF 
IF KAISER

LOS ALAMOS NMSF BUILDING EVALUATION

AS-SU1LT STRUCTURE

DESIGNED BY A. Sal liz

DATE 2.10 .95 .

CHECKED BY

DATE: $\cdots$

WALL IN-PLANE SHEAR

$$
D L=D E A D L O A D
$$

LCM = LOAD CASE $1 \quad 1.4 \mathrm{DL}+1.7$ LL.

LL = LIVE LOAD

LC = LOAD CASE $2 \quad 1.4 \mathrm{OL}+1.7 L . L+1.7 \mathrm{H}$

$H=S O I L$ PRESS.

AS-BUILT; WALL :10 (LINE C C - BASEMENT)

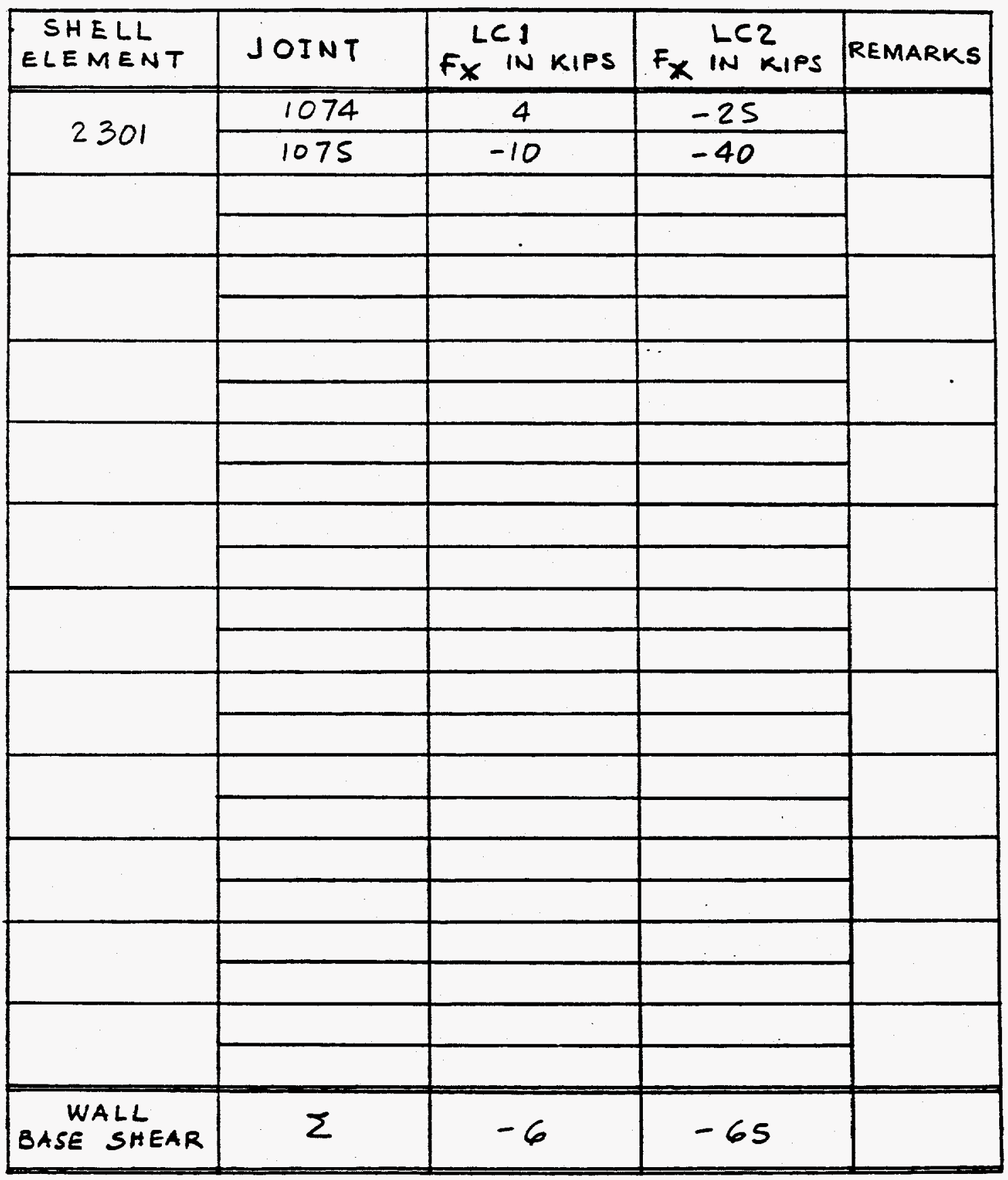

REF. COMPUTER RUN NMSF 42. FEF 


\section{LOS ALAMOS NMSF BUILDING EVALUATION} AS-SULT STEUCTUEE

DESIGNED BY A. Salhe

CHECKED BY ic

WALL IN-PLANE SHEAR

$D L=D E A D$ LOAD

LCI = LOAD CASE 1

1.4 DL. +1.7 L.L.

LCZ = LOAD CASE 2

1.4 OL. +1.7 L.L $+1.7 \mathrm{H}$

$L L=L I V E$ LOAD

$H=S O I L$ PRESS.

AS-BUILT; WALL:B ( $21^{\prime}-10^{\prime \prime}$ EAST OF LINE (3) - BASEMENT)

\begin{tabular}{|c|c|c|c|c|}
\hline $\begin{array}{l}\text { SHELL } \\
\text { ELEMENT }\end{array}$ & JOINT & $\begin{array}{l}\text { LCI } \\
F_{Y} \text { IN KIPS }\end{array}$ & $F_{Y}$ IN KIPS & REMARKS \\
\hline \multirow{2}{*}{1451} & 1075 & 16 & 14 & \\
\hline & 1085 & 1 & -2 & \\
\hline \multirow{2}{*}{1452} & 1085 & 11 & 10 & \\
\hline & 1095 & -9 & -10 & \\
\hline \multirow{2}{*}{1453} & 1095 & 1 & -12 & \\
\hline & 1105 & -17 & -26 & \\
\hline & & & - & \\
\hline & & & & \\
\hline & & & & \\
\hline & & & & \\
\hline & & & & \\
\hline & & & & \\
\hline & & & & \\
\hline & & & & \\
\hline & & & & \\
\hline & & & & \\
\hline & & & & \\
\hline & & & & \\
\hline & & & & \\
\hline \multirow[b]{2}{*}{$<$} & & & & \\
\hline & & & & \\
\hline $\begin{array}{c}\text { WALL } \\
\text { BASE SHEAR }\end{array}$ & $\Sigma$ & 3 & -26 & \\
\hline
\end{tabular}

REF. COMPUTER RUN NMSF 42. FEF 

AS-EU:Lt STRUCT

\section{DESIGNED BY A, Sahz} CHECKED BY Ј
DATE $2 \cdot 10.95$ DATE $\therefore$ - ja;

WALL IN-PLANE SHEAR

$D L=D E A D$ LOAD

LCI = LOAD CASE $1 \quad 1.4 \mathrm{DL}+1.7$ L.L.

LL = LIVE LOAD

LC2 = LUAD CASE 2

$1.4 D L .+1.7 L . L+1.7 H$

$H=S O I L$ PRESS.

AS-BUILT; WALL:19 (LINEI - ISI FLOOR)

\begin{tabular}{|c|c|c|c|c|}
\hline $\begin{array}{l}\text { SHELL } \\
\text { ELEMENT }\end{array}$ & JOINT & $\begin{array}{l}\text { LCI } \\
F_{Y} \text { IN KIPS }\end{array}$ & $F_{Y} L_{\text {IN }} \mathrm{KIPS}$ & REMARKS \\
\hline \multirow{2}{*}{3101} & 4001 & 9 & 23 & \\
\hline & 4011 & 1 & 15 & \\
\hline \multirow{2}{*}{3102} & 4011 & 5 & 3 & \\
\hline & 4021 & -.5 & -9 & \\
\hline \multirow[b]{2}{*}{3103} & 4021 & 5 & -1 & \\
\hline & 4031 & -5 & -10 & \\
\hline \multirow{2}{*}{3104} & 4031 & 5 & 1 & \\
\hline & 4041 & -5 & -9 & \\
\hline \multirow{2}{*}{3105} & 4041 & 5 & 1 & \\
\hline & 4051 & -5 & -8 & \\
\hline \multirow{2}{*}{3106} & 4051 & 6 & 11 & \\
\hline & 4061 & -5 & -2 & \\
\hline \multirow{2}{*}{3107} & 4061 & 6 & 10 & \\
\hline & 4071 & -6 & 0 & \\
\hline \multirow{2}{*}{3108} & 4071 & 6 & 4 & \\
\hline & 4081 & -5 & -8 & \\
\hline \multirow{2}{*}{3109} & 4081 & 7 & 7 & \\
\hline & 4091 & -6 & -8 & \\
\hline \multirow{2}{*}{3110} & 4091 & -1 & -29 & \\
\hline & 4101 & -13 & -39 & \\
\hline & & & & \\
\hline & & & & \\
\hline $\begin{array}{l}\text { WALL } \\
\text { BASE SHEAR }\end{array}$ & $\Sigma$ & -1 & -48 & \\
\hline
\end{tabular}

REF. COMPUTER RUN NMSF 42 . FEF 
WALL IN-PLANE SHEAR

LCI = LOAD CASE 1 1.4 DL. + 1.7 L.L.

LC2 = LOAD CASE 2

$1.4 D L .+1.7$ L.L + $1.7 H$
$D L=D E A D$ LOAD

$L L=L I V E$ LOAD

$H=S O L L$ PRESS.

AS-BUILT; WALL: 34 (LINE - IIT FLOOR)

\begin{tabular}{|c|c|c|c|c|}
\hline $\begin{array}{l}\text { SHELL } \\
\text { ELEMENT }\end{array}$ & JOINT & $\begin{array}{l}\text { LCI } \\
f_{K} \text { in KIPS }\end{array}$ & $F_{X}$ LC2 & REMARKS \\
\hline \multirow{2}{*}{4701} & 4002 & 7 & -34 & \\
\hline & 4003 & 0 & -47 & \\
\hline \multirow{2}{*}{4702} & 4003 & 4 & -19 & \\
\hline & 4004 & -4 & -25 & \\
\hline \multirow{2}{*}{4703} & 4004 & 3 & -29 & \\
\hline & 4005 & -5 & -39 & \\
\hline & & & & \\
\hline & & & & \\
\hline & & & & \\
\hline & & & & \\
\hline & & & & \\
\hline & & & & \\
\hline & & & & \\
\hline & & & & \\
\hline & & & & \\
\hline & & & & \\
\hline & & & & \\
\hline & & & & \\
\hline & & & & \\
\hline 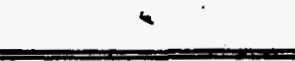 & & & & \\
\hline $\begin{array}{c}\text { WALL } \\
\text { BASE SHEAR }\end{array}$ & $\Sigma$ & 5 & -193 & \\
\hline
\end{tabular}

REF. COMPUTER RUN NMSF 42. FEF 

AS-SULLT STR:LT

SHEET 222 of $=-1$

DESIGNED BY A. Sahe DATE2.10.25. CHECKED BY Tூ

DATE

WALL IN-PLANE SHEAR

LCI = LOAD CASE $1 \quad 1.4$ DL. + 1.7 L.L.

LCZ = LUAD CASE 2

$1.4 D L .+1.7 L . L+1.7 H$

AS-BUILT; WALL: 21 (LINE - ISI FLOOR)

\begin{tabular}{|c|c|c|c|c|}
\hline $\begin{array}{l}\text { SHELL } \\
\text { ELEMENT }\end{array}$ & JOINT & $\begin{array}{l}\text { LCI } \\
\text { FY IN KIPS }\end{array}$ & $F_{Y}$ IN KIPS & REMARKS \\
\hline \multirow{2}{*}{3301} & 4014 & 5 & 2 & \\
\hline & 4024 & -1 & -4 & \\
\hline \multirow{2}{*}{3302} & 4024 & 6 & -1 & \\
\hline & 4034 & -2 & -9 & \\
\hline \multirow{2}{*}{3303} & 4034 & 5 & -2 & \\
\hline & 4044 & -4 & -10 & \\
\hline \multirow{2}{*}{3304} & 4044 & 5 & -1 & \\
\hline & 4054 & -4 & -10 & \\
\hline \multirow{2}{*}{3305} & 4054 & 5 & -1 & \\
\hline & 4064 & -5 & -10 & \\
\hline \multirow{2}{*}{3306} & 4064 & 5 & -1 & \\
\hline & 4074 & -5 & -12 & \\
\hline \multirow{2}{*}{3307} & 4074 & 6 & 1 & \\
\hline & 4084 & -5 & -8 & \\
\hline \multirow{2}{*}{3308} & 4084 & 6 & 8 & \\
\hline & 4094 & -5 & -9 & \\
\hline \multirow{2}{*}{3309} & 4094 & 0 & -61 & \\
\hline & 4104 & -11 & -69 & \\
\hline & & & & \\
\hline & & & & \\
\hline & & & & \\
\hline & & & & \\
\hline $\begin{array}{c}\text { WALL } \\
\text { BASE SHEAR }\end{array}$ & $\Sigma$ & 1 & 197 & \\
\hline
\end{tabular}

$D L=D E A D$ LOAD

LL = LIVE LOAD

$H=S O I L$ PRESS.

REF. COMPUTER RUN NMSF 42.FEF 
DESIGNED BY A. Salaz CHECKED BY S.

WALL IN-PLANE SHEAR

$D L=D E A D$ LOAD

$L C 1=\angle O A D$ CASE 1

$1.4 \mathrm{DL} .+1.7$ L.L.

LCR = LOAD CASE 2

1.4 DL. + 1.7 L.L. + $1.7 \mathrm{H}$

LL = LIVE LOAD

AS-BUILT; WALL 20 (LINE 2 - ISI FLOOR)

\begin{tabular}{|c|c|c|c|c|}
\hline $\begin{array}{c}\text { SHELL } \\
\text { ELEMENT }\end{array}$ & JOINT & $\begin{array}{l}\text { LCI } \\
\text { FY IN KIPS }\end{array}$ & $F_{Y}$ LN KIPS & REMARKS \\
\hline \multirow{2}{*}{3201} & 4003 & 4 & -6 & \\
\hline & 4013 & -4 & -14 & \\
\hline & & & & \\
\hline & & $\cdot$ & & \\
\hline & & & & \\
\hline & & & & \\
\hline & & & & \\
\hline & & & & \\
\hline & & & & \\
\hline & & & & \\
\hline & & & & \\
\hline & & & & \\
\hline & & & & \\
\hline & & & & \\
\hline & & & & \\
\hline & & & & \\
\hline & & & & \\
\hline & & & & \\
\hline & & & & \\
\hline $\begin{array}{c}\text { WALL } \\
\text { BASE SHEAR }\end{array}$ & $\Sigma$ & 0 & -20 & \\
\hline
\end{tabular}

REF. COMPUTER RUN NMSF 42. FEF 
ICF KAISER

ENGINEERS

Calc. S-0001, kev.0

SHEEt $2=4$ of 271

LOS ALAMOS NMSF BUILDING EVALUATION AS-BUILT STRUCT

WALL IN-PLANE SHEAR

$D L=D E A D$ LOAD

LCI = LOAD CASE 1

1.4 DL. + 1.7 L.L.

LL = LIVE LOAD

LC2 = LOAD CASE 2

1.4 DL. + 1.7 L.L $+1.7 \mathrm{H}$

$H=S O I L$ PRESS.

AS-BUILT; WALL 29 (LINEC - ISI FLOOR)

\begin{tabular}{|c|c|c|c|c|}
\hline $\begin{array}{l}\text { SHELL } \\
\text { ELEMENT }\end{array}$ & JOINT & $F_{x}^{L C I}$ in kips & $\begin{array}{l}\text { LC2 } \\
F_{X} \text { IN KIPS }\end{array}$ & REMARKS \\
\hline \multirow{2}{*}{4401} & 4061 & 4 & -32 & \\
\hline & 4062 & 0 & -42 & \\
\hline \multirow{2}{*}{4402} & 4062 & 3 & -7 & \\
\hline & 4063 & -2 & -9 & \\
\hline \multirow{2}{*}{4403} & 4063 & 0 & -10 & \\
\hline & 4064 & -4 & -15 & \\
\hline & & & & \\
\hline & - & & & \\
\hline & & & & \\
\hline & & & & \\
\hline & & & & \\
\hline & & 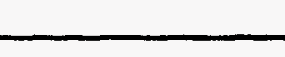 & & \\
\hline & & & & \\
\hline & & & & \\
\hline & & & & \\
\hline & & & & \\
\hline & & & & \\
\hline & & & & \\
\hline & & & & \\
\hline & & & & \\
\hline $\begin{array}{c}\text { WALL } \\
\text { BASE SHEAR }\end{array}$ & $\Sigma$ & 1 & -115 & \\
\hline
\end{tabular}

REF. COMPUTER RUN NMSF42. FEF 

ASBULLT STRUCT

WALL IN-PLANE SHEAR

DL = DEAD LOAD

LCI = LOAD CASE 1

$1.4 \mathrm{DL} .+1.7$ L.L.

LCZ = LOAD CASE 2

1.4 DL. + 1.7 L.L. $+1.7 \mathrm{H}$

LL = LIVE LOAD

AS-BUILT; WALL: 28 (LINEB-1 SI FLOOR)

\begin{tabular}{|c|c|c|c|c|}
\hline $\begin{array}{l}\text { SHELL } \\
\text { ELEMENT }\end{array}$ & JOINT & $\begin{array}{l}L C 1 \\
F_{Y} \text { IN KIPS }\end{array}$ & $F_{Y}$ IN KIPS & REMARKS \\
\hline \multirow{2}{*}{4221} & 4105 & 4 & -11 & \\
\hline & 4106 & -5 & -22 & \\
\hline \multirow{2}{*}{4222} & 4106 & 4 & -6 & \\
\hline & 4107 & -5 & -15 & \\
\hline \multirow{2}{*}{4223} & 4107 & 4 & -12 & \\
\hline & 4108 & -5 & -21 & \\
\hline \multirow{2}{*}{4224} & 4108 & 3 & -4 & \\
\hline & 4109 & -5 & -13 & \\
\hline \multirow{2}{*}{4225} & 4109 & 0 & -5 & \\
\hline & 4110 & -7 & -14 & \\
\hline & & & & \\
\hline & & & & \\
\hline & & & & \\
\hline & & & & \\
\hline & & & & \\
\hline & & & & \\
\hline & & & & \\
\hline & & & & \\
\hline $\begin{array}{l}\text { WALL } \\
\text { BASE SHEAR }\end{array}$ & $\Sigma$ & -12 & -123 & \\
\hline
\end{tabular}

REF. COMPUTER RUN NMSF 42. FEF 
ICF KAISER Cakc.

ENGINEERS S-0001, REU. O

LOS ALAMOS NMSF BUILDING EVALUATION AS-EUILT STRUCT

SMEET 226 of 271

DESIGNED BY A. Sache DATE 2.10 .25

CHECKED BY $T-C$

DATE $\div \quad \therefore$

WALL IN-PLANE SHEAR

$D L=D E A D$ LOAD

LCI = LOAD CASE $1 \quad 1.4 \mathrm{DL}+1.7$ L.L.

LCZ = LOAD CASE 2

1.4 DL. + 1.7 L.L. $+1.7 H$

LL = LIVE LOAD

$H=$ SOIL PRESS.

AS-BUILT; WALL: 25 (LINE 7 - IST FLOOR)

\begin{tabular}{|c|c|c|c|c|}
\hline $\begin{array}{c}\text { SHELL } \\
\text { ELEMENT }\end{array}$ & JOINT & $\begin{array}{l}\text { LCI } \\
\text { FY IN KIPS }^{\text {L }}\end{array}$ & $F_{Y} \stackrel{L C 2}{\text { IN KIPS }}$ & REMARKS \\
\hline \multirow{2}{*}{3701} & 4008 & 5 & -5 & \\
\hline & 4018 & 0 & -10 & \\
\hline \multirow{2}{*}{3702} & 4018 & 2 & 0 & \\
\hline & 4028 & -3 & -6 & \\
\hline \multirow{2}{*}{3703} & 4028 & 3 & -5 & \\
\hline & 4038 & -2 & -10 & \\
\hline \multirow{2}{*}{3704} & 4038 & 3 & -3 & \\
\hline & 4048 & -3 & -9 & \\
\hline \multirow{2}{*}{3705} & 4048 & 3 & -4 & \\
\hline & 4058 & -3 & -10 & \\
\hline \multirow[b]{2}{*}{3706} & 4058 & 3 & -4 & \\
\hline & 4068 & -3 & -10 & \\
\hline \multirow[b]{2}{*}{3707} & 4068 & 3 & -3 & \\
\hline & 4078 & -3 & -10 & \\
\hline \multirow{2}{*}{3708} & 4078 & 3 & -4 & \\
\hline & 4088 & -4 & -11 & \\
\hline \multirow{2}{*}{3709} & 4088 & 0 & -4 & \\
\hline & 4098 & -5 & -9 & \\
\hline & & & & \\
\hline & & & & \\
\hline & & & & \\
\hline 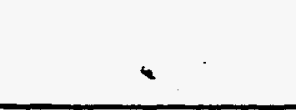 & & & & \\
\hline $\begin{array}{c}\text { WALL } \\
\text { BASE SHEAR }\end{array}$ & $\Sigma$ & -1 & -117 & \\
\hline
\end{tabular}

REF. COMPUTER RUN NMSF 42. FEF 

AS-BUILI STRKCT

SHEET 227 of =7-

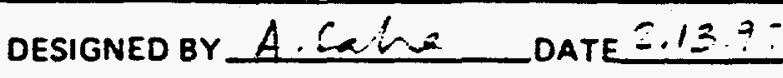

CHECKED BY TV

DATE : $=50$

WALL IN-PLANE SHEAR

LCI = LOAD CASE $1 \quad 1.4 \mathrm{DL}+1.7$ L.L.
$1.4 O L .+1.7$ L.L + $1.7 H$
$D L=D E A D$ LOAD

LL = LIVE LOAD

$H=$ SOIL PRESS.

AS-BUILT; WALL: $5 S$ (LINEG-IIT FLOOR)

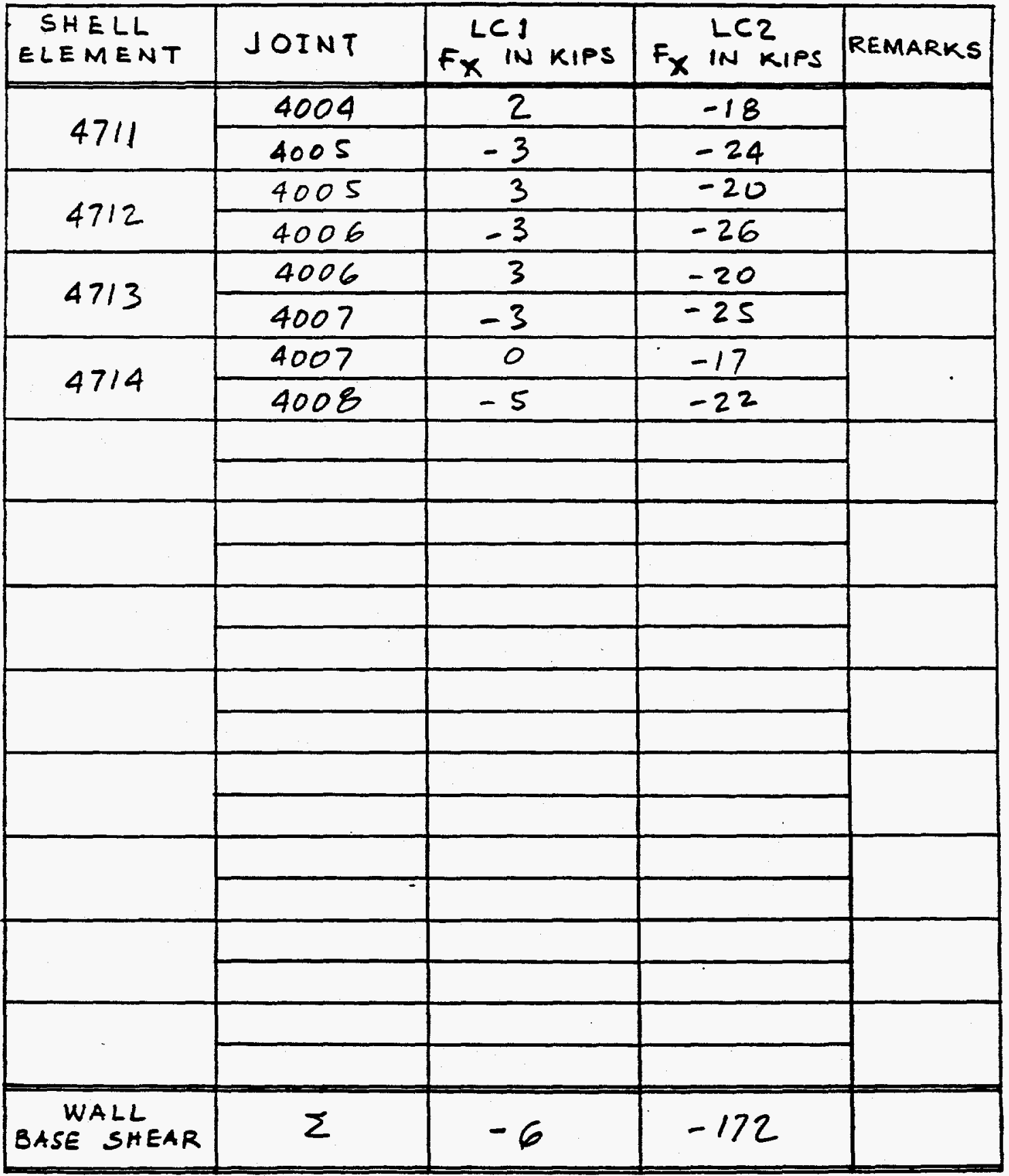

REF. COMPUTER RUN NMSF 42.FEF 

AS-EUILT STRIKT

DESIGNED BY A. fache CHECKED BY I DATE 2.13 .95

DATE:

WALL IN-PLANE SHEAR

$D L=D E A D$ LOAD

LCI = LOAD CASE 1

$1.4 \mathrm{DL} .+1.7$ L.L.

LCZ = LOAD CASE 2

1.4 DL. + 1.7 L.L + $1.7 \mathrm{H}$

LL = LIVE LOAD

$H=S O I L$ PRESS.

AS-BUILT; WALL: 38 (LINE A - IST FLOOR)

\begin{tabular}{|c|c|c|c|c|}
\hline $\begin{array}{l}\text { SHELL } \\
\text { ELEMENT }\end{array}$ & JOINT & $\begin{array}{l}\text { LCI } \\
f_{X} \text { IN KIPS }\end{array}$ & $F_{X}$ LC2 & REMARKS \\
\hline \multirow[b]{2}{*}{4101} & 4125 & 10 & -2 & \\
\hline & 4126 & 1 & -12 & \\
\hline \multirow{2}{*}{$410=$} & 4126 & 5 & -5 & \\
\hline & 4127 & -6 & -16 & \\
\hline \multirow[b]{2}{*}{4103} & 4127 & 4 & -10 & \\
\hline & 4128 & -5 & -19 & \\
\hline \multirow{2}{*}{4104} & 4128 & 4 & -3 & \\
\hline & 4129 & -6 & -13 & \\
\hline \multirow{2}{*}{4105} & 4129 & 0 & -5 & \\
\hline & 4130 & -8 & -13 & \\
\hline & & & & \\
\hline & & & & \\
\hline & & & & \\
\hline & & & & \\
\hline & & & & \\
\hline & & & & \\
\hline & & & & \\
\hline & & & & \\
\hline & & & & \\
\hline & & & & \\
\hline & & & & \\
\hline $\begin{array}{l}\text { WALL } \\
\text { BASE SHEAR }\end{array}$ & $\Sigma$ & -9 & -98 & \\
\hline
\end{tabular}

REF. COMPUTER RUN NMSF 4Z,FEF 
WALL IN-PLANE SHEAR CHECKED BY - DATE $\div / 26 \%$

LCI = LOAD CASE $1 \quad 1.4 \mathrm{DL} .+1.7 \mathrm{L.L}$.

LCZ = LOAD CASE 2

1.4 OL. + 1.7 L.L $-1.7 H$

$D L=$ DEAD LOAD

LL = LIVE LOAD

$H=$ SOIL PRESS.

AS-BUILT; WALL:32 (LINEF-I ST FLOOR)

\begin{tabular}{|c|c|c|c|c|}
\hline $\begin{array}{l}\text { SHELL } \\
\text { ELEMENT }\end{array}$ & JOINT & $F_{x}^{\text {LCI }}$ in Kips & $F_{X}$ IN KIIPS & REMARKS \\
\hline \multirow{2}{*}{4751} & 4013 & 3 & -23 & \\
\hline & 4014 & -4 & -32 & \\
\hline & & & & \\
\hline & & & & \\
\hline & & & & \\
\hline & & & & \\
\hline & & & & \\
\hline & & & & \\
\hline & & & & \\
\hline & & & & \\
\hline & & & & \\
\hline & & & & \\
\hline & & & & \\
\hline & & & & \\
\hline & & & & \\
\hline & & & & \\
\hline & & & & \\
\hline & & & & \\
\hline $\begin{array}{l}\text { WALL } \\
\text { BASE SHEAR }\end{array}$ & $\Sigma$ & -1 & -55 & \\
\hline
\end{tabular}

REF. COMPUTER RUN NMSF 42, FEF 


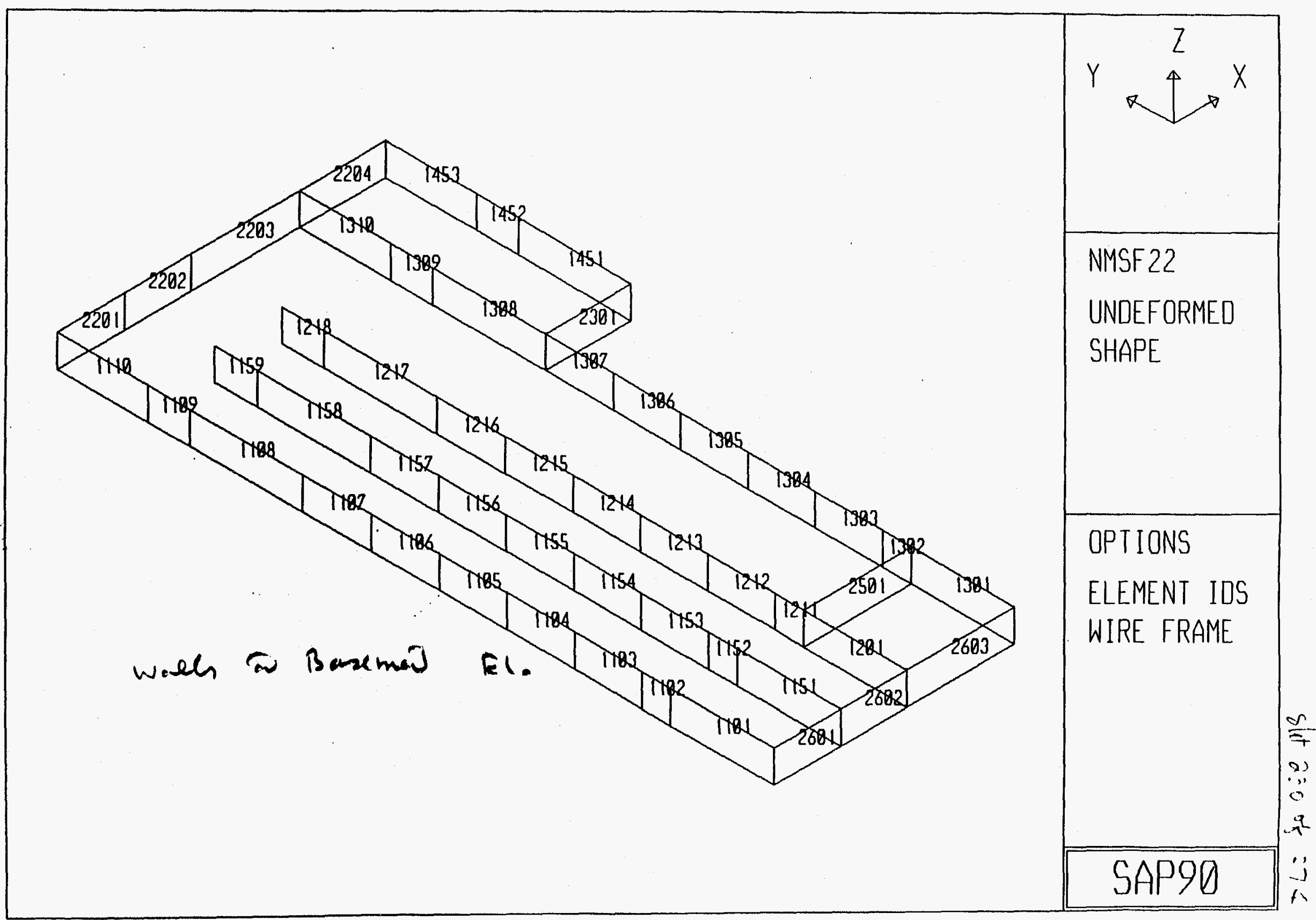




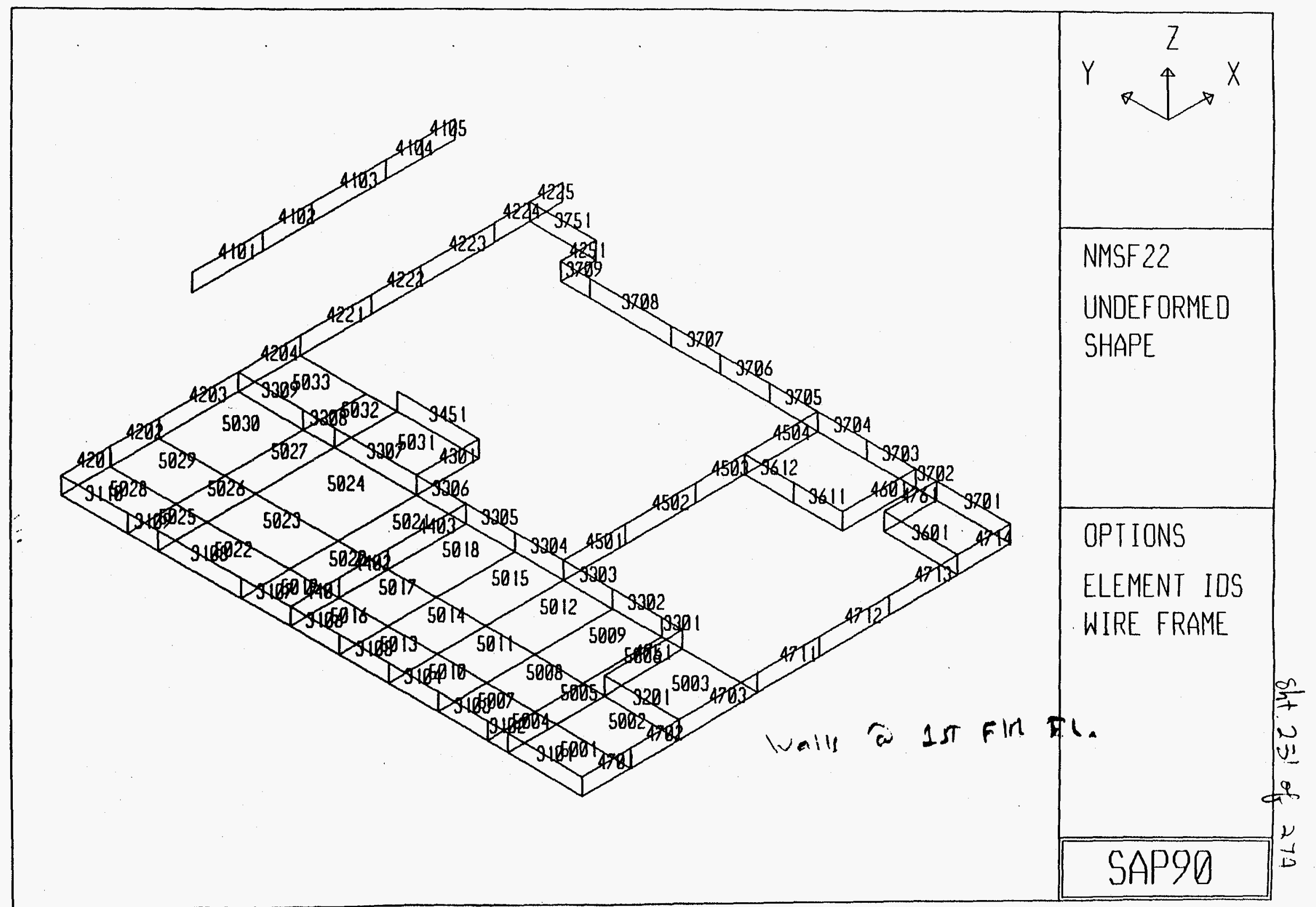




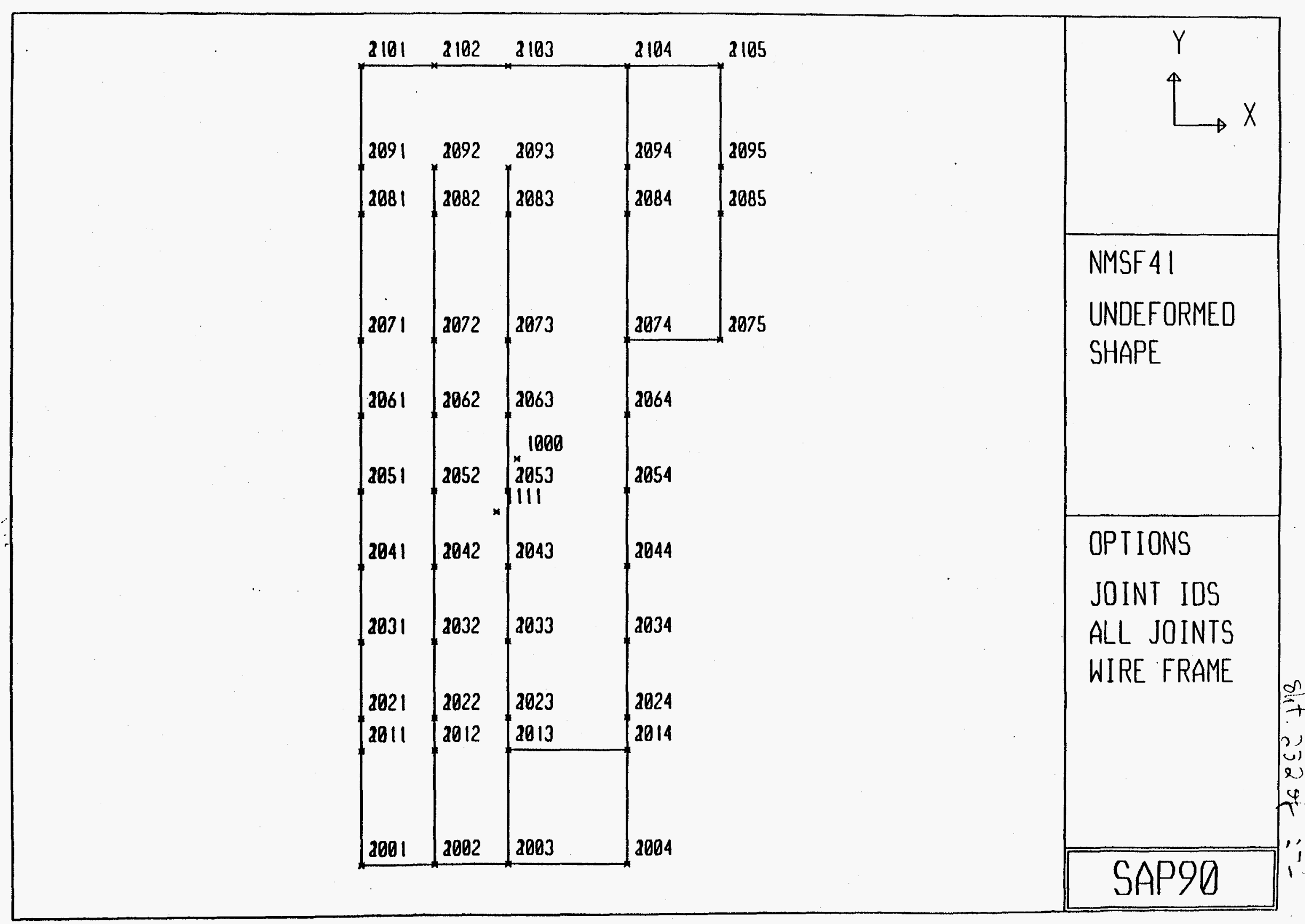


Sht. 233 of $=71$

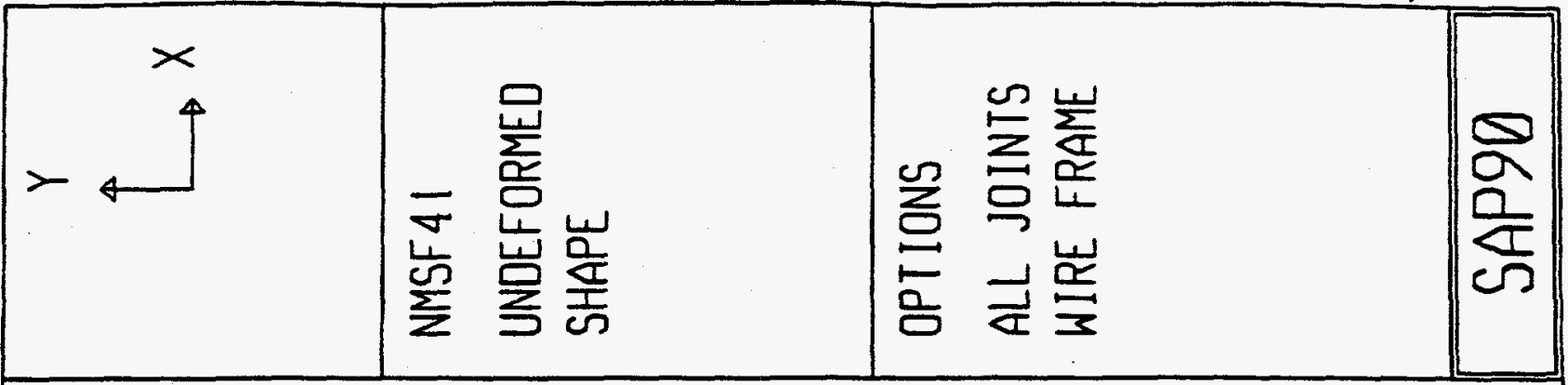

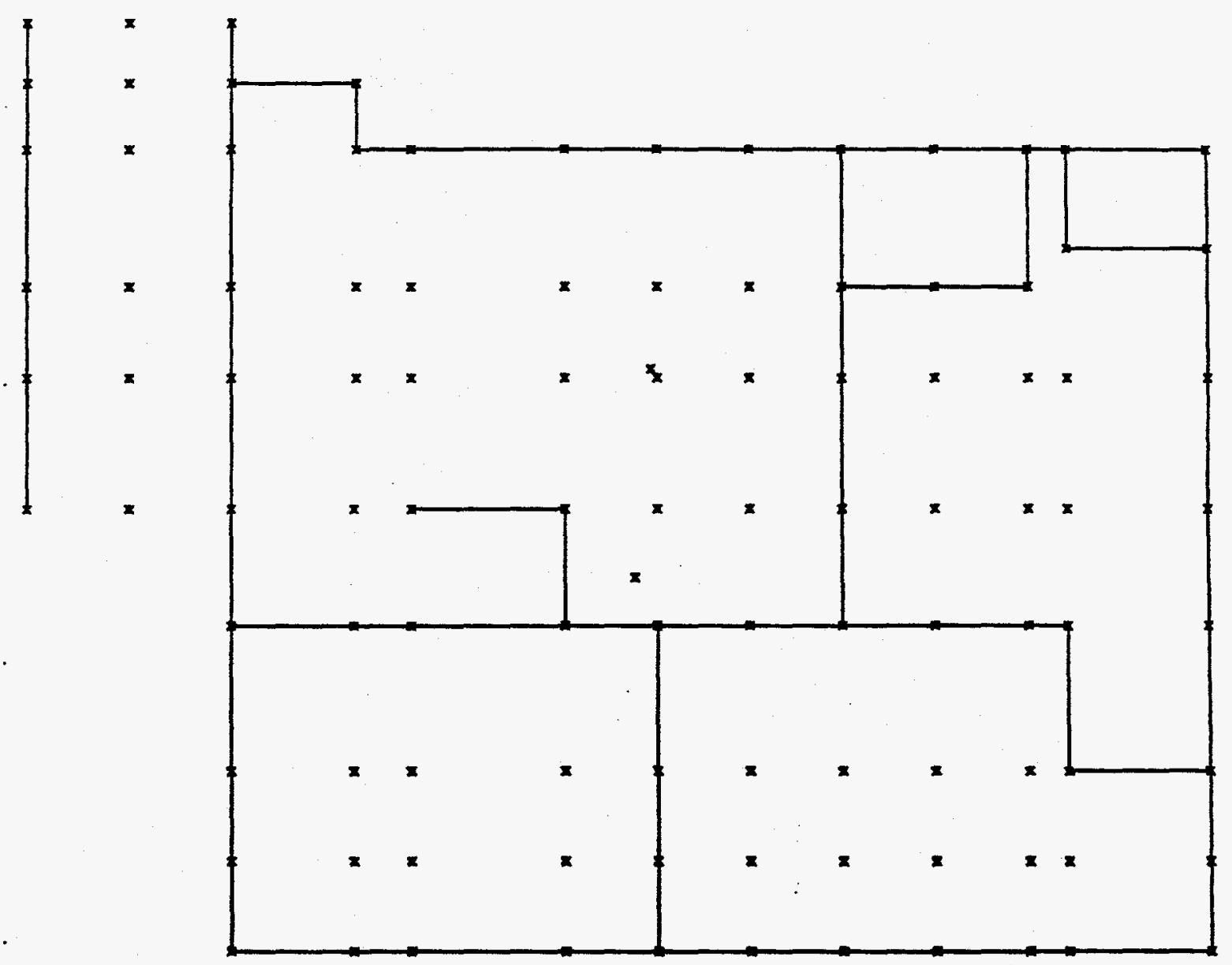



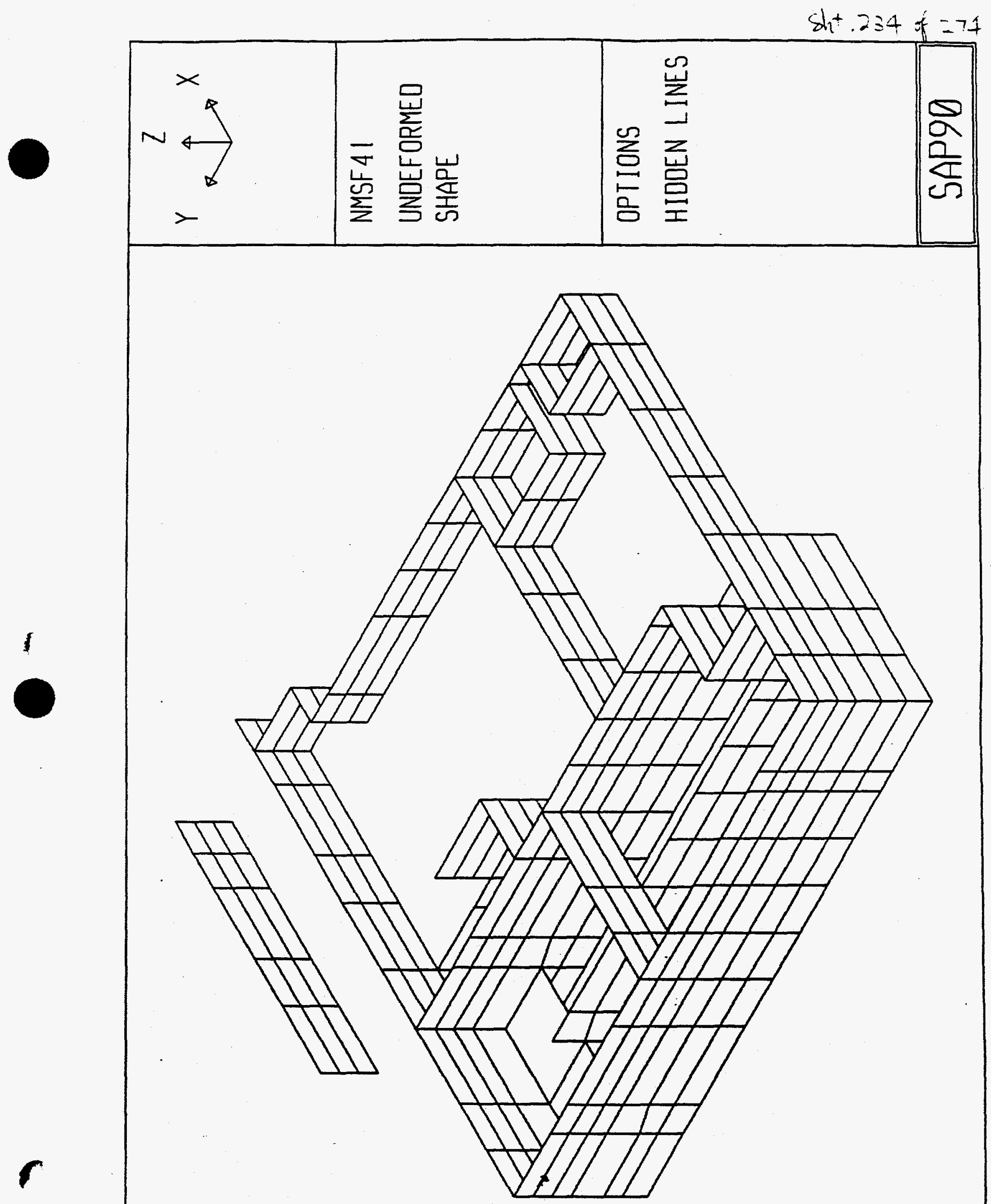
Stt. 235 of 274

\begin{tabular}{|c|c|c|c|c|c|}
\hline$N \frac{4}{\infty}$ & $\frac{}{\square}$ & 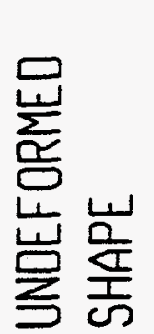 & 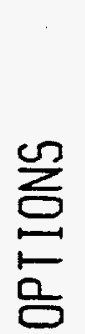 & 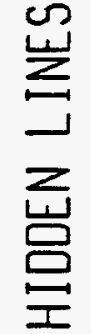 & $\frac{1}{\sigma 2}$ \\
\hline
\end{tabular}

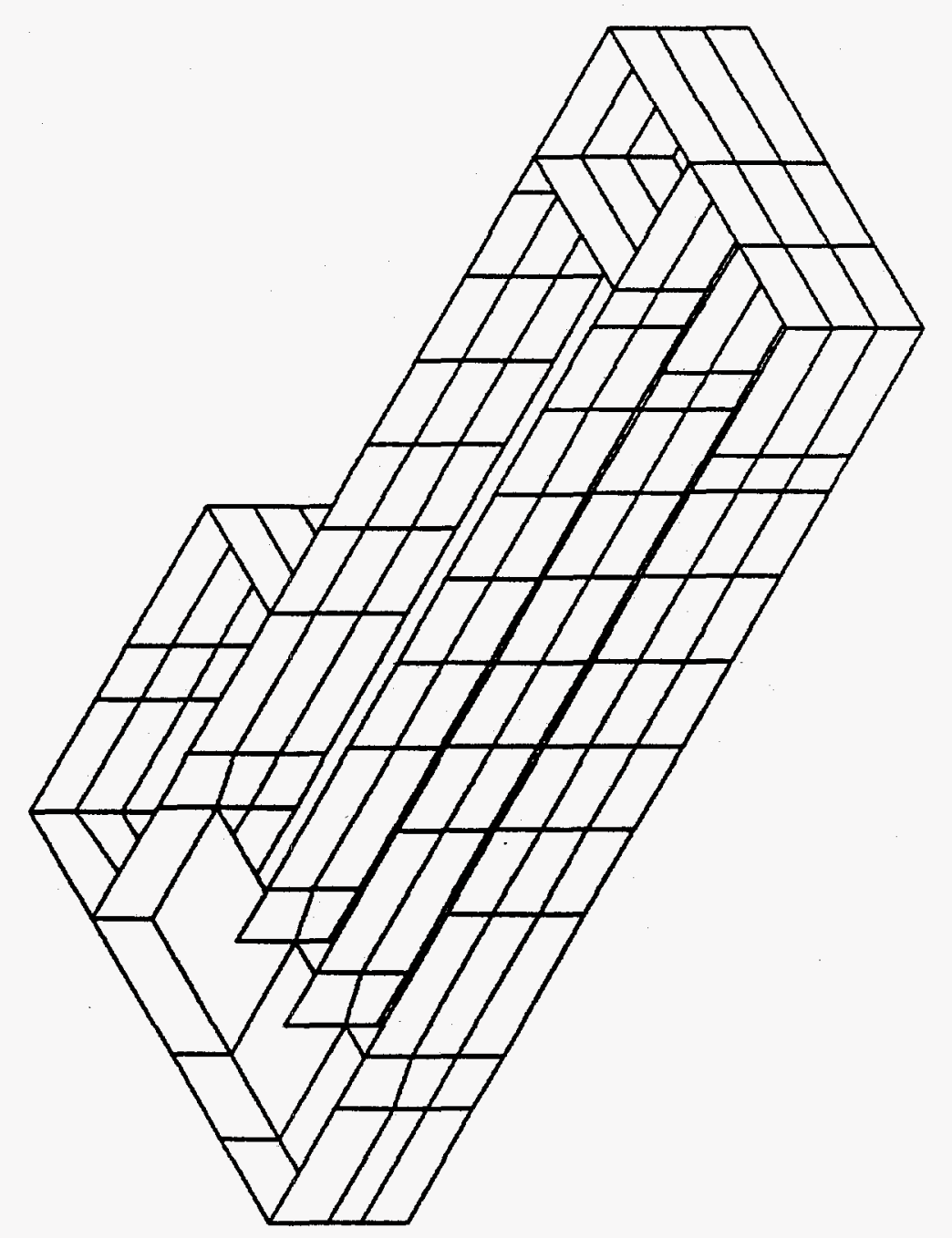


dit $230<=7 \div$

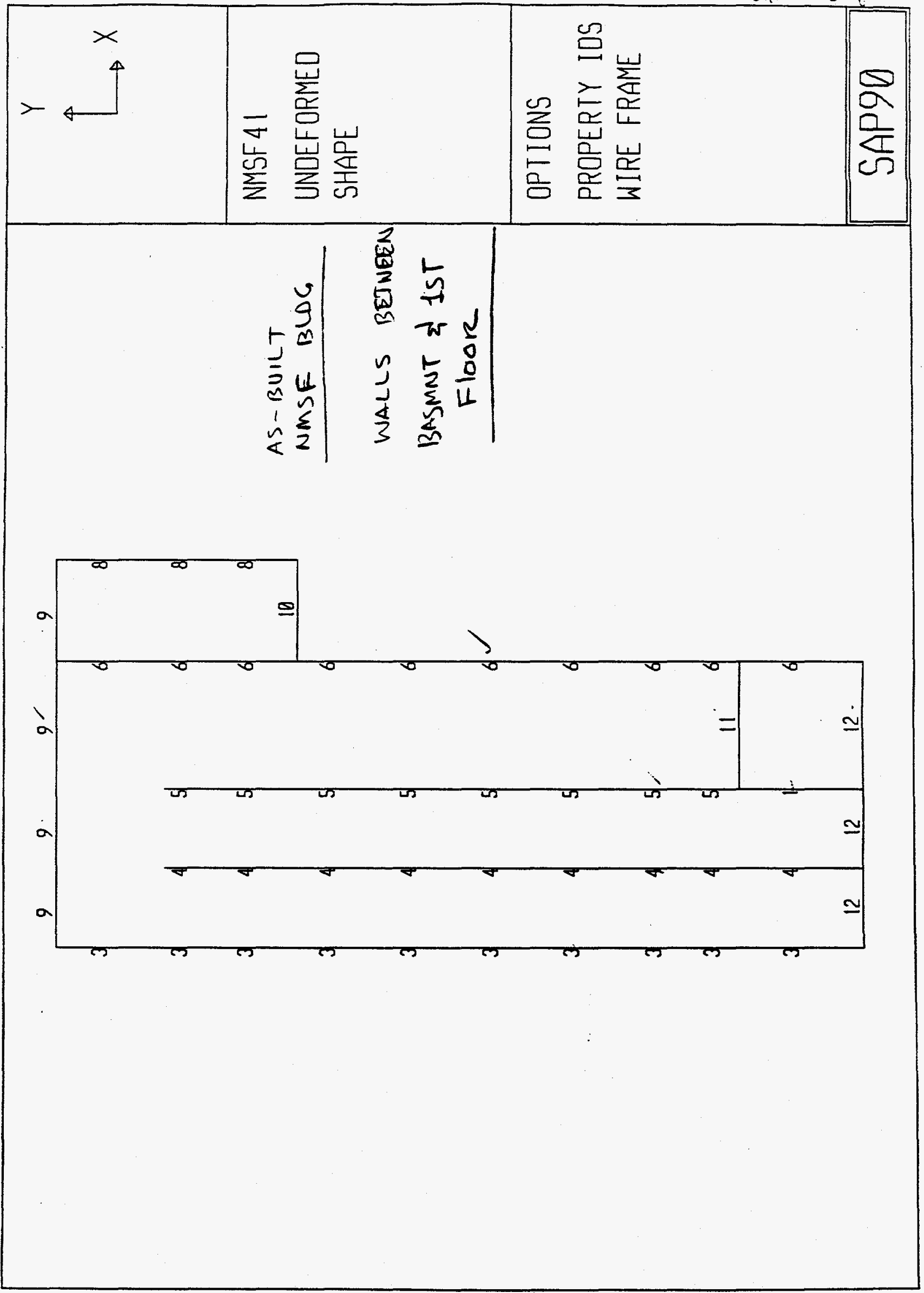


int 237 of $=74$

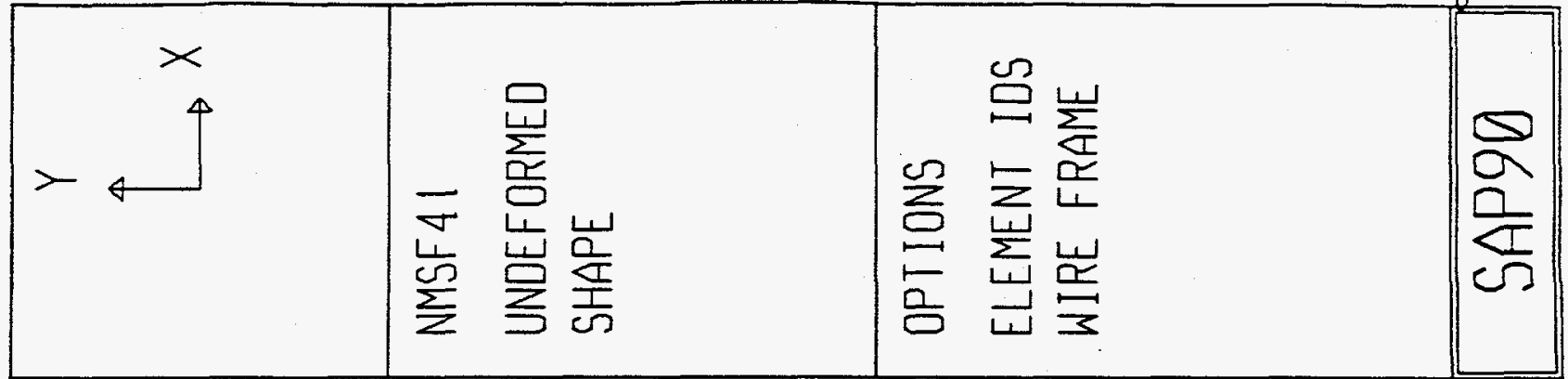

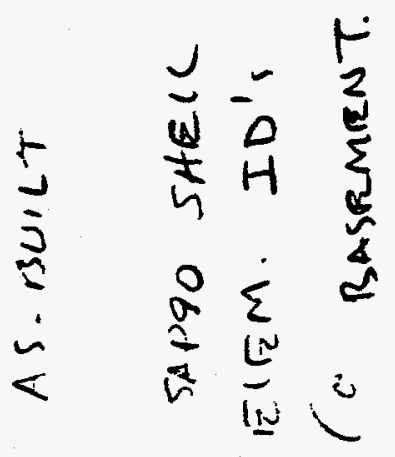

1

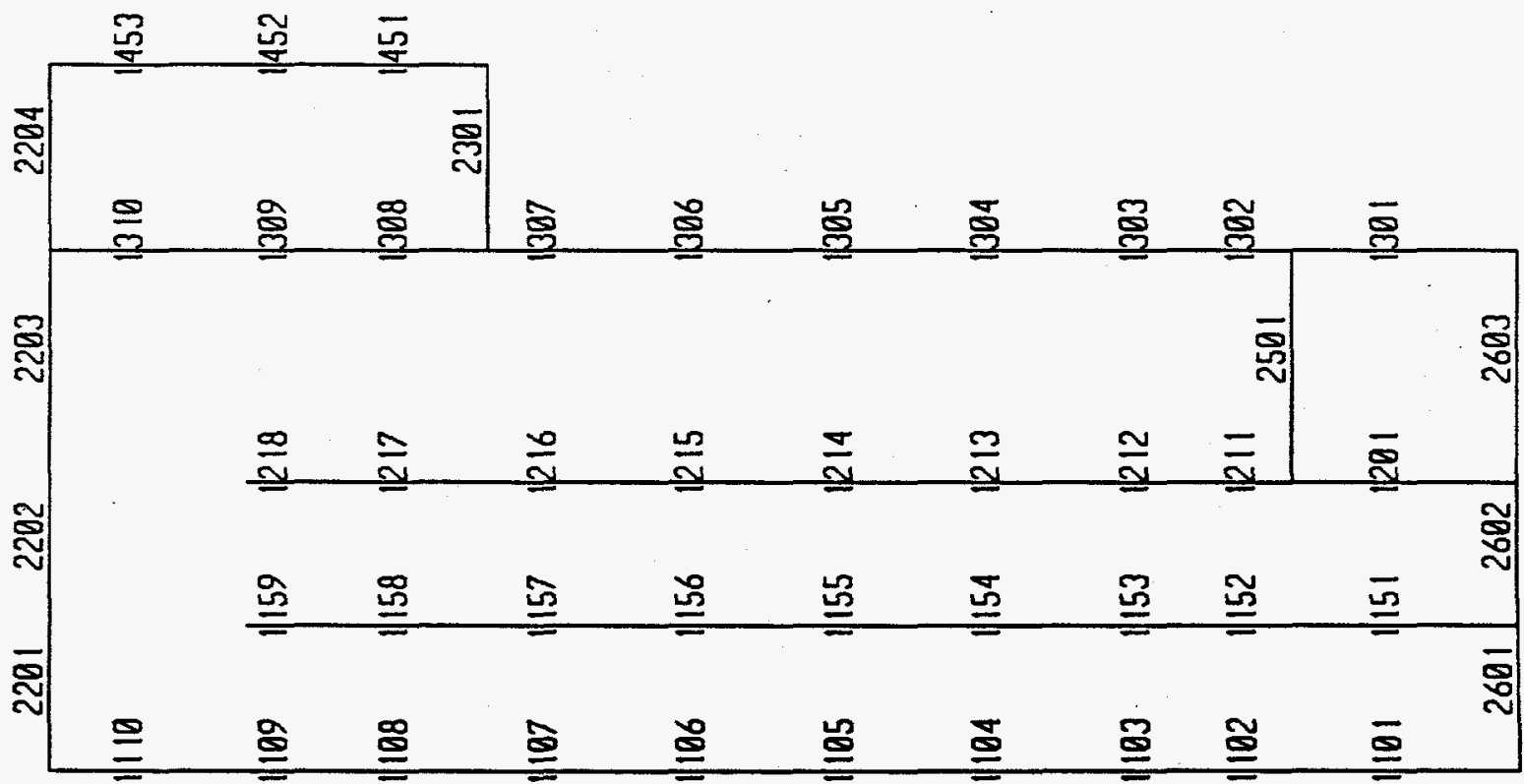


sit. $25 x=7=74$

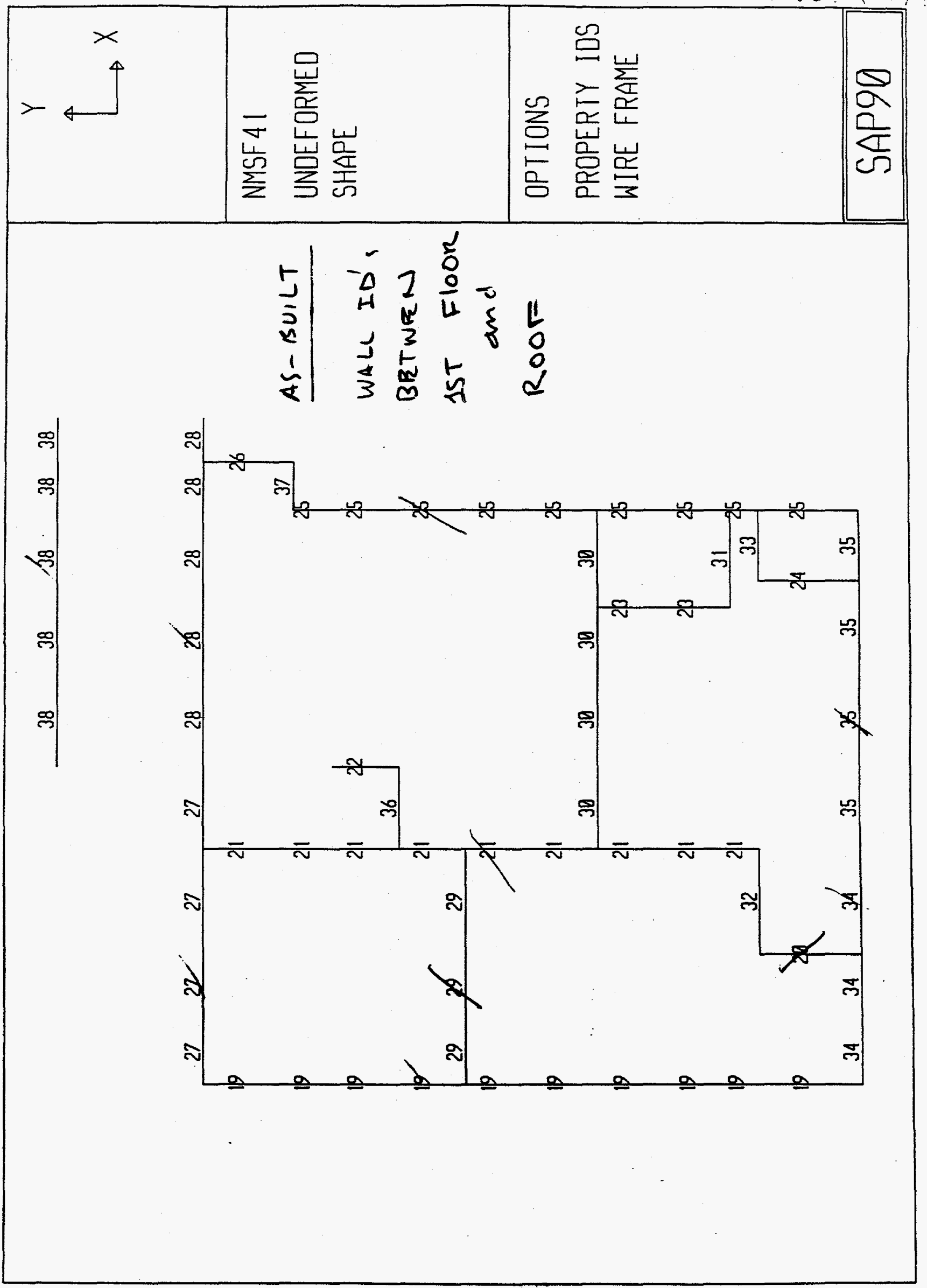


Sit. 254 st 278
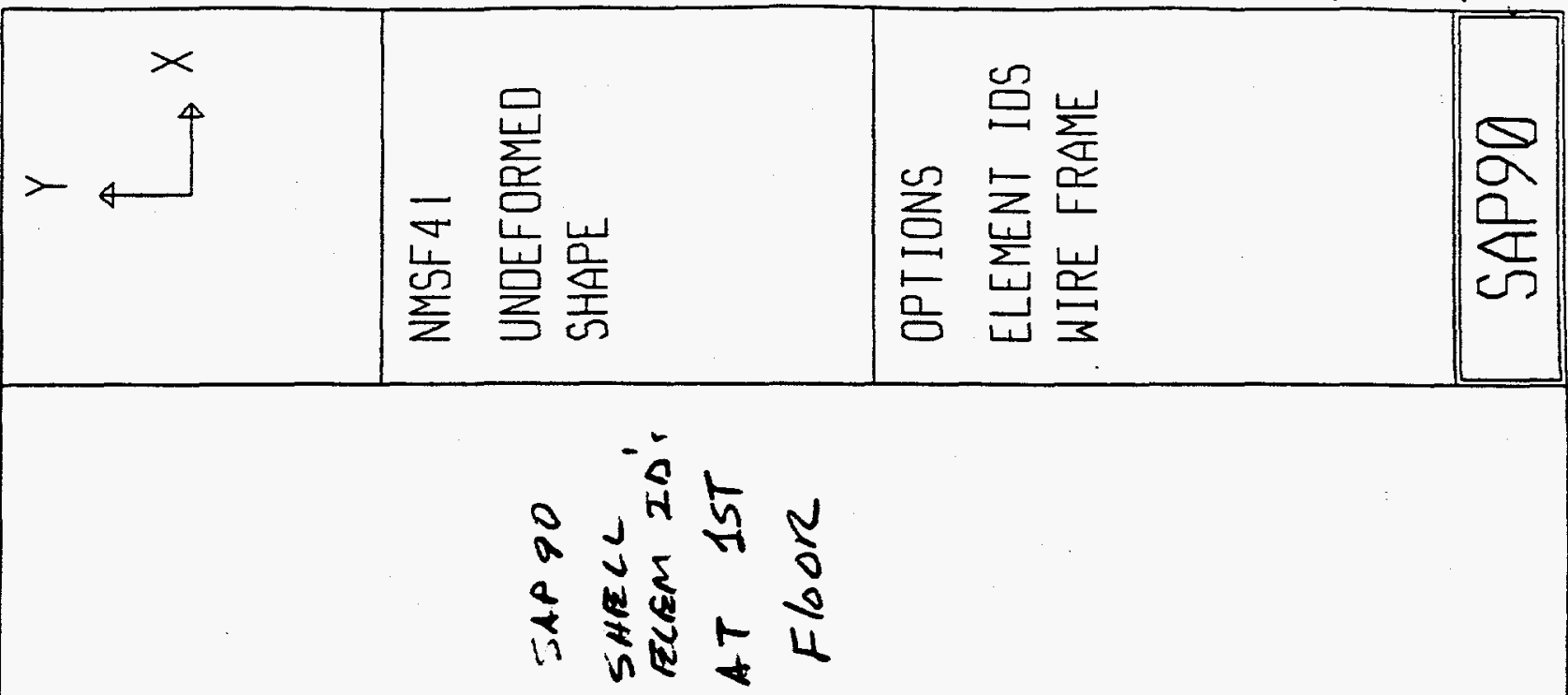

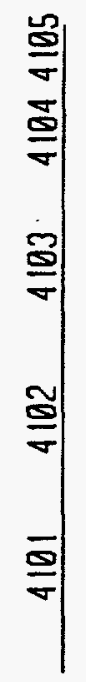

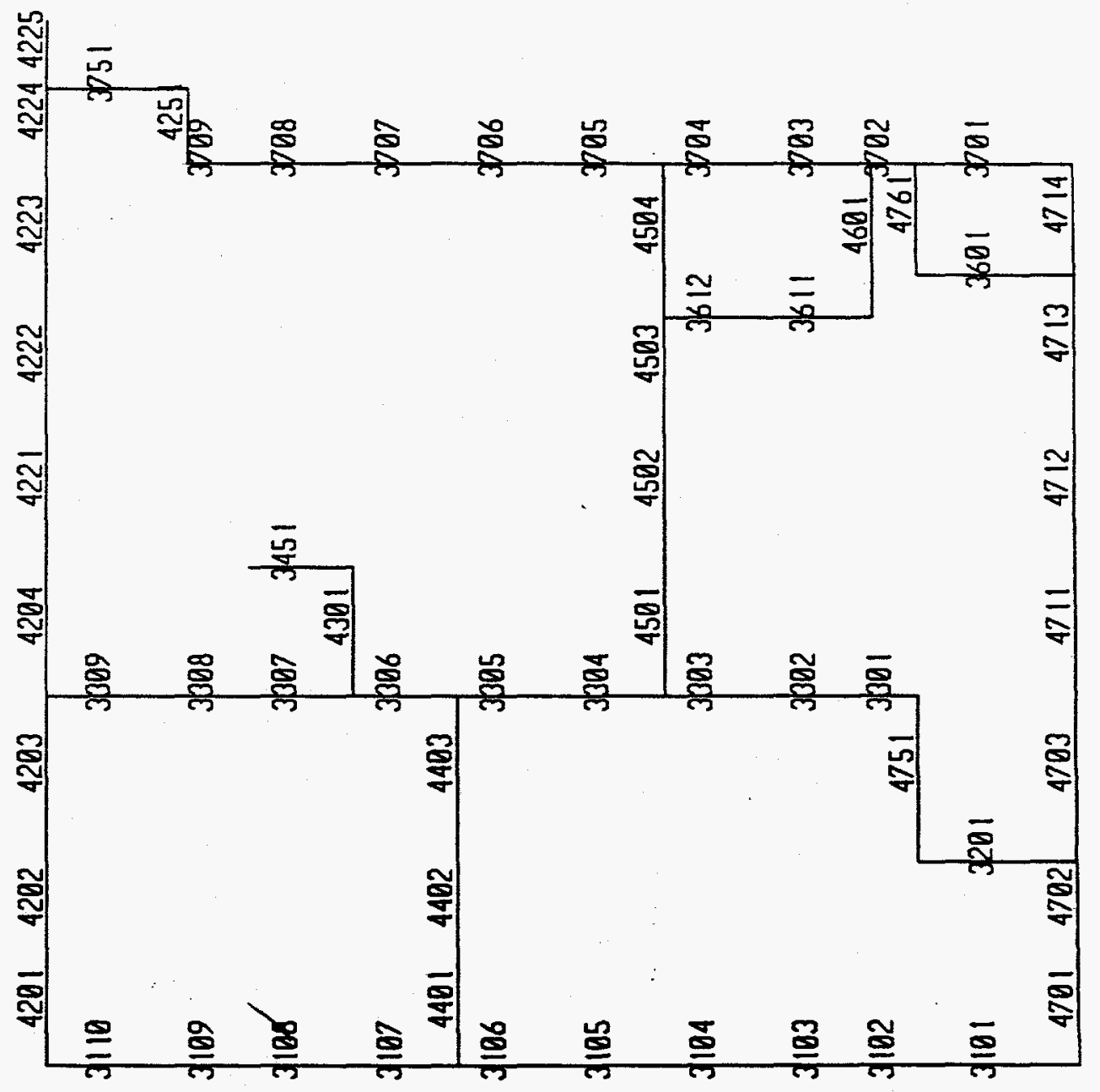




\begin{tabular}{l|}
\hline LANL \\
\hline STRUCTURAL EVALUATION OF NMSF FACILITY AT LANL
\end{tabular}

í DATE zilo's?




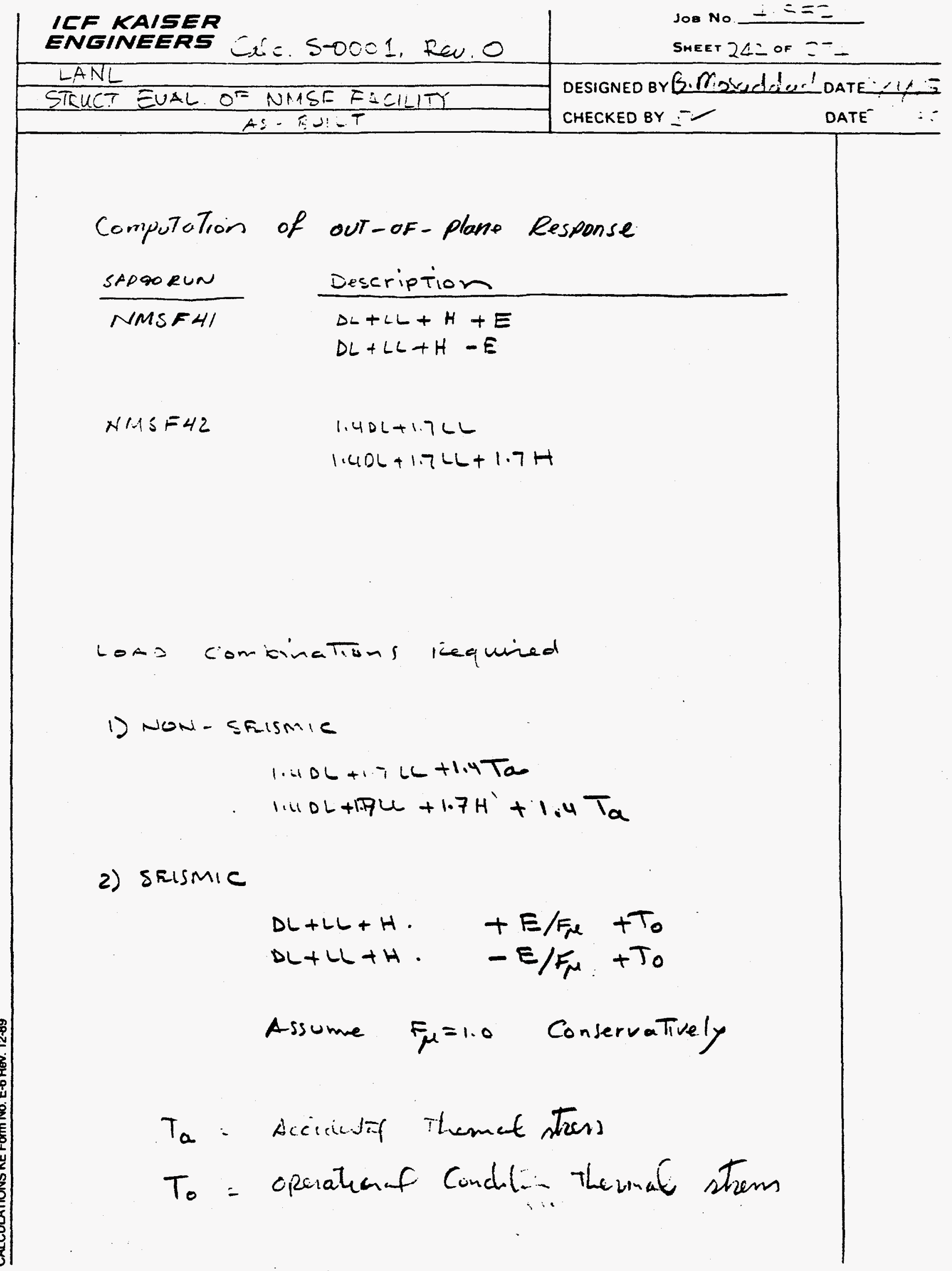




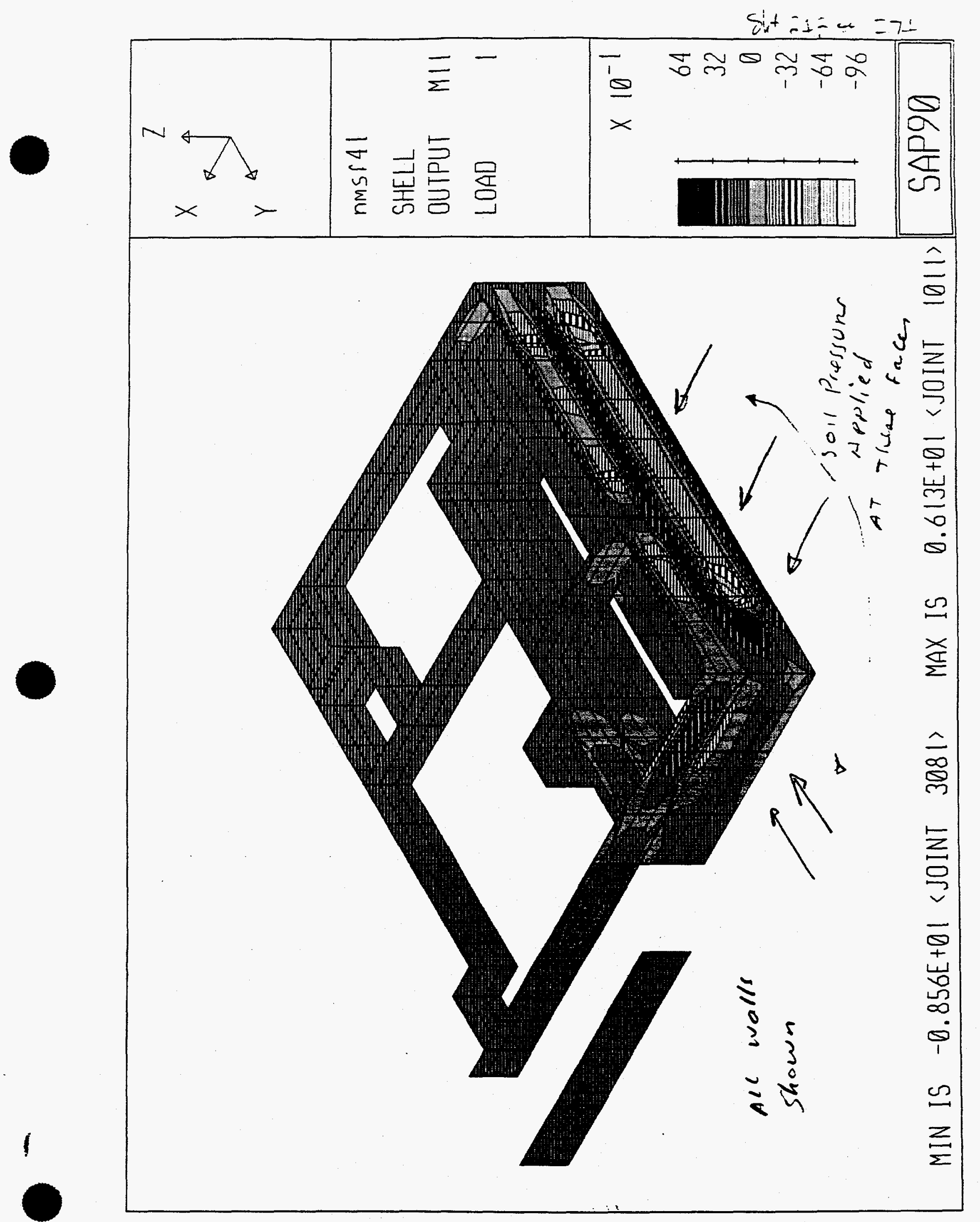




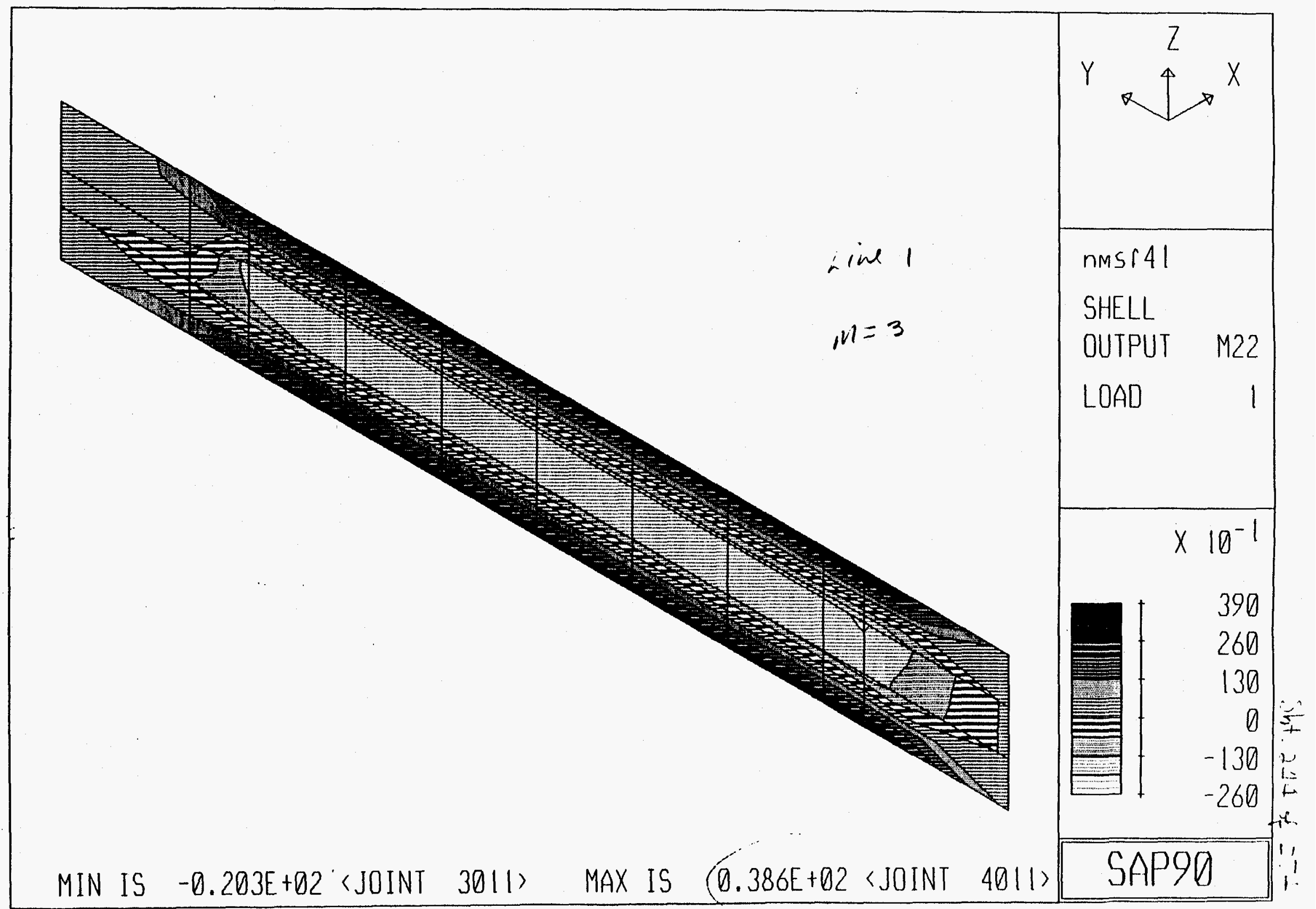




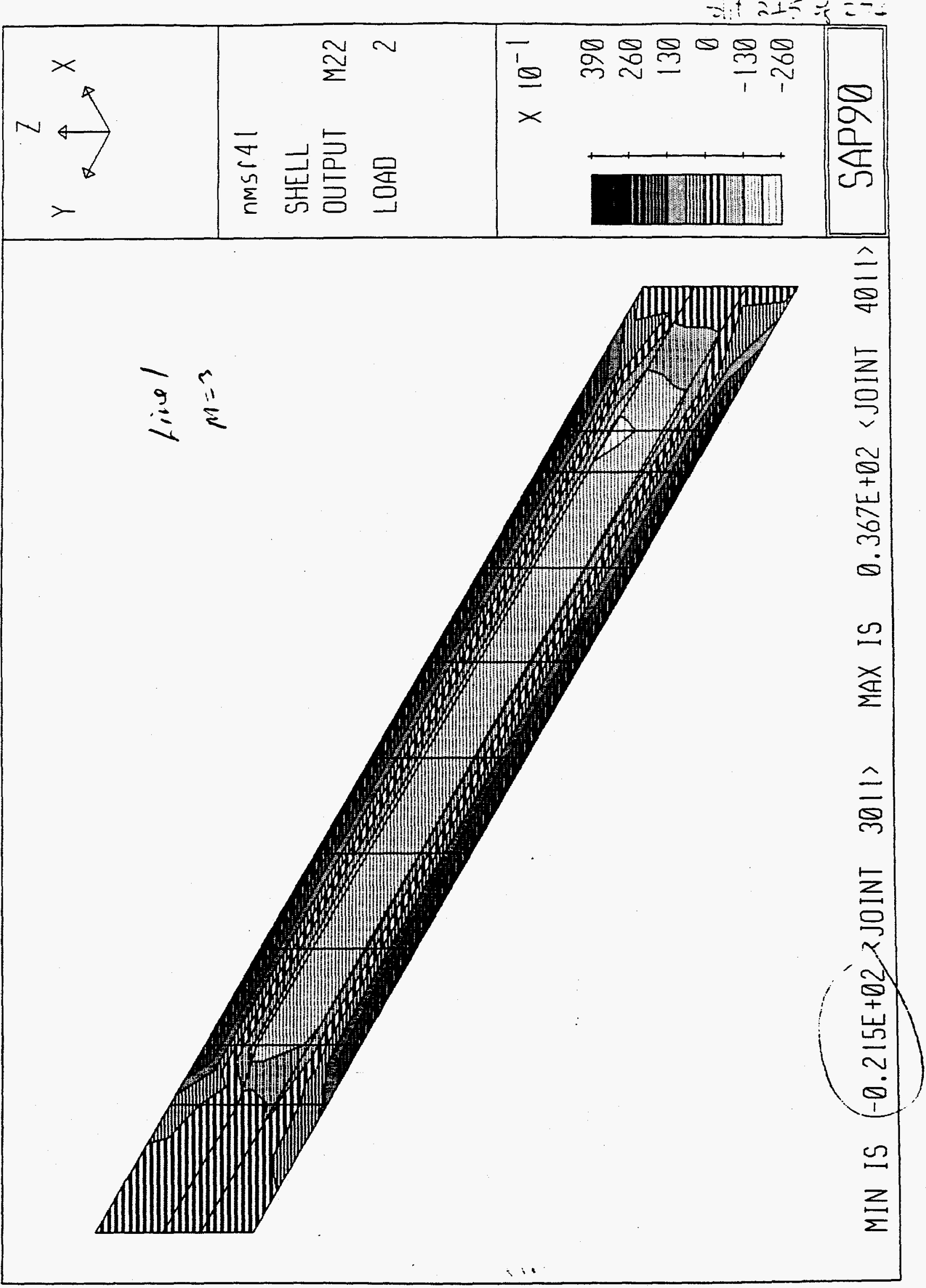


$0^{-}$

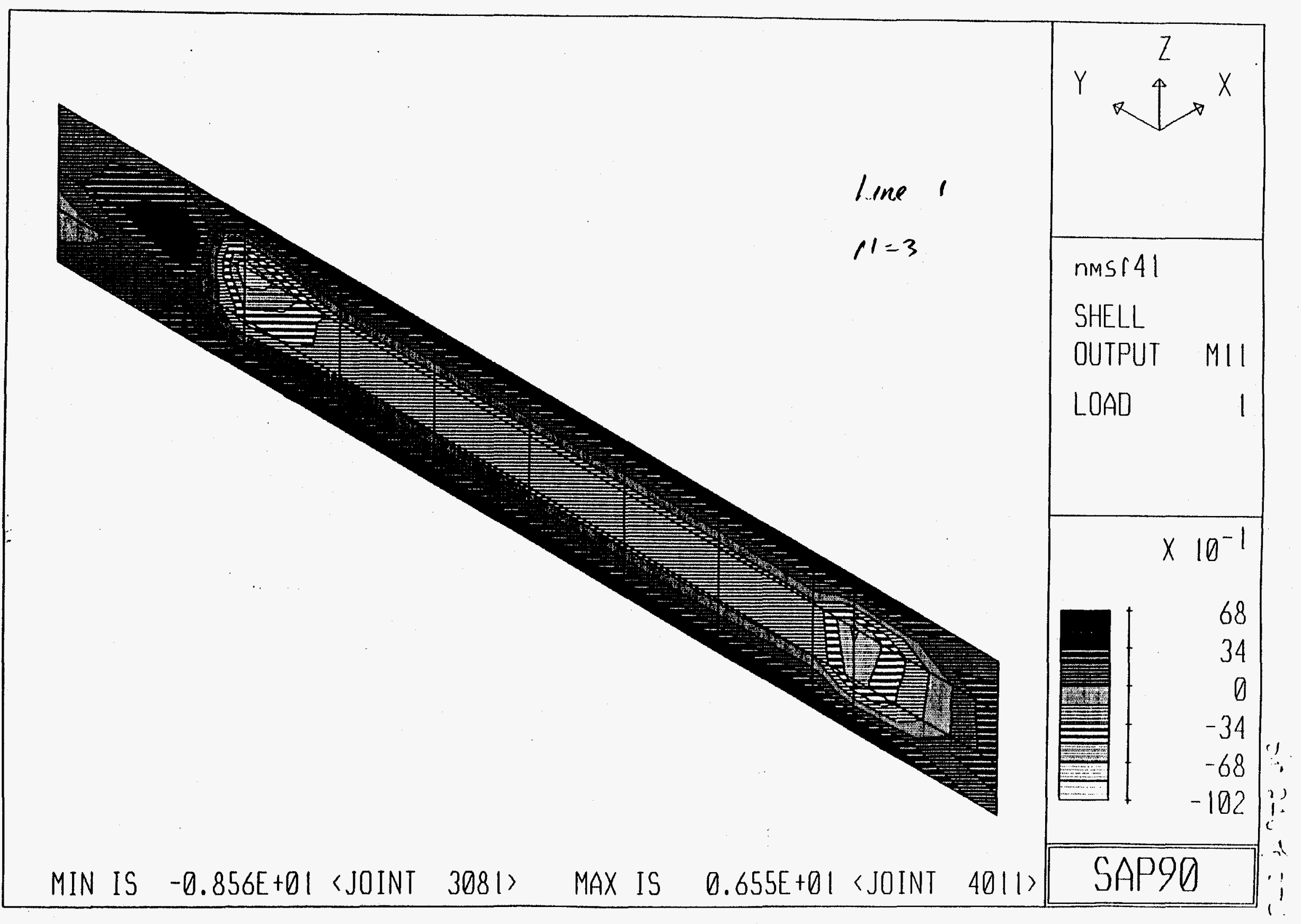




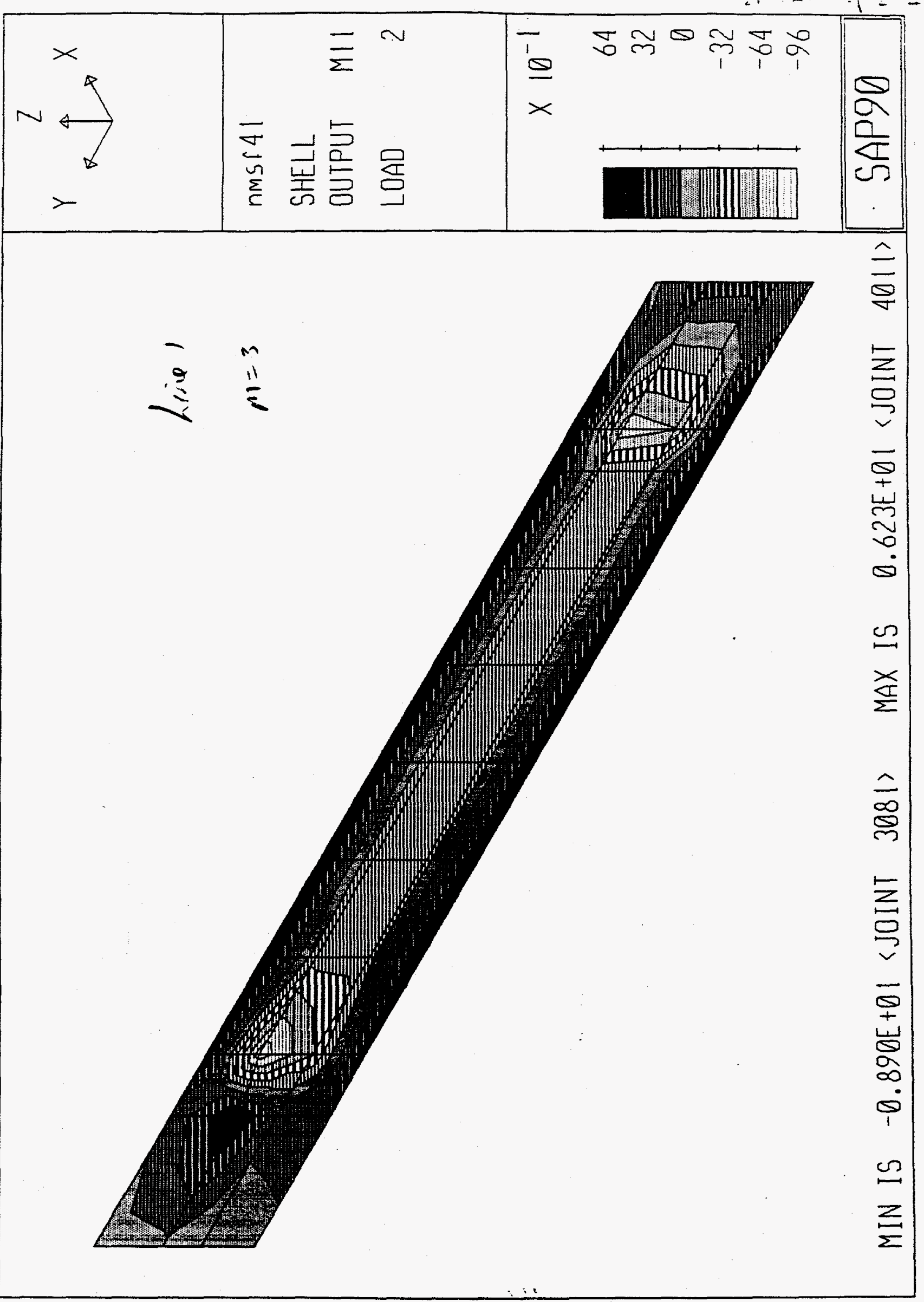




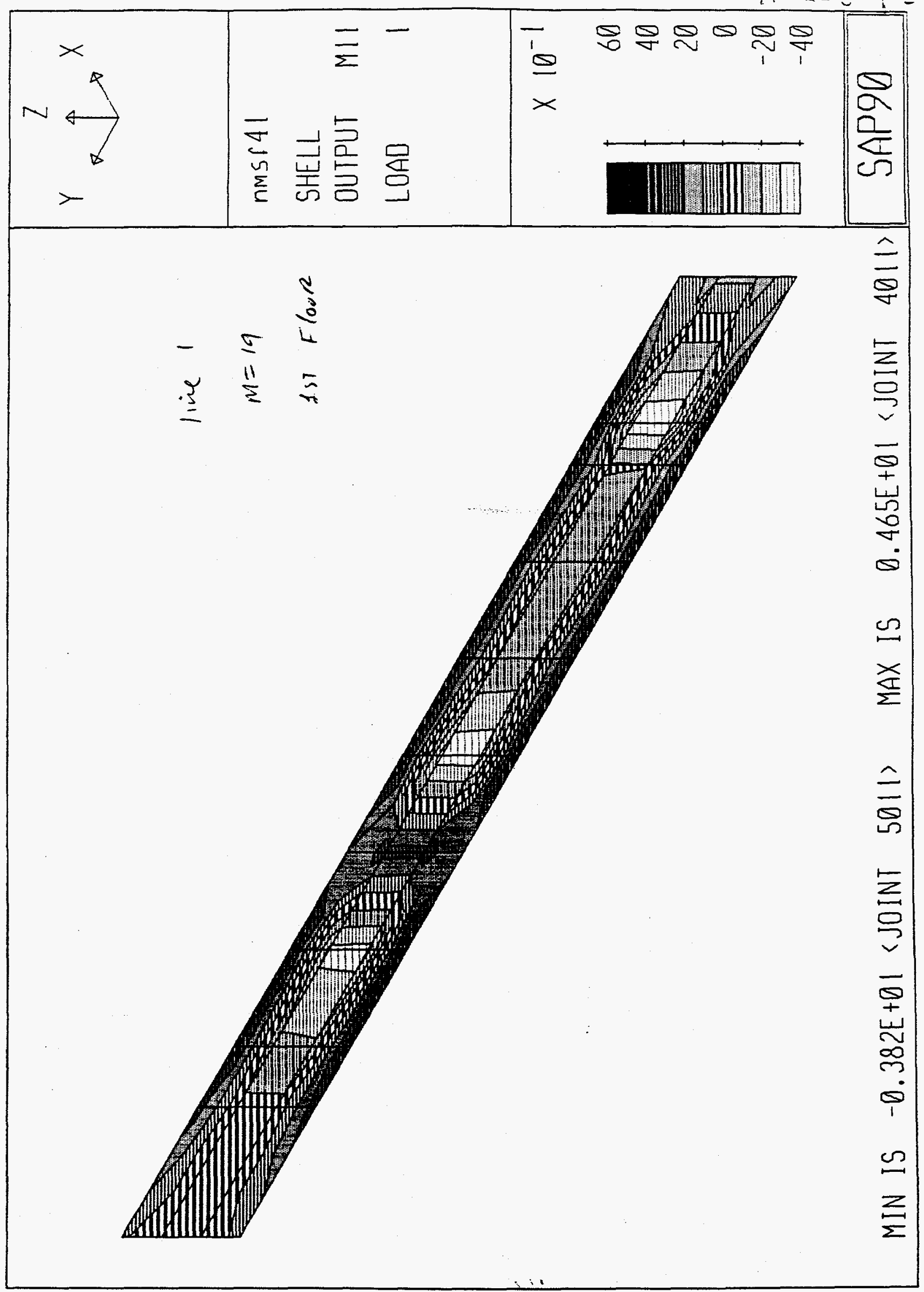




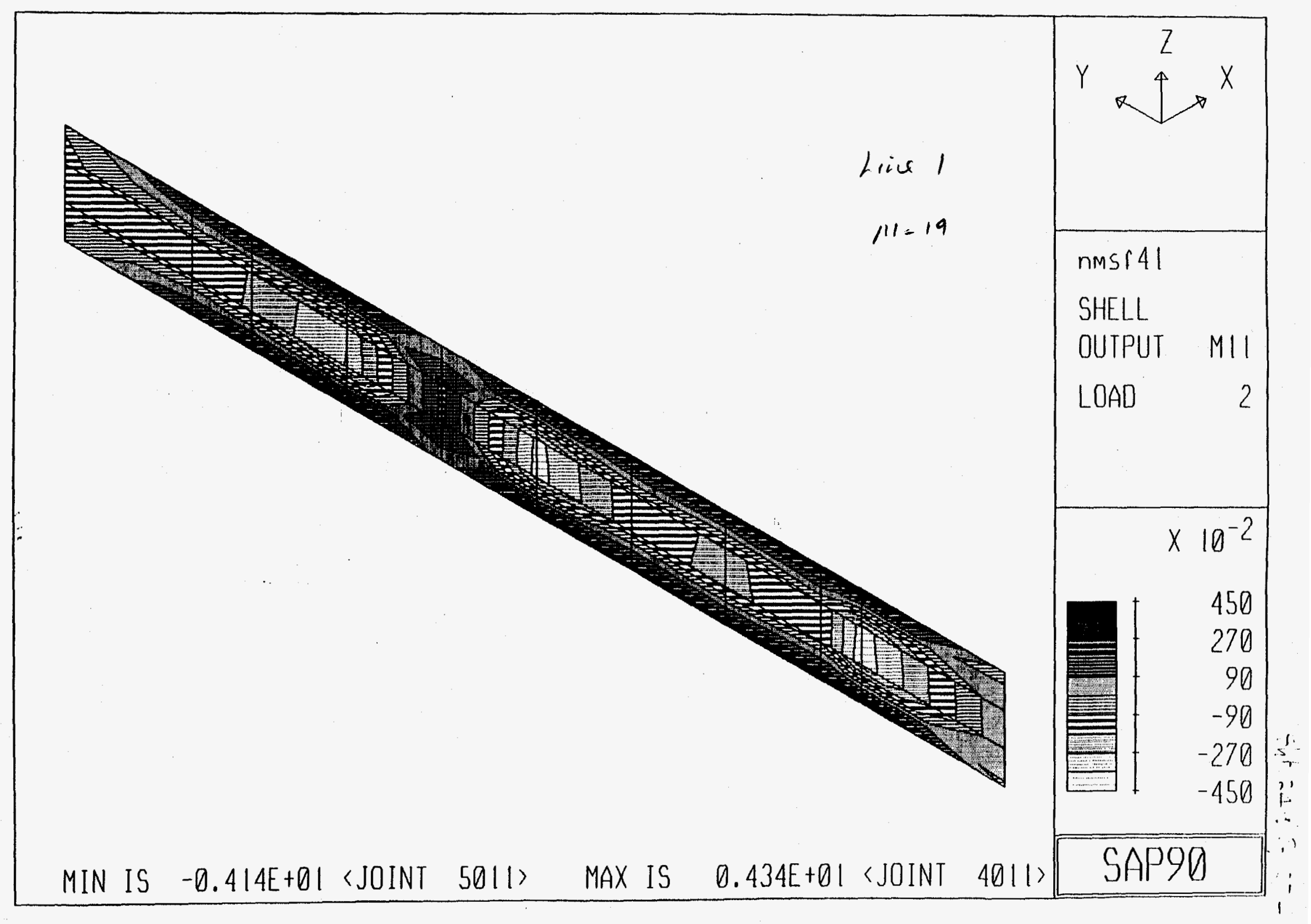


0

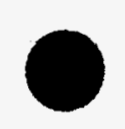

O

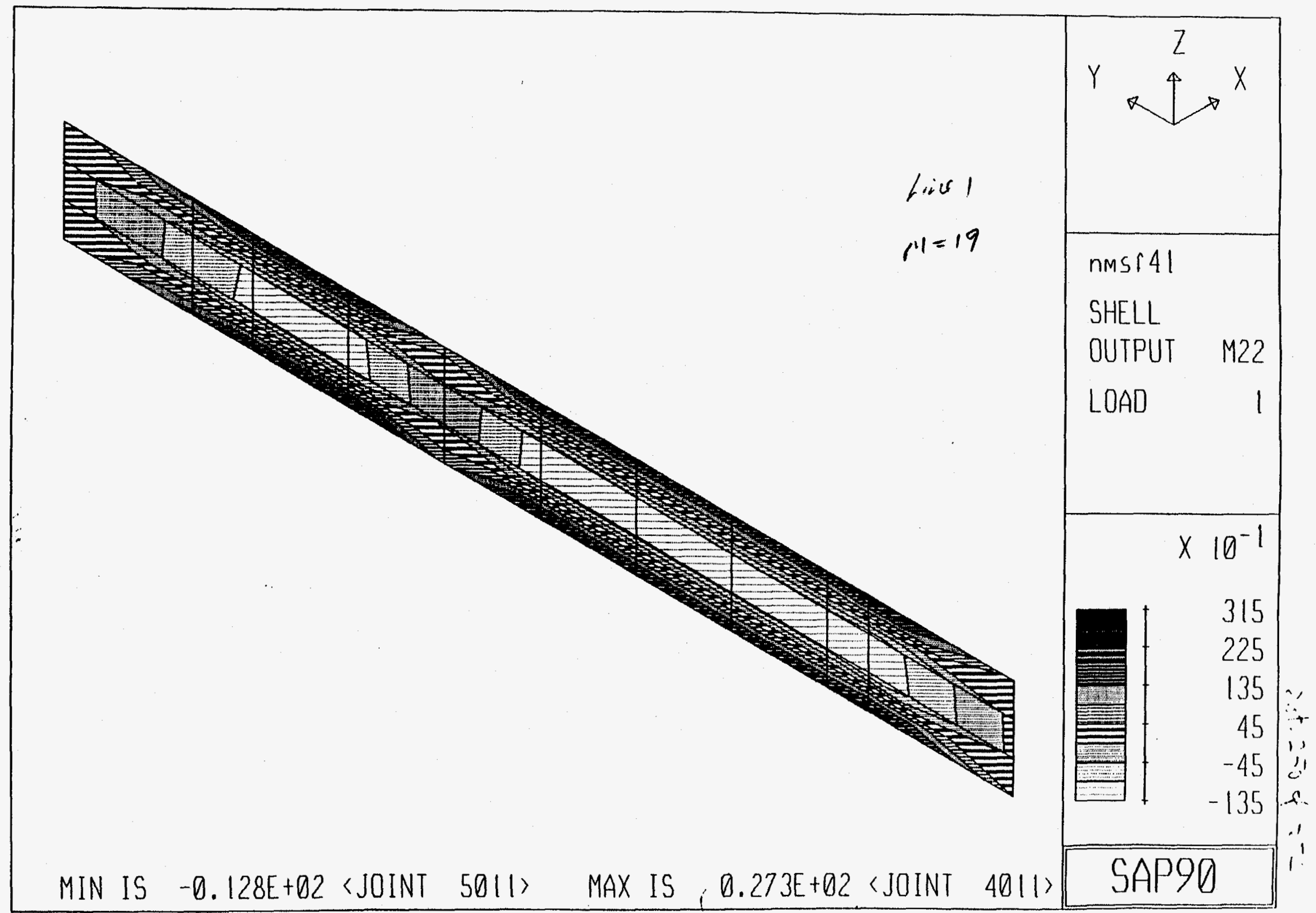




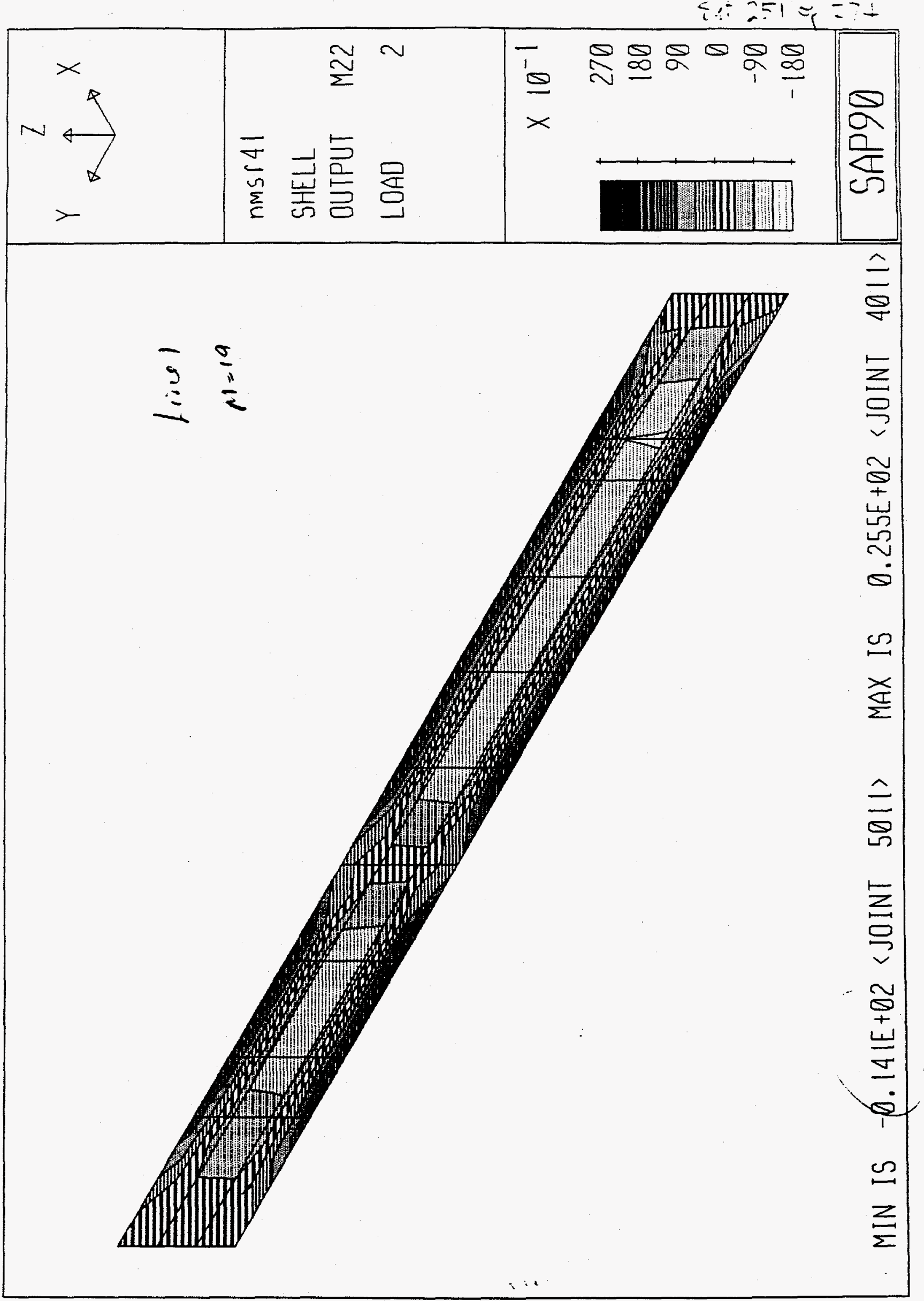



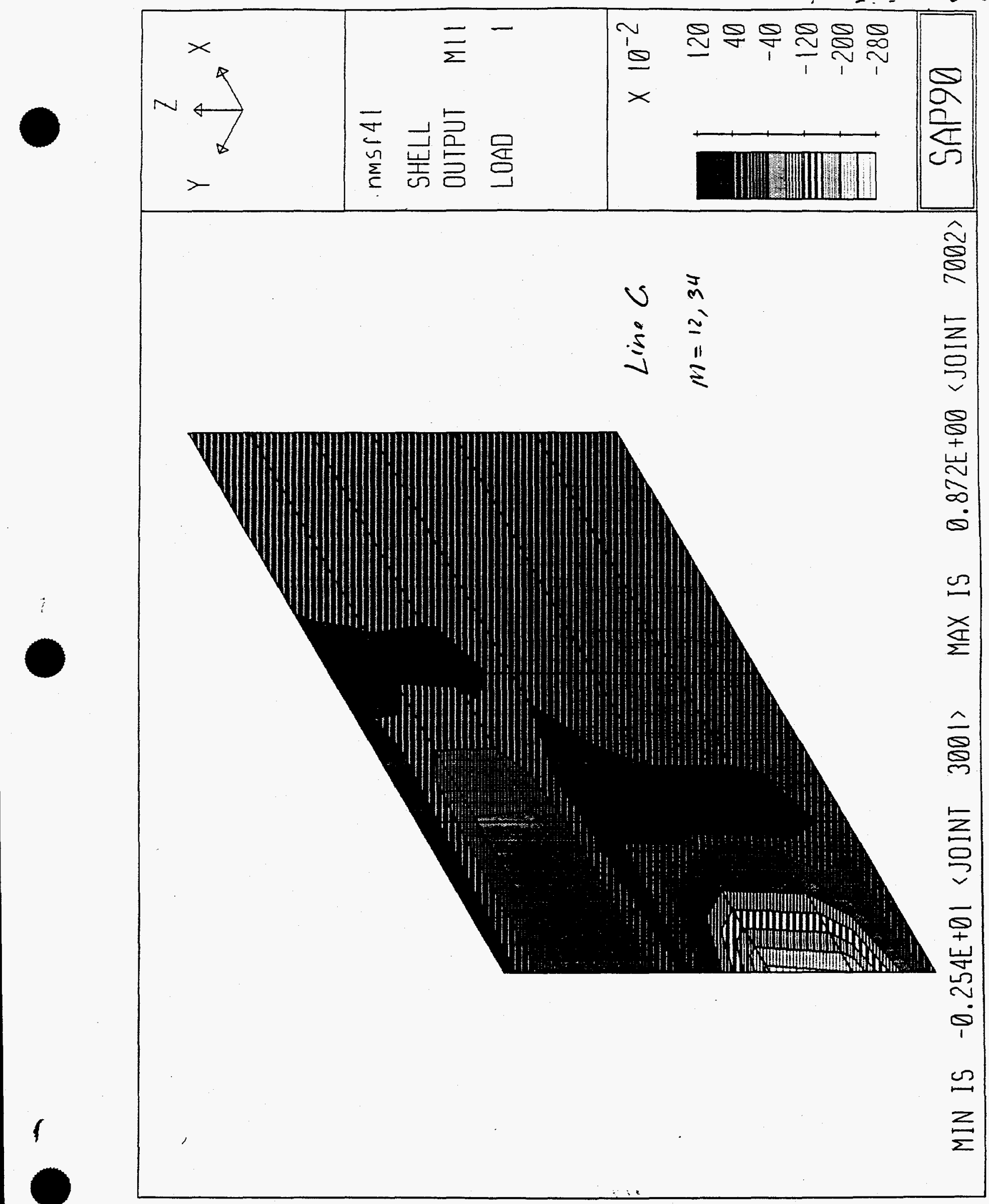


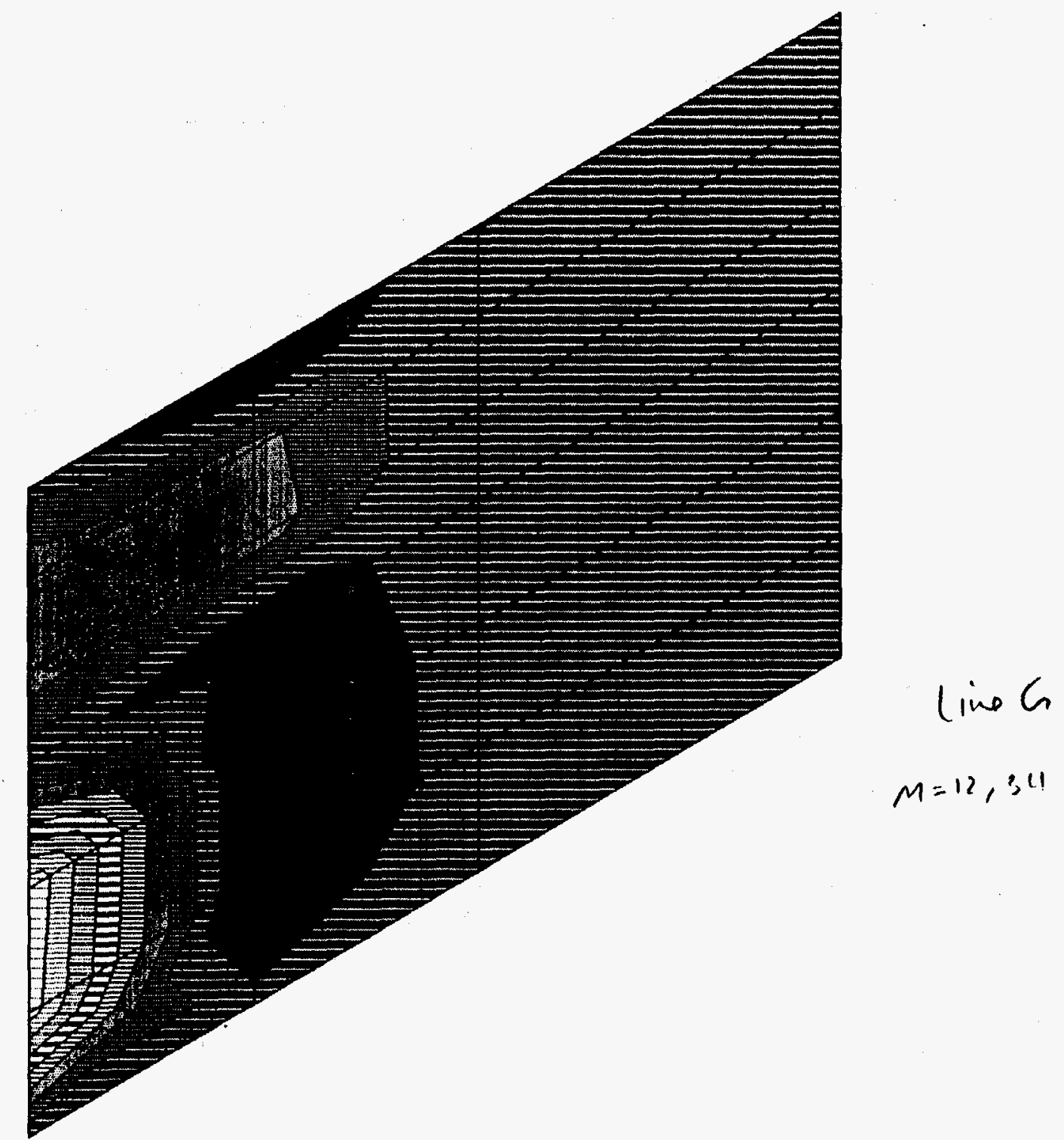

$Y \int_{\infty}^{Z} X$

nMsi4l

SHELL

OUTPUT MII

LOAD

2

MIN IS $-0.275 E+01<$ JOINT 3001〉 MAX IS $0.627 \mathrm{E}+00\langle$ JOINT 7002〉

SAPQD

70
0
-70
-140
-210
-280


प⿺辶 $=1=10=7$

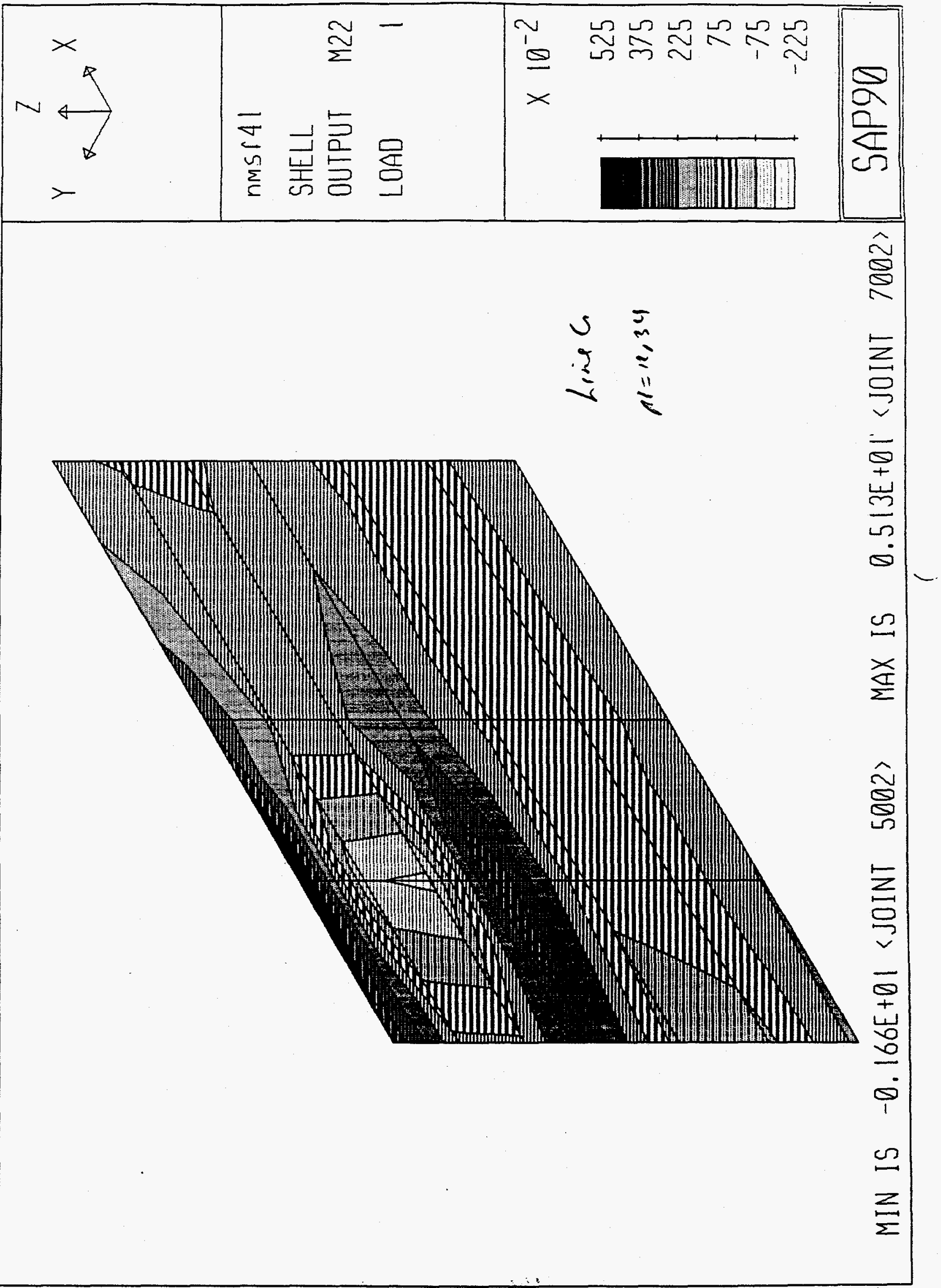




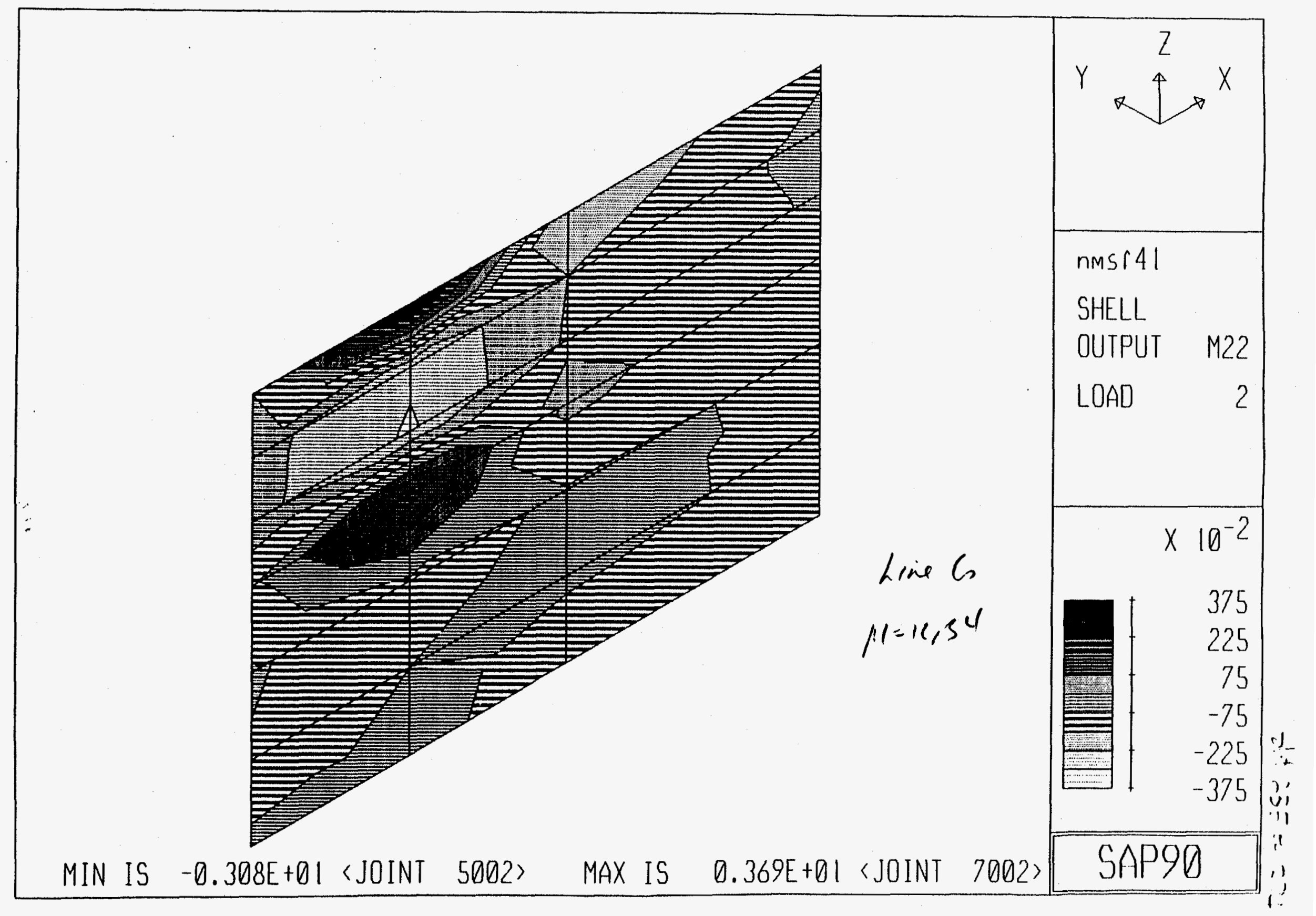




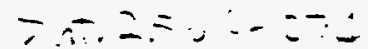
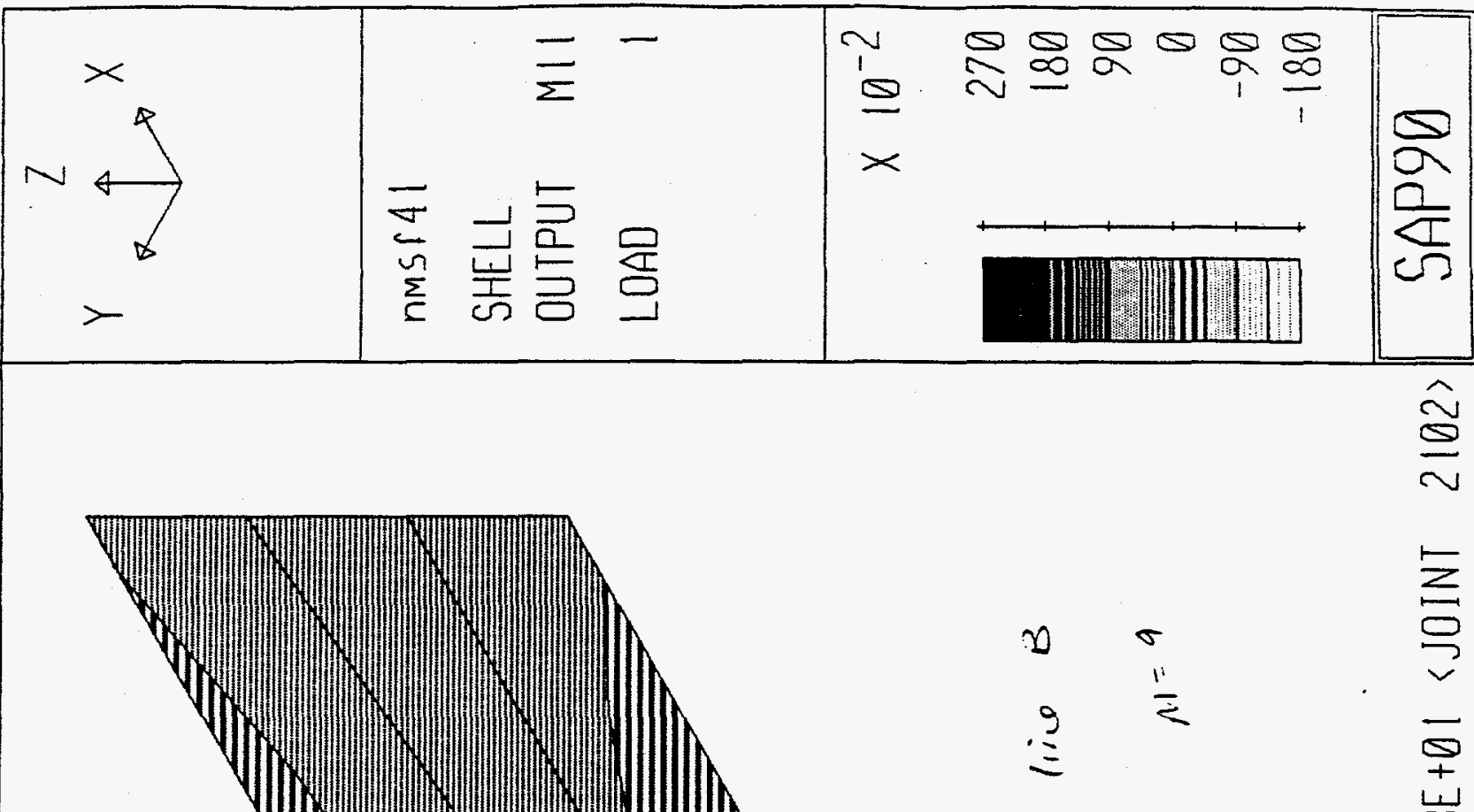

○

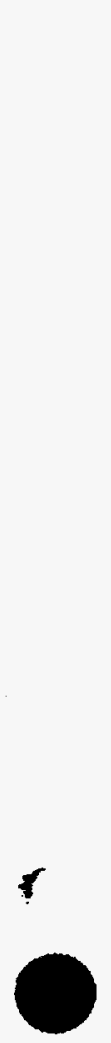




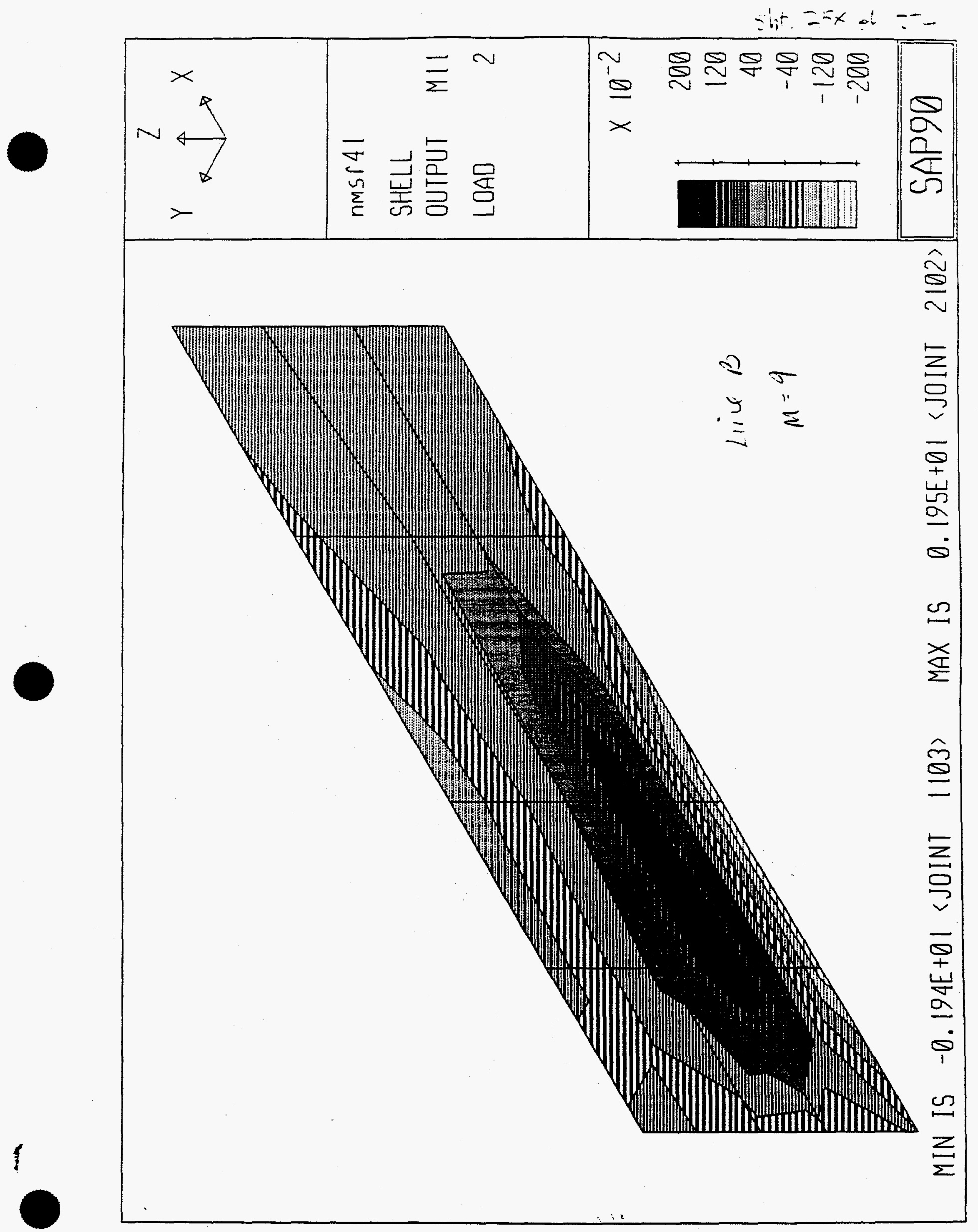




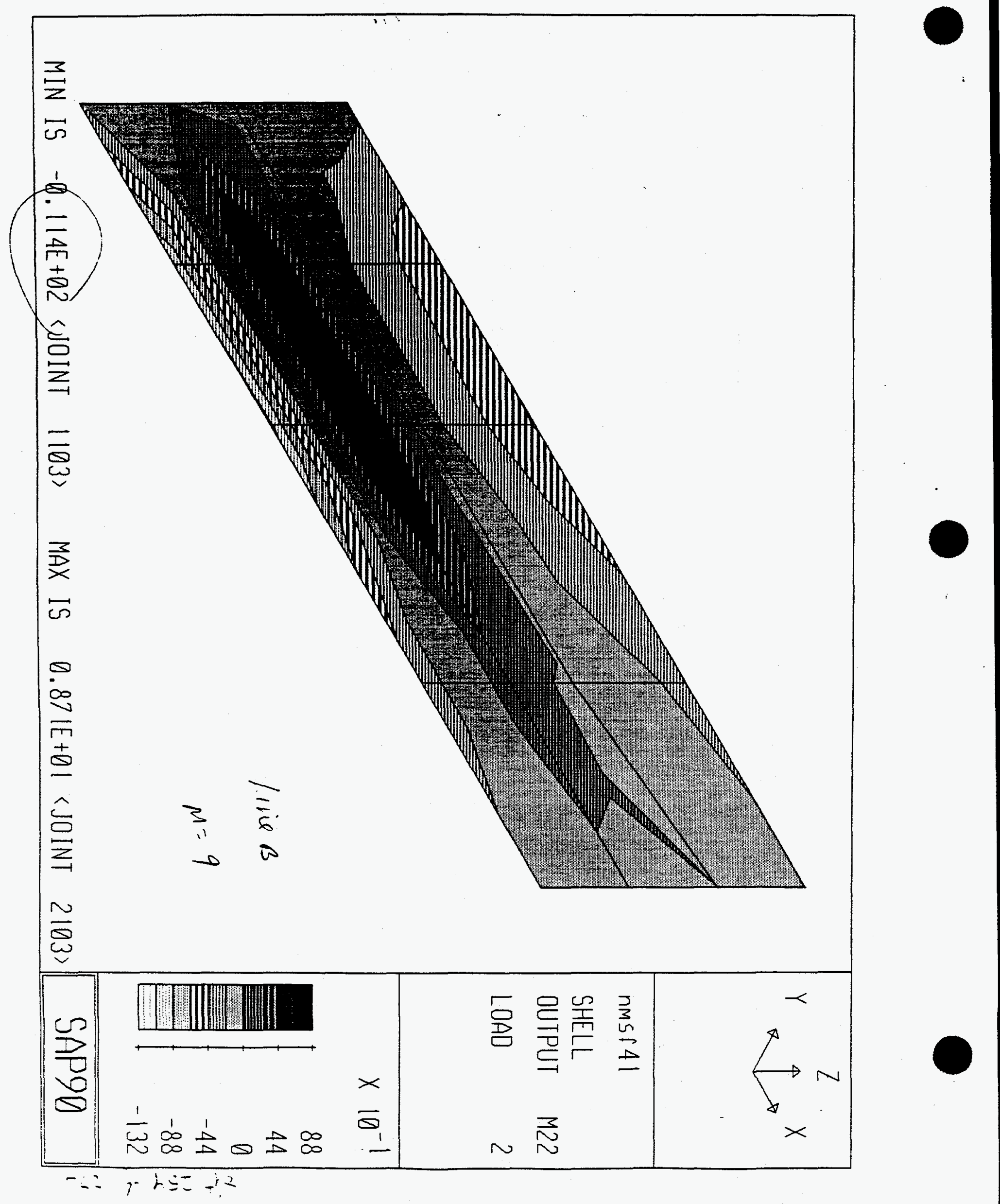




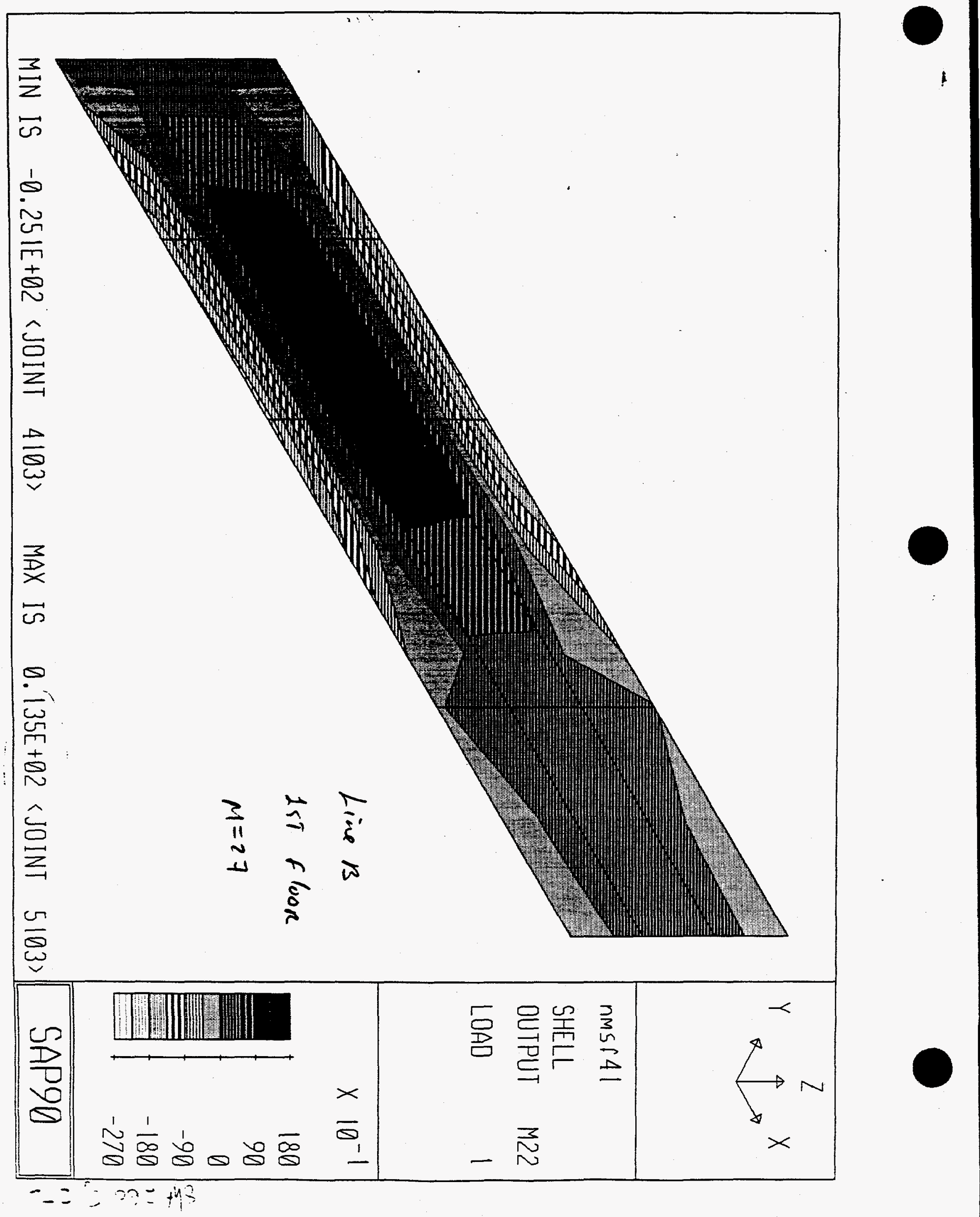




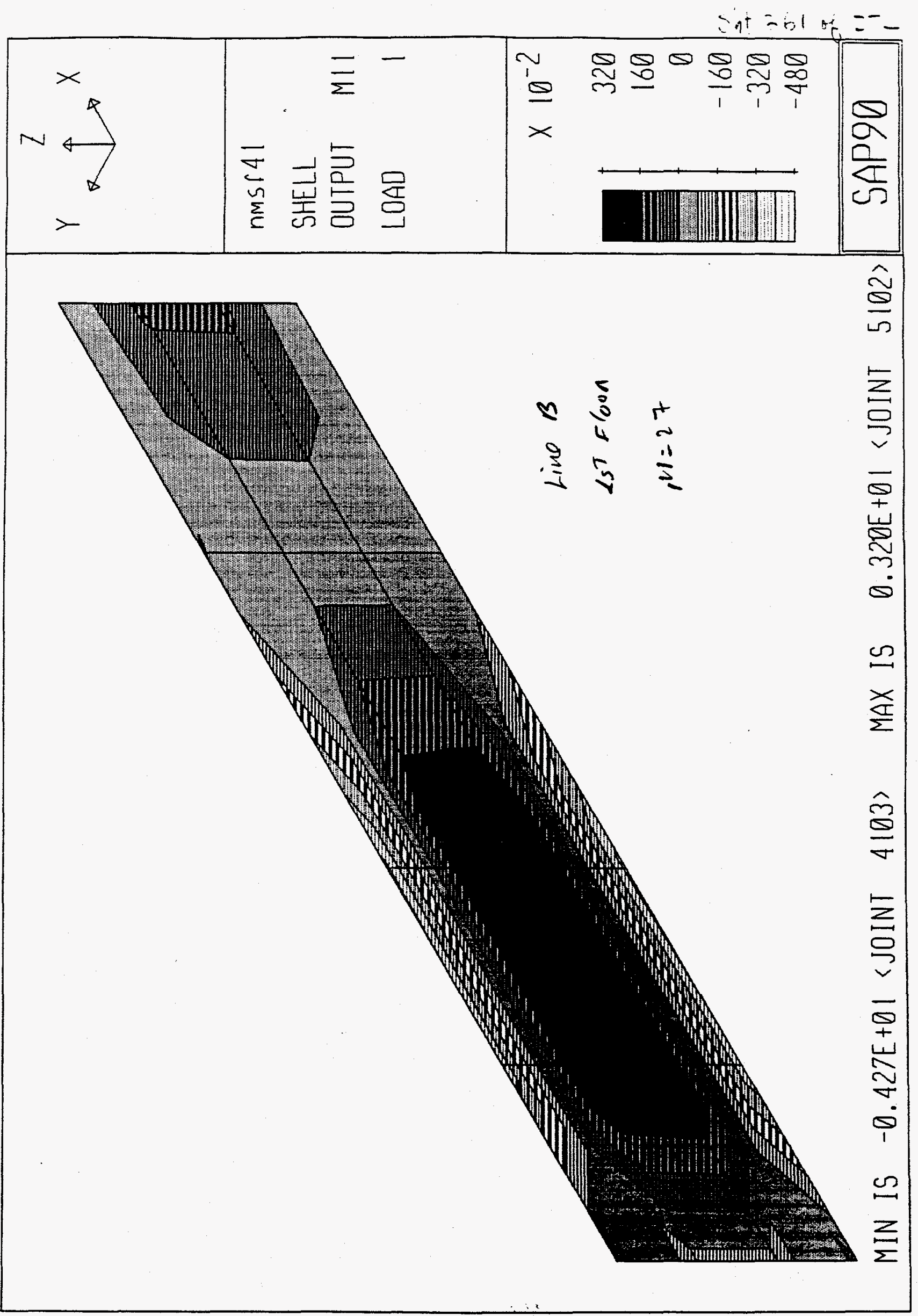




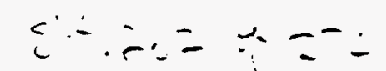

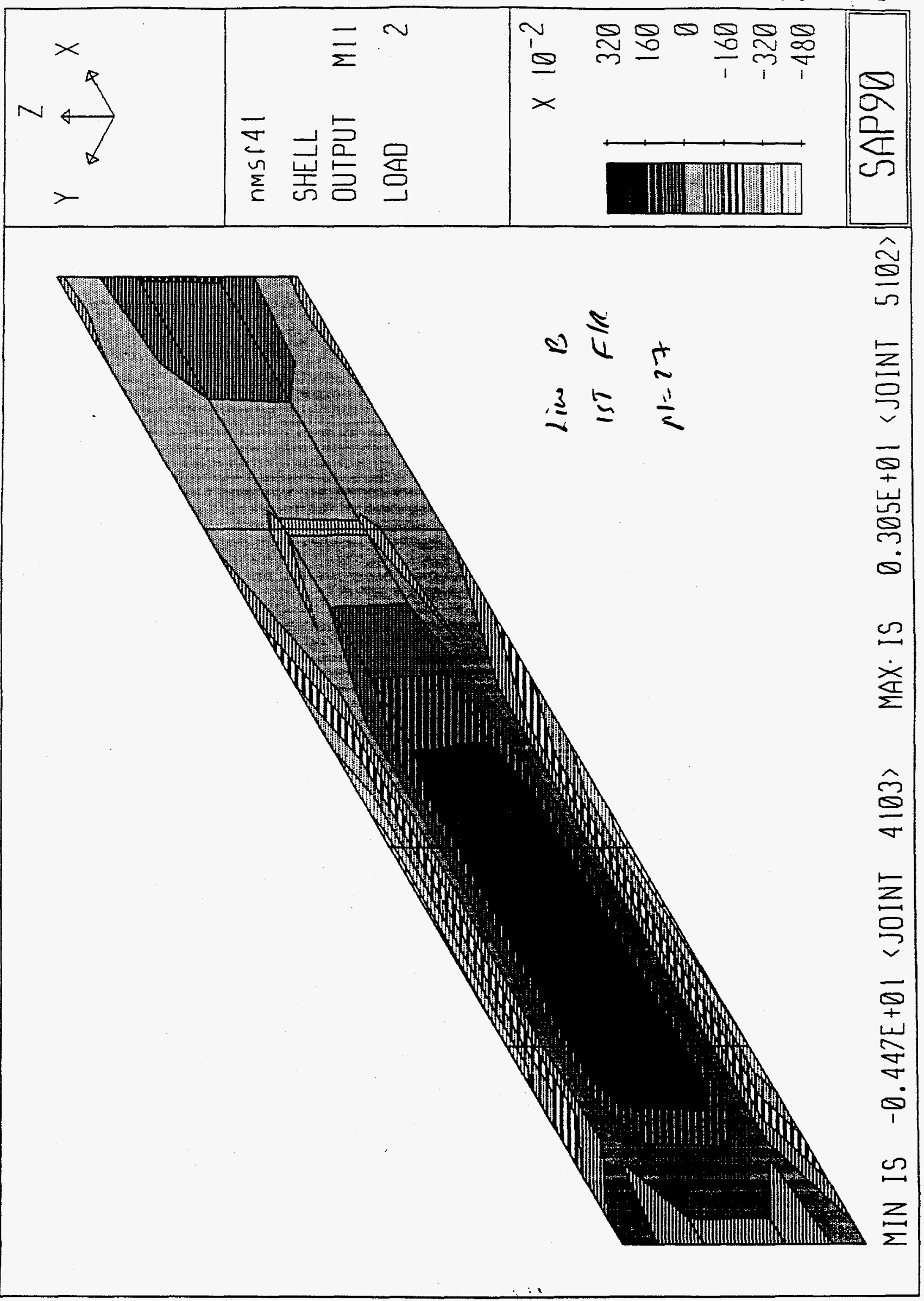


$5 t^{+}=07+0$

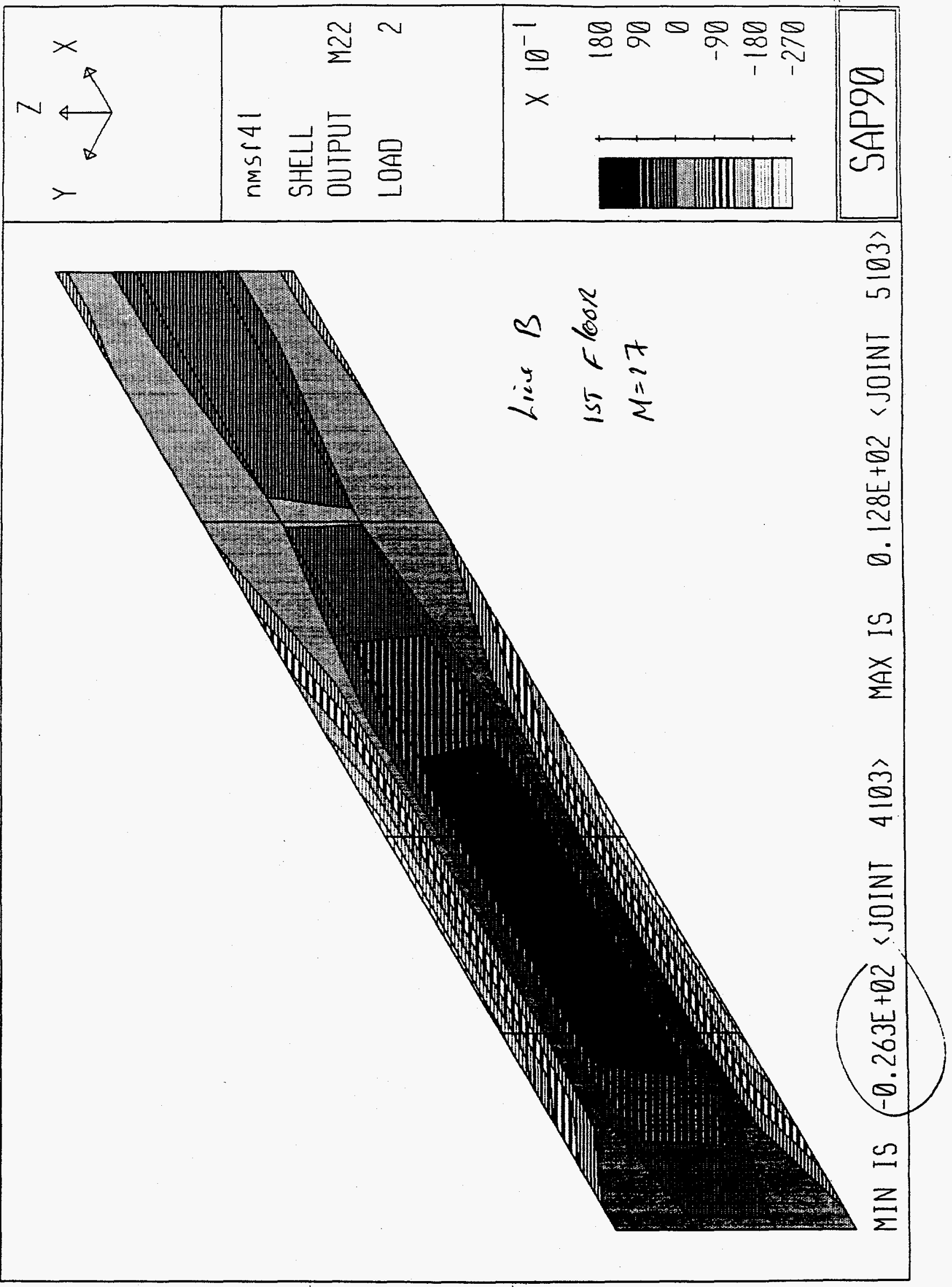




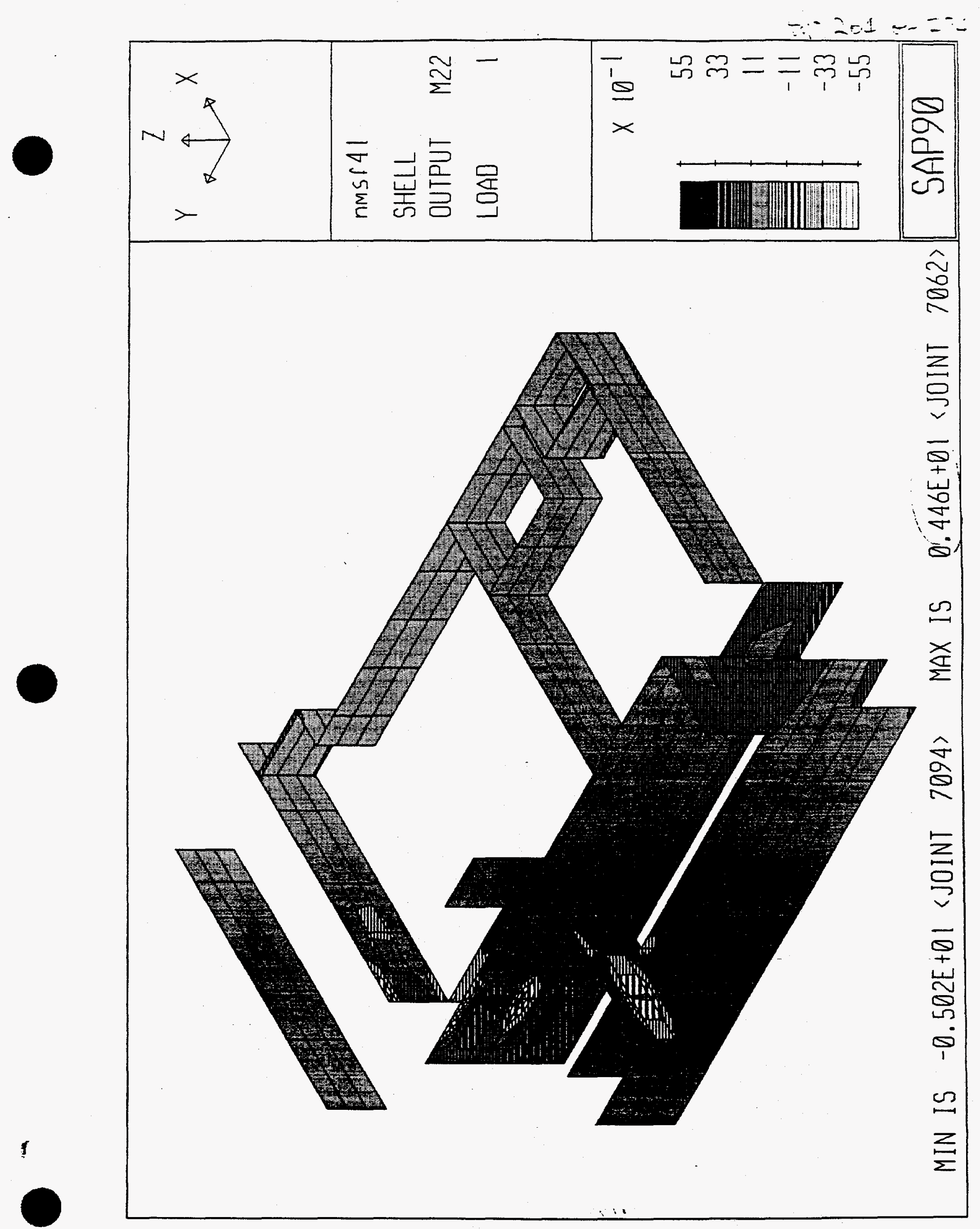




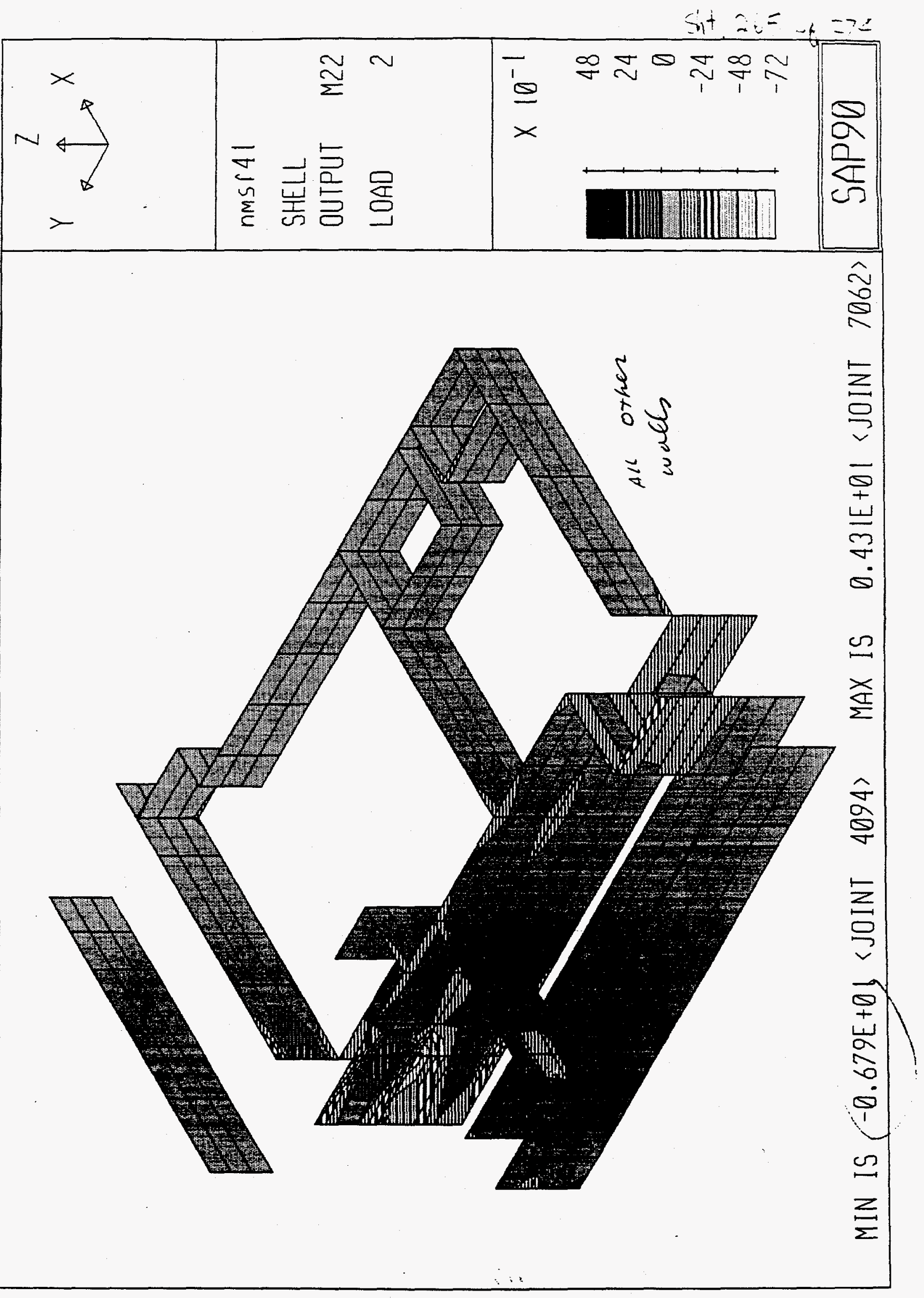



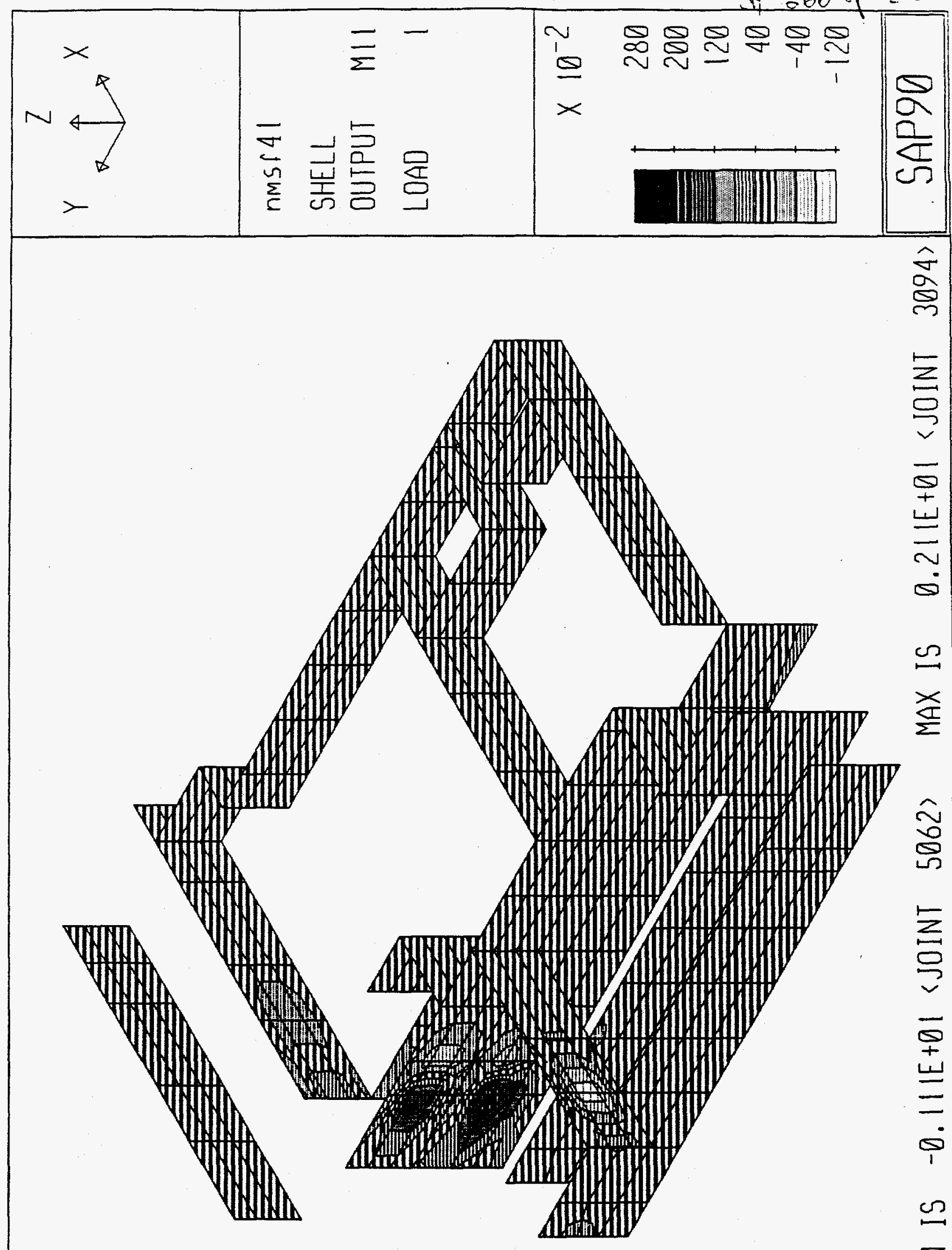

बू
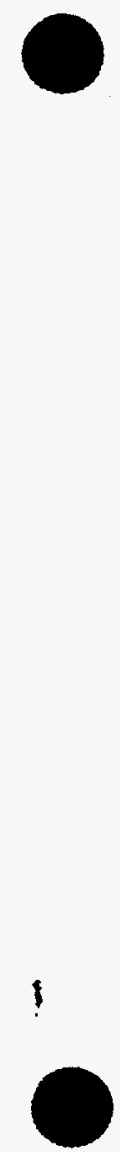


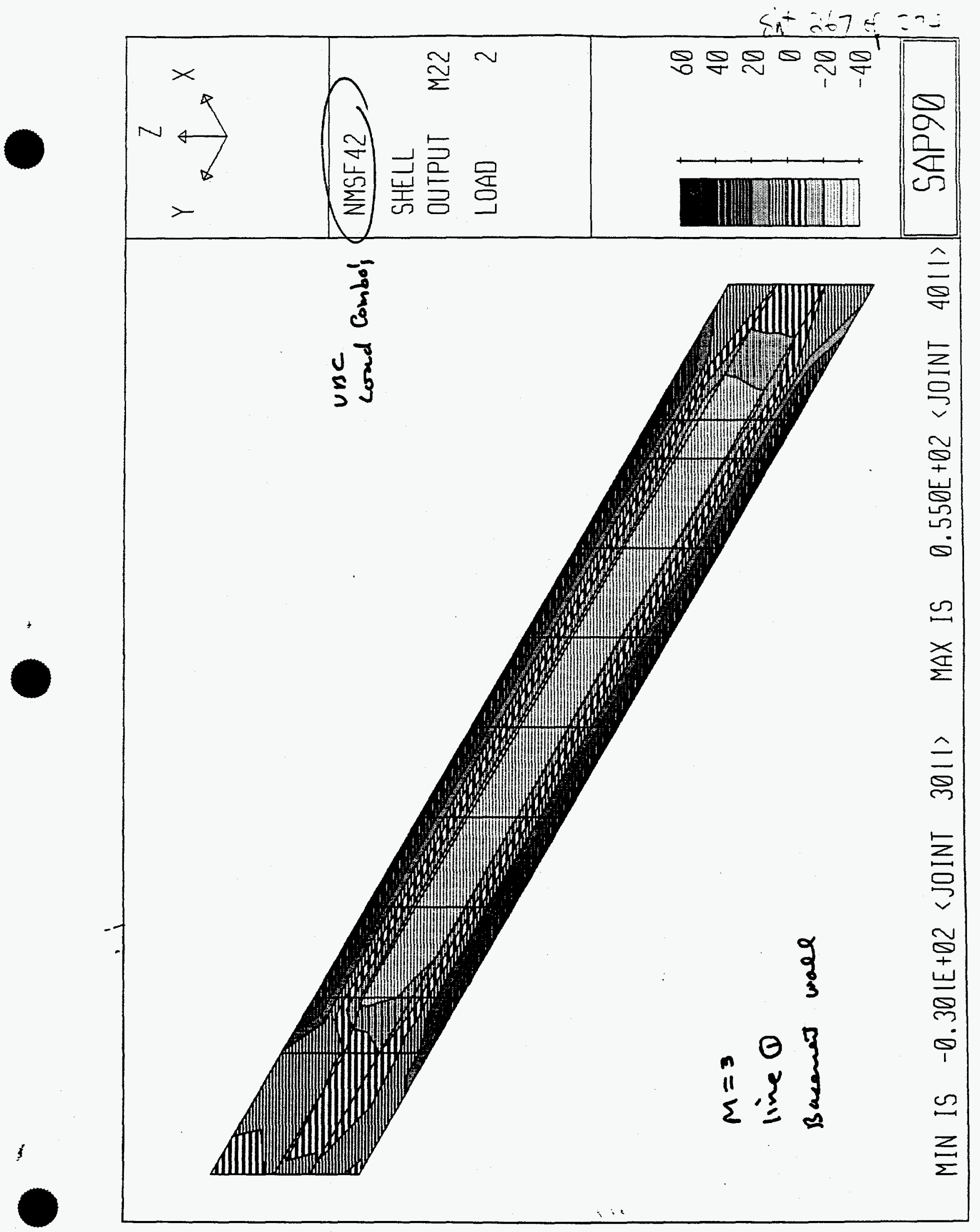


Sit 208 $\alpha=3$

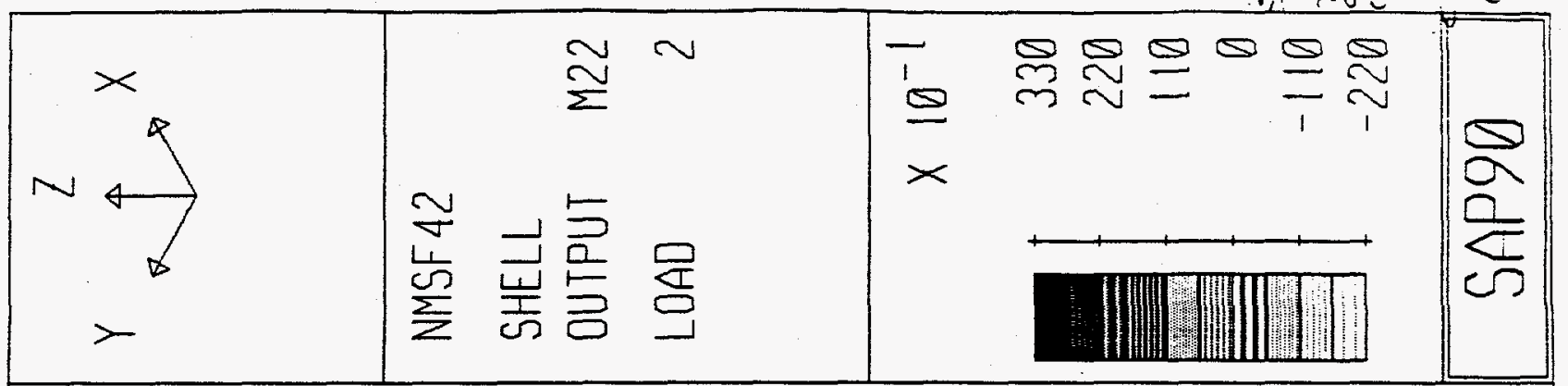

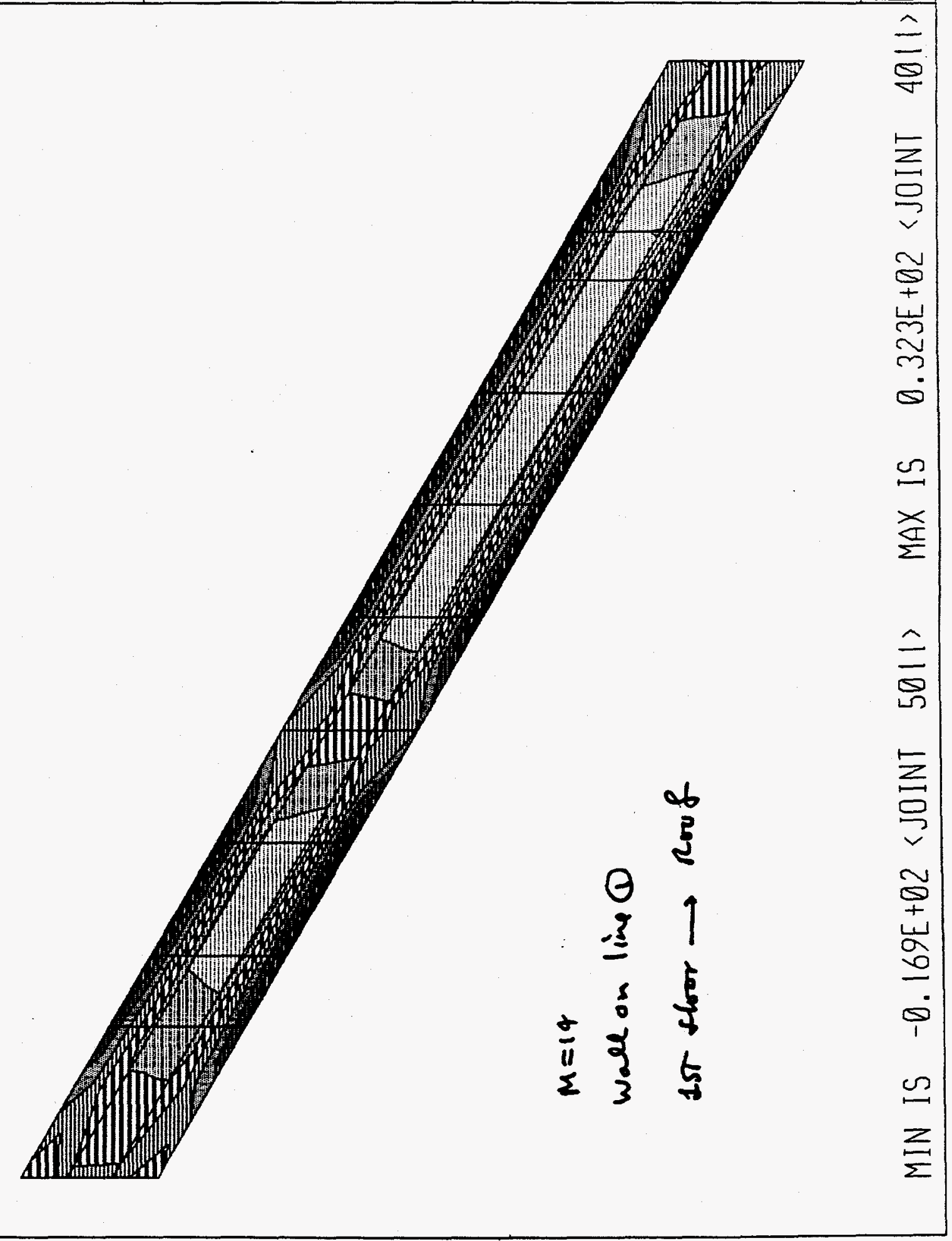




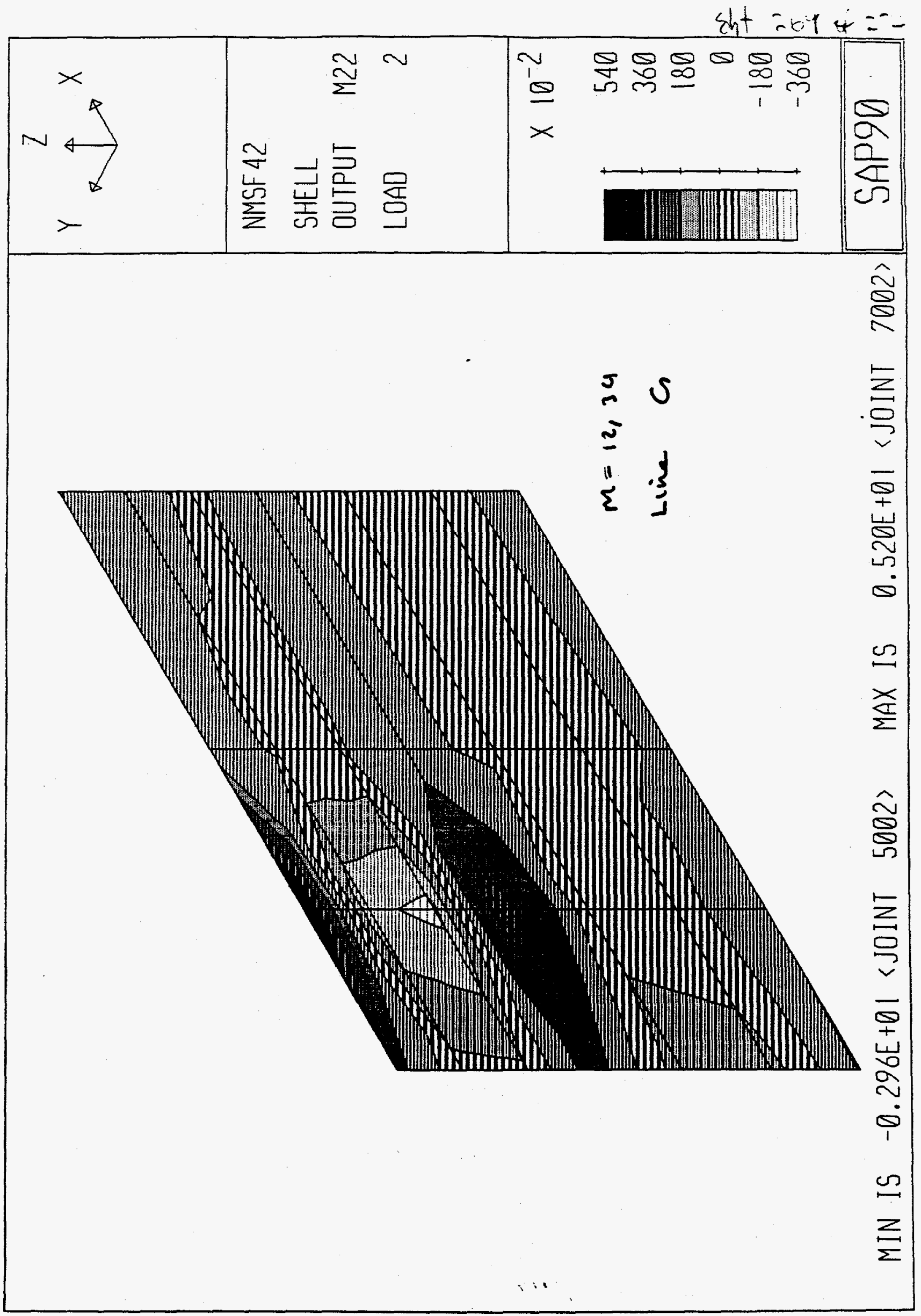


$80.706=-2$

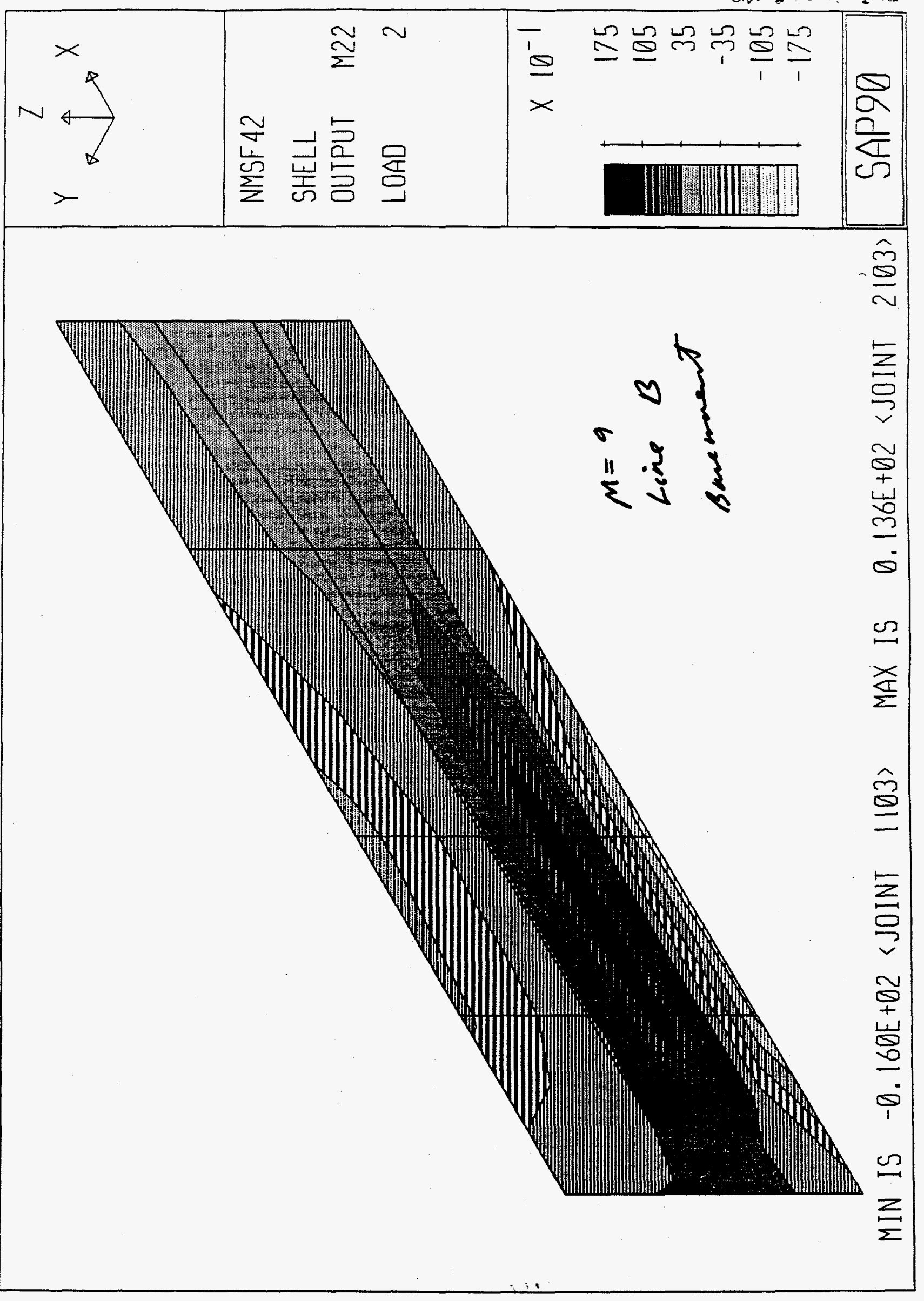


Mir s-co:1. Reen 0 Job $4155=$

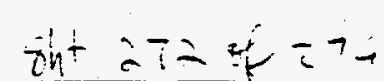

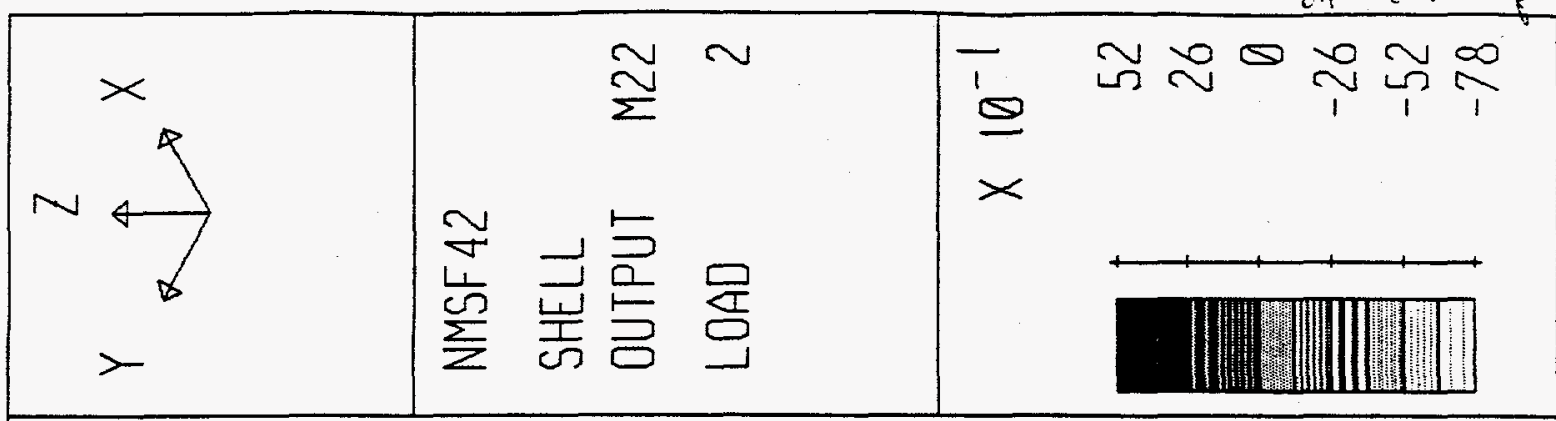

胥

$\widehat{\mathbb{V}}$

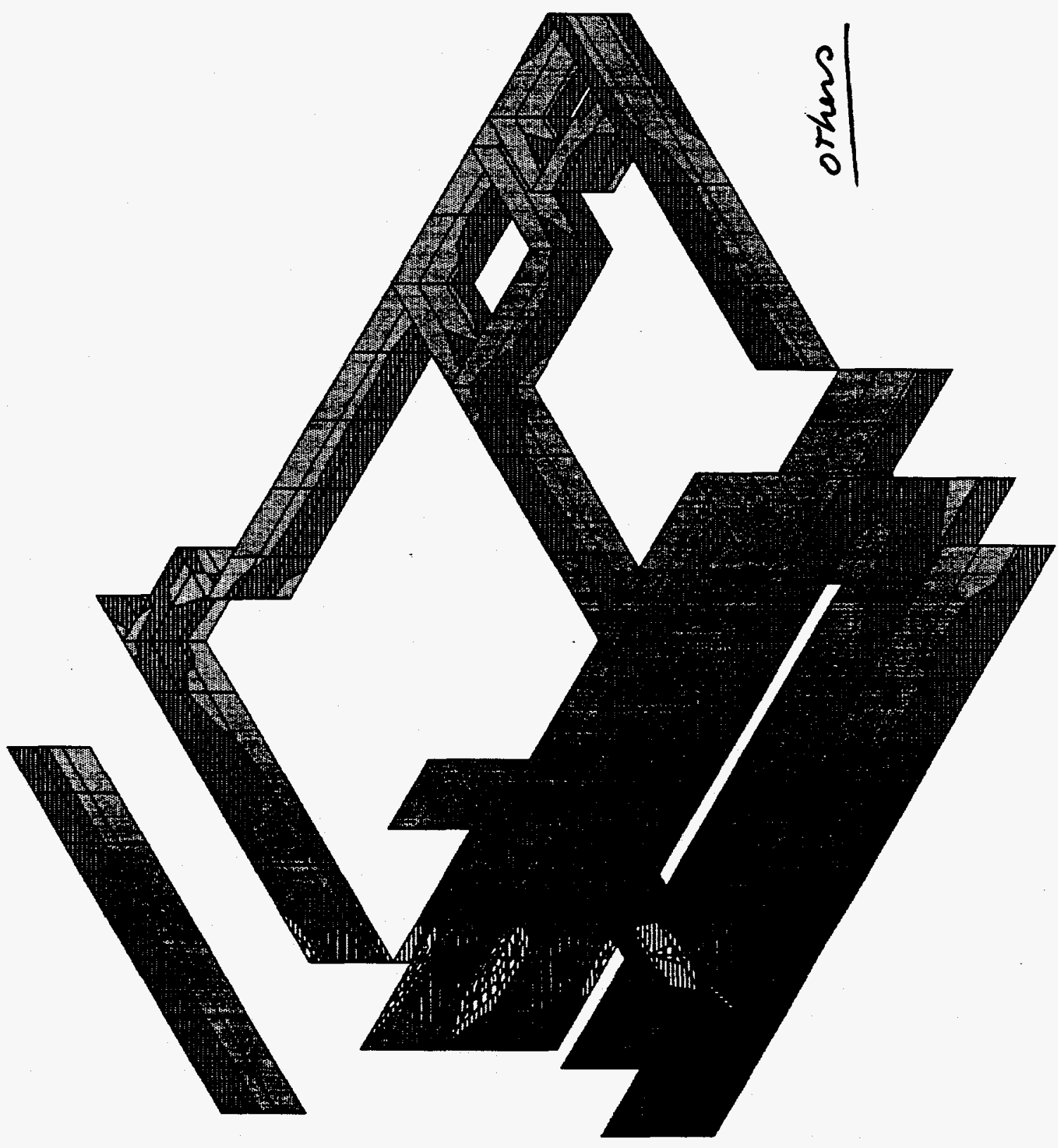

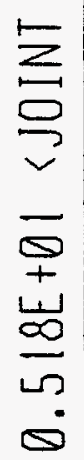

$\infty$

在

茨 


\begin{tabular}{|c|c|c|}
\hline ENGINEERS & SHEET & OF $こ \nearrow$ - \\
\hline$L A N I$ & \multirow{3}{*}{\multicolumn{2}{|c|}{$\begin{array}{l}\text { DESIGNED BY B. Mosaddad } \\
\text { CHECKED BY DATE } 5\end{array}$}} \\
\hline STRUCTURAL EVALUATION OF NMSF FACILITY AT LANL & & \\
\hline As-Built Structure & & \\
\hline
\end{tabular}

$\vdots$ 


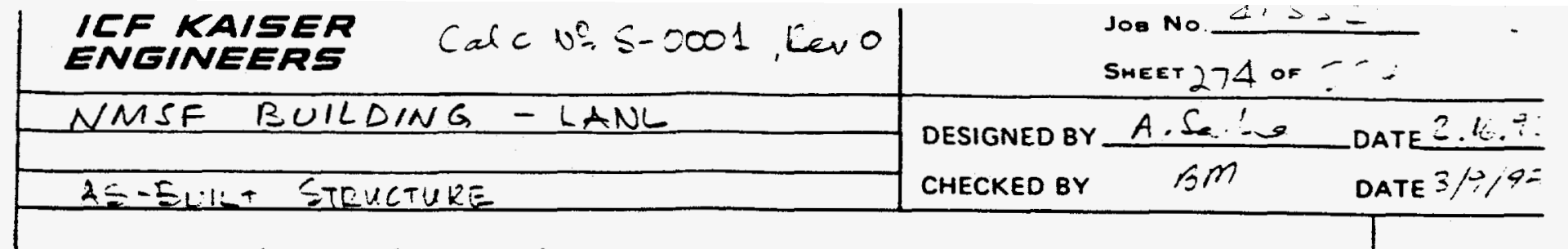

Correction of Prestressed double Tee to wall Reference Bet. (12) Darg. 5-9 and Dat. (25) Ding. S-10 width of web of Tee's not available - measured from Dat. (25) to be = 7 inches.

connection (welding) is at line (3) only.

Connection of double tee flanges at lines (B) $2(E)$ per dig. S-3 and Dat 54, dug. S-11 (shearkeys@5-00.6)

Capacity of weld @ Line (3).

assumed

Capacity parallel to line (3) $=21 \times 0.707 \times \frac{1}{4} \times 7^{\prime \prime}=26^{\mathrm{K}}$

h perpendicular to line (3) $=21 \times 0.707 \times \frac{1}{4} \times\left(5-1^{4}\right) \times 2=29.7^{\mathrm{k}}$

(Gap assumed)

$\therefore$ Capacity parallel to line (3) per ft. $=\frac{2 \times 26}{8}=6.5^{k} / \mathrm{st}$.

1 to line (3) per ft. $=\frac{2 \times 29.7}{8}=7.42 \mathrm{k} / \mathrm{ft}$

Capacity of Conn. (4) Lines (B) \& (E)

flat bar $3 / 8 \times 1 \frac{1}{2} \times 2^{n}$ in bearing $=0.375 \times\left(1.5-0.5^{\circ}\right) \times 27^{\mathrm{ksi}}=10.1^{\mathrm{k}}$ $3 / 16$ fillet

$$
=21 \times 0.707 \times \frac{3}{16} \times 2^{11} \times 2=11.1^{\mathrm{K}}
$$

Governs

$\therefore$ Conn. Capacity per ft. $=\frac{10.1}{s^{\prime}}=2 \mathrm{k} / \mathrm{ft}$.

NOTE: Wall is free at line (7).

$\$$ Assuming $3^{n}$ flange and $7^{\prime \prime} \times 25^{\prime \prime}$ web

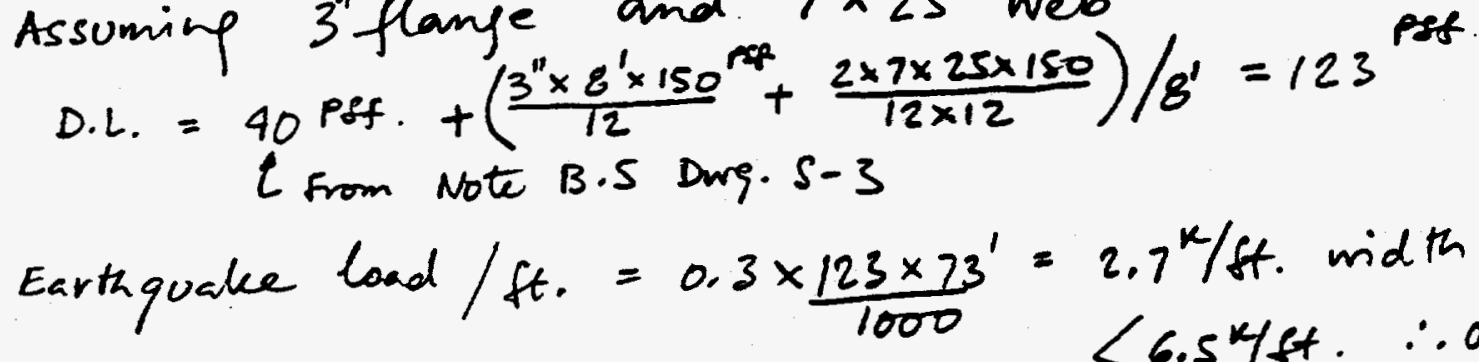

<6.5 k ft. $\therefore 0 . k$.

Load (B) \&(C) due to Torsion $=\frac{2.7 \times 92 \times 73}{2 \times 92 \times 73}=1.35 \mathrm{k} / \mathrm{ft}$.

$$
\begin{aligned}
& =1.35^{k} / f t . \\
& 2^{k} / f t . \quad \therefore 0 . k .
\end{aligned}
$$

